\title{
Article
}

Mycosphere

Doi 10.5943/mycosphere/11/1/14

\section{Fungi on wild seeds and fruits}

\section{Perera RH ${ }^{1,2,3,4}$, Hyde KD $^{2,3,4,5,6,7}$, Maharachchikumbura SSN ${ }^{8}$, Jones EBG ${ }^{9,10}$, McKenzie EHC ${ }^{11}$, Stadler $\mathbf{M}^{16}$, Lee HB $^{17}$, Samarakoon MC $^{2,12}$, Ekanayaka AH ${ }^{2}$, Camporesi $\mathrm{E}^{13,14,15}$, Liu JK ${ }^{1,8}$, Liu ZY $\mathbf{Z Y}^{1}$}

\author{
${ }^{1}$ Guizhou Key Laboratory of Agricultural Biotechnology, Guizhou Academy of Agricultural Sciences, Guiyang, \\ Guizhou 550006, P. R. China \\ ${ }^{2}$ Center of Excellence in Fungal Research, Mae Fah Luang University, Chiang Rai 57100, Thailand \\ ${ }^{3}$ School of Science, Mae Fah Luang University, Chiang Rai 57100, Thailand \\ ${ }^{4}$ Mushroom Research Foundation, 128 M.3 Ban Pa Deng T. Pa Pae, A. Mae Taeng, Chiang Mai 50150, Thailand \\ ${ }^{5}$ Key Laboratory for Plant Diversity and Biogeography of East Asia, Kunming Institute of Botany, Chinese Academy of \\ Science, Kunming 650201, Yunnan, P. R. China \\ ${ }^{6}$ World Agroforestry Centre, East and Central Asia, Kunming 650201, Yunnan, P.R. China \\ ${ }^{7}$ Innovative Institute of Plant Health, Zhongkai University of Agriculture and Engineering, Haizhu District, Guangzhou \\ 510225, P.R. China \\ ${ }^{8}$ School of Life Science and Technology, University of Electronic Science and Technology of China, Chengdu 611731, \\ P.R. China \\ ${ }^{9}$ Department of Entomology and Plant Pathology, Faculty of Agriculture, Chiang Mai University, Chiang Mai 50200, \\ Thailand \\ ${ }^{10}$ Department of Botany and Microbiology, College of Science, King Saud University, P.O Box 2455, Riyadh 11451, \\ Kingdom of Saudi Arabia \\ ${ }^{11}$ Landcare Research Manaaki Whenua, Private Bag 92170, Auckland, New Zealand \\ ${ }^{12}$ Department of Biology, Faculty of Science, Chiang Mai University, Chiang Mai 50200, Thailand \\ ${ }^{13}$ A.M.B, Circolo Micologico "Giovanni Carini”, C.P. 314, Brescia, Italy \\ ${ }^{14}$ A.M.B. Gruppo, Micologico Forlivese "Antonio Cicognani”, Via Roma 18, Forlì, Italy \\ ${ }^{15}$ Societáper gli Studi Naturalistici della Romagna, C.P. 143, Bagnacavallo, RA, Italy \\ ${ }^{16}$ Helmholtz Centre for Infection Research, Department Microbial Drugs, Inhoffenstrasse 7, 38124 Braunschweig, \\ Germany \\ ${ }^{17}$ Environmental Microbiology Lab, Department of Agricultural Biological Chemistry, College of Agriculture and Life \\ Sciences, Chonnam National University, Yongbong-Dong 300, Buk-Gu, Gwangju 61186, Korea
}

Perera RH, Hyde KD, Maharachchikumbura SSN, Jones EBG, McKenzie EHC, Stadler M, Lee HB, Samarakoon MC, Ekanayaka AH, Camporesi E, Liu JK, Liu ZY 2020 - Fungi on wild seeds and fruits. Mycosphere 11(1), 2108-2480, Doi 10.5943/mycosphere/11/1/14

\begin{abstract}
This paper reviews and determines the fungi growing on seeds and fruits of wild plants in various habitats. Such fungi colonise a wide range of substrates with most reported from cones, cupules, and leguminous pods that are high in cellulose and lignin content. There are 1348 fungal species (belonging to 230 families and 609 genera) reported from wild seeds and fruits in 84 countries, listed in this paper. Of these, 300 fungi were described from wild seeds and fruit substrates. Members of the Fabaceae support the highest number of taxa, namely $19 \%$ of the novel wild fruit fungi. Twenty-eight genera, including 5 fossil fungal genera have been described from wild seeds and fruits: Agarwalomyces, Amorocoelophoma, Anisogenispora, Archephoma, Centrolepidosporium, Cylindroaseptospora, Cylindromyces, Davidhawksworthia, Delonicicola, Discotubeufia, Glaxoa, Kionocephala, Leucaenicola, Naranus, Neolindgomyces, Pleohelicoon, Quercicola, Remotididymella, Repetoblastiella, Restilago, Soloacrosporiella, Strobiloscypha and Tainosphaeria. Archephoma, Meniscoideisporites, Palaeodiplodites, Palaeopericonia and
\end{abstract}


Xylohyphites are the new fossil fungal genera. Fungal asexual morphs predominate on wild seeds and fruits rather than the sexual morphs. The dominant fungal genera on wild seeds and fruits include Alternaria, Aspergillus, Candida, Chaetomium, Cladosporium, Colletotrichum, Curvularia, Diaporthe, Drechslera, Fusarium, Mucor, Penicillium, Pestalotiopsis, Restiosporium, Rhizopus, Talaromyces, Trichoderma and Xylaria. Certain assemblages of fungi have specific and distinct relationships with their hosts, especially Xylaria species (e.g., Xylaria magnoliae on Magnolia fruits; $X$. xanthinovelutina ( $=X$. ianthino-velutina) on Fabaceae pods; $X$. carpophila on Fagus cupules; $X$. persicaria on liquidambar fruits). Whether these species occur as endophytes and become saprobes following fruit fall requires further investigation. In this study, we also made several sexual morph collections of sordariomycetous taxa from different seed and fruit substrates mainly from Thailand, with a few from the UK. These include 15 new species, 13 new host records and 1 new geographical record. The new species are described and illustrated.

Keywords - 15 new taxa - forest floor - fructicolous - pathogens - saprobes - seminicolous

\section{Ascomycota}

Sordariomycetes O.E. Erikss. \& Winka

Amphisphaeriales D. Hawksw. \& O.E. Erikss.

Beltraniaceae Nann.

Beltraniella fertilis Heredia, R.M. Arias, M. Reyes \& R.F. Castañeda, new host record

Chaetosphaeriales Huhndorf et al.

Chaetosphaeriaceae Locq.

Dictyochaeta coryli R.H. Perera, E.B.G. Jones \& K.D. Hyde, sp. nov.

Dictyochaeta lithocarpi R.H. Perera, E.B.G. Jones \& K.D. Hyde, sp. nov.

Menisporopsis theobromae S. Hughes, new host record

Diaporthales Nannf.

Cytosporaceae Fr.

Cytospora diopuiensis Q.J. Shang, K.D. Hyde \& J.K. Liu, new host record

Diaporthaceae Höhn. ex Wehm.

Diaporthe delonicis R.H. Perera, E.B.G. Jones \& K.D. Hyde, sp. nov.

Hypocreales Lindau

Bionectriaceae Samuels \& Rossman

Clonostachys rogersoniana Schroers, new host record

Clonostachys rosea (Link) Schroers, Samuels, Seifert \& W. Gams, new host record

Clonostachys swieteniae R.H. Perera, E.B.G. Jones \& K.D. Hyde, sp. nov.

Myrotheciomycetaceae Crous

Trichothecium roseum (Pers.) Link, Mag. Gesell. naturf. Freunde, new host record

Nectriaceae Tul. \& C. Tul.

Fusarium cassiae R.H. Perera, E.B.G. Jones \& K.D. Hyde, sp. nov.

Fusarium magnoliae-champaca R.H. Perera, E.B.G. Jones \& K.D. Hyde, sp. nov.

Fusarium salinense Sand.-Den., Guarnaccia \& Polizzi, new host and new geographical record

Fusarium sp.

Fusicolla cassiae-fistulae R.H. Perera, E.B.G. Jones \& K.D. Hyde, sp. nov.

Fusicolla siamensis R.H. Perera, E.B.G. Jones \& K.D. Hyde, sp. nov.

Gliocladiopsis aquaticus Y.Z. Lu, R.H. Perera \& K.D. Hyde, new host record 
Gliocladiopsis tenuis (Bugnic.) Crous \& M.J. Wingf., new host record

Gliocladiopsis swieteniae R.H. Perera, E.B.G. Jones \& K.D. Hyde, sp. nov.

Macronectria jungneri (Henn.) Salgado \& P. Chaverri, new host record

Murinectria pseudotrichia (Schwein) M. Niranjan and V.V. Sarma, new host record

Neocosmospora magnoliae R.H. Perera, E.B.G. Jones \& K.D. Hyde, sp. nov.

Sarcopodium durantae R.H. Perera, E.B.G. Jones \& K.D. Hyde, sp. nov.

Sarcopodium flocculentum (Henn. \& E. Nyman) Pennycook \& P.M. Kirk, new host record

Volutella delonicis R.H. Perera, E.B.G. Jones \& K.D. Hyde, sp. nov.

Xylariales Nannf.

Diatrypaceae Nitschke

Allodiatrype thailandica (R.H. Perera, Jian K. Liu \& K.D. Hyde) Konta \& K.D. Hyde, new host record

Hypoxylaceae DC.

Hypoxylon delonicis R.H. Perera, E.B.G. Jones \& K.D. Hyde, sp. nov.

Xylariaceae Tul. \& C. Tul.

Xylaria arbuscula Sacc.

Xylaria fabacearum R.H. Perera, E.B.G. Jones \& K.D. Hyde, sp. nov.

Xylaria fabaceicola R.H. Perera, E.B.G. Jones \& K.D. Hyde, sp. nov.

Leotiomycetes O.E. Erikss. \& Winka

Helotiales Nannf. ex Korf \& Lizoň

Helotiales sp.

Lachnaceae Raitv.

Lachnum sp.

\section{Introduction}

Seeds are produced by spermatophytes of both angiosperms and gymnosperms. Angiosperm seeds originate from a hard or fleshy structure known as a fruit which also encloses the seeds. However, in gymnosperms, there is no specific biological structure developed to enclose the seeds (http://pediaa.com/ difference-between-fruit-and-seed/). Plants have evolved various mechanisms for the dispersal of mature seeds, which have the potential to produce new individuals (Bewle \& Black 1994).

There is a great diversity in the form and structure of seed and fruits, such as, grains, cones, acorns, berries, drupes, capsules, seed pods, nuts, nut cupules, caryopses, and nutlets (fruits of restiads or sedges). Fruit cases, such as nut cupules are considered as fruits in this paper. Cupules of beech (Fagus spp.) are a woody substrate, containing lignocellulose and similar to lignocellulose composition of coarse woody debris (Fukasawa et al. 2012). Leguminous pods also are rich in cellulose, hemicelluloses and lignin (Paula et al. 2011). Cones, which protect the seeds during development, are the reproductive organs of coniferous plants (Kilic et al. 2010). They consist of an axis with surrounding scales, which are rich in cellulose, hemicellulose (mannose, galactose, xylose) and lignin (Fogel \& Cromack 1977, Kilic et al. 2010). Nuts, kernels of chestnut, hazelnut, walnut and oak are diverse in their protein, carbohydrate and fat content, while some are rich in phosphorus (eg. hazelnut, hickory nut, and black walnut) (Wainio \& Forbes 1941). Nut shells are rich in lignin, cellulose and hemicelluloses (Vellingiri et al. 2014, Zhai et al. 2015). Fruits (achenes, pomes, berries and drupes) comprise mostly carbohydrates, some are high in lignin and cellulose, and others are rich in protein and fat, mainly because of their seeds (Wainio \& Forbes 1941). Acorns such as red oak, rock chestnut oak, and scrub oak and, some edible wild fruits (eg. 
chokeberries, sumac berries, blackhaw and mountain ash berries) are high in tannin content (Wainio \& Forbes 1941). Moreover, seeds such as those of legumes and cereals are major food sources and contain storage reserves of protein, starch and oil (Bewle \& Black 1994, Shimada 2001, Ragaee et al. 2006).

\section{History of studies of wild seed/fruit fungi}

Around the world the forest floor is seasonally covered by a carpet of native seeds and fruits, which are colonised by a wide range of endophytic, saprobic and pathogenic fungi (Rogerson \& Samuels 1992, Morais et al. 1995, Rogers et al. 2002, Somrithipol et al. 2002a, b, Udayanga et al. 2013, Perera et al. 2018a).

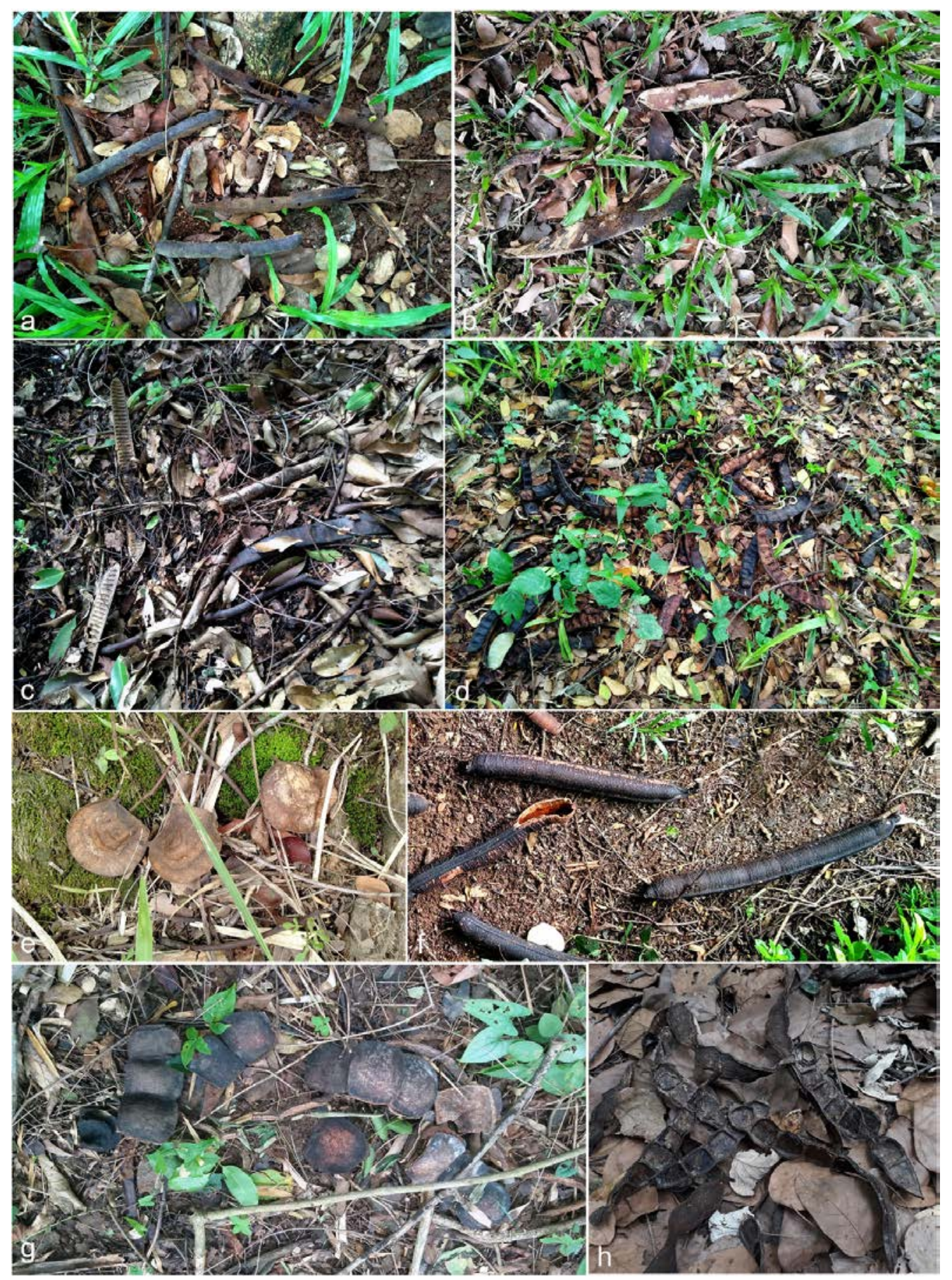

Figure 1 - Fabaceae fruits on the forest floor in Thailand (a-g) and Taiwan (h). a Cassia fistula. b Fabaceae sp. c Delonix regia. d Samanea saman. e Adenocalymma pterocarpus. f Cassia grandis. g Entada sp. h Mucuna macrocarpa. 

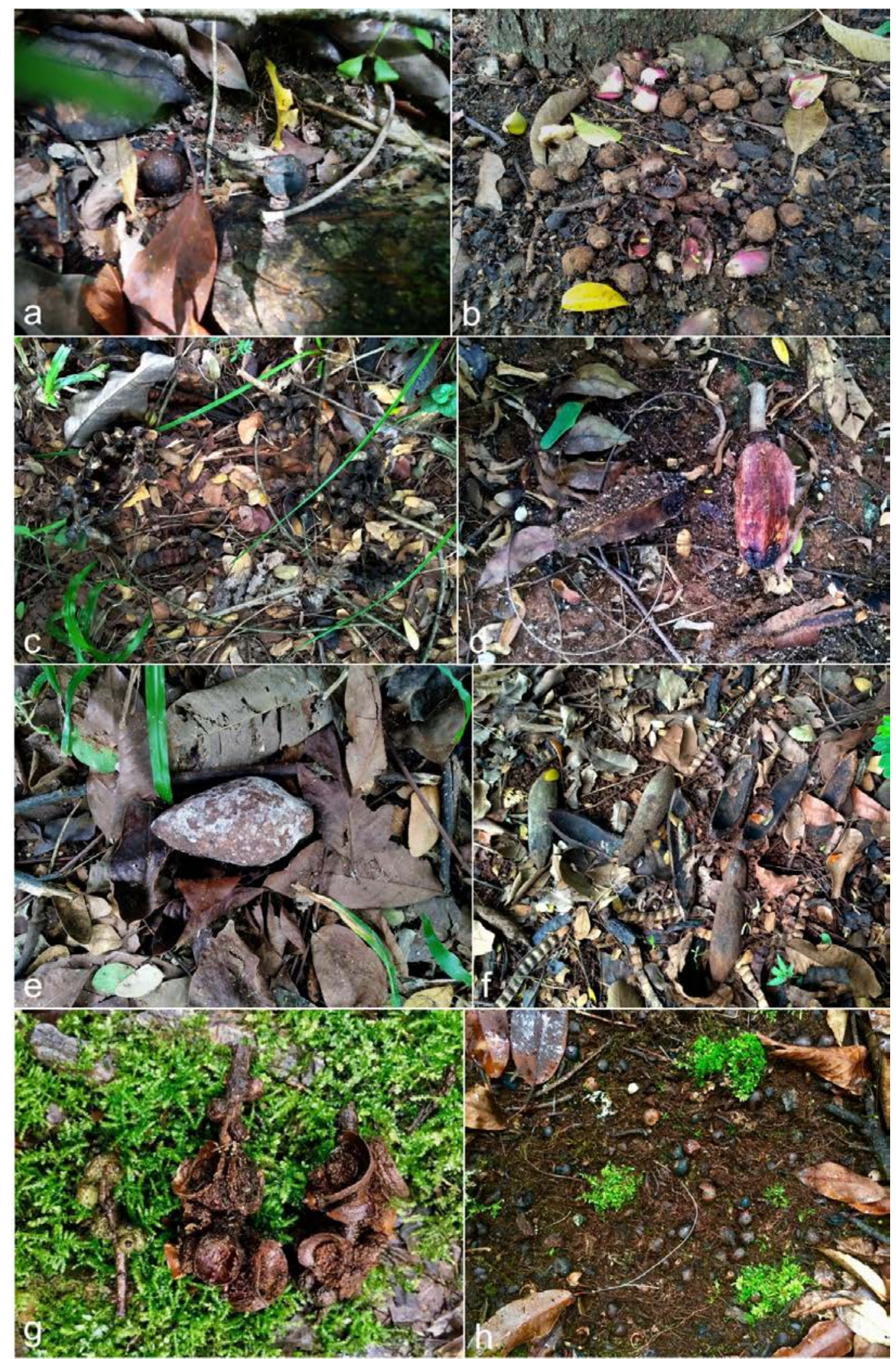

Figure 2 - Seeds and fruits on the forest floor in Thailand (a-f) and China (g, h). a Seed of Lithocarpus sp. b Immature fruits of Couroupita guianensis (cannonball tree). c Fruits of Magnolia champaca. d-f Swietenia mahagoni (d placenta with seeds, e fruit, $\mathrm{f}$ fruit outer carpels). $\mathrm{g}$ Unidentified fruits. h Unidentified seeds.

Few studies have been undertaken to document fungal groups from wild seeds and fruits, when compared to other plant substrates, e.g. wood, culms, leaves and commercial seeds and fruits (Kasai et al. 1995, Nirenberg \& Aoki 1997, Vujanovic et al. 2000, Yli-Mattila et al. 2009, Weir et 
al. 2012, Udayanga et al. 2013, Rashmi et al. 2019). Hyde et al. (2015) acknowledged that the taxonomy, ecology, and distribution of fruit and seed inhabiting fungi are poorly studied in both temperate and tropical areas. Previous research has mainly targeted postharvest fungi, those responsible for economic losses of cereals and edible fruits (Tang et al. 2003a, Neergaard 2017). However, seed and fruit inhabiting fungi of conifers and eucalyptus species has been investigated broadly as they have been widely introduced as exotic timber trees in plantations (Kasai et al. 1995, Vujanovic et al. 2000, Lupo et al. 2001, Palm 2001, Wingfield et al. 2001, Desprez-Loustau 2009). Most of the early research focused on endophytic isolations and identification of seed-borne pathogens (Anderson 1986, Mittal et al. 1990). Hence, wild seed and fruit fungi are not a wellstudied group.

An extensive study of dothideomycetous fungi associated with wild seeds and fruits in selected areas of Thailand, UK and China was carried out by Jayasiri et al. (2019), who introduced 58 new taxa. Fukasawa et al. (2012) and Carré (1964) surveyed fungi on beech cupule litter in Japan and the UK. Tang et al. (2003a) examined fruit samples from 18 native plant species yielding 101 fungal taxa in Hong Kong. Another survey in Thailand by Somrithipol et al. (2002b) investigated fungi on tropical forest fruit of Delonix regia and identified 70 fungi. Ten fungal species belonging to Ascomycota were isolated from cone scales and seeds of 28 Pinus hosts in East Asia, Europe and North America, and Canada (Vujanovic et al. 2000). In their study, Sphaeropsis sapinea, Herpotrichia juniperi, 'Phomopsis' conorum, Truncatella hartigii, Tubercularia sp. and Valsa spp. were recognised as dominant pathogens of Pinus (Vujanovic et al. 2000). A fungal succession study on Pinus densiflora cones on a forest floor investigated the early decomposition process in Japan, and identified 31 taxa (Kasai et al. 1995). Pandey \& Nimmi (1990) isolated 48 fungal species from Pinus roxburghii seeds collected from a forest in India and observed that the predominant species in ungerminated seeds are Alternaria and Fusarium spp. Anita et al. (1999) and Anita \& Sridhar (1999) reported a number of endophytic fungi from seeds and pods of mangrove wild legumes (Canavalia cathartica and Sesbania bispinosa) in Southwest coast of India. Lupo et al. (2001) studied endophytic fungi in capsules and seeds of Eucalyptus globulus in Uruguay and found 18 taxa from unopened capsules (cut off from the tree) and ten from seeds. Seed-borne fungi associated with Podocarpus falcatus and Prunus africana in Afromontane rain forests of Ethiopia were investigated by Gure (2004), and identified four Botryosphaeriaceae taxa with one new species, Diplodia rosulata. Yeasts (Candida spp., Debaryomyces spp., Kloeckera spp., Pichia spp.) colonizing the fallen ripe fruits of Amapa (Parahancornia amapa) and Clusia grandiflora in tropical forests were examined by Morais et al. (1995). They found 44 yeasts including one new species. Rogers (1979a) described a new saprobic Xylaria species, $X$. magnolia on decaying fruits of Magnolia sp. and discussed several other fruit inhabiting Xylaria species. Ju et al. (2018) studied 25 Xylaria species inhabiting fallen seeds and fruits and introduced three new species namely: Xylaria reevesiae, X. rossmanae and X. vivantii. Jankowiak (2008) investigated fungi occurring on acorns of Quercus robur, which were infested by insects in Poland. He identified 45 fungi with Alternaria alternata and yeasts as the most frequently isolated fungi (Jankowiak 2008). Although there is great diversity in fruit and seed-bearing plants, few studies been carried out especially in temperate regions (Table 2). Accordingly, the present work on fungal diversity found on seeds and fruits was undertaken.

Anderson (1986) and Mittal et al. (1990) provided checklists of micro-organisms associated with tree seeds and primarily focused on forestry trees. Seed-borne diseases of 12 host tree species namely: Acer spp., Alnus spp. Araucaria excelsa, Betula spp., Chamaecyparis sp., Fraxinus spp., Larix spp., Picea sitchensis, Pinus spp., Quercus spp., Thuja spp., Ulmus americana and U. pumila were listed by Noble et al. (1958). Watanabe (2010) listed a number of culturable seed fungi with illustrations and provided a key to species in wild and crop plants in Japan. Previous studies and check lists revealed that the fungi colonising seeds and fruits are dominated by asexual morphic fungi (Carré 1964, Anderson 1986, Mittal et al. 1990, Kasai et al. 1995, Amusa et al. 2002, Somrithipol et al. 2002b, Tang et al. 2003a, Fukasawa et al. 2012). However, a comprehensive literature review or a checklist on fungi that occur on wild seeds and fruits is lacking. 
Our approach is to provide a checklist of fungi described from seeds and fruits of noncommercial trees, shrubs and weedy plants or grasses. However, there are no details of substrates such as wood, leaves, seeds, fruits provided in most of the previous fungal lists. In Petrak's lists, fungi were listed under host names but did not mention the substrates. We also looked in the USDA host database for search by substrates, but it lists only host details. It supports for fungal hostsearch but not for other substrate details. Since 1981, Index of Fungi series and the website provided substrate details, such as wood, leaves, seeds and fruits. Our list of new fungi therefore was mainly based on Index of Fungi. Here we also list fungi reported from wild seeds and fruits based on available literature. The aim of the present study is to give an idea of the different groups of fungi occurring on wild seeds and fruits, document, discuss their role and importance, and illustrate some Sordariomycetes taxa. We also provide a brief account on fossil fungi described from seed fruit substrates.

\section{Importance of studying wild seed/fruit fungi}

Diverse fungal groups can be found generally on every part of a single plant host (Hyde 1995). Domesticated, genetically modified commercial seeds (cereals) and fruits are different from wild fruits/seeds in size, nutrient content and chemical defenses (Tang et al. 2003a, HernándezTerán et al. 2017). Disease development of those post-harvest fruits and cereals occur during transportation and storage, which is different to that in nature (Tang et al. 2003a). The few studies that have been conducted on fungi on non-commercial seeds and fruits indicate the occurrence of fungi belonging to diverse taxonomic groups such as, Ascomycota, Basidiomycota, Chytridiomycota, Mucoromycota and Blastocladiomycota (Desjardin 2000, Vujanovic et al. 2000, Somrithipol et al. 2002a, b, Nelson \& Abad 2010, Fukasawa et al. 2012, Jayasiri et al. 2019). The significance of studying wild seed/fruit fungi is further discussed below.

\section{Where do these seed/fruit fungi come from?}

There are three different ways fungi can infect or colonise wild seeds or fruits: 1) seeds become infected with endophytic fungi while in the canopy during fruit development, 2) within the soil after dispersal, and 3) by animal vectors such as fruit flies (Gallery 2007, Morais et al. 1995, Somrithipol et al. 2002b). Plant seeds carry spores of different fungi on the seed surface or inside tissues of the seed (Anderson 1986). Seed surfaces are mostly contaminated with fungal spores as they easily stick to the outer seed coat, while fungi mostly occur as mycelia inside the seed (Anderson 1986). Seeds/fruits inhabiting fungi may include endophytic, saprophytic, and pathogenic fungal species (Gallery et al. 2007). Diaporthe, Fusarium, Penicillium, Trichoderma and Xylaria-like fungi initially can occur as endophytes, but become pathogenic or saprobic under favorable conditions (Somrithipol et al. 2002b, Udayanga et al. 2013, Fukasawa et al. 2012). However, pathogenicity of most fungi associated with wild seeds/fruits has been much debated (Mittal et al. 1990). Somrithipol et al. (2002b) studied pods of Delonix regia and observed Aspergillus, Chaetomium, Penicillium, and Rhizopus species mainly occurring on the dry fruits when still attached to the tree. A different fungal community developed once seeds and fruits had been shed from the host plant (Somrithipol et al. 2002b). Lupo et al. (2001) observed Eucalyptus globulus capsules attached to the tree and shared the same endophytic fungal species with flowers, but in higher numbers. Clavicipitaceous fungi are parasitic on Poaceae hosts, and can also occur as endophytes (Rodriguez et al. 2009). During flowering of grasses, endophytic species such as Acremonium species grow into ovules and become incorporated into the seeds (Neill 1941, Clay 1990). Endophytes can be transmitted horizontally (among individuals of the same generation) or vertically (from maternal plants to offspring through seed infections) to other plants/ generations (Chung \& Schardl 1997, Saikkonen et al. 2002). Animal vectors such as common flies and fruit flies are partially responsible for fungal contamination of fruits (Morais et al. 1995), for example, Drosophila spp. are known to be the major vectors of yeasts (Morais et al. 1995). Morais et al. (1995) and Miller \& Phaff (1962) observed the coexistence of different Drosophila species in different stages of deterioration of Parahancornia amapa fruits and Ficus figs. Some studies reveal 
seeds infected by endophytic fungi contain higher concentrations of alkaloids than the normal seeds, thus they may protect seeds from vertebrate and invertebrate predators (Cheplick \& Clay 1988, Clay 1990).

\section{Fossil fungi records of seeds and fruits}

The origin of fungi is aged to the Paleoproterozoic-Mesoproterozoic eras (2500-1000 million years ago; Mya). The phylum Chytridiomycota is the earliest known group of fungi which diverged during the Mesoproterozoic (1600-1000 Mya) before the divergence of terrestrial plants (Lücking et al. 2009, Padovan et al. 2005, Torruella et al. 2012). The divergence of the phyla Ascomycota, Basidiomycota and Glomeromycota has been dated to the Neoproterozoic to Early Paleozoic (1000-485 Mya) with interaction with different groups of plants (Schüßler et al. 2001, Berney \& Pawlowski 2006, Lücking et al. 2009, Taylor et al. 2009, Beimforde et al. 2014). Selosse et al. (2015) suggested that the highly diversified plant-fungal interactions are the backbone of land ecosystems and biogeochemical cycles from the Palaeozoic era ( $<541$ Mya). Several remarkable studies revealed well-preserved fungal associations with plants mainly on leaves and different woody substrates (e.g. Cookson 1947, Dilcher 1965, Taylor et al. 1999, Mindell et al. 2007, Taylor et al. 2014, Vishnu et al. 2017). However, few fungal remains associated with fossil ovules, seeds and fruits have been described to date (Taylor 2009, Krings et al. 2012).

Renault \& Bertrand (1895) described Grilletia sphaerospermii as a chytrid fossil inhabiting the nucleus of a Carboniferous gymnosperm Sphaerospermum and dated to the upper Pennsylvanian (307-298 Mya) in France. Grilletia sphaerospermii-like organisms have been described by Oliver (1903) from seeds of Polylophospermum and Conostoma from the lower Carboniferous (358.9-323.2 Mya) in France and Scotland. Pirozynski \& Dalpé (1989) suggest that the Grilletia is close to extant Glomus, but this hypothesis needs further study. Batra et al. (1964) reported Protoascon missouriensis from a cluster of fossil fungi in a Nucellangium glabrum seed. It was preserved in Carboniferous permineralization (coal ball) collected from Tebo Coal of the Cabaniss Formation, Missouri, North America (middle Pennsylvanian, 315-307 Mya). Taylor et al. (2005) re-examined the Polylophospermum missouriensis fossil and suggested it was comparable to modern members of the Mucorales (Mucoromycota).

There are several Ascomycota fossil fungi described from seeds, fruits and ovules. Two fossil fungal species were described from India (upper Cretaceous; Maastrichtian, 72-66 Mya) associated with permineralized fruits of Viracarpon (monocotyledons). Diplodites sweetii, which is morphologically similar to extant Sphaeropsis in Botryosphaeriaceae, was described by Kalgutkar et al. (1993). Xylohyphites verrucosa, which is characterized by having verrucose conidia in chains, hence shows morphological affinities to Cladosporium (Kalgutkar \& Sigler 1995). Watanabe et al. (1999) described three fossil fungal genera: Archephoma, Meniscoideisporites and Palaeodiplodites in Japan (middle Turonian, 93.9-89.8 Mya), all associated with a bisexual cone of Cycadeoidella japonica. They suggested that the Archephoma and Palaeodiplodites are comparable to the modern phoma-like taxa and Diplodia, while Meniscoideisporites is not assignable to any extant taxon based on reliable taxonomic characters (Fig. 3). Two fossil fungal genera Palaeopericonia and Xylohyphites were described from permineralized fruit of Viracarpon sp. and fossilised cones of Araucaria mirabilis in Argentina and Canada (Kalgutkar \& Sigler 1995, Ibáñez \& Zamuner 1996).

In addition, LePage et al. (1994) described several asexual morphic fossil fungi associated with seeds and fruits from Princeton chert, British Columbia, Canada (middle Eocene, $50 \mathrm{Mya}$ ) and suggested that the fossils represent extant species from Dothideales, Alternaria and some coelomycetous asexual morphs. Stakhov et al. (2008) described the most recent fossil fungus associated with seeds of undetermined higher plants from Russia (Pleistocene 30,000 years ago).

These few fungal fossils described from seeds, fruits, pods, ovules or any other reproductive plant materials (Table 2) might be due to less attention given to microorganisms associated with reproductive structures of plants. 


\section{Materials \& methods}

\section{Sample collection, specimen examination and isolation}

Specimens were collected from seeds and fruits in Northern Thailand and United Kingdom (UK) during 2014 to 2018. Fungal fruiting structures were observed, photomicrographed and measurements were made in the laboratory as designated in Perera et al. (2016b). Fungal colonies were obtained from single spore isolation technique described in Chomnunti et al. (2014). Pure cultures were grown on potato dextrose agar (PDA) or malt extract agar (MEA) media and incubated at $25^{\circ} \mathrm{C}$ or $28^{\circ} \mathrm{C}$.

Herbarium material was preserved in the Mae Fah Luang University (MFLU) herbarium, Chiang Rai, Thailand. Living cultures were stored in the Culture Collection at Mae Fah Luang University (MFLUCC). Facesoffungi and Index Fungorum numbers were registered as outlined in Jayasiri et al. (2015) and Index Fungorum (2020). Species are delineated based on recommendations outlined by Jeewon \& Hyde (2016).

\section{DNA isolation, amplification and analyses}

Genomic DNA was extracted from fungal colonies growing on PDA or MEA or directly from the fruiting bodies as designated in Perera et al. (2016b). DNA amplifications were performed by polymerase chain reaction (PCR). Part of the large subunit nuclear rRNA gene (LSU) was amplified with primer pairs LROR (Rehner \& Samuels 1994) and LR5 (Vilgalys \& Hester 1990). The small subunit nuclear rRNA gene (SSU) was amplified with primer pairs NS1 and NS4 (White et al. 1990). Primer pairs ITS4 and ITS5 were used to amplify the 5.8S rDNA region and flanking internal transcribed spacers (ITS) (White et al. 1990). The translation elongation factor 1-alpha gene (TEF1) was amplified by using primers EF1-983F and EF1-2218R (Rehner 2001, Currie et al. 2003) or the primers EF1-728F and EF1-986R (Carbone \& Kohn 1999) or EF2 (O'Donnell et al. 1998). The RNA polymerase II second largest subunit (RPB2) gene was amplified with primers fRPB2 and fRPB2-7cR (Liu et al. 1999). The beta-tubulin (TUB2) gene was amplified by using primers T1 (O’Donnell \& Cigelnik 1997), Bt2b (Glass \& Donaldson 1995), CYLTUB1R (Crous et al. 2004) or T22 (Cigelnik 1997). Histone H3 (HIS3) region was amplified with the primers CYLH3F and CYLH3R (Lombard et al. 2012). PCR was performed following the protocols in Perera et al. (2016b). Assemblage of consensus sequences were done in ContigExpress (Vector NTI Suite 6.0). Newly generated sequences were deposited in NCBI GenBank and accession numbers are given at the end of the protologue.
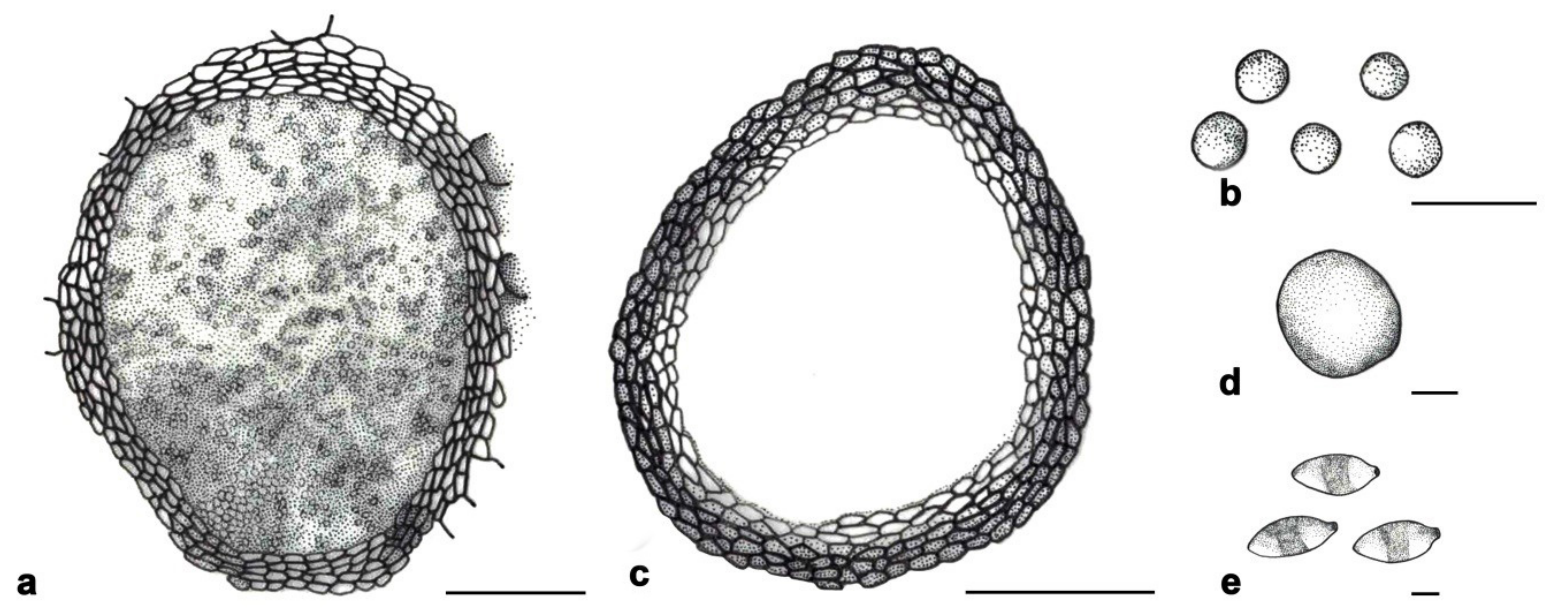

Figure 3 - Archephoma cycadeoidellae. a Mature pycnidium containing numerous globose conidia. b Conidia. Meniscoideisporites cretacea. c Pycnidium. d Conidium. Palaeodiplodia yezoensis. e Ellipsoidal conidia. Scale bars: $\mathrm{a}, \mathrm{c}-\mathrm{e}=50 \mu \mathrm{m}, \mathrm{b}=100 \mu \mathrm{m}$ (re-drawn from Watanabe et al. 1999). 

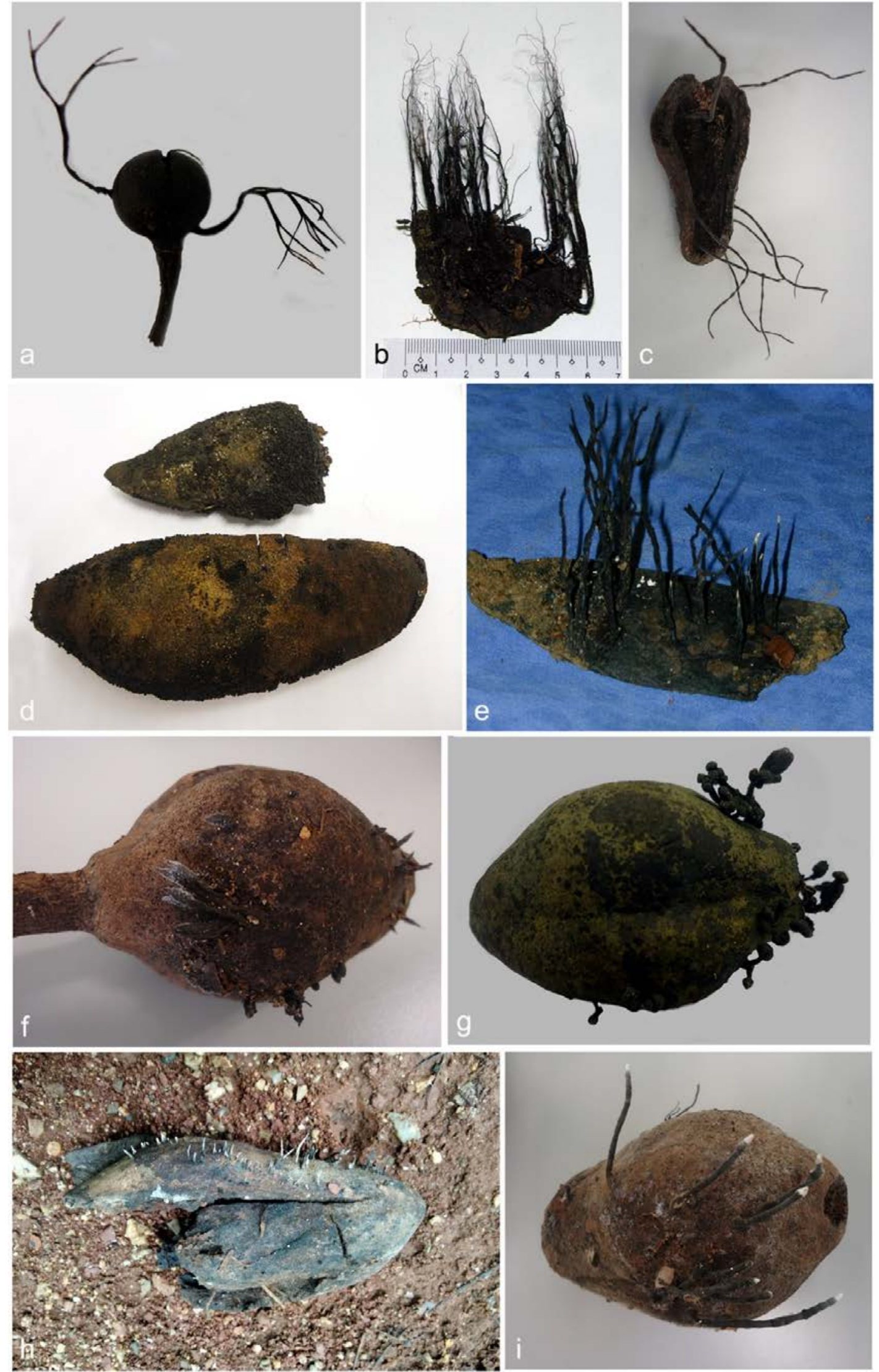

Figure 4 - a-i Xylaria species associated with forest fruits. a Xylaria sp. on an undetermined fruit. b, e Xylaria xanthinovelutina on a Fabaceae fruit. c Xylaria sp. on a Swietenia mahagoni fruit. d Xylaria sp. on a Swietenia macrophylla fruit. f, g Xylaria arbuscula on Swietenia mahagoni fruits. h, i Xylaria spp. on Swietenia mahagoni fruits. 


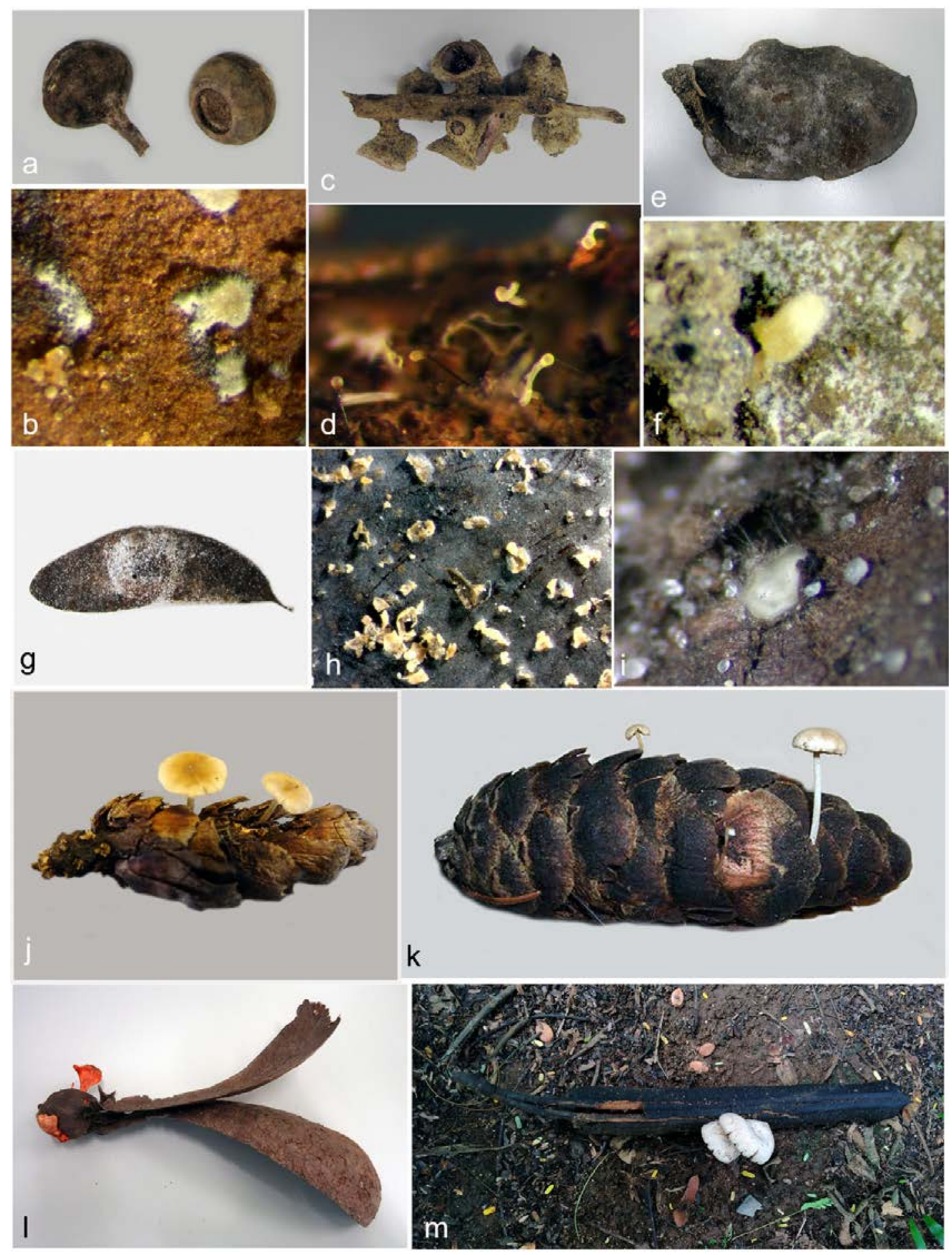

Figure 5 - a-m Fungi associated with forest fruits. a, b Thozotella lithocarpi on seed of Lithocarpus sp. c, d Parascedosporium-like fungus on fruiting rachis of Lithocarpus sp. e, $\mathrm{f}$ Thozetella fabacearum on a Fabaceae seed pod. g, h Diplodia sp. on a Fabaceae pod. i Discomycete on a Pseudotsuga menziesii cone. j, k Basidiomycete on Pseudotsuga menziesii cones. $\mathrm{l}$ Basidiomycete on Dipterocarpus fruit. m Basidiomycete on a Cassia grandis pod.

\section{Phylogenetic analyses}

The sequences obtained in this study were supplemented with the additional sequences retrieved from GenBank. The sequences were aligned using MAFFT v. 7 online server (http://mafft.cbrc.jp/alignment/server/) and, rechecked visually and improved manually using BioEdit v. 7.0.5.2 (Hall 1999). Ambiguous regions were excluded from the analyses and gaps were treated as missing data. Phylogenetic analyses were carried out with maximum likelihood analysis 
(ML), which was performed at the CIPRES web portal (Miller et al. 2010) using RAxML v. 8.2.12 as part of the "RAxML-HPC2 on TG" tool (Stamatakis 2006). Bayesian inference analysis (BI) was performed in MrBayes v. 3.2.0 (Ronquist \& Huelsenbeck 2003). Phylogenetic trees were viewed in FigTree v1.4 and modified using Microsoft PowerPoint 2016.

Before combining, single gene phylogenetic analyses were conducted for each locus to compare the tree topology and clade stability. The names of the isolates obtained in this study are printed in blue font and names of ex-type and ex-epitype taxa are printed in black bold font in the trees. Maximum likelihood bootstrap support values $\geq 75 \%$ (BT) and Bayesian posterior probabilities $\geq 0.99$ (PP) are given at the nodes respectively.

\section{Results}

\section{Taxonomy}

The classification of Sordariomycetes has been detailed in Hyde et al. (2020) and provided in the Outline of fungi and fungus-like taxa by Wijayawardene et al. (2020) and is followed here.

Amphisphaeriales D. Hawksw. \& O.E. Erikss.

Beltraniaceae Nann.

Beltraniella Subram.

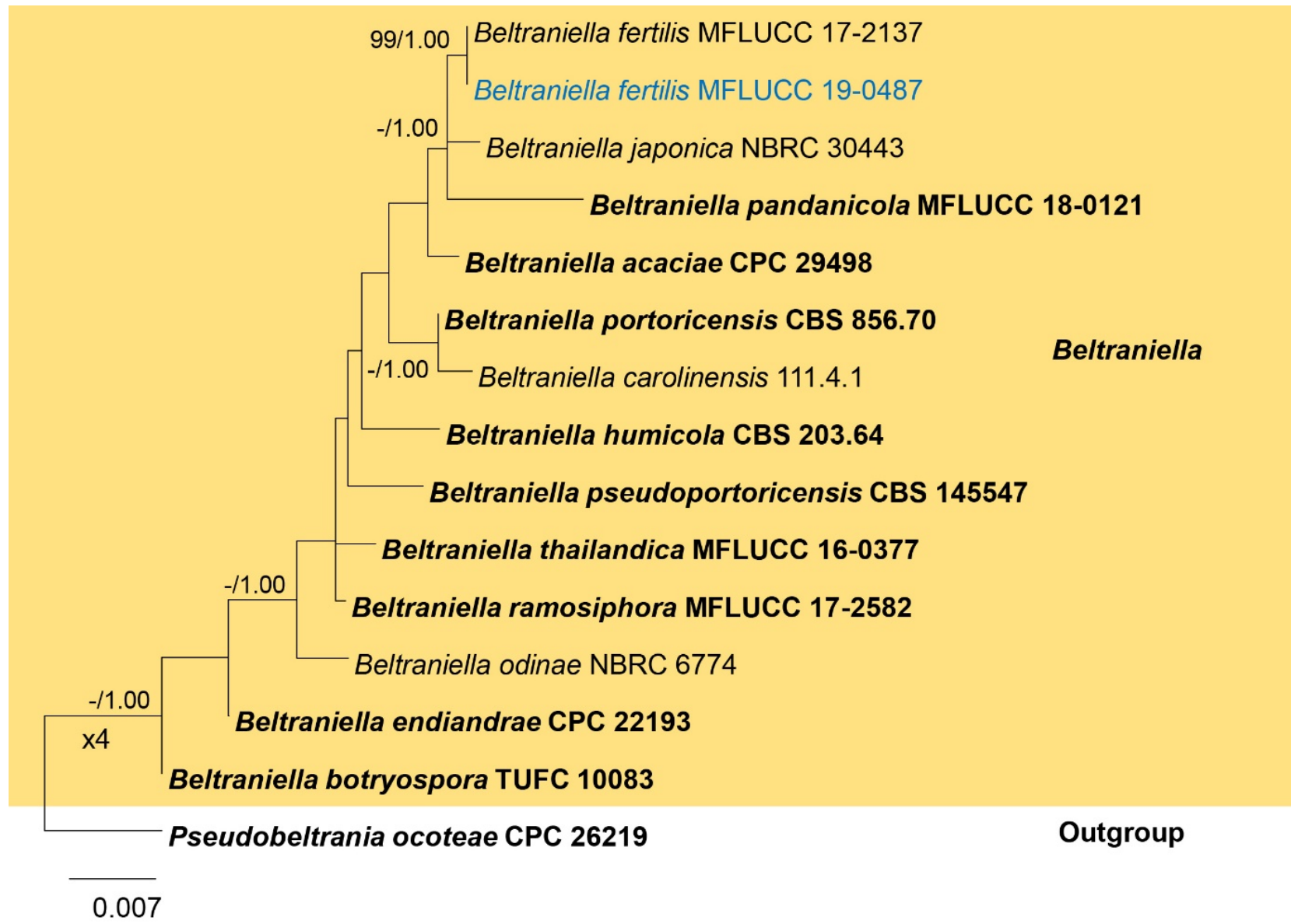

Figure 6 - Phylogram generated from RAxML analysis based on combined ITS and LSU sequence data of Beltraniella isolates. Sequences from fifteen taxa, which comprise 1311 characters including gaps, are included in the analyses. The tree was rooted to Pseudobeltrania ocoteae (CPC 26219). The scale bar indicates 0.007 nucleotide changes per site. 
Beltraniella fertilis Heredia, R.M. Arias, M. Reyes \& R.F. Castañeda, Fungal Diversity 11: 100 (2002)

Fig. 7

Saprobic on dried fruit of Lithocarpus sp. Sexual morph: Undetermined. Asexual morph: Hyphomycetous. Colony on the host greenish. Setae 115-185 × 3.5-4.5 $\mu \mathrm{m}(\mathrm{n}=10)$, erect, dark brown, thick-walled, indistinctly septate, with prominent warts, straight or flexuous, unbranched, tapering to an acute apex, arising from radially lobed basal foot cell. Long setiform conidiophores not observed. Short conidiophores 33-50 $\times 3.5-5 \mu \mathrm{m}(\bar{x}=41.8 \times 4.1 \mu \mathrm{m}, \mathrm{n}=15)$, macronematous, mononematous, single or arranged in groups around the setae, erect, straight or slightly curved, simple or branched, septate, subhyaline to pale brown, thin-walled, smooth-walled. Conidiogenous cells 6-20 × 4-6 $\mu \mathrm{m}(\bar{x}=10.4 \times 3.5 \mu \mathrm{m}, \mathrm{n}=15)$, terminal and intercalary, subcylindrical, polyblastic, apex with several flat-tipped denticles, pale brown, smooth-walled. Separating cells 7.5-10 × 3-4.1 $\mu \mathrm{m}(\bar{x}=8.6 \times 3.5 \mu \mathrm{m}, \mathrm{n}=10)$, ovoid or obovoid, fusiform, hyaline to subhyaline, thin-walled, smooth-walled, with a single flat-tipped denticle. Conidia 20-22 $\times 4.3-5.5 \mu \mathrm{m}(\bar{x}=20.8 \times 4.8 \mu \mathrm{m}, \mathrm{n}=20)$, solitary, turbinate to pyriform, distal end truncate, subhyaline, smooth, aseptate, with median transverse band of lighter pigment, base tapering to an acutely rounded tip.

Culture characteristics - Conidia germinating on PDA within 12 hours. Colonies on PDA reaching $7.5 \mathrm{~cm}$ wthin 14 days at $28^{\circ} \mathrm{C}$, medium dense, circular, flat, even margin, with sparse aerial mycelium, white from above, reverse dark brown.

Material examined - THAILAND, Chiang Mai Province, on dried fruit of Lithocarpus sp. (Fagaceae), 22 December 2017, R.H. Perera, Seed 06 (MFLU 19-0982), living culture MFLUCC 19-0487.

GenBank numbers - ITS: MT215489, LSU: MT215539.

Notes - In the phylogenetic analysis our new isolate clustered with Beltraniella fertilis (MFLUCC 17-2137) with high statistical support (99\% MLBT, 1.00 BIPP; Fig. 6). A comparison of nucleotides shows ITS and LSU loci of our isolate are identical to B. fertilis (MFLUCC 172137). Our collection is similar to $B$. fertilis by its setae, conidiophore and conidial morphology and dimensions (Heredia et al. 2002, Lin et al. 2017). Therefore, our collection is a new host record for B. fertilis.

Chaetosphaeriales Huhndorf, A.N. Mill. \& F.A. Fernández Chaetosphaeriaceae Locq.

Dictyochaeta Speg. 1923

Dictyochaeta coryli R.H. Perera, E.B.G. Jones \& K.D. Hyde, sp. nov. Fig. 9 Index Fungorum number: IF556867; Facesoffungi number: FoF07752

Etymology - Named after the host genus Corylus.

Holotype - MFLU 19-1387

Saprobic on dried Corylus avellana fruits. Sexual morph: Undetermined. Asexual morph: Hyphomycetous. Colonies on natural substrate, effuse, hairy, pale brown, with glistering white conidial masses. Setae 115-135 × 3.5-4.8 $\mu \mathrm{m}$, straight or flexuous, septate, unbranched, pale brown to brown, paler towards the apex, apex fertile with persistent collarettes, smooth-walled. Conidiophores 55-70 × 3.3-4 $\mu \mathrm{m}(\bar{x}=62.3 \times 3.8 \mu \mathrm{m}, \mathrm{n}=15)$, macronematous, mononematous, solitary or in small groups often associated with setae, straight or flexuous, cylindrical, unbranched, septate, smooth, brown to pale brown, almost hyaline at apex, smooth-walled. Conidiogenous cells $10-22 \times 2.7-3.8 \mu \mathrm{m}(\bar{x}=15.7 \times 3.3 \mu \mathrm{m}, \mathrm{n}=15)$, monophialidic, integrated, terminal, sympodially proliferating, determinate, sub-cylindrical, pale brown, with distinct, funnel-shaped collarettes, 1.2-1.6 $\mu \mathrm{m}$ high, $2.2-3 \mu \mathrm{m}$ wide. Conidia $12-19.5 \times 2.3-3.5 \mu \mathrm{m}(\bar{x}=17.4 \times 3 \mu \mathrm{m}, \mathrm{n}=30)$, solitary, aseptate, fusiform, curved, sometimes guttulate, hyaline, smooth-walled, in slimy mass, single setula at each end, $4.7-8.4 \mu \mathrm{m}$ long, $0.5-0.8 \mu \mathrm{m}$ wide, filiform, hyaline.

Culture characteristics - Conidia germinating on PDA within 12 hours. Colonies on PDA reaching up to $30 \mathrm{~cm}$ diameter after 21 days at $28^{\circ} \mathrm{C}$, circular or irregularly circular, medium dense, 
effuse, aerial mycelium forming concentric rings with cottony texture, edge entire, white from above, reverse brown.

Material examined - UK, Hampshire, Bishop Waltham, standing water, on dried fruits of Corylus avellana L. (Betulaceae), 8 December 2017, E.B.G. Jones, GJ 406 (MFLU 19-1387, holotype).

GenBank numbers - ITS: MT215494, LSU: MT215545.

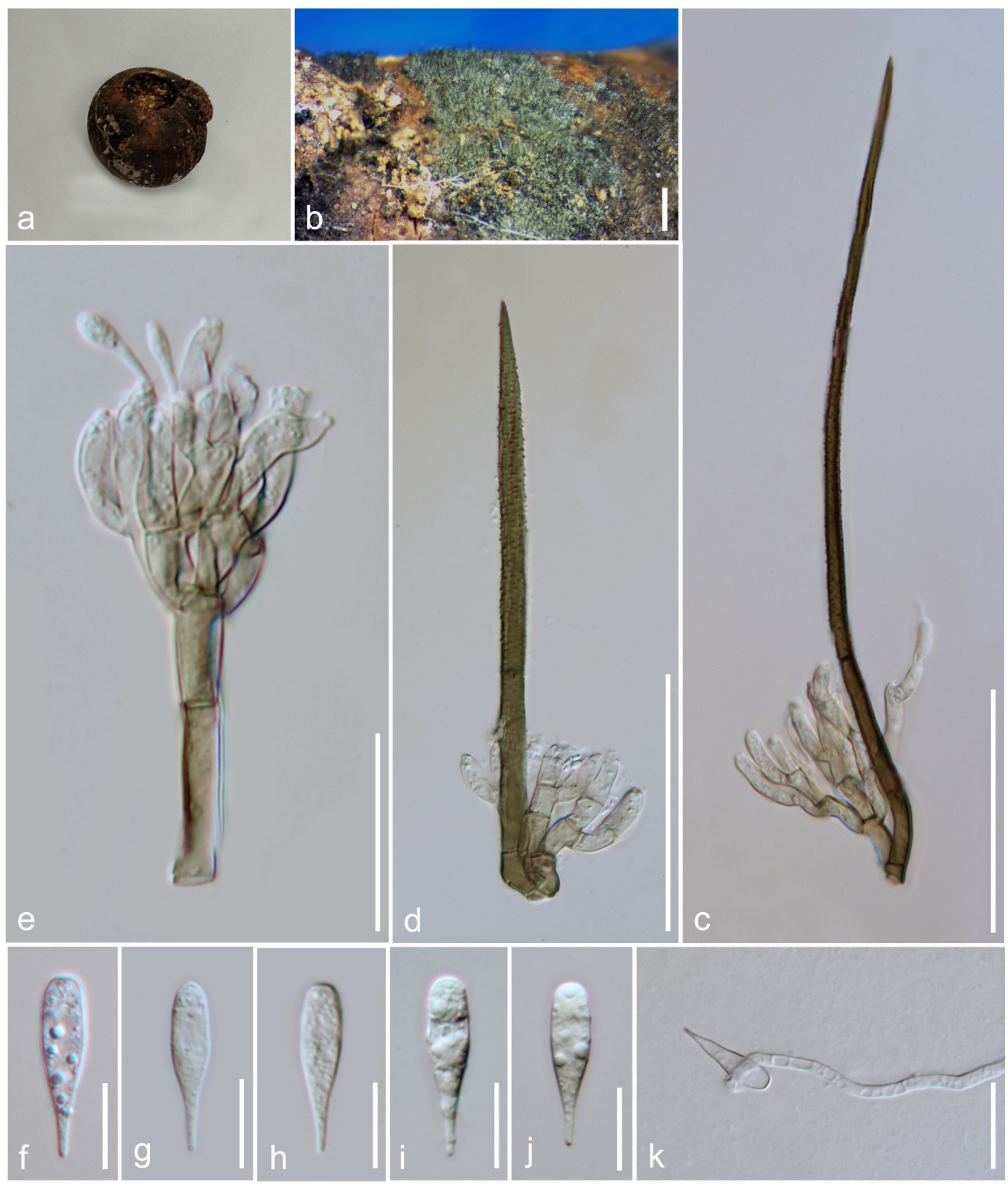

Figure 7 - Beltraniella fertilis (MFLU 19-0982). a Herbarium material. b Colony on natural substrate. c, d Setae with conidiogenous apparatus. e Conidiophores, conidiogenous cells with conidia. f-j Conidia. k Germinating conidium. Scale bars: b $=500 \mu \mathrm{m}, \mathrm{c}, \mathrm{d}=50 \mu \mathrm{m}, \mathrm{e}=20 \mu \mathrm{m}, \mathrm{f}-\mathrm{j}$ $=10 \mu \mathrm{m}, \mathrm{k}=20 \mu \mathrm{m}$. 


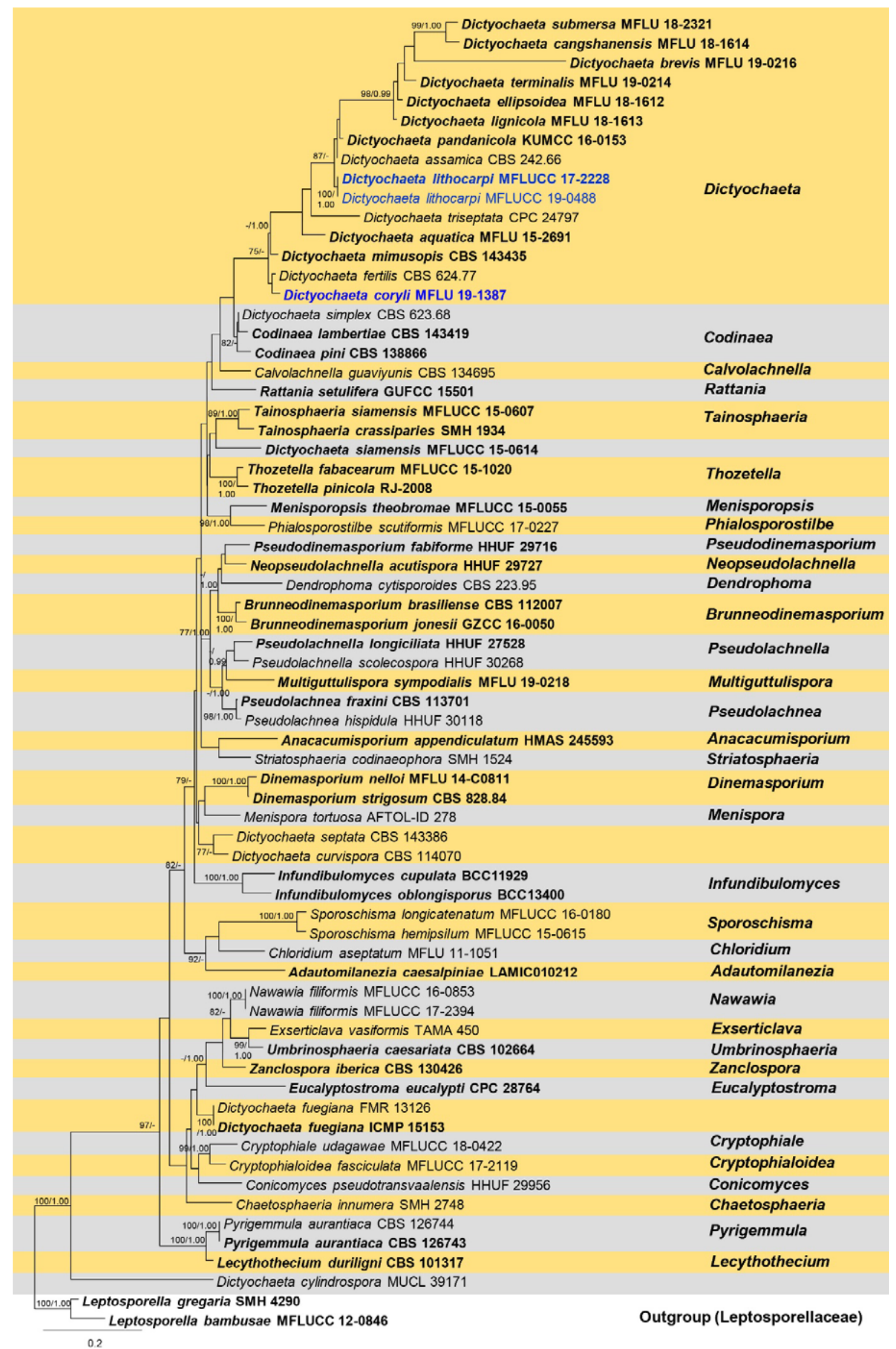

Figure 8 - Phylogram generated from RAxML analysis based on combined ITS and LSU sequence data of selected Chaetosphaeriaceae isolates. Sequences from sixty-eight taxa, which comprise 
1438 characters including gaps, are included in the analyses. The tree was rooted to Leptosporella bambusae (MFLUCC 12-846) and L. gregaria (SMH 4290). The scale bar indicates 0.2 nucleotide changes per site.

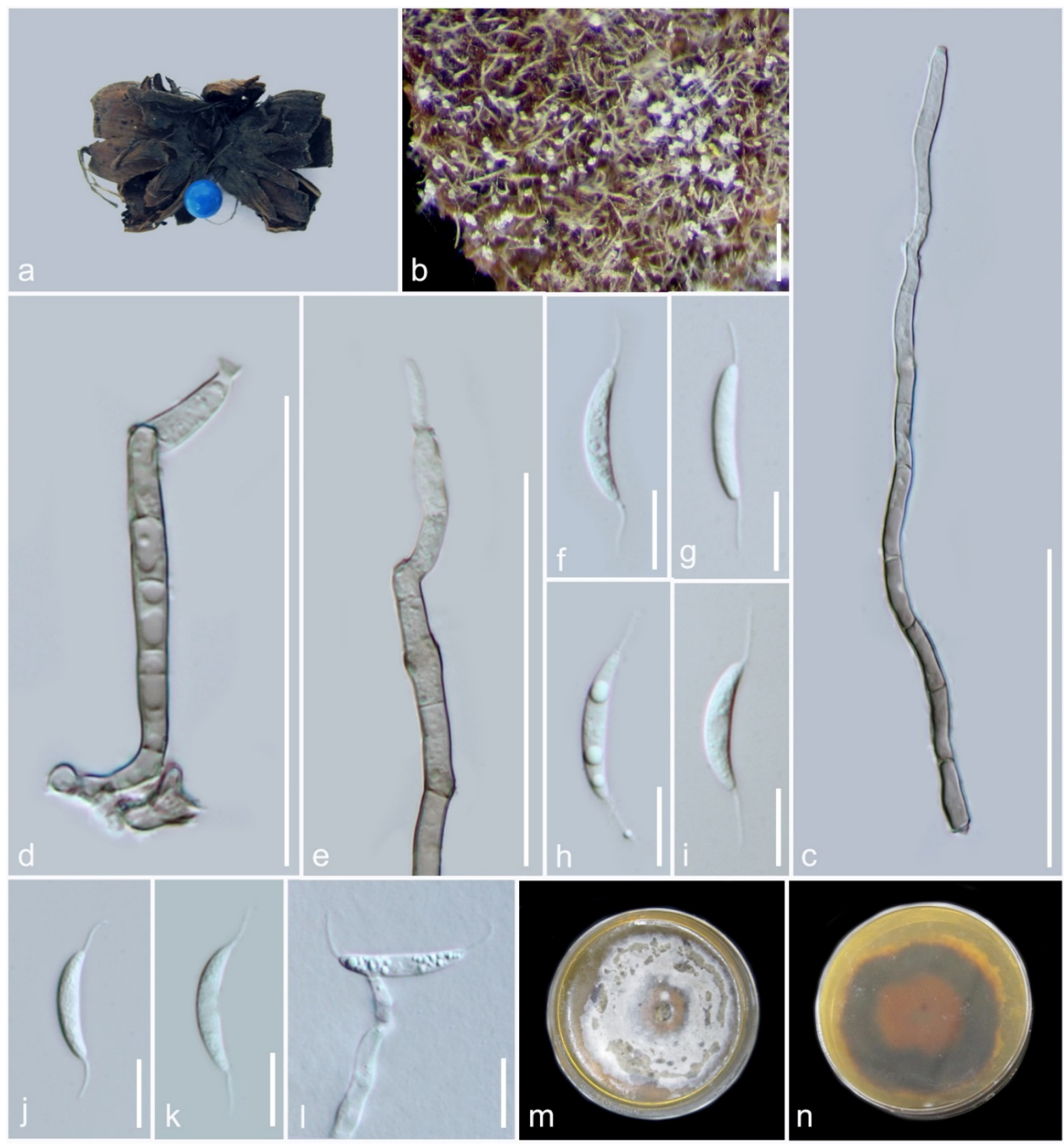

Figure 9 - Dictyochaeta coryli (MFLU 19-1387, holotype). a Herbarium material. b Colony on fruit. c Seta. d, e Conidiophores and conidiogenous cells. $\mathrm{f}-\mathrm{k}$ Conidia l Germinating conidium. m, $\mathrm{n}$ Colony on MEA. Scale bars: $\mathrm{b}=200 \mu \mathrm{m}, \mathrm{c}-\mathrm{e}=100 \mu \mathrm{m}, \mathrm{f}-\mathrm{l}=10 \mu \mathrm{m}$.

Notes - Dictyochaeta coryli groups with D. fertilis (S. Hughes \& W.B. Kendr.) Hol.-Jech. (CBS 624.77) in our phylogenetic analysis, with low statistical support (Fig. 8). Dictyochaeta coryli differs from $D$. fertilis in having aseptae conidia, while those of $D$. fertilis are 6-11 septate (Whitton et al. 2000). Dictyochaeta coryli can be distinguished from D. mimusopis Crous \& M.J. Wingf by having fertile setae, while D. assamica (Agnihothr.) Aramb., Cabello \& Mengasc. does not have setae (Arambarri et al. 1987). Dictyochaeta coryli resembles D. intermedia Gusmão \& S.M. Leão, but differs in having shorter and wider conidia $(12-19.5 \times 2.3-3.5$ vs. $18-21 \times 1.5-2.5)$ and fertile setae, while $D$. intermedia forms non-fertile setae (Cruz et al. 2009). Dictyochaeta coryli 
also cannot be assigned to any known Dictyochaeta species which lack sequence data (Kuthubutheen \& Nawawi 1991, Whitton et al. 2000, Cruz et al. 2009). We therefore identify D. coryli as a new species of Dictyochaeta.

Dictyochaeta lithocarpi R.H. Perera, E.B.G. Jones \& K.D. Hyde, sp. nov.

Fig. 10

Index Fungorum number: IF556868; Facesoffungi number: FoF07753

Etymology - Named after the host genus Lithocarpus.

Holotype - MFLU 19-1173

Saprobic on decaying Lithocarpus fruits. Sexual morph: Undetermined. Asexual morph: Hyphomycetous. Colonies on natural substrate, effuse, hairy, brown, with white conidial masses. Setae $170-275 \times 4-5 \mu \mathrm{m}(\bar{x}=209 \times 4.3 \mu \mathrm{m}, \mathrm{n}=10)$, solitary or in groups of $2-3$, straight or flexuous, septate, unbranched, apex fertile with persistent collarettes, dark brown and robust at the base, paler towards the apex, smooth-walled. Conidiophores 55-110 × 3.2-4 $\mathrm{mm}(\bar{x}=86 \times 3.7$ $\mu \mathrm{m}, \mathrm{n}=20$ ), macronematous, mononematous, aggregated, in small groups often associated with setae, rarely solitary, straight or flexuous, cylindrical, unbranched, septate, smooth, dark brown to pale brown, paler at apex, smooth-walled. Conidiogenous cells $12-22 \times 3.5-5.3 \mu \mathrm{m}(\bar{x}=16.6 \times$ $4.2 \mu \mathrm{m}, \mathrm{n}=20$ ), monophialidic, integrated, terminal, determinate, hyaline or pale brown, with distinct, sub-cylindrical, funnel-shaped collarettes, 1.8-2.1 $\mu \mathrm{m}$ high, 2.8-3.6 $\mu \mathrm{m}$ wide. Conidia 14-17 $\times 1.5-2.8 \mu \mathrm{m}(\bar{x}=15.2 \times 2.3 \mu \mathrm{m}, \mathrm{n}=30)$, solitary, aseptate, fusiform, curved, in slimy mass, hyaline, smooth-walled, single setula at each end, 6.8-10.5 $\mu \mathrm{m}$ long, 0.4-0.6 $\mu \mathrm{m}$ wide, filiform, hyaline.

Culture characteristics - Conidia germinated on PDA within 12 hours. Colonies on PDA reaching up to $35 \mathrm{~cm}$ diameter after 15 days at $28^{\circ} \mathrm{C}$, circular or irregularly circular, medium dense, flat or effuse, edge entire, white from above, reverse yellow brown.

Material examined - THAILAND, Chiang Mai Province, on dried fruits of Lithocarpus sp. (Fagaceae), 20 July 2017, R.H. Perera, Doi 05 (MFLU 19-1173, holotype), ex-type living culture MFLUCC 17-2228; ibid., 22 December 2017, Seed 08 (MFLU 18-2772), living culture MFLUCC 19-0488.

GenBank numbers - ITS: MT215492, LSU: MT215543 (MFLUCC 17-2228); ITS: MT215493, LSU: MT215544 (MFLUCC 19-0488).

Notes - Dictyochaeta lithocarpi is well separated from other Dictyochaeta species in the phylogenetic analysis (Fig. 8). Our isolate is related to D. pandanicola (KUMCC 16-0153) and D. assamica (CBS 242.66) (87\% MLBT). Dictyochaeta lithocarpi differs from $D$. pandanicola by having smaller conidia $(14-17 \times 1.5-2.8$ vs. 16-26 $\times 4-6 \mu \mathrm{m})$ and presence of setae (Tibpromma et al. 2018). Dictyochaeta lithocarpi can be distinguished from $D$. assamica by having monophialidic conidiogenous cells, while D. assamica has polyphialidic conidiogenous cells (Arambarri et al. 1987). Dictyochaeta lithocarpi also cannot be assigned to any known Dictyochaeta species, which lack sequence data (Kuthubutheen \& Nawawi 1991, Whitton et al. 2000, Cruz et al. 2009). Therefore, we identify D. lithocarpi as a novel species of Dictyochaeta.

Menisporopsis S. Hughes

Menisporopsis theobromae S. Hughes, Mycol. Pap. 48: 59 (1952)

Fig. 11

Colonies on decaying Fabaceae seed pod, scattered, superficial, effuse, white. Sexual morph: Undetermined. Asexual morph: Setae 230-280 × 5.1-6.2 $\mu \mathrm{m}$, central, solitary, erect, septate, thickwalled, lower part encased tightly by compact conidiophores and obviously wider than each conidiophore, brown. Conidiophores up to $128 \mu \mathrm{m}$ long, synnematous, macronematous, brown, cylindrical, septate, unbranched, lower part narrow, upper part wider, erect, straight or slightly flexuous, smooth, thin to thick-walled. Conidiogenous cells terminal, monophialidic, integrated, pale brown, with visible collarettes. Conidia (11-)15.7-21.6 $\times 2.1-3.8 \mu \mathrm{m}(\bar{x}=18.6 \times 2.6 \mu \mathrm{m}, \mathrm{n}=$ 30 ), aggregated in slimy masses at the apex of the synnemata, acrogenous, lunate to falcate, gently 
curved or straight, aseptate, hyaline, thin- and smooth-walled; with a single, unbranched, flexuous, tubular, hyaline setula at each end, 7.3-9.4 $\mu \mathrm{m}$ long.

Material examined - THAILAND, Chiang Mai Province, on decaying seed pods of Fabaceae plant, 5 July 2015, R.H. Perera, RHP 128 (MFLU 16-1055).

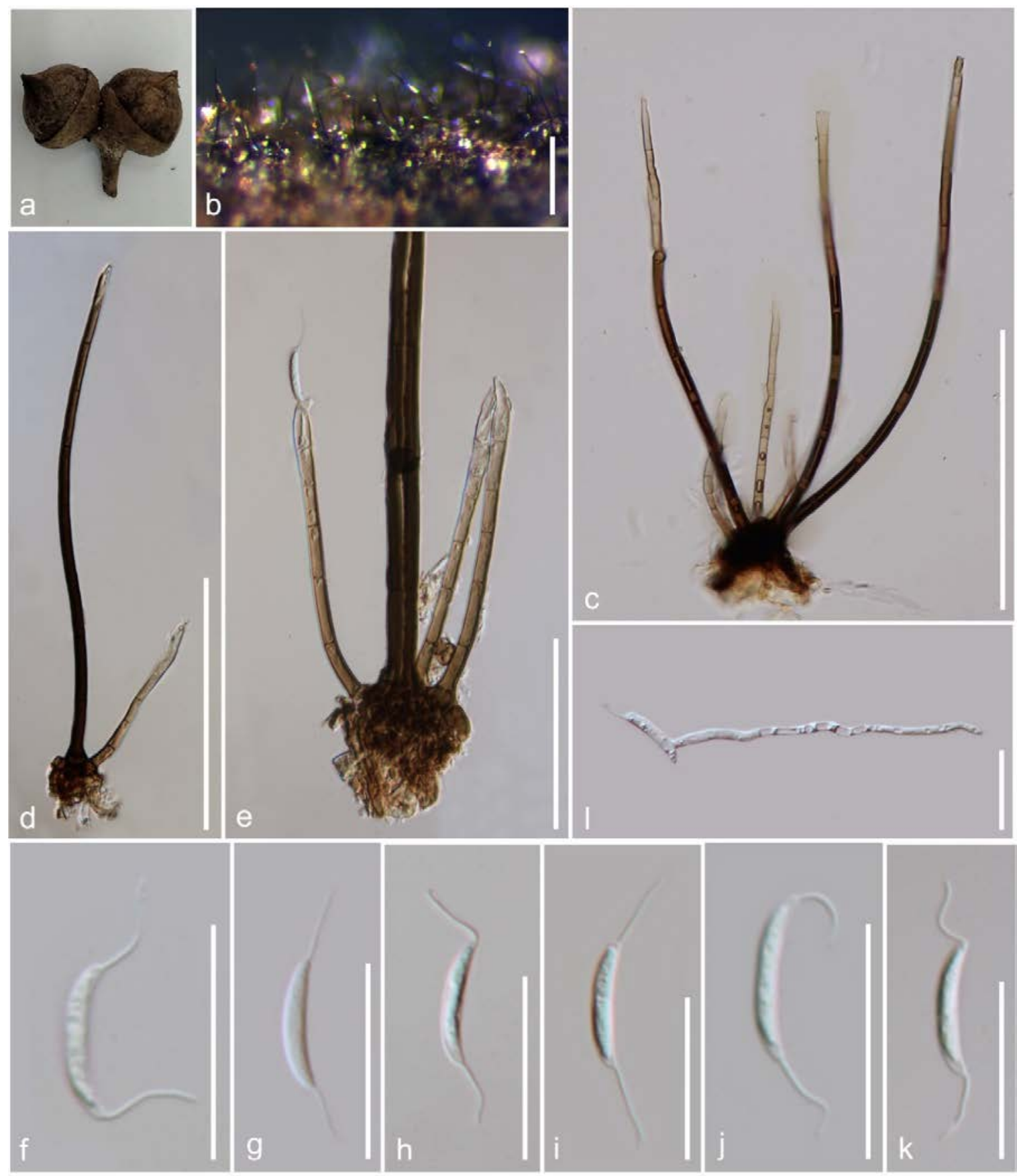

Figure 10 - Dictyochaeta lithocarpi (MFLU 19-1173, holotype). a Herbarium material. b Colony on natural substrate. c-e Setae, conidiophores and conidiogenous cells. f-k Conidia. l Germinating conidium. Scale bars: $\mathrm{b}=200 \mu \mathrm{m}, \mathrm{c}, \mathrm{d}=100 \mu \mathrm{m}, \mathrm{e}=50 \mu \mathrm{m}, \mathrm{f}-\mathrm{l}=20 \mu \mathrm{m}$.

Notes - We were unable to get a culture of this fungus. However, our collection resembles the genus Menisporopsis by its synnematous conidiophores, central, simple, dark brown setae, phialidic conidiogenous cells and lunate to falcate, $0-1$-septate conidia, with setulae at ends (Cruz et al. 2014). The newly collected fungus is morphologically similar to $M$. theobromae, which was collected in Ghana and Thailand (Hughes 1952, Liu et al. 2016). Based on morphological features we regard our fungus as $M$. theobromae (Hughes 1952, Cruz et al. 2014, Liu et al. 2016). 
Menisporopsis theobromae was previously reported from Theobroma cacao and submerged wood, and this is the first record of the fungus from seed pods of a Fabaceae plant (Hughes 1952, Liu et al. 2016).

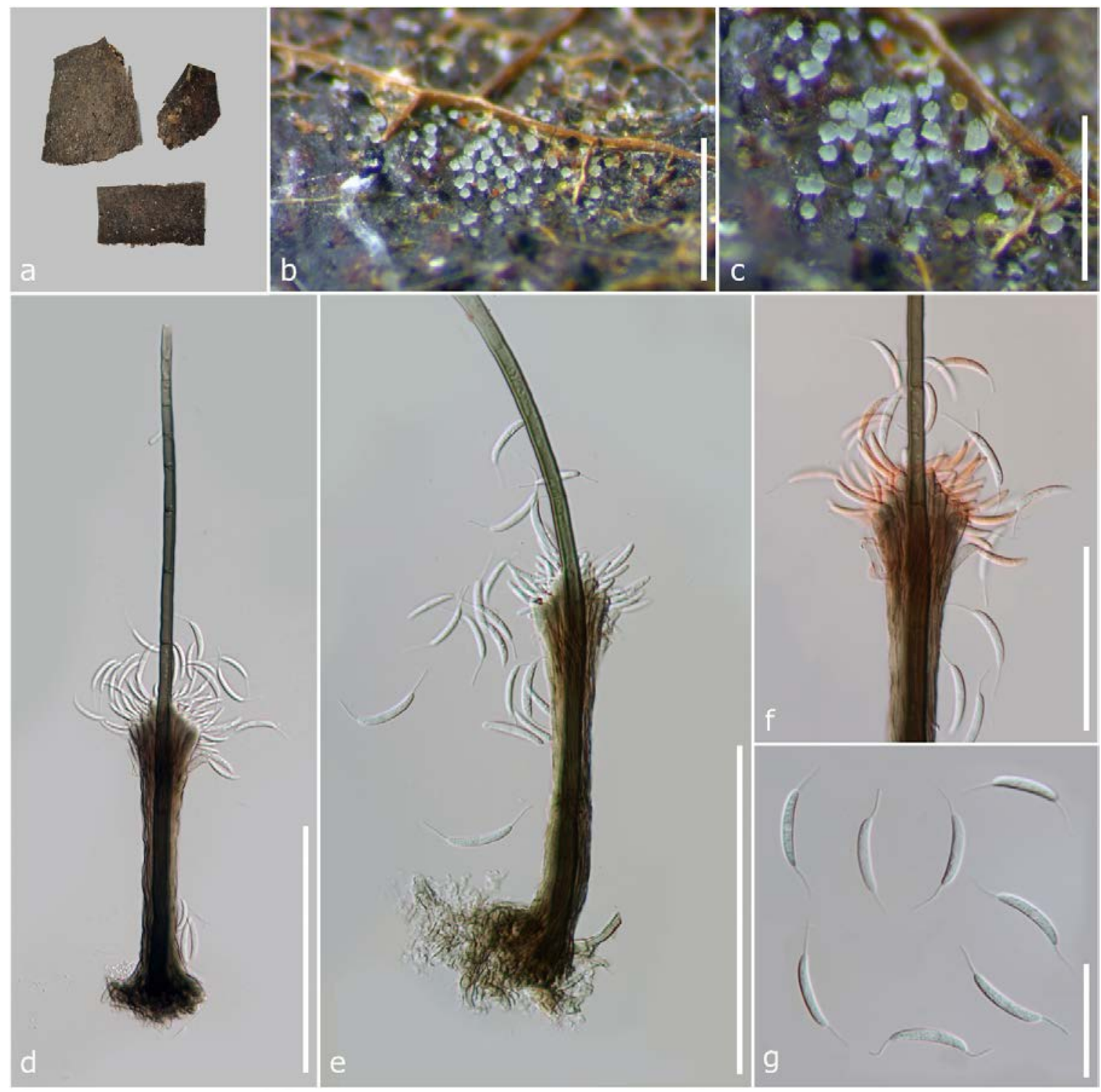

Figure 11 - Menisporopsis theobromae (MFLU 16-1055). a Herbarium material. b, c Colony on natural substrate. $\mathrm{d}$, e Conidiophores and seta. $\mathrm{f}$ Conidiogenous cells with conidia in Congo red. $\mathrm{g}$ Conidia. Scale bars: $\mathrm{b}=1 \mathrm{~mm}, \mathrm{c}=500 \mu \mathrm{m}, \mathrm{d}, \mathrm{e}=100 \mu \mathrm{m}, \mathrm{f}=50 \mu \mathrm{m}, \mathrm{g}=30 \mu \mathrm{m}$.

Diaporthales Nannf.

Cytosporaceae Fr.

Cytospora Ehrenb.

Cytospora diopuiensis Q.J. Shang, K.D. Hyde \& J.K. Liu, in Shang et al., Mycosphere 11: 203 (2020)

Figs 15, 16

Associated with dried seed pods of Cassia fistula. Sexual morph: Undetermined. Asexual morph: Stromata 125-470 $\mu \mathrm{m}$ high, 130-465 $\mu \mathrm{m}$ wide $(\mathrm{n}=10)$, with poorly developed interior, solitary to gregarious, immersed, becoming raised or erumpent through the host tissue, discoid, circular to irregular in shape, dark brown to black, glabrous. Conidiomata (excluding necks) 110 $210 \times 95-325 \mu \mathrm{m}$ diam. $(\mathrm{n}=15)$, immersed in host tissue, scattered, erumpent, circular, with 1-2 


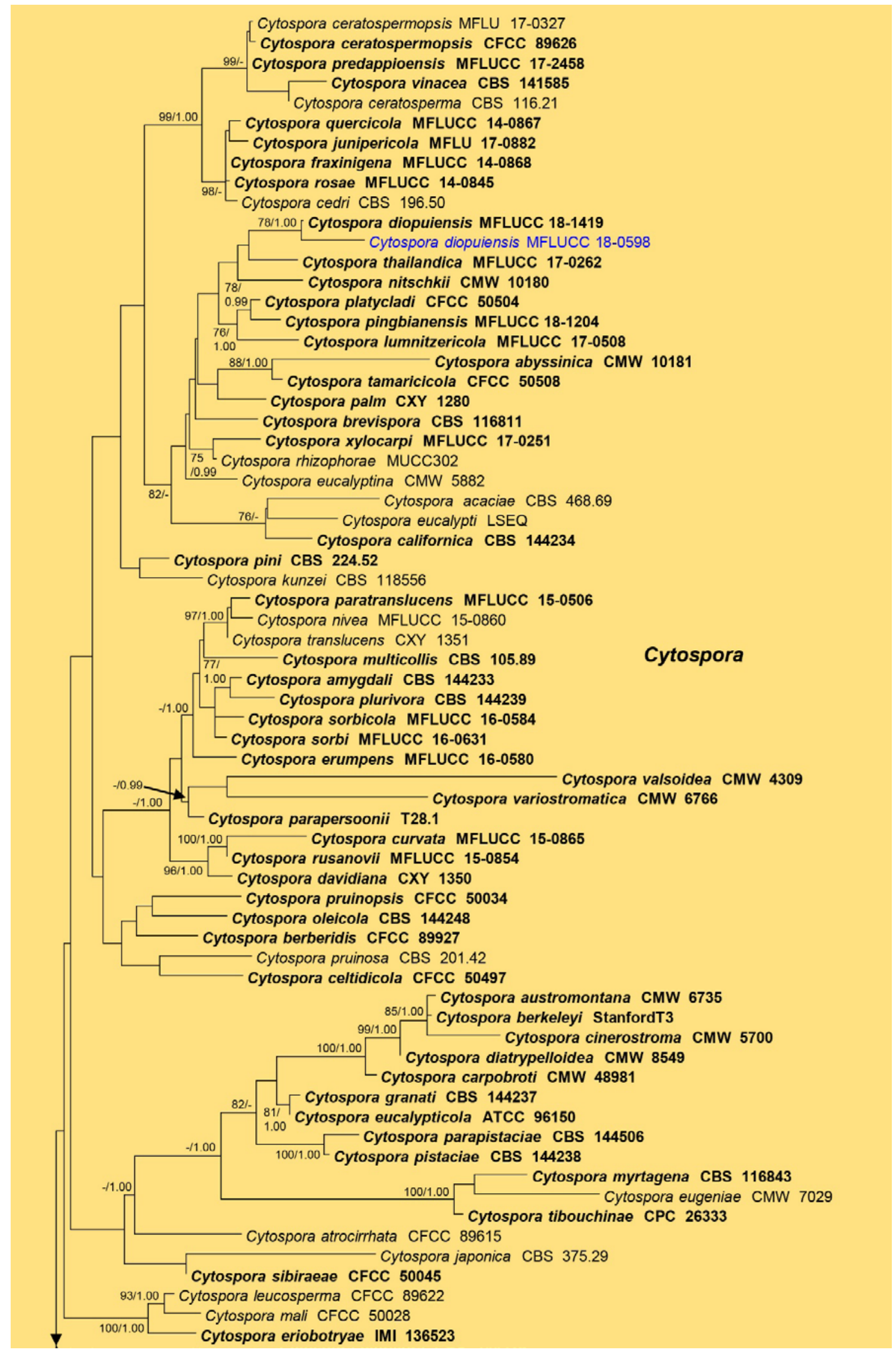

Figure 14 - Phylogram generated from RAxML analysis based on combined ITS, LSU, RPB2 and ACT sequence data of Cytospora isolates. Sequences from one hundred thirty taxa, which comprise 3352 characters including gaps, are included in the analyses. The tree was rooted to Cytospora punicae CBS 144244 . The scale bar indicates 0.04 nucleotide changes per site. 


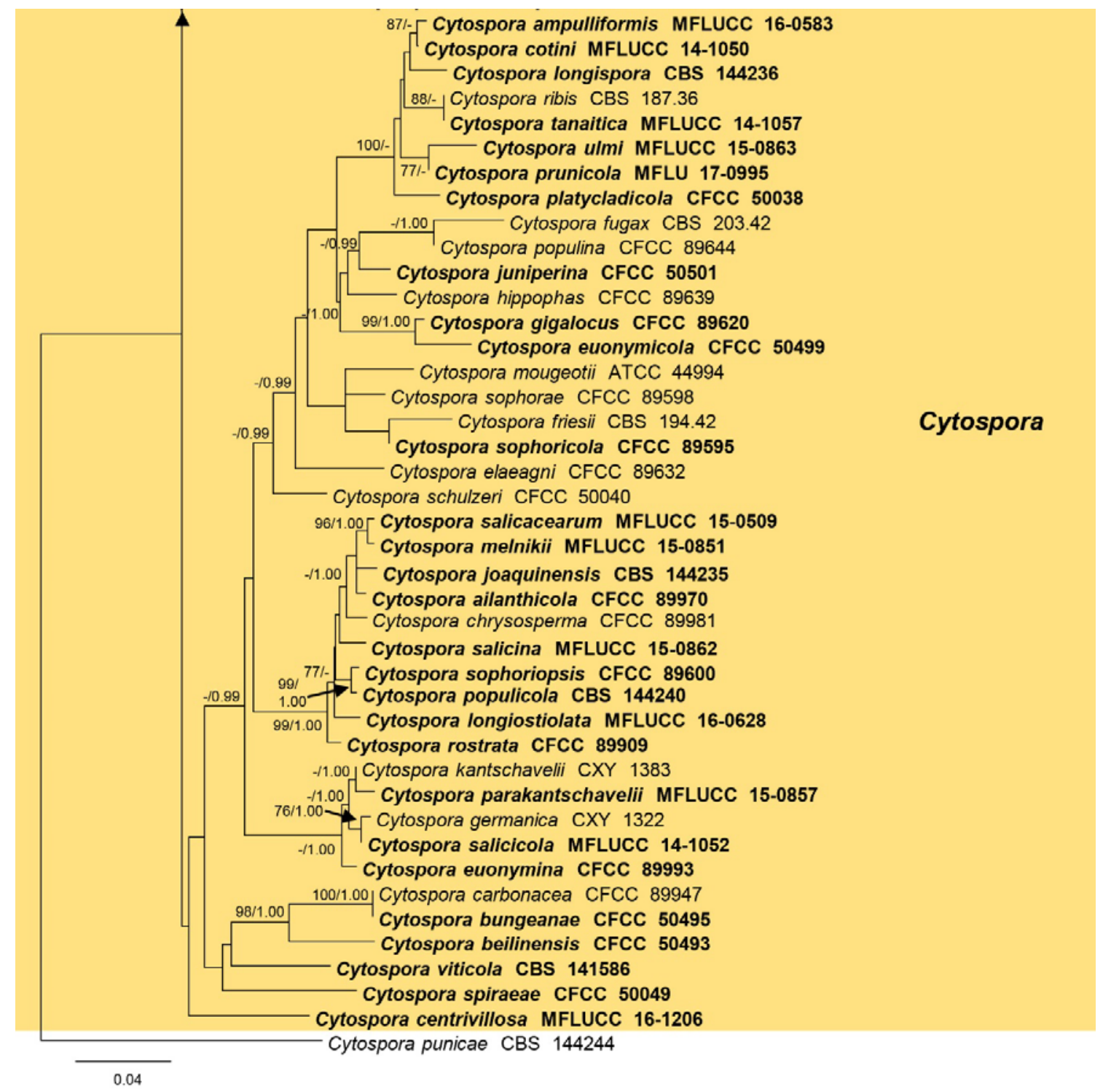

Figure 14 - Continued.

locules, ostiolate, occasionally with yellow conidial exudate. Ostioles 120-150 $\mu$ m diam. Peridium comprising a few to several layers of cells of textura angularis, inner layer thick, brown, outer later dark brown. Conidiophores branched, reduced to conidiogenous cells. Conidiogenous cells 8.4-14 $\times 1.5-2.4 \mu \mathrm{m}(\bar{x}=11 \times 1.9 \mu \mathrm{m}, \mathrm{n}=20)$, enteroblastic, phialidic, formed from the inner most layer of pycnidial wall, hyaline, smooth-walled. Conidia 4.3-6.7 × 1.4-2.3 $\mu \mathrm{m}(\bar{x}=5.3 \times 1.9 \mu \mathrm{m}, \mathrm{n}=$ 50), unicellular, allantoid, sometimes obovoid, guttulate, hyaline, smooth-walled. Chlamydospores produced on PDA, hyaline, in chains.

Culture characteristics - Conidia germinated on PDA within 12 hours. Colonies on PDA reaching $40-45 \mathrm{~mm}$ diameter after 7 days at $28^{\circ} \mathrm{C}$, colonies circular to irregular, medium dense, flat or effuse, with edge fimbriate, white from above and below, pale brown with time, sporulating on PDA within 60 days.

Material examined - Thailand, Phayao Province, dried seed pod of Cassia fistula L. (Fabaceae), 4 August 2017, R.H. Perera, PH-CAS 01 (MFLU 18-2749), dry culture MFLU 180620, living culture: MFLUCC 18-0598.

GenBank numbers - ITS: MT215491, LSU: MT215540, RPB2: MT212203.

Notes - A modern treatment of Cytospora was provided by Norphanphoun et al. (2017) and is followed here. Our new isolate MFLUCC 18-0598 groups with ex-type of Cytospora diopuiensis (MFLUCC 18-1419) in our phylogenetic analysis with low statistical support (Fig. 14). In the 
combined gene analysis of ITS and LSU, our isolate grouped with C. diopuiensis (MFLUCC 181419) with high statistical support (99\% MLBT) (data not shown). ITS and LSU data of two isolates are identical and, there is no RPB2 gene available for C. diopuiensis for comparison. However, only the sexual morph is reported for $C$. diopuiensis (Shang et al. 2020). By considering the similarities in the ITS and LSU loci, we identify our isolate as the first asexual morph record for C. diopuiensis and a new host record. Cytospora diopuiensis can be distinguished from $C$. thailandica in having larger allantoid conidia (4.3-6.7 × 1.4-2.3 vs. 3.8-4 × 1-1.3 $\mu \mathrm{m})$ and larger conidiogenous cells $(8.4-14 \times 1.5-2.4$ vs. 6-9.1 $\times 1-1.3 \mu \mathrm{m})$, while conidia of $C$. thailandica are subcylindrical (Shang et al. 2020).

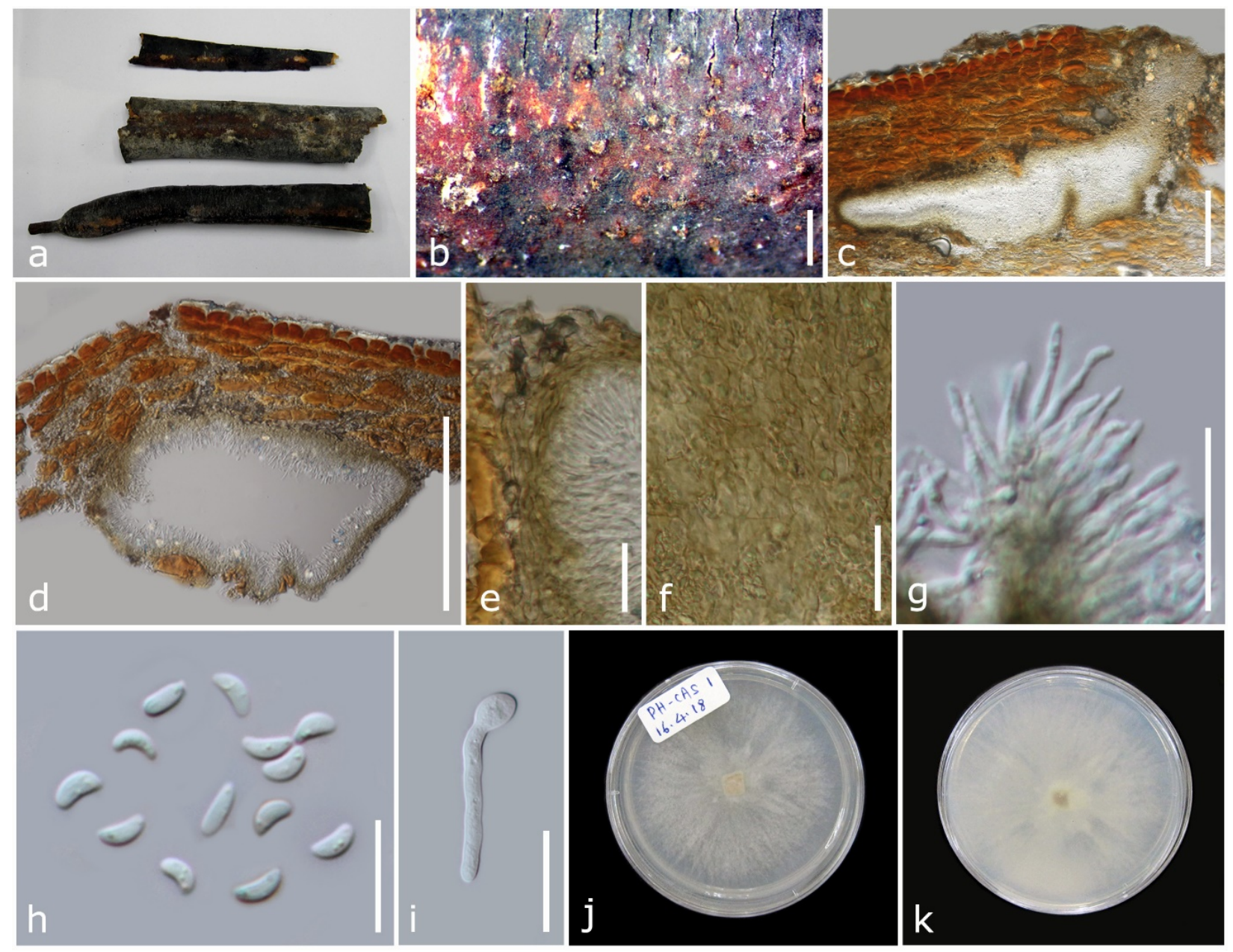

Figure 15 - Cytospora diopuiensis (MFLU 18-2749). a Herbarium material. b Conidiomata on host surface. c, d Cross section of the stroma showing conidiomata. e Peridium. f Peridium in face view. g Conidia attached to conidiogenous cells. h Conidia. i Germinating conidium. j, k Colony on PDA. Scale bars: $b=500 \mu \mathrm{m}, \mathrm{c}=100 \mu \mathrm{m}, \mathrm{d}=200 \mu \mathrm{m}, \mathrm{e}-\mathrm{g}=20 \mu \mathrm{m}, \mathrm{h}=10 \mu \mathrm{m}, \mathrm{i}=20 \mu \mathrm{m}$.

Diaporthaceae Höhn. ex Wehm.

Diaporthe Nitschke

Diaporthe delonicis R.H. Perera, E.B.G. Jones \& K.D. Hyde, sp. nov. Fig. 13 Index Fungorum number: IF556855; Facesoffungi number: FoF07754 Etymology - Named after the host genus Delonix.

Holotype - MFLU 16-1059 

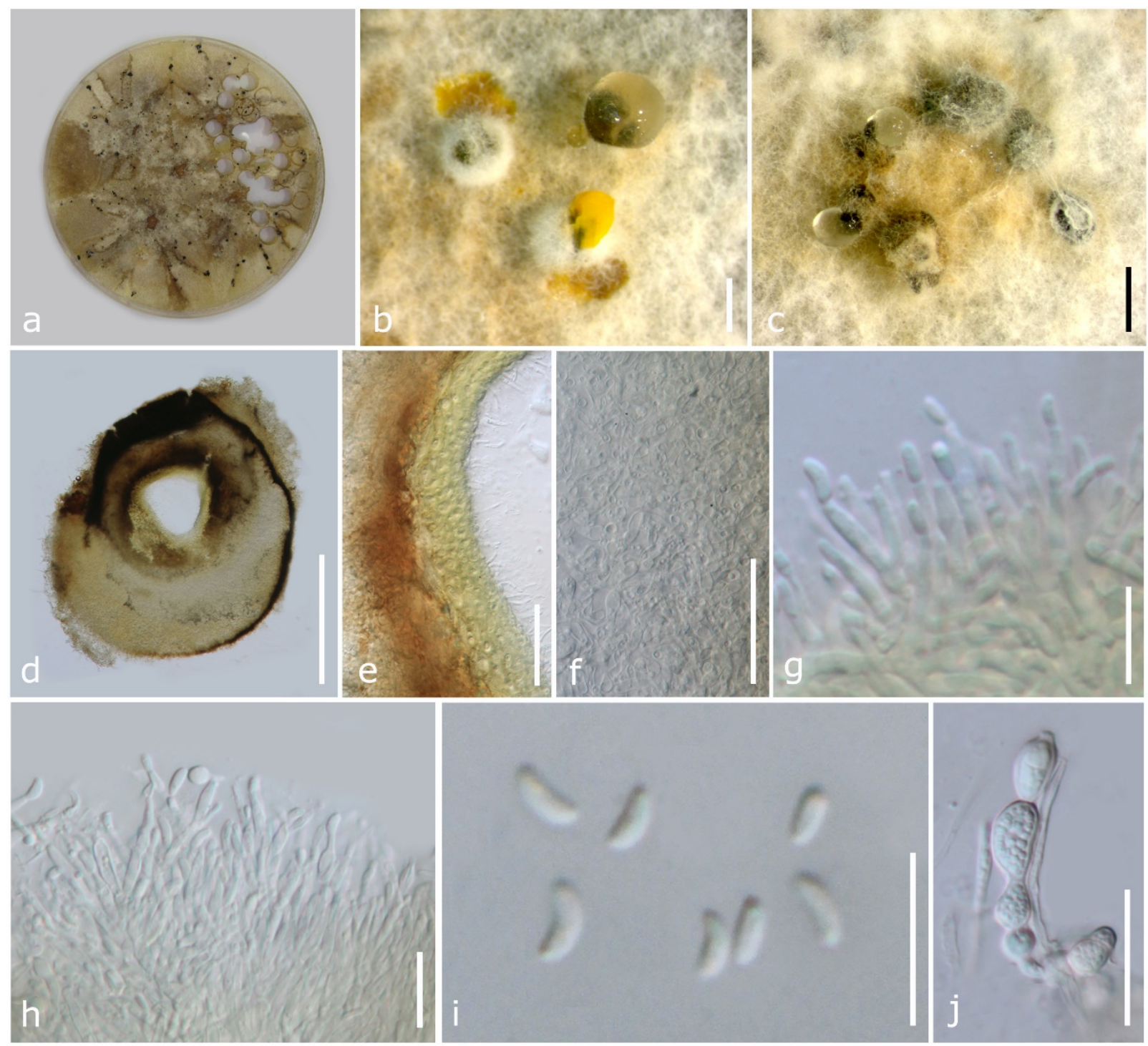

Figure 16 - Cytospora diopuiensis (MFLU 18-0620). a-c Conidiomata on PDA. d Conidioma. e Wall of the ostiolar canal. f Stromatal tissue. g, h Conidia attached to conidiogenous cells. i Conidia. j Chlamydospores. Scale bars: $b, c=1 \mathrm{~mm}, \mathrm{~d}=500 \mu \mathrm{m}, \mathrm{e}, \mathrm{f}=50 \mu \mathrm{m}, \mathrm{g}-\mathrm{j}=10 \mu \mathrm{m}, \mathrm{j}=20$ $\mu \mathrm{m}$.

Saprobic on seed pods of Delonix regia. Sexual morph: Undetermined. Asexual morph: Conidiomata $78-190 \mu \mathrm{m}(\bar{x}=120 \mu \mathrm{m})$ in diam., appearing as black raised spots on the host, scattered, globose or near-globose, brown to dark brown, conidia exuded from conidiomata in white creamy drops. Peridium $20 \mu \mathrm{m}$ thick, composed of 5-8 rows of hyaline to light brown cells of textura angularis. Conidiophores 6.4-15.2 × 1.4-2.2 $\mu \mathrm{m}(\bar{x}=11.6 \times 1.9 \mu \mathrm{m})$, sub-cylindrical, hyaline. Conidiogenous cells 5.3-10.5 $\times 1.3-2.5 \mu \mathrm{m}(\bar{x}=7.9 \times 1.9 \mu \mathrm{m})$, phialidic, tapering to the apex, collarette prominent. Alpha conidia 4.4-9 $\times 1.3-2.2 \mu \mathrm{m}(\bar{x}=7.7 \times 1.8 \mu \mathrm{m})$, aseptate, fusoid with obtuse ends, 4-guttulate, hyaline, smooth-walled. Beta conidia 16-23 $\times 1-1.7 \mu \mathrm{m}(\bar{x}=19.4 \times$ $1.2 \mu \mathrm{m})$, aseptate, filiform, slightly curved at one end and both ends rounded, hyaline, smoothwalled.

Material examined - THAILAND, Chiang Rai Province, Mae Fah Luang University premises, on dried seed pods of Delonix regia (Boj. ex Hook.) Raf. (Fabaceae), 7 May 2015, R.H. Perera, RHP 99 (MFLU 16-1059, holotype).

GenBank numbers - ITS: MT215490, TUB2: MT212209. 


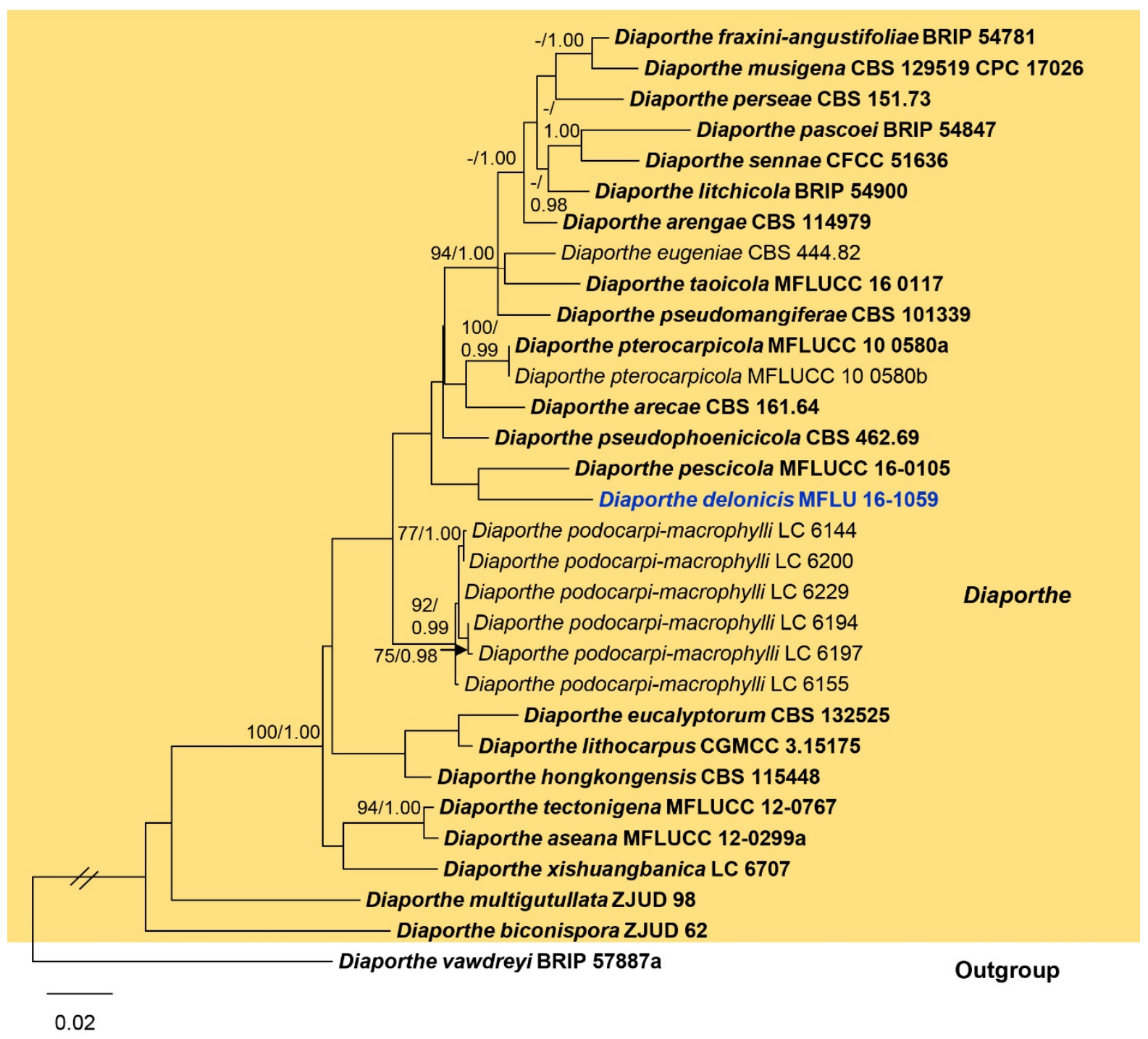

Figure 12 - Phylogram generated from RAxML analysis based on combined ITS, TEF1 and TUB2 sequence data of Diaporthe isolates. Sequences from thirty-one taxa, which comprise 1578 characters including gaps, are included in the analyses. The tree was rooted to Diaporthe vawdreyi (BRIP 57887a). The scale bar indicates 0.02 nucleotide changes per site.

Notes - The genus has been well-studied and requires ITS, TEF1 and TUB2 sequence data to resolve species (Udayanga et al. 2014, 2015, Dissanayake et al. 2017a). DNA was directly extracted from the fruiting bodies of this fungus. Diaporthe delonicis (MFLU 16-1059) groups with D. pescicola (MFLUCC 16-0105), but is unsupported within Diaporthe (Fig. 12). Diaporthe pescicola was identified as a pathogen on Prunus persica shoots in China by Dissanayake et al. (2017b). Diaporthe delonicis differs from D. pescicola by producing smaller conidiomata (78-190 vs. $300 \mu \mathrm{m})$ and conidiophores (6.4-15.2 × 1.4-2.2 vs. 21-35 × 1.5-2.5 $\mu \mathrm{m}$ ) (Dissanayake et al. 2017b). Diaporthe delonicis can also be distinguished from $D$. pescicola by smaller beta conidia (16-23 × 1-1.7 vs. 18-37 × 1-1.5 $\mu \mathrm{m}$ ) (Dissanayake et al. 2017b). Furthermore, D. delonicis produces alpha conidia with 4-guttules, while $D$. pescicola forms bi-guttulate alpha conidia (Dissanayake et al. 2017b). We also observed prominent collarettes in conidiophores of $D$. delonicis, and this character was not reported from D. pescicola (Dissanayake et al. 2017b). We therefore identified $D$. delonicis as a new species. 


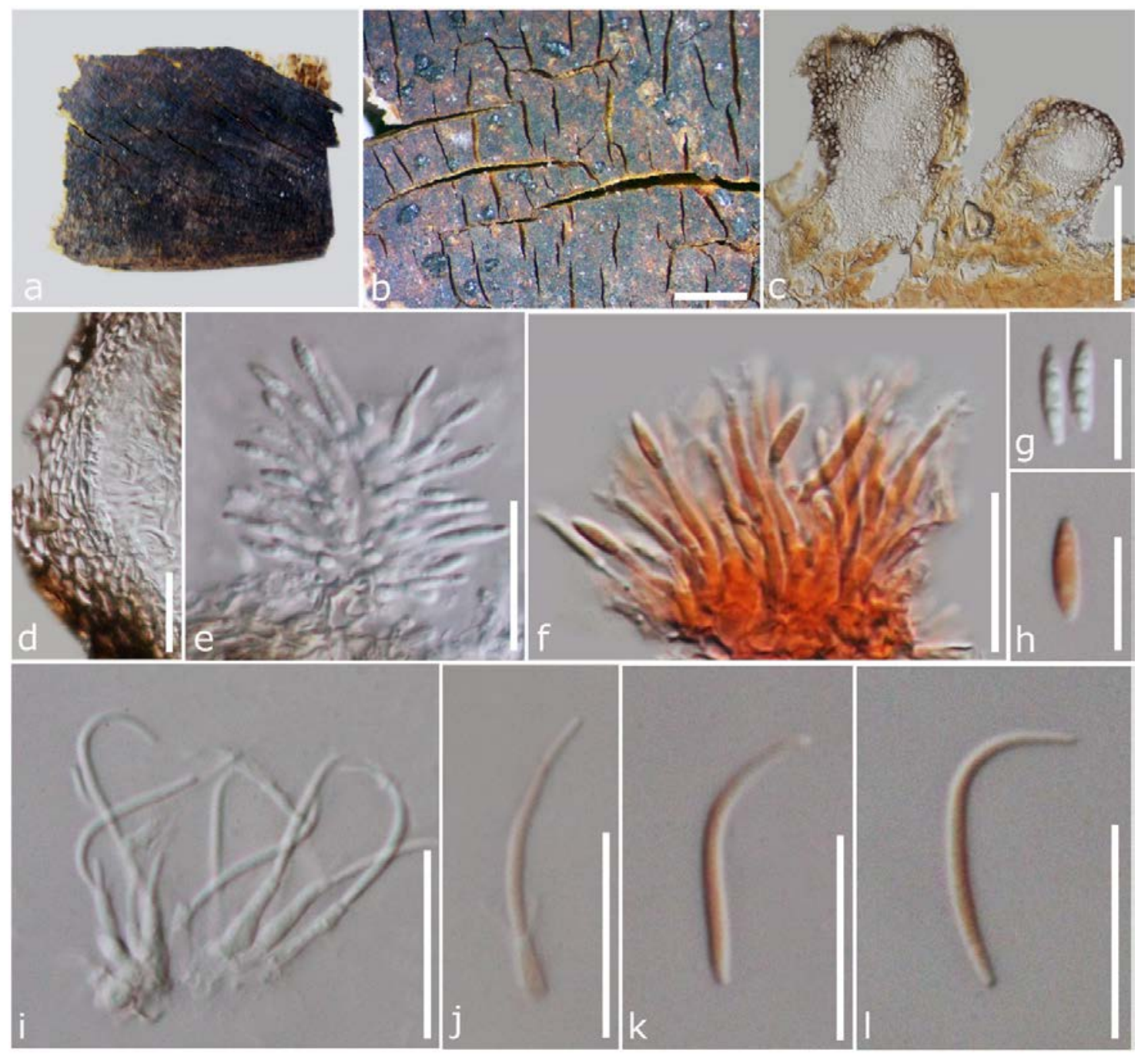

Figure 13 - Diaporthe delonicis (MFLU 16-1059, holotype). a Herbarium material. b Conidiomata on natural substrate. c Section through conidiomata. d Peridium. e, f Conidiogenous cells with alpha conidia (f in Lugol's solution). g, h Alpha conidia (h in Lugol's solution). i Conidiogenous cells giving rise to beta conidia. $\mathrm{j}-\mathrm{l}$ Beta conidia in Lugol's solution. Scale bars: $\mathrm{b}=1 \mathrm{~mm}, \mathrm{c}=100$ $\mu \mathrm{m}, \mathrm{d}-\mathrm{f}=20 \mu \mathrm{m}, \mathrm{g}, \mathrm{h}=10 \mu \mathrm{m}, \mathrm{i}-\mathrm{l}=20 \mu \mathrm{m}$.

\section{Hypocreales Lindau \\ Bionectriaceae Samuels \& Rossman Clonostachys Corda}

Clonostachys rogersoniana Schroers, Stud. Mycol. 46: 109 (2001)

Fig. 18

Facesoffungi number: FoF07755

Saprobic on decaying seed pods of Delonix regia. Sexual morph: Undetermined. Asexual morph: Hyphomycetous, colonies appearing as white patches on both inner and outer epidermis of the seed pod. Conidiophores arising from stroma, monomorphic, hyaline, smooth-walled, penicillate, stipes 70- $100 \mu \mathrm{m}$ long, 4.2-6.8 $\mu \mathrm{m}$ wide at base. Penicilli 58-80 $\mu \mathrm{m}$ high, $\times$ 50-100 $\mu \mathrm{m}$ diam. at widest point; solitary to gregarious, not sporodochial, bi- to quaterverticillate; branches of the penicillus divergent, each branch terminating in metulae and adpressed phialides. Phialides 7-12.4 $\mu \mathrm{m}(\bar{x}=10 \mu \mathrm{m})$ long, $1.8-2.9 \mu \mathrm{m}(\bar{x}=2.4 \mu \mathrm{m})$ wide at widest point, in whorls of up to 6 , narrowly flask-shaped, slightly tapering toward the apex. Intercalary phialides not observed. Conidial masses white. Conidia 4.9-9.1 $\times 1.6-2.3 \mu \mathrm{m}(\bar{x}=7.2 \times 2 \mu \mathrm{m}, \mathrm{n}=30)$, broadly ellipsoidal to oval, rarely minutely curved, ends broadly rounded, bi-guttulate, hilum laterally displaced, almost median or invisible, hyaline, smooth-walled. 


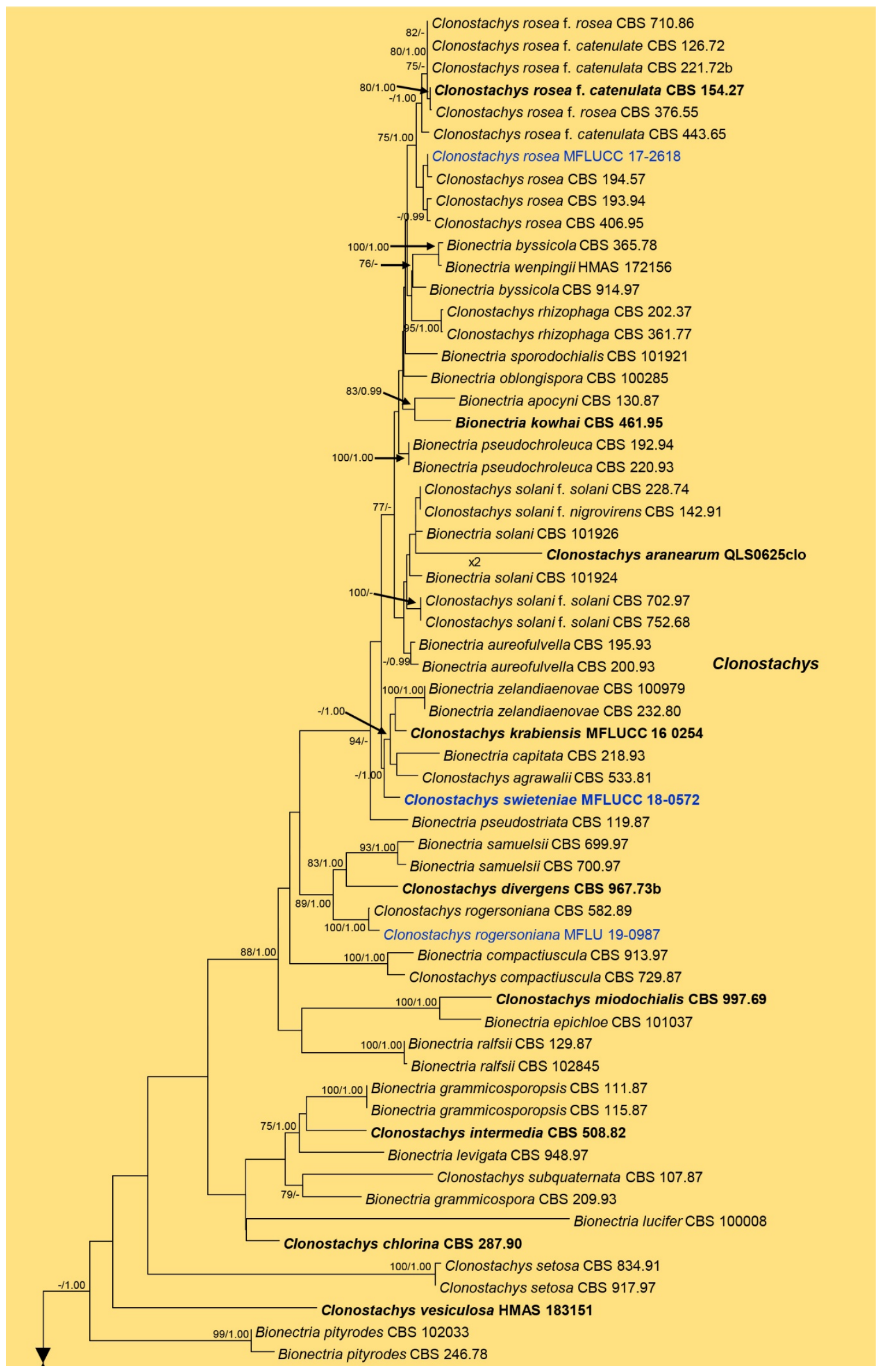

Figure 17 - Phylogram generated from RAxML analysis based on combined ITS and TUB2 sequence data of Clonostachys isolates. Sequences from seventy-two taxa, which comprise 1087 
characters including gaps, are included in the analyses. The tree was rooted to Nectria cinnabarina NRRL 20484 and Fusarium acutatum CBS 402.97. The scale bar indicates 0.07 nucleotide changes per site.

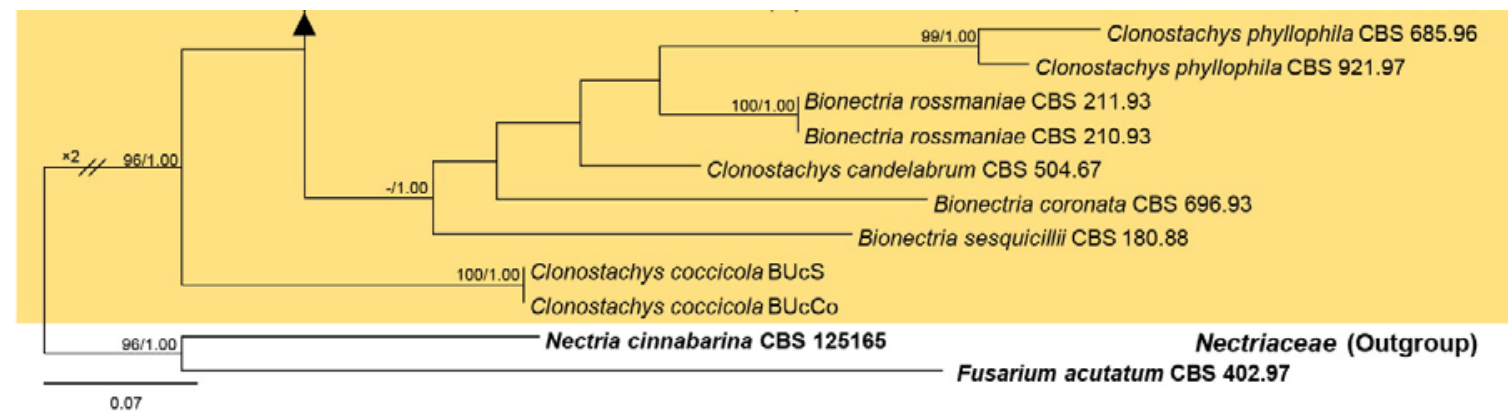

Figure 17 - Continued.

Material examined - Thailand, Chiang Mai Province, on decaying seed pods of Delonix regia (Fabaceae), 20 December 2016, R.H. Perera, Delo 01 (MFLU 19-0987).

GenBank numbers - ITS: MT215572, LSU: MT215538, TUB2: MT212208.

Notes - Our new strain clusters with Clonostachys rogersoniana (CBS 582.89) with high statistical support (100\% MLBT, 1.0 BIPP; Fig. 17) in phylogenetic analysis of ITS sequence data, and they are morphologically similar in having the same conidiophore characters and conidial dimensions (Schroers 2001). However, verticillate conidiophores were not observed in our collection (Schroers 2001). With morphological and DNA molecular analysis support, we report a new host record of Clonostachys rogersoniana from Delonix regia.

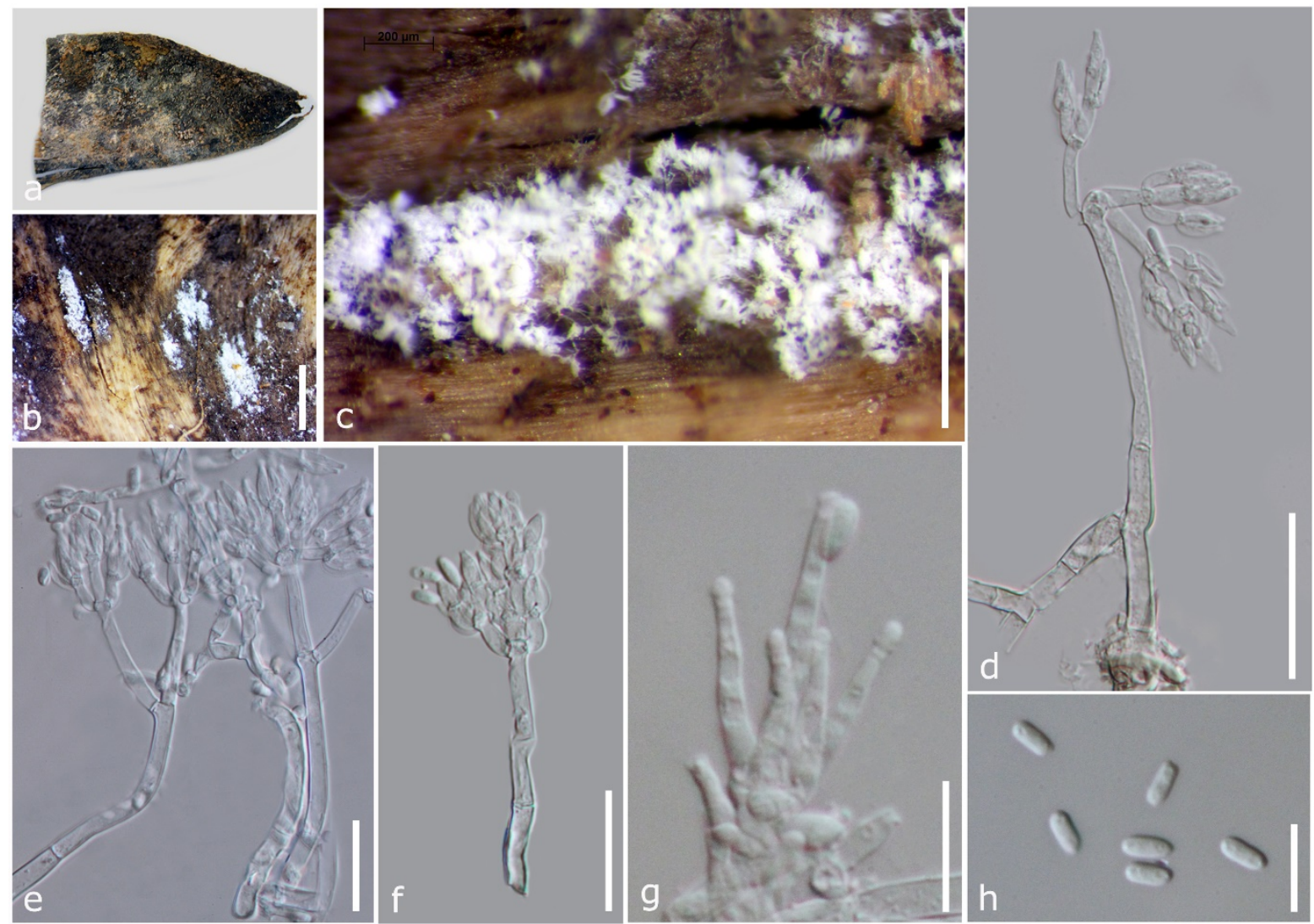

Figure 18 - Clonostachys rogersoniana (MFLU 19-0987). a Herbarium material. b, c Colonies on host. d-f Conidiophores with phialides. g Phialides with conidia. h Conidia. Scale bars: $\mathrm{b}=2 \mathrm{~mm}$, c $=1 \mathrm{~mm}, \mathrm{~d}-\mathrm{f}=20 \mu \mathrm{m}, \mathrm{g}, \mathrm{h}=10 \mu \mathrm{m}$. 
Facesoffungi number: FoF06012

Saprobic on decaying fruits of Swietenia mahagoni. Sexual morph: Stromata well developed, erumpent. Ascomata 170-215 × 160-218 $\mu \mathrm{m}(\bar{x}=185 \times 186 \mu \mathrm{m}, \mathrm{n}=7)$, perithecial, densely crowded in groups, globose to subglobose to oval, not collapsing or sometimes slightly laterally pinched when dry, light orange to yellowish orange, $\mathrm{KOH}-$, not papillate, surface smooth to slightly roughened, but not distinctly warted. Ostiole periphysate. Peridium 21-46 $\mu \mathrm{m}$ wide, wider around the ostiole, two strata: outer layer 21-30 $\mu \mathrm{m}$ wide, comprising unevenly thickened brownish yellow cells of textura angularis to textura globosa, becoming larger towards the periphery, most cells containing a vacuole; inner layer $8-14 \mu \mathrm{m}$ wide, consists of flat, hyaline cells of textura angularis. Asci 50.6-62.2 × 6-7.2 $\mu \mathrm{m}(\bar{x}=55.4 \times 6.6 \mu \mathrm{m}, \mathrm{n}=18), 8$-spored, unitunicate, narrowly clavate, apex flat with somewhat rounded edges, short stalked, apical ring J-. Ascospores 8.9-11.9 × 2.9$3.4 \mu \mathrm{m}(\bar{x}=10.7 \times 3.1 \mu \mathrm{m}, \mathrm{n}=25)$, bi-seriate above and uni-seriate below, 1 -septate, equilateral, not constricted at the septum or slightly constricted in mature ascospores, ellipsoidal to oblong ellipsoidal, 4-guttulate, hyaline, spinulose. Asexual morph: See Schroers (2001).

Culture characteristics - Ascospores germinating on PDA within 12 hours. Colonies growing on PDA, reaching up to $4.5 \mathrm{~cm}$ in 7 days at $25^{\circ} \mathrm{C}$, surface effused, smooth, margin entire to undulate, aerial mycelium rare, initially white, becoming yellowish, reverse yellowish.

Material examined - THAILAND, Chiang Rai Province, Mae Fah Luang University Garden, on decaying fruits of Swietenia mahagoni (L.) Jacq. (Meliaceae), 06 September 2017, R.H. Perera, Maho 04 (MFLU 18-2770), living culture MFLUCC 17-2618.

GenBank numbers - ITS: MT215574, LSU: MT396165, TEF: MT415234.

Notes - Our new isolate (MFLUCC 17-2618) groups with Clonostachys rosea isolates of CBS 194.57, CBS 193.94 and CBS 406.95. (Fig. 17). A comparison of nucleotides shows ITS loci of our isolate identical to C. rosea (CBS 194.57, CBS 193.94 and CBS 406.95). Our collection morphologically resembles $C$. rosea in ascomatal colour, size, asci and ascospore dimensions (Rossman et al. 1999, Schroers et al. 1999). This collection represents a new host record for $C$. rosea.

Clonostachys swieteniae R.H. Perera, E.B.G. Jones \& K.D. Hyde, sp. nov.

Fig. 20

Index Fungorum number: IF556856; Facesoffungi number: FoF07756

Etymology - Named after the host genus Swietenia.

Holotype - MFLU 18-2769

Saprobic on decaying fruits of Swietenia mahagoni. Sexual morph: Undetermined. Asexual morph: Hyphomycetous, colonies appearing as white patches on the host. Conidiophores arising from stroma, mononematous, monomorphic, hyaline, smooth-walled, penicillate, stipes 130-200 $\mu \mathrm{m}$ long, 5-8 $\mu \mathrm{m}$ wide at base. Penicilli 28-66 $\mu \mathrm{m}$ high, $\times 20-60 \mu \mathrm{m}$ diam. at widest point; solitary to gregarious, not sporodochial, bi- or quater-verticillate; branches of the penicillus divergent, each branch terminating in metulae and adpressed phialides. Phialides 9-14.1(-17) $\times$ 2.1-3.2 $\mu \mathrm{m}(\bar{x}=11.4 \times 2.6 \mu \mathrm{m}, \mathrm{n}=40)$, in whorls of 2-6, narrowly flask-shaped, slightly tapering toward the apex, with visible periclinal thickening, collarettes inconspicuous, hyaline, smoothwalled. Intercalary phialides not observed. Conidial masses white. Conidia (4.5-)5.1-6.7 × 1.9-2.6 $\mu \mathrm{m}(\bar{x}=6 \times 2.2 \mu \mathrm{m}, \mathrm{n}=40)$, broadly ellipsoidal to oval, rarely minutely curved, ends broadly rounded, straight, bi-guttulate, hilum laterally displaced, almost median or invisible, hyaline, smooth-walled.

Culture characteristics - Conidia germinating on PDA within 12 hours. Colonies growing on PDA, reaching up to $3.8 \mathrm{~cm}$ within 7 days at $25^{\circ} \mathrm{C}$, surface slightly raised, smooth, margin entire to undulate, aerial mycelium dense, initially white, becoming yellowish, reverse yellow.

Material examined - THAILAND, Chiang Rai Province, Mae Fah Luang University Garden, on decaying fruits of Swietenia mahagoni (Meliaceae), 6 September 2017, R.H. Perera, Maho 02 (MFLU 18-2769, holotype), ex-type living culture MFLUCC 18-0572. 


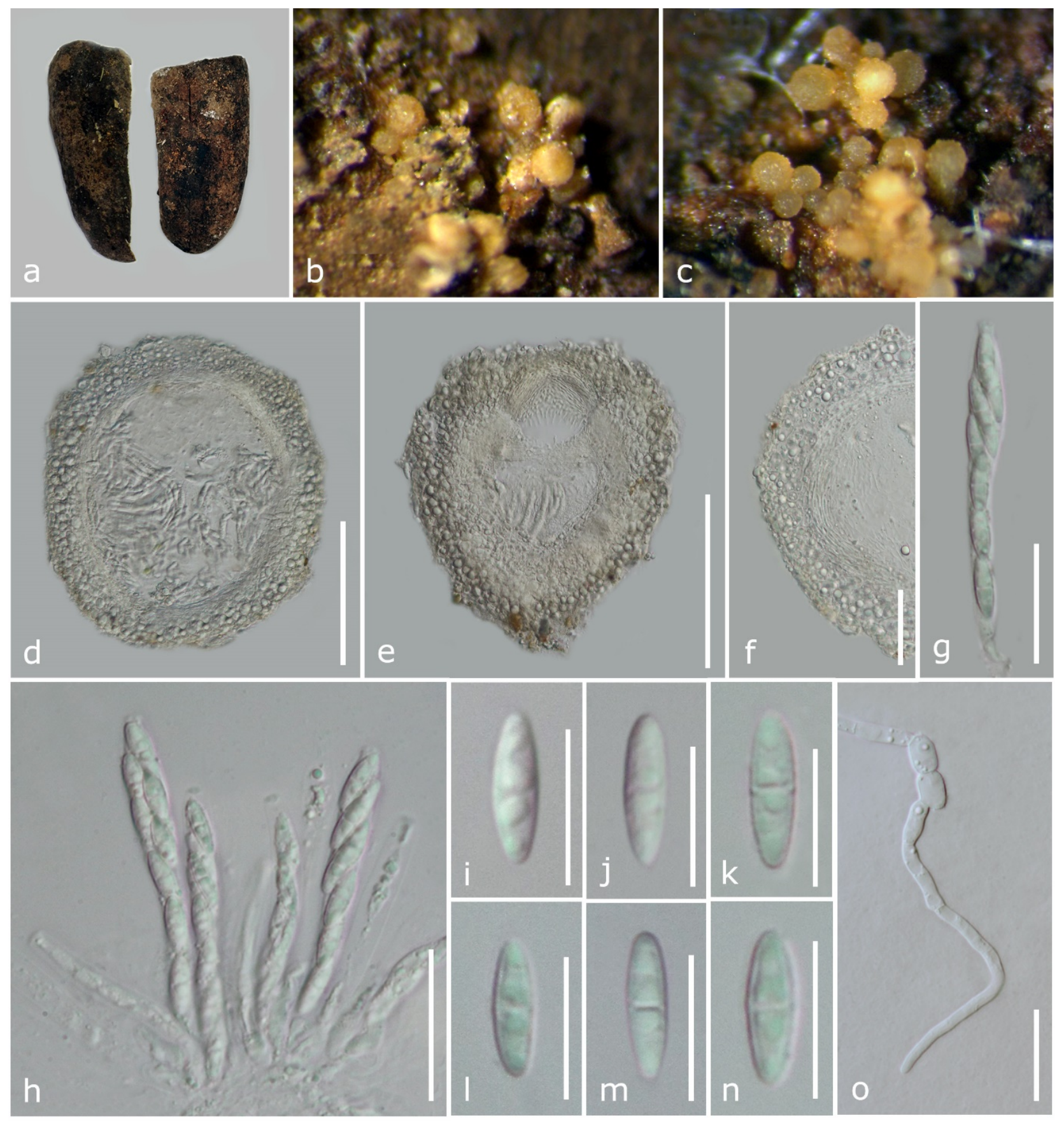

Figure 19 - Clonostachys rosea (MFLU 18-2770). a Herbarium material. b, c Ascomata on host. $\mathrm{d}$, e Section of ascomata. f Section of peridium. g, h Asci. i-n Ascospores. o Germinating ascospore. Scale bars: $\mathrm{d}$, $\mathrm{e}=100 \mu \mathrm{m}, \mathrm{f}=50 \mu \mathrm{m}, \mathrm{g}, \mathrm{h}=20 \mu \mathrm{m}, \mathrm{i}-\mathrm{n}=10 \mu \mathrm{m}, \mathrm{o}=20 \mu \mathrm{m}$.

GenBank numbers - ITS: MT215573, LSU: MT396164, TEF1: MT212204.

Notes - Clonostachys swieteniae constitutes a distinct lineage within Clonostachys (Fig. 17). A comparison of the ITS regions of C. swieteniae reveals 11 (2.1\%), 10 (2.1\%), 7 (1.5\%) and 8 (1.7\%) nucleotide differences with C. capitata, C. zelandiaenovae, C. agrawalii and C. krabiensis, respectively. Clonostachys swietenia can be discriminated from $C$. capitata by its longer and wider conidiophore stipe $(130-200 \times 5-8$ vs. $100 \times 3.5 \mu \mathrm{m})$ and straight, smaller conidia $(5.1-6.7 \times 1.9$ 2.6 vs. 6-7.2 $\times 2.8-3.4 \mu \mathrm{m}$ ), which are curved in C. capitata (Schroers 2001). Clonostachys swieteniae is different from C. krabiensis, which was also collected from Thailand, by the absence of setae and not forming sporodochial conidiophores (Tibpromma et al. 2018). We therefore identified Clonostachys swieteniae as a new taxon. 


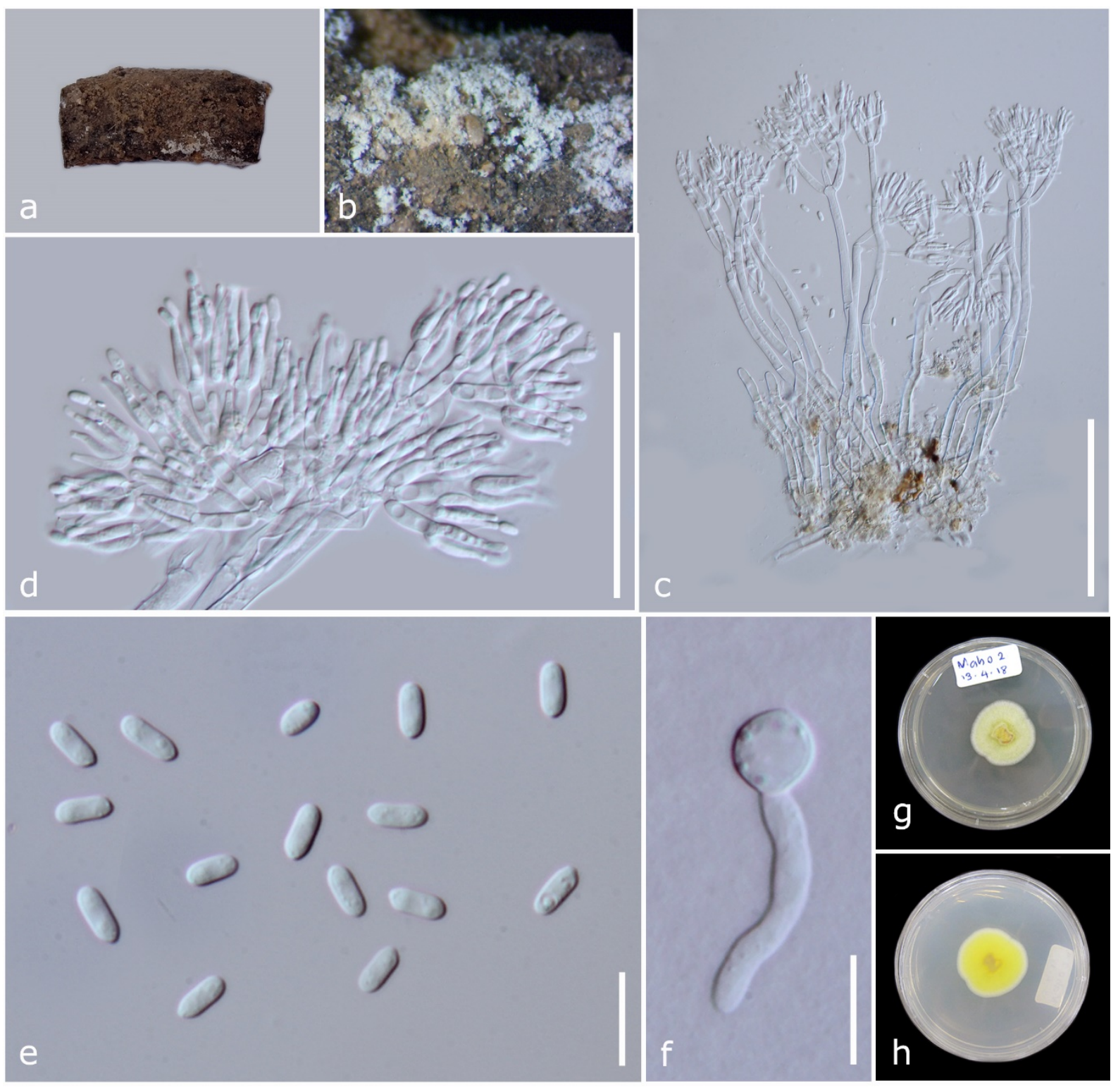

Figure 20 - Clonostachys swieteniae (MFLU 19-0987). a Herbarium material. b Colony on host. c Conidiophores. d Conidiogenous apparatus. e Conidia. f Germinating conidium. g, h Colony on PDA. Scale bars: $\mathrm{c}=100 \mu \mathrm{m}, \mathrm{d}=50 \mu \mathrm{m}$, e, $\mathrm{f}=10 \mu \mathrm{m}$.

\section{Myrotheciomycetaceae Crous Trichothecium Link}

Trichothecium roseum (Pers.) Link, Mag. Gesell. naturf. Freunde, Berlin 3(1-2): 18 (1809)

Facesoffungi number: FoF07768

Figs 22, 23

On dried pods of Delonix regia. Sexual morph: Undetermined. Asexual morph: Hyphomycetous. Conidiophores $215 \times 360 \mu \mathrm{m}$ long, 3.3-5 $\mu \mathrm{m}$ wide $(\bar{x}=270 \times 4 \mu \mathrm{m}, \mathrm{n}=20$ ), simple, long, slender, rarely branched, septate, often slightly swollen at the tip, hyaline, smoothwalled. Conidia 11.5-25.5 × 8-14.6 $\mu \mathrm{m}(\bar{x}=20 \times 11 \mu \mathrm{m}, \mathrm{n}=30)$, borne apically and singly, attached in groups or chains, pink in mass, 1-septate, ovate, hyaline, thick-walled, each with a decurved, abruptly narrowed basal hilum terminating in a distinct truncate end, often clustered. Conidiophores on MEA retrogressive, with each conidial base subsuming a portion of conidiophore apex. Conidia arise from the side of the conidiophore apex. Chlamydospores present, 7-8.8 $\mu \mathrm{m}$ wide $(\mathrm{n}=10)$, in intercalary chains, hyaline, smooth-walled. 
Culture characteristics - Conidia germinating on PDA within 12 hours. Colonies growing on PDA, reaching up to $4.5 \mathrm{~cm}$ within 7 days at $28^{\circ} \mathrm{C}$, surface effused, smooth, margin entire, aerial mycelium rare, initially white, turning salmon pink, reverse salmon pink. Colony on MEA reaching up to $3.3 \mathrm{~cm}$ in 7 days at $28^{\circ} \mathrm{C}$, sporulating within 30 days.

Materials examined - THAILAND, Chiang Rai Province, Mae Fah Luang University garden, on dried pods of Delonix regia (Fabaceae) on the ground, 1 December 2017, R.H. Perera, Delo 24 (MFLU 18-2773), living culture: MFLUCC 17-1036; ibid., on dried pods of Tamarindus indica L. (Fabaceae) on the ground, 6 December 2016, R.H. Perera, Tam 4 (MFLU 18-2735), sporulated dry culture: MFLU 18-0624, living culture: MFLUCC 17-0141.

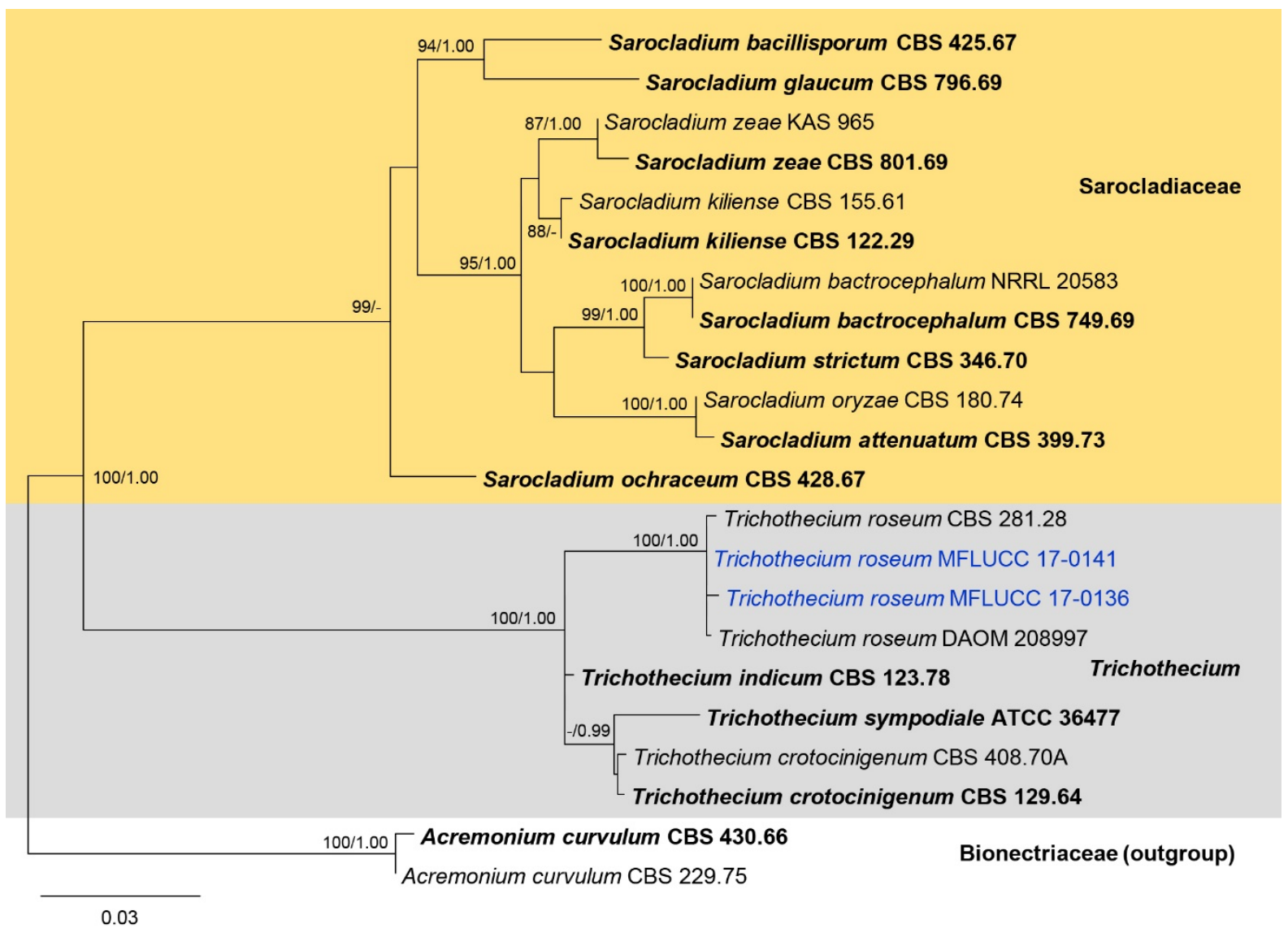

Figure 21 - Phylogram generated from RAxML analysis based on combined LSU and ITS, sequence data of Trichothecium isolates. Sequences from twenty-two taxa, which comprise 1390 characters including gaps, are included in the analyses. The tree was rooted to Acremonium curvulum CBS 430.66 and Acremonium curvulum CBS 229.75. The scale bar indicates 0.03 nucleotide changes per site.

GenBank numbers - ITS: MT215509, LSU: MT215558 (MFLUCC 17-1036); ITS: MT215510, LSU: MT215559 (MFLUCC 17-0141).

Notes - In the phylogenetic analysis our two isolates grouped within Trichothecium roseum isolates CBS 281.28 and DAOM 208997 (Fig. 21). Based on the phylogenetic analysis and morphological similarities the fungus was identified as Trichothecium roseum (Petrak 1953, Bello 2008), and is a new host record.

Nectriaceae Tul. \& C. Tul.

Fusarium Link

Fusarium cassiae R.H. Perera, E.B.G. Jones \& K.D. Hyde, sp. nov.

Fig. 25 
Index Fungorum number: IF556857; Facesoffungi number: FoF07757

Etymology - Name reflects the host genus Cassia fistula.

Holotype - MFLU 18-2751

Saprobic on decaying pods of Cassia fistula. Sexual morph: Ascomata 110-180 $\mu \mathrm{m}$ high $\times$ 110-155 $\mu \mathrm{m}$ diam. $(\bar{x}=132 \times 128 \mu \mathrm{m})$, superficial, solitary to gregarious, blue-black to dark purple, subglobose to ampulliform, or obpyriform, collapsing laterally when dry, with a slightly papillate ostiole. Peridium 16-30 $\mu \mathrm{m}$ wide, comprising dark purple to hyaline, thick-walled cells of textura angularis. Catenophyses absent. Asci 59-83 ×9-16 $\mu \mathrm{m}(\bar{x}=70.2 \times 11.8 \mu \mathrm{m}, \mathrm{n}=20), 4-8-$ spored, unitunicate, clavate, with a short pedicel, truncate at the apex, apical ring J-. Ascospores 10.3-21.2 × 5.6-8.8 $\mu \mathrm{m}(\bar{x}=15.9 \times 7 \mu \mathrm{m}, \mathrm{n}=37), 1-2$-seriate, varied in shape, ellipsoidal to oblong, or subglobose to obovoid, or fusiform, with both ends rounded, (0-)1-septate, not or slightly constricted at the septum, hyaline, pale brown when mature, guttulate, smooth-walled. Asexual morph: Undetermined.

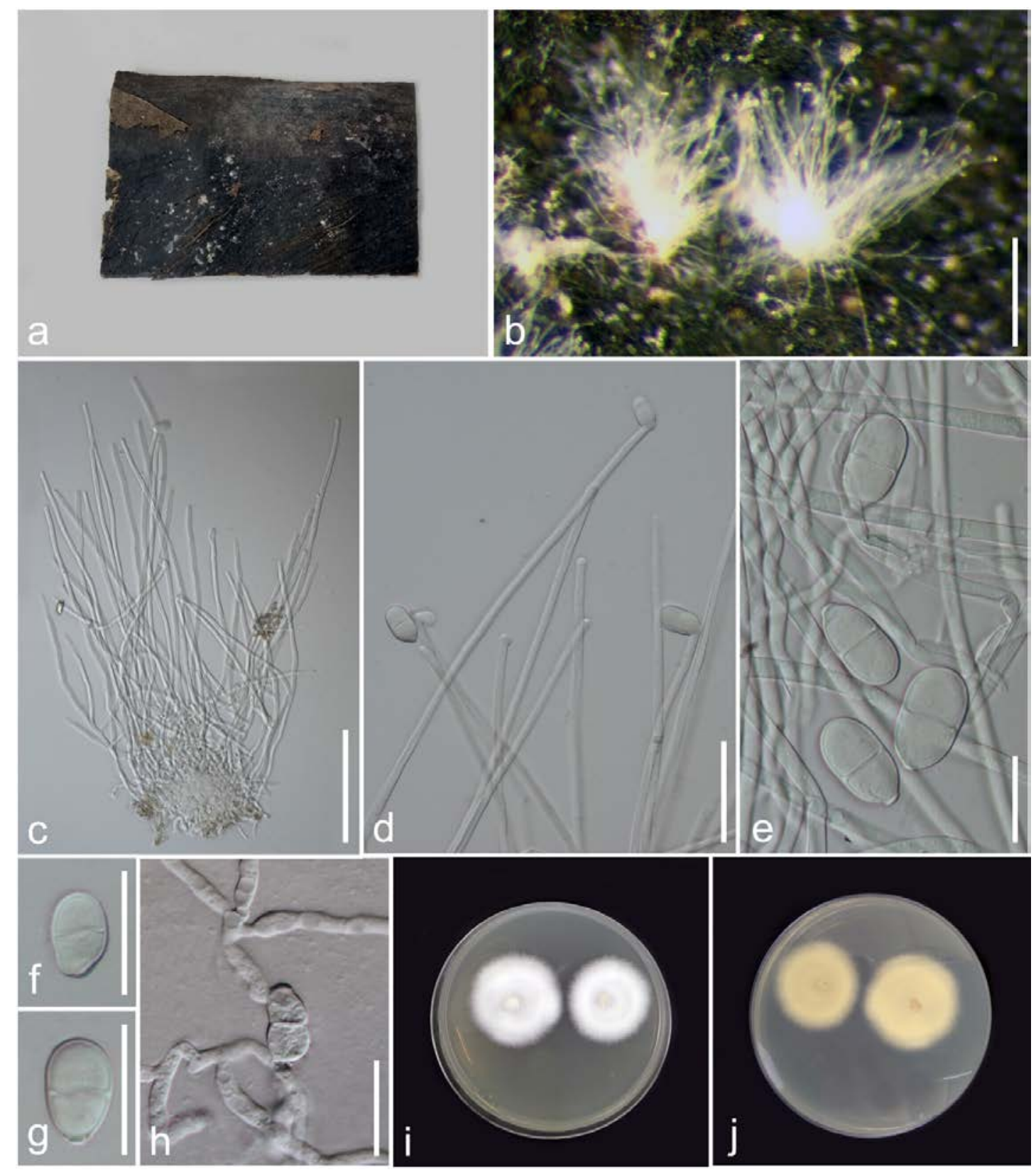

Figure 22 - Trichothecium roseum (MFLU 18-2773). a Herbarium material. b Conidiomata on host. c, d Conidiophores with conidia. e-g Conidia. h Germinating conidium. i, j Colony on PDA. Scale bars: $\mathrm{b}=200 \mu \mathrm{m}, \mathrm{c}=100 \mu \mathrm{m}, \mathrm{d}=50 \mu \mathrm{m}, \mathrm{e}-\mathrm{h}=20 \mu \mathrm{m}$. 


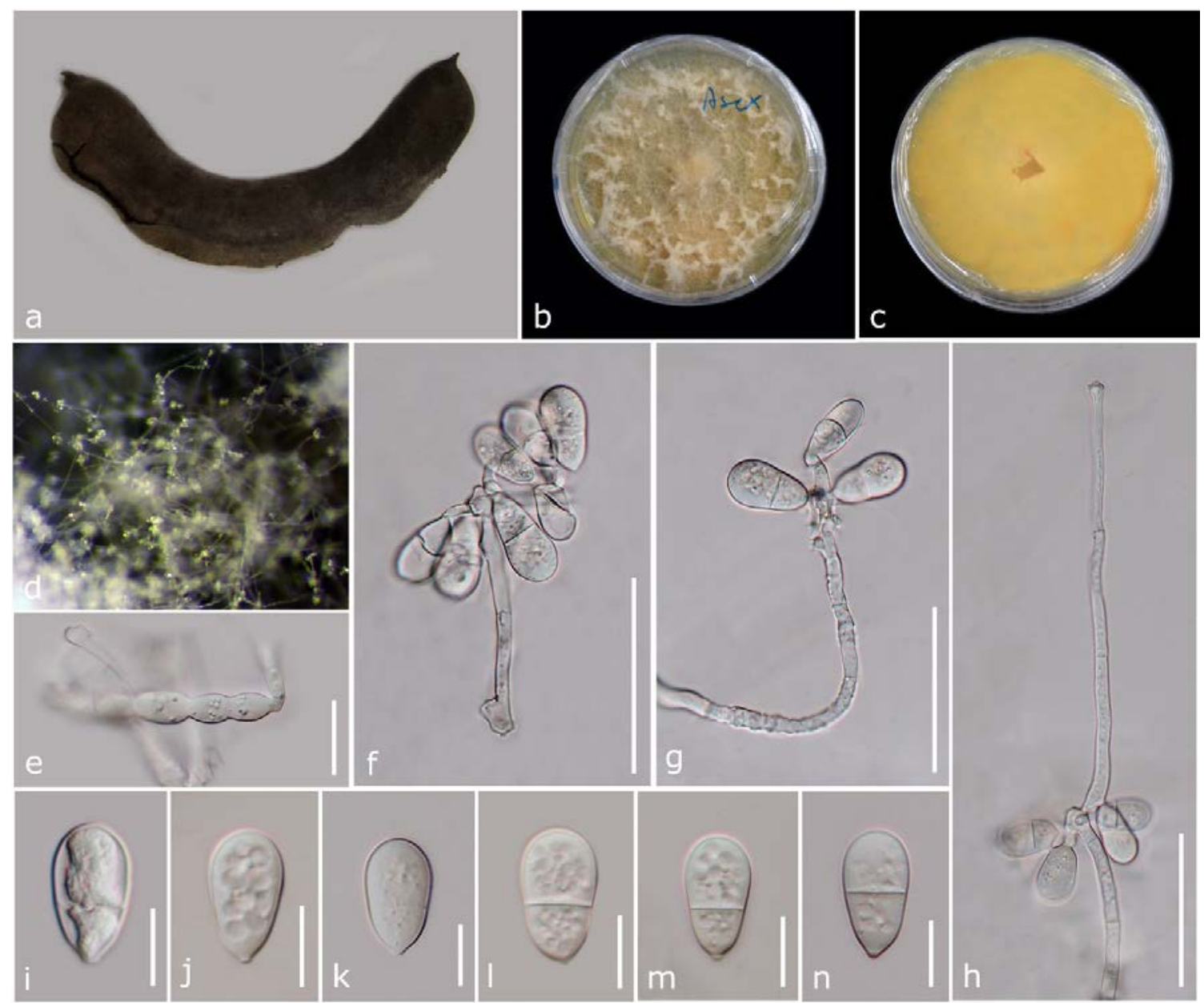

Figure 23 - Trichothecium roseum (MFLU 18-0624). a Herbarium material. b, c Sprorulating colony on MEA. d Conidiomata on MEA. e Chlamydospores. $\mathrm{f}-\mathrm{h}$ Conidiophores with conidia. $\mathrm{i}-\mathrm{n}$ Conidia. Scale bars: $\mathrm{e}-\mathrm{h}=20 \mu \mathrm{m}, \mathrm{i}-\mathrm{n}=10 \mu \mathrm{m}$.

Culture characteristics - Colonies on PDA reach 3-4.5 cm diameter within 7 days at $25^{\circ} \mathrm{C}$, circular, flat, smooth, edges crenate, zonate, whitish above, reddish at the center, reverse white.

Material examined - THAILAND, Phayao Province, decaying pods of Cassia fistula (Fabaceae) on the ground, R.H. Perera, 4 August 2017, PH-CAS 05 (MFLU 18-2751, holotype), ex-type living culture MFLUCC 18-0573.

GenBank numbers - ITS: MT215495, LSU: MT215546, RPB2: MT212197, TEF1: MT212205.

Notes - In the phylogenetic tree, Fusarium cassiae is closely related to F. stilboides (NRRL 20429) with low support (70\% ML, 0.99 BIPP; Fig. 24). A comparison of RPB2 gene region of new fungus and F. stilboides (NRRL 20429) indicates 53 (5.9\%) different nucleotides. F. cassiae also can be discriminated from $F$. stilboides in having variously shaped, (0-)1-septate ascospores vs. elliptical, 1-3 septate ascospores in F. stilboides (Booth 1971). The NCBI megablast search of ITS and TEF1 sequences show 99\% similarity (476/477) with F. lateritium (CF6), and 97\% similarity (659/682) with F. lateritium (YT2-7), respectively. However, the highest similarity $(93 \%, 801 / 858)$ obtained in the megablast search of RPB2 sequences is with $F$. lateritium strain (NRRL 13622). We therefore recognize F. cassiae as a novel species.

Fusarium magnoliae-champaca R.H. Perera, E.B.G. Jones \& K.D. Hyde, sp. nov.

Fig. 26

Index Fungorum number: IF556920; Facesoffungi number: FoF07758

Etymology - Name reflects the host plant Magnolia champaca.

Holotype - MFLU 18-2736 
Saprobic on dried fruits of Magnolia champaca. Sexual morph: Ascomata 75-215 $\mu \mathrm{m}$ high $\times$ 70-210 $\mu \mathrm{m}$ diam. $(\bar{x}=147 \times 143 \mu \mathrm{m}, \mathrm{n}=10)$, superficial, solitary to gregarious, blue-black to dark purple, subglobose to ampulliform, or obpyriform, collapsing laterally when dry. Ostioles periphysate. Peridium 30-40 $\mu \mathrm{m}$ wide, composed of two layers, inner 3-5 layers, comprising hyaline cells of textura angularis, outer 3-5 layers, comprising brown to dark purple, thick-walled cells of textura angularis. Hamathecium comprising hyaline, distinctly septate catenophyses, each cell 12.7-17.5 $\mu \mathrm{m}$ long $\times 14.2-22.5 \mu \mathrm{m}$ wide. Asci (70-)90-100(-109) $\times(7-) 9-13(-16) \mu \mathrm{m}(\bar{x}=$ $96.5 \times 11 \mu \mathrm{m}, \mathrm{n}=40)$, 8-spored, unitunicate, clavate, with short pedicel, slightly rounded to truncate at the apex. Ascospores (8-)16-20(-25) $\times(4.5-) 6-7.5(-9) \mu \mathrm{m}(\bar{x}=18 \times 6.6 \mu \mathrm{m}, \mathrm{n}=165)$, 1-2-seriate, pale brown, varied in shape, ellipsoidal to oblong, or subglobose to obovoid, or fusiform, with both ends rounded, (0-)1-septate, not constricted at the septum, smooth-walled. Asexual morph: Undetermined.

Material examined - THAILAND, Chiang Rai Province, Mae Fah Luang University garden, on ground, dried fruits of Magnolia champaca (L.) Baill. ex Pierre, (Magnoliaceae), 7 August 2017, S. Boonmee, Fruit 1 (MFLU 18-2736, holotype), ex-type living culture MFLUCC 18-0580.

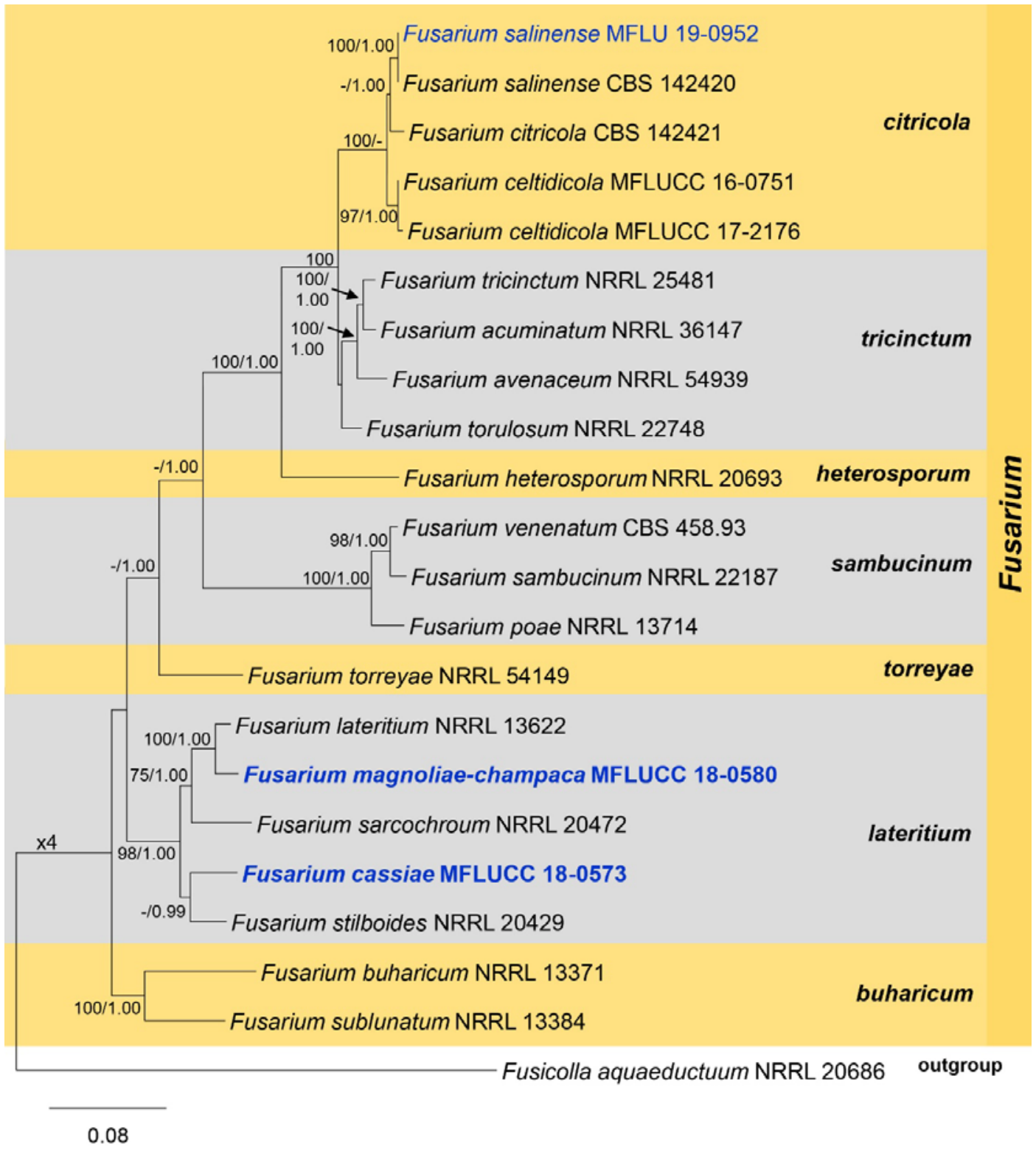

Figure 24 - Phylogram generated from RAxML analysis based on combined RPB2 and RPB1 sequence data of selected Fusarium isolates. Sequences from twenty-two taxa, which comprise 
3349 characters including gaps, are included in the analyses. The tree was rooted to Fusicolla aquaeductuum (NRRL 20696). The scale bar indicates 0.08 nucleotide changes per site.

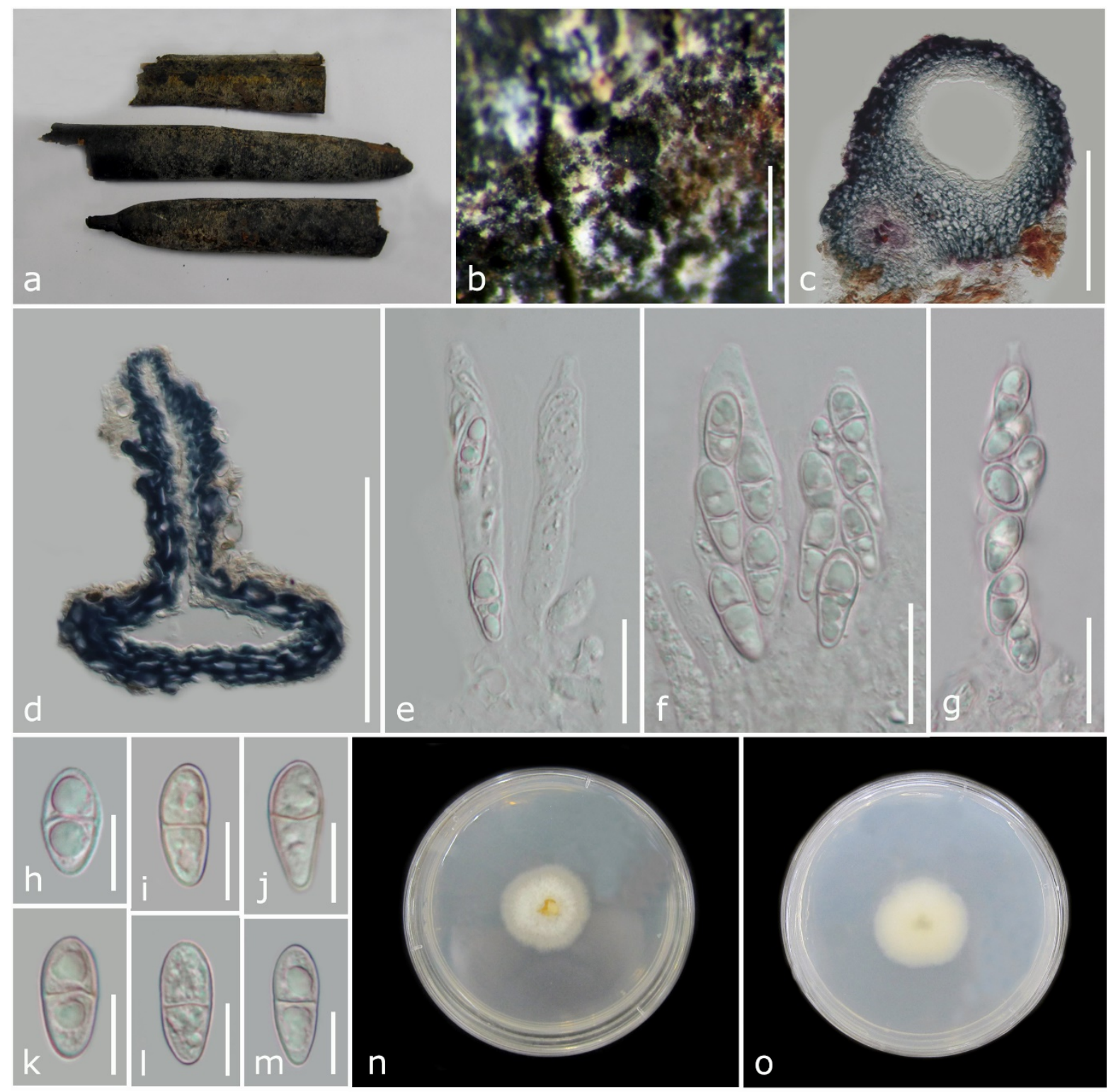

Figure 25 - Fusarium cassiae (MFLU 18-2751, holotype). a Herbarium material. b Ascomata on host. c, d Section of ascoma (d laterally collapsed). e-g Asci. h-m Ascospores. n, o Colony on PDA. Scale bars: $\mathrm{b}=500 \mu \mathrm{m}, \mathrm{c}=100 \mu \mathrm{m}, \mathrm{e}-\mathrm{g}=20 \mu \mathrm{m}, \mathrm{h}-\mathrm{m}=10 \mu \mathrm{m}$.

GenBank numbers - ITS: MT215496, LSU: MT215547, RPB2: MT212198.

Culture characteristics - Ascospores germinated on PDA within 12 hours. Colonies on PDA reaching $15-17 \mathrm{~mm}$ diameter after 7 days at $28^{\circ} \mathrm{C}$, colonies circular, medium dense, flat or effuse, with entire margin, white, becoming yellowish with time, reverse pale yellow.

Notes - In the phylogenetic analysis Fusarium magnoliae-champaca (MFLUCC 18-0580) clusters with $F$. lateritium strain (NRRL 13622) with high statistical support (100\% MLBT, 1.00 BIPP; Fig. 24). A comparison of RPB2 gene region of the new fungus and F. lateritium (NRRL 13622) indicates 27 (3.1\%) different nucleotides. Fusarium magnoliae-champaca also can be delineated from $F$. lateritium in having (0-)1 septate ascospores vs. 1-3 septate ascospores and larger asci $90-100 \times 9-13$ vs. $65-80 \times 8-11 \mu \mathrm{m}$ (Booth 1971). 

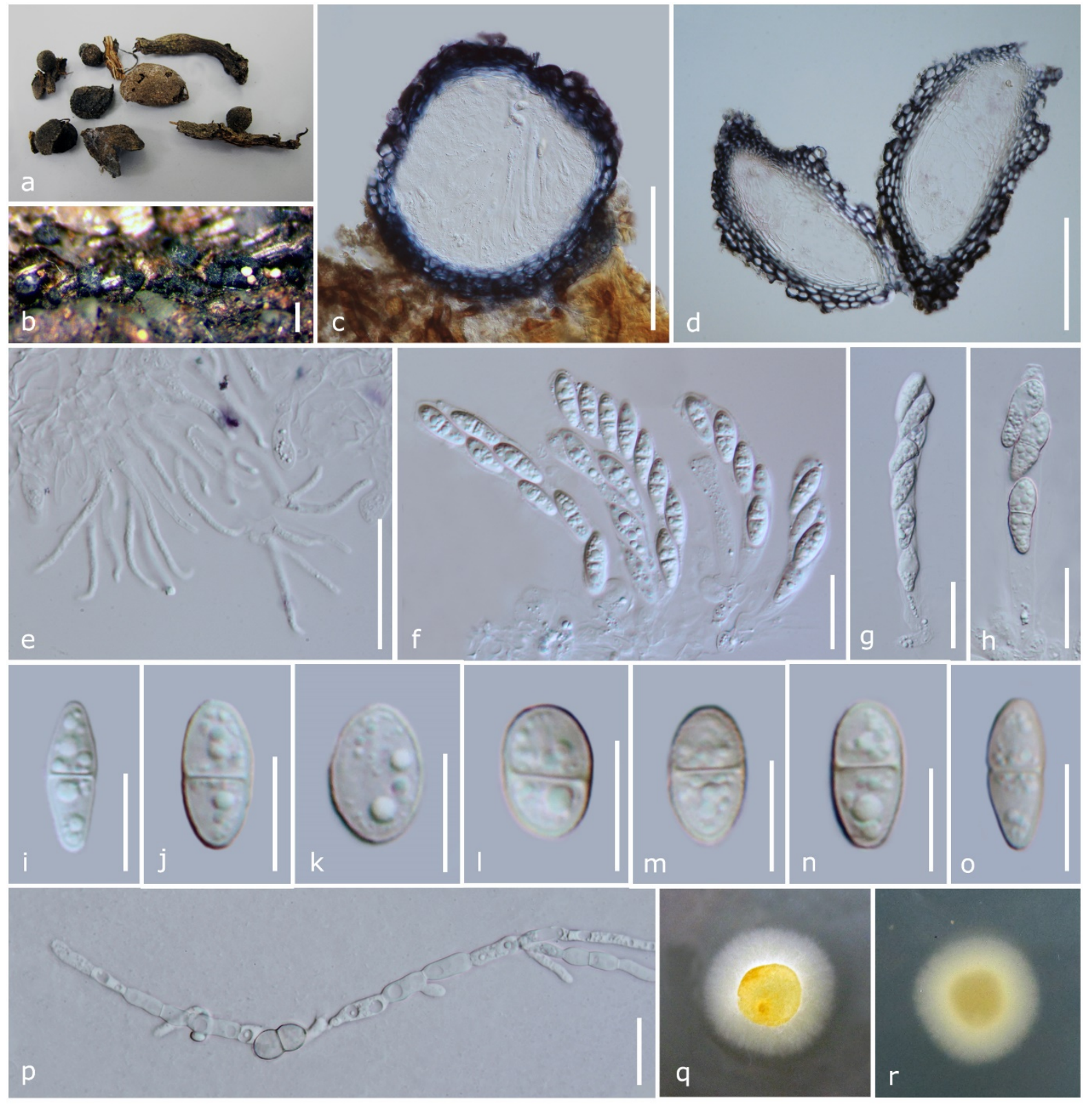

Figure 26 - Fusarium magnoliae-champaca (MFLU 18-2736, holotype). a Herbarium material. b Ascomata on host. c, d Section of ascomata. e Periphyses. f-h Asci. i-o Ascospores. p Germinating ascospore. q, r Colony on PDA. Scale bars: $b=200 \mu \mathrm{m}, \mathrm{c}, \mathrm{d}=100 \mu \mathrm{m}, \mathrm{e}-\mathrm{h}=20$ $\mu \mathrm{m}, \mathrm{i}-\mathrm{o}=10 \mu \mathrm{m}, \mathrm{p}=20 \mu \mathrm{m}$.

Fusarium salinense Sand.-Den., Guarnaccia \& Polizzi, in Sandoval-Denis, Guarnaccia, Polizzi \& Crous, Persoonia 40: 15 (2017)

Fig. 27

Facesoffungi number: FoF07759

On dead samaras of Fraxinus angustifolia. Sexual morph: Undetermined. Asexual morph: Colonies on the host appearing as pale orange coloured masses. Conidiomata sporodochial, pale orange coloured. Conidiophores 33-48 $\times 5-6.6 \mu \mathrm{m}$, densely and irregularly branched, bi- or triverticillately, bearing 1-2 terminal phialides, hyaline, smooth-walled. Phialides 7.3-12.1 × 2.9-3.6 $\mu \mathrm{m}$, subulate to subcylindrical, with a minute apical collarette, hyaline, smooth- and thin-walled. Conidia 21-42 × 3.4-4.5 $\mu \mathrm{m}(\bar{x}=40 \times 3.7 \mu \mathrm{m}, \mathrm{n}=20),(1-) 3-4$-septate, falcate, slender, curved, curvature more prominent in the upper part of the conidium, slightly tapering towards the basal end, with a foot-like basal cell, hyaline, thin- and smooth-walled. 
Material examined - ITALY, Forlì-Cesena Province, Collina - Forlì, dead aerial samaras of Fraxinus angustifolia Vahl (Oleaceae), Erio Camporesi, 22 January 2015, IT 2345 (MFLU 190952).

GenBank numbers - LSU: MT215548, RPB2: MT212199, TUB2: MT212211.

Notes - Since single spore isolation was unsuccessful, DNA was isolated directly from the fruiting bodies. In the phylogenetic analysis the new isolate (MFLU 19-0952) clusters with the extype strain of Fusarium salinense (CBS 142420) with high statistical support (100\% MLBT, 1.00 BIPP; Fig. 24). The morphology of the new collection is similar to the holotype of $F$. salinense (Sandoval-Denis et al. 2017). However, microconidia, obtained in the culture in holotype of $F$. salinense, were not observed in our collection. The DNA sequences of new strain (MFLU 19-0952) and F. salinense are similar. Hence, we identify our collection as Fusarium salinense with a new geographical record and new host for the fungus.

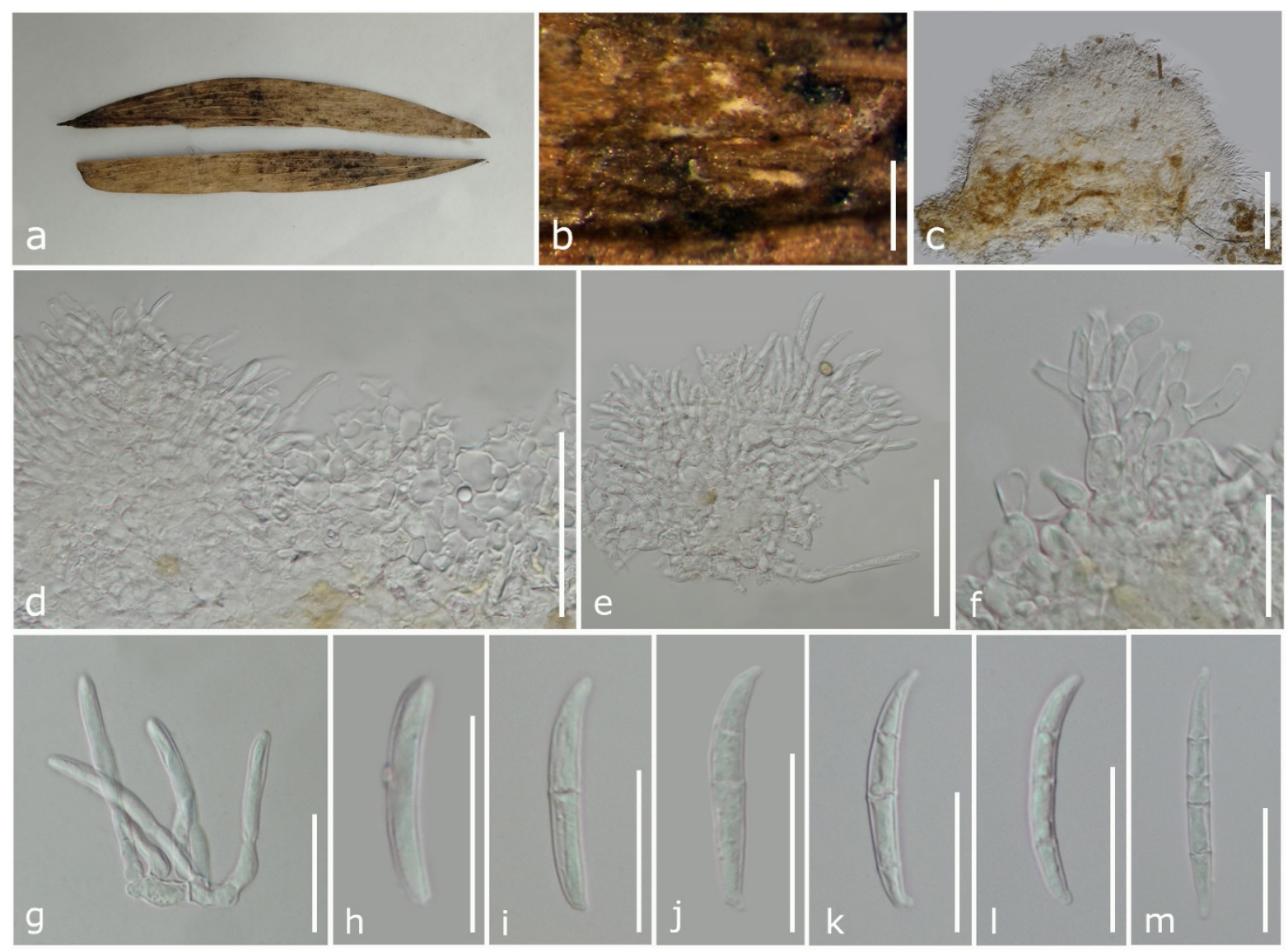

Figure 27 - Fusarium salinense (MFLU 19-0952). a Herbarium material. b Conidiomata on the host. c Conidioma mounted in water. d-g Conidiophores with conidia. h-m Conidia. Scale bars: b, $c=100 \mu \mathrm{m}, \mathrm{d}, \mathrm{e}=50 \mu \mathrm{m}, \mathrm{f}-\mathrm{m}=20 \mu \mathrm{m}$.

\section{Fusarium sp.}

Fig. 28

Saprobic on decaying seed pods of Delonix regia. Sexual morph: Ascomata 75-215 $\mu \mathrm{m}$ high $\times 70-210 \mu \mathrm{m}$ diam. $(\bar{x}=147 \times 143 \mu \mathrm{m}, \mathrm{n}=7)$, superficial, solitary to gregarious, blue-black to dark purple, subglobose to ampulliform, or obpyriform, collapsing laterally when dry. Ostioles slightly papillate, periphysate. Peridium 30-40 $\mu$ m wide, composed of two layers, inner 3-5 layers, comprising hyaline cells of textura angularis, outer 3-5 layers, comprising brown to dark purple, thick-walled cells of textura angularis. Hamathecium comprising hyaline, distinctly septate catenophyses, each cell 12.7-17.5 $\mu \mathrm{m}$ long $\times 14.2-22.5 \mu \mathrm{m}$ wide. Asci (70-)90-100(-109) $\times$ (7-)9-13(-16) $\mu \mathrm{m}(\bar{x}=89 \times 11 \mu \mathrm{m}, \mathrm{n}=40), 6-8$-spored, unitunicate, clavate, with a short pedicel, truncate at the apex, apical ring J-. Ascospores (8-)16-20(-25) $\times(4.5-) 6-7.5(-9) \mu \mathrm{m}(\bar{x}=18 \times$ 
$6.6 \mu \mathrm{m}, \mathrm{n}=165$ ), overlapping 1-2-seriate, varied in shape, ellipsoidal to oblong, or subglobose to obovoid, or fusiform, with both ends rounded, 1-septate, slightly constricted at the septum, hyaline, smooth-walled. Asexual morph: Undetermined.

Material examined - THAILAND, Chiang Mai Province, on decaying seed pods of Delonix regia (Fabaceae), 6 January 2015, R.H. Perera, M-9 (MFLU 16-1075).

GenBank numbers - LSU: MT396166, SSU: MT396180, TUB2: MT415235.

Notes - We were unable to obtain cultures of this fungus using single spore isolation. DNA was extracted from the fruiting bodies. Fungal morphology of this fungus resembles Fusarium. Only ITS and LSU sequences are available.
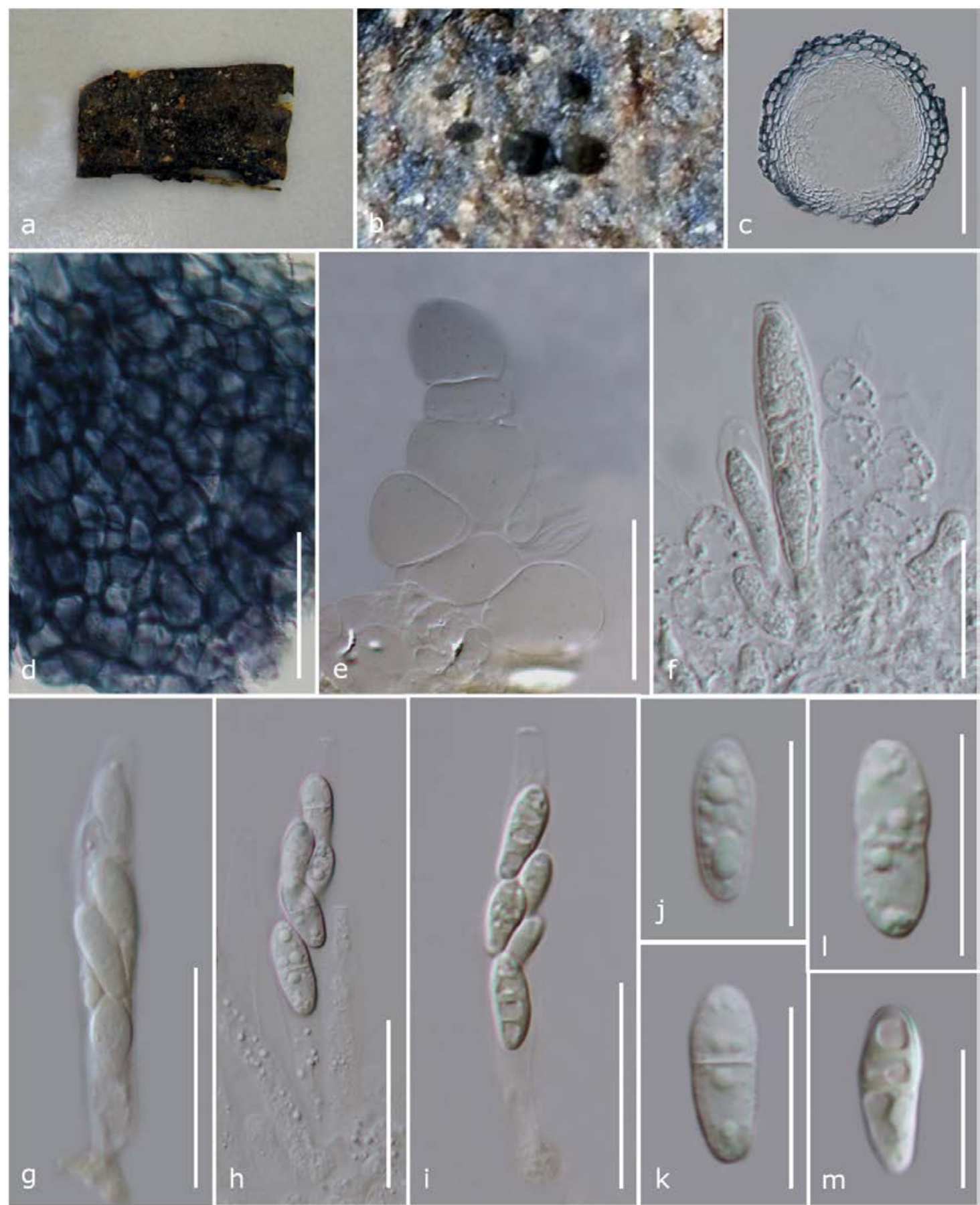

Figure 28 - Fusarium sp. (MFLU 16-1075). a Herbarium material. b Appearance of ascomata on host substrate. c Cross section through ascoma. d Peridium in face view. e, f Catenophyses. g-i Asci (i in Melzer's reagent). j-m Ascospores. Scale bars: $c=100 \mu \mathrm{m}, \mathrm{d}-\mathrm{i}=20 \mu \mathrm{m}, \mathrm{j}-\mathrm{m}=10$ $\mu \mathrm{m}$. 


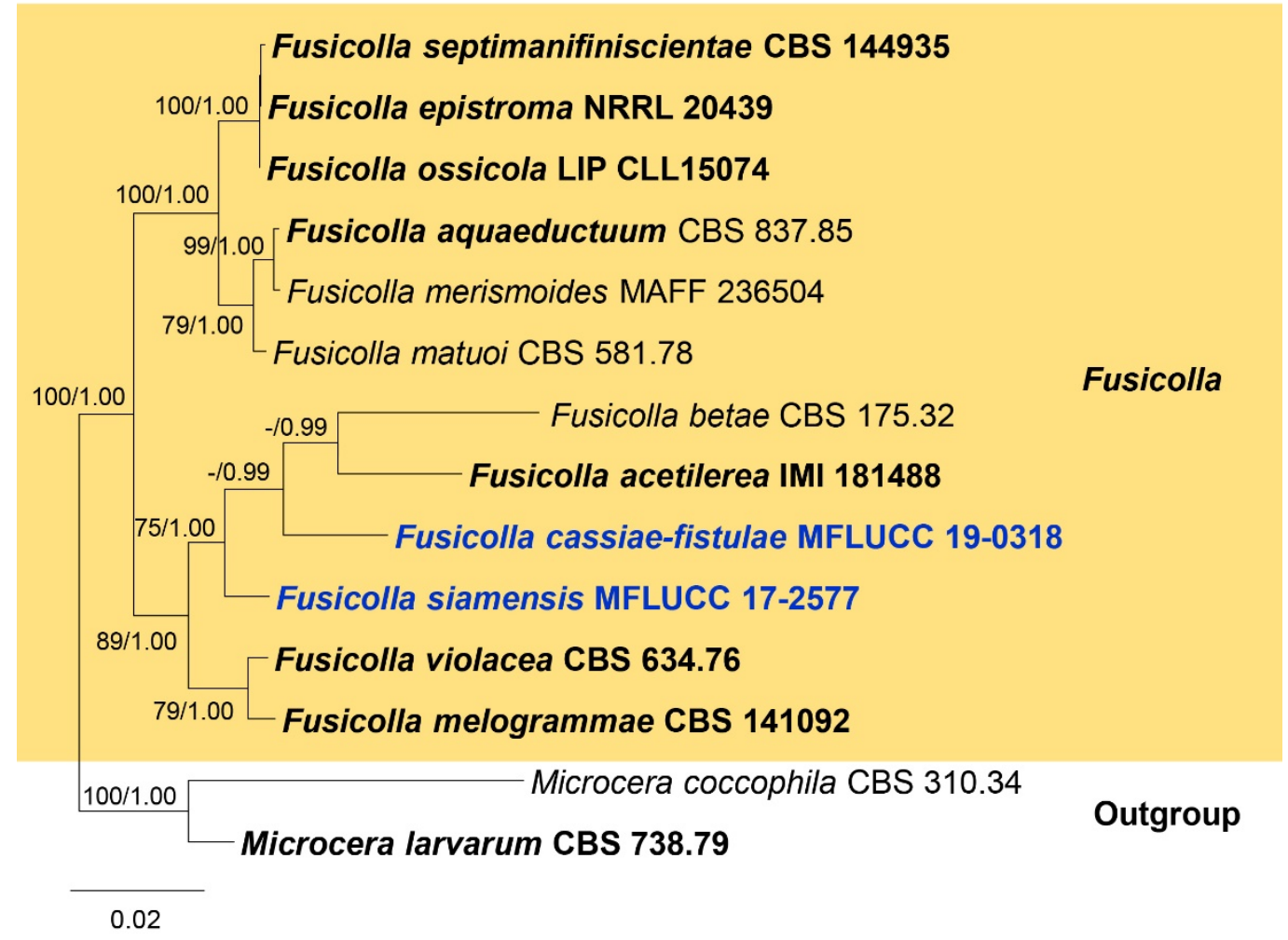

Figure 29 - Phylogram generated from RAxML analysis based on combined ITS and LSU sequence data of Fusicolla isolates. Sequences from fourteen taxa, which comprise 1337 characters including gaps, are included in the analyses. The tree was rooted to Microcera larvarum (CBS 738.79) and M. coccophila (CBS 310.34). The scale bar indicates 0.02 nucleotide changes per site.

Fusicolla cassiae-fistulae R.H. Perera, E.B.G. Jones \& K.D. Hyde, sp. nov.

Fig. 30

Index Fungorum number: IF556858; Facesoffungi number: FoF07760

Etymology - Name derived from the host plant Cassia fistula.

Holotype - MFLU 18-2753

Saprobic on decaying wood and seed pods of Cassia fistula. Sexual morph: Ascomata 150$180 \mu \mathrm{m}$ high, $130-182 \mu \mathrm{m}$ diam. $(\bar{x}=173 \times 157 \mu \mathrm{m}, \mathrm{n}=10)$, perithecial, stroma erumpent, scattered or in small groups of 3-8, globose to pyriform sometimes collapsing when dry, yellow, pale yellow to pale orange, not changing colour in $5 \% \mathrm{KOH}$, smooth walled. Ascomatal surface cells forming a textura angularis in face view, with a broadly conical, rounded ostiolar neck 40-55 $\mu \mathrm{m}$ high, $70-85 \mu \mathrm{m}$ diam. at base. Ostiole periphysate. Peridium 14-30 $\mu \mathrm{m}$ wide, two strata: outer region composed of 3-4 layers of hyaline to pale brown, thick-walled cells of textura angularis to textura globosa; inner region composed of 2-3 layers of hyaline cells of textura angularis. Asci 53$65 \times 5.8-8.2 \mu \mathrm{m}(\bar{x}=57 \times 6.7 \mu \mathrm{m}, \mathrm{n}=20)$, 8-spored, unitunicate, cylindrical to narrowly clavate, short pedicellate, apex flat, apical ring J-, lying without paraphyses. Ascospores 6.9-10.2 × 3.6-4 $\mu \mathrm{m}(\bar{x}=8.8 \times 3.8 \mu \mathrm{m}, \mathrm{n}=35)$, uniseriate, 1-septate, not constricted at the septum, ellipsoidal, rounded at ends, equilateral or inequilateral, hyaline to very pale brown, thick-walled, smooth and slightly verrucose when mature. Asexual morph: Undetermined.

Culture characteristics - Colonies on PDA at $25^{\circ} \mathrm{C}$ attaining $15-18 \mathrm{~mm}$ in 7 days. Aerial mycelium rare to absent, white to cream in centre, white at margin, margin entire, reverse pale luteous.

Material examined - THAILAND, Phayao Province, on decaying seed pods of Cassia fistula (Fabaceae), 4 August 2017, R.H. Perera, PH-CAS 08 (MFLU 18-2753, holotype), ex-type living culture MFLUCC 19-0318. 
GenBank numbers - ITS: MT215497, LSU: MT215549.

Notes - Based on phylogenetic inference of the LSU and ITS sequences Fusicolla cassiaefistulae forms an unsupported lineage within genus Fusicolla (Fig. 29). Fusicolla cassiae-fistulae resembles $F$. melogrammae and $F$. ossicola in having yellow, pale yellow to pale orange ascomata (Crous et al. 2016, Lechat \& Rossman 2017). However, F. cassiae-fistulae can be easily distinguished from F. melogrammae and F. ossicola by having smaller ascomata, asci and ascospores (Table 1). Fusicolla cassiae-fistulae and ex-type of F. melogrammae (CBS 141092) showed 15 (2.9\%, 2 gaps) different nucleotides in the ITS region and, 32 (3.8\%, 0 gaps) in LSU sequences. Furthermore, ITS region of $F$. cassiae-fistulae and ex-type of $F$. ossicola (LIP CLL15074) showed 24 (4.7\%, 1 gap) different nucleotides and 45 (5.3\%, 3 gaps) in LSU sequences. Hence, we introduce $F$. cassiae-fistulae as a novel species.

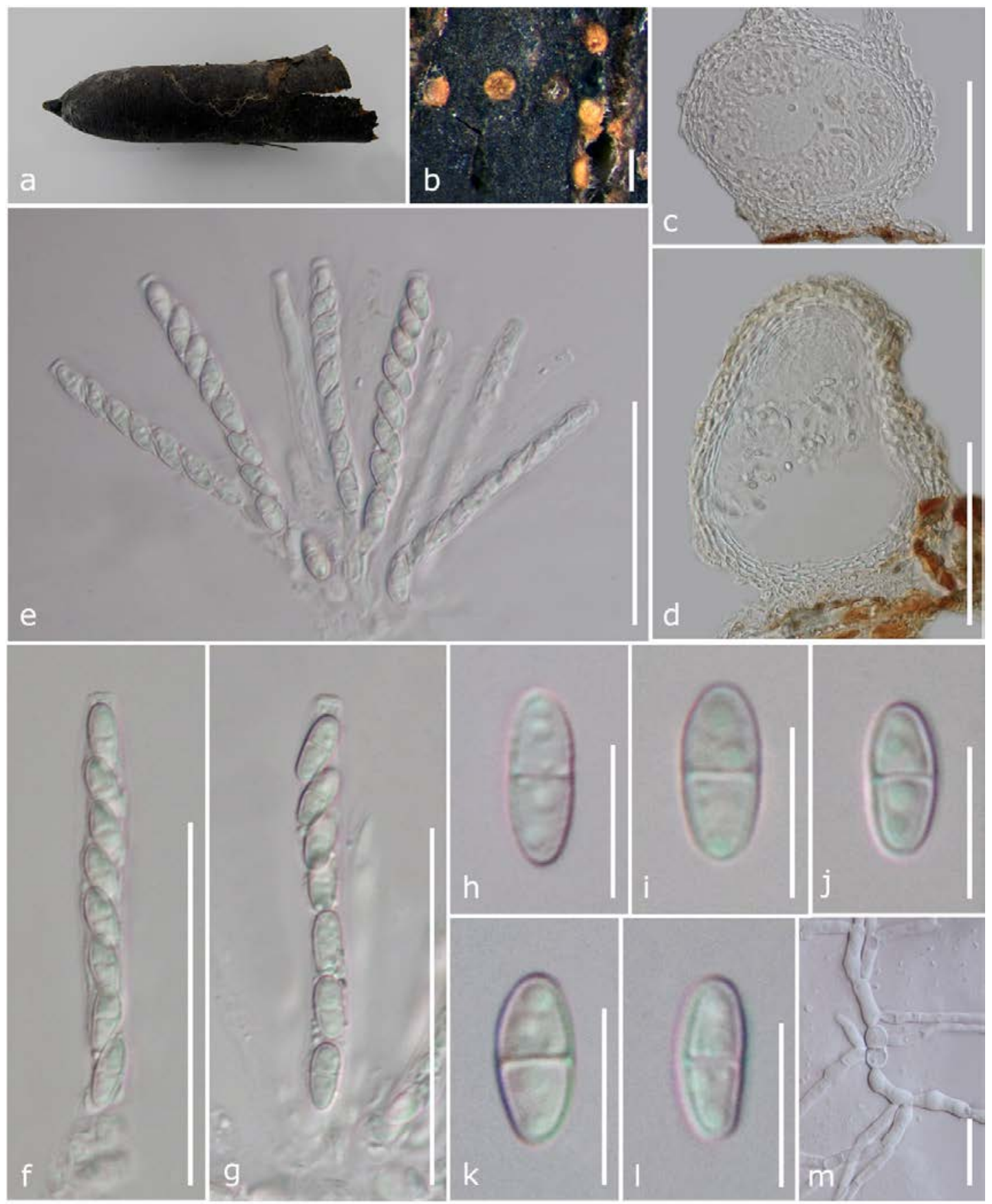

Figure 30 - Fusicolla cassiae-fistulae (MFLU 18-2753, holotype). a Herbarium material. b Ascomata on host. c, d Section of ascomata. e-g Asci. h-l Ascospores. m Germinating ascospore. Scale bars: $\mathrm{b}=200 \mu \mathrm{m}, \mathrm{c}, \mathrm{d}=100 \mu \mathrm{m}, \mathrm{e}-\mathrm{g}=50 \mu \mathrm{m}, \mathrm{h}-\mathrm{l}=10 \mu \mathrm{m}, \mathrm{m}=20 \mu \mathrm{m}$. 
Fusicolla siamensis R.H. Perera, E.B.G. Jones \& K.D. Hyde, sp. nov.

Index Fungorum number: IF556859; Facesoffungi number: FoF07761

Fig. 31

Etymology - Name refers to the country Thailand, where the specimen was collected.

Holotype - MFLU 19-1385

Associated with dried seed pods of Cassia fistula. Sexual morph: Undetermined. Asexual morph: Conidiomata sporodochial, appearing as flat wart-like, orange masses on the host surface. Conidiophores 27-46 $\mu \mathrm{m} \times 3.7-5.1 \mu \mathrm{m}(\mathrm{n}=10)$, monochasial, verticillate or penicillate, each branch terminating in one or two phialides, hyaline. Phialides 11.5-22.5 $\times 3.1-5.1 \mu \mathrm{m}(\bar{x}=14.8 \times$ $4.1 \mu \mathrm{m}, \mathrm{n}=10)$, monophialidic, cylindrical to subulate, hyaline, with periclinal thickening at the unflared tip. Microconidia absent. Macroconidia 50.5-70.5 $\times 5.1-8 \mu \mathrm{m}(\bar{x}=58.2 \times 6.8 \mu \mathrm{m}, \mathrm{n}=$ 25), 3-5(7)-septate, not constricted at the septa, sub-falcate, straight to curved, narrowing towards the ends, apical cell with a flat or sometimes rounded tip, basal cell slightly hooked with a more or less pointed end, hyaline, smooth-walled.

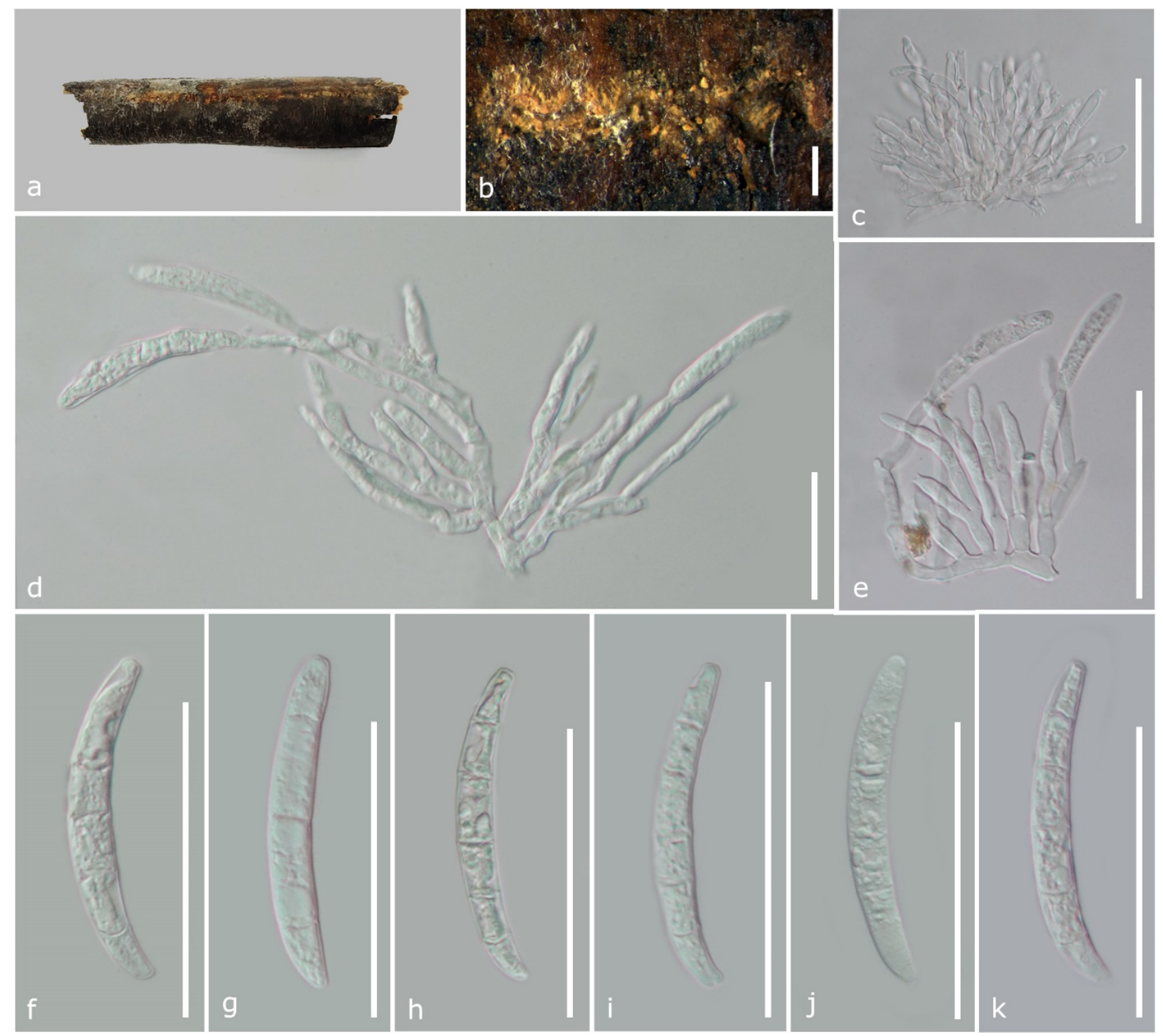

Figure 31 - Fusicolla siamensis (MFLU 19-1385, holotype). a Herbarium material. b Sporodochia on host. $\mathrm{c}-\mathrm{e}$ Conidiogenous apparatus. $\mathrm{f}-\mathrm{k}$ Conidia. Scale bars: $\mathrm{b}=500 \mu \mathrm{m}, \mathrm{c}=50 \mu \mathrm{m}, \mathrm{d}=20 \mu \mathrm{m}$, $\mathrm{e}-\mathrm{k}=50 \mu \mathrm{m}$.

Culture characteristics - Colonies on PDA at $25^{\circ} \mathrm{C}$ attaining $20-25 \mathrm{~mm}$ in 7 days. Aerial mycelium rare to absent, white to cream in centre, white at margin, reverse pale luteous, margin entire. 
Material examined - THAILAND, Phayao Province, on decaying seed pods of Cassia fistula (Fabaceae), 4 August 2017, R.H. Perera, PH-CAS 07 (MFLU 19-1385, holotype), ex-type living culture MFLUCC 17-2577.

GenBank numbers - ITS: MT215498, LSU: MT215550.

Notes - Based on phylogenetic inference of LSU and ITS sequence data Fusicolla siamensis is closely related to F. acetilerea (Tubaki, C. Booth \& T. Harada) Gräfenhan \& Seifert, F. betae Bonord. and F. cassiae-fistulae (Fig. 29). However, F. siamensis produced 3-5(7)-septate macroconidia, with basal cell slightly hooked $(50.3-70.4 \times 5.1-8 \mu \mathrm{m})$, which are larger than the 3(4) septate conidia of $F$. acetilerea (35-40 × 3-3.5 $\mu \mathrm{m}$; Tubaki et al. 1976). The conidia of $F$. betae are not falcate and 2-3-septate (Gräfenhan et al. 2011).

Table 1 Morphological comparison of related Fusicolla species.

\begin{tabular}{lllll}
\hline Species & $\begin{array}{l}\text { Ascomatal size } \\
(\boldsymbol{\mu m})\end{array}$ & Asci size $(\boldsymbol{\mu m})$ & $\begin{array}{l}\text { Ascospore size } \\
(\boldsymbol{\mu m})\end{array}$ & Reference \\
\hline $\begin{array}{l}\text { Fusicolla cassiae- } \\
\text { fistulae }\end{array}$ & $150-180 \times 130-182$ & $53-65 \times 5.8-8.2$ & $6.9-10.2 \times 3.6-4$ & This study \\
$\begin{array}{l}\text { F. melogrammae } \\
\text { F. ossicola }\end{array}$ & $250-290 \times 220-250$ & $70-80 \times 10-12$ & $12-14 \times 4.5-5$ & $\begin{array}{l}\text { Crous et al. (2016) } \\
\text { Lechat \& Rossman } \\
(2017)\end{array}$ \\
\hline
\end{tabular}

Gliocladiopsis S.B. Saksena

Gliocladiopsis aquaticus Y.Z. Lu, R.H. Perera \& K.D. Hyde, in Hyde et al., Mycosphere 9(2): 387 (2018)

Fig. 33

Saprobic on seed pods of Cassia fistula. Sexual morph: Undetermined. Asexual morph: appearing as white masses on the substrate, becoming yellowish with age. Conidiophores 90-120 $\times$ 5.1-6.5 $\mu \mathrm{m}$, penicillate, lacking stipe extensions and terminal vesicles, very pale brown. Conidiogenous apparatus with several series of hyaline to very pale brown branches; primary branches 22-27 × 3.1-5.6 $\mu \mathrm{m}$, aseptate or 1-septate; secondary branches 14.5-25 × 3-4.1 $\mu \mathrm{m}$, aseptate, hyaline; tertiary branches 9.1-15 $\times 2.2-3.4 \mu \mathrm{m}$, aseptate, hyaline. Phialides 7.5-13.1 $\times$ 1.6-2.6 $\mu \mathrm{m}(\bar{x}=11 \times 2.1 \mu \mathrm{m}, \mathrm{n}=30)$, doliiform to cymbiform to cylindrical, arranged in terminal whorls of 2-5 per branch, with periclinal thickening and minute collarettes, central phialide frequently extending above the rest. Subverticillate conidiophores absent. Conidia 13.4-20.6 × 1.7$2.6 \mu \mathrm{m}(\bar{x}=17.3 \times 2.2 \mu \mathrm{m}, \mathrm{n}=30 \mu \mathrm{m})$, cylindrical, hyaline, smooth with rounded ends, straight, $0-1$-septate, lacking a visible abscission scar.

Culture characteristics - Conidia germinating on PDA within 8 hours. Colonies growing on PDA reaching $35 \mathrm{~mm}$ within 2 weeks at $28^{\circ} \mathrm{C}$, circular, with flat to effuse surface, edge entire, aerial mycelium dense, pale brown from above and below.

Material examined - THAILAND, Phayao Province, on decaying seed pods of Cassia fistula (Fabaceae), 4 August 2017, R.H. Perera, PH-CAS 04 (MFLU 18-2750), living culture MFLUCC 19-0317).

GenBank numbers - ITS: MT215502, LSU: MT215552, HIS3: MT212195.

Notes - The new isolate (MFLUCC 19-0317) grouped with Gliocladiopsis aquaticus isolates collected from Thailand with high statistical support (Fig. 32). Our collection also shares similar morphology with G. aquaticus in having similar sporodochial morphology, forming phialides in 25 terminal whorls per branch and, similar conidiophores and conidial sizes (Hyde et al. 2018). Our isolate (MFLUCC 19-0317) and two species of G. aquaticus (MFLUCC 17-1811, MFLUCC 172028) showed 6 nucleotide differences (1.2\%, 1 gap) in the HIS3 region, and ITS region was identical. TUB2 gene region of G. aquaticus (MFLUCC 19-0317) was not available for comparison. Based on the morphological similarities and phylogenetic analysis we identify our collection as Gliocladiopsis aquaticus and a new host record from Cassia fistula. 


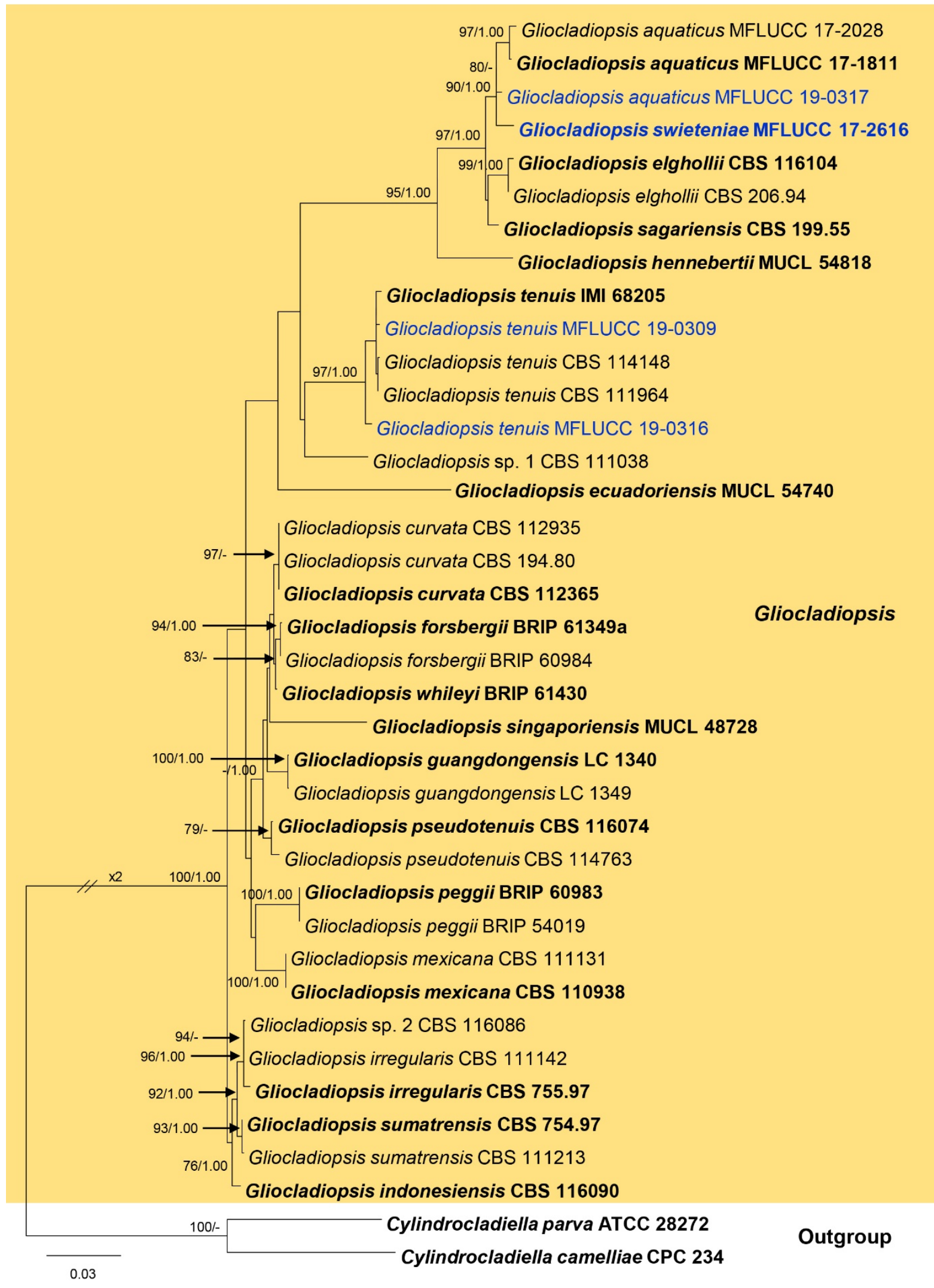

Figure 32 - Phylogram generated from RAxML analysis based on combined ITS, TUB2 and HIS3 sequence data of Gliocladiopsis isolates. Sequences from thirty-eight taxa, which comprise 1667 characters including gaps, are included in the analyses. The tree was rooted to Cylindrocladiella parva (ATCC 28272) and C. camelliae (CPS 234). The scale bar indicates 0.03 nucleotide changes per site. 


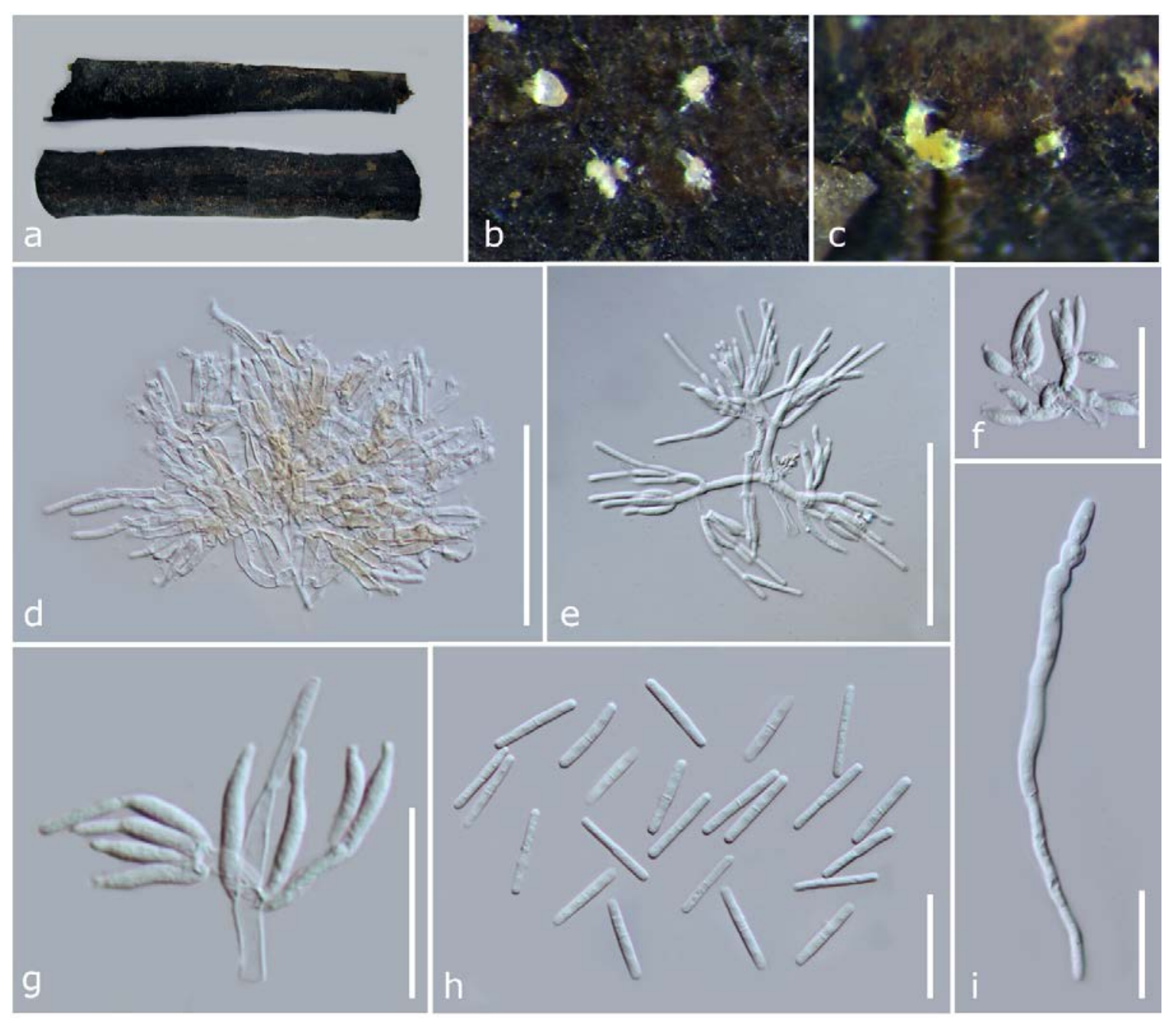

Figure 33 - Gliocladiopsis aquaticus (MFLU 18-2750). a Herbarium material. b, c Conidiomata on the host. c-g Conidiophores with conidia. h Conidia. i Germinating conidium. Scale bars: d, e = 50 $\mu \mathrm{m}, \mathrm{f}-\mathrm{i}=20 \mu \mathrm{m}$.

Gliocladiopsis tenuis (Bugnic.) Crous \& M.J. Wingf., Mycol. Res. 97(4): 446 (1993)

Fig. 34

On decaying fruits of palms and Swietenia mahagoni. Sexual morph: Undetermined. Asexual morph: Appearing as white masses on the substrate. Conidiophores penicillate, 80-105 × 4.4-8.5 $\mu \mathrm{m}$, septate, branched, hyaline, without stipe extensions and terminal vesicles. Conidiogenous apparatus with several series of hyaline branches; primary branches 15-26 × 3.8-6 $\mu \mathrm{m}$, aseptate; secondary branches 8.7-24.5 × 1.9-5.2 $\mu \mathrm{m}$, aseptate; tertiary branches 12.1-16.2 × 2.5-4.1 $\mu \mathrm{m}$, aseptate; quaternary branches absent. Conidiogenous cells phialidic. Phialides 10-14.7 × 2.2-3.7 $(\bar{x}=12.4 \times 2.8 \mu \mathrm{m}, \mathrm{n}=25)$, doliiform to cymbiform to cylindrical, arranged in terminal whorls of 2-4 per branch, sometimes with a minute collarette, central phialide frequently extending above the rest. Conidia 11.4-18 $\times$ 2.1-3.2 $\mu \mathrm{m}(\bar{x}=15.6 \times 2.5 \mu \mathrm{m}, \mathrm{n}=35)$, 1-septate, cylindrical, with rounded ends, straight, hyaline, smooth-walled, without appendages. Chlamydospores extensive, in clearly delimited chains. Conidia on MEA smaller, 9.4-14.2 $\times 1.5-2.7(\bar{x}=12 \times 2.3 \mu \mathrm{m}, \mathrm{n}=35)$.

Culture characteristics - Colonies growing on MEA, reaching $30 \mathrm{~mm}$ within 2 weeks at $28^{\circ} \mathrm{C}$, sporulation after 8 weeks, circular, surface effused, edge entire, initially yellowish white, orange brown to golden brown with age, reverse brown, mycelium superficial and partially immersed, branched, septate, hyaline to orange-brown, smooth-walled.

Material examined - THAILAND, Chiang Rai Province, personal garden, on decaying fruits of palm tree, 18 August 2017, R.H. Perera, CR-F 12 (MFLU 18-2741), living culture MFLUCC 190309; ibid. Mae Fah Luang University Garden, on decaying fruits of Swietenia mahagoni 
(Meliaceae), 6 September 2017, R.H. Perera, Maho 05 (MFLU 18-2771), living culture MFLUCC 19-0316.

GenBank numbers - ITS: MT215499, HIS3: MT212193, TEF1: MT212206, TUB2: MT212212 (MFLUCC 19-0309); ITS: MT215500, LSU: MT215551, TUB2: MT212213 (MFLUCC 19-0316)

Notes - Isolates MFLUCC 19-0309 and MFLUCC 19-0316 group with ex-type of Gliocladiopsis tenuis (IMI 68205) and other G. tenuis isolates. They were identified as Gliocladiopsis tenuis based on morphology and phylogenetic analysis (Fig. 32) (Crous \& Wingfield 1993, Crous \& Peerally 1996). Gliocladiopsis tenuis MFLU 18-2771 is a new record on Swietenia mahagoni.

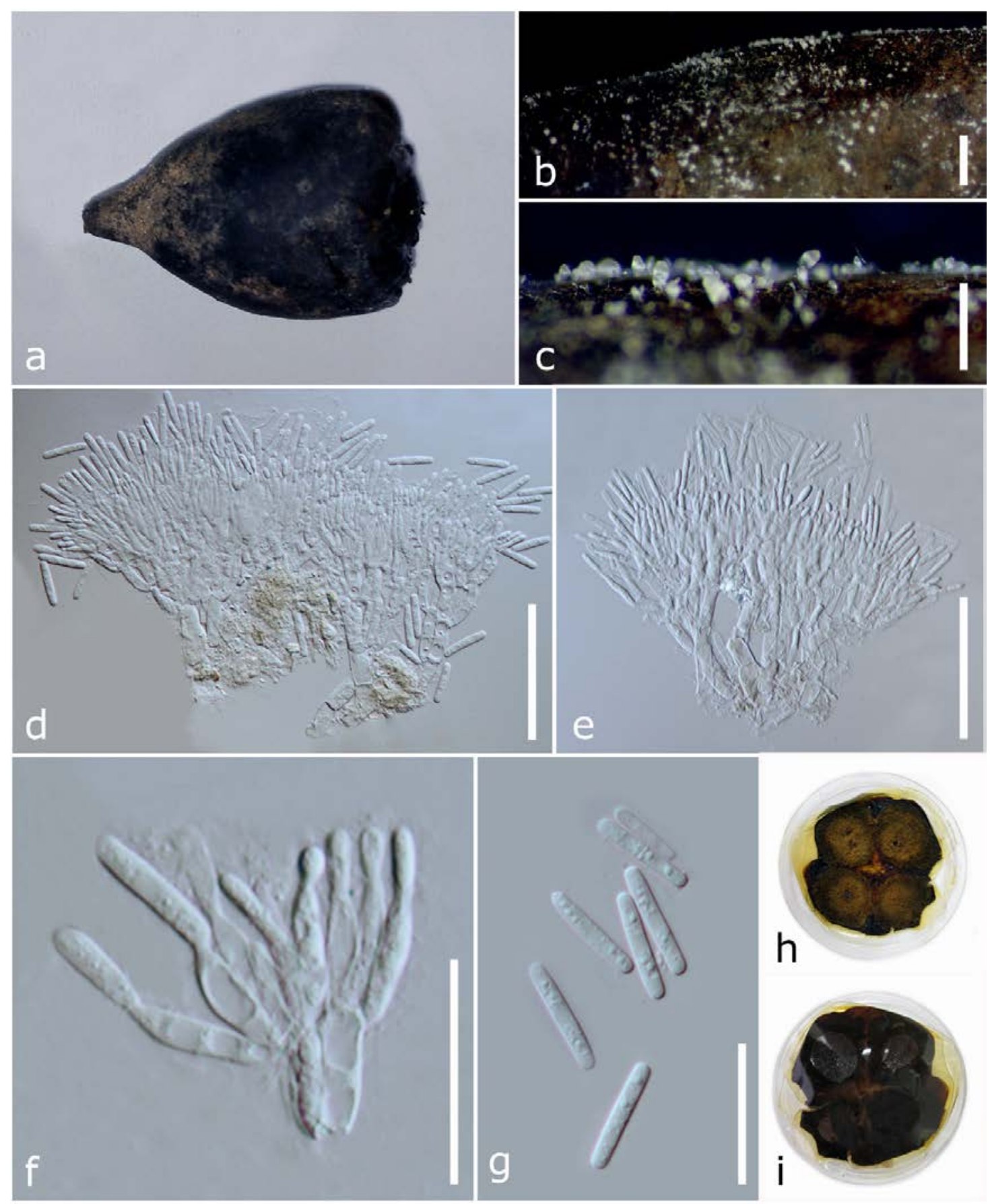

Figure 34 - Gliocladiopsis tenuis (MFLU 18-2741). a Herbarium material. b, c Conidiomata on the host. d-f Conidiophores with conidia. g Conidia. h, i Colony on MEA. Scale bars: b $=1 \mathrm{~mm}$, $c=500 \mu \mathrm{m}, \mathrm{d}, \mathrm{e}=50 \mu \mathrm{m}, \mathrm{f}, \mathrm{g}=20 \mu \mathrm{m}$. 
Gliocladiopsis swieteniae R.H. Perera, E.B.G. Jones \& K.D. Hyde, sp. nov.

Fig. 35 Index Fungorum number: IF556860; Facesoffungi number: FoF07762

Etymology - Named after the host genus Swietenia.

Holotype - MFLU 18-2767

Saprobic on decaying Swietenia mahagoni fruits. Sexual morph: Undetermined. Asexual morph: appearing as yellowish masses on the substrate. Conidiophores 70-105 × 5.2-7.6 $\mu \mathrm{m}$, penicillate without stipe extensions and terminal vesicles, very pale brown. Conidiogenous apparatus with several series of hyaline to very pale brown branches: primary branches 16.7-32 $\times$ 4.5-7 $\mu \mathrm{m}$, aseptate or 1-septate; secondary branches $11.2-20 \times 2.7-5 \mu \mathrm{m}$, aseptate, hyaline; tertiary

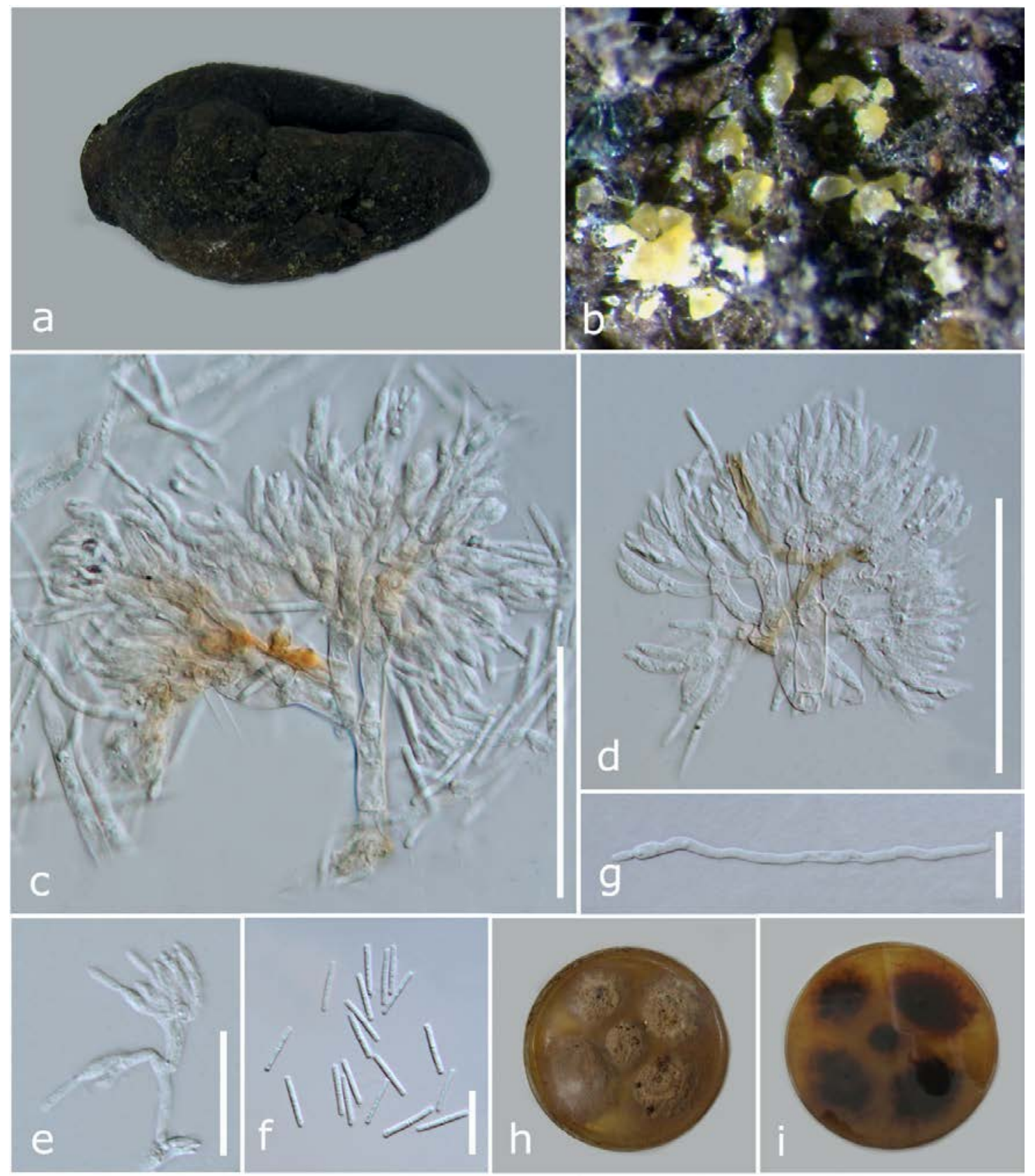

Figure 35 - Gliocladiopsis swieteniae (MFLU 18-2767, holotype). a Herbarium material. b Conidiomata on the host. c-e Conidiophores with conidia. f Conidia. g Germinating conidium. h, i Colony on MEA. Scale bars: c, $d=50 \mu \mathrm{m}$, e-g $=20 \mu \mathrm{m}$.

branches 10-14 × 2.3-3.8 $\mu \mathrm{m}$, aseptate, hyaline; quaternary branches $8.5-13 \times 1.7-2 \mu \mathrm{m}$, rare to absent, aseptate, hyaline. Phialides 10.6-17 $\times 1.9-3.8 \mu \mathrm{m}$, doliiform to cymbiform, arranged in terminal whorls of 2-6 per branch, with periclinal thickening and minute collarettes. Subverticillate 
conidiophores absent. Conidia (12.7-)14.4-17.9(-18.3) × 1.4-2 $\mu \mathrm{m}(\bar{x}=16.5 \times 1.8, \mathrm{n}=40 \mu \mathrm{m})$, cylindrical, hyaline, smooth, with rounded ends, straight, 1-septate, lacking a visible abscission scar.

Culture characteristics - Conidia germinating on PDA within 8 hours. Colonies growing on PDA reaching $30 \mathrm{~mm}$ within 2 weeks at $28^{\circ} \mathrm{C}$, circular, with effuse surface, edge entire, aerial mycelium dense, initially white, becoming sayal brown to sepia (reverse).

Material examined - THAILAND, Chiang Rai Province, Mae Fah Luang University Garden, on decaying fruits of Swietenia mahagoni (Meliaceae), 20 September 17, R.H. Perera, Maho 08 (MFLU 18-2767, holotype), ex-type living culture MFLUCC 17-2616.

GenBank numbers - ITS: MT215501, HIS3: MT212194, TUB2: MT212214.

Notes - Gliocladiopsis swieteniae is sister to Gliocladiopsis aquaticus isolates (MFLUCC 17-1811, MFLUCC 17-2028 and MFLUCC 19-0317) with high support (90\% MLBT, 1.0 BIPP; Fig. 32). Gliocladiopsis swieteniae and G. aquaticus also share similar morphology in having yellowish sporodochia and similar conidiophore size (Hyde et al. 2018). However, G. swieteniae differs from G. aquaticus in having smaller conidia $(14.4-17.9 \times 1.4-2$ vs. $16.5-21 \times 2-3 \mu \mathrm{m})$ (Hyde et al. 2018). A comparison of the ITS, TUB2 and HIS3 gene regions of G. swieteniae and ex-type of G. aquaticus (MFLUCC 17-1811) reveals 1 (0.6\%, 1 gap), 8 (1.3\%, 0 gaps) and 13 (2.8\%, 1 gap) nucleotide differences, respectively. G. swieteniae and G. aquaticus (MFLUCC 172028) showed 3 nucleotides differences (0.6\%, 2 gaps) in the ITS region, 10 different nucleotides (1.6\%, 0 gaps) in the TUB2 region and, 13 different nucleotides (2.8\%, 1 gap) in the HIS3 region. Gliocladiopsis swieteniae and G. aquaticus (MFLUCC 19-0317) showed 2 nucleotides differences (0.4\%, 0 gaps) in the ITS region, and 10 different nucleotides in the HIS3 (2.2\%, 1 gap) region. TUB2 gene region of G. aquaticus (MFLUCC 19-0317) was not available for comparison. Even though morphological differences are not strongly supported, we introduce $G$. swieteniae as a novel species considering nucleotide differences in ITS, TUB2 and HIS3 gene regions.

Macronectria Salgado \& P. Chaverri

Macronectria jungneri (Henn.) Salgado \& P. Chaverri, in Salgado-Salazar et al., Fungal Diversity 80: 448 (2016)

Fig. 37

Facesoffungi number: FoF07763

Saprobic on various plant substrates including fruits. Sexual morph: Ascomata 380-533 $\mu \mathrm{m}$ diam $(n=10)$, perithecial, gregarious in groups of many, superficial on an erumpent stroma, subglobose to pyriform, sometimes elongated and flask shaped, ostiole papillate, 128-280 $\mu \mathrm{m}$ wide, constricted below apex, not collapsing when dry, orange to sienna, with papilla same colour as perithecium, becoming dark black red when aged, red in $5 \% \mathrm{KOH}$, surface smooth. Peridium $20-40 \mu \mathrm{m}$, of two intergrading regions; outer region of intertwined hyphae with cells slightly elliptical, inner region of flattened fusoids cells. Asci 75-96 × 13-27 $\mu \mathrm{m}(\bar{x}=83 \times 21.5 \mu \mathrm{m}, \mathrm{n}=15)$, 8-spored, unitunicate, clavate, thin-walled, short pedicellate, apex simple. Ascospores 26-31 $\times 8.7-11 \mu \mathrm{m}(\bar{x}$ $=29.5 \times 9.8 \mu \mathrm{m}, \mathrm{n}=25)$, bi-seriate, fusiform, sometimes inequilateral, 1-septate, not constricted at septum, hyaline, striate. Asexual morph: See Salgado-Salazar et al. (2016).

Culture characteristics - Ascospores germinating on PDA within 12 hours. Colonies growing on PDA, reaching $2.6 \mathrm{~cm}$ in 7 days at $25^{\circ} \mathrm{C}$, surface effused, smooth, margin entire to undulate, aerial mycelium rare, initially white, becoming yellowish orange, reverse yellowish, organge at centre.

Material examined - Thailand, Chiang Rai Province, Mae Fah Luang university garden, on decaying fruits of Swietenia mahagoni (Meliaceae), 4 August 2014, R.H. Perera, RHP 09 (MFLU 16-1050), living culture MFLUCC 14-0568. 


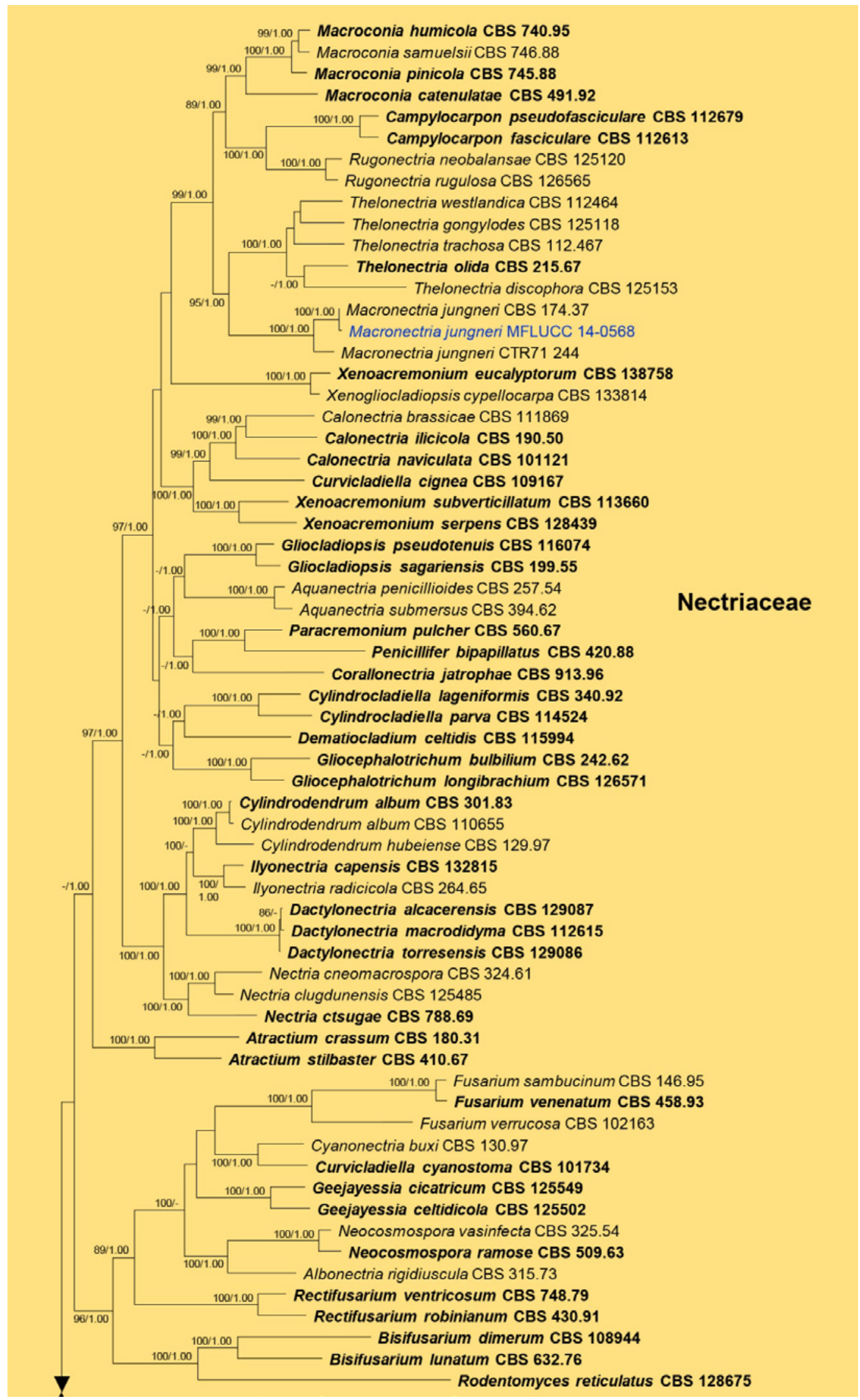

Figure 36 - Phylogram generated from RAxML analysis based on combined LSU, ITS, RPB1, RPB2, TEF1 and TUB2 sequence data of selected Nectriaceae isolates. Sequences from one hundred and twenty-nine taxa, which comprise 4456 characters including gaps, are included in the analyses. The tree was rooted to Clonostachys buxi CBS 696.93 and Verrucostoma freycinetiae MAFF 240100. The scale bar indicates 0.2 nucleotide changes per site. 


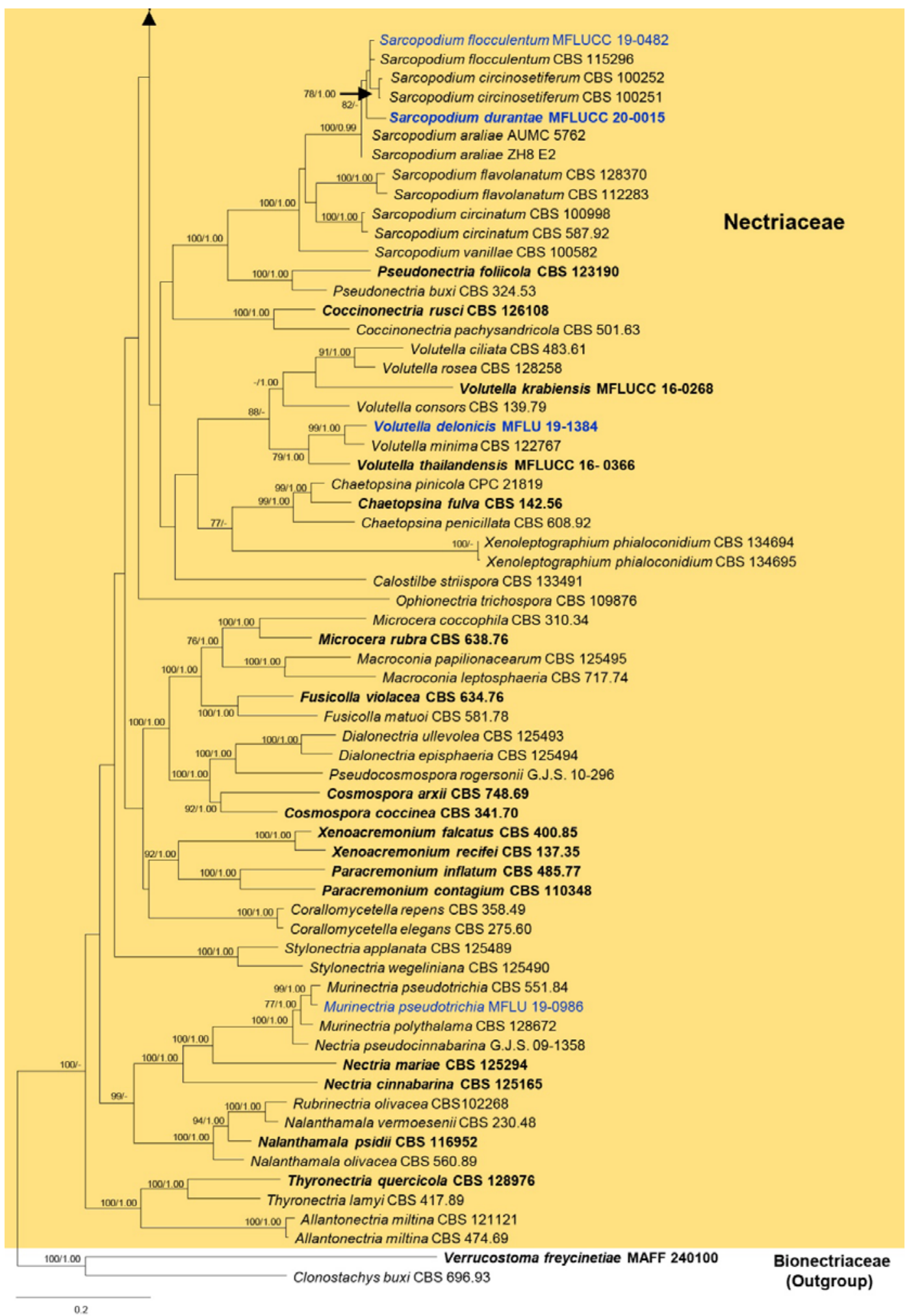

Figure 36 - Continued.

GenBank numbers - ITS: MT215505, LSU: MT215554, SSU: MT199562, TUB2: MT212217.

Notes - Our new isolate (MFLUCC 14-0568) clustered with Macronectria jungneri isolates (CBS 174.37, CTR71-244) with high bootstrap support (100\% MLBT, 1.00 BIPP; Fig. 36) and, overlapping in morphology with $M$. jungneri (Salgado-Salazar et al. 2016). ITS and TUB2 sequences of MFLUCC 14-0568 are identical with the Macronectria jungneri strain CBS 174.37 
with one nucleotide difference in LSU gene region. Therefore we identify our new isolate as $M$. jungneri and Swietenia mahagoni as a new host record for the fungus.

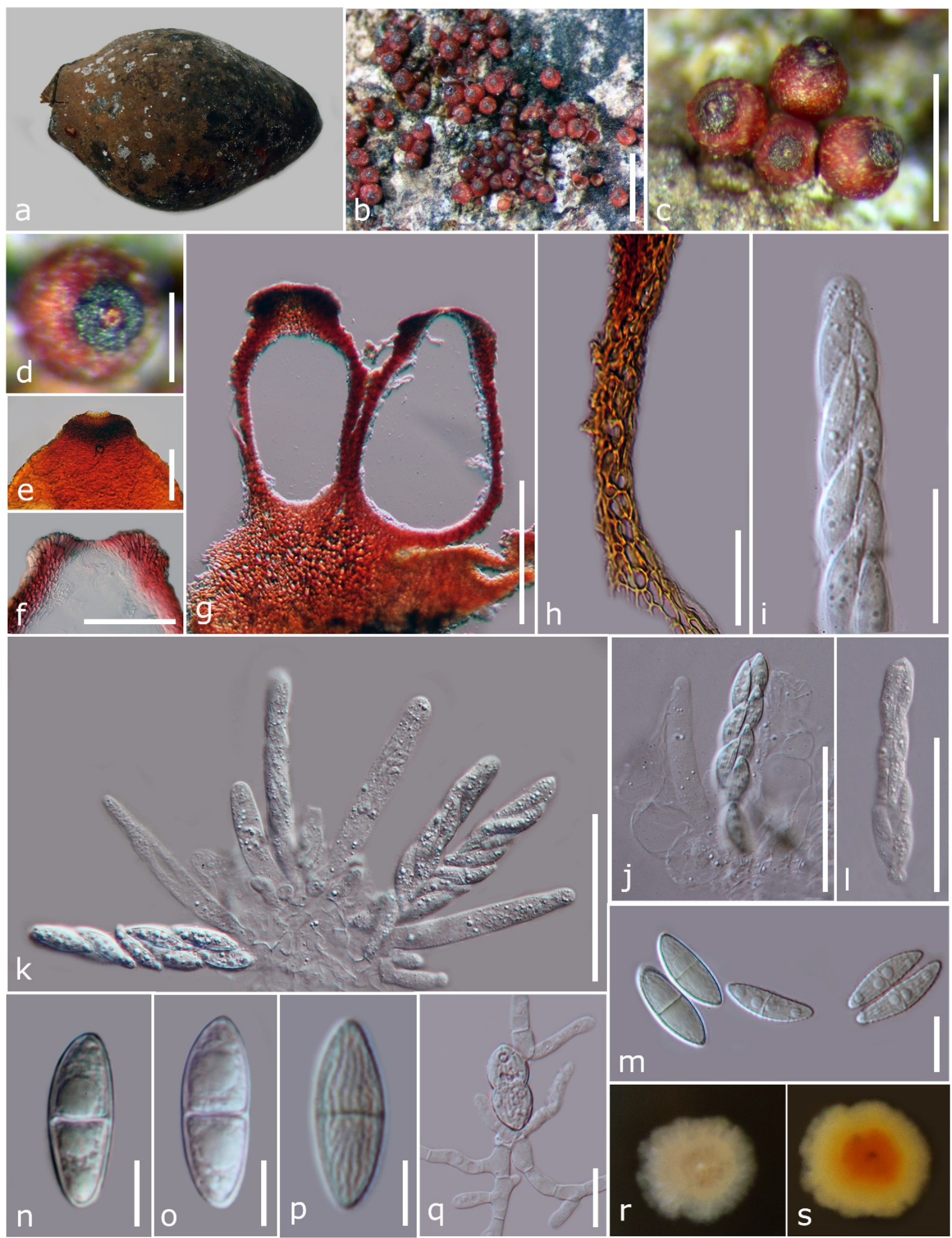

Figure 37 - Macronectria jungneri (MFLU 16-1050). a Herbarium material. b, c Ascomata on host. d Ostiole in face view. e Ostiole in side view. f Section of ostiole. g Section of ascomata. h Section of peridium. j Catenophyses. i, k, l Asci. m-p Ascospores. q Germinating ascospore. $\mathrm{r}$, s Colony on PDA. Scale bars: $\mathrm{b}=1 \mathrm{~mm}, \mathrm{c}=500 \mu \mathrm{m}, \mathrm{d}, \mathrm{e}=200 \mu \mathrm{m}, \mathrm{f}=100 \mu \mathrm{m}, \mathrm{g}, \mathrm{h}=200 \mu \mathrm{m}$, $\mathrm{i}=20 \mu \mathrm{m}, \mathrm{j}-\mathrm{l}=50 \mu \mathrm{m}, \mathrm{n}-\mathrm{p}=10 \mu \mathrm{m}, \mathrm{q}=20 \mu \mathrm{m}$. 
Murinectria M. Niranjan \& V.V. Sarma

Murinectria pseudotrichia (Schwein) M. Niranjan and V.V. Sarma

Fig. 38

On decaying seed pods of Delonix regia. Sexual morph: See Hirooka et al. (2012). Asexual morph: Synnematous. Synnemata 1000-1800 $\mu \mathrm{m}$ high including stipe, $72-115 \mu \mathrm{m}$ wide $(\mathrm{n}=20)$, erumpent through epidermis, solitary or gregarious, caespitose, cylindrical-capitate, subulatecapitate, or claviform. Stipe erect or nodding, usually unbranched or rarely up to 3 branched at base, medium to slender, distinctly hispid at base to mid-level, red-brown at base, turning dark red in 5\% KOH. Hyphae on stipe 3.4-4.5 $\mu \mathrm{m}$ wide, hyphae pigmented orange brown at base, becoming less pigmented towards apex, $\mathrm{KOH}+$. Ornamental hyphae on stipe 6-17 $\mu \mathrm{m}$ long, 2.2-4.5 $\mu \mathrm{m}$ wide ( $\mathrm{n}=16)$, cylindrical, straight, curved, sinuous or twisted, arising laterally at more or less right angles, distributed evenly over surface of synnemata, usually unbranched but occasionally dichotomously branched, aseptate. Conidiophores with sterile hyphae monoverticillate or biverticillate. Conidiogenous cells $16-22 \times 1.4-1.8 \mu \mathrm{m}(\mathrm{n}=20)$, enteroblastic, monophialidic, cylindrical to subulate, straight or curved, with periclinal thickening, collarette not conspicuous. Sterile hyphae 68-88 $\times 1.5-1.9 \mu \mathrm{m}(\mathrm{n}=16)$, mixed with phialides, acicular, usually curved or sometimes straight, unbranched or dichotomously branched, often in groups of 1-3. Conidial masses 140-285 $\mu \mathrm{m}$ wide, globose, hemispherical or more-or-less discoid, whitish yellow to sienna. Conidia 3.1-4.5 × 1.6-2.2 $\mu \mathrm{m}(\bar{x}=3.9 \times 2 \mu \mathrm{m}, \mathrm{n}=55)$, ellipsoidal, obovate or oblong, aseptate, multi-guttulate, hyaline, smooth-walled.

Material examined - THAILAND, Chiang Mai Province, on decaying seed pods of Delonix regia (Fabaceae), 1 September 2016, R.H. Perera, S-16 (MFLU 19-0986).

GenBank numbers - ITS: MT215506, LSU: MT215555, TUB2: MT212218.

Notes - DNA was directly extracted from fruiting bodies of this fungus. New isolate (MFLU 19-0986) grouped with Murinectria pseudotrichia (CBS 551.84) with good bootstrap support (96\%; Fig. 36) in our phylogenetic analysis. Our fungus was identified as Murinectria pseudotrichia based on the phylogenetic analysis and morphological similarities (Fig. 36) (Seaver 1909, Hirooka et al. 2012). This collection represents Delonix regia as a new host record for $M$. pseudotrichia.

Neocosmospora E.F. Sm.

Neocosmospora magnoliae R.H. Perera, E.B.G. Jones \& K.D. Hyde, sp. nov.

Fig. 40

Index Fungorum number: IF556861; Facesoffungi number: FoF07764

Etymology - Named after the host genus Magnolia.

Holotype - MFLU 18-2748

Associated with dried fruits of Magnolia champaca. Sexual morph: Ascomata 200-250 × 180-220 $\mu \mathrm{m}$; perithecial, astromatic, solitary or gregarious, superficial, globose to pyriform, orange to orange brown, glabrous, coarsely warted, warts $23-45 \mu \mathrm{m}$ diam, $10-50 \mu \mathrm{m}$ tall, ostiolate. Ostiole non-papillate, canal periphysate. Peridium 15-38 $\mu \mathrm{m}$ diam., inner region 1-2 layers of hyaline, flattened cells of textura angularis; outer region composed of 3-5 layers of orange brown, thickwalled cells of textura angularis; in face view a textura angularis of thick-walled, medium to dark brown cells. Hamathecium of hyaline, distinctly septate catenophyses, 14-20 $\mu \mathrm{m}$ wide. Asci 55-76 $\times$ 9.8-13 $\mu \mathrm{m}(\overline{\mathrm{x}}=67 \times 11 \mu \mathrm{m}, \mathrm{n}=25), 8$-spored, unitunicate, clavate, short-pedicellate, with a broad flattened apex, with a minute apical apparatus, J-. Ascospores (5-)8.3-11.7 × 4.5-5.8 $\mu \mathrm{m}(\overline{\mathrm{x}}$ $=10 \times 5 \mu \mathrm{m}, \mathrm{n}=30$ ), obliquely uniseriate or irregularly biseriate, broadly ellipsoid to obovoid or subfusiform, 1-septate, not constricted at the septum, 2 or 4 guttulate, hyaline to pale yellowbrown, thick-walled, finely striate when mature. Asexual morph: Undetermined.

Culture characteristics - Ascospores germinating on PDA within 12 hours. Colonies growing on PDA, reaching up to $2.6 \mathrm{~cm}$ within 7 days at $28^{\circ} \mathrm{C}$, surface effused, smooth, margin entire, aerial mycelium rare, initially white, turning rosy pink, reverse rosy pink. 


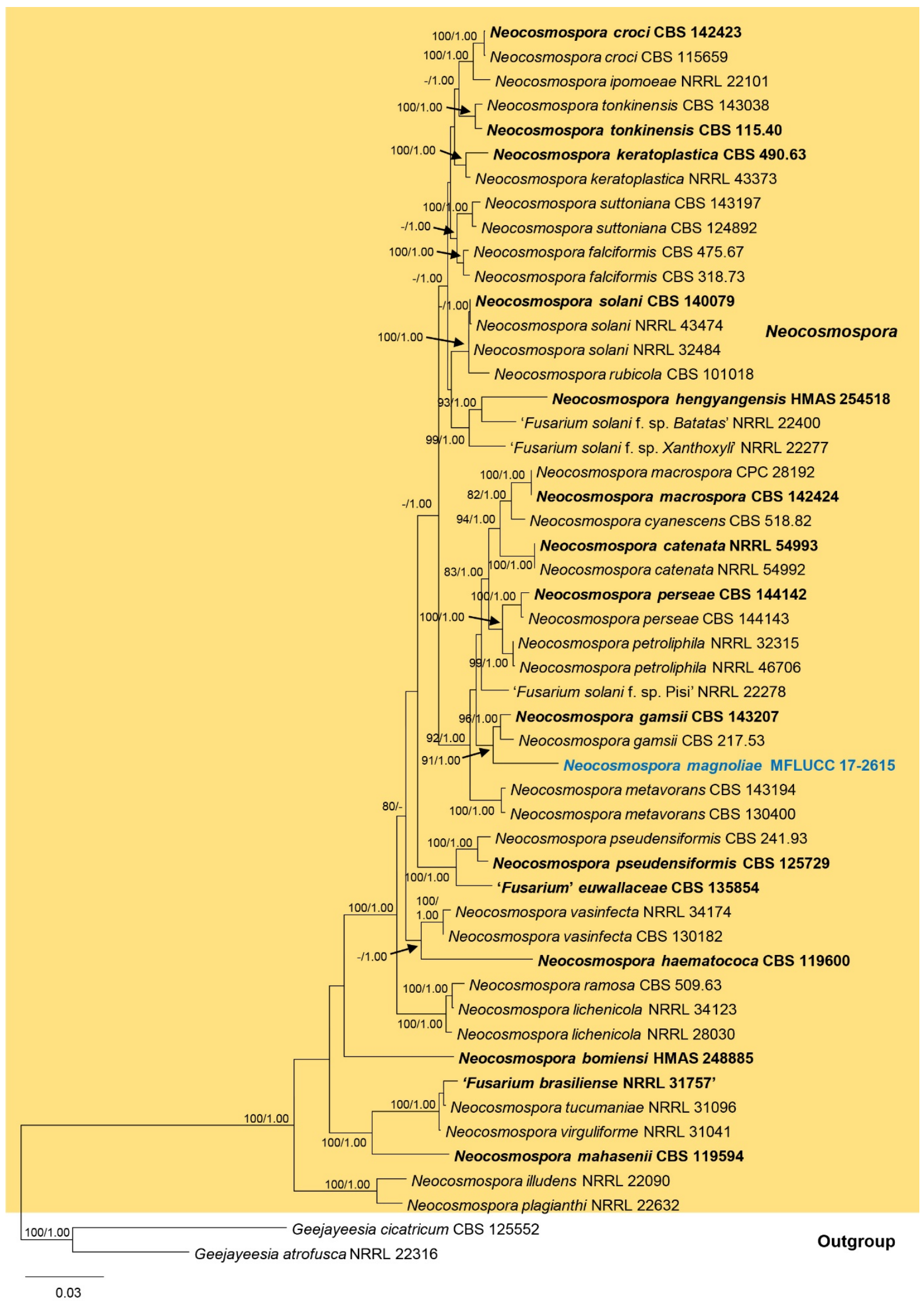

Figure 39 - Phylogram generated from RAxML analysis based on combined LSU, ITS, TEF1 and RPB2 sequence data of Neocosmospora isolates. Sequences from fifty-one taxa, which comprise 3425 characters including gaps, are included in the analyses. The tree was rooted to Geejayeesia atrofusca NRRL 22316 and Geejayeesia cicatricum CBS 125552. The scale bar indicates 0.03 nucleotide changes per site. 

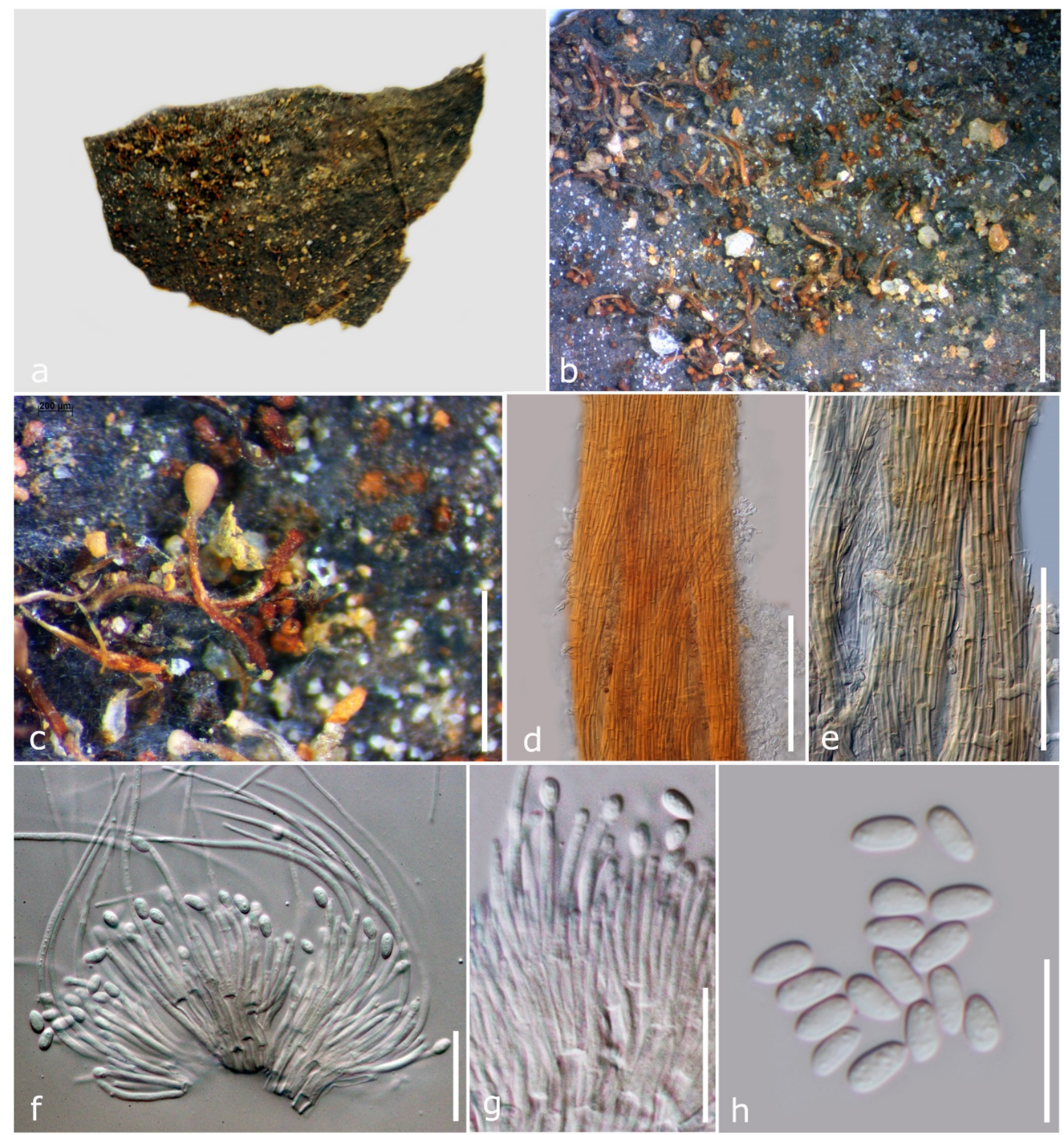

Figure 38 - Murinectria pseudotrichia (MFLU 19-0986). a Herbarium material. b, c Synnemata on host. d Stipe with ornamental hyphae on stipe. e Stipe hyphal strands. f Sterile hyphae and conidiophores. g Conidiophores with conidia. h Conidia. Scale bars: b, c $=1 \mathrm{~mm}, \mathrm{~d}, \mathrm{e}=100 \mu \mathrm{m}$, $\mathrm{f}, \mathrm{g}=20 \mu \mathrm{m}, \mathrm{h}=10 \mu \mathrm{m}$.

Material examined - THAILAND, Chiang Rai Province, Mae Fah Luang University garden, dried fruits of Magnolia champaca (Magnoliaceae) on the ground, 07 September 2017, R.H. Perera, MAG 01 (MFLU 18-2748, holotype), ex-type living culture MFLUCC 17-2615.

GenBank numbers - ITS: MT215508, LSU: MT215557, RPB2: MT212200, TEF1: MT212207.

Notes - Our new isolate (MFLUCC 17-2615) grouped sister to two isolates of Neocosmospora gamsii, CBS 143207 and CBS 217.53 with moderate bootstrap support (91\%; Fig. 39). Our isolate and ex-type $N$. gamsii (CBS 143207) showed 4 nucleotide differences (0.7\%, no gaps) in the ITS region, 12 nucleotide differences (1.8\%, 2 gaps) in the TEF1 region, and 36 different nucleotides (7.3\%, no gaps) in the RPB2 region, while their LSU sequences were 
identical. Our isolate and $N$. gamsii strain (CBS 217.53) showed 18 nucleotide differences (3.4\%, 4 gaps) in the ITS region, 8 nucleotide differences (1.2\%, no gaps) in the TEF1 region, and 36 different nucleotides $(7.3 \%$, no gaps) in the RPB2 region, while their LSU sequences were identical. Neocosmospora magnoliae can be distinguished from $N$. gamsii by its orange to orange brown ascomata and smaller asci (55-76 vs. 70-97.5 $\mu \mathrm{m}$ ), while $N$. gamsii has orange to dark brown-red ascomata (Sandoval-Denis \& Crous 2018). Hence, based on the above-mentioned differences in morphology and molecular sequence data, a new species, $N$. magnoliae is introduced.

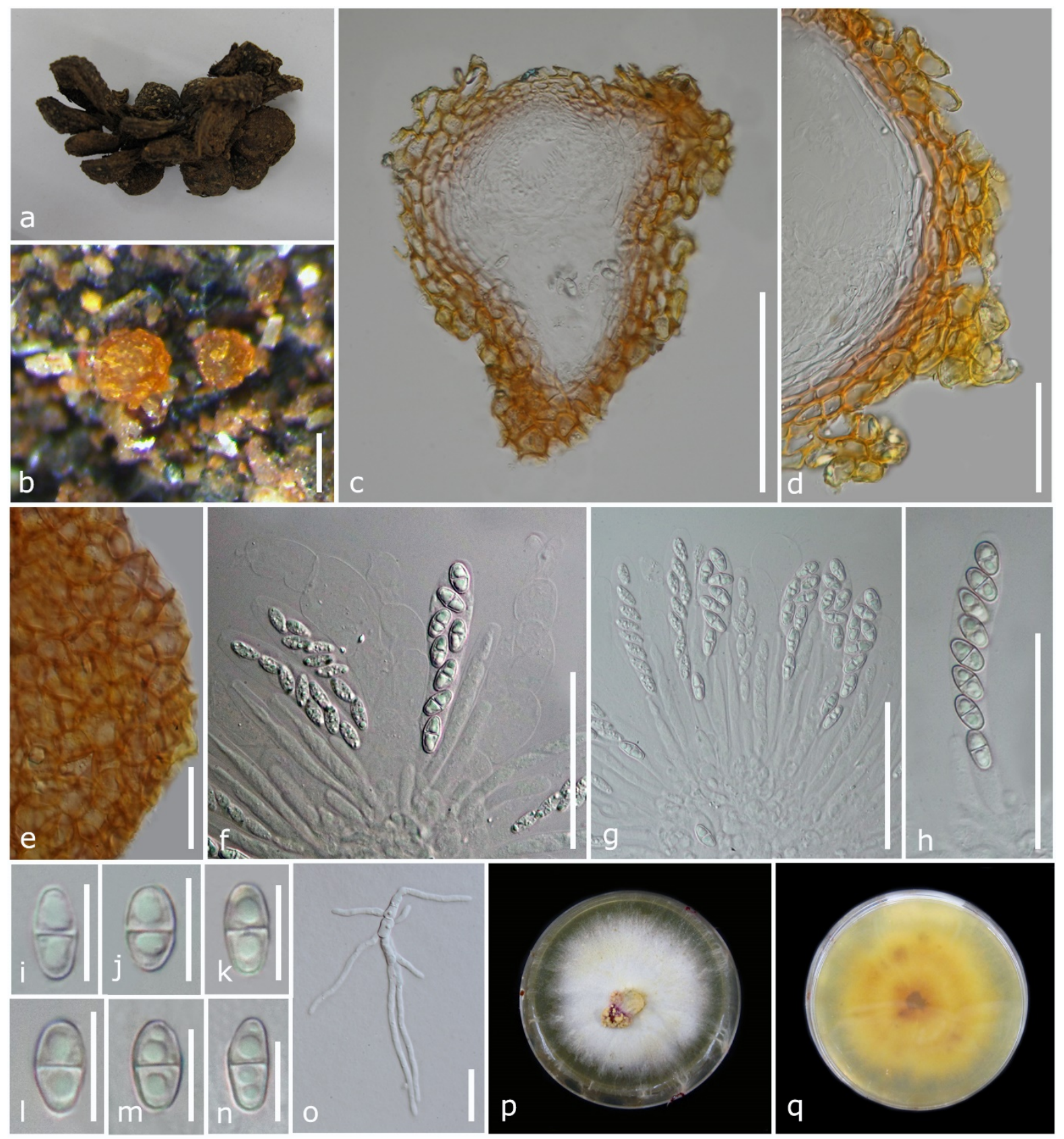

Figure 40 - Neocosmospora magnoliae (MFLU 18-2748, holotype). a Herbarium material. b Ascomata on host. c Section of ascoma. d Peridium in section. e Peridium in face view. $\mathrm{f}-\mathrm{h}$ Asci. $\mathrm{i}-\mathrm{n}$ Ascospores. o Germinating ascospore. p, q Colony on PDA. Scale bars: b, c $=100 \mu \mathrm{m}, \mathrm{d}=50$ $\mu \mathrm{m}, \mathrm{e}=20 \mu \mathrm{m}, \mathrm{f}-\mathrm{h}=50 \mu \mathrm{m}, \mathrm{i}-\mathrm{n}=10 \mu \mathrm{m}, \mathrm{o}=20 \mu \mathrm{m}$.

Sarcopodium Ehrenb. ex Schlecht.

Sarcopodium durantae R.H. Perera, E.B.G. Jones \& K.D. Hyde, sp. nov.

Fig. 41 
Index Fungorum number: IF557322; Facesoffungi number: FoF07765

Etymology - Named after the host genus Duranta.

Holotype - MFLU 18-2719

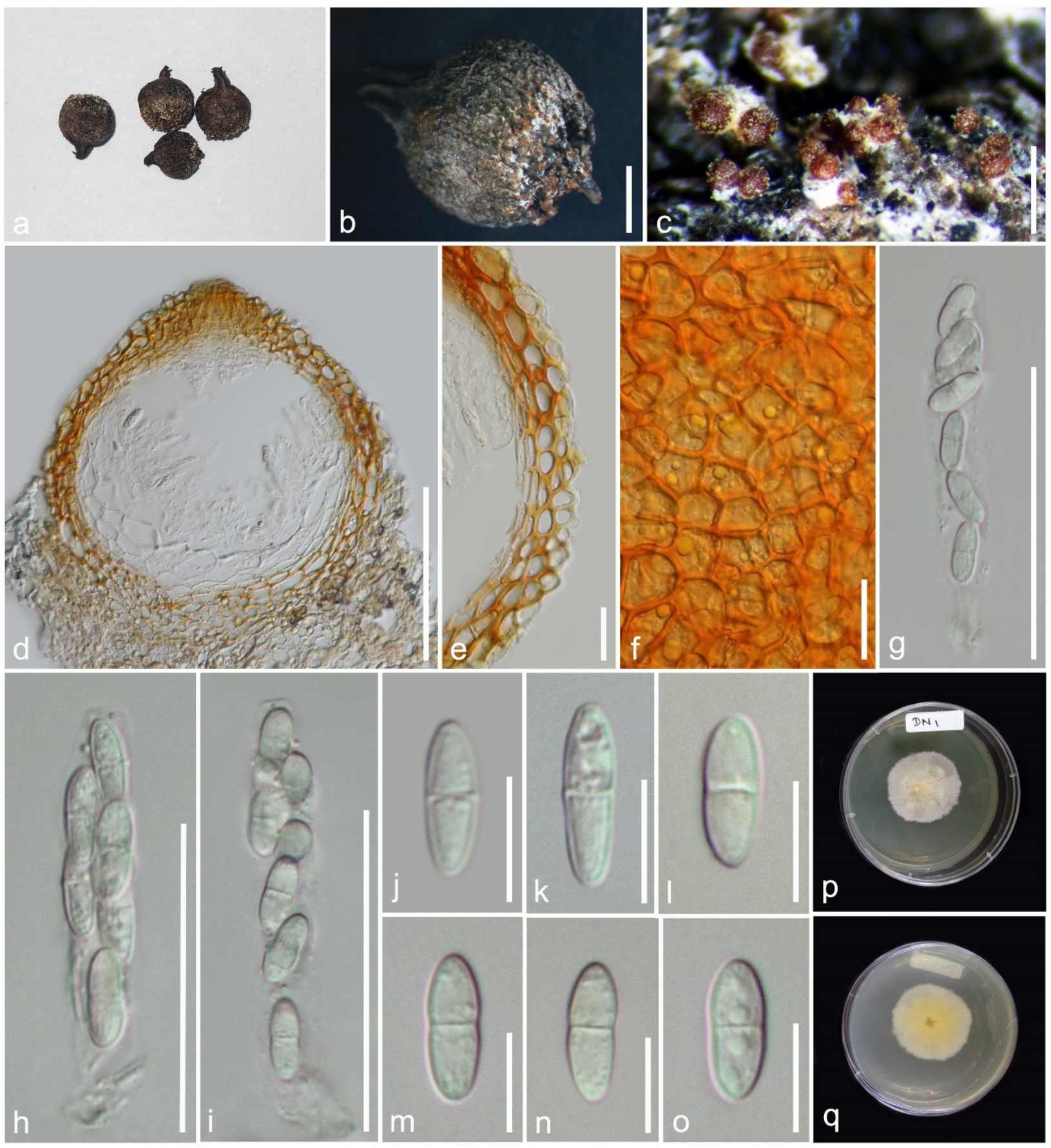

Figure 41 - Sarcopodium durantae (MFLU 18-2719, holotype). a Herbarium material. b, c Ascomata on host. d Section of ascoma. e Section of the peridium. f Peridium in face view. g-i Asci. j-o Ascospores, p, q Colony on PDA. Scale bars: $b=2 \mathrm{~mm}, \mathrm{c}=500 \mu \mathrm{m}, \mathrm{d}=100 \mu \mathrm{m}$, e, $\mathrm{f}=20 \mu \mathrm{m}, \mathrm{g}-\mathrm{i}=50 \mu \mathrm{m}, \mathrm{j}-\mathrm{O}=10 \mu \mathrm{m}$.

Saprobic on dry fruits of Duranta erecta. Sexual morph: Ascomata 175-210 $\mu \mathrm{m}$ high, 170 $210 \mu \mathrm{m}$ wide, perithecial, solitary or in groups, superficial on a minute stroma surrounded by white mycelium, globose to broadly obpyriform, not collapsing when dry, red, turning dark red in 5\% $\mathrm{KOH}$, spinulose hyphal hairs arising from the surface of the ascomatal wall, ostiole slightly papillate. Peridium 17-26 $\mu \mathrm{m}$, composed of 3-5 layers of dark orange colour cells of textura 
angularis. Hamathecium comprising hyaline, distinctly septate catenophyses, each cell $16-25 \mu \mathrm{m}$ long $\times$ 9-15 $\mu \mathrm{m}$ wide. Asci 50-75 × 7.5-10 $\mu \mathrm{m}(\bar{x}=61 \times 8.8 \mu \mathrm{m})$, unitunicate, 8 -spored, clavate to fusiform, short pedicellate, with an apical ring, J-. Ascospores $12-15 \times 4-6 \mu \mathrm{m}(\bar{x}=13.4 \times 5$ $\mu \mathrm{m})$, uni-seriate to multi-seriate, ellipsoid to fusiform, 1-septate, slightly constricted at the septum, guttulate, hyaline to pale yellow-brown, striate. Asexual morph: Undetermined.

Culture characteristics - Ascospores germinating on PDA within 12 hours. Colonies growing on PDA reaching $4 \mathrm{~cm}$ in 7 days at $28^{\circ} \mathrm{C}$, surface effused, smooth, margin entire, aerial mycelium rare, initially white, becoming pale yellowish orange, reverse yellowish, organge at centre.

Material examined - Thailand, Chiang Rai Province, on dried fruits of Duranta erecta L. (Verbenaceae), 25 November 2018, D. Sandaruwan, DN1 (MFLU 18-2719, holotype), ex-type living culture MFLUCC 20-0015.

GenBank numbers - ITS: MT386004, LSU: MT386007, TEF1: MT383671, TUB2: MT383672.

Notes - Sarcopodium durantae (MFLUCC 20-0015) groups with Sarcopodium species, forming an unsupported clade (Fig. 36). Our isolate and ex-type S. flocculentum (CBS 115296) showed 7 different nucleotides (3\%, 2 gaps) in the TEF1 region, 8 different nucleotides (1\%, 1 gap) in the TUB2 region. A comparison of the TUB2 and TEF1 gene regions of $S$. durantae (MFLUCC 20-0015) and S. circinosetiferum (CBS 100251) reveals 20 (3\%, 6 gaps) and 16 (6\%, 2 gaps) nucleotide differences, respectively. Our new isolate and S. flocculentum (CBS 115296) showed 7 different nucleotides (3\%, 2 gaps) in the TEF1 region, 8 different nucleotides (1\%, 1 gap) in the TUB2 region. A comparison of the TUB2 and TEF1 gene regions of $S$. durantae and $S$. flocculenta (MAFF 241413) reveals 9 (2\%, 1 gap) and 14 (7\%, 4 gaps) nucleotide differences, respectively. Sarcopodium mammiforme and S. raripilum do not have DNA sequence data in the GenBank for the comparison. Sarcopodium durantae can be distinguished from $S$. flocculentum by smaller ascomata $(175-210 \times 170-210$ vs. 224-314 × 215-293 $\mu \mathrm{m})$ and larger ascospores (12-15 × 4-6 vs. 10-13 × 3-4.5 $\mu \mathrm{m})$ (Rossman et al. 1999). Sarcopodium durantae differs from $S$. mammiforme by having smaller ascomata (175-210 × 170-210 vs. 300-700 $\mu \mathrm{m})$ and smaller ascospores (12-15 $\times$ 4-6 vs. 24-30 × 7-9 $\mu \mathrm{m}$ ) (Rossman et al. 1999). Sarcopodium durantae has smaller ascospores when compared to S. raripilum (12-15 × 4-6 vs. 27.5-32 × 6.5-8 $\mu \mathrm{m})$ (Rossman et al. 1999). Sarcopodium durantae differs from S. oblongisporum in having larger asci (50-75 × 7.5-10 vs. 43$59 \times 6-7.6 \mu \mathrm{m})$ and ascospores (12-15 × 4-6 vs. 9.5-12.2 × 2.2-3.9 $\mu \mathrm{m})$ (Nong \& Zhuang 2005). Based on these morphological differences, a new species, $S$. durantae is introduced in the genus Sarcopodium.

Sarcopodium flocculentum (Henn. \& E. Nyman) Pennycook \& P.M. Kirk, Index Fungorum 418: 1 (2019) Fig. 42

Facesoffungi number: FoF07766

Associated with Fabaceae hosts and Eucalyptus grandis. Sexual morph: Ascomata 150-180 $\mu \mathrm{m}$ high, 135-170 $\mu \mathrm{m}$ wide, perithecial, solitary or in groups, superficial on a minute stroma, subglobose to broadly obpyriform, sometimes collapsing apically to form cups when dry, red, turning dark red in 5\% $\mathrm{KOH}$, spinulose hyphal hairs arising from the surface of the ascomatal wall and forming around the ascomatal base, ostiole papillate. Peridium 16-34 $\mu \mathrm{m}$, composed of 2-4 layers of dark orange colour cells of textura angularis, thinner around ostiole, $2-12 \mu \mathrm{m}$ wide, composed of compactly arranged, flat cells. Hamathecium comprising hyaline, distinctly septate catenophyses, each cell 4.1-19.4 $\mu \mathrm{m}$ long $\times 6.3-12 \mu \mathrm{m}$ wide. Asci 35-63 $\times 6.2-8.3 \mu \mathrm{m}(\bar{x}=50 \times$ $7.4 \mu \mathrm{m})$, unitunicate, 8-spored, clavate to fusiform, short pedicellate, with apical ring, J-. Ascospores $7.2-12 \times 3.5-4.6 \mu \mathrm{m}(\bar{x}=9.3 \times 3.9 \mu \mathrm{m})$, uni-seriate to multi-seriate, ellipsoid to fusiform, 1-septate, slightly constricted at the septum, hyaline to pale yellow-brown, striate. Asexual morph: See Sutton (1981).

Culture characteristics - Ascospores germinating on PDA within 12 hours. Colonies growing on PDA reaching $3.8 \mathrm{~cm}$ in 7 days at $28^{\circ} \mathrm{C}$, surface effused, smooth, margin entire, aerial mycelium rare, initially white, becoming yellowish orange, reverse brownish orange. 


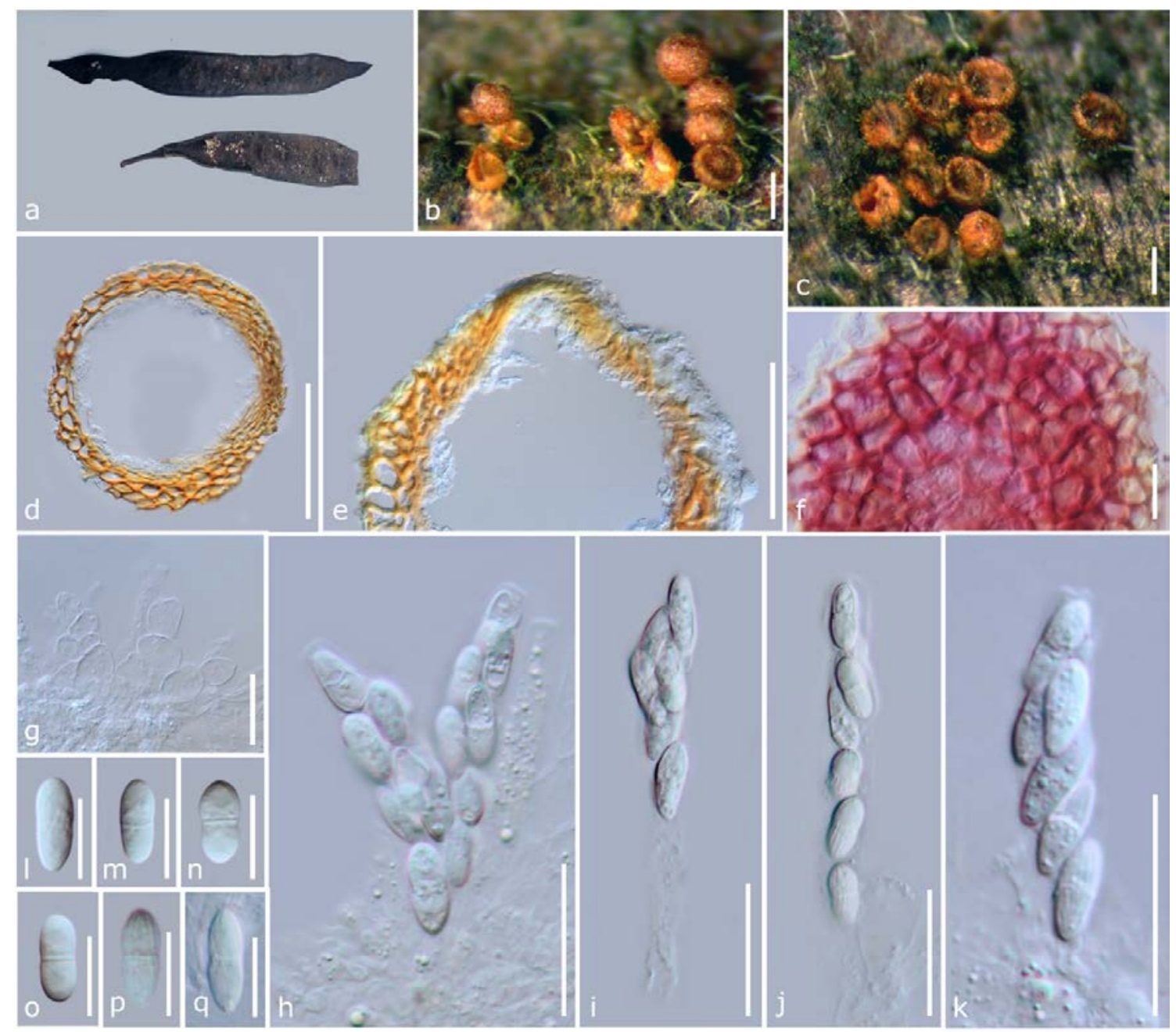

Figure 42 - Sarcopodium flocculentum (MFLU 19-0988). a Herbarium material. b, c Ascomata on host. d Section of ascoma. e Section of ostiole. $\mathrm{f}$ Peridium in face view (in $5 \% \mathrm{KOH}$ ). g Catenophyses. h-k Asci. l-q Ascospores. Scale bars: b, c $=200 \mu \mathrm{m}, \mathrm{d}=100 \mu \mathrm{m}, \mathrm{e}=50 \mu \mathrm{m}$, $\mathrm{f}-\mathrm{k}=20 \mu \mathrm{m}, \mathrm{l}-\mathrm{q}=10 \mu \mathrm{m}$.

Material examined - Thailand, Chiang Rai Province, on dried seed pods of Leucaena leucocephala (Lam.) de Wit (Fabaceae), September 2017, I.D. Goonasekara, Ipil 01 (MFLU 190988), living culture MFLUCC 19-0482).

GenBank numbers - ITS: MT215507, LSU: MT215556.

Notes - Our collection resembles the genus Sarcopodium by having red ascomata and spinulose hyphal hairs arising from the surface of the ascomatal wall and forming around the ascomatal base (Rossman et al. 1999, Lombard et al. 2015). In our phylogenetic analysis, the new isolate (MFLUCC 19-0482) clusters with S. flocculentum (CBS 115296) (Fig. 36). Sarcopodium flocculentum previously reported from two Fabaceae hosts (Pongamia pinnata and Albizia richardiana) and Eucalyptus grandis in India (Sutton 1981). Our isolate and S. flocculentum strain (CBS 115296) showed 1 nucleotide difference (no gaps) in the ITS region while, their LSU sequences were identical, which indicates that they are same species (Jeewon \& Hyde 2016). However, only the asexual morph was reported for S. flocculentum (Sutton 1981). We identified our collection as the sexual morph of S. flocculentum and Leucaena leucocephala as a new host record for the fungus.

Volutella Tode

Volutella delonicis R.H. Perera, E.B.G. Jones \& K.D. Hyde, sp. nov.

Figs 43, 44 
Index Fungorum number: IF556863; Facesoffungi number: FoF07767

Etymology - Named after the host genus Delonix.

Holotype - MFLU 19-1384

Saprobic on seed pods of Delonix regia. Sexual morph: Ascomata associated with conidiomata, 285-345 $\mu \mathrm{m}$ high, 260-280 $\mu \mathrm{m}$ wide $(\mathrm{n}=6)$, perithecial, solitary, basal stroma inconspicuous, superficial, obpyriform to pyriform, with an acute apex, orange to red, turning dark red in $5 \% \mathrm{KOH}$, surface finely roughened. Peridium 45-82 wide, two strata, outer stratum composed of 5-9 layers of orange to red, thick-walled cells of textura globosa to textura angularis; inner stratum composed of 2-4 layers of orange to red, flat, thick-walled cells of textura angularis; thinner around ostiole, composed of elongated cells. Asci not observed. Ascospores 10.7-13.1 $\times$ 2.5-3.3 $\mu \mathrm{m}(\bar{x}=11.9 \times 2.9 \mu \mathrm{m}, \mathrm{n}=20)$, 1-septate, hyaline, fusiform to biconic, smooth-walled, without appendages, germinated inside the ascomata. Asexual morph: Hyphomycetous, sporodochial, stroma inconspicuous. Sporodochia sessile, yellow. Setae 197-318 × 4.7-8 $\mu \mathrm{m}(\bar{x}=$ $258 \times 6 \mu \mathrm{m}, \mathrm{n}=10$ ), forming around the margin of conidiomata, hyaline, aseptate, stiff, cylindrical, tapering towards the apex, apex rounded. Conidiophores terverticillate, branched, septate, hyaline. Conidiogenous apparatus with subulate, conidiogenous cells, 8-9 × 1.9-2.5 $\mu \mathrm{m}(\bar{x}=8.6 \times 2.3$ $\mu \mathrm{m}, \mathrm{n}=20$ ), monophialidic, hyaline, with periclinal thickening, collarette prominent, up to $1.7 \mu \mathrm{m}$ long, $1.9 \mu \mathrm{m}$ wide. Conidia 5.2-7.4 × 1.1-1.5 $\mu \mathrm{m}(\bar{x}=6.1 \times 1.3 \mu \mathrm{m}, \mathrm{n}=30)$, slimy, aseptate, hyaline, oblong, smooth-walled, without mucilaginous sheath, forming yellow masses.

Material examined - Thailand, Chiang Mai Province, on decaying seed pods of Delonix regia (Fabaceae), 20 December 2016, R.H. Perera, Delo 02 (MFLU 19-1384, holotype).

GenBank numbers - ITS: MT215504, LSU: MT215553, RPB1: MT212196, TUB2: MT212216.

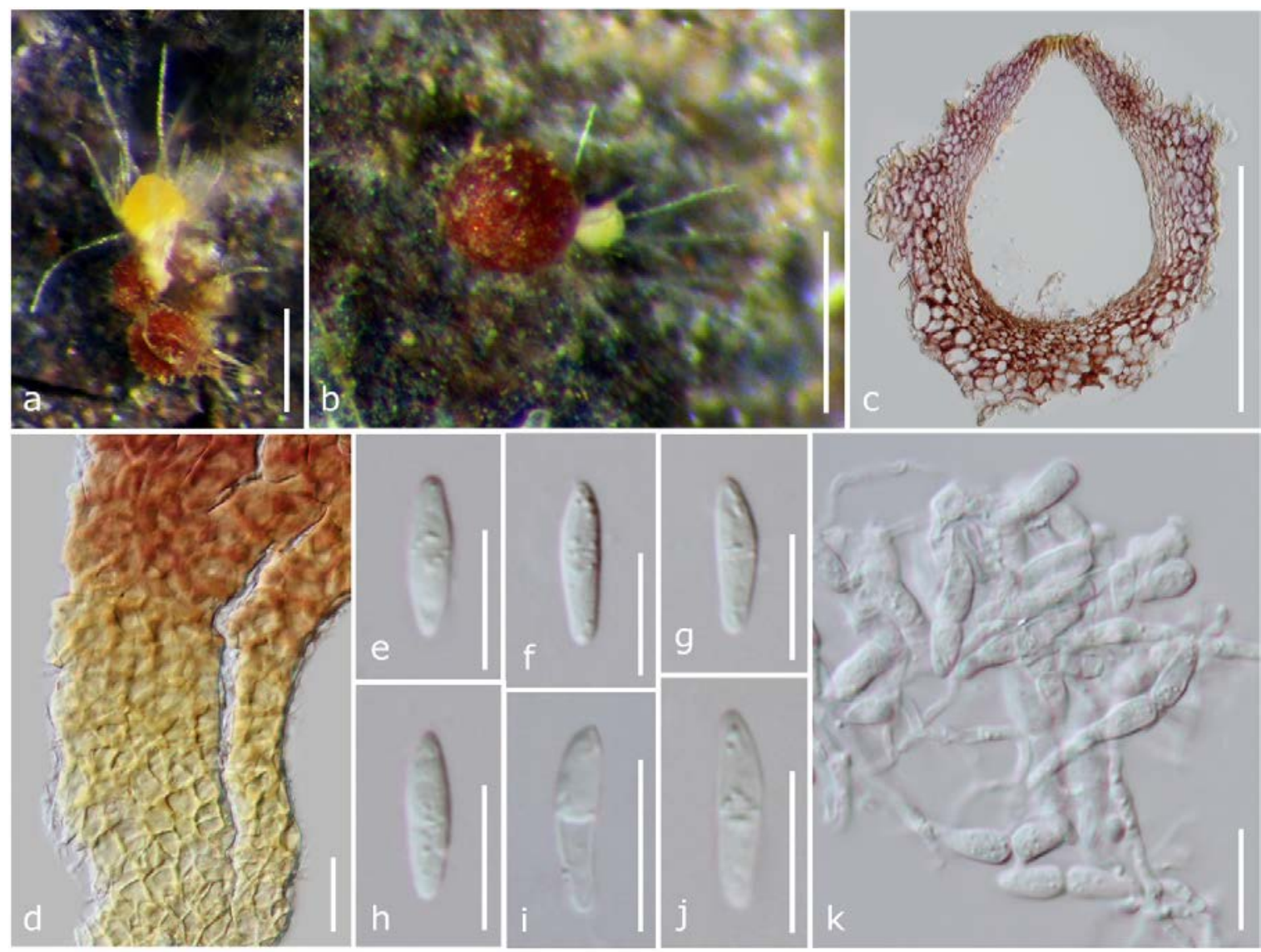

Figure 43 - Volutella delonicis (MFLU 19-1384, holotype) a, b Ascomata associated with conidiomata on host. c Section of ascoma. d Peridium in face view. e-j Ascospores. k Ascospores germinating inside the ascomata. Scale bars: $\mathrm{a}-\mathrm{c}=200 \mu \mathrm{m}, \mathrm{d}=20 \mu \mathrm{m}, \mathrm{e}-\mathrm{k}=10 \mu \mathrm{m}$. 


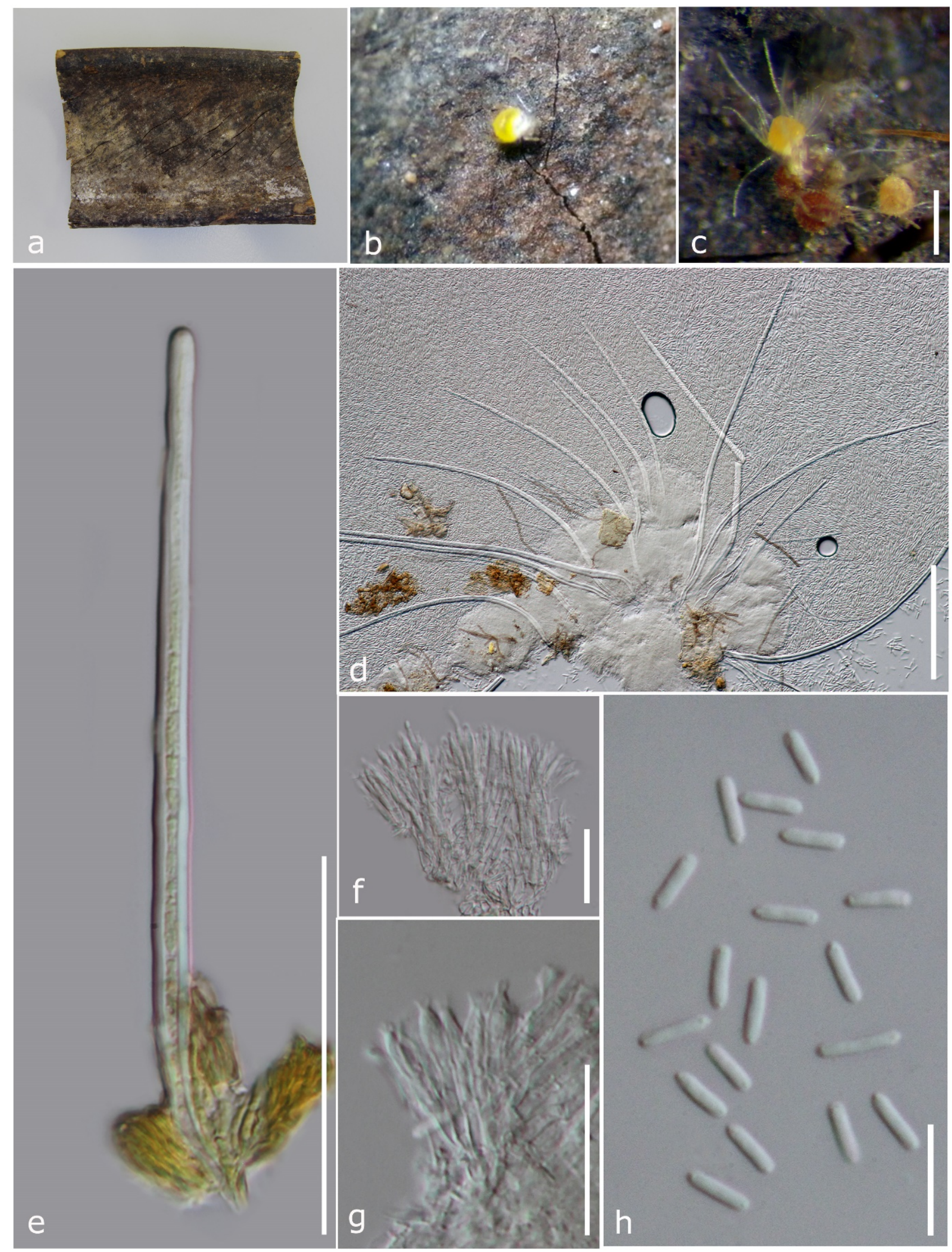

Figure 44 - Volutella delonicis (MFLU 19-1384, holotype). a Herbarium material. b, c Sporodochia (c associated with ascomata). d Sporodochium mounted in water. e Seta. $\mathrm{f}$, g Conidiogenous apparatus. h Conidia. Scale bars: $\mathrm{c}=200 \mu \mathrm{m}, \mathrm{d}=100 \mu \mathrm{m}, \mathrm{e}=50 \mu \mathrm{m}, \mathrm{f}, \mathrm{g}=20$ $\mu \mathrm{m}, \mathrm{h}=10 \mu \mathrm{m}$. 
Notes - Holomorph of Volutella delonicis was associated with decaying seed pods of Delonix regia. Volutella delonicis has a close phylogenetic affinity to V. minima CBS 122767 in multi-locus phylogenetic analysis (Fig. 36). Volutella delonicis has yellow sporodochia and aseptate setae, while V. minima produces white sporodochia and septate setae (Höhnel 1909). Furthermore, V. delonicis forms larger conidia than V. minima (5.2-7.4 $\mu \mathrm{m}$ vs. 3-5 $\mu \mathrm{m})$ (Höhnel 1909). A comparison of the LSU, ITS, RPB2 and TUB2 nucleotides of these two strains reveals $8(0.9 \%), 10$ (1.8\%), 15 (1.6\%), $44(6.2 \%)$ and 40 (7.1\%) nucleotide differences respectively in each gene region, which indicates that they are distinct taxa (Jeewon \& Hyde 2016).

\section{Xylariales Nannf. \\ Diatrypaceae Nitschke}

\section{Allodiatrype Konta \& K.D. Hyde}

Allodiatrype thailandica (R.H. Perera, Jian K. Liu \& K.D. Hyde) Konta \& K.D. Hyde 2020

Saprobic on decaying wood or seed pods of Delonix regia. Sexual morph: Stromata 540 $1050 \mu \mathrm{m}$ wide, gregarious or scattered on host, erumpent, arising through the cracks in host tissue, with 1-5 ascomata immersed in white entostroma becoming greenish yellow near the periphery, stromata outer layer black. Ascomata 280-370 $\mu \mathrm{m}$ high, $150-300 \mu \mathrm{m}$ wide $(\bar{x}=320 \times 210 \mu \mathrm{m}, \mathrm{n}=$ 20), perithecial, immersed in stromatic tissues, aggregated, globose to subglobose, narrowing towards the apex and very narrow at the base of papilla, pale brown, ostiolate, papillate. Papilla 85-150 $\mu \mathrm{m}$ high, $80-110 \mu \mathrm{m}$ wide, immersed in outer layer of stromata, conical, apex wider than base, periphysate, periphyses 17-28 $\mu \mathrm{m}$ long. Peridium 20-27 $\mu \mathrm{m}$ wide, comprising an outer, brown, thick-walled, stratum of 4-5 layers of cells of textura angularis and inner, hyaline, thickwalled, stratum of 7-10 layers of cells of textura angularis; peridium in face view a textura angularis of thin-walled, medium to dark brown cells. Hamathecium comprising long, 7.4-10.3 $\mu \mathrm{m}$ wide $(\bar{x}=8.9 \mu \mathrm{m}, \mathrm{n}=20)$, septate, paraphyses, slightly constricted at basal septa, and narrowing and tapering towards the apex. Asci 75-120 × 6.1-9.4 $\mu \mathrm{m}(\bar{x}=101 \times 8.2 \mu \mathrm{m}, \mathrm{n}=20), 4-8$-spored, unitunicate, clavate, with narrow, long, thin-walled pedicel, with thick-walled, swollen upper portion, apex flat, with J- apical apparatus. Ascospores 6.5-10.7 × 1.6-2.7 $\mu \mathrm{m}(\bar{x}=7.8 \times 2.2 \mu \mathrm{m}, \mathrm{n}$ $=50$ ), biseriate, allantoid to cylindrical, unicellular, pale brown at maturity, thick-walled, with small, fat globules at ends, smooth-walled. Asexual morph: See Li et al. (2016).

Culture characteristics - Ascospores germinated on PDA within 12 hours. Reaching $3.5 \mathrm{~cm}$ within 14 days on $\mathrm{PDA}$ at $25^{\circ} \mathrm{C}$, colonies medium dense, circular, flat, with diffuse margin, white, greenish yellow with time, below similar in colour, not zonate.

Material examined - THAILAND, Chiang Rai Province, Mae Fah Luang University premises, on dried seed pods of Delonix regia (Fabaceae), 6 January 2015, R.H. Perera, RHP 150 (MFLU 17-0735), ibid. Doi Mae Salong, on unidentified plant stem, 15 March 2014, R.H. Perera, M-1 (MFLU 16-1073), living culture MFLUCC 16-0459.

GenBank numbers - ITS: MT386002, LSU: MT215541, TUB2: MT212210 (MFLU 170735); ITS: MT386003, LSU: MT215542 (MFLUCC 16-0459).

Notes - In molecular phylogenetic analysis our new isolates clustered with putative strain of Allodiatrype thailandica (MFLUCC 15-3662) (Fig. 45). Our collection is similar to A. thailandica by greenish yellow entostroma, ascospore colour and size ( $\mathrm{Li}$ et al. 2016, Konta et al. 2020). It is therefore reported here as a new host record of $A$. thailandica and in providing TUB2 gene region sequence for the first time. 


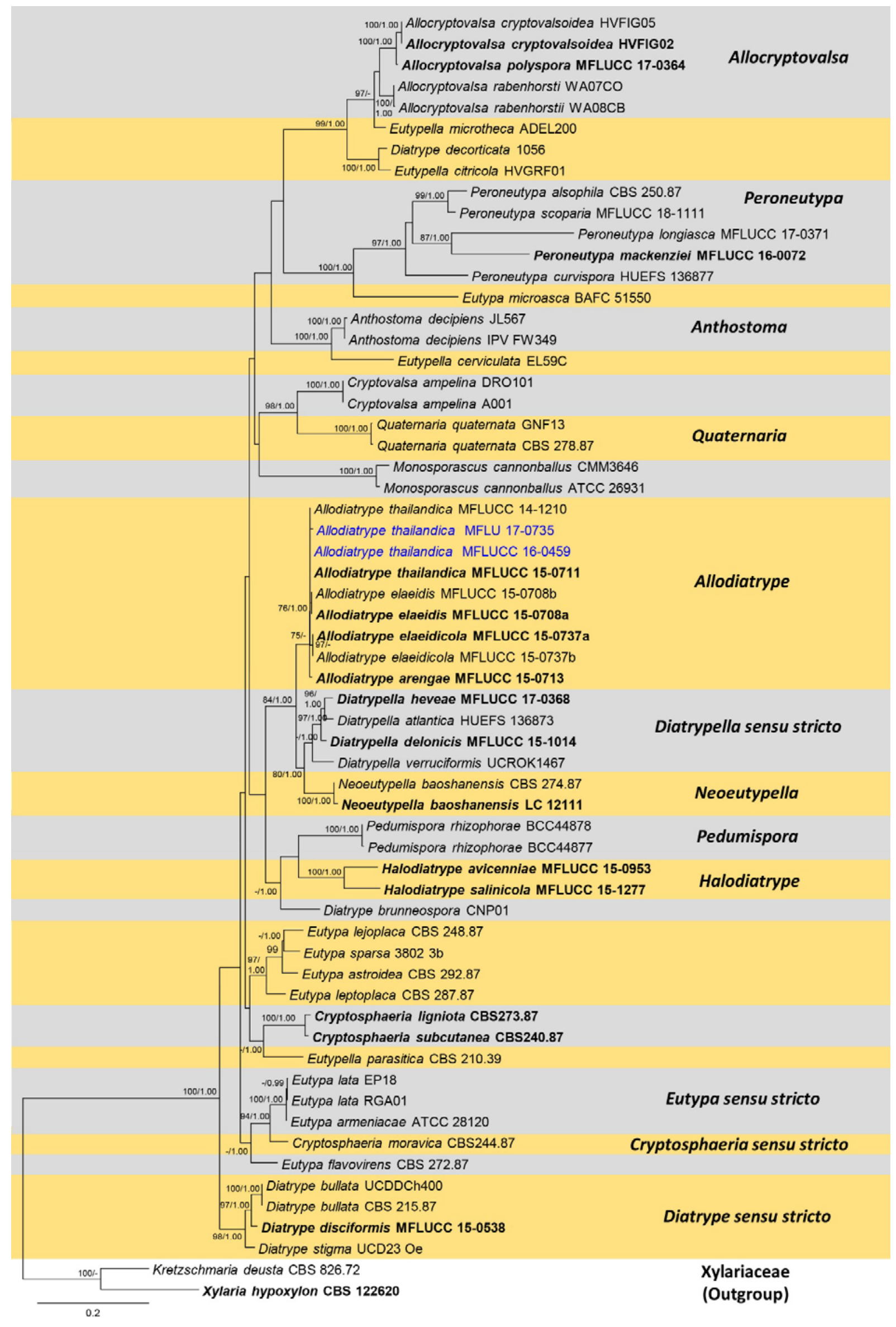

Figure 45 - Phylogram generated from RAxML analysis based on combined ITS and TUB2 sequence data of Diatrypaceae isolates. Sequences from sixty-one taxa, which comprise 993 
characters including gaps, were included in the analyses. The tree was rooted to Kretzschmaria deusta (CBS 826.72) and Xylaria hypoxylon (CBS 122620). The scale bar indicates 0.2 nucleotide changes per site.

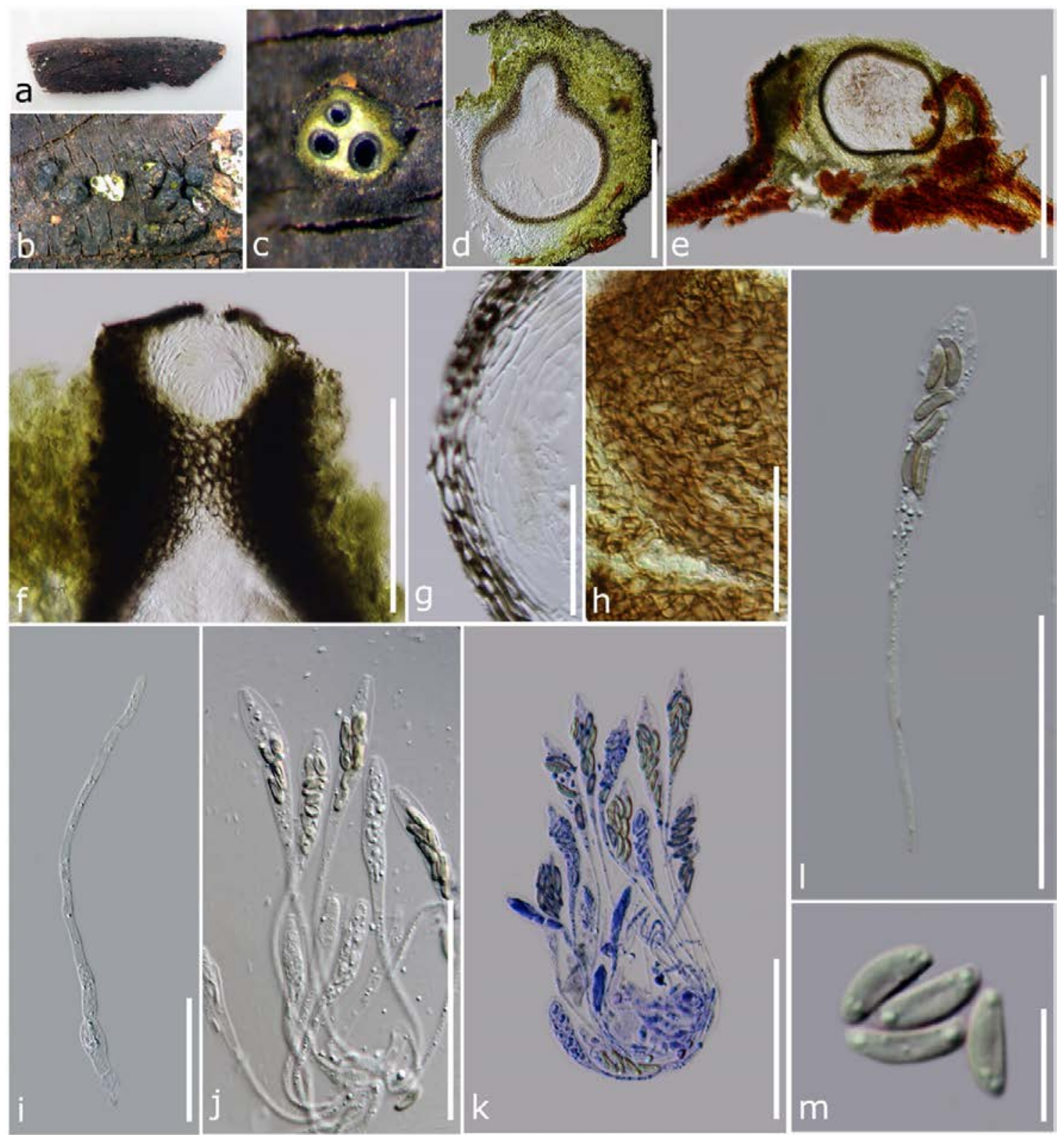

Figure 46 - Allodiatrype thailandica (MFLU 17-0735). a Herbarium material. b Appearance of ascomata on host substrate. c Cross section through stromata with ascomata. d, e Vertical section through stromata with ascomata. f Ostiole. g Peridium. h Peridium in face view. i Paraphyses. j-l Asci. m Ascospores. Scale bars: d, e $=200 \mu \mathrm{m}, \mathrm{f}-\mathrm{h}=100 \mu \mathrm{m}, \mathrm{i}=20 \mu \mathrm{m}, \mathrm{j}-\mathrm{l}=50 \mu \mathrm{m}, \mathrm{m}=10$ $\mu \mathrm{m}$.

Hypoxylaceae DC. emend. M. Stadler \& L. Wendt. Hypoxylon Bull.

Hypoxylon delonicis R.H. Perera, E.B.G. Jones \& K.D. Hyde, sp. nov.

Index Fungorum number: IF556864; Facesoffungi number: FoF07769

Etymology - Named after the host genus Delonix.

Holotype - MFLU 16-1031 


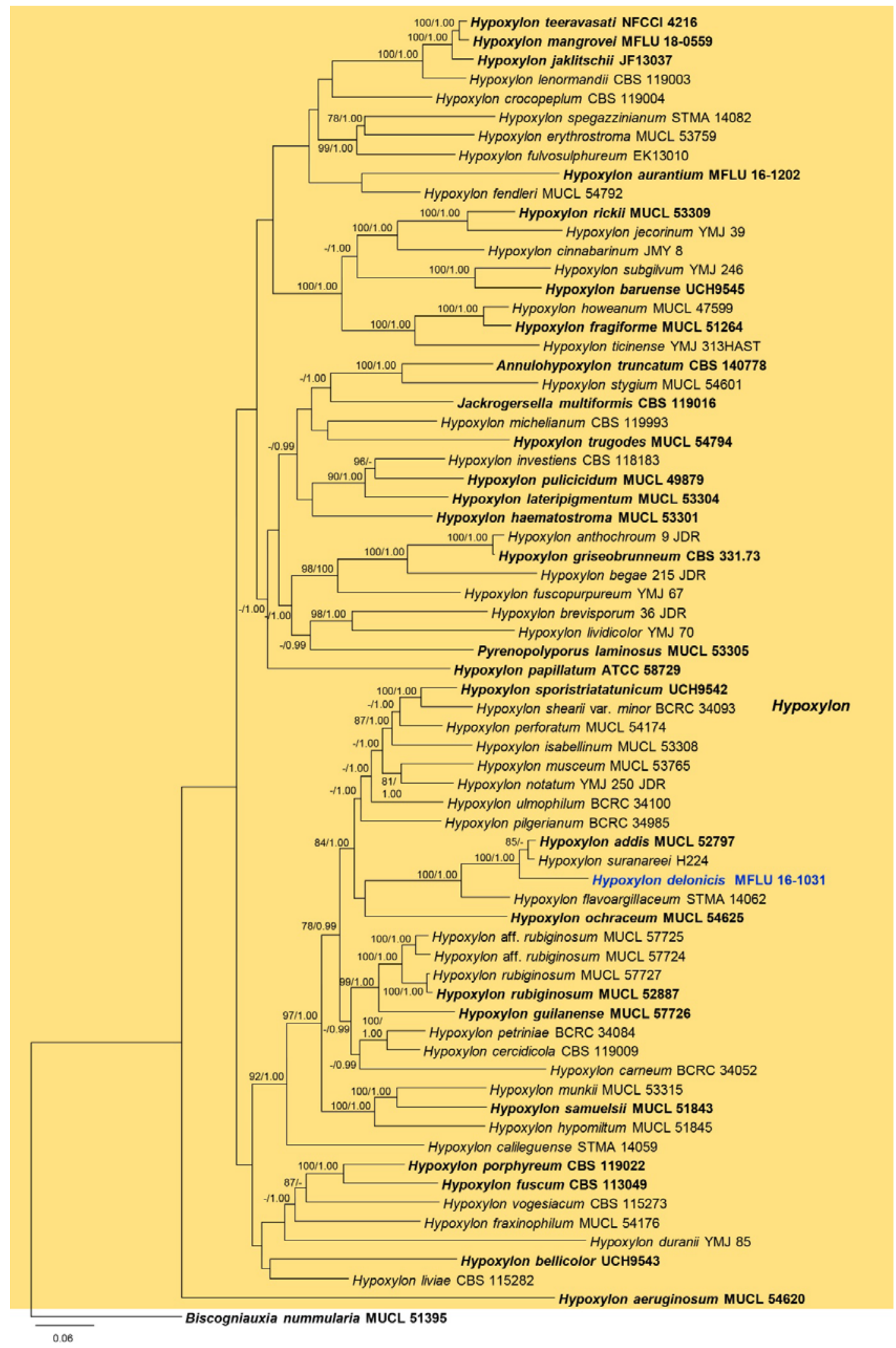

Figure 47 - Phylogram generated from RAxML analysis based on combined ITS and TUB2 sequence data of Hypoxylon isolates. Sequences from sixty-nine taxa, which comprise 2290 characters including gaps, are included in the analyses. The tree was rooted to Biscogniauxia nummularia (MUCL 51395). The scale bar indicates 0.06 nucleotide changes per site.

Notes - Colours were determined as in the previous literature of Hypoxylon species and the codes following Rayner (1970). 
Saprobic on dried seed pods of Delonix regia. Sexual morph: Ascostromata 1.3-2.3 mm diam., glomerate, effuse-pulvinate, with conspicuous perithecial mounds, surface dark Brick (60), orange red granules immediately beneath surface and between perithecia, with $\mathrm{KOH}$ extractable pigments Citrine (13). Ostioles umbilicate, opening at the centre of a raised disc. Ascomata 300$635 \times 200-485 \mu \mathrm{m}(\bar{x}=423 \times 328 \mu \mathrm{m})$, perithecial, obovoid. Peridium 20-75 $\mu \mathrm{m}$, multilayered, inner layers composed of flattened, hyaline cells of textura angularis, outer layers composed of brown cells of textura globosa. Paraphyses absent. Asci 110-140 × 8-12 $\mu \mathrm{m}(\bar{x}=126 \times 10 \mu \mathrm{m}, \mathrm{n}$ $=20$ ), 8-spored, unitunicate, cylindrical, pedicellate, apex simple. Ascospores 12.4-15 × 6.3-7.4 $\mu \mathrm{m}(\bar{x}=13.4 \times 6.8 \mu \mathrm{m}, \mathrm{n}=40)$, uniseriate, one-celled, ellipsoid-inequilateral, with narrowly rounded ends, brown to dark brown, with sigmoid germ slit spore-length, guttulate, guttules aggregated in ends when mature, perispore dehiscent in $10 \% \mathrm{KOH}$, smooth-walled, epispore smooth. Asexual morph: Undetermined.

Material examined - THAILAND, Chiang Rai Province, Mae Fah Luang University premises, on dried seed pods of Delonix regia (Fabaceae), 6 January 2015, R.H. Perera, RHP 26 (MFLU 16-1031, holotype).

GenBank numbers - ITS: MT215503, LSU: MT386008, TUB2: MT212215.

Notes - Phylogenetically Hypoxylon delonicis is related to $H$. addis and $H$. suranareei with high statistical support (100\% MLBT, 1.00 BIPP; Fig. 47). However, the holotype of $H$. suranareei was not available from the herbarium where it was reported to be deposited in the protologue, and the comparison with this species can only rely on the published data. Hypoxylon delonicis can be distinguished from $H$. suranareei by having larger ascomata 300-635 × 200-485 vs. 200-400 $\mu \mathrm{m}$, and broader asci (8-12 vs. 3.8-5 $\mu \mathrm{m}$ ) and ascospores (6.3-7.4 vs. 5-6.3 $\mu \mathrm{m}$ ) (fide Suwannasai et al. 2006). Hypoxylon delonicis differs from $H$. addis in having shorter asci lacking an apical apparatus (110-140 vs.170-190 $\mu \mathrm{m}$ ) and by its ascospores having a smooth (vs. faintly striate) perispore (Fournier et al. 2010b). Hypoxylon addis was collected from an Euphorbiaceae host in Ethiopia. Hypoxylon delonicis resembles $H$. shearii by glomerate stromata, but differs in having conspicuous raised discs around the ostioles, which are absent in H. shearii (Fournier et al. 2010b). Hypoxylon shearii has been collected from Quercus wood (Ju \& Rogers 1996, Fournier et al. 2010b). Hypoxylon delonicis is also similar to $H$. gibriacense by its glomerate stromata and lack of an apical apparatus in asci (Fournier et al. 2010b). However, $H$. delonicis differs from $H$. gibriacense in having larger ascospores $(12.4-15 \times 6.3-7.4$ vs. $11.5-13 \times 6-6.8 \mu \mathrm{m})$ and by its ascospores having a smooth (vs. fairly conspicuous striate) perispore (Fournier et al. 2010b). Hence, we introduce $H$. delonicis as a new species based on both morphological and molecular evidence.

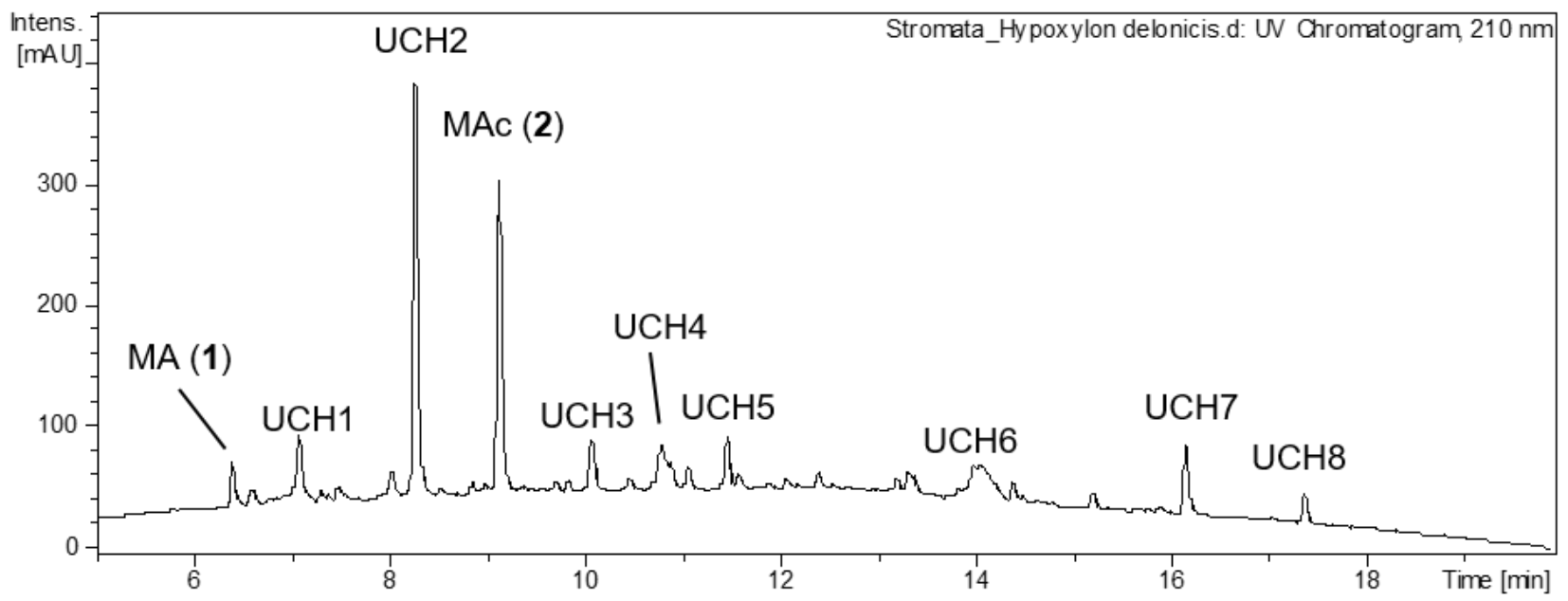

Figure 48 - HPLC-UV/VIS chromatogram of the stromatal extract of Hypoxylon delonicis at 210 nm. MA = Mitorubrinic acid (1), MAc = Mitorubrinol acetate (2), UCH1-8 = unknown compounds from $H$. delonicis. 


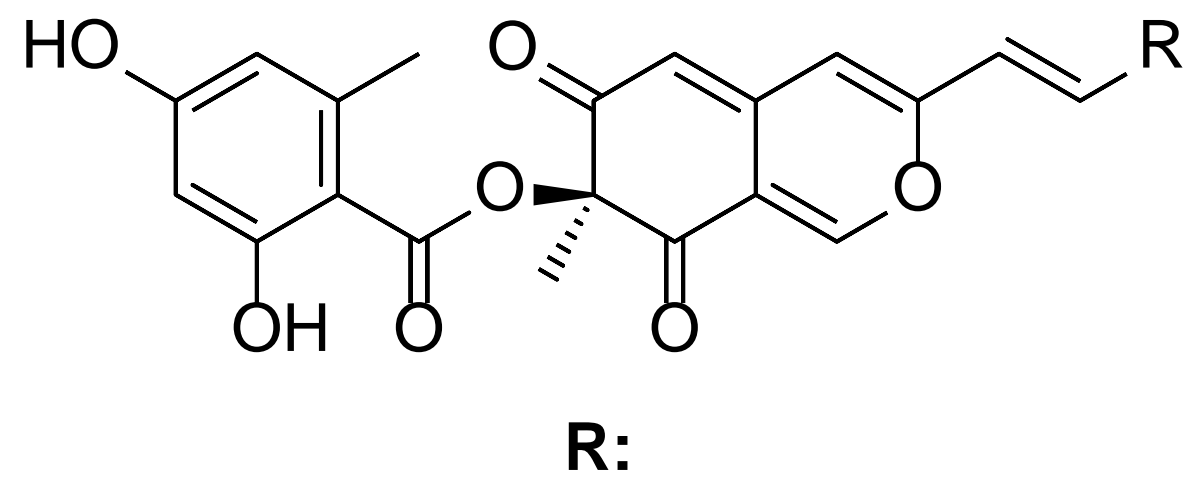

\section{$1-\mathrm{CH}_{2} \mathrm{AC}$ \\ $2-\mathrm{COOH}$}

Figure 49 - Known secondary metabolites found in stromatal extracts of Hypoxylon delonicis. 1 mitorubrinic acid. 2 mitorubrinol acetate.

Secondary metabolites: The stromatal extract was prepared and measured by HR-ESI-MS as described before (Kuhnert et al. 2017). The extract of Hypoxylon delonicis was found to contain the known azaphilone pigments mitorubrinol acetate (1) and mitorubrinic acid (2), which are widespread in Hypoxylon and form the core structures of more complicated metabolites (Surup et al. 2018) (Figs 48, 49). Besides, several peaks indicating probably representing yet unknown azaphilones were observed and labelled $\mathrm{UCH} 1-8$, with $\mathrm{UCH} 2$ even being the main peak within the extract and may accound for the citrine color of the stromatal extract in $\mathrm{KOH}$ (notably, mitorubrins give an orange colour in $\mathrm{KOH}$ ). It was of course not an option to attempt to isolate and identify these unknown components, to avoid destruction of valuable holotype specimen. However, it could be an option to watch out for the new fungus and collect more material for future mycochemical studies.

Xylariaceae Tul. \& C. Tul.

Xylaria Hill ex Grev.

Xylaria arbuscula Sacc., Michelia 1(no. 2): 249 (1878)

Figs 52, 53

Facesoffungi number: FoF07770

On decaying fruits of Swietenia mahagoni. Sexual morph: Stromata gregarious to solitary, usually unbranched or rarely branched near base, stipitate, two types: longer stromata 9.5-18 mm high $\times 2-2.3 \mathrm{~mm}$ diam., abundant, with fertile head 4-8.5 mm long, cylindrical, conical to acute at the apex; stunted stromata, 1-3 mm high $\times 0.6-1.4 \mathrm{~mm}$ diam., rare, very short-stipitate, broadly conical or flattened, fertile head conical $0.7-1.1 \mathrm{~mm}$ long, with a conical sterile apex or apically obtuse; without visible perithecial contours, surface black, glabrous, obscurely cracked and roughened; internal tissue white, brownish grey in the stipe, solid, $\mathrm{KOH}$. Ostioles obtusely papillate, black, often inconspicuous. Peridium 15-25 $\mu \mathrm{m}$ thick, composed of pale to brown cells of textura angularis. Ascomata 360-500 $\mu \mathrm{m}$ diam. $(\bar{x}=440 \mu \mathrm{m})$, completely immersed, perithecial, subglobose. Paraphyses 1.9-2.8 $\mu \mathrm{m}$ wide, hypha-like, septate, hyaline, thin-walled, embedded in gelatinous matrix. Asci $120-170 \times 6.5-7.8 \mu \mathrm{m}(\bar{x}=142 \times 7.2 \mu \mathrm{m})$, 8-spored, unitunicate, cylindrical, the spore-bearing parts $75-106 \mu \mathrm{m}$ long $(\bar{x}=90 \mu \mathrm{m})$, apical apparatus cylindrical, with a J+ apical ring, 3.2-3.6 × 2-2.6 $\mu \mathrm{m}(\bar{x}=3.4 \times 2.3 \mu \mathrm{m})$. Ascospores $12.4-18 \times 4.6-6.2 \mu \mathrm{m}$ $(\bar{x}=14.6 \times 5.4 \mu \mathrm{m})$, obliquely to transversely uniseriate, fusiform, inequilateral with narrowly rounded to subacute or slightly pinched ends, 1-2(-3) guttulate, brown, with a conspicuous, straight, germ slit slightly less than spore-length; epispore smooth, not dehiscent in $5 \% \mathrm{KOH}$. 
Asexual morph: Stromata 7-16 mm high $\times 1.3-2 \mathrm{~mm}$ diam., smaller than ascostroma, gregarious, unbranched, stipitate; head 5-7 mm in length, cylindrical, with a conical to acute apex; dark brown. Conidiophores 30-100 × 2-4 $\mu \mathrm{m}$, forming around the stromatal head, pale brown, dichotomously branched, septate. Conidia 2.5-4.7(-10) $\times 2-4.8 \mu \mathrm{m}(\bar{x}=4 \times 3 \mu \mathrm{m})$, globose, subglobose, elliptical or sometimes angular or oblong, aseptate, with a flat basal abscission scar, hyaline, thickand smooth-walled.

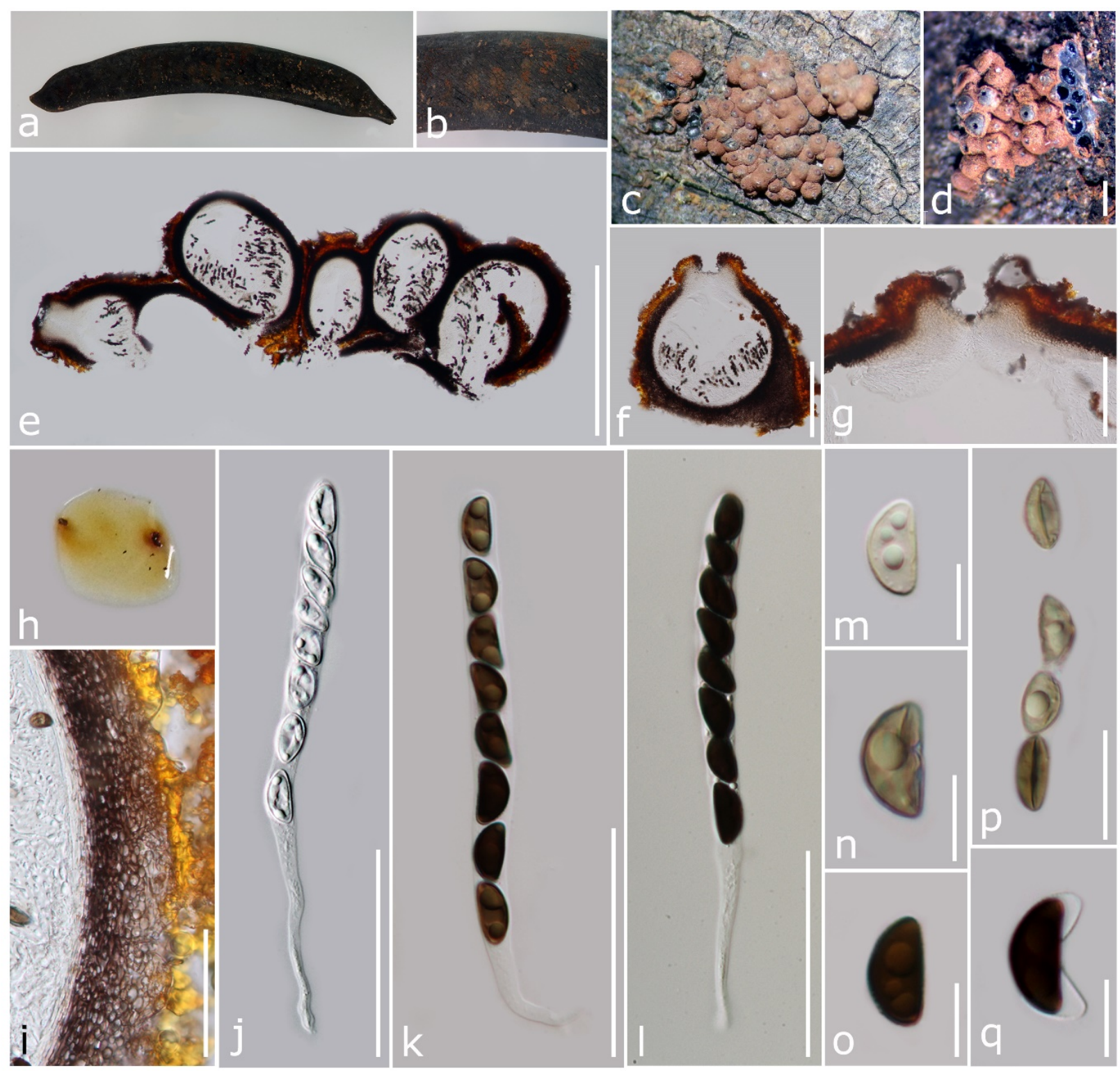

Figure 50 - Hypoxylon delonicis (MFLU 16-1031, holotype). a Herbarium material. b-d Appearance of ascomata on host substrate. e, f Vertical section through stromata with ascomata. g Ostiole. h Stromatal pigments in $10 \% \mathrm{KOH}$. i Close up of the peridium. j-l Immature and mature asci. m-o Immature and mature ascospores. p Ascospores with the germ slit. q Ascospore with released epispore in 5\% KOH. Scale bars: $d$, e $=500 \mu \mathrm{m}, \mathrm{f}=200 \mu \mathrm{m}, \mathrm{g}=100$ $\mu \mathrm{m}, \mathrm{i}-\mathrm{l}=50 \mu \mathrm{m}, \mathrm{m}-\mathrm{o}=10 \mu \mathrm{m}, \mathrm{p}=20 \mu \mathrm{m}, \mathrm{q}=10 \mu \mathrm{m}$.

Material examined - THAILAND, Chiang Rai Province, Mae Fah Luang University garden, on decaying fruits of Swietenia mahagoni (Meliaceae), 16 August 2015, R.H. Perera, RHP 21 (MFLU 16-1022); ibid., 16 December 2015, RHP 21b (MFLU 18-2721).

GenBank numbers - ITS: MT215513, LSU: MT215562 (MFLU 16-1022); ITS: MT215514, LSU: MT215563 (MFLU 18-2721). 


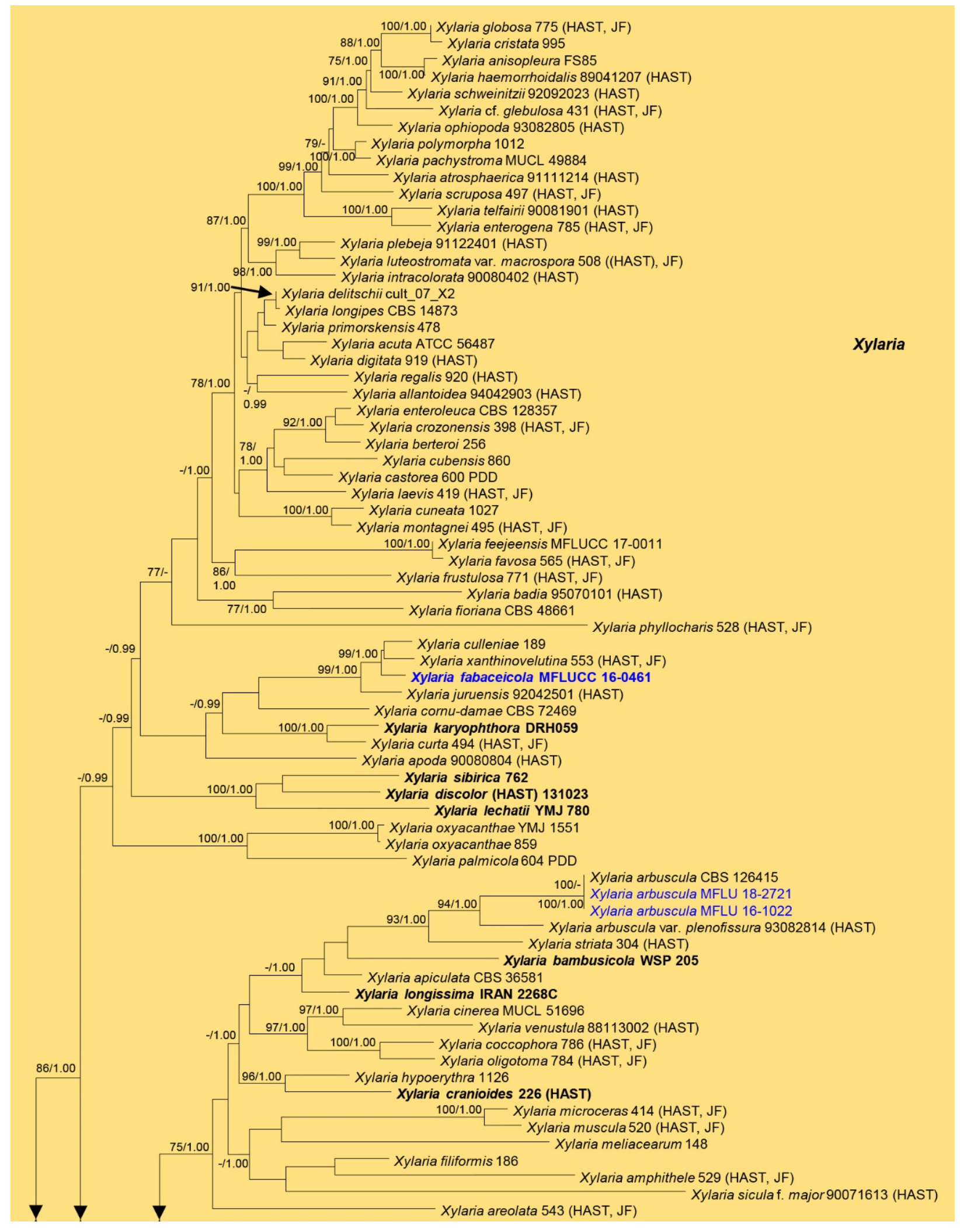

Figure 51 - Phylogram generated from RAxML analysis based on combined ITS, RPB2 and TUB2 sequence data of Xylaria isolates. Sequences from one hundred five taxa, which comprise 3476 characters including gaps, are included in the analyses. Tree was rooted to Biscogniauxia nummularia (MUCL 51395) and Hypoxylon fragiforme (MUCL 51264). The scale bar indicates 0.06 nucleotide changes per site. 


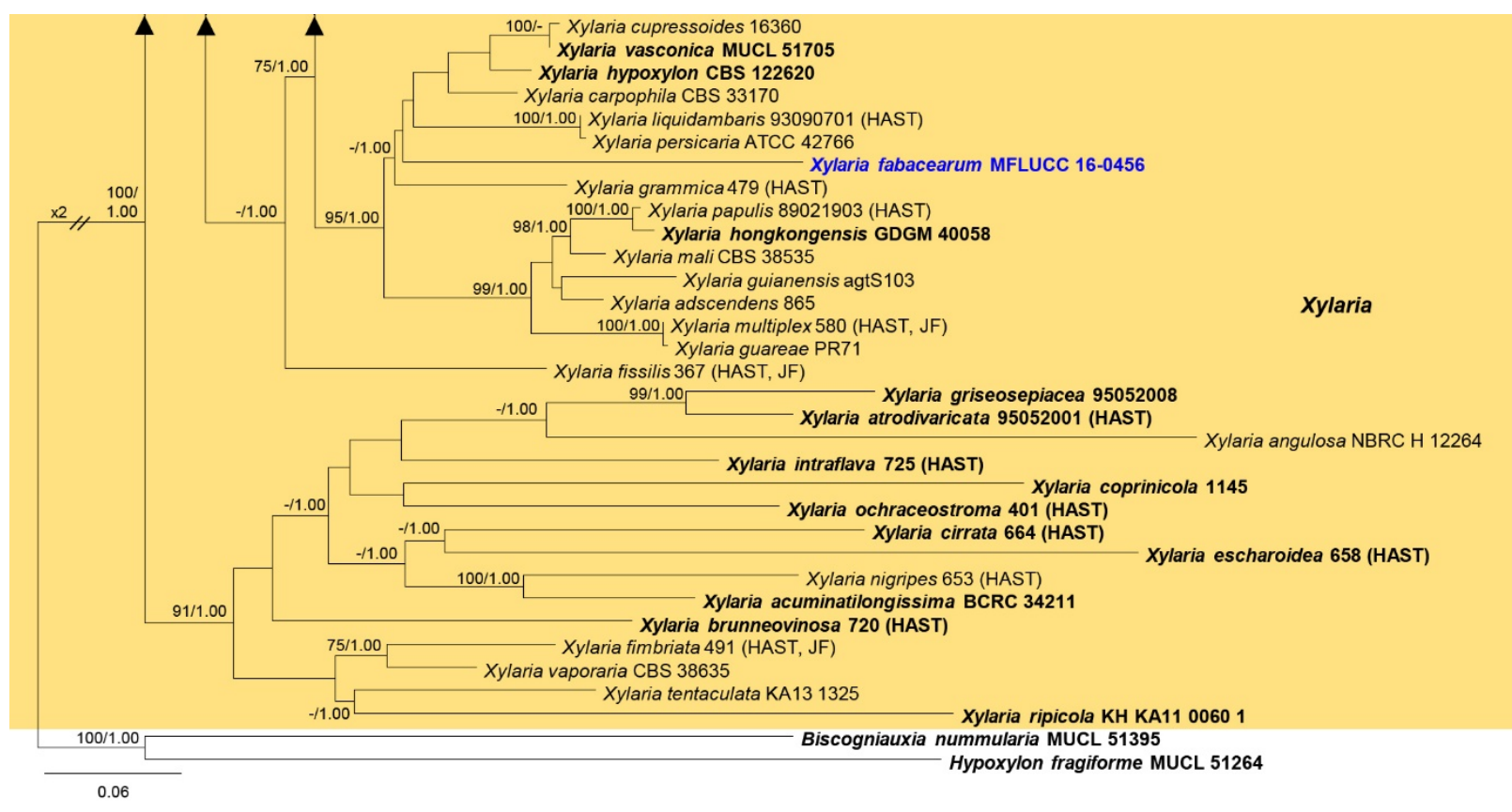

Figure 51 - Continued.

Notes - Wendt et al. (2017) and Daranagama et al. (2018) provided modern treatments of Xylariaceae which we follow here. Our new collections MFLU 16-1022 and MFLU 18-2721 grouped with Xylaria arbuscula (CBS 126415) with high statistical support in our phylogenetic analysis (Fig. 51). Specimens MFLU 16-1022 and MFLU 18-2721 belong to asexual morph and sexual morph stages, which were collected from Swietenia mahagoni fruits at the same location. The ITS and LSU gene regions of MFLU 16-1022 and MFLU 18-2721 are identical. Sexual morph of our collection was similar to $X$. arbuscula in morphology, asci and ascospore dimensions (Saccardo 1878, Fournier et al. 2010a). The holotype of $X$. arbuscula was collected from a greenhouse in Germany (Saccardo 1878). There was no asexual morph of the fungus reported so far (Saccardo 1878, Fournier et al. 2010a). By considering the morphological similarities, similarities in the ITS loci and phylogenetic analysis we identify our collection as $X$. arbuscula (Jeewon \& Hyde 2016). This is the first record of $X$. arbuscula from Swietenia mahagoni and the first report of its asexual morph. Unfortunately, we were unable to amplify the RPB2 gene region of the fungus despite several attempts.

Xylaria fabacearum R.H. Perera, E.B.G. Jones \& K.D. Hyde, sp. nov.

Fig. 54 Index Fungorum number: IF556865; Facesoffungi number: FoF07771

Etymology - Named after the host family Fabaceae.

Holotype - MFLU 16-1061

Saprobic on decaying seed pods of Fabaceae. Sexual morph: Stromata 4.5-16 mm long $\times$ 0.9-1.8 mm diam., fertile part up to $9 \mathrm{~mm}$ long $\times 1.8 \mathrm{~mm}$ diam., cylindrical, branched or unbranched, solitary or clustered, stipe, tomentose, black, with sterile apices. External surface black, non-tomentose, roughened with ascomatal contours. Internal tissue white, not becoming hollow. Ascomata 290-480 $\mu \mathrm{m}$ high $\times 270-450 \mu \mathrm{m}$ diam. $(\bar{x}=390 \times 376 \mu \mathrm{m})$, perithecial, globose to subglobose, mammiform, more less immersed, ostioles black, slightly papillate. Peridium 20-30 $\mu \mathrm{m}$ wide, composed of up to 12 layers of light brown-walled cells of textura angularis to textura prismatica, integrated with inner stroma cells. Paraphyses 1.4-2.4 $\mu \mathrm{m}$ wide, septate, hyaline. Asci 8-spored, 90-135 × 4-6 $\mu \mathrm{m}(\bar{x}=109 \times 5 \mu \mathrm{m} ; \mathrm{n}=25)$, cylindrical, long stipitate, the spore-bearing part 56-65 $\mu \mathrm{m}$ long, with an inverted hat-shaped, $\mathrm{J}+$ apical ring $2-2.5 \times$ 1.5-2 $\mu \mathrm{m}(\bar{x}=2.3 \times 1.7 \mu \mathrm{m}, \mathrm{n}=10)$. Ascospores uniseriate to overlapping uniseriate, 9-11.2 $\times 3-$ $4.3 \mu \mathrm{m}(\bar{x}=10 \times 3.6 \mu \mathrm{m}, \mathrm{n}=35)$, ellipsoid-inequilateral, brown to dark brown, aseptate, guttulate; 
germ slit conspicuous, straight, running full-length of the ascospore, perispore indehiscent in $10 \%$ $\mathrm{KOH}$; epispore smooth, lacking a hyaline sheath. Asexual morph: Undetermined.

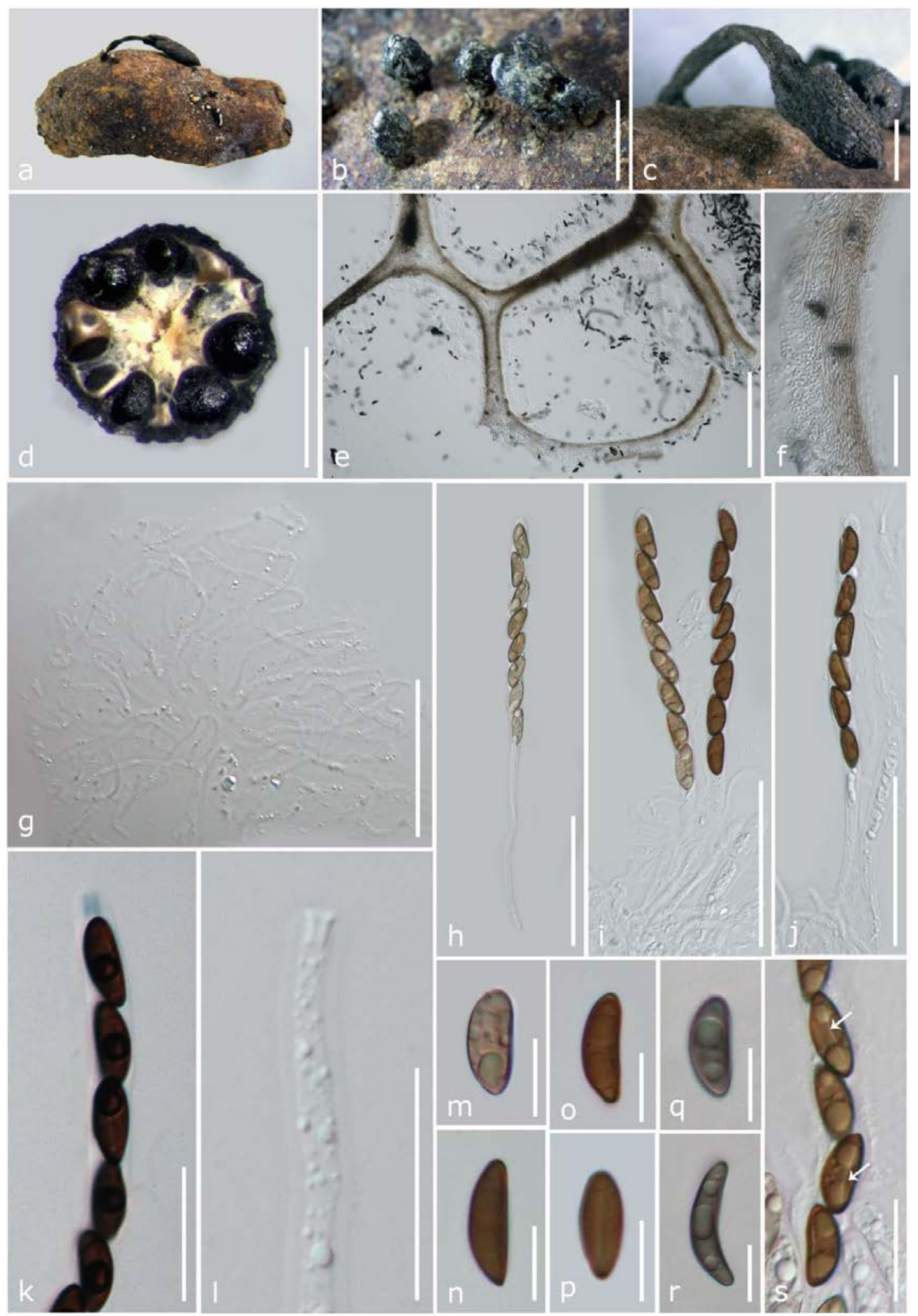

Figure 52 - Xylaria arbuscula (MFLU 18-2721). a Herbarium material. b, c Appearance of ascostromata on host substrate. d Cross section through stromata with ascomata. e Vertical section through ascomata. f Close up of the peridium. g Paraphyses. h-j Asci. k, l Close up of apical ring (k in Melzer's iodine reagent). $\mathrm{m}-\mathrm{s}$ Ascospores (q, $\mathrm{r}$ in $10 \% \mathrm{KOH}, \mathrm{s}$ arrows showing the germ slit). 
Scale bars: c, $d=2 \mathrm{~mm}, \mathrm{~d}=500 \mu \mathrm{m}, \mathrm{e}=200 \mu \mathrm{m}, \mathrm{f}-\mathrm{j}=50 \mu \mathrm{m}, \mathrm{k}, \mathrm{l}=20 \mu \mathrm{m}, \mathrm{m}-\mathrm{r}=10 \mu \mathrm{m}, \mathrm{s}=20$ $\mu \mathrm{m}$.

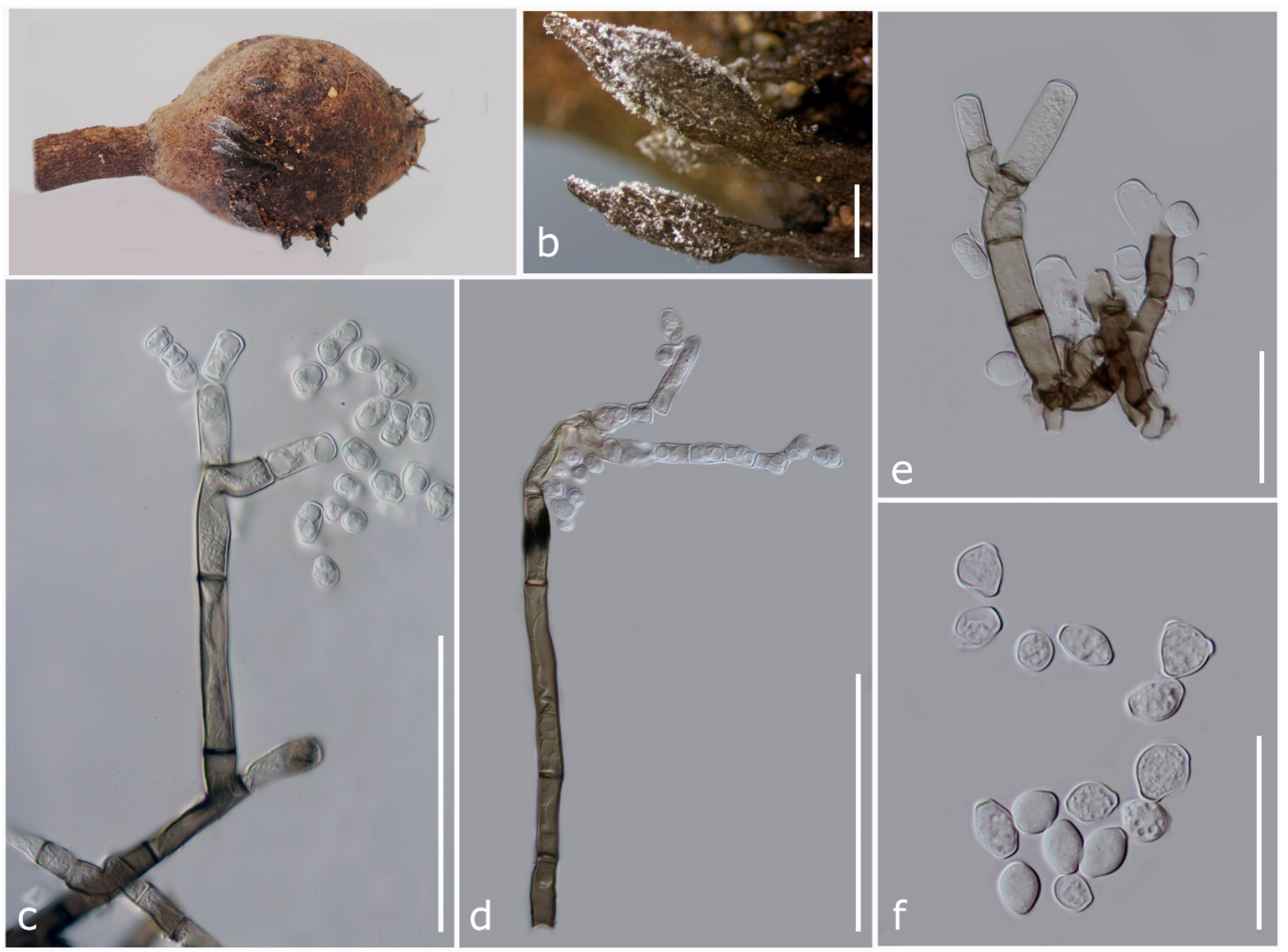

Figure 53 - Xylaria arbuscula (MFLU 16-1022). a Herbarium material. b Conidiophores on stromata. c-e Conidiophores with conidia. f Conidia. Scale bars: b $=200 \mu \mathrm{m}, \mathrm{c}, \mathrm{d}=50 \mu \mathrm{m}$, e, $\mathrm{f}=20 \mu \mathrm{m}$.

Culture characteristics - Ascospores germinating on PDA within 14 hours. Colonies growing on MEA, reaching $30 \mathrm{~mm}$ in 14 days at $28^{\circ} \mathrm{C}$, flat, initially white, aerial mycelium forming concentric rings with cottony texture, margin undulate, white to ash or black above, reverse dark brown.

Material examined - THAILAND, Chiang Mai Province, on decaying seed pods of Fabaceae, 20 December 2015, R.H. Perera, DeloL4, (MFLU 16-1061, holotype); ex-type living culture MFLUCC 16-0456.

GenBank numbers - ITS: MT215512, LSU: MT215561, RPB2: MT212202, TUB2: MT212220.

Notes - Xylaria fabacearum (MFLUCC 16-0456) groups apart from other Xylaria species in an unsupported clade (Fig. 51). Xylaria fabacearum resembles $X$. culleniae, $X$. fabaceicola and $X$. xanthinovelutina (which were mostly isolated from leguminous fruits), but differs mainly by having ascospores that lack a hyaline sheath (Ju et al. 2018). Xylaria fabacearum can be distinguished from $X$. luzonensis (which is also isolated from leguminous pods) by its larger, brown to dark brown ascospores $(9-11.2 \times 3-4.3$ vs. 8.5-9.5 $\times 3-3.5 \mu \mathrm{m})$, while $X$. luzonensis has light brown ascospores (Ju et al. 2018). Hence, we identified our collection as a new species of Xylaria. 


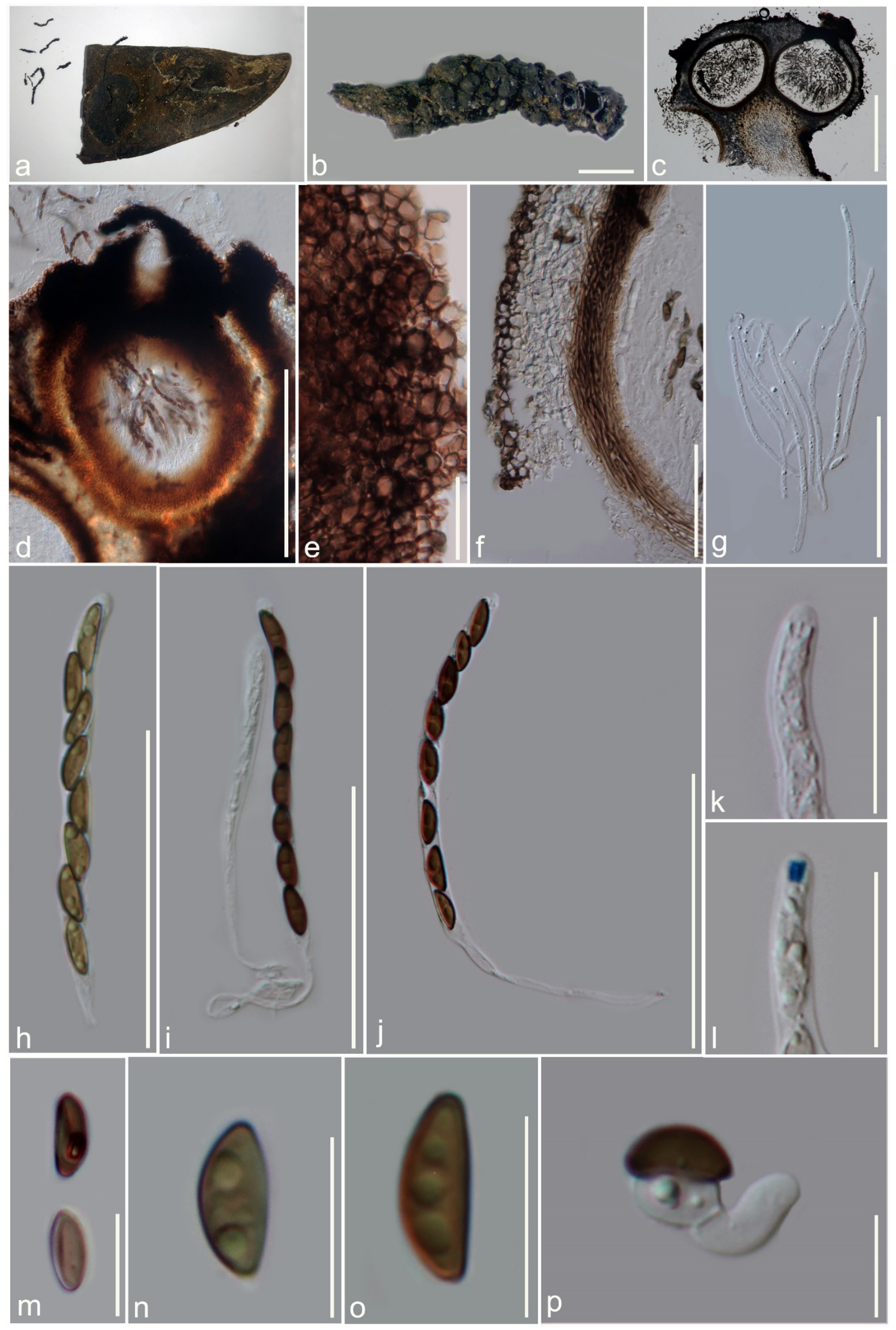

Figure 54 - Xylaria fabacearum (MFLU 16-1061, holotype). a Herbarium material. b Appearance of ascomata. c, d Vertical section through stroma with ascomata. e Face view of the peridium. 
f Section through peridium. g Paraphyses. h-l Asci. (l in Melzer's reagent). m-o Ascospores. p Germinating ascospore. Scale bars: $b=1 \mathrm{~mm}, \mathrm{c}, \mathrm{d}=200 \mu \mathrm{m}, \mathrm{e}=20 \mu \mathrm{m}, \mathrm{f}-\mathrm{j}=50 \mu \mathrm{m}, \mathrm{k}-\mathrm{l}=20$ $\mu \mathrm{m}, \mathrm{m}-\mathrm{o}=10 \mu \mathrm{m}, \mathrm{p}=5 \mu \mathrm{m}$.

Xylaria fabaceicola R.H. Perera, E.B.G. Jones \& K.D. Hyde, sp. nov.

Fig. 55

Index Fungorum number: IF556866; Facesoffungi number: FoF07772

Etymology - Named after the host family Fabaceae.

Holotype - MFLU 16-1072

Saprobic on decaying seed pods of Fabaceae. Sexual morph: Stromata 13-25 mm long $\times 0.6$ $1.5 \mathrm{~mm}$ diam., fertile part up to $12 \mathrm{~mm}$ long $\times 1.2 \mathrm{~mm}$ diam., cylindrical, branched or unbranched, solitary or clustered, with sterile apices, stipe tomentose, black. External surface black, tomentose, roughened with ascomatal contours. Internal tissue white, not becoming hollow. Ascomata 360$550 \mu \mathrm{m}$ high $\times 300-440 \mu \mathrm{m}$ diam. $(\bar{x}=480 \times 375 \mu \mathrm{m})$, perithecial, mammiform, more or less immersed, ostioles black, slightly papillate. Peridium 30-45 $\mu \mathrm{m}$ wide, two regions, outer region of 8-10 layers of hyaline to light brown, thick-walled cells of textura prismatica; inner region of 4-6 layers of hyaline cells of textura angularis. Paraphyses 3-4 $\mu \mathrm{m}$ wide, septate, hyaline, tapering towards the apex. Asci 100-130 × 4.6-6.5 $\mu \mathrm{m}(\bar{x}=117 \times 5.6 \mu \mathrm{m}, \mathrm{n}=20)$ diam., unitunicate, 8spored, cylindrical, long stipitate, the spore-bearing part 56-65 $\mu \mathrm{m}$, with an inverted hat-shaped, J+ apical ring 1.7-2.3 $\mu \mathrm{m} \times 1.3-1.8 \mu \mathrm{m}(\bar{x}=2 \times 1.5 \mu \mathrm{m}, \mathrm{n}=10)$ diam. Ascospores 7.5-10 $\times 3.4-4.8$ $\mu \mathrm{m}(\bar{x}=8.5 \times 4.2 \mu \mathrm{m} ; \mathrm{n}=25)$ diam., uniseriate to overlapping uniseriate, ellipsoid-inequilateral, pale brown to brown, aseptate, guttulate; germ slit conspicuous, straight, slightly less than the spore length; surrounded with a hyaline sheath swelling at both ends to form non-cellular appendages, perispore indehiscent in 10\% KOH, epispore smooth. Asexual morph: Undetermined.

Culture characteristics - Ascospores germinating on PDA within 12 hours. Colonies growing on MEA, reaching up to $55 \mathrm{~mm}$ in 14 days at $28^{\circ} \mathrm{C}$, flat, initially white, aerial mycelium forming concentric rings with cottony texture, margin undulate, white to ash or black, reverse dark brown.

Material examined - THAILAND, Chiang Mai Province, Mushroom Research Center, on decaying seed pods of Fabaceae, 23 December 2015, R. H. Perera, M-8 (MFLU 16-1072, holotype); ex-type living culture MFLUCC 16-0461.

GenBank numbers - ITS: MT215511, LSU: MT215560, RPB2: MT212201, TUB2: MT212219.

Notes - Xylaria fabaceicola (MFLUCC 16-0461) shows close phylogenetic relationship to $X$. xanthinovelutina, $X$. culleniae (which were mostly isolated from leguminous fruits), and $X$. juruensis (Fig. 51). Xylaria fabaceicola can be distinguished by $X$. xanthinovelutina by smaller ascospores (7.5-10 × 3.4-4.8 vs. 9-12 × 3.5-5 $\mu \mathrm{m})$, and shorter spore-bearing parts of its asci (5665 vs. 65-85 $\mu \mathrm{m}$ ) (Ju et al. 2018). A comparison of the ITS, RPB2 and TUB2 sequences of $X$. fabaceicola and $X$. xanthinovelutina (553 HAST, JF) revealed 13 (2.4\%), 13 (1.4\%) and 29 (4\%) nucleotide differences, respectively, in each DNA locus. Xylaria fabaceicola resembles $X$. culleniae, from which it differs by having a smaller stroma $(13-25 \times 0.6-1.5 \mathrm{~mm}$ vs. $55-70 \times 2-4$ $\mathrm{mm}$ ) (Ju et al. 2018). In considering morphological and, nucleotide differences in ITS, RPB2 and TUB2 regions, we identified our collection as a new Xylaria species.

There are several seed and fruit inhabiting Xylaria species, which lack DNA sequence data: Xylaria duranii, X. euphorbiicola, X. guazumae, X. heloidea, X. himalayensis, X. jaliscoensis, X. patrisiae, $X$. psidii, $X$. rhizocola, $X$. rossmanae and $X$. warburgii (Ju et al. 2018). We also compared our collections $X$. fabacearum and X. fabaceicola with these species based on morphological descriptions provided in Ju et al. (2018).

Helotiales Nannf. ex Korf \& Lizoň

Helotiales sp.

Fig. 56

Saprobic on decaying cones of Pseudotsuga menziesii. Sexual morph: 275-420 × 275-350 $\mu \mathrm{m}(\bar{x}=360 \times 300 \mu \mathrm{m}, \mathrm{n}=10)$. Ascomata apothecial, gregarious or in small groups, sessile, 
slightly erumpent from the substrate. Disc yellow, slightly concave or flat and smooth. Receptacle subspherical, concolorous, clothed with hyaline hairs. Hairs 94-105 × 5.9-6.6 $\mu \mathrm{m}(\bar{x}=98 \times 6.2$ $\mu \mathrm{m}, \mathrm{n}=20)$, tapered, 5-septate, walls usually thin. Ectal excipulum 13-18 $\mu \mathrm{m}(\bar{x}=15.0 \mu \mathrm{m}, \mathrm{n}=$ 10), composed of thin-walled, hyaline cells of textura globulosa. Medullary excipulum 76-112 $\mu \mathrm{m}$ $(\bar{x}=89 \mu \mathrm{m}, \mathrm{n}=10)$ composed of narrow, thin-walled, hyaline cells of textura epidomoidea. Hymenium hyaline. Paraphyses 0.9-1.2 $\mu \mathrm{m}$ wide $(\bar{x}=1.0 \mu \mathrm{m}, \mathrm{n}=20)$, numerous, filiform, obtuse at the apex, non-septate. Asci 91-105 × 6.6-9.6 $\mu \mathrm{m}(\bar{x}=96 \times 8.7 \mu \mathrm{m}, \mathrm{n}=30)$, 8-spored, cylindricclavate, unitunicate, tapered, with a stipitate base, croziers present at the base of asci, conical at the apex, with an amyloid ring at apex, partly blue in Meltzer's reagent. Ascospores 52-58.6 × 2.3-2.9 $\mu \mathrm{m}(\bar{x}=56.5 \times 2.6 \mu \mathrm{m}, \mathrm{n}=40)$, lying parallel in a single long fascicle within the ascus, hyaline, filiform-cylindric, slightly tapered to the lower end, rounded at the poles, 7-septate. Asexual morph: Undetermined.

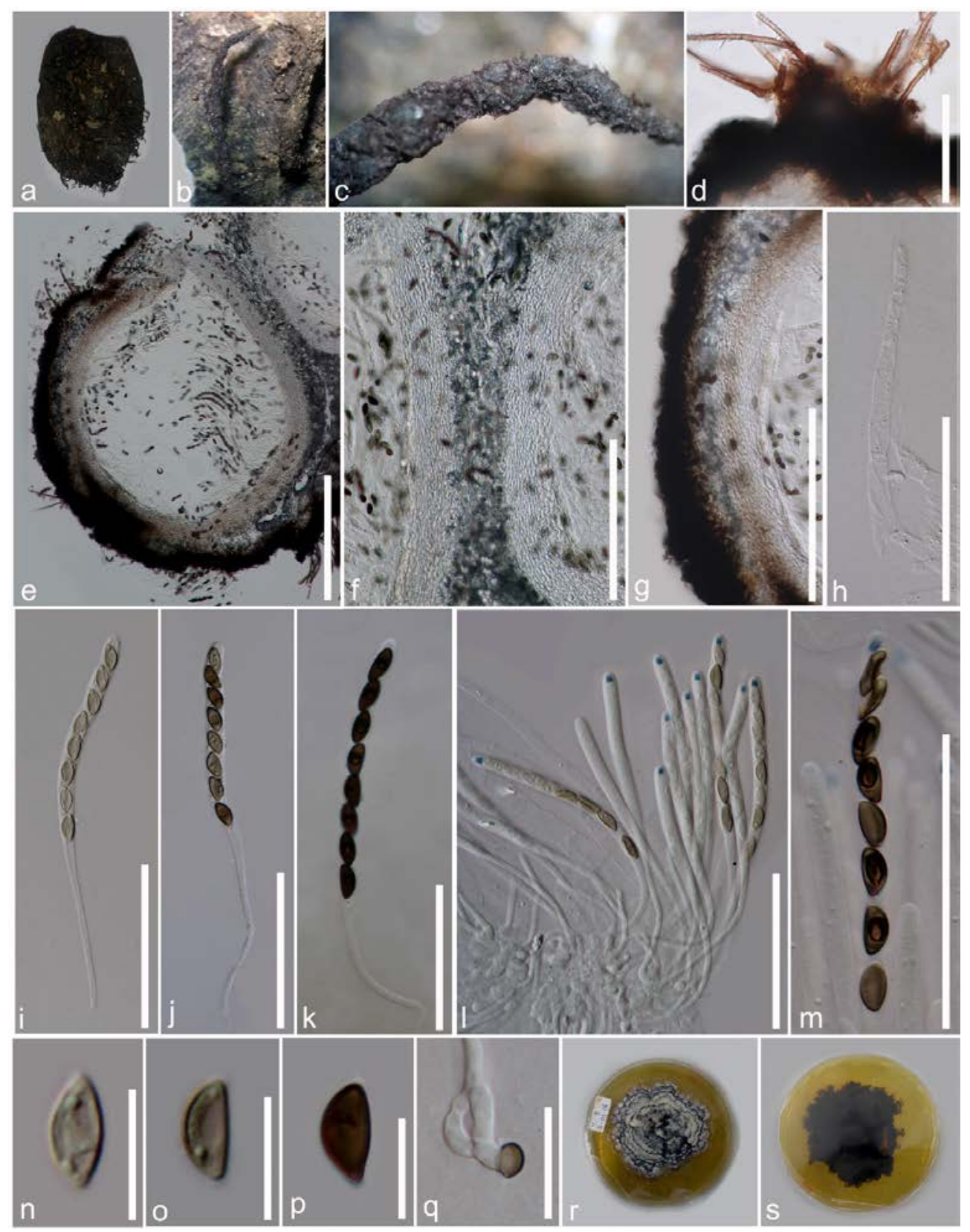

Figure 55 - Xylaria fabaceicola (MFLU 16-1072, holotype). a Herbarium material. b, c Appearance of ascomata on host substrate. d Hairs. e Vertical section through stromata with 
ascoma. f, g Peridium. h Paraphyses. i-l Asci. (l in Melzer's reagent) m Ascospores showing germ slit. n-p Ascospores. q Germinating ascospore. $r$, s Colonies on MEA. Scale bars: $d=50 \mu \mathrm{m}, \mathrm{e}=$ $200 \mu \mathrm{m}, \mathrm{f}=100 \mu \mathrm{m}, \mathrm{g}-\mathrm{m}=50 \mu \mathrm{m}, \mathrm{n}-\mathrm{q}=10 \mu \mathrm{m}$.

Material examined - UK, Hampshire, New Forest, on decaying cones of Pseudotsuga menziesii (Mirb.) Franco (Pinaceae), 24 November 2014, E.B.G. Jones, GJ076 (MFLU 16-0560).

Notes - We identified our collection as a member of Helotiales based on morphological resemblance to the order (Ekanayaka et al. 2017, 2019).

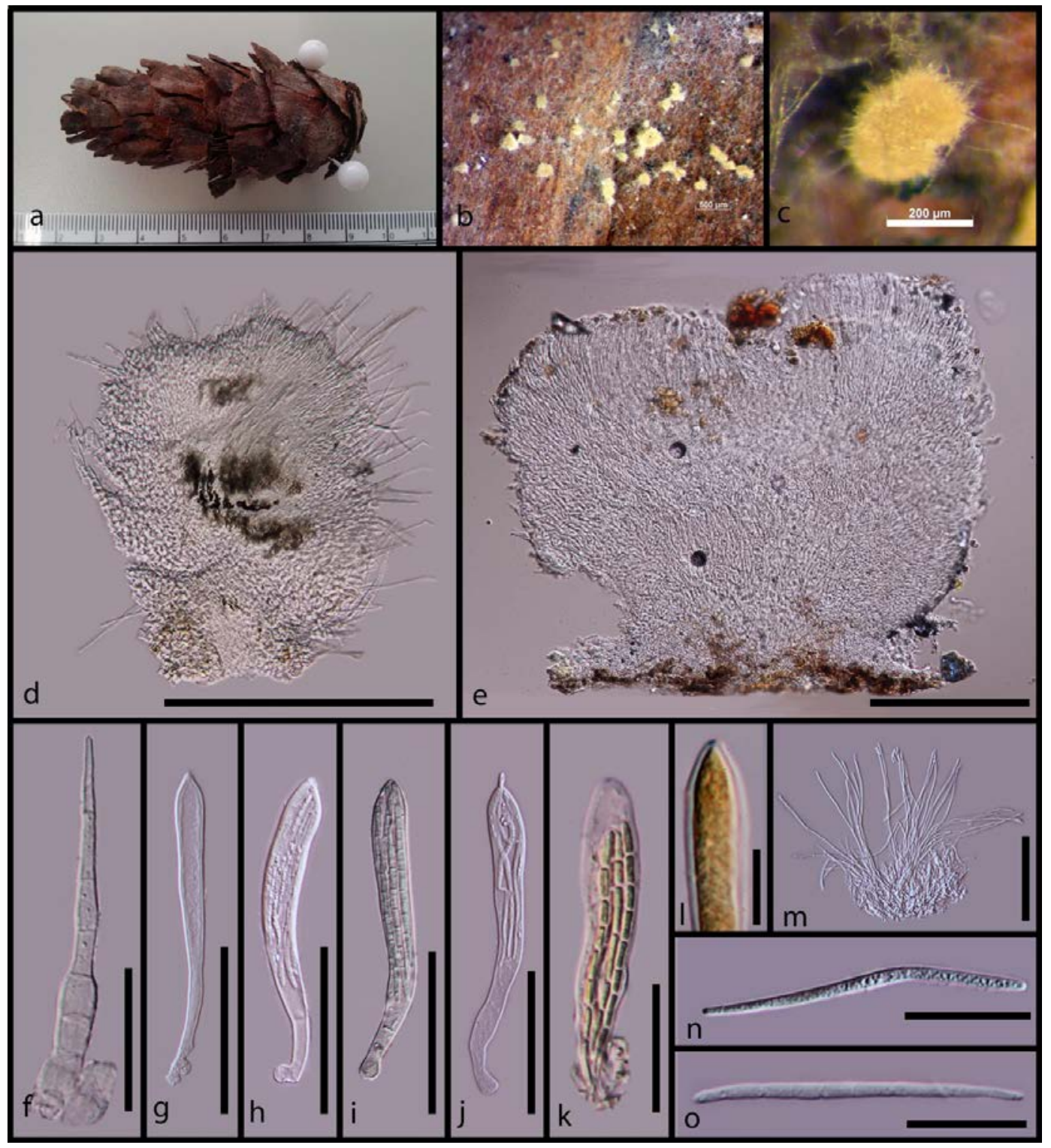

Figure 56 - Helotiales sp. (MFLU 16-0560). a Herbarium material. b, c Ascomata on host. $\mathrm{d}$, e Vertical section of ascomata. f Septate hairs. $\mathrm{g}-\mathrm{k}$ Asci. 1 Amyloid ring at the ascus apex. m Paraphyses. $\mathrm{n}$, o Ascospores. Scale bars: $\mathrm{b}=500 \mu \mathrm{m}, \mathrm{c}=200 \mu \mathrm{m}, \mathrm{d}=200 \mu \mathrm{m}, \mathrm{e}=100 \mu \mathrm{m}$, $\mathrm{f}=70 \mu \mathrm{m}, \mathrm{g}-\mathrm{k}=50 \mu \mathrm{m}, 1=20 \mu \mathrm{m}, \mathrm{m}=50 \mu \mathrm{m}, \mathrm{n}, \mathrm{o}=20 \mu \mathrm{m}$.

Lachnaceae Raitv.

Lachnum Retz.

Lachnum sp.

Fig. 57 


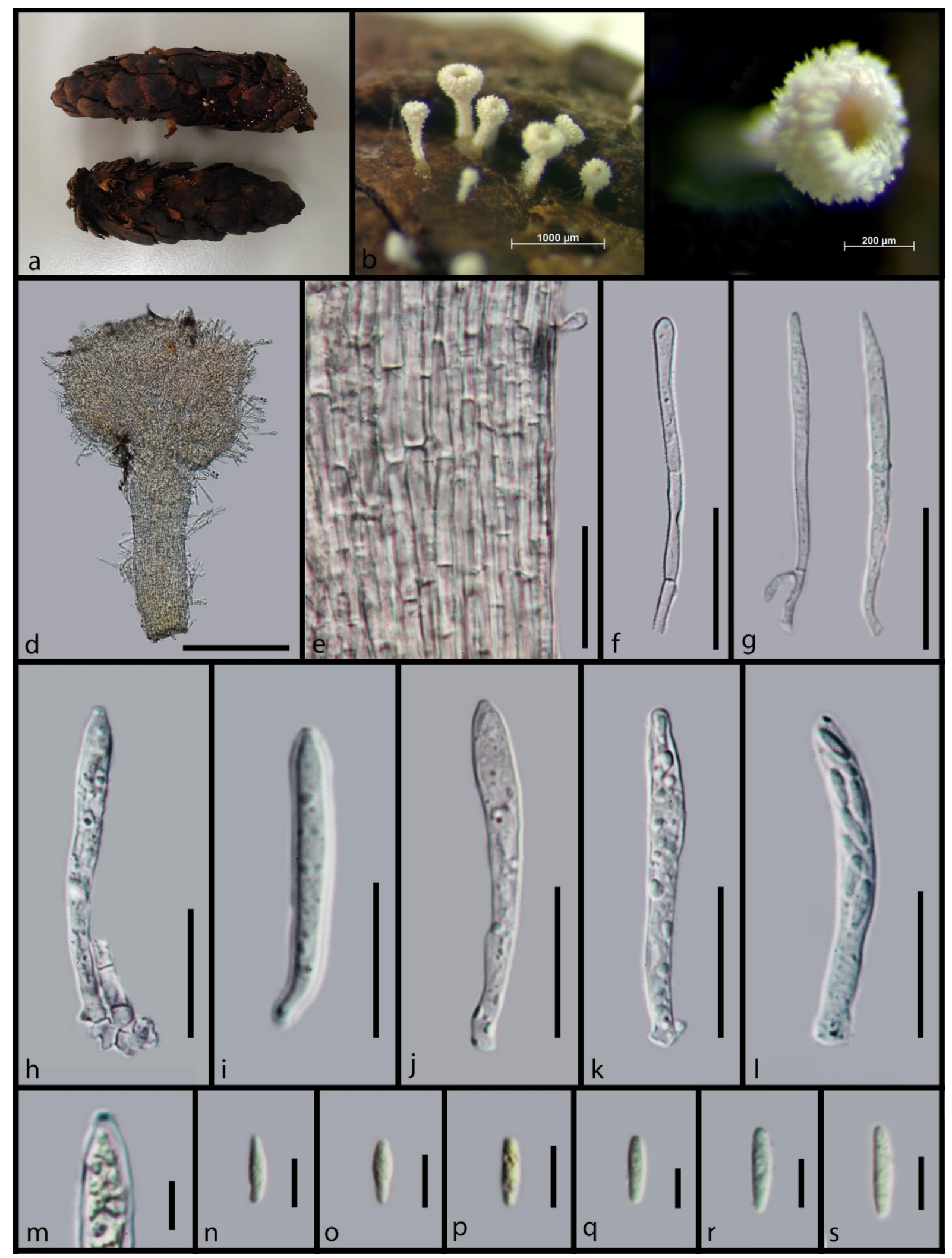

Figure 57 - Lachnum sp. (MFLU 16-1863). a Herbarium material. b, c Apothecia on host. d Vertical section of an apothecium. e Excipular cells. f Cylindric hairs. g Paraphyses. h Ascus arising from croziers. i-l Asci. m Amyloid apical ring. $\mathrm{n}-\mathrm{s}$ Ascospores. Scale bars: $\mathrm{b}=1 \mathrm{~mm}$, $\mathrm{c}=200 \mu \mathrm{m}, \mathrm{d}=300 \mu \mathrm{m}, \mathrm{e}=50 \mu \mathrm{m}, \mathrm{f}=25 \mu \mathrm{m}, \mathrm{g}=30 \mu \mathrm{m}, \mathrm{h}-\mathrm{l}=20 \mu \mathrm{m}, \mathrm{m}-\mathrm{s}=5 \mu \mathrm{m}$.

Saprobic on decaying cones of Pseudotsuga menziesii. Sexual morph: Apothecia 0.6-1 mm diam., arising singly or in small groups, stipitate, white when fresh, margins, flanks and stipe 
covered with hairs. Receptacle cupulate, disc concave, disc and the margins are white when fresh. Hairs 40-65 $\times 2.8-3.6 \mu \mathrm{m}(\bar{x}=51.2 \times 3.2 \mu \mathrm{m}, \mathrm{n}=$ 30) cylindric, septate, hyaline, walls usually thin, surface finely granulate. Ectal excipulum $8-15 \mu \mathrm{m}(\bar{x}=11 \mu \mathrm{m}, \mathrm{n}=10)$ in flanks, composed of thinwalled, long, hyaline cells of textura prismatica. Medullary excipulum in flanks, composed of thin-walled, hyaline cells of textura porrecta. Hymenium hyaline. Paraphyses $2-4 \mu \mathrm{m}$ wide $(\bar{x}=2.8 \mu \mathrm{m}, \mathrm{n}=20)$, numerous, lanceolate, septate, exceed asci in length. Asci 35-50 $\times 3.7-4.2 \mu \mathrm{m}(\bar{x}$ $=46.3 \times 4.1 \mu \mathrm{m}, \mathrm{n}=30$ ), 8-spored, unitunicate, cylindric-clavate, conical at the apex, amyloid ring present at the ascus apex, partly blue in Meltzer's reagent, stipitate base, arising from croziers. Ascospores 5.5-8.2 × 1.2-2.2 $\mu \mathrm{m}(\bar{x}=6.5 \times 1.8 \mu \mathrm{m}, \mathrm{n}=40)$, 1-2-seriate, fusoid-clavate, aseptate, hyaline. Asexual morph: Undetermined.

Material examined - UK, Hampshire, New Forest, on decaying cones of Pseudotsuga menziesii (Pinaceae), 16 May 2015, E.B.G. Jones, GJ158 (MFLU 16-1863).

Notes - We identified our collection as a Lachnum species based on morphological resemblance to the genus (Ekanayaka et al. 2017).

\section{Fungal check list}

Table 2 Fungi associated with wild seeds and fruits

Fungi are listed in alphabetical order after the Phylum.

Taxonomy based on Index Fungorum (2020) and Wijayawardene et al. (2020)

\# New species, ${ }^{* *}$ New genera

Substrate, host, country and references related to new species described from seed/fruits are in bold text.

\begin{tabular}{|c|c|c|c|c|c|}
\hline Species & Taxonomy & Substrate & Host & Country & References \\
\hline Absidia sp. & $\begin{array}{l}\text { Cunninghamellaceae, Mucoromycetes, } \\
\text { Mucoromycota }\end{array}$ & seeds & Quercus spp. & USSR & $\begin{array}{l}\text { Urosevic 1962, Mittal et } \\
\text { al. } 1990\end{array}$ \\
\hline Acremoniella atra (Corda) & Incertae sedis, Incertae sedis, & cones, seeds & Pinus strobus & Canada & Mittal \& Wang 1987 \\
\hline \multirow[t]{8}{*}{ Sacc. } & Ascomycota & seeds & Abies lasiocarpa & Norway & Talgø et al. 2010 \\
\hline & & seeds & Abies nordmanniana & Austria, Georgia & Talgø et al. 2010 \\
\hline & & seeds & Abies procera & Norway & Talgø et al. 2010 \\
\hline & & seeds & Festuca rubra & Canada & Conners 1967 \\
\hline & & seeds & Lolium perenne & Canada & Conners 1967 \\
\hline & & seeds & Picea excelsa & USSR & $\begin{array}{l}\text { Urosevic 1961, } \\
\text { Anderson 1986a }\end{array}$ \\
\hline & & seeds & Pinus sylvestris & USSR & $\begin{array}{l}\text { Urosevic 1961, } \\
\text { Anderson 1986a }\end{array}$ \\
\hline & & seeds & Quercus spp. & USSR & $\begin{array}{l}\text { Urosevic 1959, Mittal et } \\
\text { al. } 1990\end{array}$ \\
\hline
\end{tabular}


Table 2 Continued.

\begin{tabular}{|c|c|c|c|c|c|}
\hline Species & Taxonomy & Substrate & Host & Country & References \\
\hline $\begin{array}{l}\text { Acremoniella verrucosa } \\
\text { Tognini }\end{array}$ & $\begin{array}{l}\text { Incertae sedis, Incertae sedis, } \\
\text { Ascomycota }\end{array}$ & seed & Bromus inermis & Canada & Conners 1967 \\
\hline Acremonium alternatum & Bionectriaceae, Sordariomycetes, & seeds & Abies lasiocarpa & Canada & Talgø et al. 2010 \\
\hline Link & Ascomycota & seeds & Abies nordmanniana & $\begin{array}{l}\text { Austria, Georgia, } \\
\text { Russia }\end{array}$ & Talgø et al. 2010 \\
\hline & & seeds & Abies procera & Norway & Talgø et al. 2010 \\
\hline $\begin{array}{l}\text { Acremonium bacillisporum } \\
\text { (Onions \& G.L. Barron) W. } \\
\text { Gams }\end{array}$ & $\begin{array}{l}\text { Bionectriaceae, Sordariomycetes, } \\
\text { Ascomycota }\end{array}$ & acorns & Quercus robur & Poland & Jankowiak 2008 \\
\hline $\begin{array}{l}\text { Acremonium charticola } \\
\text { (Lindau) W. Gams }\end{array}$ & $\begin{array}{l}\text { Bionectriaceae, Sordariomycetes, } \\
\text { Ascomycota }\end{array}$ & acorns & Quercus robur & Poland & Jankowiak 2008 \\
\hline $\begin{array}{l}\text { Acremonium chilense } \\
\text { Morgan-Jones, J.F. White \& } \\
\text { Piont. }\end{array}$ & $\begin{array}{l}\text { Bionectriaceae, Sordariomycetes, } \\
\text { Ascomycota }\end{array}$ & seeds & Dactylis glomerata & Chile & $\begin{array}{l}\text { Morgan-Jones et al. } \\
1990\end{array}$ \\
\hline $\begin{array}{l}\text { Acremonium citrinum A. } \\
\text { Giraldo, Guarro, Cano \& } \\
\text { Gené\# }^{*}\end{array}$ & $\begin{array}{l}\text { Bionectriaceae, Sordariomycetes, } \\
\text { Ascomycota }\end{array}$ & fruit & undetermined plant & $\begin{array}{l}\text { Papua New } \\
\text { Guinea }\end{array}$ & $\begin{array}{l}\text { Giraldo-López et al. } \\
2014\end{array}$ \\
\hline Acremonium spp. & $\begin{array}{l}\text { Bionectriaceae, Sordariomycetes, } \\
\text { Ascomycota }\end{array}$ & $\begin{array}{l}\text { acorns } \\
\text { fruits } \\
\text { pods }\end{array}$ & $\begin{array}{l}\text { Quercus robur } \\
\text { Avicennia marina } \\
\text { Ilex cinerea } \\
\text { Delonix reqia }\end{array}$ & $\begin{array}{l}\text { Poland } \\
\text { India } \\
\text { Hong Kong } \\
\text { Thailand }\end{array}$ & $\begin{array}{l}\text { Jankowiak } 2008 \\
\text { Mehdi \& Saifullah } 2000 \\
\text { Tang et al. 2003a } \\
\text { Somrithipol et al. 2002b }\end{array}$ \\
\hline $\begin{array}{l}\text { Acrocalymma pterocarpi } \\
\text { Jayasiri, E.B.G. Jones \& } \\
\text { K.D. Hyde }^{\#}\end{array}$ & $\begin{array}{l}\text { Acrocalymmaceae, Dothideomycetes, } \\
\text { Ascomycota }\end{array}$ & $\begin{array}{l}\text { seed } \\
\text { pod }\end{array}$ & $\begin{array}{l}\text { Pinus thunbergii } \\
\text { Pterocarpus indicus }\end{array}$ & $\begin{array}{l}\text { Japan } \\
\text { Thailand }\end{array}$ & $\begin{array}{l}\text { Watanabe } 2010 \\
\text { Jayasiri et al. } 2019\end{array}$ \\
\hline $\begin{array}{l}\text { Acrospeira mirabilis Berk. \& } \\
\text { Broome }\end{array}$ & $\begin{array}{l}\text { Incertae sedis, Incertae sedis, } \\
\text { Ascomycota }\end{array}$ & seeds & Quercus spp. & USSR & $\begin{array}{l}\text { Urosevic 1962, } \\
\text { Mittal et al. } 1990\end{array}$ \\
\hline Acrospeira sp. & $\begin{array}{l}\text { Incertae sedis, Incertae sedis, } \\
\text { Ascomycota }\end{array}$ & seeds & Pinus taeda & USA & Anderson 1986a, b \\
\hline $\begin{array}{l}\text { Acrostalagmus luteoalbus } \\
\text { (Link) Zare, W. Gams \& } \\
\text { Schroers [as Acrostalagmus } \\
\text { cinnabarinus Corda] }\end{array}$ & $\begin{array}{l}\text { Plectosphaerellaceae, Sordariomycetes, } \\
\text { Ascomycota }\end{array}$ & seeds & Larix decidua & USSR & $\begin{array}{l}\text { Kozlowska 1968, Mittal } \\
\text { et al. } 1990\end{array}$ \\
\hline
\end{tabular}


Table 2 Continued.

\begin{tabular}{|c|c|c|c|c|c|}
\hline Species & Taxonomy & Substrate & Host & Country & References \\
\hline & & seeds & Picea excelsa & USSR & $\begin{array}{l}\text { Urosevic 1961, } \\
\text { Anderson 1986a }\end{array}$ \\
\hline & & seeds & Pinus sylvestris & USSR & $\begin{array}{l}\text { Urosevic 1961, } \\
\text { Anderson 1986a }\end{array}$ \\
\hline & & seeds & Quercus spp. & USSR & Urosevic 1959 \\
\hline $\begin{array}{l}\text { Acrostaphylus lignicola } \\
\text { Subram. }\end{array}$ & $\begin{array}{l}\text { Hypoxylaceae, Sordariomycetes, } \\
\text { Ascomycota }\end{array}$ & seeds & Eucalyptus sp. & India & $\begin{array}{l}\text { Reddy et al. 1982, Mittal } \\
\text { et al. } 1990\end{array}$ \\
\hline $\begin{array}{l}\text { Acrothecium tenebrosum } \\
\text { (Preuss) Sacc. }\end{array}$ & $\begin{array}{l}\text { Incertae sedis, Incertae sedis, } \\
\text { Ascomycota }\end{array}$ & seeds & Quercus spp. & USSR & $\begin{array}{l}\text { Urosevic 1962, Mittal et } \\
\text { al. } 1990\end{array}$ \\
\hline $\begin{array}{l}\text { Aecidium acanthocarpi J. } \\
\text { Walker \& van der Merwe }\end{array}$ & $\begin{array}{l}\text { Incertae sedis, Pucciniomycetes, } \\
\text { Basidiomycota }\end{array}$ & fruit & $\begin{array}{l}\text { Acanthocarpus } \\
\text { verticillatus }\end{array}$ & Australia & $\begin{array}{l}\text { Walker \& van der } \\
\text { Merwe } 2009\end{array}$ \\
\hline $\begin{array}{l}\text { Agarwalomyces indicus R.K. } \\
\text { Verma \& Kamal }^{* * *}\end{array}$ & $\begin{array}{l}\text { Incertae sedis, Incertae sedis, } \\
\text { Ascomycota }\end{array}$ & fruits & Lythraceae & India & Verma \& Rai 1987 \\
\hline $\begin{array}{l}\text { Akanthomyces lecanii } \\
\text { (Zimm.) Spatafora, Kepler \& } \\
\text { B. Shrestha [as Verticillium } \\
\text { lecanii (Zimm.) Viégas] }\end{array}$ & $\begin{array}{l}\text { Cordycipitaceae, Sordariomycetes, } \\
\text { Ascomycota }\end{array}$ & seed & Pinus thunbergii & Japan & Watanabe 2010 \\
\hline $\begin{array}{l}\text { Albifimbria verrucaria (Alb. } \\
\text { \& Schwein.) L. Lombard \& } \\
\text { Crous [as Myrothecium } \\
\text { verrucaria (Alb. \& Schwein.) } \\
\text { Ditmar] }\end{array}$ & $\begin{array}{l}\text { Stachybotryaceae, Sordariomycetes, } \\
\text { Ascomycota }\end{array}$ & seed & Pinus thunbergii & Japan & Watanabe 2010 \\
\hline $\begin{array}{l}\text { Albonectria rigidiuscula } \\
\text { (Berk. \& Broome) Rossman } \\
\text { \& Samuels [as Fusarium } \\
\text { rigidiusculum W.C. Snyder } \\
\text { \& H.N. Hansen] }\end{array}$ & $\begin{array}{l}\text { Nectriaceae, Sordariomycetes, } \\
\text { Ascomycota }\end{array}$ & seeds & Pinus ponderosa & USA & James 1983a \\
\hline $\begin{array}{l}\text { Allomyces anomalus } \mathrm{R} . \\
\text { Emers. }\end{array}$ & $\begin{array}{l}\text { Blastocladiaceae, Blastocladiomycetes, } \\
\text { Blastocladiomycota }\end{array}$ & seeds & Lycium cestroides & $\begin{array}{l}\text { Poland or/ } \\
\text { imported to Poland }\end{array}$ & Czeczuga et al. 2009 \\
\hline $\begin{array}{l}\text { Allophoma siamensis } \\
\text { Jayasiri, E.B.G. Jones \& } \\
\text { K.D. Hyde }^{\#}\end{array}$ & $\begin{array}{l}\text { Didymellaceae, Dothideomycetes, } \\
\text { Ascomycota }\end{array}$ & pod & $\begin{array}{l}\text { Radermachera } \\
\text { sinica }\end{array}$ & Thailand & Jayasiri et al. 2019 \\
\hline $\begin{array}{l}\text { Alternaria alternata (Fr.) } \\
\text { Keissl. }\end{array}$ & $\begin{array}{l}\text { Pleosporaceae, Dothideomycetes, } \\
\text { Ascomycota }\end{array}$ & acorns & Quercus robur & Poland & Jankowiak 2008 \\
\hline
\end{tabular}


Table 2 Continued.

\begin{tabular}{|c|c|c|c|c|c|}
\hline Species & Taxonomy & Substrate & Host & Country & References \\
\hline & & cones & Picea glauca & Canada & Mittal \& Wang 1987 \\
\hline & & cones & Pinus densiflora & Japan & Kasai et al. 1995 \\
\hline & & cones, seeds & Pinus strobus & Canada & Mittal \& Wang 1987 \\
\hline & & fruits & Diplospora dubia & Hong Kong & Tang et al. 2003a \\
\hline & & fruits & $\begin{array}{l}\text { Magnolia } \\
\text { grandiflora }\end{array}$ & Thailand & Jayasiri et al. 2019 \\
\hline & & fruits & Wikstroemia nutans & Hong Kong & Tang et al. 2003a \\
\hline & & pod & Canavalia cathartica & India & Anita \& Sridhar 2009 \\
\hline & & pod & Sesbania bispinosa & India & Anita et al. 2009 \\
\hline & & pods & Eucalyptus globulus & Uruguay & Lupo et al. 2001 \\
\hline & & seeds & Abies alba & Poland & Krol et al. 2015 \\
\hline & & seeds & Abies koreana & Korea & Cho et al. 2007 \\
\hline & & seeds & Acer pseudoplatanus & Poland & Krol et al. 2015 \\
\hline & & seeds & Alnus glutinosa & Poland & Krol et al. 2015 \\
\hline & & seeds & Betula pendula & Finland, Poland & $\begin{array}{l}\text { Lilja 1979, Mittal et al. } \\
\text { 1990, Krol et al. } 2015\end{array}$ \\
\hline & & seeds & $\begin{array}{l}\text { Callistephus } \\
\text { chinensis }\end{array}$ & $\begin{array}{l}\text { imported to } \\
\text { Taiwan }\end{array}$ & Wu et al. 2006 \\
\hline & & seeds & $\begin{array}{l}\text { Casuarina } \\
\text { equisetifolia }\end{array}$ & India & Anju et al. 2012 \\
\hline & & seeds & Cosmos sulphureus & $\begin{array}{l}\text { imported to } \\
\text { Taiwan }\end{array}$ & Wu et al. 2006 \\
\hline & & seed & $\begin{array}{l}\text { Cryptomeria } \\
\text { japonica }\end{array}$ & Japan & Watanabe 2010 \\
\hline & & seeds & $\begin{array}{l}\text { Cupressus } \\
\text { sempervirens }\end{array}$ & Egypt & $\begin{array}{l}\text { Farag et al. 1977, Mittal } \\
\text { et al. } 1990\end{array}$ \\
\hline & & seeds & Dahlia $\times$ hybrida & $\begin{array}{l}\text { imported to } \\
\text { Taiwan }\end{array}$ & Wu et al. 2006 \\
\hline & & seeds & Dalbergia sissoo & India & $\begin{array}{l}\text { Kumar 2014, Naz et al. } \\
2015\end{array}$ \\
\hline & & seeds & Delonix regia & India & Sahu et al. 2003 \\
\hline & & seeds & $\begin{array}{l}\text { Dianthus } \\
\text { caryophyllus }\end{array}$ & Taiwan & Li \& Wu 2002 \\
\hline
\end{tabular}


Table 2 Continued.

\begin{tabular}{|c|c|c|c|c|c|}
\hline Species & Taxonomy & Substrate & Host & Country & References \\
\hline & & seeds & Dianthus spp. & Taiwan & Li \& Wu 2002 \\
\hline & & seeds & Eucalyptus sp. & India & $\begin{array}{l}\text { Saxena 1985, Mittal et } \\
\text { al. } 1990\end{array}$ \\
\hline & & seeds & Fagus sylvatica & Poland & Krol et al. 2015 \\
\hline & & seeds & Fraxinus excelsior & Sweden & Cleary et al. 2013 \\
\hline & & seeds & Larix decidua & Poland & Krol et al. 2015 \\
\hline & & seeds & Larix occidentalis & USA & James et al. 1996 \\
\hline & & seed & Larix sp. & Japan & Watanabe 2010 \\
\hline & & seed & $\begin{array}{l}\text { Leucaena } \\
\text { leucocephala }\end{array}$ & Australia & Nik \& Parbery 1977 \\
\hline & & seed & $\begin{array}{l}\text { Macroptilium } \\
\text { atropurpureum }\end{array}$ & Australia & Nik \& Parbery 1977 \\
\hline & & seed & $\begin{array}{l}\text { Macroptilium } \\
\text { lathyroides }\end{array}$ & Australia & Nik \& Parbery 1977 \\
\hline & & seed & $\begin{array}{l}\text { Macrotyloma } \\
\text { axillare }\end{array}$ & Australia & Nik \& Parbery 1977 \\
\hline & & seed & Medicago scutellata & Australia & Nik \& Parbery 1977 \\
\hline & & seeds & Picea abies & Poland & Krol et al. 2015 \\
\hline & & seeds & Picea glauca & Canada & $\begin{array}{l}\text { Mittal \& Wang } 1986, \\
\text { 1987, Mittal et al. } 1990\end{array}$ \\
\hline & & seed & Pinus densiflora & Japan & Watanabe 2010 \\
\hline & & seeds & Pinus elliottii & South Africa & Cilliers et al. 1995 \\
\hline & & seeds & Pinus ponderosa & USA & James \& Genz 1982 \\
\hline & & seeds & Pinus roxburghii & India & $\begin{array}{l}\text { Munjal \& Sharma 1975, } \\
\text { Mittal et al. } 1990\end{array}$ \\
\hline & & seeds & Pinus sylvestris & Poland & Krol et al. 2015 \\
\hline & & seed & Pinus thunbergii & Japan & Watanabe 2010 \\
\hline & & seeds & Pinus wallichiana & Taiwan & $\begin{array}{l}\text { Munjal \& Sharma 1975, } \\
\text { Mittal et al. } 1990\end{array}$ \\
\hline & & seeds & Primula $\times$ polyantha & $\begin{array}{l}\text { imported to } \\
\text { Taiwan }\end{array}$ & Wu et al. 2006 \\
\hline
\end{tabular}


Table 2 Continued.

\begin{tabular}{|c|c|c|c|c|c|}
\hline Species & Taxonomy & Substrate & Host & Country & References \\
\hline & & seed & Prunus serrulata & Japan & Watanabe 2010 \\
\hline & & seeds & $\begin{array}{l}\text { Quercus } \\
\text { castaneifolia }\end{array}$ & Iran & Kavosi et al. 2013 \\
\hline & & seeds & Quercus robur & Poland & Krol et al. 2015 \\
\hline & & seeds & Quercus rubra & Poland & Krol et al. 2015 \\
\hline & & seeds & $\begin{array}{l}\text { Robinia } \\
\text { pseudoacacia }\end{array}$ & Hungary & $\begin{array}{l}\text { Hangyal-Balul 1983, } \\
\text { Mittal et al. } 1990\end{array}$ \\
\hline & & seed & Sesbania bispinosa & India & Anita et al. 2009 \\
\hline & & seeds & Shorea robusta & India & $\begin{array}{l}\text { Mittal \& Sharma 1982b, } \\
\text { Mittal et al. } 1990\end{array}$ \\
\hline & & seed & $\begin{array}{l}\text { Stylosanthes } \\
\text { guianensis }\end{array}$ & Australia & Nik \& Parbery 1977 \\
\hline & & seed & Stylosanthes humilis & Australia & Nik \& Parbery 1977 \\
\hline & & seeds & Tagetes patula & $\begin{array}{l}\text { imported to } \\
\text { Taiwan }\end{array}$ & Wu et al. 2006 \\
\hline & & seeds & Tectona grandis & India & Mohanan et al. 2005 \\
\hline & & seed & $\begin{array}{l}\text { Trifolium } \\
\text { semipilosum }\end{array}$ & Australia & Nik \& Parbery 1977 \\
\hline & & seeds & Viola wittrockiana & $\begin{array}{l}\text { imported to } \\
\text { Taiwan }\end{array}$ & Wu et al. 2006 \\
\hline $\begin{array}{l}\text { Alternaria arbusti E.G. } \\
\text { Simmons }\end{array}$ & $\begin{array}{l}\text { Pleosporaceae, Dothideomycetes, } \\
\text { Ascomycota }\end{array}$ & pod & Sesbania bispinosa & India & Anita et al. 2009 \\
\hline Alternaria atra (Preuss) & Pleosporaceae, Dothideomycetes, & seeds & Picea excelsa & USSR & Urosevic 1961, \\
\hline Woudenb. \& Crous [as & Ascomycota & & & & Anderson 1986a \\
\hline Stemphylium atrum (Preuss) & & seeds & Pinus sylvestris & USSR & Urosevic 1961, \\
\hline Sacc.] & & & & & Anderson 1986a \\
\hline Alternaria atra (Preuss) & & seeds & Acer pseudoplatanus & Poland & Krol et al. 2015 \\
\hline Woudenb. \& Crous [as & & seeds & Alnus glutinosa & Poland & Krol et al. 2015 \\
\hline \multirow[t]{3}{*}{ Ulocladium atrum Preuss] } & & seeds & $\begin{array}{l}\text { Callistephus } \\
\text { chinensis }\end{array}$ & $\begin{array}{l}\text { imported to } \\
\text { Taiwan }\end{array}$ & Wu et al. 2006 \\
\hline & & seeds & Cosmos sulphureus & imported to & Wu et al. 2006 \\
\hline & & seeds & Dahlia $\times$ hybrida & $\begin{array}{l}\text { imported to } \\
\text { Taiwan }\end{array}$ & Wu et al. 2006 \\
\hline
\end{tabular}


Table 2 Continued.

\begin{tabular}{|c|c|c|c|c|c|}
\hline Species & Taxonomy & Substrate & Host & Country & References \\
\hline \multirow{9}{*}{$\begin{array}{l}\text { Alternaria botrytis (Preuss) } \\
\text { Woudenb. \& Crous } \\
\text { [as Ulocladium botrytis } \\
\text { Preuss] } \\
\text { Alternaria brassicae (Berk.) } \\
\text { Sacc. }\end{array}$} & \multirow{7}{*}{$\begin{array}{l}\text { Pleosporaceae, Dothideomycetes, } \\
\text { Ascomycota }\end{array}$} & seeds & Fagus sylvatica & Poland & Krol et al. 2015 \\
\hline & & seed & Medicago sativa & Australia & Nik \& Parbery 1977 \\
\hline & & seeds & Primula malacoides & imported to & Wu et al. 2006 \\
\hline & & seeds & Tagetes patula & $\begin{array}{l}\text { Taiwan } \\
\text { imported to } \\
\text { Taiwan }\end{array}$ & Wu et al. 2006 \\
\hline & & seeds & Casuarina spp. & India & Sahai \& Otra 1982 \\
\hline & & seeds & Cosmos sulphureus & $\begin{array}{l}\text { imported to } \\
\text { Taiwan }\end{array}$ & Wu et al. 2006 \\
\hline & & seeds & Pyrus spp. & India & Sahai \& Otra 1982 \\
\hline & \multirow{2}{*}{$\begin{array}{l}\text { Pleosporaceae, Dothideomycetes, } \\
\text { Ascomycota }\end{array}$} & seeds & Picea excelsa & USSR & Urosevic 1961, \\
\hline & & seeds & Pinus sylvestris & USSR & $\begin{array}{l}\text { Anderson 1986a } \\
\text { Urosevic 1961, } \\
\text { Anderson 1986a }\end{array}$ \\
\hline $\begin{array}{l}\text { Alternaria chartarum Preuss } \\
\text { [as Ulocladium chartarum } \\
\text { (Preuss) E.G. Simmons] }\end{array}$ & $\begin{array}{l}\text { Pleosporaceae, Dothideomycetes, } \\
\text { Ascomycota }\end{array}$ & seed & Pinus densiflora & Japan & Watanabe 2010 \\
\hline \multirow[t]{4}{*}{$\begin{array}{l}\text { Alternaria circinans (Berk. } \\
\text { \& M.A. Curtis) P.C. Bolle }\end{array}$} & \multirow[t]{4}{*}{$\begin{array}{l}\text { Pleosporaceae, Dothideomycetes, } \\
\text { Ascomycota }\end{array}$} & seeds & Abies sibirica & USSR & $\begin{array}{l}\text { Prisyazhnyuk } 1960 \text {, } \\
\text { Mittal et al. } 1990\end{array}$ \\
\hline & & seeds & Larix sibirica & USSR & $\begin{array}{l}\text { Prisyazhnyuk } 1960 \text {, } \\
\text { Mittal et al. } 1990\end{array}$ \\
\hline & & seeds & Picea abies & USSR & $\begin{array}{l}\text { Prisyazhnyuk } 1960 \text {, } \\
\text { Mittal et al. } 1990\end{array}$ \\
\hline & & seeds & Pinus sylvestris & USSR & $\begin{array}{l}\text { Prisyazhnyuk } 1960 \text {, } \\
\text { Mittal et al. } 1990\end{array}$ \\
\hline \multirow{6}{*}{$\begin{array}{l}\text { Alternaria citri (Penz.) } \\
\text { Mussat } \\
\text { Alternaria consortialis } \\
\text { (Thüm.) J.W. Groves \& S. } \\
\text { Hughes }\end{array}$} & \multirow{6}{*}{$\begin{array}{l}\text { Pleosporaceae, Dothideomycetes, } \\
\text { Ascomycota } \\
\text { Pleosporaceae, Dothideomycetes, } \\
\text { Ascomycota }\end{array}$} & seeds & Eucalyptus globulus & Uruguay & Lupo et al. 2001 \\
\hline & & seeds & Agropyron cristatum & Canada & Conners 1967 \\
\hline & & seeds & Alnus glutinosa & Poland & Krol et al. 2015 \\
\hline & & seeds & Apium graveolens & Canada & Conners 1967 \\
\hline & & seeds & Medicago sativa & Canada & Conners 1967 \\
\hline & & seed & $\begin{array}{l}\text { Pseudotsuga } \\
\text { menziesii }\end{array}$ & USA & $\begin{array}{l}\text { Gordon 1967, Mittal et } \\
\text { al. } 1990\end{array}$ \\
\hline
\end{tabular}


Table 2 Continued.

\begin{tabular}{|c|c|c|c|c|c|}
\hline Species & Taxonomy & Substrate & Host & Country & References \\
\hline & & seed & Quercus robur & Poland & Krol et al. 2015 \\
\hline & & seed & Quercus rubra & Poland & Krol et al. 2015 \\
\hline \multirow{2}{*}{\multicolumn{2}{|c|}{$\begin{array}{l}\text { Alternaria consortialis } \\
\text { (Thüm.) J.W. Groves \& S. }\end{array}$}} & seeds & Acer rubrum & USA & Friedrich et al. 1971, \\
\hline & & & & & Mittal et al. 1990 \\
\hline \multirow{2}{*}{\multicolumn{2}{|c|}{$\begin{array}{l}\text { Hughes [as Stemphylium } \\
\text { consortiale (Thüm.) J.W. } \\
\text { Groves \& Skolko] }\end{array}$}} & seeds & Betula & USA & Friedrich et al. 1971, \\
\hline & & & alleghaniensis & & Mittal et al. 1990 \\
\hline $\begin{array}{l}\text { Alternaria cosmosa Y.S. Li } \\
\text { \& W.S. Wu }\end{array}$ & $\begin{array}{l}\text { Pleosporaceae, Dothideomycetes, } \\
\text { Ascomycota }\end{array}$ & seeds & Cosmos sulphureus & $\begin{array}{l}\text { imported to } \\
\text { Taiwan, Taiwan }\end{array}$ & $\begin{array}{l}\text { Wu \& Li 2005, Wu et } \\
\text { al. } 2006\end{array}$ \\
\hline $\begin{array}{l}\text { Alternaria danida E.G. } \\
\text { Simmons }^{\#}\end{array}$ & $\begin{array}{l}\text { Pleosporaceae, Dothideomycetes, } \\
\text { Ascomycota }\end{array}$ & seeds & $\begin{array}{l}\text { Ageratum } \\
\text { houstonianum }\end{array}$ & Italy & Simmons 1997 \\
\hline Alternaria dianthi J.V. & Pleosporaceae, Dothideomycetes, & pod & Canavalia cathartica & India & Anita \& Sridhar 2009 \\
\hline Almeida \& Sousa da Câmara & Ascomycota & pod & Sesbania bispinosa & India & Anita et al. 2009 \\
\hline $\begin{array}{l}\text { Alternaria dianthicola } \\
\text { Neerg. }\end{array}$ & $\begin{array}{l}\text { Pleosporaceae, Dothideomycetes, } \\
\text { Ascomycota }\end{array}$ & seeds & $\begin{array}{l}\text { Dianthus } \\
\text { caryophyllus }\end{array}$ & Taiwan & Li \& Wu 2002 \\
\hline $\begin{array}{l}\text { Alternaria ellipsoidea E.G. } \\
\text { Simmons }^{\#}\end{array}$ & $\begin{array}{l}\text { Pleosporaceae, Dothideomycetes, } \\
\text { Ascomycota }\end{array}$ & $\begin{array}{l}\text { seeds } \\
\text { seeds }\end{array}$ & $\begin{array}{l}\text { Dianthus spp. } \\
\text { Dianthus barbatus }\end{array}$ & $\begin{array}{l}\text { Taiwan } \\
\text { USA }\end{array}$ & $\begin{array}{l}\text { Li \& Wu } 2002 \\
\text { Simmons } 2002\end{array}$ \\
\hline $\begin{array}{l}\text { Alternaria eryngii (Pers.) S. } \\
\text { Hughes \& E.G. Simmons }\end{array}$ & $\begin{array}{l}\text { Pleosporaceae, Dothideomycetes, } \\
\text { Ascomycota }\end{array}$ & pod & Sesbania bispinosa & India & Anita et al. 2009 \\
\hline $\begin{array}{l}\text { Alternaria graminicola E.G. } \\
\text { Simmons }{ }^{\#}\end{array}$ & $\begin{array}{l}\text { Pleosporaceae, Dothideomycetes, } \\
\text { Ascomycota }\end{array}$ & grain & $\begin{array}{l}\text { Poaceae plant [as } \\
\text { Gramineae] }\end{array}$ & UK & Simmons 2007 \\
\hline Alternaria humicola Oudem. & $\begin{array}{l}\text { Pleosporaceae, Dothideomycetes, } \\
\text { Ascomycota }\end{array}$ & seeds & Quercus spp. & USSR & $\begin{array}{l}\text { Urosevic 1962, Mittal et } \\
\text { al. } 1990\end{array}$ \\
\hline $\begin{array}{l}\text { Alternaria infectoria E.G. } \\
\text { Simmons }\end{array}$ & $\begin{array}{l}\text { Pleosporaceae, Dothideomycetes, } \\
\text { Ascomycota }\end{array}$ & seeds & Fraxinus excelsior & Sweden & Hayatgheibi 2013 \\
\hline \multicolumn{2}{|l|}{$\begin{array}{l}\text { Alternaria infectoria E.G. } \\
\text { Simmons [as Lewia } \\
\text { infectoria (Fuckel) M.E. Barr } \\
\text { \& E.G. Simmons] }\end{array}$} & seeds & Fraxinus excelsior & Sweden & Cleary et al. 2013 \\
\hline \multirow[t]{2}{*}{$\begin{array}{l}\text { Alternaria iridicola (Ellis \& } \\
\text { Everh.) J.A. Elliott }\end{array}$} & $\begin{array}{l}\text { Pleosporaceae, Dothideomycetes, } \\
\text { Ascomycota }\end{array}$ & seeds & Larix sibirica & USSR & $\begin{array}{l}\text { Prisyazhnyuk 1960, } \\
\text { Mittal et al. } 1990\end{array}$ \\
\hline & & seeds & Picea abies & USSR & $\begin{array}{l}\text { Prisyazhnyuk 1960, } \\
\text { Mittal et al. } 1990\end{array}$ \\
\hline
\end{tabular}


Table 2 Continued.

\begin{tabular}{|c|c|c|c|c|c|}
\hline Species & Taxonomy & Substrate & Host & Country & References \\
\hline & & seeds & Pinus sylvestris & USSR & $\begin{array}{l}\text { Prisyazhnyuk } 1960, \\
\text { Mittal et al. } 1990\end{array}$ \\
\hline $\begin{array}{l}\text { Alternaria jesenskae } \\
\text { Lzbuda, P. Elias \& Sterfl.\# }\end{array}$ & $\begin{array}{l}\text { Pleosporaceae, Dothideomycetes, } \\
\text { Ascomycota }\end{array}$ & seeds & $\begin{array}{l}\text { Fumana } \\
\text { procumbens }\end{array}$ & Slovakia & Labuda et al. 2008 \\
\hline $\begin{array}{l}\text { Alternaria linicola J.W. } \\
\text { Groves \& Skolko }\end{array}$ & $\begin{array}{l}\text { Pleosporaceae, Dothideomycetes, } \\
\text { Ascomycota }\end{array}$ & seeds & $\begin{array}{l}\text { Linum } \\
\text { usitatissimum }\end{array}$ & Canada & $\begin{array}{l}\text { Groves \& Skolko 1944, } \\
\text { Conners } 1967\end{array}$ \\
\hline \multirow[t]{2}{*}{$\begin{array}{l}\text { Alternaria longipes (Ellis \& } \\
\text { Everh.) E.W. Mason }\end{array}$} & $\begin{array}{l}\text { Pleosporaceae, Dothideomycetes, } \\
\text { Ascomycota }\end{array}$ & seeds & $\begin{array}{l}\text { Dianthus } \\
\text { caryophyllus }\end{array}$ & Taiwan & Li \& Wu 2002 \\
\hline & & seeds & Dianthus spp. & Taiwan & Li \& Wu 2002 \\
\hline $\begin{array}{l}\text { Alternaria longissima } \\
\text { Deighton \& MacGarvie }\end{array}$ & $\begin{array}{l}\text { Pleosporaceae, Dothideomycetes, } \\
\text { Ascomycota }\end{array}$ & seeds & Bauhinia sp. & Thailand & $\begin{array}{l}\text { Chalermpongse et al. } \\
\text { 1984, Mittal et al. } 1990\end{array}$ \\
\hline $\begin{array}{l}\text { Alternaria maritima G.K. } \\
\text { Sutherl. }\end{array}$ & $\begin{array}{l}\text { Pleosporaceae, Dothideomycetes, } \\
\text { Ascomycota }\end{array}$ & fruits & Avicennia marina & India & Mehdi \& Saifullah 2000 \\
\hline $\begin{array}{l}\text { Alternaria patula H.C. Wu } \\
\& \text { W.S. Wu }\end{array}$ & $\begin{array}{l}\text { Pleosporaceae, Dothideomycetes, } \\
\text { Ascomycota }\end{array}$ & seeds & Tagetes patula & $\begin{array}{l}\text { imported to } \\
\text { Taiwan, Taiwan }\end{array}$ & $\begin{array}{l}\text { Wu \& Wu 2005, Wu et } \\
\text { al. } 2006\end{array}$ \\
\hline \multirow[t]{3}{*}{ Alternaria peponis Yatel } & $\begin{array}{l}\text { Pleosporaceae, Dothideomycetes, } \\
\text { Ascomycota }\end{array}$ & seeds & Larix sibirica & USSR & $\begin{array}{l}\text { Prisyazhnyuk } 1960 \text {, } \\
\text { Mittal et al. } 1990\end{array}$ \\
\hline & & seeds & Picea abies & USSR & $\begin{array}{l}\text { Prisyazhnyuk 1960, } \\
\text { Mittal et al. } 1990\end{array}$ \\
\hline & & seeds & Pinus sylvestris & USSR & $\begin{array}{l}\text { Prisyazhnyuk } 1960 \text {, } \\
\text { Mittal et al. } 1990\end{array}$ \\
\hline $\begin{array}{l}\text { Alternaria oudemansii (E.G. } \\
\text { Simmons) Woudenb. \& } \\
\text { Crous [as Ulocladium } \\
\text { oudemansii E.G. Simmons] }\end{array}$ & $\begin{array}{l}\text { Pleosporaceae, Dothideomycetes, } \\
\text { Ascomycota }\end{array}$ & seed & Pinus densiflora & Japan & Watanabe 2010 \\
\hline \multirow[t]{3}{*}{$\begin{array}{l}\text { Alternaria radicina Meier, } \\
\text { Drechsler \& E.D. Eddy }\end{array}$} & $\begin{array}{l}\text { Pleosporaceae, Dothideomycetes, } \\
\text { Ascomycota }\end{array}$ & seeds & Larix sibirica & USSR & $\begin{array}{l}\text { Prisyazhnyuk } 1960 \text {, } \\
\text { Mittal et al. } 1990\end{array}$ \\
\hline & & seeds & Picea abies & USSR & $\begin{array}{l}\text { Prisyazhnyuk } 1960 \text {, } \\
\text { Mittal et al. } 1990\end{array}$ \\
\hline & & seeds & Pinus sylvestris & USSR & $\begin{array}{l}\text { Prisyazhnyuk } 1960, \\
\text { Mittal et al. } 1990\end{array}$ \\
\hline $\begin{array}{l}\text { Alternaria ranunculi E.G. } \\
\text { Simmons }{ }^{\#}\end{array}$ & $\begin{array}{l}\text { Pleosporaceae, Dothideomycetes, } \\
\text { Ascomycota }\end{array}$ & seeds & Ranunculus sp. & Israel & Simmons 2007 \\
\hline
\end{tabular}


Table 2 Continued.

\begin{tabular}{|c|c|c|c|c|c|}
\hline Species & Taxonomy & Substrate & Host & Country & References \\
\hline $\begin{array}{l}\text { Alternaria raphani J.W. } \\
\text { Groves \& Skolko }\end{array}$ & $\begin{array}{l}\text { Pleosporaceae, Dothideomycetes, } \\
\text { Ascomycota }\end{array}$ & acorns & Quercus robur & Poland & Jankowiak 2008 \\
\hline Alternaria resedae Neerg." & $\begin{array}{l}\text { Pleosporaceae, Dothideomycetes, } \\
\text { Ascomycota }\end{array}$ & seeds & Reseda odorata & Denmark & Neergaard 1945 \\
\hline \multirow[t]{2}{*}{ Alternaria spp. } & Pleosporaceae, Dothideomycetes, & cones, seeds & Pinus nigra & Canada & Vujanovic et al. 2000 \\
\hline & Ascomycota & cones, seeds & $\begin{array}{l}\text { Pinus nigra ssp. } \\
\text { nigra }\end{array}$ & Canada & Vujanovic et al. 2000 \\
\hline Alternaria spp. [as & & cones, seeds & Pinus spp. & Canada & Vujanovic et al. 2000 \\
\hline \multirow[t]{4}{*}{ Ulocladium spp.] } & & fruits & Microcos paniculata & Hong Kong & Tang et al. 2003a \\
\hline & & seeds & Acer saccharum & USA & $\begin{array}{l}\text { Mittal et al. } 1990 \text {, } \\
\text { Janerette } 1979\end{array}$ \\
\hline & & seed & Larix kaempferi & Japan & Watanabe 2010 \\
\hline & & seeds & Pinus ponderosa & USA & $\begin{array}{l}\text { James \& Genz 1982, } \\
\text { Anderson 1986a }\end{array}$ \\
\hline \multirow[t]{12}{*}{ Alternaria sp. [as Lewia sp.] } & & seeds & Fraxinus excelsior & Sweden & Cleary et al. 2013 \\
\hline & & cones, seeds & $\begin{array}{l}\text { Pinus sylvestris } \\
\text { 'Fastigiata' }\end{array}$ & Canada & Vujanovic et al. 2000 \\
\hline & & pod & $\begin{array}{l}\text { Colophospermum } \\
\text { mopane }\end{array}$ & Southern Africa & Jordaan et al. 2006 \\
\hline & & seed capsules & Eucalyptus grandis & South Africa & Jimu et al. 2016 \\
\hline & & seeds & Abies lasiocarpa & Canada, Norway & Talgø et al. 2010 \\
\hline & & seeds & Abies nordmanniana & $\begin{array}{l}\text { Austria, Georgia, } \\
\text { Russia }\end{array}$ & Talgø et al. 2010 \\
\hline & & seeds & Abies procera & Norway & Talgø et al. 2010 \\
\hline & & seeds & Acer saccharum & USA & $\begin{array}{l}\text { Janerette 1979, Mittal et } \\
\text { al. } 1990\end{array}$ \\
\hline & & seeds & Albizia lebbeck & India & Natarajan et al. 2003 \\
\hline & & seeds & $\begin{array}{l}\text { Araucaria } \\
\text { angustifolia }\end{array}$ & Australia & $\begin{array}{l}\text { Kamara et al. } 1981 \text {, } \\
\text { Mittal et al. } 1990\end{array}$ \\
\hline & & seeds & $\begin{array}{l}\text { Araucaria } \\
\text { cunninghamii }\end{array}$ & Australia & $\begin{array}{l}\text { Kamara et al. } 1981 \text {, } \\
\text { Mittal et al. } 1990\end{array}$ \\
\hline & & seeds & $\begin{array}{l}\text { Araucaria } \\
\text { heterophylla }\end{array}$ & Egypt & $\begin{array}{l}\text { Kamara et al. } 1981 \text {, } \\
\text { Mittal et al. } 1990\end{array}$ \\
\hline
\end{tabular}


Table 2 Continued.

\begin{tabular}{|c|c|c|c|c|c|}
\hline Species & Taxonomy & Substrate & Host & Country & References \\
\hline & & seeds & Betula & USA & Shigo \& Yelenosky \\
\hline & & & alleghaniensis & & 1963, Mittal et al. 1990 \\
\hline & & seeds & Betula papyrifera & USA & $\begin{array}{l}\text { Smoot \& Segall 1963, } \\
\text { Mittal et al. } 1990\end{array}$ \\
\hline & & seeds & Cupressus arizonica & Uruguay & Anderson 1986a \\
\hline & & seeds & Cupressus & Uruguay & Anderson 1986a \\
\hline & & & macrocarpa & & \\
\hline & & seeds & Cupressus torulosa & Uruguay & Anderson 1986a \\
\hline & & seeds & Dalbergia sissoides & India & Mohanan et al. 2005 \\
\hline & & seeds & $\begin{array}{l}\text { Enterolobium } \\
\text { contortisiliquum }\end{array}$ & Uruguay & Anderson 1986a \\
\hline & & seeds & Eucalyptus grandis & $\begin{array}{l}\text { South Africa, } \\
\text { Uruguay }\end{array}$ & $\begin{array}{l}\text { Anderson 1986a, Jimu et } \\
\text { al. } 2016\end{array}$ \\
\hline & & seeds & Eucalyptus maidenii & Uruguay & Anderson 1986a \\
\hline & & seeds & Fraxinus excelsior & Sweden & Cleary et al. 2013 \\
\hline & & seeds & Fraxinus excelsior & Sweden & Hayatgheibi 2013 \\
\hline & & seeds & Phyllostachys edulis & China & Shen et al. 2014 \\
\hline & & seeds & Picea engelmannii & USA & $\begin{array}{l}\text { Fuller \& Hildebrand } \\
\text { 1985, Mittal et al. } 1990\end{array}$ \\
\hline & & seeds & Picea sitchensis & UK & $\begin{array}{l}\text { Salt 1964, Mittal et al. } \\
1990\end{array}$ \\
\hline & & seeds & Pinus contorta & USA & $\begin{array}{l}\text { Fuller \& Hildebrand } \\
\text { 1985, Mittal et al. } 1990\end{array}$ \\
\hline & & seeds & $\begin{array}{l}\text { Pinus elliottii var. } \\
\text { elliottii }\end{array}$ & Uruguay, USA & $\begin{array}{l}\text { Anderson 1986a, } \\
\text { Fraedrich \& Miller } 1995\end{array}$ \\
\hline & & seeds & Pinus nigra & Hungary & $\begin{array}{l}\text { Hangyal 1973, Mittal et } \\
\text { al. } 1990\end{array}$ \\
\hline & & seeds & Pinus ponderosa & USA & $\begin{array}{l}\text { Fuller \& Hildebrand } \\
\text { 1985, Mittal et al. } 1990\end{array}$ \\
\hline & & seeds & Pinus sylvestris & Hungary & $\begin{array}{l}\text { Hangyal 1973, Mittal et } \\
\text { al. } 1990\end{array}$ \\
\hline & & seeds & Pinus taeda & Georgia, USA & $\begin{array}{l}\text { Anderson 1986a, b, } \\
\text { Huang \& Kuhlman } 1990\end{array}$ \\
\hline
\end{tabular}


Table 2 Continued.

\begin{tabular}{|c|c|c|c|c|c|}
\hline Species & Taxonomy & Substrate & Host & Country & References \\
\hline & & seeds & $\begin{array}{l}\text { Platanus } \\
\text { occidentalis }\end{array}$ & USA & $\begin{array}{l}\text { Fakir et al. 1971, Mittal } \\
\text { et al. } 1990\end{array}$ \\
\hline & & seeds & $\begin{array}{l}\text { Pseudotsuga } \\
\text { menziesii }\end{array}$ & Canada & $\begin{array}{l}\text { Salisbury 1955, Mittal et } \\
\text { al. } 1990\end{array}$ \\
\hline & & seeds & Quercus robur & Turkey & Oskay et al. 2018 \\
\hline & & seeds & Tectona grandis & India & Anderson 1986a \\
\hline \multirow[t]{17}{*}{ Alternaria tenuis Nees } & $\begin{array}{l}\text { Pleosporaceae, Dothideomycetes, } \\
\text { Ascomycota }\end{array}$ & seeds & Acer rubrum & USA & $\begin{array}{l}\text { Friedrich et al. } 1971 \text {, } \\
\text { Mittal et al. } 1990\end{array}$ \\
\hline & & seeds & Acer saccharinum & USA & $\begin{array}{l}\text { Friedrich et al. } 1971 \text {, } \\
\text { Mittal et al. } 1990\end{array}$ \\
\hline & & seeds & Acer saccharum & USA & $\begin{array}{l}\text { Friedrich et al. } 1971 \text {, } \\
\text { Mittal et al. } 1990\end{array}$ \\
\hline & & seed & Agropyron cristatum & Canada & Conners 1967 \\
\hline & & seed & Agrostis gigantea & Canada & Conners 1967 \\
\hline & & seeds & Albizia falcataria & Philippines & $\begin{array}{l}\text { Agmata 1979, Mittal et } \\
\text { al. } 1990\end{array}$ \\
\hline & & seeds & Albizia lebbeck & India & Natarajan et al. 2003 \\
\hline & & seed & Anethum graveolens & Canada & Conners 1967 \\
\hline & & seed & $\begin{array}{l}\text { Apium graveolens } \\
\text { var. dulce }\end{array}$ & Canada & Conners 1967 \\
\hline & & seeds & $\begin{array}{l}\text { Araucaria } \\
\text { angustifolia }\end{array}$ & Australia & $\begin{array}{l}\text { Kamara et al. } 1981 \text {, } \\
\text { Mittal et al. } 1990\end{array}$ \\
\hline & & seeds & $\begin{array}{l}\text { Araucaria } \\
\text { heterophylla }\end{array}$ & Egypt & $\begin{array}{l}\text { El-Kady et al. } 1986 \text {, } \\
\text { Mittal et al. } 1990\end{array}$ \\
\hline & & seed & $\begin{array}{l}\text { Asparagus officinalis } \\
\text { var. altilis }\end{array}$ & Canada & Conners 1967 \\
\hline & & seeds & $\begin{array}{l}\text { Betula } \\
\text { alleghaniensis }\end{array}$ & USA & $\begin{array}{l}\text { Friedrich et al. } 1971 \text {, } \\
\text { Mittal et al. } 1990\end{array}$ \\
\hline & & seeds & Betula papyrifera & USA & $\begin{array}{l}\text { Friedrich et al. } 1971 \text {, } \\
\text { Mittal et al. } 1990\end{array}$ \\
\hline & & seeds & Betula verrucosa & USSR & $\begin{array}{l}\text { Kozlowska 1968, Mittal } \\
\text { et al. } 1990\end{array}$ \\
\hline & & seeds & Bombax ceiba & Bangladesh & Mittal et al. 1990 \\
\hline & & seed & Bromus inermis & Canada & Conners 1967 \\
\hline
\end{tabular}


Table 2 Continued.

\begin{tabular}{|c|c|c|c|c|c|}
\hline Species & Taxonomy & Substrate & Host & Country & References \\
\hline & & seeds & $\begin{array}{l}\text { Callistephus } \\
\text { chinensis }\end{array}$ & China & $\begin{array}{l}\text { Gloyer } 1931 \text {, Crosier \& } \\
\text { Heit } 1948\end{array}$ \\
\hline & & seeds & Cassia bakeriana & Thailand & $\begin{array}{l}\text { Chalermpongse et al. } \\
\text { 1984, Mittal et al. } 1990\end{array}$ \\
\hline & & seeds & Cassia fistula & India & $\begin{array}{l}\text { Mittal \& Sharma 1981a, } \\
\text { Mittal et al. } 1990\end{array}$ \\
\hline & & seeds & Cedrus deodara & India, Uruguay & Munjal \& Sharma 1975 \\
\hline & & seed & Dactylis glomerata & Canada & Conners 1967 \\
\hline & & seeds & Dalbergia sissoo & India & $\begin{array}{l}\text { Mittal \& Sharma 1981b, } \\
\text { Mittal et al. } 1990\end{array}$ \\
\hline & & seed & Elymus virginicus & Canada & Conners 1967 \\
\hline & & seeds & $\begin{array}{l}\text { Eucalyptus } \\
\text { camaldulensis }\end{array}$ & Thailand & $\begin{array}{l}\text { Chalermpongse et al. } \\
\text { 1984, Mittal et al. } 1990\end{array}$ \\
\hline & & seeds & Fagus sylvatica & Hungary & Mittal et al. 1990 \\
\hline & & seed & Festuca elatior & Canada & Conners 1967 \\
\hline & & seed & Festuca rubra & Canada & Conners 1967 \\
\hline & & seeds & Fraxinus americana & USA & $\begin{array}{l}\text { Friedrich et al. } 1971 \text {, } \\
\text { Mittal et al. } 1990\end{array}$ \\
\hline & & seeds & Gmelina arborea & Thailand & $\begin{array}{l}\text { Chalermpongse et al. } \\
\text { 1984, Mittal et al. } 1990\end{array}$ \\
\hline & & seeds & Larix decidua & USSR & $\begin{array}{l}\text { Kozlowska 1968, Mittal } \\
\text { et al. } 1990\end{array}$ \\
\hline & & seeds & Larix sibirica & USSR & $\begin{array}{l}\text { Prisyazhnyuk 1960, } \\
\text { Mittal et al. } 1990\end{array}$ \\
\hline & & seeds & Lobelia erinus & UK & $\begin{array}{l}\text { Hall \& Taylor } 1983 \text {, } \\
\text { Mittal et al. } 1990\end{array}$ \\
\hline & & seed & Lolium perenne & Canada & Conners 1967 \\
\hline & & seed & Medicago sativa & Canada & Conners 1967 \\
\hline & & seed & Phleum pratense & Canada & Conners 1967 \\
\hline & & seeds & Picea abies & USSR & $\begin{array}{l}\text { Prisyazhnyuk 1960, } \\
\text { Mittal et al. } 1990\end{array}$ \\
\hline & & seeds & Picea excelsa & USSR & $\begin{array}{l}\text { Urosevic 1961, } \\
\text { Anderson 1986a } \\
\end{array}$ \\
\hline
\end{tabular}


Table 2 Continued.

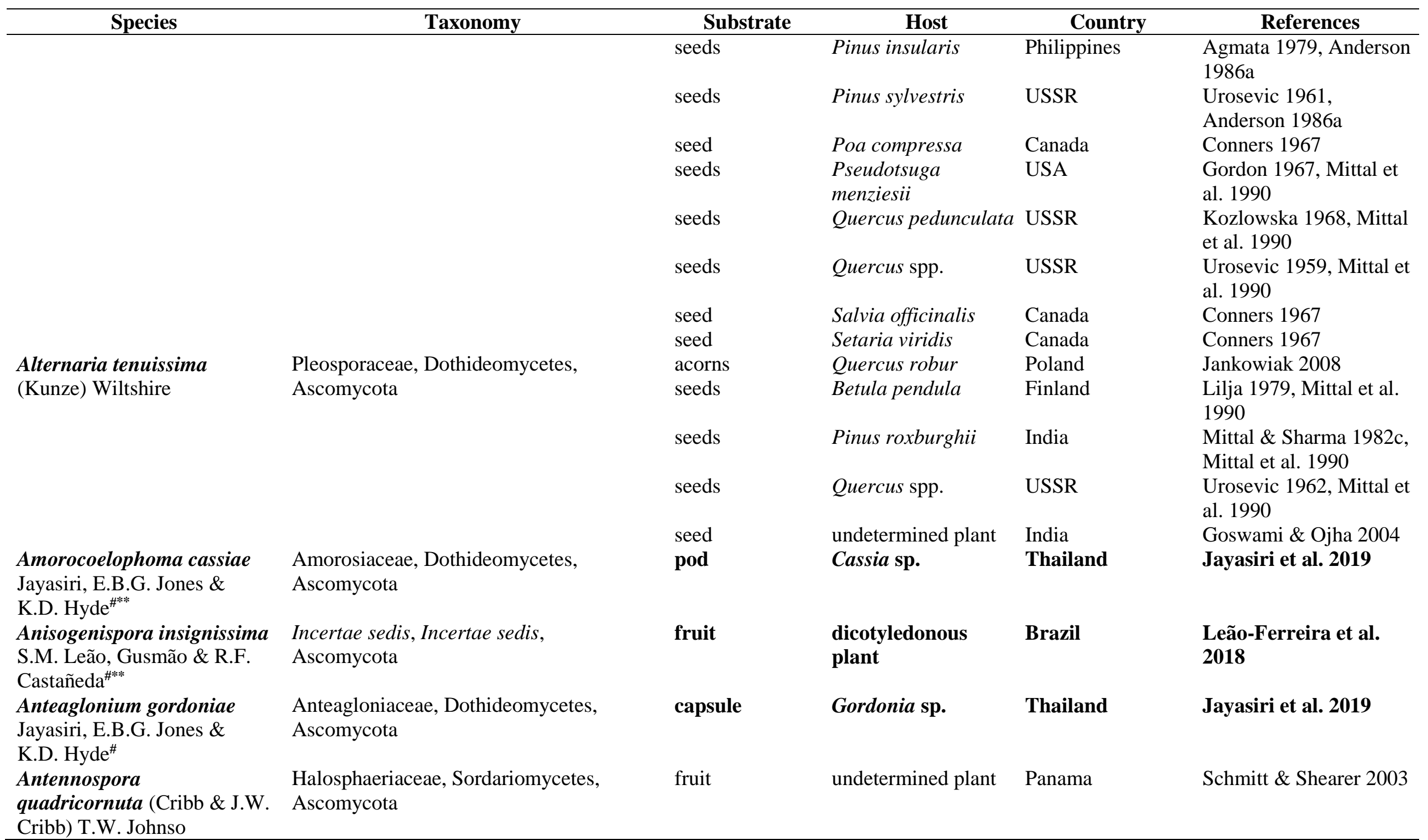


Table 2 Continued.

\begin{tabular}{|c|c|c|c|c|c|}
\hline Species & Taxonomy & Substrate & Host & Country & References \\
\hline $\begin{array}{l}\text { Anthostomella clypeata (De } \\
\text { Not.) Sacc. }\end{array}$ & $\begin{array}{l}\text { Xylariaceae, Sordariomycetes, } \\
\text { Ascomycota }\end{array}$ & fruits & Microcos paniculata & Hong Kong & Tang et al. 2003a \\
\hline $\begin{array}{l}\text { Anthracoidea multicaulis } \\
\text { Vánky \& Salo\# }\end{array}$ & $\begin{array}{l}\text { Anthracoideaceae, Ustilaginomycetes, } \\
\text { Basidiomycota }\end{array}$ & nutlets & Carex multicaulis & USA & Vánky \& Salo 2011 \\
\hline Apiocarpella sp. & $\begin{array}{l}\text { Incertae sedis, Incertae sedis, } \\
\text { Ascomycota }\end{array}$ & seed & Prunus serrulata & Japan & Watanabe 2010 \\
\hline $\begin{array}{l}\text { Aplosporella dryobalonopis } \\
\text { S.N.S. Srivast. }\end{array}$ & $\begin{array}{l}\text { Aplosporellaceae, Dothideomycetes, } \\
\text { Ascomycota }\end{array}$ & fruit, seeds & $\begin{array}{l}\text { Dryobalanops } \\
\text { aromatica }\end{array}$ & Malaysia & $\begin{array}{l}\text { Srivastava } \mathbf{1 9 5 6 a} \\
\text { Mittal et al. } 1990\end{array}$ \\
\hline Aposphaeria sp. & $\begin{array}{l}\text { Melanommataceae, Dothideomycetes, } \\
\text { Ascomycota }\end{array}$ & acorns & Quercus robur & Poland & Jankowiak 2008 \\
\hline $\begin{array}{l}\text { Arachnophora fagicola } \\
\text { Hennebert }^{\#}\end{array}$ & $\begin{array}{l}\text { Incertae sedis, Incertae sedis, } \\
\text { Ascomycota }\end{array}$ & cupules & Fagus sylvatica & Belgium & Hennebert 1963 \\
\hline $\begin{array}{l}\text { Arachnophora hughesii R.F. } \\
\text { Castañeda \& Guarro }^{\#}\end{array}$ & $\begin{array}{l}\text { Incertae sedis, Incertae sedis, } \\
\text { Ascomycota }\end{array}$ & pod & Samanea saman & Cuba & $\begin{array}{l}\text { Castañeda-Ruíz \& } \\
\text { Guarro } 1998\end{array}$ \\
\hline $\begin{array}{l}\text { Arcopilus aureus (Chivers) } \\
\text { X.Wei Wang \& Samson [as } \\
\text { Chaetomium aureum } \\
\text { Chivers] }\end{array}$ & $\begin{array}{l}\text { Chaetomiaceae, Sordariomycetes, } \\
\text { Ascomycota }\end{array}$ & seeds & Gossypium sp. & Pakistan & Lodhi \& Naeem 1955 \\
\hline $\begin{array}{l}\text { Archephoma cycadeoidellae } \\
\text { K. Watan., H. Nishida \& } \\
\text { Tak. Kobay }{ }^{\# * *}\end{array}$ & Fossil fungus & cone & $\begin{array}{l}\text { Cycadeoidella } \\
\text { japonica }\end{array}$ & Japan & Watanabe et al. 1999 \\
\hline \multirow[t]{6}{*}{ Arthrinium spp. } & $\begin{array}{l}\text { Apiosporaceae, Sordariomycetes, } \\
\text { Ascomycota }\end{array}$ & fruits & $\begin{array}{l}\text { Rhodomyrtus } \\
\text { tomentosa }\end{array}$ & Hong Kong & Tang et al. 2003a \\
\hline & & seed & Cercis chinensis & Japan & Watanabe 2010 \\
\hline & & seed & Gentiana sp. & Japan & Watanabe 2010 \\
\hline & & seed & Pinus densiflora & Japan & Watanabe 2010 \\
\hline & & seed & Prunus serrulata & Japan & Watanabe 2010 \\
\hline & & seeds & Picea jezoensis & Japan & Cheng \& Igarashi 1987 \\
\hline
\end{tabular}


Table 2 Continued.

\begin{tabular}{|c|c|c|c|c|c|}
\hline Species & Taxonomy & Substrate & Host & Country & References \\
\hline & & seeds & $\begin{array}{l}\text { Chamaecyparis } \\
\text { obtusa }\end{array}$ & South Korea & $\begin{array}{l}\text { Mathur 1974, Anderson } \\
\text { 1986a }\end{array}$ \\
\hline & & seeds & Abies koreana & Korea & Cho et al. 2007 \\
\hline & & seeds & Phyllostachys edulis & China & Shen et al. 2014 \\
\hline $\begin{array}{l}\text { Arthrobotrys } \\
\text { arthrobotryoides (Berl.) } \\
\text { Lindau }\end{array}$ & Orbiliaceae, Orbiliomycetes, Ascomycota & seeds & Quercus spp. & USSR & $\begin{array}{l}\text { Urosevic 1959, Mittal et } \\
\text { al. } 1990\end{array}$ \\
\hline $\begin{array}{l}\text { Arthrobotrys oligosporus } \\
\text { Fresen. }\end{array}$ & Orbiliaceae, Orbiliomycetes, Ascomycota & seed & Prunus serrulata & Japan & Watanabe 2010 \\
\hline Arthrobotrys superbus Corda & Orbiliaceae, Orbiliomycetes, Ascomycota & seeds & Quercus spp. & USSR & $\begin{array}{l}\text { Urosevic 1959, Mittal et } \\
\text { al. } 1990\end{array}$ \\
\hline $\begin{array}{l}\text { Arthrobotrys superbus var. } \\
\text { oligosporus (Fresen.) Coem. }\end{array}$ & Orbiliaceae, Orbiliomycetes, Ascomycota & seeds & Quercus spp. & USSR & $\begin{array}{l}\text { Urosevic 1959, Mittal et } \\
\text { al. } 1990\end{array}$ \\
\hline $\begin{array}{l}\text { Arxotrichum succineum } \\
\text { (L.M. Ames) A. Nováková \& } \\
\text { M. Kolařík [as Chaetomium } \\
\text { succineum L.M. Ames] }\end{array}$ & $\begin{array}{l}\text { Chaetomiaceae, Sordariomycetes, } \\
\text { Ascomycota }\end{array}$ & seed & Agropyron cristatum & Canada & Conners 1967 \\
\hline $\begin{array}{l}\text { Ascochyta agropyrina } \\
\text { (Fairm.) Trotter }\end{array}$ & $\begin{array}{l}\text { Didymellaceae, Dothideomycetes, } \\
\text { Ascomycota }\end{array}$ & seed & Agropyron cristatum & Canada & Conners 1967 \\
\hline Ascochyta conorum Henn. & $\begin{array}{l}\text { Didymellaceae, Dothideomycetes, } \\
\text { Ascomycota }\end{array}$ & seeds & Picea abies & USSR & $\begin{array}{l}\text { Prisyazhnyuk 1960, } \\
\text { Mittal et al. } 1990\end{array}$ \\
\hline Ascochyta fagi Woron. & $\begin{array}{l}\text { Didymellaceae, Dothideomycetes, } \\
\text { Ascomycota }\end{array}$ & fruit cupules & Fagus crenata & Japan & $\begin{array}{l}\text { Fukasawa et al. 2012, } \\
\text { Tateno et al. } 2015\end{array}$ \\
\hline \multirow[t]{2}{*}{ Ascochyta spp. } & $\begin{array}{l}\text { Didymellaceae, Dothideomycetes, } \\
\text { Ascomycota }\end{array}$ & fruits & Microcos paniculata & Hong Kong & Tang et al. 2003a \\
\hline & & seeds & Acer palmatum & South Korea & $\begin{array}{l}\text { Mathur 1974, Anderson } \\
\text { 1986a }\end{array}$ \\
\hline \multirow[t]{4}{*}{ Ascomycetes } & Ascomycota & pods & Delonix regia & Thailand & Somrithipol et al. 2002b \\
\hline & & seed capsules & Eucalyptus grandis & South Africa & Jimu et al. 2016 \\
\hline & & seeds & Eucalyptus grandis & South Africa & Jimu et al. 2016 \\
\hline & & seeds & Fraxinus excelsior & Sweden & Cleary et al. 2013 \\
\hline Ascotricha sp. & $\begin{array}{l}\text { Xylariaceae, Sordariomycetes, } \\
\text { Ascomycota }\end{array}$ & acorns & Quercus robur & Poland & Jankowiak 2008 \\
\hline
\end{tabular}


Table 2 Continued.

\begin{tabular}{|c|c|c|c|c|c|}
\hline Species & Taxonomy & Substrate & Host & Country & References \\
\hline Aspergillus aculeatus lizuka & $\begin{array}{l}\text { Aspergillaceae, Eurotiomycetes, } \\
\text { Ascomycota }\end{array}$ & seeds & Delonix regia & India & Sahu et al. 2003 \\
\hline $\begin{array}{l}\text { Aspergillus aculeatus [as } \\
\text { 'occuleatus'] }\end{array}$ & $\begin{array}{l}\text { Aspergillaceae, Eurotiomycetes, } \\
\text { Ascomycota }\end{array}$ & seeds & $\begin{array}{l}\text { Cupressus } \\
\text { sempervirens }\end{array}$ & Egypt & $\begin{array}{l}\text { Farag et al. 1977, Mittal } \\
\text { et al. } 1990\end{array}$ \\
\hline $\begin{array}{l}\text { Aspergillus amstelodami } \\
\text { Thom \& Church }\end{array}$ & $\begin{array}{l}\text { Aspergillaceae, Eurotiomycetes, } \\
\text { Ascomycota }\end{array}$ & seeds & $\begin{array}{l}\text { Platanus } \\
\text { occidentalis }\end{array}$ & USA & $\begin{array}{l}\text { Fakir et al. 1971, Mittal } \\
\text { et al. } 1990\end{array}$ \\
\hline $\begin{array}{l}\text { Aspergillus caespitosus } \\
\text { Raper \& Thom }\end{array}$ & $\begin{array}{l}\text { Aspergillaceae, Eurotiomycetes, } \\
\text { Ascomycota }\end{array}$ & seeds & Pinus wallichiana & India & $\begin{array}{l}\text { Mittal \& Sharma 1982b, } \\
\text { Mittal et al. } 1990\end{array}$ \\
\hline \multirow[t]{12}{*}{ Aspergillus candidus Link } & Aspergillaceae, Eurotiomycetes, & pods & Delonix regia & Thailand & Somrithipol et al. 2002b \\
\hline & Ascomycota & seeds & Bauhinia sp. & Thailand & $\begin{array}{l}\text { Chalermpongse et al. } \\
\text { 1984, Mittal et al. } 1990\end{array}$ \\
\hline & & seeds & Cedrus deodara & India & Mittal 1983 \\
\hline & & seeds & $\begin{array}{l}\text { Eucalyptus } \\
\text { citriodora }\end{array}$ & India & $\begin{array}{l}\text { Mittal \& Sharma 1984, } \\
\text { Mittal et al. } 1990\end{array}$ \\
\hline & & seeds & Eucalyptus sp. & India & $\begin{array}{l}\text { Reddy et al. 1982, Mittal } \\
\text { et al. } 1990\end{array}$ \\
\hline & & seeds & Larix sibirica & USSR & $\begin{array}{l}\text { Prisyazhnyuk } 1960 \text {, } \\
\text { Mittal et al. } 1990\end{array}$ \\
\hline & & seeds & $\begin{array}{l}\text { Leucaena } \\
\text { leucocephala }\end{array}$ & Thailand & $\begin{array}{l}\text { Chalermpongse et al. } \\
\text { 1984, Mittal et al. } 1990\end{array}$ \\
\hline & & seeds & Picea abies & USSR & $\begin{array}{l}\text { Prisyazhnyuk } 1960 \text {, } \\
\text { Mittal et al. } 1990\end{array}$ \\
\hline & & seeds & Pinus caribaea & Thailand & $\begin{array}{l}\text { Chalermpongse et al. } \\
\text { 1984, Mittal et al. } 1990\end{array}$ \\
\hline & & seeds & Pinus roxburghii & India & $\begin{array}{l}\text { Mittal \& Sharma 1982c, } \\
\text { Mittal et al. } 1990\end{array}$ \\
\hline & & seeds & Pinus wallichiana & India & $\begin{array}{l}\text { Mittal \& Sharma 1982c, } \\
\text { Mittal et al. } 1990\end{array}$ \\
\hline & & seed & $\begin{array}{l}\text { Stylosanthes } \\
\text { guianensis }\end{array}$ & Australia & Nik \& Parbery 1977 \\
\hline \multirow[t]{2}{*}{$\begin{array}{l}\text { Aspergillus candidus Link } \\
\text { [as Aspergillus candidum] }\end{array}$} & & seeds & Abies sibirica & USSR & $\begin{array}{l}\text { Prisyazhnyuk } 1960 \text {, } \\
\text { Mittal et al. } 1990\end{array}$ \\
\hline & & seeds & Dalbergia sissoo & India & Kumar 2014 \\
\hline
\end{tabular}


Table 2 Continued.

\begin{tabular}{|c|c|c|c|c|c|}
\hline Species & Taxonomy & Substrate & Host & Country & References \\
\hline & & seeds & Pinus sylvestris & USSR & $\begin{array}{l}\text { Prisyazhnyuk } 1960 \text {, } \\
\text { Mittal et al. } 1990\end{array}$ \\
\hline Aspergillus carneus & Aspergillaceae, Eurotiomycetes, & seeds & Pyrus spp. & India & Sahai \& Otra 1982 \\
\hline Blochwitz & Ascomycota & seeds & Sapium spp. & India & Sahai \& Otra 1982 \\
\hline $\begin{array}{l}\text { Aspergillus chevalieri Thom } \\
\text { \& Church }\end{array}$ & $\begin{array}{l}\text { Aspergillaceae, Eurotiomycetes, } \\
\text { Ascomycota }\end{array}$ & seeds & $\begin{array}{l}\text { Pseudotsuga } \\
\text { menziesii }\end{array}$ & USA & $\begin{array}{l}\text { Gordon 1967, Mittal et } \\
\text { al. } 1990\end{array}$ \\
\hline \multirow[t]{5}{*}{ Aspergillus clavatus Desm. } & $\begin{array}{l}\text { Aspergillaceae, Eurotiomycetes, } \\
\text { Ascomycota }\end{array}$ & seeds & Albizia lebbeck & India & $\begin{array}{l}\text { Mittal \& Sharma 1982a, } \\
\text { Mittal et al. } 1990 \text {, } \\
\text { Natarajan et al. } 2003\end{array}$ \\
\hline & & seeds & Melia spp. & India & Sahai \& Otra 1982 \\
\hline & & seeds & Quercus spp. & India & Sahai \& Otra 1982 \\
\hline & & seeds & Sapium spp. & India & Sahai \& Otra 1982 \\
\hline & & seeds & Thuja spp. & India & Sahai \& Otra 1982 \\
\hline $\begin{array}{l}\text { Aspergillus delicatus H.Z. } \\
\text { Kong }^{\#}\end{array}$ & $\begin{array}{l}\text { Aspergillaceae, Eurotiomycetes, } \\
\text { Ascomycota }\end{array}$ & fruit & undetermined plant & China & Kong 1997 \\
\hline $\begin{array}{l}\text { Aspergillus flavipes (Bainier } \\
\text { \& R. Sartory) Thom \& }\end{array}$ & $\begin{array}{l}\text { Aspergillaceae, Eurotiomycetes, } \\
\text { Ascomycota }\end{array}$ & seeds & Betula verrucosa & USSR & $\begin{array}{l}\text { Kozlowska 1968, Mittal } \\
\text { et al. } 1990\end{array}$ \\
\hline Church & & seeds & Cupressus spp. & India & Sahai \& Otra 1982 \\
\hline & & seeds & Eucalyptus sp. & India & $\begin{array}{l}\text { Reddy et al. 1982, Mittal } \\
\text { et al. } 1990\end{array}$ \\
\hline \multirow{9}{*}{ Aspergillus flavus Link } & & seeds & Melia spp. & India & Sahai \& Otra 1982 \\
\hline & $\begin{array}{l}\text { Aspergillaceae, Eurotiomycetes, } \\
\text { Ascomycota }\end{array}$ & fruit & $\begin{array}{l}\text { Artocarpus } \\
\text { communis }\end{array}$ & Nigeria & Amusa et al. 2002 \\
\hline & & fruits & Avicennia marina & India & Mehdi \& Saifullah 2000 \\
\hline & & pod & Canavalia cathartica & India & Anita \& Sridhar 2009 \\
\hline & & pods & Delonix regia & Thailand & Somrithipol et al. 2002b \\
\hline & & pod & Sesbania bispinosa & India & Anita et al. 2009 \\
\hline & & seeds & Abies sibirica & USSR & $\begin{array}{l}\text { Prisyazhnyuk } 1960 \text {, } \\
\text { Mittal et al. } 1990\end{array}$ \\
\hline & & seeds & $\begin{array}{l}\text { Acacia } \\
\text { auriculiformis }\end{array}$ & $\begin{array}{l}\text { Philippines, } \\
\text { Thailand }\end{array}$ & $\begin{array}{l}\text { Quiniones 1985, 1987, } \\
\text { Anderson 1986a, Mittal } \\
\text { et al. } 1990\end{array}$ \\
\hline & & seeds & $\begin{array}{l}\text { Agathis } \\
\text { philippinensis }\end{array}$ & Philippines & Quiniones 1987 \\
\hline
\end{tabular}


Table 2 Continued.

\begin{tabular}{|c|c|c|c|c|c|}
\hline Species & Taxonomy & Substrate & Host & Country & References \\
\hline & & seeds & Agathis robusta & Philippines & $\begin{array}{l}\text { Quiniones 1985, 1987, } \\
\text { Anderson 1986a }\end{array}$ \\
\hline & & seeds & Albizia falcataria & Philippines & $\begin{array}{l}\text { Quiniones 1985, 1987, } \\
\text { Mittal et al. } 1990\end{array}$ \\
\hline & & seeds & Albizia julibrissin & Philippines & $\begin{array}{l}\text { Quiniones 1985, 1987, } \\
\text { Mittal et al. } 1990\end{array}$ \\
\hline & & seeds & Albizia lebbeck & India, Philippines & $\begin{array}{l}\text { Quiniones 1985, 1987, } \\
\text { Mittal et al. 1990, } \\
\text { Natarajan et al. } 2003\end{array}$ \\
\hline & & seeds & Albizia procera & Philippines & $\begin{array}{l}\text { Dayan 1986, Quiniones } \\
\text { 1987, Mittal et al. } 1990\end{array}$ \\
\hline & & seeds & $\begin{array}{l}\text { Alstonia } \\
\text { macrophylla }\end{array}$ & Philippines & $\begin{array}{l}\text { Quiniones 1985, 1987, } \\
\text { Mittal et al. } 1990\end{array}$ \\
\hline & & seeds & $\begin{array}{l}\text { Anthocephalus } \\
\text { chinensis }\end{array}$ & Philippines & Quiniones 1987 \\
\hline & & seeds & $\begin{array}{l}\text { Antidesma } \\
\text { ghaesembilla }\end{array}$ & Philippines & $\begin{array}{l}\text { Dayan 1986, Mittal et al. } \\
1990\end{array}$ \\
\hline & & seeds & Bauhinia acuminata & Philippines & $\begin{array}{l}\text { Quiniones 1985, 1987, } \\
\text { Mittal et al. } 1990\end{array}$ \\
\hline & & seeds & Bauhinia sp. & Thailand & $\begin{array}{l}\text { Chalermpongse et al. } \\
\text { 1984, Mittal et al. } 1990\end{array}$ \\
\hline & & seeds & Bombax anceps & Thailand & $\begin{array}{l}\text { Chalermpongse et al. } \\
\text { 1984, Mittal et al. } 1990\end{array}$ \\
\hline & & seeds & Bombax ceiba & Bangladesh & Mittal et al. 1990 \\
\hline & & seeds & Calamus ornatus & Philippines & $\begin{array}{l}\text { Quiniones 1985, 1987, } \\
\text { Mittal et al. } 1990\end{array}$ \\
\hline & & seed & Canavalia cathartica & India & Anita \& Sridhar 2009 \\
\hline & & seeds & Cassia fistula & India, Thailand & $\begin{array}{l}\text { Mittal \& Sharma 1981a, } \\
\text { Mittal et al. } 1990\end{array}$ \\
\hline & & seeds & Cassia siamea & $\begin{array}{l}\text { Philippines, } \\
\text { Thailand }\end{array}$ & $\begin{array}{l}\text { Quiniones 1985, 1987, } \\
\text { Mittal et al. } 1990\end{array}$ \\
\hline & & seeds & $\begin{array}{l}\text { Casuarina } \\
\text { equisetifolia }\end{array}$ & Philippines & $\begin{array}{l}\text { Quiniones 1985, 1987, } \\
\text { Anderson 1986a }\end{array}$ \\
\hline & & seeds & Cedrus deodara & India & Munjal \& Sharma 1975 \\
\hline
\end{tabular}


Table 2 Continued.

\begin{tabular}{|c|c|c|c|c|c|}
\hline Species & Taxonomy & Substrate & Host & Country & References \\
\hline & & seeds & Cupressus spp. & India & Sahai \& Otra 1982 \\
\hline & & seeds & $\begin{array}{l}\text { Dalbergia } \\
\text { cochinchinensis }\end{array}$ & Thailand & $\begin{array}{l}\text { Chalermpongse et al. } \\
\text { 1984, Mittal et al. } 1990\end{array}$ \\
\hline & & seeds & Dalbergia sissoo & India & $\begin{array}{l}\text { Mittal \& Sharma 1981b, } \\
\text { Mittal et al. 1990, } \\
\text { Kumar } 2014\end{array}$ \\
\hline & & seeds & Delonix regia & India & Sahu et al. 2003 \\
\hline & & seeds & Eucalyptus alba & Thailand & $\begin{array}{l}\text { Chalermpongse et al. } \\
\text { 1984, Mittal et al. } 1990\end{array}$ \\
\hline & & seeds & $\begin{array}{l}\text { Eucalyptus } \\
\text { citriodora }\end{array}$ & India & $\begin{array}{l}\text { Mittal \& Sharma 1984, } \\
\text { Mittal et al. } 1990\end{array}$ \\
\hline & & seeds & Eucalyptus deglupta & Thailand & $\begin{array}{l}\text { Chalermpongse et al. } \\
\text { 1984, Mittal et al. } 1990\end{array}$ \\
\hline & & seeds & Eucalyptus hybrid & India & $\begin{array}{l}\text { Mittal 1986, Mittal et al. } \\
1990\end{array}$ \\
\hline & & seeds & Eucalyptus sp. & India & $\begin{array}{l}\text { Saxena 1985, Mittal et } \\
\text { al. } 1990\end{array}$ \\
\hline & & seeds & Gliricidia sepium & Philippines & $\begin{array}{l}\text { Dayan 1986, } 1987, \\
\text { Mittal et al. } 1990\end{array}$ \\
\hline & & seeds & Gmelina arborea & $\begin{array}{l}\text { Philippines, } \\
\text { Thailand }\end{array}$ & $\begin{array}{l}\text { Quiniones 1985, 1987, } \\
\text { Mittal et al. } 1990\end{array}$ \\
\hline & & seeds & $\begin{array}{l}\text { Lagerstroemia } \\
\text { speciosa }\end{array}$ & Philippines & $\begin{array}{l}\text { Dayan 1986, 1987, } \\
\text { Anderson 1986a }\end{array}$ \\
\hline & & seeds & Larix sibirica & USSR & $\begin{array}{l}\text { Prisyazhnyuk 1960, } \\
\text { Mittal et al. } 1990\end{array}$ \\
\hline & & seed & $\begin{array}{l}\text { Leucaena } \\
\text { leucocephala }\end{array}$ & $\begin{array}{l}\text { Australia, } \\
\text { Bangladesh, } \\
\text { Philippines }\end{array}$ & $\begin{array}{l}\text { Nik \& Parbery 1977, } \\
\text { Quiniones 1985, 1987, } \\
\text { Mittal et al. 1990, Islam } \\
\text { et al. } 2008\end{array}$ \\
\hline & & seed & Medicago truncatula & Australia & Nik \& Parbery 1977 \\
\hline & & seeds & Melia azedarach & Thailand & $\begin{array}{l}\text { Chalermpongse et al. } \\
\text { 1984, Mittal et al. } 1990\end{array}$ \\
\hline & & seeds & Melia spp. & India & Sahai \& Otra 1982 \\
\hline
\end{tabular}


Table 2 Continued.

\begin{tabular}{|c|c|c|c|c|c|}
\hline Species & Taxonomy & Substrate & Host & Country & References \\
\hline & & seeds & Parkia roxburghii & Philippines & $\begin{array}{l}\text { Dayan 1986, Anderson } \\
\text { 1986a }\end{array}$ \\
\hline & & seeds & Parkia roxburghii & Philippines & Quiniones 1987 \\
\hline & & seeds & Picea abies & USSR & $\begin{array}{l}\text { Prisyazhnyuk } 1960 \text {, } \\
\text { Mittal et al. } 1990\end{array}$ \\
\hline & & seeds & Picea excelsa & USSR & $\begin{array}{l}\text { Urosevic 1961, } \\
\text { Anderson 1986a }\end{array}$ \\
\hline & & seeds & $\begin{array}{l}\text { Piliostigma } \\
\text { malabaricum }\end{array}$ & Philippines & $\begin{array}{l}\text { Quiniones 1985, } \\
\text { Anderson 1986a }\end{array}$ \\
\hline & & seeds & $\begin{array}{l}\text { Piliostigma } \\
\text { malabaricum }\end{array}$ & Philippines & Quiniones 1987 \\
\hline & & seeds & Pinus caribaea & Thailand & $\begin{array}{l}\text { Chalermpongse et al. } \\
\text { 1984, Mittal et al. } 1990\end{array}$ \\
\hline & & seeds & Pinus merkusii & Thailand & $\begin{array}{l}\text { Chalermpongse et al. } \\
\text { 1984, Mittal et al. } 1990\end{array}$ \\
\hline & & seeds & Pinus roxburghii & India & $\begin{array}{l}\text { Munjal \& Sharma 1975, } \\
\text { Mittal et al. } 1990\end{array}$ \\
\hline & & seeds & Pinus sylvestris & Poland, USSR & $\begin{array}{l}\text { Garbowski 1936, Mittal } \\
\text { et al. } 1990\end{array}$ \\
\hline & & seeds & Pinus wallichiana & India, Pakistan & $\begin{array}{l}\text { Munjal \& Sharma 1975, } \\
\text { Mittal et al. 1990, } \\
\text { Ashaeer } 2000\end{array}$ \\
\hline & & seeds & $\begin{array}{l}\text { Pittosporum } \\
\text { resiniferum }\end{array}$ & Philippines & $\begin{array}{l}\text { Quiniones 1985, 1987, } \\
\text { Anderson 1986a }\end{array}$ \\
\hline & & seeds & $\begin{array}{l}\text { Pseudotsuga } \\
\text { menziesii }\end{array}$ & USA & $\begin{array}{l}\text { Gordon 1967, Mittal et } \\
\text { al. } 1990\end{array}$ \\
\hline & & seeds & Pterocarpus indicus & Philippines & Quiniones 1987 \\
\hline & & seeds & Pyrus spp. & India & Sahai \& Otra 1982 \\
\hline & & seeds & $\begin{array}{l}\text { Quercus } \\
\text { castaneifolia }\end{array}$ & Iran & Kavosi et al. 2013 \\
\hline & & seeds & Quercus spp. & India & Sahai \& Otra 1982 \\
\hline & & seeds & Samanea saman & $\begin{array}{l}\text { Philippines, } \\
\text { Thailand }\end{array}$ & $\begin{array}{l}\text { Quiniones 1985, 1987, } \\
\text { Mittal et al. } 1990\end{array}$ \\
\hline
\end{tabular}


Table 2 Continued.

\begin{tabular}{|c|c|c|c|c|c|}
\hline Species & Taxonomy & Substrate & Host & Country & References \\
\hline & & seeds & Serialbizzia acle & Philippines & $\begin{array}{l}\text { Quiniones 1985, 1987, } \\
\text { Anderson 1986a }\end{array}$ \\
\hline & & seed & Sesbania bispinosa & India & Anita et al. 2009 \\
\hline & & seeds & Sesbania grandiflora & Philippines & $\begin{array}{l}\text { Quiniones 1985, 1987, } \\
\text { Mittal et al. } 1990\end{array}$ \\
\hline & & seeds & Shorea robusta & India, Thailand & $\begin{array}{l}\text { Mittal \& Sharma 1982b, } \\
\text { Mittal et al. } 1990\end{array}$ \\
\hline & & seed & $\begin{array}{l}\text { Stylosanthes } \\
\text { guianensis }\end{array}$ & Australia & Nik \& Parbery 1977 \\
\hline & & seeds & $\begin{array}{l}\text { Swietenia } \\
\text { macrophylla }\end{array}$ & Philippines & $\begin{array}{l}\text { Dayan 1986, Quiniones } \\
\text { 1987, Mittal et al. } 1990\end{array}$ \\
\hline & & seeds & Thuja spp. & India & Sahai \& Otra 1982 \\
\hline & & seeds & Toona calantas & Philippines & Quiniones 1987 \\
\hline & & seeds & Triplaris cumingiana & Philippines & $\begin{array}{l}\text { Quiniones 1985, 1987, } \\
\text { Anderson 1986a }\end{array}$ \\
\hline & & seeds & $\begin{array}{l}\text { Wallaceodendron } \\
\text { celibcum }\end{array}$ & Philippines & $\begin{array}{l}\text { Dayan 1986, Quiniones } \\
\text { 1987, Mittal et al. } 1990\end{array}$ \\
\hline & & seeds & $\begin{array}{l}\text { Xylia xylocarpa var. } \\
\text { kerrii }\end{array}$ & Thailand & $\begin{array}{l}\text { Chalermpongse et al. } \\
\text { 1984, Mittal et al. } 1990\end{array}$ \\
\hline $\begin{array}{l}\text { Aspergillus flavus var. } \\
\text { oryzae (Ahlb.) Kurtzman, } \\
\text { M.J. Smiley, Robnett \& } \\
\text { Wicklow }\end{array}$ & $\begin{array}{l}\text { Aspergillaceae, Eurotiomycetes, } \\
\text { Ascomycota }\end{array}$ & seeds & Pinus kesiya & Thailand & $\begin{array}{l}\text { Chalermpongse et al. } \\
\text { 1984, Mittal et al. } 1990\end{array}$ \\
\hline Aspergillus fumigatus & Aspergillaceae, Eurotiomycetes, & fruits & Avicennia marina & India & Mehdi \& Saifullah 2000 \\
\hline \multirow[t]{8}{*}{ Fresen. } & Ascomycota & pod & Canavalia cathartica & India & Anita \& Sridhar 2009 \\
\hline & & pod & Sesbania bispinosa & India & Anita et al. 2009 \\
\hline & & seeds & Albizia lebbeck & India & Natarajan et al. 2003 \\
\hline & & seeds & $\begin{array}{l}\text { Atropa belladonna } \\
\text { var. lutea }\end{array}$ & $\begin{array}{l}\text { Poland or/ } \\
\text { imported to Poland }\end{array}$ & Czeczuga et al. 2009 \\
\hline & & seeds & Cassia fistula & India & $\begin{array}{l}\text { Mittal \& Sharma 1981a, } \\
\text { Mittal et al. } 1990\end{array}$ \\
\hline & & seeds & Casuarina spp. & India & Sahai \& Otra 1982 \\
\hline & & seeds & Cedrus deodara & India & Mittal 1983 \\
\hline & & seeds & Cupressus spp. & India & Sahai \& Otra 1982 \\
\hline
\end{tabular}


Table 2 Continued.

\begin{tabular}{|c|c|c|c|c|c|}
\hline Species & Taxonomy & Substrate & Host & Country & References \\
\hline & & seeds & Delonix regia & India & Sahu et al. 2003 \\
\hline & & seeds & $\begin{array}{l}\text { Eucalyptus } \\
\text { citriodora }\end{array}$ & India & $\begin{array}{l}\text { Mittal \& Sharma 1984, } \\
\text { Mittal et al. } 1990\end{array}$ \\
\hline & & seeds & Eucalyptus deglupta & Thailand & $\begin{array}{l}\text { Chalermpongse et al. } \\
\text { 1984, Mittal et al. } 1990\end{array}$ \\
\hline & & seeds & Eucalyptus hybrid & India & $\begin{array}{l}\text { Mittal 1986, Mittal et al. } \\
1990\end{array}$ \\
\hline & & seeds & $\begin{array}{l}\text { Leucaena } \\
\text { leucocephala }\end{array}$ & Thailand & $\begin{array}{l}\text { Chalermpongse et al. } \\
\text { 1984, Mittal et al. } 1990\end{array}$ \\
\hline & & seeds & Melia spp. & India & Sahai \& Otra 1982 \\
\hline & & seed & $\begin{array}{l}\text { Peltophorum } \\
\text { pterocarpum } \\
\text { [= Peltophorum } \\
\text { ferrugineum] }\end{array}$ & India & Goswami \& Ojha 2004 \\
\hline & & seeds & Pinus merkusii & Thailand & $\begin{array}{l}\text { Chalermpongse et al. } \\
\text { 1984, Mittal et al. } 1990\end{array}$ \\
\hline & & seeds & Pinus wallichiana & India & $\begin{array}{l}\text { Mittal \& Sharma 1982b, } \\
\text { Mittal et al. } 1990\end{array}$ \\
\hline & & seeds & $\begin{array}{l}\text { Pseudotsuga } \\
\text { menziesii }\end{array}$ & USA & $\begin{array}{l}\text { Gordon 1967, Mittal et } \\
\text { al. } 1990\end{array}$ \\
\hline & & seeds & Pyrus spp. & India & Sahai \& Otra 1982 \\
\hline & & $\begin{array}{l}\text { seeds } \\
\text { seeds }\end{array}$ & $\begin{array}{l}\text { Quercus spp. } \\
\text { Rhamnus purshiana }\end{array}$ & $\begin{array}{l}\text { India } \\
\text { Poland or/ } \\
\text { imported to Poland }\end{array}$ & $\begin{array}{l}\text { Sahai \& Otra } 1982 \\
\text { Czeczuga et al. } 2009\end{array}$ \\
\hline & & seeds & Sapium spp. & India & Sahai \& Otra 1982 \\
\hline & & seed & Sesbania bispinosa & India & Anita et al. 2009 \\
\hline & & seeds & Shorea robusta & Thailand & $\begin{array}{l}\text { Mittal \& Sharma 1982b, } \\
\text { Mittal et al. } 1990\end{array}$ \\
\hline \multirow[t]{2}{*}{$\begin{array}{l}\text { Aspergillus funiculosus G. } \\
\text { Sm. }\end{array}$} & \multirow[t]{2}{*}{$\begin{array}{l}\text { Aspergillaceae, Eurotiomycetes, } \\
\text { Ascomycota }\end{array}$} & seeds & Pinus roxburghii & India & $\begin{array}{l}\text { Mittal \& Sharma 1982c, } \\
\text { Mittal et al. } 1990\end{array}$ \\
\hline & & seeds & Pinus wallichiana & India & $\begin{array}{l}\text { Mittal \& Sharma 1982b, } \\
\text { Mittal et al. } 1990\end{array}$ \\
\hline
\end{tabular}


Table 2 Continued.

\begin{tabular}{|c|c|c|c|c|c|}
\hline Species & Taxonomy & Substrate & Host & Country & References \\
\hline & & seeds & Shorea robusta & India & $\begin{array}{l}\text { Mittal \& Sharma 1982b, } \\
\text { Mittal et al. } 1990\end{array}$ \\
\hline \multirow[t]{11}{*}{$\begin{array}{l}\text { Aspergillus glaucus (L.) } \\
\text { Link }\end{array}$} & $\begin{array}{l}\text { Aspergillaceae, Eurotiomycetes, } \\
\text { Ascomycota }\end{array}$ & seeds & Abies sibirica & USSR & $\begin{array}{l}\text { Prisyazhnyuk } 1960 \text {, } \\
\text { Mittal et al. } 1990\end{array}$ \\
\hline & & seeds & Bombax ceiba & Bangladesh & Mittal et al. 1990 \\
\hline & & seed & $\begin{array}{l}\text { Leucaena } \\
\text { leucocephala }\end{array}$ & Australia & Nik \& Parbery 1977 \\
\hline & & seed & Lotononis angolensis & Australia & Nik \& Parbery 1977 \\
\hline & & seed & Medicago truncatula & Australia & Nik \& Parbery 1977 \\
\hline & & seeds & Picea abies & USSR & $\begin{array}{l}\text { Prisyazhnyuk } 1960 \text {, } \\
\text { Mittal et al. } 1990\end{array}$ \\
\hline & & seeds & Pinus sylvestris & USSR & $\begin{array}{l}\text { Prisyazhnyuk } 1960 \text {, } \\
\text { Mittal et al. } 1990\end{array}$ \\
\hline & & seeds & $\begin{array}{l}\text { Pseudotsuga } \\
\text { menziesii }\end{array}$ & Canada & $\begin{array}{l}\text { Salisbury 1955, Mittal et } \\
\text { al. } 1990\end{array}$ \\
\hline & & seed & Stylosanthes gracilis & Australia & Nik \& Parbery 1977 \\
\hline & & seed & $\begin{array}{l}\text { Stylosanthes } \\
\text { guianensis }\end{array}$ & Australia & Nik \& Parbery 1977 \\
\hline & & seed & Stylosanthes humilis & Australia & Nik \& Parbery 1977 \\
\hline $\begin{array}{l}\text { Aspergillus glaucus (L.) } \\
\text { Link [as Aspergillus glauca] }\end{array}$ & & seeds & Pinus caribaea & $\begin{array}{l}\text { Belize [as British } \\
\text { Honduras] }\end{array}$ & Hocking 1968 \\
\hline $\begin{array}{l}\text { Aspergillus humicola } \\
\text { Chaudhuri \& Sachar }\end{array}$ & $\begin{array}{l}\text { Aspergillaceae, Eurotiomycetes, } \\
\text { Ascomycota }\end{array}$ & seeds & Pinus roxburghii & India & $\begin{array}{l}\text { Munjal \& Sharma 1975, } \\
\text { Mittal et al. } 1990\end{array}$ \\
\hline $\begin{array}{l}\text { Aspergillus janus Raper \& } \\
\text { Thom }\end{array}$ & $\begin{array}{l}\text { Aspergillaceae, Eurotiomycetes, } \\
\text { Ascomycota }\end{array}$ & seeds & Pinus wallichiana & Pakistan & Ashaeer 2000 \\
\hline Aspergillus koningii Oudem. & Aspergillaceae, Eurotiomycetes, & seeds & Cedrus deodara & India & Mittal 1983 \\
\hline $\begin{array}{l}\text { Aspergillus koningii Oudem. } \\
\text { [as‘koninge’] }\end{array}$ & Ascomycota & seeds & Shorea robusta & India & $\begin{array}{l}\text { Mittal \& Sharma 1982b, } \\
\text { Mittal et al. } 1990\end{array}$ \\
\hline \multirow[t]{3}{*}{ Aspergillus luchuensis Inui } & $\begin{array}{l}\text { Aspergillaceae, Eurotiomycetes, } \\
\text { Ascomycota }\end{array}$ & seeds & Cedrus deodara & India & Mittal 1983 \\
\hline & & seeds & $\begin{array}{l}\text { Eucalyptus } \\
\text { citriodora }\end{array}$ & India & $\begin{array}{l}\text { Mittal \& Sharma 1984, } \\
\text { Mittal et al. } 1990\end{array}$ \\
\hline & & seeds & Pinus wallichiana & India & $\begin{array}{l}\text { Mittal \& Sharma 1982b, } \\
\text { Mittal et al. } 1990\end{array}$ \\
\hline
\end{tabular}


Table 2 Continued.

\begin{tabular}{|c|c|c|c|c|c|}
\hline Species & Taxonomy & Substrate & Host & Country & References \\
\hline \multirow{5}{*}{$\begin{array}{l}\text { Aspergillus nidulans } \\
\text { (Eidam) G. Winter }\end{array}$} & Aspergillaceae, Eurotiomycetes, & fruits & Avicennia marina & India & Mehdi \& Saifullah 2000 \\
\hline & Ascomycota & seeds & Bauhinia sp. & Thailand & $\begin{array}{l}\text { Chalermpongse et al. } \\
\text { 1984, Mittal et al. } 1990\end{array}$ \\
\hline & & seeds & Cassia bakeriana & Thailand & $\begin{array}{l}\text { Chalermpongse et al. } \\
\text { 1984, Mittal et al. } 1990\end{array}$ \\
\hline & & seeds & Eucalyptus sp. & India & $\begin{array}{l}\text { Reddy et al. 1982, Mittal } \\
\text { et al. } 1990\end{array}$ \\
\hline & & seed & Linum usitatissimum & Canada & Conners 1967 \\
\hline \multirow[t]{16}{*}{ Aspergillus niger Tiegh. } & $\begin{array}{l}\text { Aspergillaceae, Eurotiomycetes, } \\
\text { Ascomycota }\end{array}$ & acorns & Quercus robur & Poland & $\begin{array}{l}\text { Jankowiak 2008, Krol et } \\
\text { al. } 2015\end{array}$ \\
\hline & & fruit & $\begin{array}{l}\text { Artocarpus } \\
\text { communis }\end{array}$ & Nigeria & Amusa et al. 2002 \\
\hline & & fruits & Psychotria asiatica & Hong Kong & Tang et al. 2003a \\
\hline & & fruits & $\begin{array}{l}\text { Rhodomyrtus } \\
\text { tomentosa }\end{array}$ & Hong Kong & Tang et al. 2003a \\
\hline & & fruits & Sarcandra glabra & Hong Kong & Tang et al. 2003a \\
\hline & & pod, seed & Canavalia cathartica & India & Anita \& Sridhar 2009 \\
\hline & & pod, seed & Sesbania bispinosa & India & Anita et al. 2009 \\
\hline & & pods & Delonix regia & Thailand & Somrithipol et al. 2002b \\
\hline & & seeds & Abies lasiocarpa & Canada, Norway & Talgø et al. 2010 \\
\hline & & seeds & Abies nordmanniana & $\begin{array}{l}\text { Austria, Georgia, } \\
\text { Russia }\end{array}$ & Talgø et al. 2010 \\
\hline & & seeds & Abies procera & Norway & Talgø et al. 2010 \\
\hline & & seeds & Abies sibirica & USSR & $\begin{array}{l}\text { Prisyazhnyuk 1960, } \\
\text { Mittal et al. } 1990\end{array}$ \\
\hline & & seeds & $\begin{array}{l}\text { Acacia } \\
\text { auriculiformis }\end{array}$ & $\begin{array}{l}\text { Philippines, } \\
\text { Thailand }\end{array}$ & $\begin{array}{l}\text { Quiniones 1985, 1987, } \\
\text { Anderson 1986a, Mittal } \\
\text { et al. } 1990\end{array}$ \\
\hline & & seeds & Albizia falcataria & Philippines & $\begin{array}{l}\text { Dayan 1986, Quiniones } \\
\text { 1987, Mittal et al. } 1990\end{array}$ \\
\hline & & seeds & Albizia julibrissin & Philippines & $\begin{array}{l}\text { Quiniones 1985, 1987, } \\
\text { Mittal et al. } 1990\end{array}$ \\
\hline & & seeds & Albizia lebbeck & India, Philippines & $\begin{array}{l}\text { Dayan 1986, Quiniones } \\
\text { 1987, Mittal et al. 1990, }\end{array}$ \\
\hline
\end{tabular}


Table 2 Continued.

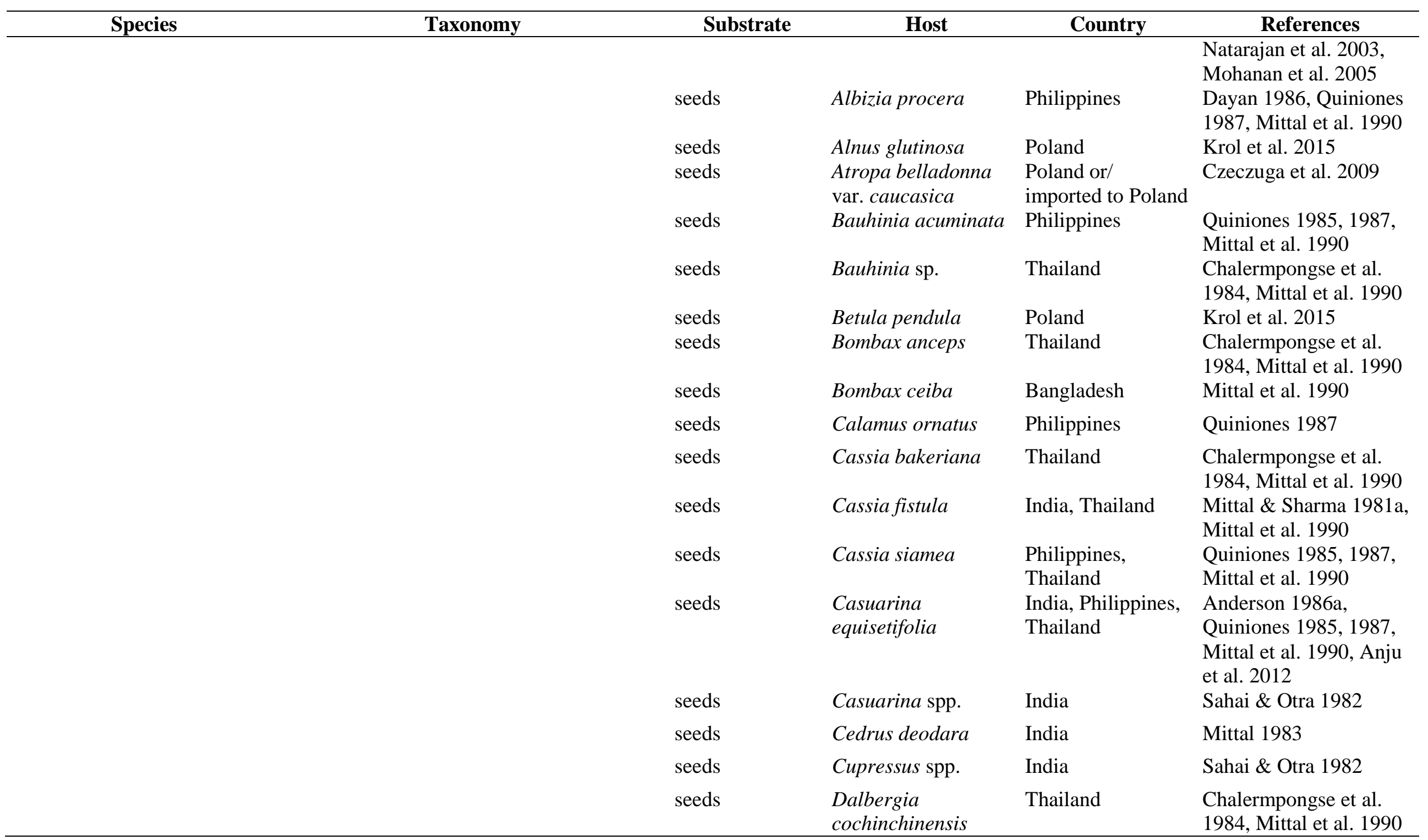


Table 2 Continued.

\begin{tabular}{|c|c|c|c|c|c|}
\hline Species & Taxonomy & Substrate & Host & Country & References \\
\hline & & seeds & Dalbergia sissoo & India & $\begin{array}{l}\text { Kumar 2014, Mittal \& } \\
\text { Sharma 1981b, Mittal et } \\
\text { al. 1990, Mohanan et al. } \\
2005\end{array}$ \\
\hline & & seeds & Eucalyptus alba & Thailand & $\begin{array}{l}\text { Chalermpongse et al. } \\
\text { 1984, Mittal et al. } 1990\end{array}$ \\
\hline & & seeds & $\begin{array}{l}\text { Eucalyptus } \\
\text { citriodora }\end{array}$ & India & $\begin{array}{l}\text { Mittal \& Sharma 1984, } \\
\text { Mittal et al. } 1990\end{array}$ \\
\hline & & seeds & Eucalyptus deglupta & Thailand & $\begin{array}{l}\text { Chalermpongse et al. } \\
\text { 1984, Mittal et al. } 1990\end{array}$ \\
\hline & & seeds & Eucalyptus hybrid & India & $\begin{array}{l}\text { Mittal 1986, Mittal et al. } \\
1990\end{array}$ \\
\hline & & seeds & Eucalyptus sp. & India & $\begin{array}{l}\text { Reddy et al. 1982, Mittal } \\
\text { et al. } 1990\end{array}$ \\
\hline & & seeds & Fagus sylvatica & Poland & Krol et al. 2015 \\
\hline & & seeds & Gliricidia sepium & Philippines & $\begin{array}{l}\text { Dayan 1986, Quiniones } \\
\text { 1987, Mittal et al. } 1990\end{array}$ \\
\hline & & seeds & Gmelina arborea & $\begin{array}{l}\text { Philippines, } \\
\text { Thailand }\end{array}$ & $\begin{array}{l}\text { Quiniones 1985, 1987, } \\
\text { Mittal et al. } 1990\end{array}$ \\
\hline & & seeds & $\begin{array}{l}\text { Holarrhena } \\
\text { antidysenterica }\end{array}$ & Thailand & $\begin{array}{l}\text { Chalermpongse et al. } \\
\text { 1984, Mittal et al. } 1990\end{array}$ \\
\hline & & seeds & $\begin{array}{l}\text { Lagerstroemia } \\
\text { calyculata }\end{array}$ & Thailand & $\begin{array}{l}\text { Chalermpongse et al. } \\
\text { 1984, Mittal et al. } 1990\end{array}$ \\
\hline & & seeds & Larix decidua & USSR & $\begin{array}{l}\text { Kozlowska 1968, Mittal } \\
\text { et al. } 1990\end{array}$ \\
\hline & & seeds & Larix sibirica & USSR & $\begin{array}{l}\text { Prisyazhnyuk 1960, } \\
\text { Mittal et al. } 1990\end{array}$ \\
\hline & & seeds & $\begin{array}{l}\text { Leucaena } \\
\text { leucocephala }\end{array}$ & $\begin{array}{l}\text { Bangladesh, } \\
\text { Philippines, } \\
\text { Thailand }\end{array}$ & $\begin{array}{l}\text { Quiniones 1985, 1987, } \\
\text { Mittal et al. 1990, Islam } \\
\text { et al. } 2008\end{array}$ \\
\hline & & seed & Linum usitatissimum & Canada & Conners 1967 \\
\hline & & seed & $\begin{array}{l}\text { Macroptilium } \\
\text { atropurpureum }\end{array}$ & Australia & Nik \& Parbery 1977 \\
\hline
\end{tabular}


Table 2 Continued.

\begin{tabular}{|c|c|c|c|c|c|}
\hline Species & Taxonomy & Substrate & Host & Country & References \\
\hline & & seeds & Melia azedarach & Thailand & $\begin{array}{l}\text { Chalermpongse et al. } \\
\text { 1984, Mittal et al. } 1990\end{array}$ \\
\hline & & seeds & Parkia roxburghii & Philippines & Quiniones 1987 \\
\hline & & seeds & Picea abies & Poland, USSR & $\begin{array}{l}\text { Prisyazhnyuk 1960, } \\
\text { Mittal et al. 1990, Krol } \\
\text { et al. } 2015\end{array}$ \\
\hline & & seeds & Picea excelsa & USSR & $\begin{array}{l}\text { Urosevic 1961, } \\
\text { Anderson 1986a }\end{array}$ \\
\hline & & seeds & $\begin{array}{l}\text { Piliostigma } \\
\text { malabaricum }\end{array}$ & Philippines & $\begin{array}{l}\text { Quiniones 1985, } \\
\text { Anderson 1986a, } 1987\end{array}$ \\
\hline & & seeds & Pinus caribaea & Thailand & $\begin{array}{l}\text { Chalermpongse et al. } \\
\text { 1984, Mittal et al. } 1990\end{array}$ \\
\hline & & seed & Pinus densiflora & Japan & Watanabe 2010 \\
\hline & & seeds & Pinus elliottii & South Africa & Cilliers et al. 1995 \\
\hline & & seeds & $\begin{array}{l}\text { Pinus elliottii var. } \\
\text { elliottii }\end{array}$ & Georgia & Huang \& Kuhlman 1990 \\
\hline & & seeds & Pinus kesiya & Philippines & $\begin{array}{l}\text { Quiniones 1985, 1987, } \\
\text { Anderson 1986a }\end{array}$ \\
\hline & & seeds & Pinus oocarpa & Honduras & Mittal et al. 1990 \\
\hline & & seeds & Pinus roxburghii & India & $\begin{array}{l}\text { Mittal \& Sharma 1982c, } \\
\text { Mittal et al. } 1990\end{array}$ \\
\hline & & seeds & Pinus sylvestris & Poland, USSR & $\begin{array}{l}\text { Urosevic 1961, } \\
\text { Anderson 1986a, Krol et } \\
\text { al. } 2015\end{array}$ \\
\hline & & seeds & Pinus taeda & Georgia & Huang \& Kuhlman 1990 \\
\hline & & seeds & Pinus wallichiana & India & $\begin{array}{l}\text { Munjal \& Sharma 1975, } \\
\text { Mittal et al. } 1990\end{array}$ \\
\hline & & seeds & $\begin{array}{l}\text { Pittosporum } \\
\text { resiniferum }\end{array}$ & Philippines & $\begin{array}{l}\text { Dayan 1986, Quiniones } \\
\text { 1987, Mittal et al. } 1990\end{array}$ \\
\hline & & seeds & $\begin{array}{l}\text { Platanus } \\
\text { occidentalis }\end{array}$ & USA & $\begin{array}{l}\text { Fakir et al. 1971, Mittal } \\
\text { et al. } 1990\end{array}$ \\
\hline & & seeds & $\begin{array}{l}\text { Pseudotsuga } \\
\text { menziesii }\end{array}$ & Canada & $\begin{array}{l}\text { Salisbury 1955, Mittal et } \\
\text { al. } 1990\end{array}$ \\
\hline
\end{tabular}


Table 2 Continued.

\begin{tabular}{|c|c|c|c|c|c|}
\hline Species & Taxonomy & Substrate & Host & Country & References \\
\hline & & seeds & Pterocarpus indicus & Philippines & Quiniones 1987 \\
\hline & & seeds & $\begin{array}{l}\text { Quercus } \\
\text { castaneifolia }\end{array}$ & Iran & Kavosi et al. 2013 \\
\hline & & seeds & Quercus pedunculata & USSR & $\begin{array}{l}\text { Kozlowska 1968, Mittal } \\
\text { et al. } 1990\end{array}$ \\
\hline & & seeds & Quercus spp. & USSR & $\begin{array}{l}\text { Urosevic 1959, Mittal et } \\
\text { al. } 1990\end{array}$ \\
\hline & & seeds & Ribes aureus & $\begin{array}{l}\text { Poland or/ } \\
\text { imported to Poland }\end{array}$ & Czeczuga et al. 2009 \\
\hline & & seeds & Rosa mollis & $\begin{array}{l}\text { Poland or/ } \\
\text { imported to Poland }\end{array}$ & Czeczuga et al. 2009 \\
\hline & & seeds & Samanea saman & $\begin{array}{l}\text { Philippines, } \\
\text { Thailand }\end{array}$ & $\begin{array}{l}\text { Quiniones 1985, } \\
\text { Anderson 1986a, Mittal } \\
\text { et al. 1990, } 1987\end{array}$ \\
\hline & & seeds & Sapium spp. & India & Sahai \& Otra 1982 \\
\hline & & seeds & Sesbania grandiflora & Philippines & $\begin{array}{l}\text { Quiniones 1985, 1987, } \\
\text { Mittal et al. } 1990\end{array}$ \\
\hline & & seeds & Shorea robusta & India, Thailand & $\begin{array}{l}\text { Chalermpongse et al. } \\
\text { 1984, Mittal et al. } 1990\end{array}$ \\
\hline & & seeds & Shorea spp. & India & $\begin{array}{l}\text { Singh et al. 1979, Mittal } \\
\text { et al. } 1990\end{array}$ \\
\hline & & seeds & $\begin{array}{l}\text { Swietenia } \\
\text { macrophylla }\end{array}$ & Philippines & $\begin{array}{l}\text { Dayan 1986, Quiniones } \\
\text { 1987, Mittal et al. } 1990\end{array}$ \\
\hline & & seeds & Tectona grandis & India & Mohanan et al. 2005 \\
\hline & & seeds & Toona calantas & Philippines & Quiniones 1987 \\
\hline & & seeds & Triplaris cumingiana & Philippines & $\begin{array}{l}\text { Quiniones 1985, } \\
\text { Anderson 1986a, } 1987\end{array}$ \\
\hline & & seeds & $\begin{array}{l}\text { Xylia xylocarpa var. } \\
\text { kerrii }\end{array}$ & Thailand & $\begin{array}{l}\text { Chalermpongse et al. } \\
\text { 1984, Mittal et al. } 1990\end{array}$ \\
\hline $\begin{array}{l}\text { Aspergillus niger var. niger } \\
\text { Tiegh. }\end{array}$ & $\begin{array}{l}\text { Aspergillaceae, Eurotiomycetes, } \\
\text { Ascomycota }\end{array}$ & seeds & Delonix regia & India & Sahu et al. 2003 \\
\hline \multirow[t]{2}{*}{$\begin{array}{l}\text { Aspergillus ochraceus G. } \\
\text { Wilh. }\end{array}$} & $\begin{array}{l}\text { Aspergillaceae, Eurotiomycetes, } \\
\text { Ascomycota }\end{array}$ & pod & Canavalia cathartica & India & Anita \& Sridhar 2009 \\
\hline & & seed & Abies balsamea & Canada & Conners 1967 \\
\hline
\end{tabular}


Table 2 Continued.

\begin{tabular}{|c|c|c|c|c|c|}
\hline Species & Taxonomy & Substrate & Host & Country & References \\
\hline & & seeds & Albizia lebbeck & India & $\begin{array}{l}\text { Mittal \& Sharma 1982a, } \\
\text { Mittal et al. } 1990\end{array}$ \\
\hline & & seeds & Bombax ceiba & Bangladesh & Mittal et al. 1990 \\
\hline & & seed & Canavalia cathartica & India & Anita \& Sridhar 2009 \\
\hline & & seeds & Cassia fistula & India & $\begin{array}{l}\text { Mittal \& Sharma 1981a, } \\
\text { Mittal et al. } 1990\end{array}$ \\
\hline & & seeds & Dalbergia sissoo & India & $\begin{array}{l}\text { Mittal \& Sharma 1981b, } \\
\text { Mittal et al. } 1990\end{array}$ \\
\hline & & seeds & Larix sibirica & USSR & $\begin{array}{l}\text { Prisyazhnyuk } 1960 \text {, } \\
\text { Mittal et al. } 1990\end{array}$ \\
\hline & & seeds & Picea abies & USSR & $\begin{array}{l}\text { Prisyazhnyuk } 1960 \text {, } \\
\text { Mittal et al. } 1990\end{array}$ \\
\hline & & seeds & Pinus kesiya & Thailand & $\begin{array}{l}\text { Chalermpongse et al. } \\
\text { 1984, Mittal et al. } 1990\end{array}$ \\
\hline & & seeds & Pinus sylvestris & USSR & $\begin{array}{l}\text { Prisyazhnyuk } 1960 \text {, } \\
\text { Mittal et al. } 1990\end{array}$ \\
\hline & & seeds & $\begin{array}{l}\text { Platanus } \\
\text { occidentalis }\end{array}$ & USA & $\begin{array}{l}\text { Fakir et al. 1971, Mittal } \\
\text { et al. } 1990\end{array}$ \\
\hline $\begin{array}{l}\text { Aspergillus ochraceus G. } \\
\text { Wilh. [as 'ochraceum'] }\end{array}$ & & seeds & Abies sibirica & USSR & $\begin{array}{l}\text { Prisyazhnyuk } 1960 \text {, } \\
\text { Mittal et al. } 1990\end{array}$ \\
\hline \multirow{5}{*}{$\begin{array}{l}\text { Aspergillus oryzae (Ahlb.) } \\
\text { Cohn }\end{array}$} & & seeds & Casuarina spp. & India & Sahai \& Otra 1982 \\
\hline & $\begin{array}{l}\text { Aspergillaceae, Eurotiomycetes, } \\
\text { Ascomycota }\end{array}$ & pod, seed & Sesbania bispinosa & India & Anita et al. 2009 \\
\hline & & seeds & Picea excelsa & USSR & $\begin{array}{l}\text { Urosevic 1961, } \\
\text { Anderson 1986a }\end{array}$ \\
\hline & & seeds & Pinus sylvestris & USSR & $\begin{array}{l}\text { Urosevic 1961, } \\
\text { Anderson 1986a }\end{array}$ \\
\hline & & seeds & $\begin{array}{l}\text { Pseudotsuga } \\
\text { menziesii }\end{array}$ & USA & $\begin{array}{l}\text { Gordon 1967, Mittal et } \\
\text { al. } 1990\end{array}$ \\
\hline $\begin{array}{l}\text { Aspergillus parasiticus } \\
\text { Speare }\end{array}$ & $\begin{array}{l}\text { Aspergillaceae, Eurotiomycetes, } \\
\text { Ascomycota }\end{array}$ & pod & Canavalia cathartica & India & Anita \& Sridhar 2009 \\
\hline $\begin{array}{l}\text { Aspergillus phoenicis } \\
\text { (Corda) Thom \& Currie }\end{array}$ & $\begin{array}{l}\text { Aspergillaceae, Eurotiomycetes, } \\
\text { Ascomycota }\end{array}$ & $\begin{array}{l}\text { seed } \\
\text { seeds }\end{array}$ & $\begin{array}{l}\text { Sesbania bispinosa } \\
\text { Albizia lebbeck }\end{array}$ & $\begin{array}{l}\text { India } \\
\text { India }\end{array}$ & $\begin{array}{l}\text { Anita et al. } 2009 \\
\text { Mittal \& Sharma 1982a, } \\
\text { Mittal et al. } 1990\end{array}$ \\
\hline
\end{tabular}


Table 2 Continued.

\begin{tabular}{|c|c|c|c|c|c|}
\hline Species & Taxonomy & Substrate & Host & Country & References \\
\hline \multirow{4}{*}{$\begin{array}{l}\text { Aspergillus repens (Corda) } \\
\text { Sacc. }\end{array}$} & \multirow{4}{*}{$\begin{array}{l}\text { Aspergillaceae, Eurotiomycetes, } \\
\text { Ascomycota }\end{array}$} & seeds & Dalbergia sissoo & India & Kumar 2014 \\
\hline & & seeds & $\begin{array}{l}\text { Pseudotsuga } \\
\text { menziesii }\end{array}$ & USA & $\begin{array}{l}\text { Gordon 1967, Mittal et } \\
\text { al. } 1990\end{array}$ \\
\hline & & seeds & $\begin{array}{l}\text { Platanus } \\
\text { occidentalis }\end{array}$ & USA & $\begin{array}{l}\text { Fakir et al. 1971, Mittal } \\
\text { et al. } 1990\end{array}$ \\
\hline & & seed & Setaria viridis & Canada & Conners 1967 \\
\hline \multirow[t]{4}{*}{ Aspergillus restrictus G. Sm. } & \multirow[t]{4}{*}{$\begin{array}{l}\text { Aspergillaceae, Eurotiomycetes, } \\
\text { Ascomycota }\end{array}$} & seeds & Bauhinia sp. & Thailand & $\begin{array}{l}\text { Chalermpongse et al. } \\
\text { 1984, Mittal et al. } 1990\end{array}$ \\
\hline & & seeds & Cassia bakeriana & Thailand & $\begin{array}{l}\text { Chalermpongse et al. } \\
\text { 1984, Mittal et al. } 1990\end{array}$ \\
\hline & & seeds & $\begin{array}{l}\text { Holarrhena } \\
\text { antidysenterica }\end{array}$ & Thailand & $\begin{array}{l}\text { Chalermpongse et al. } \\
\text { 1984, Mittal et al. } 1990\end{array}$ \\
\hline & & seeds & $\begin{array}{l}\text { Lagerstroemia } \\
\text { calyculata }\end{array}$ & Thailand & $\begin{array}{l}\text { Chalermpongse et al. } \\
\text { 1984, Mittal et al. } 1990\end{array}$ \\
\hline \multirow{2}{*}{$\begin{array}{l}\text { Aspergillus ruber (Jos. } \\
\text { König, Spieck. \& W. } \\
\text { Bremer) Thom \& Church }\end{array}$} & \multirow[t]{2}{*}{$\begin{array}{l}\text { Aspergillaceae, Eurotiomycetes, } \\
\text { Ascomycota }\end{array}$} & seed & $\begin{array}{l}\text { Macroptilium } \\
\text { atropurpureum }\end{array}$ & Australia & Nik \& Parbery 1977 \\
\hline & & seeds & $\begin{array}{l}\text { Platanus } \\
\text { occidentalis }\end{array}$ & USA & $\begin{array}{l}\text { Fakir et al. 1971, Mittal } \\
\text { et al. } 1990\end{array}$ \\
\hline $\begin{array}{l}\text { Aspergillus spinulosporus } \\
\text { Hubka, S.W. Peterson \& M. } \\
\text { Kolař́k [as Aspergillus } \\
\text { nidulans var. echinulatus } \\
\text { Fennell \& Raper] }\end{array}$ & $\begin{array}{l}\text { Aspergillaceae, Eurotiomycetes, } \\
\text { Ascomycota }\end{array}$ & seeds & $\begin{array}{l}\text { Casuarina spp., } \\
\text { Pyrus spp. }\end{array}$ & India & Sahai \& Otra 1982 \\
\hline \multirow[t]{4}{*}{ Aspergillus spp. } & \multirow{4}{*}{$\begin{array}{l}\text { Aspergillaceae, Eurotiomycetes, } \\
\text { Ascomycota }\end{array}$} & acorns & Quercus robur & Poland & Jankowiak 2008 \\
\hline & & seed capsules & Eucalyptus grandis & South Africa & Jimu et al. 2016 \\
\hline & & seeds & Acacia confusa & Philippines & $\begin{array}{l}\text { Agmata 1979, Anderson } \\
\text { 1986a }\end{array}$ \\
\hline & & seeds & Acer rubrum & USA & $\begin{array}{l}\text { Friedrich et al. } 1971 \text {, } \\
\text { Mittal et al. } 1990\end{array}$ \\
\hline
\end{tabular}


Table 2 Continued.

\begin{tabular}{|c|c|c|c|c|c|}
\hline Species & Taxonomy & Substrate & Host & Country & References \\
\hline & & seeds & Acer saccharinum & USA & $\begin{array}{l}\text { Friedrich et al. } 1971 \text {, } \\
\text { Mittal et al. } 1990\end{array}$ \\
\hline & & seeds & Albizia falcataria & Philippines & $\begin{array}{l}\text { Agmata 1979, Mittal et } \\
\text { al. } 1990\end{array}$ \\
\hline & & seeds & Albizia lebbeck & India & Mohanan et al. 2005 \\
\hline & & seeds & Albizia procera & Philippines & $\begin{array}{l}\text { Agmata 1979, Anderson } \\
\text { 1986a, Mittal et al. } 1990\end{array}$ \\
\hline & & seeds & $\begin{array}{l}\text { Anthocephalus } \\
\text { chinensis }\end{array}$ & Philippines & $\begin{array}{l}\text { Agmata 1979, Mittal et } \\
\text { al. } 1990\end{array}$ \\
\hline & & seeds & $\begin{array}{l}\text { Araucaria } \\
\text { heterophylla }\end{array}$ & Egypt & $\begin{array}{l}\text { Kamara et al. } 1981 \text {, } \\
\text { Mittal et al. } 1990\end{array}$ \\
\hline & & seeds & Azadirachta indica & Malaysia & $\begin{array}{l}\text { Mittal \& Sharma 1982b, } \\
\text { Mittal et al. } 1990\end{array}$ \\
\hline & & seeds & $\begin{array}{l}\text { Betula } \\
\text { alleghaniensis }\end{array}$ & USA & $\begin{array}{l}\text { Smoot \& Segall 1963, } \\
\text { Mittal et al. } 1990\end{array}$ \\
\hline & & seeds & Betula papyrifera & USA & $\begin{array}{l}\text { Friedrich 1969, Mittal et } \\
\text { al. } 1990\end{array}$ \\
\hline & & seeds & Bombax ceiba & India & Mittal et al. 1990 \\
\hline & & seeds & Canavalia cathartica & India & $\begin{array}{l}\text { Anita \& Sridhar 2009, } \\
\text { Seena \& Sridhar } 2004\end{array}$ \\
\hline & & seed & $\begin{array}{l}\text { Cryptomeria } \\
\text { japonica }\end{array}$ & Japan & Watanabe 2010 \\
\hline & & seeds & Dalbergia sissoides & India & Mohanan et al. 2005 \\
\hline & & seeds & Delonix regia & Brazil & $\begin{array}{l}\text { Mathur 1974, Anderson } \\
\text { 1986a }\end{array}$ \\
\hline & & seeds & $\begin{array}{l}\text { Dianthus } \\
\text { caryophyllus }\end{array}$ & Taiwan & Li \& Wu 2002 \\
\hline & & seeds & Dianthus spp. & Taiwan & Li \& Wu 2002 \\
\hline & & seeds & $\begin{array}{l}\text { Eucalyptus } \\
\text { citriodora }\end{array}$ & India & Anderson 1986a \\
\hline & & seeds & Eucalyptus globulus & India & Anderson 1986a \\
\hline & & seeds & Eucalyptus grandis & India & Anderson 1986a \\
\hline & & seeds & $\begin{array}{l}\text { Eucalyptus } \\
\text { tereticornis }\end{array}$ & India & Anderson 1986a \\
\hline
\end{tabular}


Table 2 Continued.

\begin{tabular}{|c|c|c|c|c|c|}
\hline Species & Taxonomy & Substrate & Host & Country & References \\
\hline & & seeds & Fraxinus americana & USA & $\begin{array}{l}\text { Friedrich et al. } 1971 \text {, } \\
\text { Mittal et al. } 1990\end{array}$ \\
\hline & & seeds & Gmelina arborea & Philippines & $\begin{array}{l}\text { Agmata 1979, Anderson } \\
\text { 1986a }\end{array}$ \\
\hline & & seeds & Larix occidentalis & USA & James et al. 1996 \\
\hline & & seeds & $\begin{array}{l}\text { Leucaena } \\
\text { leucocephala }\end{array}$ & Philippines & $\begin{array}{l}\text { Agmata 1979, Anderson } \\
\text { 1986a }\end{array}$ \\
\hline & & seeds & Manilkara bidentata & USA & Bayman et al. 1998 \\
\hline & & seeds & Picea engelmannii & USA & $\begin{array}{l}\text { Fuller \& Hildebrand } \\
\text { 1985, Mittal et al. } 1990\end{array}$ \\
\hline & & seeds & Picea sitchensis & UK & $\begin{array}{l}\text { Salt 1964, Mittal et al. } \\
1990\end{array}$ \\
\hline & & seeds & $\begin{array}{l}\text { Pinus armandii var. } \\
\text { mastersiana }\end{array}$ & Taiwan & $\begin{array}{l}\text { Jong \& Chen } 1966 \text {, } \\
\text { Mittal et al. } 1990\end{array}$ \\
\hline & & seeds & Pinus caribaea & $\begin{array}{l}\text { Belize [as British } \\
\text { Honduras] }\end{array}$ & Hocking 1968 \\
\hline & & seeds & Pinus contorta & USA & $\begin{array}{l}\text { Fuller \& Hildebrand } \\
\text { 1985, Mittal et al. } 1990\end{array}$ \\
\hline & & seed & Pinus densiflora & Japan & Watanabe 2010 \\
\hline & & seeds & Pinus elliottii & Taiwan, USA & $\begin{array}{l}\text { Jong \& Chen 1966, } \\
\text { Mittal et al. } 1990\end{array}$ \\
\hline & & seeds & $\begin{array}{l}\text { Pinus elliottii var. } \\
\text { elliottii }\end{array}$ & Uruguay & Anderson 1986a \\
\hline & & seeds & Pinus luchuensis & Taiwan & $\begin{array}{l}\text { Jong \& Chen 1966, } \\
\text { Mittal et al. } 1990\end{array}$ \\
\hline & & seeds & Pinus massoniana & Taiwan & $\begin{array}{l}\text { Jong \& Chen } 1966 \text {, } \\
\text { Mittal et al. } 1990\end{array}$ \\
\hline & & seeds & Pinus merkusii & Philippines & $\begin{array}{l}\text { Agmata 1979, Anderson } \\
\text { 1986a }\end{array}$ \\
\hline & & seeds & Pinus nigra & Hungary & $\begin{array}{l}\text { Hangyal 1973, Mittal et } \\
\text { al. } 1990\end{array}$ \\
\hline & & seeds & Pinus patula & East Africa & $\begin{array}{l}\text { Gibson 1957, Mittal et } \\
\text { al. } 1990\end{array}$ \\
\hline
\end{tabular}


Table 2 Continued.

\begin{tabular}{|c|c|c|c|c|c|}
\hline Species & Taxonomy & Substrate & Host & Country & References \\
\hline & & seeds & Pinus ponderosa & USA & $\begin{array}{l}\text { James \& Genz 1982, } \\
\text { Anderson 1986a }\end{array}$ \\
\hline & & seeds & Pinus sylvestris & Hungary, UK & $\begin{array}{l}\text { Hangyal 1973, Whittle } \\
\text { 1977, Mittal et al. } 1990\end{array}$ \\
\hline & & seeds & Pinus taeda & USA & $\begin{array}{l}\text { Mason \& van Arsdel } \\
\text { 1978, Anderson 1986a }\end{array}$ \\
\hline & & seeds & Pinus taiwanensis & Taiwan & $\begin{array}{l}\text { Jong \& Chen } 1966 \text {, } \\
\text { Mittal et al. } 1990\end{array}$ \\
\hline & & seed & Pinus thunbergii & Japan & Watanabe 2010 \\
\hline & & seeds & $\begin{array}{l}\text { Pinus thunbergii } \\
{[=\text { Pinus }} \\
\text { thunbergiana }]\end{array}$ & Taiwan & $\begin{array}{l}\text { Jong \& Chen } 1966 \text {, } \\
\text { Mittal et al. } 1990\end{array}$ \\
\hline & & seeds & $\begin{array}{l}\text { Pseudotsuga } \\
\text { menziesii }\end{array}$ & Canada, USA & $\begin{array}{l}\text { Bloomberg 1966, } \\
\text { Gordon 1967, Mittal et } \\
\text { al. } 1990\end{array}$ \\
\hline & & seeds & $\begin{array}{l}\text { Pseudotsuga } \\
\text { menziesii }\end{array}$ & Canada & Bloomberg 1966 \\
\hline & & seeds & Sesbania grandiflora & Philippines & $\begin{array}{l}\text { Quiniones 1985, } \\
\text { Anderson 1986a }\end{array}$ \\
\hline & & seeds & Tectona grandis & India & $\begin{array}{l}\text { Anderson 1986a, } \\
\text { Mohanan et al. } 2005\end{array}$ \\
\hline & & seeds & Vitex parviflora & Philippines & $\begin{array}{l}\text { Agmata 1979, Anderson } \\
\text { 1986a }\end{array}$ \\
\hline $\begin{array}{l}\text { Aspergillus sulphureus } \\
\text { Desm. }\end{array}$ & $\begin{array}{l}\text { Aspergillaceae, Eurotiomycetes, } \\
\text { Ascomycota }\end{array}$ & seeds & $\begin{array}{l}\text { Eucalyptus } \\
\text { citriodora }\end{array}$ & India & $\begin{array}{l}\text { Mittal \& Sharma 1984, } \\
\text { Mittal et al. } 1990\end{array}$ \\
\hline \multirow[t]{2}{*}{$\begin{array}{l}\text { Aspergillus sulphureus } \\
\text { Desm. [as 'sulphureous'] }\end{array}$} & & seeds & Albizia lebbeck & India & $\begin{array}{l}\text { Mittal \& Sharma 1982a, } \\
\text { Mittal et al. } 1990\end{array}$ \\
\hline & & seeds & Cassia fistula & India & $\begin{array}{l}\text { Mittal \& Sharma 1981a, } \\
\text { Mittal et al. } 1990\end{array}$ \\
\hline \multirow[t]{2}{*}{$\begin{array}{l}\text { Aspergillus sydowii (Bainier } \\
\text { \& Sartory) Thom \& Church }\end{array}$} & $\begin{array}{l}\text { Aspergillaceae, Eurotiomycetes, } \\
\text { Ascomycota }\end{array}$ & pods & Delonix regia & Thailand & Somrithipol et al. 2002b \\
\hline & & seeds & Pinus wallichiana & India & $\begin{array}{l}\text { Mittal \& Sharma 1982b, } \\
\text { Mittal et al. } 1990\end{array}$ \\
\hline
\end{tabular}


Table 2 Continued.

\begin{tabular}{|c|c|c|c|c|c|}
\hline Species & Taxonomy & Substrate & Host & Country & References \\
\hline \multirow{8}{*}{$\begin{array}{l}\text { Aspergillus sydowii (Bainier } \\
\text { \& Sartory) Thom \& Church } \\
\text { [as 'sydowi'] }\end{array}$} & & pod & Sesbania bispinosa & India & Anita et al. 2009 \\
\hline & & seeds & Albizia lebbeck & India & $\begin{array}{l}\text { Mittal \& Sharma 1982a, } \\
\text { Mittal et al. } 1990\end{array}$ \\
\hline & & seeds & Cedrus deodara & India & Mittal 1983 \\
\hline & & seeds & Dalbergia sissoo & India & Kumar 2014 \\
\hline & & seeds & $\begin{array}{l}\text { Eucalyptus } \\
\text { citriodora }\end{array}$ & India & $\begin{array}{l}\text { Mittal \& Sharma 1984, } \\
\text { Mittal et al. } 1990\end{array}$ \\
\hline & & seeds & Eucalyptus hybrid & India & $\begin{array}{l}\text { Mittal 1986, Mittal et al. } \\
1990\end{array}$ \\
\hline & & seeds & Eucalyptus sp. & India & $\begin{array}{l}\text { Reddy et al. 1982, Mittal } \\
\text { et al. } 1990\end{array}$ \\
\hline & & seed & Stylosanthes gracilis & Australia & Nik \& Parbery 1977 \\
\hline \multirow[t]{8}{*}{ Aspergillus tamarii Kita } & Aspergillaceae, Eurotiomycetes, & pod & Canavalia cathartica & India & Anita \& Sridhar 2009 \\
\hline & Ascomycota & seed & Canavalia cathartica & India & Anita \& Sridhar 2009 \\
\hline & & seeds & Cassia floribunda & Thailand & $\begin{array}{l}\text { Chalermpongse et al. } \\
\text { 1984, Mittal et al. } 1990\end{array}$ \\
\hline & & seeds & Dalbergia sissoo & India & Kumar 2014 \\
\hline & & seeds & $\begin{array}{l}\text { Eucalyptus } \\
\text { citriodora }\end{array}$ & India & $\begin{array}{l}\text { Mittal \& Sharma 1984, } \\
\text { Mittal et al. } 1990\end{array}$ \\
\hline & & seeds & $\begin{array}{l}\text { Leucaena } \\
\text { leucocephala }\end{array}$ & Thailand & $\begin{array}{l}\text { Chalermpongse et al. } \\
\text { 1984, Mittal et al. } 1990\end{array}$ \\
\hline & & seeds & Pinus patula & Kenya & $\begin{array}{l}\text { Gibson 1957, Mittal et } \\
\text { al. } 1990\end{array}$ \\
\hline & & seed & Sesbania bispinosa & India & Anita et al. 2009 \\
\hline \multirow[t]{4}{*}{ Aspergillus terreus Thom } & $\begin{array}{l}\text { Aspergillaceae, Eurotiomycetes, } \\
\text { Ascomycota }\end{array}$ & pod & Sesbania bispinosa & India & Anita et al. 2009 \\
\hline & & seeds & $\begin{array}{l}\text { Acacia } \\
\text { auriculiformis }\end{array}$ & Thailand & $\begin{array}{l}\text { Chalermpongse et al. } \\
\text { 1984, Mittal et al. } 1990\end{array}$ \\
\hline & & seeds & Bauhinia sp. & Thailand & $\begin{array}{l}\text { Chalermpongse et al. } \\
\text { 1984, Mittal et al. } 1990\end{array}$ \\
\hline & & seeds & Bombax anceps & Thailand & $\begin{array}{l}\text { Chalermpongse et al. } \\
\text { 1984, Mittal et al. } 1990\end{array}$ \\
\hline
\end{tabular}


Table 2 Continued.

\begin{tabular}{|c|c|c|c|c|c|}
\hline Species & Taxonomy & Substrate & Host & Country & References \\
\hline & & seeds & Cassia siamea & Thailand & $\begin{array}{l}\text { Quiniones 1985, Mittal } \\
\text { et al. } 1990\end{array}$ \\
\hline & & seeds & $\begin{array}{l}\text { Casuarina } \\
\text { equisetifolia }\end{array}$ & India & Anju et al. 2012 \\
\hline & & seeds & Casuarina spp. & India & Sahai \& Otra 1982 \\
\hline & & seeds & Dalbergia sissoo & India & Kumar 2014 \\
\hline & & seeds & Eucalyptus sp. & India & $\begin{array}{l}\text { Reddy et al. 1982, Mittal } \\
\text { et al. } 1990\end{array}$ \\
\hline & & seeds & Melia spp. & India & Sahai \& Otra 1982 \\
\hline & & seeds & $\begin{array}{l}\text { Pinus elliottii var. } \\
\text { elliottii }\end{array}$ & Georgia & Huang \& Kuhlman 1990 \\
\hline & & seeds & Quercus spp. & India & Sahai \& Otra 1982 \\
\hline & & seeds & Shorea robusta & India & $\begin{array}{l}\text { Mittal \& Sharma 1982b, } \\
\text { Mittal et al. } 1990\end{array}$ \\
\hline & & seeds & Thuja spp. & India & Sahai \& Otra 1982 \\
\hline \multirow[t]{2}{*}{$\begin{array}{l}\text { Aspergillus terricola } \\
\text { Marchal \& É.J. Marchal }\end{array}$} & \multirow[t]{2}{*}{$\begin{array}{l}\text { Aspergillaceae, Eurotiomycetes, } \\
\text { Ascomycota }\end{array}$} & seeds & Pinus roxburghii & India & $\begin{array}{l}\text { Mittal \& Sharma 1982c, } \\
\text { Mittal et al. } 1990\end{array}$ \\
\hline & & seeds & Pinus wallichiana & India & $\begin{array}{l}\text { Mittal \& Sharma 1982b, } \\
\text { Mittal et al. } 1990\end{array}$ \\
\hline $\begin{array}{l}\text { Aspergillus unguis (Émile- } \\
\text { Weill \& L. Gaudin) Thom \& } \\
\text { Raper }\end{array}$ & $\begin{array}{l}\text { Aspergillaceae, Eurotiomycetes, } \\
\text { Ascomycota }\end{array}$ & seeds & Eucalyptus sp. & India & $\begin{array}{l}\text { Reddy et al. 1982, Mittal } \\
\text { et al. } 1990\end{array}$ \\
\hline Aspergillus ustus (Bainier) & Aspergillaceae, Eurotiomycetes, & seeds & Delonix regia & India & Sahu et al. 2003 \\
\hline Thom \& Church & Ascomycota & seeds & Sapium spp. & India & Sahai \& Otra 1982 \\
\hline \multirow[t]{7}{*}{$\begin{array}{l}\text { Aspergillus variecolor (Berk. } \\
\text { \& Broome) Thom \& Raper }\end{array}$} & $\begin{array}{l}\text { Aspergillaceae, Eurotiomycetes, } \\
\text { Ascomycota }\end{array}$ & seeds & Albizia lebbeck & India & $\begin{array}{l}\text { Mittal \& Sharma 1982a, } \\
\text { Mittal et al. } 1990\end{array}$ \\
\hline & & seeds & Casuarina spp. & India & Sahai \& Otra 1982 \\
\hline & & seeds & Cedrus deodara & India & Mittal 1983 \\
\hline & & seed & $\begin{array}{l}\text { Cupressus } \\
\text { sempervirens }\end{array}$ & Egypt & $\begin{array}{l}\text { Farag et al. 1977, Mittal } \\
\text { et al. } 1990\end{array}$ \\
\hline & & seeds & Cupressus spp. & India & Sahai \& Otra 1982 \\
\hline & & seeds & Pinus densiflora & Japan & Watanabe 2010 \\
\hline & & seeds & Pinus kesiya & Thailand & $\begin{array}{l}\text { Chalermpongse et al. } \\
\text { 1984, Mittal et al. } 1990\end{array}$ \\
\hline
\end{tabular}


Table 2 Continued.

\begin{tabular}{|c|c|c|c|c|c|}
\hline Species & Taxonomy & Substrate & Host & Country & References \\
\hline & & seeds & Pinus roxburghii & India & $\begin{array}{l}\text { Mittal \& Sharma 1982c, } \\
\text { Mittal et al. } 1990\end{array}$ \\
\hline & & seeds & Pinus wallichiana & India & $\begin{array}{l}\text { Mittal \& Sharma 1982b, } \\
\text { Mittal et al. } 1990\end{array}$ \\
\hline & & seeds & Thuja spp. & India & Sahai \& Otra 1982 \\
\hline & & seeds & $\begin{array}{l}\text { Xylia xylocarpa var. } \\
\text { kerrii }\end{array}$ & Thailand & $\begin{array}{l}\text { Chalermpongse et al. } \\
\text { 1984, Mittal et al. } 1990\end{array}$ \\
\hline \multirow[t]{2}{*}{ Aspergillus wentii Wehmer } & $\begin{array}{l}\text { Aspergillaceae, Eurotiomycetes, } \\
\text { Ascomycota }\end{array}$ & seeds & Albizia lebbeck & India & $\begin{array}{l}\text { Mittal \& Sharma 1982a, } \\
\text { Mittal et al. } 1990\end{array}$ \\
\hline & & seeds & Shorea robusta & India & $\begin{array}{l}\text { Mittal \& Sharma 1982b, } \\
\text { Mittal et al. } 1990\end{array}$ \\
\hline \multirow[t]{2}{*}{ Asteroma sp. } & \multirow{2}{*}{$\begin{array}{l}\text { Gnomoniaceae, Sordariomycetes, } \\
\text { Ascomycota }\end{array}$} & fruits & Ardisia qinquegona & Hong Kong & Tang et al. 2003a \\
\hline & & fruits & Litsea rotundifolia & Hong Kong & Tang et al. 2003a \\
\hline Asteromella sp. & $\begin{array}{l}\text { Incertae sedis, Dothideomycetes, } \\
\text { Ascomycota }\end{array}$ & seeds & Pinus taeda & USA & Anderson 1986a, b \\
\hline $\begin{array}{l}\text { Atrocalyx krabiensis } \\
\text { Jayasiri, E.B.G. Jones \& } \\
\text { K.D. Hyde }^{\#}\end{array}$ & $\begin{array}{l}\text { Lophiotremataceae, Dothideomycetes, } \\
\text { Ascomycota }\end{array}$ & pods & Acacia sp. & Thailand & Jayasiri et al. 2019 \\
\hline $\begin{array}{l}\text { Aureobasidium } \\
\text { melanogenum (Herm.-Nijh.) } \\
\text { Zalar, Gostinčar \& Gunde- } \\
\text { Cim. [as Aureobasidium } \\
\text { pullulans var. melanogenum } \\
\text { Herm.-Nijh.] }\end{array}$ & $\begin{array}{l}\text { Saccotheciaceae, Dothideomycetes, } \\
\text { Ascomycota }\end{array}$ & pods & Eucalyptus globulus & Uruguay & Lupo et al. 2001 \\
\hline \multirow{9}{*}{$\begin{array}{l}\text { Aureobasidium pullulans } \\
\text { (de Bary \& Löwenthal) G. } \\
\text { Arnaud }\end{array}$} & \multirow{9}{*}{$\begin{array}{l}\text { Saccotheciaceae, Dothideomycetes, } \\
\text { Ascomycota }\end{array}$} & acorns & Quercus robur & Poland & Jankowiak 2008 \\
\hline & & cones & Picea glauca & Canada & Mittal \& Wang 1987 \\
\hline & & cones & Pinus densiflora & Japan & Kasai et al. 1995 \\
\hline & & cones & Pinus strobus & Canada & Mittal \& Wang 1987 \\
\hline & & seeds & Acer pseudoplatanus & Poland & Krol et al. 2015 \\
\hline & & seed & Agropyron cristatum & Canada & Conners 1967 \\
\hline & & seed & Agrostis gigantea & Canada & Conners 1967 \\
\hline & & seed & Anethum graveolens & USA & Conners 1967 \\
\hline & & seed & $\begin{array}{l}\text { Apium graveolens } \\
\text { var. dulce }\end{array}$ & Canada & Conners 1967 \\
\hline
\end{tabular}


Table 2 Continued.

\begin{tabular}{|c|c|c|c|c|c|}
\hline Species & Taxonomy & Substrate & Host & Country & References \\
\hline & & seed & $\begin{array}{l}\text { Asparagus officinalis } \\
\text { var. altilis }\end{array}$ & Canada & Conners 1967 \\
\hline & & seeds & Betula pendula & Finland & $\begin{array}{l}\text { Lilja 1979, Mittal et al. } \\
1990\end{array}$ \\
\hline & & seed & Cercis chinensis & Japan & Watanabe 2010 \\
\hline & & seed & $\begin{array}{l}\text { Cryptomeria } \\
\text { japonica }\end{array}$ & Japan & Watanabe 2010 \\
\hline & & seed & Dactylis glomerata & Canada & Conners 1967 \\
\hline & & seed & Festuca elatior & Canada & Conners 1967 \\
\hline & & seed & Festuca rubra & Canada & Conners 1967 \\
\hline & & seeds & Fraxinus excelsior & Sweden & Cleary et al. 2013 \\
\hline & & seed & Linum usitatissimum & Canada & Conners 1967 \\
\hline & & seed & Lolium perenne & Canada & Conners 1967 \\
\hline & & seed & Medicago sativa & Canada & Conners 1967 \\
\hline & & seeds & Picea glauca & Canada & Mittal \& Wang 1987 \\
\hline & & seed & Pinus densiflora & Japan & Watanabe 2010 \\
\hline & & seeds & Pinus ponderosa & USA & $\begin{array}{l}\text { Fuller \& Hildebrand } \\
\text { 1985, Anderson 1986a }\end{array}$ \\
\hline & & seeds & Pinus strobus & Canada & Mittal \& Wang 1987 \\
\hline & & seeds & Pinus sylvestris & Poland & Krol et al. 2015 \\
\hline & & seeds & $\begin{array}{l}\text { Platanus } \\
\text { occidentalis }\end{array}$ & USA & $\begin{array}{l}\text { Fakir et al. 1971, Mittal } \\
\text { et al. } 1990\end{array}$ \\
\hline & & seed & Prunus serrulata & Japan & Watanabe 2010 \\
\hline & & seeds & $\begin{array}{l}\text { Pseudotsuga } \\
\text { menziesii }\end{array}$ & USA & $\begin{array}{l}\text { Gordon 1967, Mittal et } \\
\text { al. } 1990\end{array}$ \\
\hline & & seed & $\begin{array}{l}\text { Stylosanthes } \\
\text { guianensis }\end{array}$ & Australia & Nik \& Parbery 1977 \\
\hline & & seed & Stylosanthes humilis & Australia & Nik \& Parbery 1977 \\
\hline $\begin{array}{l}\text { Aureobasidium pullulans } \\
\text { var. aubasidani Yurlova }\end{array}$ & $\begin{array}{l}\text { Saccotheciaceae, Dothideomycetes, } \\
\text { Ascomycota }\end{array}$ & pods & Eucalyptus globulus & Uruguay & Lupo et al. 2001 \\
\hline \multirow[t]{2}{*}{ Aureobasidium spp. } & $\begin{array}{l}\text { Saccotheciaceae, Dothideomycetes, } \\
\text { Ascomycota }\end{array}$ & seeds & Acer saccharum & USA & $\begin{array}{l}\text { Janerette 1979, Mittal et } \\
\text { al. } 1990\end{array}$ \\
\hline & & seed & $\begin{array}{l}\text { Cryptomeria } \\
\text { japonica }\end{array}$ & Japan & Watanabe 2010 \\
\hline
\end{tabular}


Table 2 Continued.

\begin{tabular}{|c|c|c|c|c|c|}
\hline Species & Taxonomy & Substrate & Host & Country & References \\
\hline & & seeds & Fraxinus excelsior & Sweden & $\begin{array}{l}\text { Cleary et al. 2013, } \\
\text { Hayatgheibi } 2013\end{array}$ \\
\hline & & seeds & Phyllostachys edulis & China & Shen et al. 2014 \\
\hline & & seeds & Picea engelmannii & USA & $\begin{array}{l}\text { Fuller \& Hildebrand } \\
\text { 1985, Mittal et al. } 1990\end{array}$ \\
\hline & & seeds & Pinus contorta & USA & $\begin{array}{l}\text { Fuller \& Hildebrand } \\
\text { 1985, Mittal et al. } 1990\end{array}$ \\
\hline & & seed & Pinus densiflora & Japan & Watanabe 2010 \\
\hline & & seeds & $\begin{array}{l}\text { Pinus elliottii var. } \\
\text { elliottii }\end{array}$ & USA & Fraedrich \& Miller 1995 \\
\hline & & seeds & Pinus ponderosa & USA & $\begin{array}{l}\text { Fuller \& Hildebrand } \\
\text { 1985, Mittal et al. } 1990\end{array}$ \\
\hline & & seed & Prunus serrulata & Japan & Watanabe 2010 \\
\hline & & seeds & $\begin{array}{l}\text { Pseudotsuga } \\
\text { menziesii }\end{array}$ & Canada & $\begin{array}{l}\text { Salisbury 1955, Mittal et } \\
\text { al. } 1990\end{array}$ \\
\hline $\begin{array}{l}\text { Austropleospora keteleeriae } \\
\text { Jayasiri, E.B.G. Jones \& } \\
\text { K.D. Hyde }^{\#}\end{array}$ & $\begin{array}{l}\text { Didymosphaeriaceae, Dothideomycetes, } \\
\text { Ascomycota }\end{array}$ & cone & Keteleeria fortunei & China & Jayasiri et al. 2019 \\
\hline \multirow[t]{7}{*}{ Basidiomycete } & Basidiomycota & cones & Pinus densiflora & Japan & Kasai et al. 1995 \\
\hline & & seed capsules & Eucalyptus grandis & South Africa & Jimu et al. 2016 \\
\hline & & seeds & Corallorhiza striata & Canada & Zelmer et al. 1996 \\
\hline & & seeds & Delonix regia & Thailand & Somrithipol et al. 2002b \\
\hline & & seeds & Eucalyptus grandis & South Africa & Jimu et al. 2016 \\
\hline & & seeds & $\begin{array}{l}\text { Platanthera } \\
\text { hyperborea }\end{array}$ & Canada & Zelmer et al. 1996 \\
\hline & & seeds & Quercus robur & Turkey & Oskay et al. 2018 \\
\hline $\begin{array}{l}\text { Basipetospora rubra G.T. } \\
\text { Cole \& W.B. Kendr. }\end{array}$ & $\begin{array}{l}\text { Monascaceae, Eurotiomycetes, } \\
\text { Ascomycota }\end{array}$ & seed & Pinus thunbergii & Japan & Watanabe 2010 \\
\hline Beauveria sp. & $\begin{array}{l}\text { Cordycipitaceae, Sordariomycetes, } \\
\text { Ascomycota }\end{array}$ & seed & Pinus monticola & USA & $\begin{array}{l}\text { Ganley \& Newcombe } \\
2006\end{array}$ \\
\hline Beltrania rhombica Penz. & $\begin{array}{l}\text { Beltraniaceae, Sordariomycetes, } \\
\text { Ascomycota }\end{array}$ & pods & Delonix regia & Thailand & Somrithipol et al. 2002b \\
\hline $\begin{array}{l}\text { Beltrania santapaui Piroz. \& } \\
\text { S.D. Patil }\end{array}$ & $\begin{array}{l}\text { Beltraniaceae, Sordariomycetes, } \\
\text { Ascomycota }\end{array}$ & seeds & $\begin{array}{l}\text { Quercus } \\
\text { castaneifolia }\end{array}$ & Iran & Kavosi et al. 2013 \\
\hline
\end{tabular}


Table 2 Continued.

\begin{tabular}{|c|c|c|c|c|c|}
\hline Species & Taxonomy & Substrate & Host & Country & References \\
\hline Beltrania spp. & $\begin{array}{l}\text { Beltraniaceae, Sordariomycetes, } \\
\text { Ascomycota }\end{array}$ & $\begin{array}{l}\text { fruits } \\
\text { seeds }\end{array}$ & $\begin{array}{l}\text { Psychotria asiatica } \\
\text { Albizia lebbeck }\end{array}$ & $\begin{array}{l}\text { Hong Kong } \\
\text { India }\end{array}$ & $\begin{array}{l}\text { Tang et al. 2003a } \\
\text { Mohanan et al. } 2005\end{array}$ \\
\hline $\begin{array}{l}\text { Beltraniella vateriae K. } \\
\text { Swapna, Nagaveni, Kunwar } \\
\text { \& Manohar.\# }\end{array}$ & $\begin{array}{l}\text { Beltraniaceae, Sordariomycetes, } \\
\text { Ascomycota }\end{array}$ & seeds & Vateria indica & India & Priya et al. 2011 \\
\hline $\begin{array}{l}\text { Bhadradriella hyalina } \\
\text { Nagaraju, Kunwar \& } \\
\text { Manohar. }^{\text {Mana }}\end{array}$ & $\begin{array}{l}\text { Incertae sedis, Incertae sedis, } \\
\text { Ascomycota }\end{array}$ & pods & Roystonea regia & India & Nagaraju et al. 2011a \\
\hline $\begin{array}{l}\text { Biflagellosporella } \\
\text { amazonensis Matsush. }\end{array}$ & $\begin{array}{l}\text { Incertae sedis, Incertae sedis, } \\
\text { Ascomycota }\end{array}$ & fruit & Inga sp. & Peru & Matsushima 1993 \\
\hline $\begin{array}{l}\text { Bipolaris setariae } \\
\text { Shoemaker }\end{array}$ & $\begin{array}{l}\text { Pleosporaceae, Dothideomycetes, } \\
\text { Ascomycota }\end{array}$ & seed & Setaria viridis & Canada & Conners 1967 \\
\hline Bipolaris sorokiniana & Pleosporaceae, Dothideomycetes, & seed & Agropyron cristatum & Canada & Conners 1967 \\
\hline Shoemaker & Ascomycota & seed & Elymus virginicus & Canada & Conners 1967 \\
\hline & & seed & Festuca elatior & Canada & Conners 1967 \\
\hline & & seed & Linum usitatissimum & Canada & Conners 1967 \\
\hline & & seed & Lolium perenne & Canada & Conners 1967 \\
\hline & & seed & Setaria viridis & Canada & Conners 1967 \\
\hline Bipolaris sp. & Pleosporaceae, Dothideomycetes, & seeds & Eucalyptus grandis & South Africa & Jimu et al. 2016 \\
\hline & Ascomycota & seeds & $\begin{array}{l}\text { Platanus } \\
\text { occidentalis }\end{array}$ & USA & $\begin{array}{l}\text { Fakir et al. 1971, Mittal } \\
\text { et al. } 1990\end{array}$ \\
\hline $\begin{array}{l}\text { Bispora antennata (Pers.) } \\
\text { E.W. Mason }\end{array}$ & $\begin{array}{l}\text { Incertae sedis, Incertae sedis, } \\
\text { Ascomycota }\end{array}$ & seeds & Pinus kesiya & Thailand & $\begin{array}{l}\text { Chalermpongse et al. } \\
\text { 1984, Mittal et al. } 1990\end{array}$ \\
\hline $\begin{array}{l}\text { Bispora betulina (Corda) S. } \\
\text { Hughes }\end{array}$ & $\begin{array}{l}\text { Incertae sedis, Incertae sedis, } \\
\text { Ascomycota }\end{array}$ & seed & Gentiana sp. & Japan & Watanabe 2010 \\
\hline Bispora sp. & $\begin{array}{l}\text { Incertae sedis, Incertae sedis, } \\
\text { Ascomycota }\end{array}$ & seeds & Pinus taeda & USA & Anderson 1986a, b \\
\hline \multirow[t]{3}{*}{ Black sterile mycelium } & Fungi & seeds & Albizia lebbeck & India & Mohanan et al. 2005 \\
\hline & & seeds & Dalbergia sissoides & India & Mohanan et al. 2005 \\
\hline & & seeds & Tectona grandis & India & Mohanan et al. 2005 \\
\hline Blakeslea trispora Thaxt. & $\begin{array}{l}\text { Choanephoraceae, Mucoromycetes, } \\
\text { Mucoromycota }\end{array}$ & seeds & Crataegus azarolus & $\begin{array}{l}\text { Poland or/ } \\
\text { imported to Poland }\end{array}$ & Czeczuga et al. 2009 \\
\hline
\end{tabular}


Table 2 Continued.

\begin{tabular}{|c|c|c|c|c|c|}
\hline Species & Taxonomy & Substrate & Host & Country & References \\
\hline \multirow[t]{4}{*}{$\begin{array}{l}\text { Blastocladia angusta A. } \\
\text { Lund }\end{array}$} & \multirow[t]{4}{*}{$\begin{array}{l}\text { Blastocladiaceae, Blastocladiomycetes, } \\
\text { Blastocladiomycota }\end{array}$} & seeds & Celtis tenuifolia & $\begin{array}{l}\text { Poland or/ } \\
\text { imported to Poland }\end{array}$ & Czeczuga et al. 2009 \\
\hline & & seeds & $\begin{array}{l}\text { Cephalotaxus } \\
\text { drupacea }\end{array}$ & $\begin{array}{l}\text { Poland or/ } \\
\text { imported to Poland }\end{array}$ & Czeczuga et al. 2009 \\
\hline & & seeds & Cornus sanguinea & $\begin{array}{l}\text { Poland or/ } \\
\text { imported to Poland }\end{array}$ & Czeczuga et al. 2009 \\
\hline & & seeds & Lonicera caprifolium & $\begin{array}{l}\text { Poland or/ } \\
\text { imported to Poland }\end{array}$ & Czeczuga et al. 2009 \\
\hline $\begin{array}{l}\text { Blastocladia arborata S.N. } \\
\text { Dasgupta \& R. John } \#\end{array}$ & $\begin{array}{l}\text { Blastocladiaceae, Blastocladiomycetes, } \\
\text { Blastocladiomycota }\end{array}$ & fruits & myrtaceous plant & India & $\begin{array}{l}\text { Das-Gupta \& John } \\
1988\end{array}$ \\
\hline \multirow[t]{8}{*}{$\begin{array}{l}\text { Blastocladia globosa } \\
\text { Kanouse }\end{array}$} & \multirow[t]{8}{*}{$\begin{array}{l}\text { Blastocladiaceae, Blastocladiomycetes, } \\
\text { Blastocladiomycota }\end{array}$} & seeds & Arum korolkowii & $\begin{array}{l}\text { Poland or/ } \\
\text { imported to Poland }\end{array}$ & Czeczuga et al. 2009 \\
\hline & & seeds & Bryonia cretica & $\begin{array}{l}\text { Poland or/ } \\
\text { imported to Poland }\end{array}$ & Czeczuga et al. 2009 \\
\hline & & seeds & Juniperus communis & $\begin{array}{l}\text { Poland or/ } \\
\text { imported to Poland }\end{array}$ & Czeczuga et al. 2009 \\
\hline & & seeds & Juniperus oxycedrus & $\begin{array}{l}\text { Poland or/ } \\
\text { imported to Poland }\end{array}$ & Czeczuga et al. 2009 \\
\hline & & seeds & Opuntia ficus-indica & $\begin{array}{l}\text { Poland or/ } \\
\text { imported to Poland }\end{array}$ & Czeczuga et al. 2009 \\
\hline & & seeds & Sorbus torminalis & $\begin{array}{l}\text { Poland or/ } \\
\text { imported to Poland }\end{array}$ & Czeczuga et al. 2009 \\
\hline & & seeds & Trifolium pratense & $\begin{array}{l}\text { Poland or/ } \\
\text { imported to Poland }\end{array}$ & Czeczuga et al. 2009 \\
\hline & & seeds & Vicia sativa & Poland & Kiziewicz 2005 \\
\hline \multirow[t]{4}{*}{$\begin{array}{l}\text { Blastocladia gracilis } \\
\text { Kanouse }\end{array}$} & \multirow[t]{4}{*}{$\begin{array}{l}\text { Blastocladiaceae, Blastocladiomycetes, } \\
\text { Blastocladiomycota }\end{array}$} & seeds & Cornus capitata & $\begin{array}{l}\text { Poland or/ } \\
\text { imported to Poland }\end{array}$ & Czeczuga et al. 2009 \\
\hline & & seeds & Lonicera etrusca & $\begin{array}{l}\text { Poland or/ } \\
\text { imported to Poland }\end{array}$ & Czeczuga et al. 2009 \\
\hline & & seeds & $\begin{array}{l}\text { Lonicera } \\
\text { periclymenum }\end{array}$ & $\begin{array}{l}\text { Poland or/ } \\
\text { imported to Poland }\end{array}$ & Czeczuga et al. 2009 \\
\hline & & seeds & Rhamnus purshiana & $\begin{array}{l}\text { Poland or/ } \\
\text { imported to Poland }\end{array}$ & Czeczuga et al. 2009 \\
\hline
\end{tabular}


Table 2 Continued.

\begin{tabular}{|c|c|c|c|c|c|}
\hline Species & Taxonomy & Substrate & Host & Country & References \\
\hline & & seeds & Rhus copallina & $\begin{array}{l}\text { Poland or/ } \\
\text { imported to Poland }\end{array}$ & Czeczuga et al. 2009 \\
\hline & & seeds & Rosa mollis & $\begin{array}{l}\text { Poland or/ } \\
\text { imported to Poland }\end{array}$ & Czeczuga et al. 2009 \\
\hline \multirow[t]{3}{*}{$\begin{array}{l}\text { Blastocladia pringsheimii } \\
\text { Reinsch }\end{array}$} & $\begin{array}{l}\text { Blastocladiaceae, Blastocladiomycetes, } \\
\text { Blastocladiomycota }\end{array}$ & seeds & Celtis tenuifolia & $\begin{array}{l}\text { Poland or/ } \\
\text { imported to Poland }\end{array}$ & Czeczuga et al. 2009 \\
\hline & & seeds & Juniperus communis & $\begin{array}{l}\text { Poland or/ } \\
\text { imported to Poland }\end{array}$ & Czeczuga et al. 2009 \\
\hline & & seeds & Lycium chinense & $\begin{array}{l}\text { Poland or/ } \\
\text { imported to Poland }\end{array}$ & Czeczuga et al. 2009 \\
\hline \multirow[t]{6}{*}{ Blastocladia ramosa Thaxt. } & $\begin{array}{l}\text { Blastocladiaceae, Blastocladiomycetes, } \\
\text { Blastocladiomycota }\end{array}$ & seeds & Ginkgo biloba & $\begin{array}{l}\text { Poland or/ } \\
\text { imported to Poland }\end{array}$ & Czeczuga et al. 2009 \\
\hline & & seeds & Humulus lupulus & $\begin{array}{l}\text { Poland or/ } \\
\text { imported to Poland }\end{array}$ & Czeczuga et al. 2009 \\
\hline & & seeds & Humulus scandens & $\begin{array}{l}\text { Poland or/ } \\
\text { imported to Poland }\end{array}$ & Czeczuga et al. 2009 \\
\hline & & seeds & Ilex verticillata & $\begin{array}{l}\text { Poland or/ } \\
\text { imported to Poland }\end{array}$ & Czeczuga et al. 2009 \\
\hline & & seeds & Lonicera etrusca & $\begin{array}{l}\text { Poland or/ } \\
\text { imported to Poland }\end{array}$ & Czeczuga et al. 2009 \\
\hline & & seeds & $\begin{array}{l}\text { Rhododendron } \\
\text { nipponicum }\end{array}$ & $\begin{array}{l}\text { Poland or/ } \\
\text { imported to Poland }\end{array}$ & Czeczuga et al. 2009 \\
\hline $\begin{array}{l}\text { Blastocladia sessilis S.N. } \\
\text { Dasgupta \& R. John\# }\end{array}$ & $\begin{array}{l}\text { Blastocladiaceae, Blastocladiomycetes, } \\
\text { Blastocladiomycota }\end{array}$ & $\begin{array}{l}\text { various } \\
\text { submerged } \\
\text { fruits }\end{array}$ & $\begin{array}{l}\text { undetermined } \\
\text { plants }\end{array}$ & India & $\begin{array}{l}\text { Das-Gupta \& John } \\
1988\end{array}$ \\
\hline $\begin{array}{l}\text { Blastocladiella emersonii } \\
\text { Cantino \& Hyatt }\end{array}$ & $\begin{array}{l}\text { Blastocladiaceae, Blastocladiomycetes, } \\
\text { Blastocladiomycota }\end{array}$ & seeds & $\begin{array}{l}\text { Zantedeschia albo- } \\
\text { maculata }\end{array}$ & $\begin{array}{l}\text { Poland or/ } \\
\text { imported to Poland }\end{array}$ & Czeczuga et al. 2009 \\
\hline $\begin{array}{l}\text { Blastocladiella } \\
\text { microcystogena Whiffen }\end{array}$ & $\begin{array}{l}\text { Blastocladiaceae, Blastocladiomycetes, } \\
\text { Blastocladiomycota }\end{array}$ & seeds & Bryonia dioica & $\begin{array}{l}\text { Poland or/ } \\
\text { imported to Poland }\end{array}$ & Czeczuga et al. 2009 \\
\hline \multirow[t]{2}{*}{$\begin{array}{l}\text { Blastocladiella stuebenii } \\
\text { Couch \& Whiffen }\end{array}$} & $\begin{array}{l}\text { Blastocladiaceae, Blastocladiomycetes, } \\
\text { Blastocladiomycota }\end{array}$ & seeds & Daphne gnidium & $\begin{array}{l}\text { Poland or/ } \\
\text { imported to Poland }\end{array}$ & Czeczuga et al. 2009 \\
\hline & & seeds & Opuntia ficus-indica & $\begin{array}{l}\text { Poland or/ } \\
\text { imported to Poland }\end{array}$ & Czeczuga et al. 2009 \\
\hline
\end{tabular}


Table 2 Continued.

\begin{tabular}{|c|c|c|c|c|c|}
\hline Species & Taxonomy & Substrate & Host & Country & References \\
\hline $\begin{array}{l}\text { Blastocladiopsis parva } \\
\text { Whiffen ex Sparrow }\end{array}$ & $\begin{array}{l}\text { Blastocladiaceae, Blastocladiomycetes, } \\
\text { Blastocladiomycota }\end{array}$ & seeds & Atropa komarovii & $\begin{array}{l}\text { Poland or/ } \\
\text { imported to Poland }\end{array}$ & Czeczuga et al. 2009 \\
\hline & & seeds & Bryonia dioica & $\begin{array}{l}\text { Poland or/ } \\
\text { imported to Poland }\end{array}$ & Czeczuga et al. 2009 \\
\hline & & seeds & Conium maculatum & $\begin{array}{l}\text { Poland or/ } \\
\text { imported to Poland }\end{array}$ & Czeczuga et al. 2009 \\
\hline & & seeds & Humulus lupulus & $\begin{array}{l}\text { Poland or/ } \\
\text { imported to Poland }\end{array}$ & Czeczuga et al. 2009 \\
\hline & & seeds & Humulus scandens & $\begin{array}{l}\text { Poland or/ } \\
\text { imported to Poland }\end{array}$ & Czeczuga et al. 2009 \\
\hline & & seeds & Sambucus ebulus & $\begin{array}{l}\text { Poland or/ } \\
\text { imported to Poland }\end{array}$ & Czeczuga et al. 2009 \\
\hline & & seeds & Vicia sativa & Poland & Kiziewicz 2005 \\
\hline Blyttiomyces laevis Sparrow & $\begin{array}{l}\text { Incertae sedis, Chytridiomycetes, } \\
\text { Chytridiomycota }\end{array}$ & seeds & Atropa belladonna & $\begin{array}{l}\text { Poland or/ } \\
\text { imported to Poland }\end{array}$ & Czeczuga et al. 2009 \\
\hline $\begin{array}{l}\text { Boeremia linicola (Naumov } \\
\text { \& Vassiljevsky) } \\
\text { Jayawardena, Jayasiri \& K.D. } \\
\text { Hyde [as Ascochyta linicola } \\
\text { Naumov \& Vassiljevsky] }\end{array}$ & $\begin{array}{l}\text { Didymellaceae, Dothideomycetes, } \\
\text { Ascomycota }\end{array}$ & seed & Linum usitatissimum & Canada & Conners 1967 \\
\hline $\begin{array}{l}\text { Boothiomyces macroporosus } \\
\text { (Karling) Letcher }\end{array}$ & $\begin{array}{l}\text { Terramycetaceae, Rhizophydiomycetes, } \\
\text { Chytridiomycota }\end{array}$ & seeds & Asparagus officinalis & $\begin{array}{l}\text { Poland or/ } \\
\text { imported to Poland }\end{array}$ & Czeczuga et al. 2009 \\
\hline $\begin{array}{l}\text { [as Rhizophydium } \\
\text { macroporosum Karling] }\end{array}$ & & seeds & Paris quadrifolia & $\begin{array}{l}\text { Poland or/ } \\
\text { imported to Poland }\end{array}$ & Czeczuga et al. 2009 \\
\hline $\begin{array}{l}\text { Botryocrea sclerotioides } \\
\text { (Höhn.) Petr. }\end{array}$ & $\begin{array}{l}\text { Nectriaceae, Sordariomycetes, } \\
\text { Ascomycota }\end{array}$ & seeds & Pinus elliottii & South Africa & Cilliers et al. 1995 \\
\hline $\begin{array}{l}\text { Botryodiplodia acicola } \\
\text { (Sacc.) Petr. }\end{array}$ & $\begin{array}{l}\text { Incertae sedis, Sordariomycetes, } \\
\text { Ascomycota }\end{array}$ & seeds & Pinus sylvestris & UK & Whittle 1977 \\
\hline $\begin{array}{l}\text { Botryodiplodia palmarum } \\
\text { (Cooke) Petr. \& Syd. }\end{array}$ & $\begin{array}{l}\text { Incertae sedis, Sordariomycetes, } \\
\text { Ascomycota }\end{array}$ & seeds & $\begin{array}{l}\text { Cupressus } \\
\text { sempervirens }\end{array}$ & Egypt & $\begin{array}{l}\text { Farag et al. 1977, Mittal } \\
\text { et al. } 1990\end{array}$ \\
\hline $\begin{array}{l}\text { Botryodiplodia ribis } \\
\text { (Schulzer \& Sacc.) Namysł. }\end{array}$ & $\begin{array}{l}\text { Incertae sedis, Sordariomycetes, } \\
\text { Ascomycota }\end{array}$ & seeds & Pinus oocarpa & UK & $\begin{array}{l}\text { Rees 1982, Mittal et al. } \\
1990\end{array}$ \\
\hline
\end{tabular}


Table 2 Continued.

\begin{tabular}{|c|c|c|c|c|c|}
\hline Species & Taxonomy & Substrate & Host & Country & References \\
\hline & & seeds & Pinus pseudostrobus & UK & $\begin{array}{l}\text { Rees 1982, Mittal et al. } \\
1990\end{array}$ \\
\hline \multirow[t]{17}{*}{ Botryodiplodia spp. } & $\begin{array}{l}\text { Incertae sedis, Sordariomycetes, } \\
\text { Ascomycota }\end{array}$ & seeds & $\begin{array}{l}\text { Adenanthera } \\
\text { microsperma }\end{array}$ & India & $\begin{array}{l}\text { Mathur 1974, Anderson } \\
\text { 1986a }\end{array}$ \\
\hline & & seeds & $\begin{array}{l}\text { Araucaria } \\
\text { angustifolia }\end{array}$ & Australia & $\begin{array}{l}\text { Kamara et al. } 1981 \text {, } \\
\text { Mittal et al. } 1990\end{array}$ \\
\hline & & seeds & Araucaria bidwillii & Egypt & $\begin{array}{l}\text { Kamara et al. 1981, } \\
\text { Mittal et al. } 1990\end{array}$ \\
\hline & & seeds & $\begin{array}{l}\text { Araucaria } \\
\text { cunninghamii }\end{array}$ & Australia & $\begin{array}{l}\text { Kamara et al. 1981, } \\
\text { Mittal et al. } 1990\end{array}$ \\
\hline & & seeds & Azadirachta indica & India & $\begin{array}{l}\text { Mathur 1974, Mittal et } \\
\text { al. } 1990\end{array}$ \\
\hline & & seeds & Bombax ceiba & India & Mittal et al. 1990 \\
\hline & & seeds & $\begin{array}{l}\text { Casuarina } \\
\text { equisetifolia }\end{array}$ & Philippines & $\begin{array}{l}\text { Mathur 1974, Mittal et } \\
\text { al. } 1990\end{array}$ \\
\hline & & seed & Cercis chinensis & Japan & Watanabe 2010 \\
\hline & & seeds & Eucalyptus grandis & Uruguay & $\begin{array}{l}\text { Mathur 1974, Anderson } \\
\text { 1986a }\end{array}$ \\
\hline & & seeds & Ficus benjamina & India & $\begin{array}{l}\text { Mathur 1974, Anderson } \\
\text { 1986a }\end{array}$ \\
\hline & & seeds & Leucaena spp. & Philippines & $\begin{array}{l}\text { Mathur 1974, Anderson } \\
\text { 1986a }\end{array}$ \\
\hline & & seeds & Pinus caribaea & Madagascar & $\begin{array}{l}\text { Mathur 1974, Anderson } \\
\text { 1986a }\end{array}$ \\
\hline & & seeds & Pinus elliottii & USA & $\begin{array}{l}\text { Mathur 1974, Mittal et } \\
\text { al. } 1990\end{array}$ \\
\hline & & seeds & Pinus khasya & Madagascar & $\begin{array}{l}\text { Mathur 1974, Anderson } \\
\text { 1986a }\end{array}$ \\
\hline & & seeds & Pinus taeda & USA & $\begin{array}{l}\text { Covington et al. } 1982 \text {, } \\
\text { Mittal et al. } 1990\end{array}$ \\
\hline & & seeds & Shorea assamica & Malaysia & $\begin{array}{l}\text { Hong 1981, Mittal et al. } \\
1990\end{array}$ \\
\hline & & seeds & Tectona grandis & India & Anderson 1986a \\
\hline
\end{tabular}


Table 2 Continued.

\begin{tabular}{|c|c|c|c|c|c|}
\hline Species & Taxonomy & Substrate & Host & Country & References \\
\hline \multirow{19}{*}{$\begin{array}{l}\text { Botryodiplodia theobromae } \\
\text { Pat. = Lasiodiplodia } \\
\text { theobromae (Pat.) Griffon \& } \\
\text { Maubl. }\end{array}$} & \multirow[t]{19}{*}{$\begin{array}{l}\text { Botryosphaeriaceae, Dothideomycetes, } \\
\text { Ascomycota }\end{array}$} & fruit & $\begin{array}{l}\text { Artocarpus } \\
\text { communis }\end{array}$ & Nigeria & Amusa et al. 2002 \\
\hline & & fruits & Alocasia odora & Hong Kong & Tang et al. 2003a \\
\hline & & fruits & Ardisia punctata & Hong Kong & Tang et al. 2003a \\
\hline & & fruits & Bridelia tomentosa & Hong Kong & Tang et al. 2003a \\
\hline & & fruits & Ilex asprella & Hong Kong & Tang et al. 2003a \\
\hline & & fruits & Ilex pubescens & Hong Kong & Tang et al. 2003a \\
\hline & & fruits & Microcos paniculata & Hong Kong & Tang et al. 2003a \\
\hline & & fruits & $\begin{array}{l}\text { Viburnum } \\
\text { sempervirens }\end{array}$ & Hong Kong & Tang et al. 2003a \\
\hline & & seeds & $\begin{array}{l}\text { Acacia } \\
\text { auriculiformis }\end{array}$ & Thailand & $\begin{array}{l}\text { Chalermpongse et al. } \\
\text { 1984, Mittal et al. } 1990\end{array}$ \\
\hline & & seeds & Acacia confusa & Philippines & $\begin{array}{l}\text { Agmata 1979, Anderson } \\
\text { 1986a }\end{array}$ \\
\hline & & seeds & $\begin{array}{l}\text { Acrocarpus } \\
\text { fraxinifolius }\end{array}$ & Rwanda & $\begin{array}{l}\text { Mathur 1974, Anderson } \\
\text { 1986a }\end{array}$ \\
\hline & & seeds & Albizia falcataria & Philippines & $\begin{array}{l}\text { Dayan 1986, Mittal et al. } \\
1990\end{array}$ \\
\hline & & seeds & $\begin{array}{l}\text { Araucaria } \\
\text { cunninghamii }\end{array}$ & Australia & $\begin{array}{l}\text { Kamara et al. } 1981 \text {, } \\
\text { Mittal et al. } 1990\end{array}$ \\
\hline & & seeds & $\begin{array}{l}\text { Casuarina } \\
\text { equisetifolia }\end{array}$ & Philippines & $\begin{array}{l}\text { Quiniones 1985, } \\
\text { Anderson 1986a }\end{array}$ \\
\hline & & seeds & Cordia alliodora & Colombia & $\begin{array}{l}\text { Mathur 1974, Anderson } \\
\text { 1986a }\end{array}$ \\
\hline & & seeds & $\begin{array}{l}\text { Dalbergia } \\
\text { cochinchinensis }\end{array}$ & Thailand & $\begin{array}{l}\text { Chalermpongse et al. } \\
\text { 1984, Mittal et al. } 1990\end{array}$ \\
\hline & & seeds & Grevillea robusta & Rwanda & $\begin{array}{l}\text { Mathur 1974, Anderson } \\
\text { 1986a }\end{array}$ \\
\hline & & seeds & Hevea sp. & Malaysia & $\begin{array}{l}\text { Edwards \& Sutherland } \\
\text { 1979, Mittal et al. } 1990\end{array}$ \\
\hline & & seeds & Hevea brasiliensis & India & $\begin{array}{l}\text { Srivastava 1964, Mittal } \\
\text { et al. } 1990\end{array}$ \\
\hline
\end{tabular}


Table 2 Continued.

\begin{tabular}{|c|c|c|c|c|c|}
\hline Species & Taxonomy & Substrate & Host & Country & References \\
\hline & & seeds & $\begin{array}{l}\text { Holarrhena } \\
\text { antidysenterica }\end{array}$ & Thailand & $\begin{array}{l}\text { Chalermpongse et al. } \\
\text { 1984, Mittal et al. } 1990\end{array}$ \\
\hline & & seeds & $\begin{array}{l}\text { Leucaena } \\
\text { leucocephala }\end{array}$ & Thailand & $\begin{array}{l}\text { Quiniones 1985, Mittal } \\
\text { et al. } 1990\end{array}$ \\
\hline & & seeds & Melia azedarach & Thailand & $\begin{array}{l}\text { Chalermpongse et al. } \\
\text { 1984, Mittal et al. } 1990\end{array}$ \\
\hline & & seeds & Pinus spp. & Nicaragua & $\begin{array}{l}\text { Richardson 1983, } \\
\text { Anderson 1986a }\end{array}$ \\
\hline & & seeds & Pinus caribaea & $\begin{array}{l}\text { Guatemala, } \\
\text { Nicaragua, } \\
\text { Honduras }\end{array}$ & Mathur 1974 \\
\hline & & seeds & Pinus merkusii & $\begin{array}{l}\text { Philippines, } \\
\text { Thailand }\end{array}$ & $\begin{array}{l}\text { Agmata 1979, Anderson } \\
\text { 1986a, Mittal et al. } 1990\end{array}$ \\
\hline & & seeds & Pinus oocarpa & $\begin{array}{l}\text { Central America, } \\
\text { UK }\end{array}$ & $\begin{array}{l}\text { Rees 1982, Anderson } \\
\text { 1986a, Mittal et al. } 1990\end{array}$ \\
\hline & & seeds & Pinus pseudostrobus & UK & $\begin{array}{l}\text { Rees 1982, Mittal et al. } \\
1990\end{array}$ \\
\hline & & seeds & $\begin{array}{l}\text { Swietenia } \\
\text { macrophylla }\end{array}$ & Philippines & $\begin{array}{l}\text { Agmata 1979, Anderson } \\
\text { 1986a }\end{array}$ \\
\hline & & seeds & Tectona grandis & - & $\begin{array}{l}\text { Mathur 1974, Anderson } \\
\text { 1986a }\end{array}$ \\
\hline & & seeds & Triplaris cumingiana & Philippines & $\begin{array}{l}\text { Quiniones 1985, } \\
\text { Anderson 1986a }\end{array}$ \\
\hline & & seeds & $\begin{array}{l}\text { Acacia } \\
\text { auriculiformis }\end{array}$ & Philippines & Quiniones 1987 \\
\hline & & seeds & Albizia falcataria & Philippines & Quiniones 1987 \\
\hline & & seeds & Albizia julibrissin & Philippines & Quiniones 1987 \\
\hline & & seeds & $\begin{array}{l}\text { Leucaena } \\
\text { leucocephala }\end{array}$ & $\begin{array}{l}\text { Bangladesh, } \\
\text { Philippines }\end{array}$ & $\begin{array}{l}\text { Quiniones 1987, Islam et } \\
\text { al. } 2008\end{array}$ \\
\hline & & seeds & $\begin{array}{l}\text { Casuarina } \\
\text { equisetifolia }\end{array}$ & Philippines & Quiniones 1987 \\
\hline \multirow{3}{*}{$\begin{array}{l}\text { Botryosphaeria dothidea } \\
\text { (Moug.) Ces. \& De Not. }\end{array}$} & \multirow{3}{*}{$\begin{array}{l}\text { Botryosphaeriaceae, Dothideomycetes, } \\
\text { Ascomycota }\end{array}$} & seeds & Tectona grandis & India & Mohanan et al. 2005 \\
\hline & & cones & Pinus patula & South Africa & Smith et al. 1996 \\
\hline & & cones & Pinus radiate & South Africa & Smith et al. 1996 \\
\hline
\end{tabular}


Table 2 Continued.

\begin{tabular}{|c|c|c|c|c|c|}
\hline Species & Taxonomy & Substrate & Host & Country & References \\
\hline \multirow[t]{2}{*}{ Botryosphaeria spp. } & \multirow{2}{*}{$\begin{array}{l}\text { Botryosphaeriaceae, Dothideomycetes, } \\
\text { Ascomycota }\end{array}$} & fruits & Ardisia punctata & Hong Kong & Tang et al. 2003a \\
\hline & & seeds & Eucalyptus grandis & South Africa & Jimu et al. 2016 \\
\hline $\begin{array}{l}\text { Botryosphaeria stevensii } \\
\text { Shoemaker }\end{array}$ & $\begin{array}{l}\text { Botryosphaeriaceae, Dothideomycetes, } \\
\text { Ascomycota }\end{array}$ & seeds & Fraxinus excelsior & Sweden & Cleary et al. 2013 \\
\hline $\begin{array}{l}\text { Botryotinia fuckeliana (de } \\
\text { Bary) Whetzel }\end{array}$ & $\begin{array}{l}\text { Sclerotiniaceae, Leotiomycetes, } \\
\text { Ascomycota }\end{array}$ & seeds & Fraxinus excelsior & Sweden & Hayatgheibi 2013 \\
\hline Botryotrichum sp. & $\begin{array}{l}\text { Chaetomiaceae, Sordariomycetes, } \\
\text { Ascomycota, }\end{array}$ & seeds & $\begin{array}{l}\text { Eucalyptus } \\
\text { citriodora }\end{array}$ & India & $\begin{array}{l}\text { Mittal \& Sharma } 1984 \text {, } \\
\text { Mittal et al. } 1990\end{array}$ \\
\hline \multirow[t]{2}{*}{ Botrytis allii Munn } & \multirow[t]{2}{*}{$\begin{array}{l}\text { Sclerotiniaceae, Leotiomycetes, } \\
\text { Ascomycota }\end{array}$} & seeds & Picea excelsa & USSR & $\begin{array}{l}\text { Urosevic 1961, } \\
\text { Anderson 1986a }\end{array}$ \\
\hline & & seeds & Pinus sylvestris & USSR & $\begin{array}{l}\text { Urosevic 1961, } \\
\text { Anderson 1986a }\end{array}$ \\
\hline \multirow[t]{13}{*}{ Botrytis cinerea Pers. } & \multirow[t]{13}{*}{$\begin{array}{l}\text { Sclerotiniaceae, Leotiomycetes, } \\
\text { Ascomycota }\end{array}$} & cones & Pinus ponderosa & USA & $\begin{array}{l}\text { James 1983a, James } \\
1995\end{array}$ \\
\hline & & cones & Pinus sylvestris & UK & Whittle 1977 \\
\hline & & seeds & Abies alba & Poland & Krol et al. 2015 \\
\hline & & seeds & Abies amabilis & South Korea & $\begin{array}{l}\text { Mathur 1974, Anderson } \\
\text { 1986a }\end{array}$ \\
\hline & & seeds & Abies nordmanniana & Austria, Georgia & Talgø et al. 2010 \\
\hline & & seeds & Abies procera & Norway & Talgø et al. 2010 \\
\hline & & seeds & Acer ginolamax & South Korea & $\begin{array}{l}\text { Mathur 1974, Anderson } \\
\text { 1986a }\end{array}$ \\
\hline & & seeds & Acer rubrum & USA & $\begin{array}{l}\text { Friedrich et al. } 1971 \text {, } \\
\text { Mittal et al. } 1990\end{array}$ \\
\hline & & seeds & Acer saccharinum & USA & $\begin{array}{l}\text { Friedrich et al. } 1971 \text {, } \\
\text { Mittal et al. } 1990\end{array}$ \\
\hline & & seeds & Acer saccharum & USA & $\begin{array}{l}\text { Friedrich et al. } 1971 \text {, } \\
\text { Mittal et al. } 1990\end{array}$ \\
\hline & & seeds & Acer sp. & South Korea & $\begin{array}{l}\text { Mathur 1974, Mittal et } \\
\text { al. } 1990\end{array}$ \\
\hline & & seeds & $\begin{array}{l}\text { Acrocarpus } \\
\text { fraxinifolius }\end{array}$ & India & $\begin{array}{l}\text { Mathur 1974, Anderson } \\
\text { 1986a }\end{array}$ \\
\hline & & seed & Agropyron cristatum & Canada & Conners 1967 \\
\hline
\end{tabular}


Table 2 Continued.

\begin{tabular}{|c|c|c|c|c|c|}
\hline Species & Taxonomy & Substrate & Host & Country & References \\
\hline & & seeds & Alnus glutinosa & Poland & Krol et al. 2015 \\
\hline & & seed & $\begin{array}{l}\text { Apium graveolens } \\
\text { var. dulce }\end{array}$ & Canada & Conners 1967 \\
\hline & & seeds & $\begin{array}{l}\text { Betula } \\
\text { alleghaniensis }\end{array}$ & USA & $\begin{array}{l}\text { Friedrich et al. } 1971 \text {, } \\
\text { Mittal et al. } 1990\end{array}$ \\
\hline & & seeds & Betula papyrifera & USA & $\begin{array}{l}\text { Friedrich et al. } 1971 \text {, } \\
\text { Mittal et al. } 1990\end{array}$ \\
\hline & & seeds & Betula pendula & Poland & Krol et al. 2015 \\
\hline & & seeds & $\begin{array}{l}\text { Callistephus } \\
\text { chinensis }\end{array}$ & China & $\begin{array}{l}\text { Gloyer 1931, Crosier \& } \\
\text { Heit } 1948\end{array}$ \\
\hline & & seeds & Carpinus eximia & South Korea & $\begin{array}{l}\text { Mathur 1974, Mittal et } \\
\text { al. } 1990\end{array}$ \\
\hline & & seeds & Cassia fistula & India & $\begin{array}{l}\text { Mathur 1974, Mittal et } \\
\text { al. } 1990\end{array}$ \\
\hline & & seeds & Cedrela toona & India & $\begin{array}{l}\text { Mathur 1974, Anderson } \\
\text { 1986a }\end{array}$ \\
\hline & & seeds & Centaurea cyanus & China & $\begin{array}{l}\text { Gloyer 1931, Crosier \& } \\
\text { Heit } 1948\end{array}$ \\
\hline & & seeds & Eucalyptus globulus & Uruguay & Lupo et al. 2001 \\
\hline & & seeds & Eucalyptus sp. & India & $\begin{array}{l}\text { Saxena 1985, Mittal et } \\
\text { al. } 1990\end{array}$ \\
\hline & & seeds & Fagus sylvatica & Poland & Krol et al. 2015 \\
\hline & & seeds & Fraxinus americana & USA & $\begin{array}{l}\text { Friedrich et al. } 1971 \text {, } \\
\text { Mittal et al. } 1990\end{array}$ \\
\hline & & seeds & Gmelina arborea & Philippines & $\begin{array}{l}\text { Mathur 1974, Anderson } \\
\text { 1986a }\end{array}$ \\
\hline & & seeds & $\begin{array}{l}\text { Jacaranda } \\
\text { mimosifolia }\end{array}$ & India & $\begin{array}{l}\text { Mathur 1974, Anderson } \\
\text { 1986a }\end{array}$ \\
\hline & & seeds & Larix decidua & Poland, USSR & $\begin{array}{l}\text { Kozlowska 1968, Mittal } \\
\text { et al. 1990, Krol et al. } \\
2015\end{array}$ \\
\hline & & seeds & Larix occidentalis & USA & James et al. 1996 \\
\hline & & seed & Linum usitatissimum & Canada & Conners 1967 \\
\hline
\end{tabular}


Table 2 Continued.

\begin{tabular}{|c|c|c|c|c|c|}
\hline Species & Taxonomy & Substrate & Host & Country & References \\
\hline & & seed & Lolium perenne & Canada & Conners 1967 \\
\hline & & seed & $\begin{array}{l}\text { Nierembergia } \\
\text { frutescens }\end{array}$ & Canada & Conners 1967 \\
\hline & & seeds & Picea excelsa & USSR & $\begin{array}{l}\text { Urosevic 1961, } \\
\text { Anderson 1986a }\end{array}$ \\
\hline & & seeds & Pinus caribaea & Cuba & $\begin{array}{l}\text { Mathur 1974, Anderson } \\
\text { 1986a }\end{array}$ \\
\hline & & seeds & Pinus nigra & Hungary & $\begin{array}{l}\text { Turchetti 1982, Mittal et } \\
\text { al. } 1990\end{array}$ \\
\hline & & seeds & Pinus ponderosa & USA & $\begin{array}{l}\text { James \& Genz 1982, } \\
\text { Anderson 1986a, James } \\
1995\end{array}$ \\
\hline & & seeds & Pinus sylvestris & Poland, UK, USSR & $\begin{array}{l}\text { Urosevic 1961, Whittle } \\
\text { 1977, Anderson, 1986a } \\
\text { Krol et al. 2015, }\end{array}$ \\
\hline & & seed & Prunus serrulata & Japan & Watanabe 2010 \\
\hline & & seeds & Quercus pedunculata & USSR & $\begin{array}{l}\text { Kozlowska 1968, Mittal } \\
\text { et al. } 1990\end{array}$ \\
\hline & & seeds & Quercus spp. & USSR & $\begin{array}{l}\text { Urosevic 1959, Mittal et } \\
\text { al. } 1990\end{array}$ \\
\hline & & seed & Salvia officinalis & Canada & Conners 1967 \\
\hline Botrytis sp. & $\begin{array}{l}\text { Sclerotiniaceae, Leotiomycetes, } \\
\text { Ascomycota }\end{array}$ & seeds & $\begin{array}{l}\text { Pseudotsuga } \\
\text { menziesii }\end{array}$ & Canada & $\begin{array}{l}\text { Salisbury 1955, Mittal et } \\
\text { al. } 1990\end{array}$ \\
\hline $\begin{array}{l}\text { Botrytis viciae-hirsutae X.Y. } \\
\text { Wang, L.X. Zhang \& Z.Y. } \\
\text { Zhang }^{\#}\end{array}$ & $\begin{array}{l}\text { Sclerotiniaceae, Leotiomycetes, } \\
\text { Ascomycota }\end{array}$ & fruit & Vicia hirsuta & China & Wang et al. 1995 \\
\hline $\begin{array}{l}\text { Brachydesmiella brasiliensis } \\
\text { R.F. Castaňeda, Gusmăo \& } \\
\text { Heredia\# }\end{array}$ & $\begin{array}{l}\text { Incertae sedis, Incertae sedis, } \\
\text { Ascomycota }\end{array}$ & pods & Fabaceae plant & Brazil & $\begin{array}{l}\text { Castañeda-Ruiz et al. } \\
2006\end{array}$ \\
\hline Brown, sterile mycelia & Fungi & seeds & Shorea robusta & India & $\begin{array}{l}\text { Mittal \& Sharma 1982b, } \\
\text { Mittal et al. } 1990\end{array}$ \\
\hline $\begin{array}{l}\text { Brunneiperidium } \\
\text { involucratum Daranag., } \\
\text { Camporesi \& K.D. Hyde }^{\#}\end{array}$ & $\begin{array}{l}\text { Xylariaceae, Sordariomycetes, } \\
\text { Ascomycota }\end{array}$ & cone & Pinus sylvestris & Italy & Daranagama et al. 2015 \\
\hline
\end{tabular}


Table 2 Continued.

\begin{tabular}{|c|c|c|c|c|c|}
\hline Species & Taxonomy & Substrate & Host & Country & References \\
\hline $\begin{array}{l}\text { Buerenia myrrhidendri } \\
\text { Döbbeler }^{\#}\end{array}$ & $\begin{array}{l}\text { Protomycetaceae, Taphrinomycetes, } \\
\text { Ascomycota }\end{array}$ & fruits & $\begin{array}{l}\text { Myrridendron } \\
\text { donnell-smithii }\end{array}$ & Costa Rica & Döbbeler 1995 \\
\hline $\begin{array}{l}\text { Cadophora fastigiata } \\
\text { Lagerb. \& Melin [as } \\
\text { Phialophora fastigiata } \\
\text { (Lagerb. \& Melin) Conant] }\end{array}$ & $\begin{array}{l}\text { Ploettnerulaceae, Leotiomycetes, } \\
\text { Ascomycota }\end{array}$ & seed & Pinus densiflora & Japan & Watanabe 2010 \\
\hline $\begin{array}{l}\text { Cadophora malorum (Kidd } \\
\text { \& Beaumont) W. Gams [as } \\
\text { Phialophora malorum (Kidd } \\
\text { \& Beaumont) McColloch] }\end{array}$ & $\begin{array}{l}\text { Ploettnerulaceae, Leotiomycetes, } \\
\text { Ascomycota }\end{array}$ & $\begin{array}{l}\text { seeds } \\
\text { seed }\end{array}$ & $\begin{array}{l}\text { Pinus elliottii } \\
\text { Pinus densiflora }\end{array}$ & $\begin{array}{l}\text { South Africa } \\
\text { Japan }\end{array}$ & $\begin{array}{l}\text { Cilliers et al. } 1995 \\
\text { Watanabe } 2010\end{array}$ \\
\hline Calcarisporium sp. & $\begin{array}{l}\text { Calcarisporiaceae, Sordariomycetes, } \\
\text { Ascomycota }\end{array}$ & seeds & $\begin{array}{l}\text { Platanus } \\
\text { occidentalis }\end{array}$ & USA & $\begin{array}{l}\text { Fakir et al. 1971, Mittal } \\
\text { et al. } 1990\end{array}$ \\
\hline $\begin{array}{l}\text { Caldariomyces fumago } \\
\text { Woron. }\end{array}$ & $\begin{array}{l}\text { Capnodiaceae, Dothideomycetes, } \\
\text { Ascomycota, }\end{array}$ & fruit & Gustavia superba & Panama & Goos \& Pirozynski 1975 \\
\hline $\begin{array}{l}\text { Calonectria brasiliensis } \\
\text { (Bat. \& Cif.) L. Lombard, } \\
\text { M.J. Wingf. \& Crous [as } \\
\text { Cylindrocladium brasiliense } \\
\text { (Bat. \& Cif.) Peerally] }\end{array}$ & $\begin{array}{l}\text { Nectriaceae, Sordariomycetes, } \\
\text { Ascomycota }\end{array}$ & seeds & Eucalyptus sp. & Brazil & $\begin{array}{l}\text { Cruz \& Figueiredo 1961, } \\
\text { Anderson 1986a }\end{array}$ \\
\hline $\begin{array}{l}\text { Calonectria gracilis Crous, } \\
\text { M.J. Wingf. \& Alfenas } \\
\text { [as Cylindrocarpon gracile }\end{array}$ & $\begin{array}{l}\text { Nectriaceae, Sordariomycetes, } \\
\text { Ascomycota }\end{array}$ & $\begin{array}{l}\text { cones } \\
\text { cones }\end{array}$ & $\begin{array}{l}\text { Pinus ponderosa } \\
\text { Pseudotsuga } \\
\text { menziesii var. glauca }\end{array}$ & $\begin{array}{l}\text { USA } \\
\text { USA }\end{array}$ & $\begin{array}{l}\text { James } 1995 \\
\text { James } 1995\end{array}$ \\
\hline Bugnic.] & & seeds & $\begin{array}{l}\text { Pseudotsuga } \\
\text { menziesii var. glauca }\end{array}$ & USA & James 1995 \\
\hline $\begin{array}{l}\text { Calonectria kyotensis } \\
\text { Terash. }\end{array}$ & $\begin{array}{l}\text { Nectriaceae, Sordariomycetes, } \\
\text { Ascomycota }\end{array}$ & fruit & undetermined plant & Panama & $\begin{array}{l}\text { Samuels \& Dumon } \\
\text { 1982, Piepenbring } 2006\end{array}$ \\
\hline $\begin{array}{l}\text { Calonectria morganii Crous, } \\
\text { Alfenas \& M.J. Wingf. }\end{array}$ & $\begin{array}{l}\text { Nectriaceae, Sordariomycetes, } \\
\text { Ascomycota }\end{array}$ & seeds & Horsfieldia sp. & Malaysia & $\begin{array}{l}\text { Lee \& Ahmad } 1982 \text {, } \\
\text { Mittal et al. } 1990\end{array}$ \\
\hline $\begin{array}{l}\text { [as Cylindrocladium } \\
\text { scoparium Morgan] }\end{array}$ & & seeds & Maesopsis eminii & Malaysia & $\begin{array}{l}\text { Lee \& Ahmad } 1982 \text {, } \\
\text { Mittal et al. } 1990\end{array}$ \\
\hline & & seeds & Shorea roxburghii & Malaysia & $\begin{array}{l}\text { Lee \& Ahmad } 1982 \text {, } \\
\text { Mittal et al. } 1990\end{array}$ \\
\hline
\end{tabular}


Table 2 Continued.

\begin{tabular}{|c|c|c|c|c|c|}
\hline Species & Taxonomy & Substrate & Host & Country & References \\
\hline & & seeds & Shorea talura & Malaysia & $\begin{array}{l}\text { Lee \& Ahmad 1982, } \\
\text { Mittal et al. } 1990\end{array}$ \\
\hline \multirow[t]{15}{*}{$\begin{array}{l}\text { Caloscypha fulgens (Pers.) } \\
\text { Boud. }\end{array}$} & $\begin{array}{l}\text { Caloscyphaceae, Pezizomycetes, } \\
\text { Ascomycota }\end{array}$ & seeds & Abies amabilis & Canada & $\begin{array}{l}\text { Sutherland 1979, Mittal } \\
\text { et al. } 1990\end{array}$ \\
\hline & & seeds & Abies grandis & Canada & $\begin{array}{l}\text { Sutherland 1979, Mittal } \\
\text { et al. } 1990\end{array}$ \\
\hline & & seeds & Abies lasiocarpa & Canada & Talgø et al. 2010 \\
\hline & & seeds & Coniferae plant & Canada & Anderson 1986a \\
\hline & & seeds & Picea abies & Canada, UK, USA & $\begin{array}{l}\text { Gordon et al. } 1976 \text {, } \\
\text { Mittal et al. } 1990\end{array}$ \\
\hline & & seeds & Picea engelmannii & Canada, USA & $\begin{array}{l}\text { Sutherland 1979, } \\
\text { Wicklow-Howard \& } \\
\text { Skujins 1980, Mittal et } \\
\text { al. } 1990\end{array}$ \\
\hline & & seeds & Picea glauca & Canada & $\begin{array}{l}\text { Epners 1964, Sutherland } \\
\text { 1979, Mittal et al. } 1990\end{array}$ \\
\hline & & seeds & $\begin{array}{l}\text { Picea glauca } \times P \text {. } \\
\text { engelmannii }\end{array}$ & Canada & $\begin{array}{l}\text { Sutherland 1979, Mittal } \\
\text { et al. } 1990\end{array}$ \\
\hline & & seeds & Picea sitchensis & Canada , UK, USA & $\begin{array}{l}\text { Paden et al. 1978, } \\
\text { Harvey Jr 1980, Woods } \\
\text { et al. 1982, Mittal et al. } \\
1990\end{array}$ \\
\hline & & seeds & Picea spp. & Canada, USA & $\begin{array}{l}\text { Epners 1964, Anderson } \\
\text { 1986a }\end{array}$ \\
\hline & & seeds & Pinus contorta & Canada & $\begin{array}{l}\text { Sutherland 1979, Mittal } \\
\text { et al. } 1990\end{array}$ \\
\hline & & seeds & Pinus monticola & Canada & $\begin{array}{l}\text { Sutherland 1979, Mittal } \\
\text { et al. } 1990\end{array}$ \\
\hline & & seeds & Pinus ponderosa & Canada & $\begin{array}{l}\text { Sutherland 1979, Mittal } \\
\text { et al. } 1990\end{array}$ \\
\hline & & seeds & $\begin{array}{l}\text { Pseudotsuga } \\
\text { menziesii }\end{array}$ & Canada & $\begin{array}{l}\text { Sutherland 1979, Mittal } \\
\text { et al. } 1990\end{array}$ \\
\hline & & seeds & Tsuga heterophylla & Canada & $\begin{array}{l}\text { Sutherland 1979, Mittal } \\
\text { et al. } 1990\end{array}$ \\
\hline
\end{tabular}


Table 2 Continued.

\begin{tabular}{|c|c|c|c|c|c|}
\hline Species & Taxonomy & Substrate & Host & Country & References \\
\hline & & seeds & Tsuga mertensiana & Canada & $\begin{array}{l}\text { Sutherland 1979, Mittal } \\
\text { et al. } 1990\end{array}$ \\
\hline $\begin{array}{l}\text { Calycellina ochracea (Grelet } \\
\text { \& Croz.) Dennis }\end{array}$ & $\begin{array}{l}\text { Pezizellaceae, Leotiomycetes, } \\
\text { Ascomycota }\end{array}$ & fruit cupules & Fagus sylvatica & UK & Carré 1964 \\
\hline $\begin{array}{l}\text { Calycina cupulincola } \\
\text { [as Cystopezizella } \\
\text { cupulincola } \text { Svrček] }\end{array}$ & $\begin{array}{l}\text { Pezizellaceae, Leotiomycetes, } \\
\text { Ascomycota }\end{array}$ & cupule & Quercus robur & $\begin{array}{l}\text { former } \\
\text { Czechoslovakia }\end{array}$ & Svrcek 1987 \\
\hline \multirow[t]{10}{*}{ Camarosporium sp. } & $\begin{array}{l}\text { Camarosporiaceae, Dothideomycetes, } \\
\text { Ascomycota }\end{array}$ & seeds & $\begin{array}{l}\text { Chamaecyparis } \\
\text { lawsoniana }\end{array}$ & France, Italy & $\begin{array}{l}\text { Motta \& Saponaro 1983, } \\
\text { Mittal et al. } 1990\end{array}$ \\
\hline & & seeds & $\begin{array}{l}\text { Cupressus } \\
\text { abramsiana }\end{array}$ & France, Italy & $\begin{array}{l}\text { Saponaro \& Motta 1984, } \\
\text { Mittal et al. } 1990\end{array}$ \\
\hline & & seeds & Cupressus arizonica & France, Italy & $\begin{array}{l}\text { Saponaro \& Motta 1984, } \\
\text { Mittal et al. } 1990\end{array}$ \\
\hline & & seeds & Cupressus glabra & France, Italy & $\begin{array}{l}\text { Saponaro \& Motta 1984, } \\
\text { Mittal et al. } 1990\end{array}$ \\
\hline & & seeds & $\begin{array}{l}\text { Cupressus } \\
\text { goveniana }\end{array}$ & France, Italy & $\begin{array}{l}\text { Saponaro \& Motta 1984, } \\
\text { Mittal et al. } 1990\end{array}$ \\
\hline & & seeds & Cupressus lusitanica & France, Italy & $\begin{array}{l}\text { Saponaro \& Motta 1984, } \\
\text { Mittal et al. } 1990\end{array}$ \\
\hline & & seeds & $\begin{array}{l}\text { Cupressus lusitanica } \\
\text { var. benthamii }\end{array}$ & France, Italy & $\begin{array}{l}\text { Saponaro \& Motta 1984, } \\
\text { Mittal et al. } 1990\end{array}$ \\
\hline & & seeds & $\begin{array}{l}\text { Cupressus } \\
\text { sempervirens }\end{array}$ & France, Italy & $\begin{array}{l}\text { Motta \& Saponaro 1983, } \\
\text { Mittal et al. } 1990\end{array}$ \\
\hline & & seeds & Cupressus torulosa & France, Italy & $\begin{array}{l}\text { Saponaro \& Motta 1984, } \\
\text { Mittal et al. } 1990\end{array}$ \\
\hline & & seeds & Thuja orientalis & Italy, France & $\begin{array}{l}\text { Motta \& Saponaro 1983, } \\
\text { Mittal et al. } 1990\end{array}$ \\
\hline $\begin{array}{l}\text { Candida albicans (C.P. } \\
\text { Robin) Berkhout }\end{array}$ & $\begin{array}{l}\text { Incertae sedis, Saccharomycetes, } \\
\text { Ascomycota }\end{array}$ & seeds & Ficus pumila & $\begin{array}{l}\text { Poland or/ } \\
\text { imported to Poland }\end{array}$ & Czeczuga et al. 2009 \\
\hline $\begin{array}{l}\text { Candida asparagi F.Y. Bai } \\
\& \text { H.Z. Lu }\end{array}$ & $\begin{array}{l}\text { Incertae sedis, Saccharomycetes, } \\
\text { Ascomycota }\end{array}$ & fruit & Asparagus filicinus & China & Lu et al. 2004 \\
\hline $\begin{array}{l}\text { Candida berthetii Boidin, } \\
\text { Pignal, Mermiér \& Arpin }\end{array}$ & $\begin{array}{l}\text { Incertae sedis, Saccharomycetes, } \\
\text { Ascomycota }\end{array}$ & fruits & $\begin{array}{l}\text { Parahancornia } \\
\text { amapa }\end{array}$ & Brazil & Morais et al. 1995 \\
\hline
\end{tabular}


Table 2 Continued.

\begin{tabular}{|c|c|c|c|c|c|}
\hline Species & Taxonomy & Substrate & Host & Country & References \\
\hline Candida blankii-like & $\begin{array}{l}\text { Incertae sedis, Saccharomycetes, } \\
\text { Ascomycota }\end{array}$ & fruits & $\begin{array}{l}\text { Parahancornia } \\
\text { amapa }\end{array}$ & Brazil & Morais et al. 1995 \\
\hline $\begin{array}{l}\text { Candida caryicola } \\
\text { Kurtzman }^{\#}\end{array}$ & $\begin{array}{l}\text { Incertae sedis, Saccharomycetes, } \\
\text { Ascomycota }\end{array}$ & nut & Carya glabra & USA & Kurtzman 2001 \\
\hline Candida citrea Nakase & $\begin{array}{l}\text { Incertae sedis, Saccharomycetes, } \\
\text { Ascomycota }\end{array}$ & fruits & $\begin{array}{l}\text { Parahancornia } \\
\text { aтара }\end{array}$ & Brazil & Morais et al. 1995 \\
\hline $\begin{array}{l}\text { Candida fructus (Nakase) } \\
\text { S.A. Mey. \& Yarrow }\end{array}$ & $\begin{array}{l}\text { Incertae sedis, Saccharomycetes, } \\
\text { Ascomycota }\end{array}$ & fruits & $\begin{array}{l}\text { Parahancornia } \\
\text { amapa }\end{array}$ & Brazil & Morais et al. 1995 \\
\hline $\begin{array}{l}\text { Candida insectamans D.B. } \\
\text { Scott, Van der Walt \& Klift }\end{array}$ & $\begin{array}{l}\text { Incertae sedis, Saccharomycetes, } \\
\text { Ascomycota }\end{array}$ & fruits & $\begin{array}{l}\text { Parahancornia } \\
\text { amapa }\end{array}$ & Brazil & Morais et al. 1995 \\
\hline $\begin{array}{l}\text { Candida krusei (Castell.) } \\
\text { Berkhout }\end{array}$ & $\begin{array}{l}\text { Incertae sedis, Saccharomycetes, } \\
\text { Ascomycota }\end{array}$ & fruits & $\begin{array}{l}\text { Parahancornia } \\
\text { amapa }\end{array}$ & Brazil & Morais et al. 1995 \\
\hline $\begin{array}{l}\text { Candida leandrae Ruivo, } \\
\text { Pagnocca, Lachane \& C.A. } \\
\text { Rosa }\end{array}$ & $\begin{array}{l}\text { Incertae sedis, Saccharomycetes, } \\
\text { Ascomycota }\end{array}$ & fruit & Leandra reversa & Brazil & Ruivo et al. 2004 \\
\hline $\begin{array}{l}\text { Candida linzhiensis F.Y. Bai } \\
\& \mathrm{Z} . \mathrm{W} . \mathrm{Wu}^{\#}\end{array}$ & $\begin{array}{l}\text { Incertae sedis, Saccharomycetes, } \\
\text { Ascomycota }\end{array}$ & fruit & undetermined plant & China & Wu \& Bai 2006 \\
\hline Candida lipolytica-like & $\begin{array}{l}\text { Incertae sedis, Saccharomycetes, } \\
\text { Ascomycota }\end{array}$ & fruits & $\begin{array}{l}\text { Parahancornia } \\
\text { amapa }\end{array}$ & Brazil & Morais et al. 1995 \\
\hline $\begin{array}{l}\text { Candida norvegensis } \\
\text { Dietrichson ex Uden \& H.R. } \\
\text { Buckley }\end{array}$ & $\begin{array}{l}\text { Incertae sedis, Saccharomycetes, } \\
\text { Ascomycota }\end{array}$ & fruits & $\begin{array}{l}\text { Parahancornia } \\
\text { amapa }\end{array}$ & Brazil & Morais et al. 1995 \\
\hline $\begin{array}{l}\text { Candida parapsilosis } \\
\text { (Ashford) Langeron \& Talice }\end{array}$ & $\begin{array}{l}\text { Incertae sedis, Saccharomycetes, } \\
\text { Ascomycota }\end{array}$ & fruits & $\begin{array}{l}\text { Parahancornia } \\
\text { amapa }\end{array}$ & Brazil & Morais et al. 1995 \\
\hline Candida quercitrusa-like & $\begin{array}{l}\text { Incertae sedis, Saccharomycetes, } \\
\text { Ascomycota }\end{array}$ & fruits & $\begin{array}{l}\text { Parahancornia } \\
\text { amapa }\end{array}$ & Brazil & Morais et al. 1995 \\
\hline $\begin{array}{l}\text { Candida rugopelliculosa- } \\
\text { like }\end{array}$ & $\begin{array}{l}\text { Incertae sedis, Saccharomycetes, } \\
\text { Ascomycota }\end{array}$ & fruits & $\begin{array}{l}\text { Parahancornia } \\
\text { amapa }\end{array}$ & Brazil & Morais et al. 1995 \\
\hline $\begin{array}{l}\text { Candida sorbosa-like } \\
\text { complex }\end{array}$ & $\begin{array}{l}\text { Incertae sedis, Saccharomycetes, } \\
\text { Ascomycota }\end{array}$ & fruits & $\begin{array}{l}\text { Parahancornia } \\
\text { amapa }\end{array}$ & Brazil & Morais et al. 1995 \\
\hline $\begin{array}{l}\text { Candida sorboxylosa-like } \\
\text { complex }\end{array}$ & $\begin{array}{l}\text { Incertae sedis, Saccharomycetes, } \\
\text { Ascomycota }\end{array}$ & fruits & $\begin{array}{l}\text { Parahancornia } \\
\text { amapa }\end{array}$ & Brazil & Morais et al. 1995 \\
\hline Candida spp. & $\begin{array}{l}\text { Incertae sedis, Saccharomycetes, } \\
\text { Ascomycota }\end{array}$ & seeds & Acer saccharum & USA & $\begin{array}{l}\text { Janerette 1979, Mittal et } \\
\text { al. } 1990\end{array}$ \\
\hline
\end{tabular}


Table 2 Continued.

\begin{tabular}{|c|c|c|c|c|c|}
\hline Species & Taxonomy & Substrate & Host & Country & References \\
\hline & & seed & Pinus densiflora & Japan & Watanabe 2010 \\
\hline & & seeds & Pinus taeda & USA & $\begin{array}{l}\text { Covington et al. 1982, } \\
\text { Anderson 1986a }\end{array}$ \\
\hline & & seed & Pinus thunbergii & Japan & Watanabe 2010 \\
\hline & & seed & Prunus serrulata & Japan & Watanabe 2010 \\
\hline $\begin{array}{l}\text { Candida uthaithanina } \\
\text { Limtong, Jindam., Am- }{ }^{\#}\end{array}$ & $\begin{array}{l}\text { Incertae sedis, Saccharomycetes, } \\
\text { Ascomycota }\end{array}$ & $\begin{array}{l}\text { fruit in } \\
\text { dipterocarp } \\
\text { forest }\end{array}$ & undetermined plant & Thailand & Limtong et al. 2011 \\
\hline Capnodiales spp. & Dothideomycetes, Ascomycota & seeds & Fraxinus excelsior & Sweden & Cleary et al. 2013 \\
\hline $\begin{array}{l}\text { Caryospora quercus Jayasiri, } \\
\text { E.B.G. Jones \& K.D. Hyde }\end{array}$ & $\begin{array}{l}\text { Caryosporaceae, Dothideomycetes, } \\
\text { Ascomycota }\end{array}$ & fruit & Quercus sp. & Thailand & Jayasiri et al. 2019 \\
\hline \multirow[t]{4}{*}{$\begin{array}{l}\text { Catenaria anguillulae } \\
\text { Sorokīn }\end{array}$} & $\begin{array}{l}\text { Catenariaceae, Blastocladiomycetes, } \\
\text { Blastocladiomycota }\end{array}$ & seeds & $\begin{array}{l}\text { Atropa belladonna } \\
\text { var. caucasica }\end{array}$ & $\begin{array}{l}\text { Poland or/ } \\
\text { imported to Poland }\end{array}$ & Czeczuga et al. 2009 \\
\hline & & seeds & $\begin{array}{l}\text { Prunella grandiflora } \\
\text { ssp. pyrenaia }\end{array}$ & $\begin{array}{l}\text { Poland or/ } \\
\text { imported to Poland }\end{array}$ & Czeczuga et al. 2009 \\
\hline & & seeds & Sambucus racemosa & $\begin{array}{l}\text { Poland or/ } \\
\text { imported to Poland }\end{array}$ & Czeczuga et al. 2009 \\
\hline & & seeds & Vicia sativa & Poland & Kiziewicz 2005 \\
\hline Catenaria verrucosa Karling & $\begin{array}{l}\text { Catenariaceae, Blastocladiomycetes, } \\
\text { Blastocladiomycota }\end{array}$ & seeds & Vicia sativa & Poland & Kiziewicz 2005 \\
\hline $\begin{array}{l}\text { Catenochytridium } \\
\text { carolinianum Berdan }\end{array}$ & $\begin{array}{l}\text { Catenochytridiaceae, } \\
\text { Cladochytriomycetes, Chytridiomycota }\end{array}$ & seeds & $\begin{array}{l}\text { Crataegus azarolus, } \\
\text { Sambucus ebulus }\end{array}$ & $\begin{array}{l}\text { Poland or/ } \\
\text { imported to Poland }\end{array}$ & Czeczuga et al. 2009 \\
\hline $\begin{array}{l}\text { Catenophlyctis variabilis } \\
\text { (Karling) Karling }\end{array}$ & $\begin{array}{l}\text { Catenariaceae, Blastocladiomycetes, } \\
\text { Blastocladiomycota }\end{array}$ & seeds & Sambucus ebulus & $\begin{array}{l}\text { Poland or/ } \\
\text { imported to Poland }\end{array}$ & Czeczuga et al. 2009 \\
\hline Catenularia sp. & $\begin{array}{l}\text { Chaetosphaeriaceae, Sordariomycetes, } \\
\text { Ascomycota }\end{array}$ & pods & Delonix regia & Thailand & Somrithipol et al. 2002b \\
\hline $\begin{array}{l}\text { Centrolepidosporium } \\
\text { sclerodermum R.G. Shivas } \\
\& \text { Vánky }^{* * *}\end{array}$ & $\begin{array}{l}\text { Incertae sedis, Ustilaginomycetes, } \\
\text { Basidiomycota }\end{array}$ & nutlets & Centrolepis exserta & Australia & Shivas \& Vánky 2007 \\
\hline $\begin{array}{l}\text { Cephaliophora tropica } \\
\text { Thaxt. }\end{array}$ & $\begin{array}{l}\text { Ascodesmidaceae, Pezizomycetes, } \\
\text { Ascomycota }\end{array}$ & seed & $\begin{array}{l}\text { Macroptilium } \\
\text { lathyroides }\end{array}$ & Australia & Nik \& Parbery 1977 \\
\hline $\begin{array}{l}\text { Cephalosporium curtipes } \\
\text { Sacc. }\end{array}$ & $\begin{array}{l}\text { Incertae sedis, Sordariomycetes, } \\
\text { Ascomycota }\end{array}$ & seeds & Melia spp. & India & Sahai \& Otra 1982 \\
\hline
\end{tabular}


Table 2 Continued.

\begin{tabular}{|c|c|c|c|c|c|}
\hline Species & Taxonomy & Substrate & Host & Country & References \\
\hline \multirow[t]{3}{*}{$\begin{array}{l}\text { Cephalosporium roseum } \\
\text { Oudem. }\end{array}$} & $\begin{array}{l}\text { Incertae sedis, Sordariomycetes, } \\
\text { Ascomycota }\end{array}$ & seeds & Betula verrucosa & USSR & $\begin{array}{l}\text { Kozlowska 1968, Mittal } \\
\text { et al. } 1990\end{array}$ \\
\hline & & seeds & Pinus sylvestris & USSR & $\begin{array}{l}\text { Kozlowska 1968, Mittal } \\
\text { et al. } 1990\end{array}$ \\
\hline & & seeds & Quercus pedunculata & USSR & $\begin{array}{l}\text { Kozlowska 1968, Mittal } \\
\text { et al. } 1990\end{array}$ \\
\hline \multirow[t]{14}{*}{ Cephalosporium spp. } & $\begin{array}{l}\text { Incertae sedis, Sordariomycetes, } \\
\text { Ascomycota }\end{array}$ & seeds & $\begin{array}{l}\text { Acrocarpus } \\
\text { fraxinifolius }\end{array}$ & Rwanda & $\begin{array}{l}\text { Mathur 1974, Anderson } \\
\text { 1986a }\end{array}$ \\
\hline & & seeds & Albizia falcataria & Philippines & $\begin{array}{l}\text { Mathur 1974, Mittal et } \\
\text { al. } 1990\end{array}$ \\
\hline & & seeds & Alnus maximowiczii & South Korea & $\begin{array}{l}\text { Mathur 1974, Anderson } \\
\text { 1986a }\end{array}$ \\
\hline & & seeds & Azadirachta indica & India & $\begin{array}{l}\text { Mathur 1974, Mittal et } \\
\text { al. } 1990\end{array}$ \\
\hline & & seeds & Delonix regia & Philippines & $\begin{array}{l}\text { Dayan 1986, Mittal et al. } \\
1990\end{array}$ \\
\hline & & seeds & $\begin{array}{l}\text { Endospermum } \\
\text { peltatum }\end{array}$ & Philippines & $\begin{array}{l}\text { Agmata 1979, Anderson } \\
\text { 1986a }\end{array}$ \\
\hline & & seeds & $\begin{array}{l}\text { Eucalyptus } \\
\text { citriodora }\end{array}$ & India & Anderson 1986a \\
\hline & & seeds & Eucalyptus deglupta & Philippines & $\begin{array}{l}\text { Mathur 1974, Anderson } \\
\text { 1986a }\end{array}$ \\
\hline & & seeds & Eucalyptus globulus & India & Anderson 1986a \\
\hline & & seeds & Ficus krishnae & India & $\begin{array}{l}\text { Mathur 1974, Anderson } \\
\text { 1986a }\end{array}$ \\
\hline & & seeds & Fraxinus americana & USA & $\begin{array}{l}\text { Friedrich et al. } 1971 \text {, } \\
\text { Mittal et al. } 1990\end{array}$ \\
\hline & & seeds & Gmelina arborea & India & $\begin{array}{l}\text { Mathur 1974, Anderson } \\
\text { 1986a }\end{array}$ \\
\hline & & seeds & Gmelina moluccana & Solomon Islands & $\begin{array}{l}\text { Mathur 1974, Anderson } \\
\text { 1986a }\end{array}$ \\
\hline & & seeds & $\begin{array}{l}\text { Lagerstroemia } \\
\text { speciosa }\end{array}$ & Philippines & $\begin{array}{l}\text { Quiniones 1985, } \\
\text { Anderson 1986a }\end{array}$ \\
\hline
\end{tabular}


Table 2 Continued.

\begin{tabular}{|c|c|c|c|c|c|}
\hline Species & Taxonomy & Substrate & Host & Country & References \\
\hline & & seeds & $\begin{array}{l}\text { Leucaena } \\
\text { leucocephala }\end{array}$ & Philippines & $\begin{array}{l}\text { Quiniones 1987, Mathur } \\
\text { 1974, Anderson 1986a }\end{array}$ \\
\hline & & seeds & $\begin{array}{l}\text { Mimosa } \\
\text { caesalpiniafolia }\end{array}$ & Brazil & $\begin{array}{l}\text { Mathur 1974, Anderson } \\
\text { 1986a }\end{array}$ \\
\hline & & seeds & Panax ginseng & South Korea & Anderson 1986a \\
\hline & & seeds & Pinus elliottii & Taiwan, USA & $\begin{array}{l}\text { Rowan \& Debarr 1974, } \\
\text { Anderson 1986a }\end{array}$ \\
\hline & & seeds & $\begin{array}{l}\text { Pinus elliottii var. } \\
\text { elliottii }\end{array}$ & Uruguay & Anderson 1986a \\
\hline & & seeds & Pinus merkusii & Philippines & $\begin{array}{l}\text { Agmata 1979, Anderson } \\
\text { 1986a }\end{array}$ \\
\hline & & seeds & Pinus ponderosa & USA & $\begin{array}{l}\text { James \& Genz 1982, } \\
\text { Anderson 1986a }\end{array}$ \\
\hline & & seeds & Pinus taeda & Georgia, USA & $\begin{array}{l}\text { Anderson 1986a, b, } \\
\text { Huang \& Kuhlman } 1990\end{array}$ \\
\hline & & seeds & Pinus wallichiana & India & $\begin{array}{l}\text { Munjal \& Sharma 1975, } \\
\text { Mittal et al. } 1990\end{array}$ \\
\hline & & seeds & $\begin{array}{l}\text { Pseudotsuga } \\
\text { menziesii }\end{array}$ & Canada & $\begin{array}{l}\text { Bloomberg 1969, Mittal } \\
\text { et al. } 1990\end{array}$ \\
\hline & & seeds & Tectona grandis & India & $\begin{array}{l}\text { Mathur 1974, Anderson } \\
\text { 1986a }\end{array}$ \\
\hline \multirow{3}{*}{$\begin{array}{l}\text { Cephalosporium } \\
\text { subverticillatum Schulzer \& } \\
\text { Sacc. }\end{array}$} & $\begin{array}{l}\text { Incertae sedis, Sordariomycetes, } \\
\text { Ascomycota }\end{array}$ & seeds & Picea excelsa & USSR & $\begin{array}{l}\text { Urosevic 1961, } \\
\text { Anderson 1986a }\end{array}$ \\
\hline & & seeds & Pinus sylvestris & USSR & $\begin{array}{l}\text { Urosevic 1961, } \\
\text { Anderson 1986a }\end{array}$ \\
\hline & & seeds & Quercus spp. & USSR & $\begin{array}{l}\text { Urosevic 1959, Mittal et } \\
\text { al. } 1990\end{array}$ \\
\hline $\begin{array}{l}\text { Cephalothecium roseum } \\
\text { Corda }\end{array}$ & $\begin{array}{l}\text { Incertae sedis, Sordariomycetes, } \\
\text { Ascomycota }\end{array}$ & seeds & Larix decidua & USSR & $\begin{array}{l}\text { Kozlowska 1968, Mittal } \\
\text { et al. } 1990\end{array}$ \\
\hline Ceratobasidium spp. & $\begin{array}{l}\text { Ceratobasidiaceae, Agaricomycetes, } \\
\text { Basidiomycota }\end{array}$ & seeds & Picea sitchensis & UK & $\begin{array}{l}\text { Salt 1964, Mittal et al. } \\
1990\end{array}$ \\
\hline $\begin{array}{l}\text { Ceratocystis ethacetica } \\
\text { (Went) Mbenoun \& Z.W. de } \\
\text { Beer }^{\#} \text { [as Thielaviopsis }\end{array}$ & $\begin{array}{l}\text { Ceratocystidaceae, Sordariomycetes, } \\
\text { Ascomycota }\end{array}$ & fruits & Saccharum sp. & Indonesia & Mbenoun et al. 2014 \\
\hline
\end{tabular}


Table 2 Continued.

\begin{tabular}{|c|c|c|c|c|c|}
\hline Species & Taxonomy & Substrate & Host & Country & References \\
\hline \multicolumn{6}{|l|}{ ethacetica Went] } \\
\hline $\begin{array}{l}\text { Ceratocystis fagacearum } \\
\text { (Bretz) J. Hunt }\end{array}$ & $\begin{array}{l}\text { Ceratocystidaceae, Sordariomycetes, } \\
\text { Ascomycota }\end{array}$ & seeds & Quercus spp. & USA & Mittal et al. 1990 \\
\hline Ceratocystis spp. & $\begin{array}{l}\text { Ceratocystidaceae, Sordariomycetes, } \\
\text { Ascomycota }\end{array}$ & seeds & Pinus taeda & Georgia & Huang \& Kuhlman 1990 \\
\hline \multirow[t]{4}{*}{ Ceratorhiza sp. } & $\begin{array}{l}\text { Ceratobasidiaceae, Agaricomycetes, } \\
\text { Basidiomycota }\end{array}$ & seeds & $\begin{array}{l}\text { Corallorhiza } \\
\text { maculata }\end{array}$ & Canada & Zelmer et al. 1996 \\
\hline & & seeds & Platanthera dilatata & Canada & Zelmer et al. 1996 \\
\hline & & seeds & $\begin{array}{l}\text { Platanthera } \\
\text { hyperborea }\end{array}$ & Canada & Zelmer et al. 1996 \\
\hline & & seeds & Spiranthes lacera & Canada & Zelmer et al. 1996 \\
\hline \multirow[t]{2}{*}{ Cercospora spp. } & $\begin{array}{l}\text { Mycosphaerellaceae, Dothideomycetes, } \\
\text { Ascomycota }\end{array}$ & $\begin{array}{l}\text { seeds, seed } \\
\text { capsules }\end{array}$ & Eucalyptus grandis & South Africa & Jimu et al. 2016 \\
\hline & & seeds & Tectona grandis & India & Anderson 1986a \\
\hline \multirow[t]{3}{*}{ Ceuthospora sp. } & Phacidiaceae, Leotiomycetes, & cones, seeds & Pinus albicaulis & Canada & Vujanovic et al. 2000 \\
\hline & Ascomycota & cones, seeds & Pinus sylvestris & Canada & Vujanovic et al. 2000 \\
\hline & & cones, seeds & Pinus tabulaeformis & Canada & Vujanovic et al. 2000 \\
\hline $\begin{array}{l}\text { Chaetocladium brefeldii } \\
\text { Tiegh. \& G. Le Monn. }\end{array}$ & $\begin{array}{l}\text { Mucoraceae, Mucoromycetes, } \\
\text { Mucoromycota }\end{array}$ & seeds & Sorbus torminalis & $\begin{array}{l}\text { Poland or/ } \\
\text { imported to Poland }\end{array}$ & Czeczuga et al. 2009 \\
\hline Chaetomella sp. & $\begin{array}{l}\text { Chaetomellaceae, Leotiomycetes, } \\
\text { Ascomycota }\end{array}$ & seeds & $\begin{array}{l}\text { Pinus armandii var. } \\
\text { mastersiana }\end{array}$ & Taiwan & $\begin{array}{l}\text { Jong \& Chen } 1966 \text {, } \\
\text { Mittal et al. } 1990\end{array}$ \\
\hline $\begin{array}{l}\text { Chaetomium affine Corda } \\
\text { [as 'offine'] }\end{array}$ & $\begin{array}{l}\text { Chaetomiaceae, Sordariomycetes, } \\
\text { Ascomycota }\end{array}$ & seeds & Quercus spp. & USSR & Mittal et al. 1990 \\
\hline \multirow[t]{4}{*}{$\begin{array}{l}\text { Chaetomium cochliodes } \\
\text { Palliser }\end{array}$} & $\begin{array}{l}\text { Chaetomiaceae, Sordariomycetes, } \\
\text { Ascomycota }\end{array}$ & seed & $\begin{array}{l}\text { Platanus } \\
\text { occidentalis }\end{array}$ & USA & Mittal et al. 1990 \\
\hline & & seed & $\begin{array}{l}\text { Apium graveolens } \\
\text { var. dulce }\end{array}$ & Canada & Conners 1967 \\
\hline & & seed & Linum usitatissimum & Canada & Conners 1967 \\
\hline & & seeds & Medicago sativa & Canada & Conners 1967 \\
\hline \multirow[t]{2}{*}{ Chaetomium elatum Kunze } & Chaetomiaceae, Sordariomycetes, & seed & Pinus densiflora & Japan & Watanabe 2010 \\
\hline & Ascomycota & seed & Pinus patula & Kenya & $\begin{array}{l}\text { Gibson 1957, Mittal et } \\
\text { al. } 1990\end{array}$ \\
\hline $\begin{array}{l}\text { Chaetomium gangligerum } \\
\text { L.M. Ames }\end{array}$ & $\begin{array}{l}\text { Chaetomiaceae, Sordariomycetes, } \\
\text { Ascomycota }\end{array}$ & seeds & Erysimum cheiri & Pakistan & Lodhi \& Naeem 1955 \\
\hline
\end{tabular}


Table 2 Continued.

\begin{tabular}{|c|c|c|c|c|c|}
\hline Species & Taxonomy & Substrate & Host & Country & $\begin{array}{l}\text { References } \\
\end{array}$ \\
\hline & & seeds & $\begin{array}{l}\text { Pseudotsuga } \\
\text { menziesii }\end{array}$ & Canada & $\begin{array}{l}\text { Gordon 1967, Mittal et } \\
\text { al. } 1990\end{array}$ \\
\hline & & seeds & Verbena sp. & Pakistan & Lodhi \& Naeem 1955 \\
\hline \multirow[t]{23}{*}{$\begin{array}{l}\text { Chaetomium globosum } \\
\text { Kunze }\end{array}$} & $\begin{array}{l}\text { Chaetomiaceae, Sordariomycetes, } \\
\text { Ascomycota }\end{array}$ & pods & Delonix regia & Thailand & Somrithipol et al. 2002b \\
\hline & & pods & Eucalyptus globulus & Uruguay & Lupo et al. 2001 \\
\hline & & seeds & Abies alba & Poland & Krol et al. 2015 \\
\hline & & seed & Abies balsamea & Canada & Conners 1967 \\
\hline & & seeds & Acer saccharinum & USA & $\begin{array}{l}\text { Friedrich et al. } 1971 \text {, } \\
\text { Mittal et al. } 1990\end{array}$ \\
\hline & & seed & Agrostis gigantea & Canada & Conners 1967 \\
\hline & & seeds & Alnus glutinosa & Poland & Krol et al. 2015 \\
\hline & & seeds & $\begin{array}{l}\text { Betula } \\
\text { alleghaniensis }\end{array}$ & USA & $\begin{array}{l}\text { Friedrich et al. } 1971 \text {, } \\
\text { Mittal et al. } 1990\end{array}$ \\
\hline & & seeds & Betula pendula & Poland & Krol et al. 2015 \\
\hline & & seeds & Betula verrucosa & USSR & $\begin{array}{l}\text { Kozlowska 1968, Mittal } \\
\text { et al. } 1990\end{array}$ \\
\hline & & seeds & Cedrus deodara & India & Munjal \& Sharma 1975 \\
\hline & & seed & Cercis chinensis & Japan & Watanabe 2010 \\
\hline & & seed & Desmodium intortum & Australia & Nik \& Parbery 1977 \\
\hline & & seeds & Eucalyptus sp. & India & $\begin{array}{l}\text { Saxena 1985, Mittal et } \\
\text { al. } 1990\end{array}$ \\
\hline & & seed & Festuca elatior & Canada & Conners 1967 \\
\hline & & seed & Festuca rubra & Canada & Conners 1967 \\
\hline & & seeds & Fraxinus americana & USA & $\begin{array}{l}\text { Friedrich et al. } 1971 \text {, } \\
\text { Mittal et al. } 1990\end{array}$ \\
\hline & & seeds & Larix occidentalis & USA & James et al. 1996 \\
\hline & & seed & Linum usitatissimum & Canada & Conners 1967 \\
\hline & & seed & Lolium perenne & Canada & Conners 1967 \\
\hline & & seed & $\begin{array}{l}\text { Macroptilium } \\
\text { atropurpureum }\end{array}$ & Australia & Nik \& Parbery 1977 \\
\hline & & seed & Phleum pratense & Canada & Conners 1967 \\
\hline & & seeds & Picea excelsa & USSR & $\begin{array}{l}\text { Urosevic 1961, } \\
\text { Anderson 1986a }\end{array}$ \\
\hline
\end{tabular}


Table 2 Continued.

\begin{tabular}{|c|c|c|c|c|c|}
\hline Species & Taxonomy & Substrate & Host & Country & References \\
\hline & & seed & Pinus densiflora & Japan & Watanabe 2010 \\
\hline & & seeds & $\begin{array}{l}\text { Pinus elliottii var. } \\
\text { elliottii }\end{array}$ & Uruguay & Anderson 1986a \\
\hline & & seeds & Pinus pinaster & Uruguay & Anderson 1986a \\
\hline & & seeds & Pinus roxburghii & India & $\begin{array}{l}\text { Munjal \& Sharma 1975, } \\
\text { Mittal et al. } 1990\end{array}$ \\
\hline & & seeds & Pinus sylvestris & Poland, USSR & $\begin{array}{l}\text { Urosevic 1961, } \\
\text { Anderson 1986a, Krol et } \\
\text { al. } 2015\end{array}$ \\
\hline & & seed & Pinus thunbergii & Japan & Watanabe 2010 \\
\hline & & seeds & Pinus wallichiana & India & $\begin{array}{l}\text { Munjal \& Sharma 1975, } \\
\text { Mittal et al. } 1990\end{array}$ \\
\hline & & seed & Poa compressa & Canada & Conners 1967 \\
\hline & & seeds & Quercus pedunculata & USSR & $\begin{array}{l}\text { Kozlowska 1968, Mittal } \\
\text { et al. } 1990\end{array}$ \\
\hline & & seed & Stylosanthes gracilis & Australia & Nik \& Parbery 1977 \\
\hline & & seed & $\begin{array}{l}\text { Stylosanthes } \\
\text { guianensis }\end{array}$ & Australia & Nik \& Parbery 1977 \\
\hline & & seed & Stylosanthes humilis & Australia & Nik \& Parbery 1977 \\
\hline & & seed & Teramnus uncinatus & Australia & Nik \& Parbery 1977 \\
\hline & & seed & Trifolium repens & Australia & Nik \& Parbery 1977 \\
\hline Chaetomium murorum & Chaetomiaceae, Sordariomycetes, & seed & Linum usitatissimum & Canada & Conners 1967 \\
\hline Corda & Ascomycota & seeds & Pinus wallichiana & India & $\begin{array}{l}\text { Mittal \& Sharma 1982b, } \\
\text { Mittal et al. } 1990\end{array}$ \\
\hline $\begin{array}{l}\text { Chaetomium oblatum } \\
\text { Dreyfuss \& Arx }{ }^{\#}\end{array}$ & $\begin{array}{l}\text { Chaetomiaceae, Sordariomycetes, } \\
\text { Ascomycota }\end{array}$ & seed & undetermined plant & Israel & von Arx et al. 1986 \\
\hline $\begin{array}{l}\text { Chaetomium spinulosum } \\
\text { Sörgel }\end{array}$ & $\begin{array}{l}\text { Chaetomiaceae, Sordariomycetes, } \\
\text { Ascomycota }\end{array}$ & seed & $\begin{array}{l}\text { Stylosanthes } \\
\text { guianensis }\end{array}$ & Australia & Nik \& Parbery 1977 \\
\hline \multirow[t]{2}{*}{ Chaetomium spirale Zopf } & $\begin{array}{l}\text { Chaetomiaceae, Sordariomycetes, } \\
\text { Ascomycota }\end{array}$ & seeds & Pinus roxburghii & India & $\begin{array}{l}\text { Munjal \& Sharma 1975, } \\
\text { Mittal et al. } 1990\end{array}$ \\
\hline & & seeds & Pinus sylvestris & Poland & $\begin{array}{l}\text { Garbowski 1936, Mittal } \\
\text { et al. } 1990\end{array}$ \\
\hline Chaetomium spp. & $\begin{array}{l}\text { Chaetomiaceae, Sordariomycetes, } \\
\text { Ascomycota }\end{array}$ & fruits & Avicennia marina & India & Mehdi \& Saifullah 2000 \\
\hline
\end{tabular}


Table 2 Continued.

\begin{tabular}{|c|c|c|c|c|c|}
\hline Species & Taxonomy & Substrate & Host & Country & References \\
\hline & & seeds & $\begin{array}{l}\text { Acacia } \\
\text { auriculiformis }\end{array}$ & Philippines & $\begin{array}{l}\text { Quiniones 1985, 1987, } \\
\text { Anderson 1986a }\end{array}$ \\
\hline & & seeds & Albizia falcataria & Philippines & $\begin{array}{l}\text { Agmata 1979, Mittal et } \\
\text { al. } 1990\end{array}$ \\
\hline & & seeds & Albizia lebbeck & India & $\begin{array}{l}\text { Natarajan et al. 2003, } \\
\text { Mohanan et al. } 2005\end{array}$ \\
\hline & & seeds & $\begin{array}{l}\text { Araucaria } \\
\text { cunninghamii }\end{array}$ & Australia & $\begin{array}{l}\text { Kamara et al. } 1981 \text {, } \\
\text { Mittal et al. } 1990\end{array}$ \\
\hline & & seeds & Bombax ceiba & India & Mittal et al. 1990 \\
\hline & & seeds & Cassia siamea & $\begin{array}{l}\text { Philippines, } \\
\text { Thailand }\end{array}$ & $\begin{array}{l}\text { Quiniones 1985, Mittal } \\
\text { et al. } 1990\end{array}$ \\
\hline & & seeds & $\begin{array}{l}\text { Casuarina } \\
\text { equisetifolia }\end{array}$ & Philippines & $\begin{array}{l}\text { Quiniones 1985, 1987, } \\
\text { Anderson 1986a }\end{array}$ \\
\hline & & seeds & Cedrus deodara & India & Mittal 1983 \\
\hline & & seeds & $\begin{array}{l}\text { Dalbergia } \\
\text { cochinchinensis }\end{array}$ & Thailand & $\begin{array}{l}\text { Chalermpongse et al. } \\
\text { 1984, Mittal et al. } 1990\end{array}$ \\
\hline & & seeds & Dalbergia sissoides & India & Mohanan et al. 2005 \\
\hline & & seeds & Delonix regia & Philippines & $\begin{array}{l}\text { Quiniones 1985, } \\
\text { Anderson 1986a }\end{array}$ \\
\hline & & seeds & Delonix regia & Philippines & Quiniones 1987 \\
\hline & & seeds & $\begin{array}{l}\text { Eucalyptus } \\
\text { citriodora }\end{array}$ & India & Anderson 1986a \\
\hline & & seeds & Eucalyptus globulus & India & Anderson 1986a \\
\hline & & seeds & $\begin{array}{l}\text { Holarrhena } \\
\text { antidysenterica }\end{array}$ & Thailand & $\begin{array}{l}\text { Chalermpongse et al. } \\
\text { 1984, Mittal et al. } 1990\end{array}$ \\
\hline & & seeds & Parkia roxburghii & Philippines & $\begin{array}{l}\text { Dayan 1986, Anderson } \\
\text { 1986a }\end{array}$ \\
\hline & & seeds & Parkia roxburghii & Philippines & Quiniones 1987 \\
\hline & & seeds & Picea jezoensis & Japan & Cheng \& Igarashi 1987 \\
\hline & & seeds & Picea sitchensis & UK & $\begin{array}{l}\text { Salt 1964, Mittal et al. } \\
1990\end{array}$ \\
\hline & & seeds & $\begin{array}{l}\text { Piliostigma } \\
\text { malabaricum }\end{array}$ & Philippines & $\begin{array}{l}\text { Quiniones 1985, } \\
\text { Anderson 1986a }\end{array}$ \\
\hline
\end{tabular}


Table 2 Continued.

\begin{tabular}{|c|c|c|c|c|c|}
\hline Species & Taxonomy & Substrate & Host & Country & References \\
\hline & & seeds & $\begin{array}{l}\text { Piliostigma } \\
\text { malabaricum }\end{array}$ & Philippines & Quiniones 1987 \\
\hline & & seeds & Pinus caribaea & Central America & $\begin{array}{l}\text { Mathur 1974, Anderson } \\
\text { 1986a }\end{array}$ \\
\hline & & seeds & Pinus contorta & USA & $\begin{array}{l}\text { Sutherland 1979, Mittal } \\
\text { et al. } 1990\end{array}$ \\
\hline & & seeds & Pinus elliottii & Taiwan, USA & $\begin{array}{l}\text { Rowan \& Debarr 1974, } \\
\text { Mittal et al. } 1990\end{array}$ \\
\hline & & seeds & $\begin{array}{l}\text { Pinus elliottii var. } \\
\text { elliottii }\end{array}$ & USA & Fraedrich \& Miller 1995 \\
\hline & & seeds & Pinus kesiya & Thailand & $\begin{array}{l}\text { Chalermpongse et al. } \\
\text { 1984, Mittal et al. } 1990\end{array}$ \\
\hline & & seeds & Pinus massoniana & Taiwan & $\begin{array}{l}\text { Jong \& Chen } 1966 \text {, } \\
\text { Mittal et al. } 1990\end{array}$ \\
\hline & & seeds & Pinus oocarpa & Central America & $\begin{array}{l}\text { Mathur 1974, Anderson } \\
\text { 1986a }\end{array}$ \\
\hline & & seeds & Pinus sylvestris & Poland & $\begin{array}{l}\text { Garbowski 1936, Mittal } \\
\text { et al. } 1990\end{array}$ \\
\hline & & seeds & Pinus taeda & Georgia, USA & $\begin{array}{l}\text { Anderson 1986a, b, } \\
\text { Huang \& Kuhlman } 1990\end{array}$ \\
\hline & & seeds & Polyscias nodosa & Philippines & $\begin{array}{l}\text { Agmata 1979, Mittal et } \\
\text { al. } 1990\end{array}$ \\
\hline & & seeds & $\begin{array}{l}\text { Pseudotsuga } \\
\text { menziesii }\end{array}$ & Canada & $\begin{array}{l}\text { Salisbury 1955, Mittal et } \\
\text { al. } 1990\end{array}$ \\
\hline & & seeds & Pterocarpus indicus & Philippines & $\begin{array}{l}\text { Agmata 1979, Anderson } \\
\text { 1986a }\end{array}$ \\
\hline & & seeds & Samanea saman & Thailand & $\begin{array}{l}\text { Chalermpongse et al. } \\
\text { 1984, Mittal et al. } 1990\end{array}$ \\
\hline & & seeds & Serialbizia acle & Philippines & $\begin{array}{l}\text { Quiniones 1985, } 1987 \\
\text { Anderson 1986a }\end{array}$ \\
\hline & & seeds & Shorea robusta & India & $\begin{array}{l}\text { Mittal \& Sharma 1982b, } \\
\text { Mittal et al. } 1990\end{array}$ \\
\hline & & seeds & $\begin{array}{l}\text { Swietenia } \\
\text { macrophylla }\end{array}$ & Philippines & $\begin{array}{l}\text { Agmata 1979, Anderson } \\
\text { 1986a }\end{array}$ \\
\hline
\end{tabular}


Table 2 Continued.

\begin{tabular}{|c|c|c|c|c|c|}
\hline Species & Taxonomy & Substrate & Host & Country & References \\
\hline & & seeds & Tectona grandis & India & $\begin{array}{l}\text { Anderson 1986a, } \\
\text { Mohanan et al. } 2005\end{array}$ \\
\hline $\begin{array}{l}\text { Chaetomium trilaterale } \\
\text { Chivers }\end{array}$ & $\begin{array}{l}\text { Chaetomiaceae, Sordariomycetes, } \\
\text { Ascomycota }\end{array}$ & seed & $\begin{array}{l}\text { Stylosanthes } \\
\text { guianensis }\end{array}$ & Australia & Nik \& Parbery 1977 \\
\hline \multirow[t]{2}{*}{ Chaetophoma sp. } & $\begin{array}{l}\text { Incertae sedis, Dothideomycetes, } \\
\text { Ascomycota }\end{array}$ & seeds & Acer saccharum & USA & $\begin{array}{l}\text { Friedrich et al. } 1971 \text {, } \\
\text { Mittal et al. } 1990\end{array}$ \\
\hline & & seeds & Pinus taeda & USA & Covington et al. 1982 \\
\hline Chaetopsina fulva Rambelli & $\begin{array}{l}\text { Nectriaceae, Sordariomycetes, } \\
\text { Ascomycota }\end{array}$ & pods & Delonix regia & Thailand & Somrithipol et al. 2002b \\
\hline $\begin{array}{l}\text { Chaetopsina indica } \\
\text { Nagaraju, Kunwar, Sureshk. } \\
\text { \& Manohar.\# }\end{array}$ & $\begin{array}{l}\text { Nectriaceae, Sordariomycetes, } \\
\text { Ascomycota }\end{array}$ & fruit & Albizia lebbeck & India & Nagaraju et al. 2011b \\
\hline $\begin{array}{l}\text { Chaetosphaeria } \\
\text { garethjonesii R.H. Perera, } \\
\text { Maharachch. \& K.D. Hyde }\end{array}$ & $\begin{array}{l}\text { Chaetosphaeriaceae, Sordariomycetes, } \\
\text { Ascomycota }\end{array}$ & pod & Fabaceae plant & Thailand & Perera et al. 2016b \\
\hline $\begin{array}{l}\text { Chaetostylum fresenii Tiegh. } \\
\text { \& G. Le Monn. }\end{array}$ & $\begin{array}{l}\text { Mucoraceae, Mucoromycetes, } \\
\text { Mucoromycota }\end{array}$ & seeds & Picea abies & USSR & $\begin{array}{l}\text { Prisyazhnyuk } 1960 \text {, } \\
\text { Mittal et al. } 1990\end{array}$ \\
\hline Chalastospora gossypii & Pleosporaceae, Dothideomycetes, & seed & Agropyron cristatum & Canada & Conners 1967 \\
\hline (Jacz.) U. Braun \& Crous & Ascomycota & seed & Bromus inermis & Canada & Conners 1967 \\
\hline [as Cladosporium malorum & & seed & Linum usitatissimum & Canada & Conners 1967 \\
\hline Heald] & & seed & Medicago sativa & Canada & Conners 1967 \\
\hline Cheilaria sp. & $\begin{array}{l}\text { Incertae sedis, Incertae sedis, } \\
\text { Ascomycota }\end{array}$ & fruits & Litsea rotundifolia & Hong Kong & Tang et al. 2003a \\
\hline Chlamydomyces sp. & $\begin{array}{l}\text { Incertae sedis, Incertae sedis, } \\
\text { Ascomycota }\end{array}$ & seeds & Pinus taeda & USA & Anderson 1986a, b \\
\hline $\begin{array}{l}\text { Chloridium chloroconium } \\
\text { (W. Gams \& Hol.-Jech.) } \\
\text { Réblová \& Seifert } \\
\text { [as Gonytrichum } \\
\text { chlamydosporium G.L. } \\
\text { Barron \& G.C. Bhatt] }\end{array}$ & $\begin{array}{l}\text { Chaetosphaeriaceae, Sordariomycetes, } \\
\text { Ascomycota }\end{array}$ & fruits & Alocasia odora & Hong Kong & Tang et al. 2003a \\
\hline Chloridium sp. & $\begin{array}{l}\text { Chaetosphaeriaceae, Sordariomycetes, } \\
\text { Ascomycota }\end{array}$ & $\begin{array}{l}\text { pods } \\
\text { cones }\end{array}$ & $\begin{array}{l}\text { Delonix regia } \\
\text { Pinus densiflora }\end{array}$ & $\begin{array}{l}\text { Thailand } \\
\text { Japan }\end{array}$ & $\begin{array}{l}\text { Somrithipol et al. 2002b } \\
\text { Kasai et al. } 1995\end{array}$ \\
\hline
\end{tabular}


Table 2 Continued.

\begin{tabular}{|c|c|c|c|c|c|}
\hline Species & Taxonomy & Substrate & Host & Country & References \\
\hline Chrysomyxa ledi (Alb. \& & Coleosporiaceae, Pucciniomycetes, & cones & Picea abies & Finland & Kaitera et al. 2014 \\
\hline \multirow[t]{2}{*}{ Schwein.) de Bary } & Basidiomycota & cones & Picea glauca & Finland & Kaitera et al. 2014 \\
\hline & & cones & Picea omorika & Finland & Kaitera et al. 2014 \\
\hline Chrysomyxa monesis Ziller & $\begin{array}{l}\text { Coleosporiaceae, Pucciniomycetes, } \\
\text { Basidiomycota }\end{array}$ & cones & Picea sitchensis & Canada & Wood 1986 \\
\hline \multirow[t]{5}{*}{$\begin{array}{l}\text { Chrysomyxa pyrolata } \\
\text { (Körn.) G. Winter }\end{array}$} & $\begin{array}{l}\text { Coleosporiaceae, Pucciniomycetes, } \\
\text { Basidiomycota }\end{array}$ & cones & Picea abies & Finland & $\begin{array}{l}\text { Kaitera 2013, Kaitera et } \\
\text { al. 2009, } 2014\end{array}$ \\
\hline & & cones & Picea glauca & Canada & Sutherland et al. 1984 \\
\hline & & cones & Picea pungens & USA & $\begin{array}{l}\text { Nelson \& Krebill 1970, } \\
1982\end{array}$ \\
\hline & & cones & Picea spp. & Canada & Wood 1986 \\
\hline & & seeds & Picea abies & Finland, USSR & $\begin{array}{l}\text { Prisyazhnyuk 1960, } \\
\text { Mittal et al. 1990, } \\
\text { Tillman-Sutela et al. } \\
2004\end{array}$ \\
\hline $\begin{array}{l}\text { Chrysomyxa woroninii } \\
\text { Tranzschel }\end{array}$ & $\begin{array}{l}\text { Coleosporiaceae, Pucciniomycetes, } \\
\text { Basidiomycota }\end{array}$ & seeds & Picea abies & USSR & $\begin{array}{l}\text { Prisyazhnyuk } 1960 \text {, } \\
\text { Mittal et al. } 1990\end{array}$ \\
\hline Chrysosporium sp. & $\begin{array}{l}\text { Onygenaceae, Eurotiomycetes, } \\
\text { Ascomycota }\end{array}$ & acorns & Quercus robur & Poland & Jankowiak 2008 \\
\hline \multirow[t]{7}{*}{$\begin{array}{l}\text { Chytridium xylophilum } \\
\text { Cornu }\end{array}$} & $\begin{array}{l}\text { Chytridiaceae, Chytridiomycetes, } \\
\text { Chytridiomycota }\end{array}$ & seeds & $\begin{array}{l}\text { Armeria } \\
\text { transmontana }\end{array}$ & $\begin{array}{l}\text { Poland or/ } \\
\text { imported to Poland }\end{array}$ & Czeczuga et al. 2009 \\
\hline & & seeds & Atropa komarovii & $\begin{array}{l}\text { Poland or/ } \\
\text { imported to Poland }\end{array}$ & Czeczuga et al. 2009 \\
\hline & & seeds & Bryonia cretica & $\begin{array}{l}\text { Poland or/ } \\
\text { imported to Poland }\end{array}$ & Czeczuga et al. 2009 \\
\hline & & seeds & Cornus capitata & $\begin{array}{l}\text { Poland or/ } \\
\text { imported to Poland }\end{array}$ & Czeczuga et al. 2009 \\
\hline & & seeds & Datura stramonium & $\begin{array}{l}\text { Poland or/ } \\
\text { imported to Poland }\end{array}$ & Czeczuga et al. 2009 \\
\hline & & seeds & Hyoscyamus aureus & $\begin{array}{l}\text { Poland or/ } \\
\text { imported to Poland }\end{array}$ & Czeczuga et al. 2009 \\
\hline & & seeds & $\begin{array}{l}\text { Polygonum } \\
\text { amphibium }\end{array}$ & $\begin{array}{l}\text { Poland or/ } \\
\text { imported to Poland }\end{array}$ & Czeczuga et al. 2009 \\
\hline
\end{tabular}


Table 2 Continued.

\begin{tabular}{|c|c|c|c|c|c|}
\hline Species & Taxonomy & Substrate & Host & Country & References \\
\hline \multirow[t]{2}{*}{$\begin{array}{l}\text { Chytriomyces aureus } \\
\text { Karling }\end{array}$} & \multirow[t]{2}{*}{$\begin{array}{l}\text { Chytriomycetaceae, Chytridiomycetes, } \\
\text { Chytridiomycota }\end{array}$} & seeds & Atropa komarovii & $\begin{array}{l}\text { Poland or/ } \\
\text { imported to Poland }\end{array}$ & Czeczuga et al. 2009 \\
\hline & & seeds & $\begin{array}{l}\text { Hyoscyamus } \\
\text { gyoerffyi }\end{array}$ & $\begin{array}{l}\text { Poland or/ } \\
\text { imported to Poland }\end{array}$ & Czeczuga et al. 2009 \\
\hline Ciboria alni (Maul) Whetzel & $\begin{array}{l}\text { Sclerotiniaceae, Leotiomycetes, } \\
\text { Ascomycota }\end{array}$ & $\begin{array}{l}\text { seed } \\
\text { seeds }\end{array}$ & $\begin{array}{l}\text { Alnus rugosa } \\
\text { Alnus sp. }\end{array}$ & $\begin{array}{l}\text { Canada } \\
\text { former } \\
\text { Czechoslovakia }\end{array}$ & $\begin{array}{l}\text { Conners } 1967 \\
\text { Anderson } 1986 a\end{array}$ \\
\hline \multirow{5}{*}{$\begin{array}{l}\text { Ciboria batschiana (Zopf) } \\
\text { N.F. Buchw. }\end{array}$} & \multirow{5}{*}{$\begin{array}{l}\text { Sclerotiniaceae, Leotiomycetes, } \\
\text { Ascomycota }\end{array}$} & acorns & Quercus robur & Poland & Jankowiak 2008 \\
\hline & & seeds & Quercus petraea & France & $\begin{array}{l}\text { Delatour \& Morelet } \\
\text { 1979, Mittal et al. } 1990\end{array}$ \\
\hline & & seeds & Quercus robur & France , Germany & $\begin{array}{l}\text { Delatour \& Morelet } \\
\text { 1979, Mittal et al. } 1990 \text {, } \\
\text { Schroder et al. } 2004\end{array}$ \\
\hline & & seeds & Quercus rubra & France & Anderson 1986a \\
\hline & & seeds & Quercus spp. & France & Anderson 1986a \\
\hline $\begin{array}{l}\text { Ciboria betulae (Woronin) } \\
\text { W.L. White }\end{array}$ & $\begin{array}{l}\text { Sclerotiniaceae, Leotiomycetes, } \\
\text { Ascomycota }\end{array}$ & seeds & Betula spp. & $\begin{array}{l}\text { Asia, Europe, } \\
\text { North America, } \\
\text { former } \\
\text { Czechoslovakia }\end{array}$ & $\begin{array}{l}\text { Buchwald 1947, Mittal } \\
\text { et al. } 1990\end{array}$ \\
\hline $\begin{array}{l}\text { Ciboria rufofusca (O. } \\
\text { Weberb.) Sacc. }\end{array}$ & $\begin{array}{l}\text { Sclerotiniaceae, Leotiomycetes, } \\
\text { Ascomycota }\end{array}$ & cone & Picea sp. & Canada & Conners 1967 \\
\hline \multirow{4}{*}{$\begin{array}{l}\text { Cirrenalia nigrospora } \\
\text { Somrith. Chatmala \& E.B.G. } \\
\text { Jones }^{\#}\end{array}$} & \multirow{4}{*}{$\begin{array}{l}\text { Halosphaeriaceae, Sordariomycetes, } \\
\text { Ascomycota }\end{array}$} & pod & Delonix regia & Thailand & Somrithipol et al. 2002a \\
\hline & & pods & Hevea brasiliensis & Thailand & Somrithipol et al. 2002b \\
\hline & & seeds & $\begin{array}{l}\text { Borassus } \\
\text { machadonis }\end{array}$ & Thailand & Somrithipol et al. $2002 b$ \\
\hline & & seeds & $\begin{array}{l}\text { Choerospondias } \\
\text { axillaris }\end{array}$ & Thailand & $\begin{array}{l}\text { Somrithipol et al. } \\
\text { 2002b }\end{array}$ \\
\hline Cirrenalia sp. & $\begin{array}{l}\text { Halosphaeriaceae, Sordariomycetes, } \\
\text { Ascomycota }\end{array}$ & pods & Delonix regia & Thailand & Somrithipol et al. 2002b \\
\hline Cistella sp. & Calloriaceae, Leotiomycetes, Ascomycota & fruit cupules & Fagus sylvatica & UK & Carré 1964 \\
\hline $\begin{array}{l}\text { Cladochytrium crassum } \\
\text { Hillegas }\end{array}$ & $\begin{array}{l}\text { Cladochytriaceae, Chytridiomycetes, } \\
\text { Chytridiomycota }\end{array}$ & seeds & $\begin{array}{l}\text { Polygonum } \\
\text { amphibium }\end{array}$ & $\begin{array}{l}\text { Poland or/ } \\
\text { imported to Poland }\end{array}$ & Czeczuga et al. 2009 \\
\hline
\end{tabular}


Table 2 Continued.

\begin{tabular}{|c|c|c|c|c|c|}
\hline Species & Taxonomy & Substrate & Host & Country & References \\
\hline \multirow[t]{2}{*}{$\begin{array}{l}\text { Cladochytrium hyalinum } \\
\text { Berdan }\end{array}$} & $\begin{array}{l}\text { Cladochytriaceae, Chytridiomycetes, } \\
\text { Chytridiomycota }\end{array}$ & seeds & $\begin{array}{l}\text { Armeria maritima } \\
\text { ssp. maritima }\end{array}$ & $\begin{array}{l}\text { Poland or/ } \\
\text { imported to Poland }\end{array}$ & Czeczuga et al. 2009 \\
\hline & & seeds & Sambucus ebulus & $\begin{array}{l}\text { Poland or/ } \\
\text { imported to Poland }\end{array}$ & Czeczuga et al. 2009 \\
\hline $\begin{array}{l}\text { Cladochytrium taianum S.C. } \\
\text { Shen \& Siang }\end{array}$ & $\begin{array}{l}\text { Cladochytriaceae, Chytridiomycetes, } \\
\text { Chytridiomycota }\end{array}$ & seeds & Ginkgo biloba & $\begin{array}{l}\text { Poland or/ } \\
\text { imported to Poland }\end{array}$ & Czeczuga et al. 2009 \\
\hline \multirow[t]{4}{*}{$\begin{array}{l}\text { Cladochytrium tenue } \\
\text { Nowak. }\end{array}$} & $\begin{array}{l}\text { Cladochytriaceae, Chytridiomycetes, } \\
\text { Chytridiomycota }\end{array}$ & seeds & Cicuta virosa & $\begin{array}{l}\text { Poland or/ } \\
\text { imported to Poland }\end{array}$ & Czeczuga et al. 2009 \\
\hline & & seeds & Ficus pumila & $\begin{array}{l}\text { Poland or/ } \\
\text { imported to Poland }\end{array}$ & Czeczuga et al. 2009 \\
\hline & & seeds & Juniperus oxycedrus & $\begin{array}{l}\text { Poland or/ } \\
\text { imported to Poland }\end{array}$ & Czeczuga et al. 2009 \\
\hline & & seeds & Lonicera caprifolium & $\begin{array}{l}\text { Poland or/ } \\
\text { imported to Poland }\end{array}$ & Czeczuga et al. 2009 \\
\hline $\begin{array}{l}\text { Cladonia lacryma S. } \\
\text { Hammer." }\end{array}$ & $\begin{array}{l}\text { Cladoniaceae, Lecanoromycetes, } \\
\text { Ascomycota }\end{array}$ & cones & $\begin{array}{l}\text { cone producing } \\
\text { evergreen plant }\end{array}$ & USA & Hammer 2001 \\
\hline $\begin{array}{l}\text { Cladophialophora } \\
\text { chaetospira (Grove) Crous \& } \\
\text { Arzanlou [as Septonema } \\
\text { chaetospira (Grove) S. } \\
\text { Hughes] }\end{array}$ & $\begin{array}{l}\text { Herpotrichiellaceae, Eurotiomycetes, } \\
\text { Ascomycota }\end{array}$ & seed & Cercis chinensis & Japan & Watanabe 2010 \\
\hline $\begin{array}{l}\text { Cladosporium aphidis } \\
\text { Thüm. }\end{array}$ & $\begin{array}{l}\text { Cladosporiaceae, Dothideomycetes, } \\
\text { Ascomycota }\end{array}$ & pod & $\begin{array}{l}\text { Laburnum } \\
\text { anagyroides }\end{array}$ & UK & Jayasiri et al. 2019 \\
\hline $\begin{array}{l}\text { Cladosporium aterrimum } \\
\text { Ellis \& Everh. }\end{array}$ & $\begin{array}{l}\text { Cladosporiaceae, Dothideomycetes, } \\
\text { Ascomycota }\end{array}$ & seeds & Picea excelsa & USSR & $\begin{array}{l}\text { Urosevic 1961, } \\
\text { Anderson 1986a }\end{array}$ \\
\hline $\begin{array}{l}\text { Cladosporium } \\
\text { austrohemisphaericum } \\
\text { Bensch, Crous \& U. Braun }{ }^{\#}\end{array}$ & $\begin{array}{l}\text { Cladosporiaceae, Dothideomycetes, } \\
\text { Ascomycota }\end{array}$ & fruit & $\begin{array}{l}\text { Lagunaria } \\
\text { patersonia }\end{array}$ & New Zealand & Bensch et al. 2015 \\
\hline $\begin{array}{l}\text { Cladosporium bauhiniana } \\
\text { Arv.Kumar, Anju Kumar \& } \\
\text { KHarwar }^{\#}\end{array}$ & $\begin{array}{l}\text { Cladosporiaceae, Dothideomycetes, } \\
\text { Ascomycota }\end{array}$ & pods & Bauhinia varirgata & India & Kumar et al. 2006 \\
\hline $\begin{array}{l}\text { Cladosporium } \\
\text { chlorocephalum (Fresen.) } \\
\text { E.W. Mason \& M.B. Ellis }\end{array}$ & $\begin{array}{l}\text { Cladosporiaceae, Dothideomycetes, } \\
\text { Ascomycota }\end{array}$ & seeds & Shorea robusta & India & $\begin{array}{l}\text { Mittal \& Sharma 1982b, } \\
\text { Mittal et al. } 1990\end{array}$ \\
\hline
\end{tabular}


Table 2 Continued.

\begin{tabular}{|c|c|c|c|c|c|}
\hline Species & Taxonomy & Substrate & Host & Country & References \\
\hline \multirow{26}{*}{$\begin{array}{l}\text { Cladosporium } \\
\text { cladosporioides (Fresen.) } \\
\text { G.A. de Vries }\end{array}$} & $\begin{array}{l}\text { Cladosporiaceae, Dothideomycetes, } \\
\text { Ascomycota }\end{array}$ & acorns & Quercus robur & Poland & Jankowiak 2008 \\
\hline & & cones & Picea glauca & Canada & Mittal \& Wang 1987 \\
\hline & & cones & Pinus densiflora & Japan & Kasai et al. 1995 \\
\hline & & cones, seeds & Pinus strobus & Canada & Mittal \& Wang 1987 \\
\hline & & fruits & Ardisia punctata & Hong Kong & Tang et al. 2003a \\
\hline & & fruits & Bridelia tomentosa & Hong Kong & Tang et al. 2003a \\
\hline & & fruits & Diplospora dubia & Hong Kong & Tang et al. 2003a \\
\hline & & fruits & Ilex asprella & Hong Kong & Tang et al. 2003a \\
\hline & & fruits & Ilex cinerea & Hong Kong & Tang et al. 2003a \\
\hline & & fruits & Ilex pubescens & Hong Kong & Tang et al. 2003a \\
\hline & & fruits & Litsea rotundifolia & Hong Kong & Tang et al. 2003a \\
\hline & & fruits & Psychotria asiatica & Hong Kong & Tang et al. 2003a \\
\hline & & fruits & Sarcandra glabra & Hong Kong & Tang et al. 2003a \\
\hline & & fruits & $\begin{array}{l}\text { Viburnum } \\
\text { sempervirens }\end{array}$ & Hong Kong & Tang et al. 2003a \\
\hline & & pod & Canavalia cathartica & India & Anita \& Sridhar 2009 \\
\hline & & pods & Eucalyptus globulus & Uruguay & Lupo et al. 2001 \\
\hline & & seeds & Acacia confusa & Philippines & $\begin{array}{l}\text { Agmata 1979, Anderson } \\
\text { 1986a }\end{array}$ \\
\hline & & seeds & Acer pseudoplatanus & Poland & Krol et al. 2015 \\
\hline & & seed & Agropyron cristatum & Canada & Conners 1967 \\
\hline & & seeds & Alnus glutinosa & Poland & Krol et al. 2015 \\
\hline & & seed & Anethum graveolens & Canada & Conners 1967 \\
\hline & & seed & $\begin{array}{l}\text { Apium graveolens } \\
\text { var. dulce }\end{array}$ & Canada & Conners 1967 \\
\hline & & seed & $\begin{array}{l}\text { Asparagus officinalis } \\
\text { var. altilis }\end{array}$ & Canada & Conners 1967 \\
\hline & & seeds & Betula pendula & Poland & Krol et al. 2015 \\
\hline & & seeds & Cassia siamea & Philippines & $\begin{array}{l}\text { Quiniones 1985, Mittal } \\
\text { et al. } 1990\end{array}$ \\
\hline & & seeds & Cassia siamea & Philippines & Quiniones 1987 \\
\hline
\end{tabular}


Table 2 Continued.

\begin{tabular}{|c|c|c|c|c|c|}
\hline Species & Taxonomy & Substrate & Host & Country & References \\
\hline & & seeds & $\begin{array}{l}\text { Casuarina } \\
\text { equisetifolia }\end{array}$ & Philippines & $\begin{array}{l}\text { Quiniones 1985, 1987, } \\
\text { Anderson 1986a }\end{array}$ \\
\hline & & seeds & Casuarina spp. & India & Sahai \& Otra 1982 \\
\hline & & seeds & Cedrus deodara & India & Mittal 1983 \\
\hline & & seeds & $\begin{array}{l}\text { Cupressus } \\
\text { sempervirens }\end{array}$ & Egypt & $\begin{array}{l}\text { Farag et al. 1977, Mittal } \\
\text { et al. } 1990\end{array}$ \\
\hline & & seeds & Cupressus spp. & India & Sahai \& Otra 1982 \\
\hline & & seeds & Delonix regia & Philippines & $\begin{array}{l}\text { Quiniones 1985, 1987, } \\
\text { Anderson 1986a }\end{array}$ \\
\hline & & seed & Elymus virginicus & Canada & Conners 1967 \\
\hline & & seeds & Eucalyptus hybrid & India & $\begin{array}{l}\text { Mittal 1986, Mittal et al. } \\
1990\end{array}$ \\
\hline & & seeds & Eucalyptus sp. & India & $\begin{array}{l}\text { Saxena 1985, Mittal et } \\
\text { al. } 1990\end{array}$ \\
\hline & & seeds & Fagus sylvatica & Poland & Krol et al. 2015 \\
\hline & & seed & Festuca elatior & Canada & Conners 1967 \\
\hline & & seeds & $\begin{array}{l}\text { Leucaena } \\
\text { leucocephala }\end{array}$ & Philippines & $\begin{array}{l}\text { Quiniones 1985, 1987, } \\
\text { Mittal et al. } 1990\end{array}$ \\
\hline & & seed & Linum usitatissimum & Canada & Conners 1967 \\
\hline & & seed & Medicago sativa & Canada & Conners 1967 \\
\hline & & seeds & Melia spp. & India & Sahai \& Otra 1982 \\
\hline & & seeds & $\begin{array}{l}\text { Musanga } \\
\text { cecropioides }\end{array}$ & Philippines & $\begin{array}{l}\text { Agmata 1979, Anderson } \\
\text { 1986a }\end{array}$ \\
\hline & & seeds & Picea glauca & Canada & Mittal \& Wang 1987 \\
\hline & & seeds & Pinus caribaea & $\begin{array}{l}\text { Belize [as British } \\
\text { Honduras] }\end{array}$ & Hocking 1968 \\
\hline & & seed & Pinus densiflora & Japan & Watanabe 2010 \\
\hline & & seeds & Pinus insularis & Philippines & $\begin{array}{l}\text { Agmata 1979, Anderson } \\
\text { 1986a }\end{array}$ \\
\hline & & seeds & Pinus roxburghii & India & $\begin{array}{l}\text { Mittal \& Sharma 1982c, } \\
\text { Mittal et al. } 1990\end{array}$ \\
\hline & & seeds & Pinus sylvestris & Poland & Krol et al. 2015 \\
\hline & & seed & Pinus thunbergii & Japan & Watanabe 2010 \\
\hline
\end{tabular}


Table 2 Continued.

\begin{tabular}{|c|c|c|c|c|c|}
\hline Species & Taxonomy & Substrate & Host & Country & References \\
\hline & & seeds & $\begin{array}{l}\text { Pittosporum } \\
\text { resiniferum }\end{array}$ & Philippines & $\begin{array}{l}\text { Quiniones 1985, } \\
\text { Anderson 1986a }\end{array}$ \\
\hline & & seeds & $\begin{array}{l}\text { Platanus } \\
\text { occidentalis }\end{array}$ & USA & $\begin{array}{l}\text { Fakir et al. 1971, Mittal } \\
\text { et al. } 1990\end{array}$ \\
\hline & & seeds & Pterocarpus indicus & Philippines & $\begin{array}{l}\text { Agmata 1979, Anderson } \\
\text { 1986a }\end{array}$ \\
\hline & & seeds & Pyrus spp. & India & Sahai \& Otra 1982 \\
\hline & & seeds & Quercus robur & Poland & Krol et al. 2015 \\
\hline & & seeds & Quercus spp. & India & Sahai \& Otra 1982 \\
\hline & & seed & Salvia officinalis & Canada & Conners 1967 \\
\hline & & seeds & Shorea robusta & India & $\begin{array}{l}\text { Mittal \& Sharma 1982b, } \\
\text { Mittal et al. } 1990\end{array}$ \\
\hline & & seeds & Thuja spp. & India & Sahai \& Otra 1982 \\
\hline & & seeds & Triplaris cumingiana & Philippines & $\begin{array}{l}\text { Quiniones 1985, } \\
\text { Anderson 1986a }\end{array}$ \\
\hline $\begin{array}{l}\text { Cladosporium cucumerinum } \\
\text { Ellis \& Arthur }\end{array}$ & $\begin{array}{l}\text { Cladosporiaceae, Dothideomycetes, } \\
\text { Ascomycota }\end{array}$ & seeds & Pinus ponderosa & USA & $\begin{array}{l}\text { James \& Genz 1982, } \\
\text { Anderson 1986a }\end{array}$ \\
\hline $\begin{array}{l}\text { Cladosporium dominicanum } \\
\text { Zalar, de Hoog \& Gunde- } \\
\text { Cim. }\end{array}$ & $\begin{array}{l}\text { Cladosporiaceae, Dothideomycetes, } \\
\text { Ascomycota }\end{array}$ & pod & Delonix regia & Thailand & Jayasiri et al. 2019 \\
\hline Cladosporium elegans Penz. & $\begin{array}{l}\text { Cladosporiaceae, Dothideomycetes, } \\
\text { Ascomycota }\end{array}$ & seed & Linum usitatissimum & Canada & Conners 1967 \\
\hline $\begin{array}{l}\text { Cladosporium elegantulum } \\
\text { Pidopl. \& Deniak }\end{array}$ & $\begin{array}{l}\text { Cladosporiaceae, Dothideomycetes, } \\
\text { Ascomycota }\end{array}$ & seeds & Quercus spp. & USSR & $\begin{array}{l}\text { Urosevic 1959, Mittal et } \\
\text { al. } 1990\end{array}$ \\
\hline $\begin{array}{l}\text { Cladosporium entadae } \\
\text { Jayasiri, E.B.G. Jones \& } \\
\text { K.D. Hyde }^{\#}\end{array}$ & $\begin{array}{l}\text { Cladosporiaceae, Dothideomycetes, } \\
\text { Ascomycota }\end{array}$ & pod & Entada phaseoloides & Thailand & Jayasiri et al. 2019 \\
\hline \multirow[t]{2}{*}{$\begin{array}{l}\text { Cladosporium epiphyllum } \\
\text { (Pers.) Nees }\end{array}$} & \multirow[t]{2}{*}{$\begin{array}{l}\text { Cladosporiaceae, Dothideomycetes, } \\
\text { Ascomycota }\end{array}$} & seeds & Picea excelsa & USSR & $\begin{array}{l}\text { Urosevic 1961, } \\
\text { Anderson 1986a }\end{array}$ \\
\hline & & seeds & Pinus sylvestris & USSR & $\begin{array}{l}\text { Prisyazhnyuk 1960, } \\
\text { Anderson 1986a }\end{array}$ \\
\hline \multirow{2}{*}{$\begin{array}{l}\text { Cladosporium herbarum } \\
\text { (Pers.) Link }\end{array}$} & \multirow{2}{*}{$\begin{array}{l}\text { Cladosporiaceae, Dothideomycetes, } \\
\text { Ascomycota }\end{array}$} & acorns & Quercus robur & Poland & Jankowiak 2008 \\
\hline & & cones & Picea glauca & Canada & Mittal \& Wang 1987 \\
\hline
\end{tabular}


Table 2 Continued.

\begin{tabular}{|c|c|c|c|c|c|}
\hline Species & Taxonomy & Substrate & Host & Country & References \\
\hline & & cones, seeds & Pinus spp. & Canada & Vujanovic et al. 2000 \\
\hline & & cones, seeds & Pinus strobus & Canada & Mittal \& Wang 1987 \\
\hline & & fruits & Avicennia marina & India & Mehdi \& Saifullah 2000 \\
\hline & & seeds & Abies sibirica & USSR & $\begin{array}{l}\text { Prisyazhnyuk 1960, } \\
\text { Mittal et al. } 1990\end{array}$ \\
\hline & & seed & $\begin{array}{l}\text { Apium graveolens } \\
\text { var. dulce }\end{array}$ & USA & Conners 1967 \\
\hline & & seeds & Betula pendula & Finland & $\begin{array}{l}\text { Lilja 1979, Mittal et al. } \\
1990\end{array}$ \\
\hline & & seed & Bromus inermis & Canada & Conners 1967 \\
\hline & & seeds & Cedrus deodara & India & Mittal 1983 \\
\hline & & seeds & Eucalyptus sp. & India & $\begin{array}{l}\text { Saxena 1985, Mittal et } \\
\text { al. } 1990\end{array}$ \\
\hline & & seed & Festuca elatior & Canada & Conners 1967 \\
\hline & & seeds & Larix decidua & Poland & Krol et al. 2015 \\
\hline & & seeds & Larix sibirica & USSR & $\begin{array}{l}\text { Prisyazhnyuk } 1960 \text {, } \\
\text { Mittal et al. } 1990\end{array}$ \\
\hline & & seed & Linum usitatissimum & Canada & Conners 1967 \\
\hline & & seeds & Picea abies & USSR & $\begin{array}{l}\text { Prisyazhnyuk } 1960 \text {, } \\
\text { Mittal et al. } 1990\end{array}$ \\
\hline & & seeds & Picea excelsa & USSR & $\begin{array}{l}\text { Urosevic 1961, } \\
\text { Anderson 1986a }\end{array}$ \\
\hline & & seeds & Picea glauca & Canada & Mittal \& Wang 1987 \\
\hline & & seeds & Pinus nigra & Hungary & $\begin{array}{l}\text { Hangyal 1973, Mittal et } \\
\text { al. } 1990\end{array}$ \\
\hline & & seeds & Pinus sylvestris & $\begin{array}{l}\text { Hungary, UK, } \\
\text { USSR }\end{array}$ & $\begin{array}{l}\text { Urosevic 1961, Whittle } \\
\text { 1977, Anderson 1986a }\end{array}$ \\
\hline & & seeds & Quercus robur & Poland & Krol et al. 2015 \\
\hline Cladosporium hordei Pass. & $\begin{array}{l}\text { Cladosporiaceae, Dothideomycetes, } \\
\text { Ascomycota }\end{array}$ & seeds & Quercus spp. & USSR & $\begin{array}{l}\text { Urosevic 1959, Mittal et } \\
\text { al. } 1990\end{array}$ \\
\hline $\begin{array}{l}\text { Cladosporium magnoliigena } \\
\text { Jayasiri, E.B.G. Jones \& } \\
\text { K.D. Hyde }\end{array}$ & $\begin{array}{l}\text { Cladosporiaceae, Dothideomycetes, } \\
\text { Ascomycota }\end{array}$ & cone & $\begin{array}{l}\text { Magnolia } \\
\text { grandiflora }\end{array}$ & China & Jayasiri et al. 2019 \\
\hline
\end{tabular}


Table 2 Continued.

\begin{tabular}{|c|c|c|c|c|c|}
\hline Species & Taxonomy & Substrate & Host & Country & References \\
\hline \multirow{3}{*}{$\begin{array}{l}\text { Cladosporium oxysporum } \\
\text { Berk. \& M.A. Curtis }\end{array}$} & Cladosporiaceae, Dothideomycetes, & seeds & Dianthus spp. & Taiwan & Li \& Wu 2002 \\
\hline & Ascomycota & seed & Pinus wallichiana & India & $\begin{array}{l}\text { Mittal \& Sharma 1982b, } \\
\text { Mittal et al. } 1990\end{array}$ \\
\hline & & seeds & Sesbania bispinosa & India & Anita et al. 2009 \\
\hline \multirow[t]{17}{*}{ Cladosporium spp. } & Cladosporiaceae, Dothideomycetes, & pod & Canavalia cathartica & India & Anita \& Sridhar 2009 \\
\hline & Ascomycota & seed capsules & Eucalyptus grandis & South Africa & Jimu et al. 2016 \\
\hline & & seeds & Abies lasiocarpa & Canada, Norway & Talgø et al. 2010 \\
\hline & & seeds & Abies nordmanniana & $\begin{array}{l}\text { Austria, Georgia, } \\
\text { Russia }\end{array}$ & Talgø et al. 2010 \\
\hline & & seeds & Abies procera & Norway & Talgø et al. 2010 \\
\hline & & seeds & $\begin{array}{l}\text { Acacia } \\
\text { auriculiformis }\end{array}$ & Philippines & $\begin{array}{l}\text { Dayan 1986, Mittal et al. } \\
1990\end{array}$ \\
\hline & & seeds & Acer saccharum & USA & $\begin{array}{l}\text { Janerette 1979, Mittal et } \\
\text { al. } 1990\end{array}$ \\
\hline & & seeds & $\begin{array}{l}\text { Araucaria } \\
\text { heterophylla }\end{array}$ & Egypt & $\begin{array}{l}\text { Kamara et al. } 1981 \text {, } \\
\text { Mittal et al. } 1990\end{array}$ \\
\hline & & seeds & Bauhinia sp. & Thailand & $\begin{array}{l}\text { Chalermpongse et al. } \\
\text { 1984, Mittal et al. } 1990\end{array}$ \\
\hline & & seeds & $\begin{array}{l}\text { Callistephus } \\
\text { chinensis }\end{array}$ & China & $\begin{array}{l}\text { Gloyer } 1931 \text {, Crosier \& } \\
\text { Heit } 1948\end{array}$ \\
\hline & & seed & Canavalia cathartica & India & Anita \& Sridhar 2009 \\
\hline & & seeds & Cassia bakeriana & Thailand & $\begin{array}{l}\text { Chalermpongse et al. } \\
\text { 1984, Mittal et al. } 1990\end{array}$ \\
\hline & & seeds & Cassia siamea & Philippines & Quiniones 1987 \\
\hline & & seeds & $\begin{array}{l}\text { Casuarina } \\
\text { equisetifolia }\end{array}$ & $\begin{array}{l}\text { Philippines, } \\
\text { Thailand }\end{array}$ & $\begin{array}{l}\text { Chalermpongse et al. } \\
\text { 1984, Mittal et al. } 1990\end{array}$ \\
\hline & & seeds & Delonix regia & Philippines & $\begin{array}{l}\text { Dayan 1986, Mittal et al. } \\
1990\end{array}$ \\
\hline & & seeds & $\begin{array}{l}\text { Dianthus } \\
\text { caryophyllus }\end{array}$ & Taiwan & Li \& Wu 2002 \\
\hline & & seeds & $\begin{array}{l}\text { Endospermum } \\
\text { peltatum }\end{array}$ & Philippines & $\begin{array}{l}\text { Agmata 1979, Anderson } \\
\text { 1986a }\end{array}$ \\
\hline
\end{tabular}


Table 2 Continued.

\begin{tabular}{|c|c|c|c|c|c|}
\hline Species & Taxonomy & Substrate & Host & Country & References \\
\hline & & seeds & $\begin{array}{l}\text { Eucalyptus } \\
\text { citriodora }\end{array}$ & India & $\begin{array}{l}\text { Mittal \& Sharma 1984, } \\
\text { Mittal et al. } 1990\end{array}$ \\
\hline & & seeds & Eucalyptus grandis & South Africa & Jimu et al. 2016 \\
\hline & & seeds & Fraxinus excelsior & Sweden & $\begin{array}{l}\text { Cleary et al. 2013, } \\
\text { Hayatgheibi } 2013\end{array}$ \\
\hline & & seeds & $\begin{array}{l}\text { Lagerstroemia } \\
\text { speciosa }\end{array}$ & Philippines & $\begin{array}{l}\text { Quiniones 1985, } \\
\text { Anderson 1986a }\end{array}$ \\
\hline & & seeds & Larix occidentalis & USA & James et al. 1996 \\
\hline & & seeds & $\begin{array}{l}\text { Leucaena } \\
\text { leucocephala }\end{array}$ & Philippines & $\begin{array}{l}\text { Agmata 1979, Anderson } \\
\text { 1986a }\end{array}$ \\
\hline & & seeds & Phyllostachys edulis & China & Shen et al. 2014 \\
\hline & & seeds & Picea engelmannii & USA & $\begin{array}{l}\text { Fuller \& Hildebrand } \\
\text { 1985, Mittal et al. } 1990\end{array}$ \\
\hline & & seeds & Picea jezoensis & Japan & Cheng \& Igarashi 1987 \\
\hline & & seeds & Pinus contorta & USA & $\begin{array}{l}\text { Fuller \& Hildebrand } \\
\text { 1985, Mittal et al. } 1990\end{array}$ \\
\hline & & seeds & Pinus kesiya & Thailand & $\begin{array}{l}\text { Chalermpongse et al. } \\
\text { 1984, Mittal et al. } 1990\end{array}$ \\
\hline & & seed & Pinus monticola & USA & $\begin{array}{l}\text { Ganley \& Newcombe } \\
2006\end{array}$ \\
\hline & & seeds & Pinus patula & Kenya & $\begin{array}{l}\text { Gibson 1957, Mittal et } \\
\text { al. } 1990\end{array}$ \\
\hline & & seeds & Pinus ponderosa & USA & $\begin{array}{l}\text { Fuller \& Hildebrand } \\
\text { 1985, Mittal et al. } 1990\end{array}$ \\
\hline & & seeds & Pinus sylvestris & USSR & $\begin{array}{l}\text { Dolejs 1964, Mittal et al. } \\
1990\end{array}$ \\
\hline & & Seeds & Pinus taeda & USA & Anderson 1986a, b \\
\hline & & seeds & Polyscias nodosa & Philippines & $\begin{array}{l}\text { Agmata 1979, Anderson } \\
\text { 1986a }\end{array}$ \\
\hline & & seed & Prunus serrulata & Japan & Watanabe 2010 \\
\hline & & seeds & Quercus robur & Germany & Schroder et al. 2004 \\
\hline & & seeds & $\begin{array}{l}\text { Swietenia } \\
\text { macrophylla }\end{array}$ & Philippines & $\begin{array}{l}\text { Agmata 1979, Anderson } \\
\text { 1986a }\end{array}$ \\
\hline
\end{tabular}


Table 2 Continued.

\begin{tabular}{|c|c|c|c|c|c|}
\hline Species & Taxonomy & Substrate & Host & Country & References \\
\hline \multirow[t]{10}{*}{$\begin{array}{l}\text { Cladosporium } \\
\text { sphaerospermum Penz. }\end{array}$} & $\begin{array}{l}\text { Cladosporiaceae, Dothideomycetes, } \\
\text { Ascomycota }\end{array}$ & acorns & Quercus robur & Poland & Jankowiak 2008 \\
\hline & & pods & Delonix regia & Thailand & Somrithipol et al. 2002b \\
\hline & & seeds & $\begin{array}{l}\text { Callistephus } \\
\text { chinensis }\end{array}$ & $\begin{array}{l}\text { imported to } \\
\text { Taiwan }\end{array}$ & Wu et al. 2006 \\
\hline & & seeds & Casuarina spp. & India & Sahai \& Otra 1982 \\
\hline & & seeds & Cosmos sulphureus & $\begin{array}{l}\text { imported to } \\
\text { Taiwan }\end{array}$ & Wu et al. 2006 \\
\hline & & seeds & Picea excelsa & USSR & $\begin{array}{l}\text { Urosevic 1961, } \\
\text { Anderson 1986a }\end{array}$ \\
\hline & & seeds & Pinus sylvestris & USSR & $\begin{array}{l}\text { Urosevic 1961, } \\
\text { Anderson 1986a }\end{array}$ \\
\hline & & seeds & Pyrus spp. & India & Sahai \& Otra 1982 \\
\hline & & seeds & Quercus spp. & India & Sahai \& Otra 1982 \\
\hline & & seeds & Sapium spp. & India & Sahai \& Otra 1982 \\
\hline $\begin{array}{l}\text { Cladosporium tenuissimum } \\
\text { Cooke }\end{array}$ & $\begin{array}{l}\text { Cladosporiaceae, Dothideomycetes, } \\
\text { Ascomycota }\end{array}$ & seeds & Eucalyptus sp. & India & $\begin{array}{l}\text { Reddy et al. 1982, Mittal } \\
\text { et al. } 1990\end{array}$ \\
\hline $\begin{array}{l}\text { Claviceps bothriochloae } \\
\text { Tanda \& Y. Muray }\end{array}$ & $\begin{array}{l}\text { Clavicipitaceae, Sordariomycetes, } \\
\text { Ascomycota }\end{array}$ & seeds & $\begin{array}{l}\text { Bothriochloa } \\
\text { parviflora }\end{array}$ & Japan & Tanda 1991 \\
\hline Claviceps microspora Tanda & $\begin{array}{l}\text { Clavicipitaceae, Sordariomycetes, } \\
\text { Ascomycota }\end{array}$ & seeds & Arundinella hirta & Japan & Tanda 1985 \\
\hline $\begin{array}{l}\text { Claviceps panicoidearum } \\
\text { Tanda \& Y. Harada }{ }^{\#}\end{array}$ & $\begin{array}{l}\text { Clavicipitaceae, Sordariomycetes, } \\
\text { Ascomycota }\end{array}$ & seeds & Isachne globose & Japan & Tanda \& Harada 1989 \\
\hline \multirow{5}{*}{$\begin{array}{l}\text { Clavochytridium simplex } \\
\text { Doweld [as Blastocladiella } \\
\text { simplex V.D. Matthews] }\end{array}$} & $\begin{array}{l}\text { Blastocladiaceae, Blastocladiomycetes, } \\
\text { Blastocladiomycota }\end{array}$ & seeds & Hyoscyamus aureus & $\begin{array}{l}\text { Poland or/ } \\
\text { imported to Poland }\end{array}$ & Czeczuga et al. 2009 \\
\hline & & seeds & $\begin{array}{l}\text { Hyoscyamus } \\
\text { gyoerffyi }\end{array}$ & $\begin{array}{l}\text { Poland or/ } \\
\text { imported to Poland }\end{array}$ & Czeczuga et al. 2009 \\
\hline & & seeds & $\begin{array}{l}\text { Hyoscyamus niger } \\
\text { var. vernalis }\end{array}$ & $\begin{array}{l}\text { Poland or/ } \\
\text { imported to Poland }\end{array}$ & Czeczuga et al. 2009 \\
\hline & & seeds & Lonicera etrusca & $\begin{array}{l}\text { Poland or/ } \\
\text { imported to Poland }\end{array}$ & Czeczuga et al. 2009 \\
\hline & & seeds & Lycium chinense & $\begin{array}{l}\text { Poland or/ } \\
\text { imported to Poland }\end{array}$ & Czeczuga et al. 2009 \\
\hline
\end{tabular}


Table 2 Continued.

\begin{tabular}{|c|c|c|c|c|c|}
\hline Species & Taxonomy & Substrate & Host & Country & References \\
\hline $\begin{array}{l}\text { Clavochytridium variabile } \\
\text { Doweld [as Blastocladiella } \\
\text { variabilis Harder \& Sörgel] }\end{array}$ & $\begin{array}{l}\text { Blastocladiaceae, } \\
\text { BlastocladiomycetesBlastocladiomycota }\end{array}$ & seeds & Cornus capitata & $\begin{array}{l}\text { Poland or/ } \\
\text { imported to Poland }\end{array}$ & Czeczuga et al. 2009 \\
\hline Clohiesia sp. & $\begin{array}{l}\text { Incertae sedis, Sordariomycetes, } \\
\text { Ascomycota }\end{array}$ & fruits & $\begin{array}{l}\text { Viburnum } \\
\text { sempervirens }\end{array}$ & Hong Kong & Tang et al. 2003a \\
\hline $\begin{array}{l}\text { Clonostachys candelabrum } \\
\text { (Bonord.) Schroers } \\
\text { [as Verticillium candelabrum } \\
\text { Bonord.] }\end{array}$ & $\begin{array}{l}\text { Bionectriaceae, Sordariomycetes, } \\
\text { Ascomycota }\end{array}$ & seeds & Quercus spp. & USSR & Mittal et al. 1990 \\
\hline $\begin{array}{l}\text { Clonostachys } \\
\text { compactiuscula (Sacc.) D. } \\
\text { Hawksw. \& W. Gams [as } \\
\text { Bionectria compactiuscula] }\end{array}$ & $\begin{array}{l}\text { Bionectriaceae, Sordariomycetes, } \\
\text { Ascomycota }\end{array}$ & seeds & Fraxinus excelsior & Sweden & Hayatgheibi 2013 \\
\hline $\begin{array}{l}\text { Schroers [as Verticillium } \\
\text { compactiusculum Sacc.] }\end{array}$ & & seeds & Quercus spp. & USSR & $\begin{array}{l}\text { Urosevic 1962, Mittal et } \\
\text { al. } 1990\end{array}$ \\
\hline $\begin{array}{l}\text { Clonostachys rosea (Link) } \\
\text { Schroers, Samuels, Seifert \& } \\
\text { W. Gams }\end{array}$ & $\begin{array}{l}\text { Bionectriaceae, Sordariomycetes, } \\
\text { Ascomycota }\end{array}$ & seeds & Quercus robur & Turkey & Oskay et al. 2018 \\
\hline $\begin{array}{l}\text { Clonostachys sp. } \\
\text { [as Sesquicillium] }\end{array}$ & $\begin{array}{l}\text { Bionectriaceae, Sordariomycetes, } \\
\text { Ascomycota }\end{array}$ & pods & Delonix regia & Thailand & Somrithipol et al. 2002b \\
\hline $\begin{array}{l}\text { Coccomyces strobilicola } \\
\text { Spooner }\end{array}$ & $\begin{array}{l}\text { Rhytismataceae, Leotiomycetes, } \\
\text { Ascomycota }\end{array}$ & cone & Picea sp. & Austria & Spooner 1987 \\
\hline Cochliobolus spp. & $\begin{array}{l}\text { Pleosporaceae, Dothideomycetes, } \\
\text { Ascomycota }\end{array}$ & seeds & Eucalyptus grandis & South Africa & Jimu et al. 2016 \\
\hline \multirow{4}{*}{$\begin{array}{l}\text { Collariella bostrychodes } \\
\text { (Zopf) X.Wei Wang \& } \\
\text { Samson [as Chaetomium } \\
\text { bostrychodes Zopf] }\end{array}$} & $\begin{array}{l}\text { Chaetomiaceae, Sordariomycetes, } \\
\text { Ascomycota }\end{array}$ & seeds & Cedrus deodara & India & Munjal \& Sharma 1975 \\
\hline & & seeds & Pinus roxburghii & India & $\begin{array}{l}\text { Mittal \& Sharma 1982c, } \\
\text { Mittal et al. } 1990\end{array}$ \\
\hline & & seeds & Pinus sylvestris & USSR & $\begin{array}{l}\text { Dolejs 1964, Mittal et al. } \\
1990\end{array}$ \\
\hline & & seeds & Pinus wallichiana & India & $\begin{array}{l}\text { Munjal \& Sharma 1975, } \\
\text { Mittal et al. } 1990\end{array}$ \\
\hline
\end{tabular}


Table 2 Continued.

\begin{tabular}{|c|c|c|c|c|c|}
\hline Species & Taxonomy & Substrate & Host & Country & References \\
\hline Colletotrichum aotearoa & Glomerellaceae, Sordariomycetes, & berries & Coprosma sp. & New Zealand & Weir et al. 2012 \\
\hline B.S. Weir \& P.R. Johnst.\# & Ascomycota & fruits & $\begin{array}{l}\text { Dysoxylum } \\
\text { spectabile }\end{array}$ & New Zealand & Weir et al. 2012 \\
\hline \multirow[b]{2}{*}{$\begin{array}{l}\text { Colletotrichum capsici (Syd. } \\
\text { \& P. Syd.) E.J. Butler \& } \\
\text { Bisby }\end{array}$} & \multirow[b]{2}{*}{$\begin{array}{l}\text { Glomerellaceae, Sordariomycetes, } \\
\text { Ascomycota }\end{array}$} & fruits & Vitex lucens & New Zealand & Weir et al. 2012 \\
\hline & & seeds & Sesbania grandiflora & India & $\begin{array}{l}\text { Anderson 1986a, } \\
\text { Srinivasan } 1952\end{array}$ \\
\hline $\begin{array}{l}\text { Colletotrichum cosmi } \\
\text { Damm, P.F. Cannon \& } \\
\text { Crous }^{\#}\end{array}$ & $\begin{array}{l}\text { Glomerellaceae, Sordariomycetes, } \\
\text { Ascomycota }\end{array}$ & seed & Cosmos sp. & The Netherlands & Damm et al. 2012 \\
\hline $\begin{array}{l}\text { Colletotrichum dematium } \\
\text { (Pers.) Grove }\end{array}$ & $\begin{array}{l}\text { Glomerellaceae, Sordariomycetes, } \\
\text { Ascomycota }\end{array}$ & seeds & Viola wittrockiana & $\begin{array}{l}\text { imported to } \\
\text { Taiwan }\end{array}$ & Wu et al. 2006 \\
\hline \multirow{17}{*}{$\begin{array}{l}\text { Colletotrichum } \\
\text { gloeosporioides (Penz.) Penz. } \\
\text { \& Sacc. }\end{array}$} & \multirow{17}{*}{$\begin{array}{l}\text { Glomerellaceae, Sordariomycetes, } \\
\text { Ascomycota }\end{array}$} & fruits & Alocasia odora & Hong Kong & Tang et al. 2003a \\
\hline & & fruits & Ardisia punctata & Hong Kong & Tang et al. 2003a \\
\hline & & fruits & Ardisia qinquegona & Hong Kong & Tang et al. 2003a \\
\hline & & fruits & $\begin{array}{l}\text { Cleistocalyx } \\
\text { operculatus }\end{array}$ & Hong Kong & Tang et al. 2003a \\
\hline & & fruits & Dichroa febrifuga & Hong Kong & Tang et al. 2003a \\
\hline & & fruits & Ilex asprella & Hong Kong & Tang et al. 2003a \\
\hline & & fruits & Ilex cinerea & Hong Kong & Tang et al. 2003a \\
\hline & & fruits & Ilex pubescens & Hong Kong & Tang et al. 2003a \\
\hline & & fruits & Litsea rotundifolia & Hong Kong & Tang et al. 2003a \\
\hline & & fruits & Microcos paniculata & Hong Kong & Tang et al. 2003a \\
\hline & & fruits & Psychotria asiatica & Hong Kong & Tang et al. 2003a \\
\hline & & fruits & $\begin{array}{l}\text { Rhodomyrtus } \\
\text { tomentosa }\end{array}$ & Hong Kong & Tang et al. 2003a \\
\hline & & fruits & Sarcandra glabra & Hong Kong & Tang et al. 2003a \\
\hline & & fruits & $\begin{array}{l}\text { Viburnum } \\
\text { sempervirens }\end{array}$ & Hong Kong & Tang et al. 2003a \\
\hline & & seeds & Agathis macrophylla & Philippines & $\begin{array}{l}\text { Quiniones 1985, 1987, } \\
\text { Anderson 1986a }\end{array}$ \\
\hline & & seeds & $\begin{array}{l}\text { Agathis } \\
\text { philippinensis }\end{array}$ & Philippines & Quiniones 1987 \\
\hline & & seeds & Albizia lebbeck & India & Mohanan et al. 2005 \\
\hline
\end{tabular}


Table 2 Continued.

\begin{tabular}{|c|c|c|c|c|c|}
\hline Species & Taxonomy & Substrate & Host & Country & References \\
\hline & & seeds & Alnus glutinosa & Poland & Krol et al. 2015 \\
\hline & & seeds & $\begin{array}{l}\text { Dalbergia } \\
\text { cochinchinensis }\end{array}$ & Thailand & $\begin{array}{l}\text { Chalermpongse et al. } \\
\text { 1984, Mittal et al. } 1990\end{array}$ \\
\hline & & seeds & Fagus sylvatica & Poland & Krol et al. 2015 \\
\hline & & seeds & $\begin{array}{l}\text { Leucaena } \\
\text { leucocephala }\end{array}$ & $\begin{array}{l}\text { Malaysia, } \\
\text { Philippines }\end{array}$ & $\begin{array}{l}\text { Quiniones 1985, 1987, } \\
\text { Mittal et al. } 1990\end{array}$ \\
\hline & & seeds & Pinus sylvestris & Poland & Krol et al. 2015 \\
\hline & & seeds & Pterocarpus indicus & Philippines & $\begin{array}{l}\text { Mathur 1974, Anderson } \\
\text { 1986a, Quiniones } 1987\end{array}$ \\
\hline & & seeds & Pongamia pinnata & India & $\begin{array}{l}\text { Jamaluddin et al. } 1983 \text {, } \\
\text { Mittal et al. } 1990\end{array}$ \\
\hline & & seeds & Shorea acuminata & Malaysia & Mittal et al. 1990 \\
\hline & & seeds & Shorea materialis & Malaysia & Mittal et al. 1990 \\
\hline & & seeds & Tectona grandis & India & Mohanan et al. 2005 \\
\hline Colletotrichum & & fruits & Alocasia odora & Hong Kong & Tang et al. 2003a \\
\hline gloeosporioides (Penz.) Penz. & & fruits & Ardisia crenata & Hong Kong & Tang et al. 2003a \\
\hline \& Sacc. [as Glomerella & & fruits & Ilex asprella & Hong Kong & Tang et al. 2003a \\
\hline cingulata (Stoneman) & & fruits & Sarcandra glabra & Hong Kong & Tang et al. 2003a \\
\hline Spauld. \& H. Schrenk] & & & & & \\
\hline $\begin{array}{l}\text { Colletotrichum godetiae } \\
\text { Neerg. }\end{array}$ & $\begin{array}{l}\text { Glomerellaceae, Sordariomycetes, } \\
\text { Ascomycota }\end{array}$ & seed & $\begin{array}{l}\text { Clarkia hybrida, cv. } \\
\text { kelvon glory }\end{array}$ & Denmark & Damm et al. 2012 \\
\hline & & fruits & Sambucus nigra & The Netherlands & Damm et al. 2012 \\
\hline $\begin{array}{l}\text { Colletotrichum graminicola } \\
\text { (Ces.) G.W. Wilson }\end{array}$ & $\begin{array}{l}\text { Glomerellaceae, Sordariomycetes, } \\
\text { Ascomycota }\end{array}$ & seeds & $\begin{array}{l}\text { Leucaena } \\
\text { leucocephala }\end{array}$ & Philippines & $\begin{array}{l}\text { Quiniones 1985, 1987, } \\
\text { Mittal et al. } 1990\end{array}$ \\
\hline $\begin{array}{l}\text { Colletotrichum } \\
\text { lindemuthianum (Sacc. \& } \\
\text { Magnus) Briosi \& Cavara }\end{array}$ & $\begin{array}{l}\text { Glomerellaceae, Sordariomycetes, } \\
\text { Ascomycota }\end{array}$ & pod & Canavalia cathartica & India & Anita \& Sridhar 2009 \\
\hline $\begin{array}{l}\text { Colletotrichum lini } \\
\text { (Westerd.) Tochinai }\end{array}$ & $\begin{array}{l}\text { Glomerellaceae, Sordariomycetes, } \\
\text { Ascomycota }\end{array}$ & seed & Linum usitatissimum & Canada & Conners 1967 \\
\hline \multirow[t]{4}{*}{$\begin{array}{l}\text { Colletotrichum musae (Berk. } \\
\text { \& M.A. Curtis) Arx }\end{array}$} & $\begin{array}{l}\text { Glomerellaceae, Sordariomycetes, } \\
\text { Ascomycota }\end{array}$ & fruits & Ardisia qinquegona & Hong Kong & Tang et al. 2003a \\
\hline & & fruits & Diplospora dubia & Hong Kong & Tang et al. 2003a \\
\hline & & fruits & Ilex asprella & Hong Kong & Tang et al. 2003a \\
\hline & & fruits & Ilex cinerea & Hong Kong & Tang et al. 2003a \\
\hline
\end{tabular}


Table 2 Continued.

\begin{tabular}{|c|c|c|c|c|c|}
\hline Species & Taxonomy & Substrate & Host & Country & References \\
\hline & & fruits & Wikstroemia nutans & Hong Kong & Tang et al. 2003a \\
\hline $\begin{array}{l}\text { Colletotrichum orbiculare } \\
\text { Damm, P.F. Cannon \& Crous }\end{array}$ & $\begin{array}{l}\text { Glomerellaceae, Sordariomycetes, } \\
\text { Ascomycota }\end{array}$ & fruits & Diplospora dubia & Hong Kong & Tang et al. 2003a \\
\hline \multirow[t]{11}{*}{ Colletotrichum spp. } & Glomerellaceae, Sordariomycetes, & seed & Sesbania bispinosa & India & Anita et al. 2009 \\
\hline & Ascomycota & fruits & Ilex pubescens & Hong Kong & Tang et al. 2003a \\
\hline & & fruits & Litsea rotundifolia & Hong Kong & Tang et al. 2003a \\
\hline & & seeds & Acer palmatum & South Korea & $\begin{array}{l}\text { Mathur 1974, Anderson } \\
\text { 1986a }\end{array}$ \\
\hline & & seeds & $\begin{array}{l}\text { Acrocarpus } \\
\text { fraxinifolius }\end{array}$ & Rwanda & $\begin{array}{l}\text { Mathur 1974, Anderson } \\
\text { 1986a }\end{array}$ \\
\hline & & seeds & Cedrela odorata & Colombia & $\begin{array}{l}\text { Mathur 1974, Anderson } \\
\text { 1986a }\end{array}$ \\
\hline & & seeds & $\begin{array}{l}\text { Eucalyptus } \\
\text { citriodora }\end{array}$ & India & Anderson 1986a \\
\hline & & seeds & Phyllostachys edulis & China & Shen et al. 2014 \\
\hline & & seeds & Pinus caribaea & UK & $\begin{array}{l}\text { Hocking 1968, Mittal et } \\
\text { al. } 1990\end{array}$ \\
\hline & & seeds & Pinus oocarpa & UK & $\begin{array}{l}\text { Rees 1982, Mittal et al. } \\
1990\end{array}$ \\
\hline & & seeds & Pinus pseudostrobus & UK & $\begin{array}{l}\text { Rees 1982, Mittal et al. } \\
1990\end{array}$ \\
\hline $\begin{array}{l}\text { Colletotrichum spp. } \\
\text { [as Glomerella] }\end{array}$ & & seeds & Eucalyptus grandis & South Africa & Jimu et al. 2016 \\
\hline $\begin{array}{l}\text { Colletotrichum truncatum } \\
\text { (Schwein.) Andrus \& W.D. } \\
\text { Moore }\end{array}$ & $\begin{array}{l}\text { Glomerellaceae, Sordariomycetes, } \\
\text { Ascomycota }\end{array}$ & seeds & $\begin{array}{l}\text { Leucaena } \\
\text { leucocephala }\end{array}$ & Philippines & Quiniones 1987 \\
\hline Comminutispora spp. & $\begin{array}{l}\text { Incertae sedis, Dothideomycetes, } \\
\text { Ascomycota }\end{array}$ & seed capsules & Eucalyptus grandis & South Africa & Jimu et al. 2016 \\
\hline \multirow[t]{4}{*}{ Conidioxyphium sp. } & $\begin{array}{l}\text { Trichomeriaceae, Eurotiomycetes, } \\
\text { Ascomycota }\end{array}$ & fruits & Litsea rotundifolia & Hong Kong & Tang et al. 2003a \\
\hline & & fruits & Microcos paniculata & Hong Kong & Tang et al. 2003a \\
\hline & & fruits & Psychotria asiatica & Hong Kong & Tang et al. 2003a \\
\hline & & fruits & $\begin{array}{l}\text { Viburnum } \\
\text { sempervirens }\end{array}$ & Hong Kong & Tang et al. 2003a \\
\hline
\end{tabular}


Table 2 Continued.

\begin{tabular}{|c|c|c|c|c|c|}
\hline Species & Taxonomy & Substrate & Host & Country & References \\
\hline Conioscypha varia Shearer & $\begin{array}{l}\text { Conioscyphaceae, Sordariomycetes, } \\
\text { Ascomycota }\end{array}$ & fruit & Scheelea zonensis & Panama & Goos \& Pirozynski 1975 \\
\hline \multirow[t]{2}{*}{$\begin{array}{l}\text { Coniosporium aterrimum } \\
\text { (Corda) Sacc. }\end{array}$} & \multirow[t]{2}{*}{$\begin{array}{l}\text { Incertae sedis, Eurotiomycetes, } \\
\text { Ascomycota }\end{array}$} & seeds & Pinus sylvestris & USSR & $\begin{array}{l}\text { Urosevic 1961, } \\
\text { Anderson 1986a }\end{array}$ \\
\hline & & seeds & Quercus spp. & USSR & $\begin{array}{l}\text { Urosevic 1962, Mittal et } \\
\text { al. } 1990\end{array}$ \\
\hline \multirow[t]{2}{*}{ Coniothecium atrum Corda } & \multirow{2}{*}{$\begin{array}{l}\text { Incertae sedis, Leotiomycetes, } \\
\text { Ascomycota }\end{array}$} & seeds & Cedrus deodara & India & Munjal \& Sharma 1975 \\
\hline & & seeds & Pinus roxburghii & India & $\begin{array}{l}\text { Munjal \& Sharma 1975, } \\
\text { Mittal et al. } 1990\end{array}$ \\
\hline \multirow[t]{2}{*}{ Coniothecium sp. } & \multirow{2}{*}{$\begin{array}{l}\text { Incertae sedis, Leotiomycetes, } \\
\text { Ascomycota }\end{array}$} & seeds & Cedrus deodara & India & Munjal \& Sharma 1975 \\
\hline & & seeds & Pinus roxburghii & India & $\begin{array}{l}\text { Munjal \& Sharma 1975, } \\
\text { Mittal et al. } 1990\end{array}$ \\
\hline \multirow[t]{2}{*}{$\begin{array}{l}\text { Coniothyrium conicola } \\
\text { Vestergr. }\end{array}$} & \multirow[t]{2}{*}{$\begin{array}{l}\text { Coniothyriaceae, Dothideomycetes, } \\
\text { Ascomycota }\end{array}$} & seeds & Picea abies & USSR & $\begin{array}{l}\text { Prisyazhnyuk } 1960, \\
\text { Mittal et al. } 1990\end{array}$ \\
\hline & & seeds & Pinus sylvestris & USSR & $\begin{array}{l}\text { Prisyazhnyuk } 1960 \text {, } \\
\text { Mittal et al. } 1990\end{array}$ \\
\hline $\begin{array}{l}\text { Coniothyrium leprosum } \\
\text { Fairm." }\end{array}$ & $\begin{array}{l}\text { Coniothyriaceae, Dothideomycetes, } \\
\text { Ascomycota }\end{array}$ & fruits & Eucalyptus sp. & USA & $\begin{array}{l}\text { Millspaugh \& Nuttall } \\
1923\end{array}$ \\
\hline \multirow[t]{2}{*}{$\begin{array}{l}\text { Coniothyrium olivaceum } \\
\text { Bonord. }\end{array}$} & \multirow[t]{2}{*}{$\begin{array}{l}\text { Coniothyriaceae, Dothideomycetes, } \\
\text { Ascomycota }\end{array}$} & seed & $\begin{array}{l}\text { Betula } \\
\text { alleghaniensis }\end{array}$ & USA & $\begin{array}{l}\text { Smoot \& Segall 1963, } \\
\text { Mittal et al. } 1990\end{array}$ \\
\hline & & seeds & Melilotus sp. & Canada & Conners 1967 \\
\hline \multirow{4}{*}{$\begin{array}{l}\text { Coniothyrium quercinum } \\
\text { (Bonord.) Sacc. }\end{array}$} & \multirow{4}{*}{$\begin{array}{l}\text { Coniothyriaceae, Dothideomycetes, } \\
\text { Ascomycota }\end{array}$} & acorns & Quercus robur & Poland & Jankowiak 2008 \\
\hline & & seeds & Picea excelsa & USSR & $\begin{array}{l}\text { Urosevic 1961, } \\
\text { Anderson 1986a }\end{array}$ \\
\hline & & seeds & Pinus sylvestris & USSR & $\begin{array}{l}\text { Urosevic 1961, } \\
\text { Anderson 1986a }\end{array}$ \\
\hline & & seeds & Quercus spp. & USSR & $\begin{array}{l}\text { Urosevic 1964, Mittal et } \\
\text { al. } 1990\end{array}$ \\
\hline \multirow[t]{3}{*}{ Coniothyrium spp. } & \multirow[t]{3}{*}{$\begin{array}{l}\text { Coniothyriaceae, Dothideomycetes, } \\
\text { Ascomycota }\end{array}$} & seeds & Acer rubrum & USA & $\begin{array}{l}\text { Friedrich et al. } 1971 \text {, } \\
\text { Mittal et al. } 1990\end{array}$ \\
\hline & & seeds & Acer saccharinum & USA & $\begin{array}{l}\text { Friedrich et al. } 1971 \text {, } \\
\text { Mittal et al. } 1990\end{array}$ \\
\hline & & seeds & Acer saccharum & USA & $\begin{array}{l}\text { Friedrich et al. } 1971 \text {, } \\
\text { Mittal et al. } 1990\end{array}$ \\
\hline
\end{tabular}


Table 2 Continued.

\begin{tabular}{|c|c|c|c|c|c|}
\hline Species & Taxonomy & Substrate & Host & Country & References \\
\hline & & seeds & $\begin{array}{l}\text { Betula } \\
\text { alleghaniensis }\end{array}$ & USA & $\begin{array}{l}\text { Friedrich et al. } 1971 \text {, } \\
\text { Mittal et al. } 1990\end{array}$ \\
\hline & & seeds & Betula papyrifera & USA & $\begin{array}{l}\text { Smoot \& Segall 1963, } \\
\text { Mittal et al. } 1990\end{array}$ \\
\hline & & seeds & $\begin{array}{l}\text { Chamaecyparis } \\
\text { lawsoniana }\end{array}$ & France, Italy & $\begin{array}{l}\text { Motta \& Saponaro 1983, } \\
\text { Mittal et al. } 1990\end{array}$ \\
\hline & & seeds & $\begin{array}{l}\text { Cupressus } \\
\text { abramsiana }\end{array}$ & France, Italy & $\begin{array}{l}\text { Saponaro \& Motta 1984, } \\
\text { Mittal et al. } 1990\end{array}$ \\
\hline & & seeds & Cupressus arizonica & France, Italy & $\begin{array}{l}\text { Saponaro \& Motta 1984, } \\
\text { Mittal et al. } 1990\end{array}$ \\
\hline & & seeds & Cupressus glabra & France, Italy & $\begin{array}{l}\text { Saponaro \& Motta 1984, } \\
\text { Mittal et al. } 1990\end{array}$ \\
\hline & & seeds & $\begin{array}{l}\text { Cupressus } \\
\text { goveniana }\end{array}$ & France, Italy & $\begin{array}{l}\text { Saponaro \& Motta 1984, } \\
\text { Mittal et al. } 1990\end{array}$ \\
\hline & & seeds & Cupressus lusitanica & France, Italy & $\begin{array}{l}\text { Saponaro \& Motta 1984, } \\
\text { Mittal et al. } 1990\end{array}$ \\
\hline & & seeds & $\begin{array}{l}\text { Cupressus lusitanica } \\
\text { var. benthamii }\end{array}$ & France, Italy & $\begin{array}{l}\text { Saponaro \& Motta 1984, } \\
\text { Mittal et al. } 1990\end{array}$ \\
\hline & & seeds & $\begin{array}{l}\text { Cupressus } \\
\text { sempervirens }\end{array}$ & France, Italy & $\begin{array}{l}\text { Motta \& Saponaro 1983, } \\
\text { Mittal et al. } 1990\end{array}$ \\
\hline & & seeds & Cupressus torulosa & France, Italy & $\begin{array}{l}\text { Saponaro \& Motta 1984, } \\
\text { Mittal et al. } 1990\end{array}$ \\
\hline & & seeds & Fraxinus americana & USA & $\begin{array}{l}\text { Friedrich et al. } 1971 \text {, } \\
\text { Mittal et al. } 1990\end{array}$ \\
\hline & & seeds & $\begin{array}{l}\text { Platanus } \\
\text { occidentalis }\end{array}$ & USA & $\begin{array}{l}\text { Fakir et al. 1971, Mittal } \\
\text { et al. } 1990\end{array}$ \\
\hline & & seeds & $\begin{array}{l}\text { Sequoia } \\
\text { sempervirens }\end{array}$ & USA & $\begin{array}{l}\text { Davidson 1970, Mittal et } \\
\text { al. } 1990\end{array}$ \\
\hline & & seeds & Thuja orientalis & Italy, France & $\begin{array}{l}\text { Motta \& Saponaro 1983, } \\
\text { Mittal et al. } 1990\end{array}$ \\
\hline $\begin{array}{l}\text { Coniozyma leucospermi } \\
\text { (Crous \& Denman) Crous }\end{array}$ & $\begin{array}{l}\text { Incertae sedis, Dothideomycetes, } \\
\text { Ascomycota }\end{array}$ & seeds & Fraxinus excelsior & Sweden & Hayatgheibi 2013 \\
\hline $\begin{array}{l}\text { Cophinforma atrovirens } \\
\text { (Mehl \& Slippers) A. Alves }\end{array}$ & $\begin{array}{l}\text { Botryosphaeriaceae, Dothideomycetes, } \\
\text { Ascomycota }\end{array}$ & pod & Ailanthus sp. & Thailand & Jayasiri et al. 2019 \\
\hline
\end{tabular}


Table 2 Continued.

\begin{tabular}{|c|c|c|c|c|c|}
\hline Species & Taxonomy & Substrate & Host & Country & References \\
\hline \multicolumn{6}{|l|}{ \& A.J.L. Phillips } \\
\hline Coprinopsis spp. & $\begin{array}{l}\text { Psathyrellaceae, Agaricomycetes, } \\
\text { Basidiomycota }\end{array}$ & seed & Prunus serrulata & Japan & Watanabe 2010 \\
\hline Coprinus sp. & $\begin{array}{l}\text { Agaricaceae, Agaricomycetes, } \\
\text { Basidiomycota }\end{array}$ & seeds & Cedrus deodara & India & Munjal \& Sharma 1975 \\
\hline $\begin{array}{l}\text { Cordana terrestris (Timonin) } \\
\text { Hern.-Restr., Gené \& Guarro } \\
\text { [as Pseudobotrytis terrestris } \\
\text { (Timonin) Subram.] }\end{array}$ & $\begin{array}{l}\text { Cordanaceae, Sordariomycetes, } \\
\text { Ascomycota }\end{array}$ & pods & Delonix regia & Thailand & Somrithipol et al. 2002b \\
\hline $\begin{array}{l}\text { Cordyceps farinosa } \\
\text { (Holmsk.) Kepler, B. } \\
\text { Shrestha \& Spatafora } \\
\text { [as Paecilomyces farinosus } \\
\text { (Holmsk.) A.H.S. Br. \& G. } \\
\text { Sm.] }\end{array}$ & $\begin{array}{l}\text { Cordycipitaceae, Sordariomycetes, } \\
\text { Ascomycota }\end{array}$ & acorns & Quercus robur & Poland & Jankowiak 2008 \\
\hline $\begin{array}{l}\text { Corollospora novofusca } \\
\text { Kohlm. \& Volkm.-Kohlm." }\end{array}$ & $\begin{array}{l}\text { Halosphaeriaceae, Sordariomycetes, } \\
\text { Ascomycota }\end{array}$ & fruit & Casuarina sp. & USA & $\begin{array}{l}\text { Kohlmeyer \& } \\
\text { Volkmann-Kohlmeyer } \\
1991\end{array}$ \\
\hline $\begin{array}{l}\text { Corynelia fructigena A.R. } \\
\text { Wood, van der Linde, } \\
\text { Cheew. \& Crous }{ }^{\#}\end{array}$ & $\begin{array}{l}\text { Coryneliaceae, Eurotiomycetes, } \\
\text { Ascomycota }\end{array}$ & fruit & $\begin{array}{l}\text { Podocarpus } \\
\text { latifolius }\end{array}$ & South Africa & Wood et al. 2015 \\
\hline $\begin{array}{l}\text { Corynespora sp. } \\
\text { [as 'Corynospora'] }\end{array}$ & $\begin{array}{l}\text { Corynesporascaceae, Dothideomycetes, } \\
\text { Ascomycota }\end{array}$ & seeds & Cassia siamea & Thailand & $\begin{array}{l}\text { Chalermpongse et al. } \\
\text { 1984, Mittal et al. } 1990\end{array}$ \\
\hline Cronartium conigenum & Cronartiaceae, Pucciniomycetes, & cones & Pinus maximinoi & Guatemala & Rayachhltry et al. 1995b \\
\hline Hedgc. \& N.R. Hunt & Basidiomycota & $\begin{array}{l}\text { cones } \\
\text { cones }\end{array}$ & $\begin{array}{l}\text { Pinus oocarpa } \\
\text { Pinus pseudostrobus }\end{array}$ & $\begin{array}{l}\text { Guatemala } \\
\text { Guatemala }\end{array}$ & $\begin{array}{l}\text { Rayachhltry et al. 1995a } \\
\text { Rayachhltry et al. 1995a }\end{array}$ \\
\hline $\begin{array}{l}\text { Cronartium harknessii E. } \\
\text { Meinecke [as Peridermium } \\
\text { harknessii J.P. Moore] }\end{array}$ & $\begin{array}{l}\text { Cronartiaceae, Pucciniomycetes, } \\
\text { Basidiomycota }\end{array}$ & cones & Pinus contorta & USA & Byler \& Platt 1972 \\
\hline $\begin{array}{l}\text { Cryptococcus foliicola Q.M. } \\
\text { Wang \& F.Y. Bai }\end{array}$ & $\begin{array}{l}\text { Cryptococcaceae, Tremellomycetes, } \\
\text { Basidiomycota }\end{array}$ & $\begin{array}{l}\text { cones } \\
\text { seeds }\end{array}$ & $\begin{array}{l}\text { Pinus muricata } \\
\text { Fraxinus excelsior }\end{array}$ & $\begin{array}{l}\text { USA } \\
\text { Sweden }\end{array}$ & $\begin{array}{l}\text { Byler \& Platt } 1972 \\
\text { Hayatgheibi } 2013\end{array}$ \\
\hline Cryptococcus sp. & $\begin{array}{l}\text { Cryptococcaceae, Tremellomycetes, } \\
\text { Basidiomycota }\end{array}$ & seeds & Fraxinus excelsior & Sweden & Hayatgheibi 2013 \\
\hline
\end{tabular}


Table 2 Continued.

\begin{tabular}{|c|c|c|c|c|c|}
\hline Species & Taxonomy & Substrate & Host & Country & References \\
\hline $\begin{array}{l}\text { Cryptophiale udagawae } \\
\text { Piroz. \& Ichinoe }\end{array}$ & $\begin{array}{l}\text { Chaetosphaeriaceae, Sordariomycetes, } \\
\text { Ascomycota }\end{array}$ & cones & Pinus densiflora & Japan & Kasai et al. 1995 \\
\hline $\begin{array}{l}\text { Cryptospora longispora } \\
\text { Servazzi }\end{array}$ & $\begin{array}{l}\text { Gnomoniaceae, Sordariomycetes, } \\
\text { Ascomycota }\end{array}$ & seeds & Araucaria excelsa & USA & $\begin{array}{l}\text { Kahn et al. 1965, Mittal } \\
\text { et al. } 1990\end{array}$ \\
\hline $\begin{array}{l}\text { Cunninghamella echinulata } \\
\text { (Thaxt.) Thaxt. ex Blakeslee }\end{array}$ & $\begin{array}{l}\text { Cunninghamellaceae, Mucoromycetes, } \\
\text { Mucoromycota }\end{array}$ & seed & $\begin{array}{l}\text { Apium graveolens } \\
\text { var. dulce }\end{array}$ & Canada & Conners 1967 \\
\hline $\begin{array}{l}\text { Cunninghamella elegans } \\
\text { Lendn. }\end{array}$ & $\begin{array}{l}\text { Cunninghamellaceae, Mucoromycetes, } \\
\text { Mucoromycota }\end{array}$ & $\begin{array}{l}\text { seed } \\
\text { seed }\end{array}$ & $\begin{array}{l}\text { Linum usitatissimum } \\
\text { Apium graveolens } \\
\text { var. dulce }\end{array}$ & $\begin{array}{l}\text { Canada } \\
\text { Canada }\end{array}$ & $\begin{array}{l}\text { Conners } 1967 \\
\text { Conners } 1967\end{array}$ \\
\hline $\begin{array}{l}\text { Curreya conorum (Fuckel) } \\
\text { Sacc. } \\
\text { Curreya spp. }\end{array}$ & $\begin{array}{l}\text { Didymosphaeriaceae, Dothideomycetes, } \\
\text { Ascomycota } \\
\text { Didymosphaeriaceae, Dothideomycetes, } \\
\text { Ascomycota }\end{array}$ & $\begin{array}{l}\text { seeds } \\
\text { seeds }\end{array}$ & $\begin{array}{l}\text { Pinus sylvestris } \\
\text { Eucalyptus grandis }\end{array}$ & $\begin{array}{l}\text { USSR } \\
\text { South Africa }\end{array}$ & $\begin{array}{l}\text { Dolejs 1964, Mittal et al. } \\
1990 \\
\text { Jimu et al. } 2016\end{array}$ \\
\hline $\begin{array}{l}\text { Curvularia australiensis } \\
\text { (Bugnic. ex M.B. Ellis) } \\
\text { Manamgoda, L. Cai \& K.D. } \\
\text { Hyde [as Drechslera } \\
\text { australiensis Bugnic. ex } \\
\text { M.B. Ellis] }\end{array}$ & $\begin{array}{l}\text { Pleosporaceae, Dothideomycetes, } \\
\text { Ascomycota }\end{array}$ & $\begin{array}{l}\text { seeds } \\
\text { seeds } \\
\text { seeds } \\
\text { seeds }\end{array}$ & $\begin{array}{l}\text { Cupressus spp. } \\
\text { Eucalyptus sp. } \\
\text { Quercus spp. } \\
\text { Viola wittrockiana }\end{array}$ & $\begin{array}{l}\text { India } \\
\text { India } \\
\text { India } \\
\text { imported to } \\
\text { Taiwan }\end{array}$ & $\begin{array}{l}\text { Sahai \& Otra } 1982 \\
\text { Saxena 1985, Mittal et } \\
\text { al. } 1990 \\
\text { Sahai \& Otra } 1982 \\
\text { Wu et al. } 2006\end{array}$ \\
\hline $\begin{array}{l}\text { Curvularia hawaiiensis } \\
\text { (Bugnic. ex M.B. Ellis) } \\
\text { Manamgoda, L. Cai \& K.D. } \\
\text { Hyde [as Drechslera } \\
\text { hawaiiensis Bugnic. ex M.B. } \\
\text { Ellis] }\end{array}$ & $\begin{array}{l}\text { Pleosporaceae, Dothideomycetes, } \\
\text { Ascomycota }\end{array}$ & seeds & $\begin{array}{l}\text { Leucaena } \\
\text { leucocephala }\end{array}$ & Thailand & $\begin{array}{l}\text { Chalermpongse et al. } \\
\text { 1984, Mittal et al. } 1990\end{array}$ \\
\hline $\begin{array}{l}\text { Curvularia pallescens } \\
\text { Boedijn }\end{array}$ & $\begin{array}{l}\text { Pleosporaceae, Dothideomycetes, } \\
\text { Ascomycota }\end{array}$ & seeds & Pinus wallichiana & India & $\begin{array}{l}\text { Munjal \& Sharma 1975, } \\
\text { Mittal et al. } 1990\end{array}$ \\
\hline \multirow[t]{3}{*}{ Curvularia affinis Boedijn } & $\begin{array}{l}\text { Pleosporaceae, Dothideomycetes, } \\
\text { Ascomycota }\end{array}$ & seeds & Cassia siamea & Thailand & $\begin{array}{l}\text { Chalermpongse et al. } \\
\text { 1984, Mittal et al. } 1990\end{array}$ \\
\hline & & seeds & $\begin{array}{l}\text { Holarrhena } \\
\text { antidysenterica }\end{array}$ & Thailand & $\begin{array}{l}\text { Chalermpongse et al. } \\
\text { 1984, Mittal et al. } 1990\end{array}$ \\
\hline & & seeds & $\begin{array}{l}\text { Quercus } \\
\text { castaneifolia }\end{array}$ & Iran & Kavosi et al. 2013 \\
\hline
\end{tabular}


Table 2 Continued.

\begin{tabular}{|c|c|c|c|c|c|}
\hline Species & Taxonomy & Substrate & Host & Country & References \\
\hline \multirow[t]{2}{*}{$\begin{array}{l}\text { Curvularia brachyspora } \\
\text { Boedijn }\end{array}$} & $\begin{array}{l}\text { Pleosporaceae, Dothideomycetes, } \\
\text { Ascomycota }\end{array}$ & seeds & $\begin{array}{l}\text { Acacia } \\
\text { auriculiformis }\end{array}$ & Thailand & $\begin{array}{l}\text { Chalermpongse et al. } \\
\text { 1984, Mittal et al. } 1990\end{array}$ \\
\hline & & seeds & $\begin{array}{l}\text { Casuarina } \\
\text { equisetifolia }\end{array}$ & Philippines & $\begin{array}{l}\text { Quiniones 1985, } \\
\text { Anderson 1986a, } 1987\end{array}$ \\
\hline Curvularia clavata B.L. Jain & $\begin{array}{l}\text { Pleosporaceae, Dothideomycetes, } \\
\text { Ascomycota }\end{array}$ & seeds & Delonix regia & India & Sahu et al. 2003 \\
\hline \multirow{10}{*}{$\begin{array}{l}\text { Curvularia eragrostidis } \\
\text { (Henn.) J.A. Mey. }\end{array}$} & Pleosporaceae, Dothideomycetes, & pod & Canavalia cathartica & India & Anita \& Sridhar 2009 \\
\hline & Ascomycota & pod & Sesbania bispinosa & India & Anita et al. 2009 \\
\hline & & seed & Canavalia cathartica & India & Anita \& Sridhar 2009 \\
\hline & & seeds & Cosmos sulphureus & $\begin{array}{l}\text { imported to } \\
\text { Taiwan }\end{array}$ & Wu et al. 2006 \\
\hline & & seed & $\begin{array}{l}\text { Desmodium } \\
\text { uncinatum }\end{array}$ & Australia & Nik \& Parbery 1977 \\
\hline & & seed & $\begin{array}{l}\text { Macroptilium } \\
\text { atropurpureum }\end{array}$ & Australia & Nik \& Parbery 1977 \\
\hline & & seed & $\begin{array}{l}\text { Macroptilium } \\
\text { lathyroides }\end{array}$ & Australia & Nik \& Parbery 1977 \\
\hline & & seed & $\begin{array}{l}\text { Stylosanthes } \\
\text { guianensis }\end{array}$ & Australia & Nik \& Parbery 1977 \\
\hline & & seed & Stylosanthes humilis & Australia & Nik \& Parbery 1977 \\
\hline & & seed & Teramnus uncinatus & Australia & Nik \& Parbery 1977 \\
\hline \multirow[t]{5}{*}{$\begin{array}{l}\text { Curvularia geniculata } \\
\text { (Tracy \& Earle) Boedijn }\end{array}$} & $\begin{array}{l}\text { Pleosporaceae, Dothideomycetes, } \\
\text { Ascomycota }\end{array}$ & seed & Eucalyptus sp. & India & $\begin{array}{l}\text { Reddy et al. 1982, Mittal } \\
\text { et al. } 1990\end{array}$ \\
\hline & & seeds & $\begin{array}{l}\text { Holarrhena } \\
\text { antidysenterica }\end{array}$ & Thailand & $\begin{array}{l}\text { Chalermpongse et al. } \\
\text { 1984, Mittal et al. } 1990\end{array}$ \\
\hline & & seeds & Linum usitatissimum & Canada & Conners 1967 \\
\hline & & seeds & Pinus caribaea & $\begin{array}{l}\text { Belize [as British } \\
\text { Honduras] }\end{array}$ & Hocking 1968 \\
\hline & & seeds & Samanea saman & Thailand & $\begin{array}{l}\text { Chalermpongse et al. } \\
\text { 1984, Mittal et al. } 1990\end{array}$ \\
\hline \multirow[t]{2}{*}{$\begin{array}{l}\text { Curvularia inaequalis } \\
\text { (Shear) Boedijn }\end{array}$} & $\begin{array}{l}\text { Pleosporaceae, Dothideomycetes, } \\
\text { Ascomycota }\end{array}$ & seed & Acer rubrum & USA & $\begin{array}{l}\text { Friedrich et al. } 1971 \text {, } \\
\text { Mittal et al. } 1990\end{array}$ \\
\hline & & seed & Acer saccharinum & USA & Friedrich et al. 1971, \\
\hline
\end{tabular}


Table 2 Continued.

\begin{tabular}{|c|c|c|c|c|c|}
\hline Species & Taxonomy & Substrate & Host & Country & References \\
\hline \multirow{22}{*}{$\begin{array}{l}\text { Curvularia intermedia } \\
\text { Boedijn } \\
\text { Curvularia lunata (Wakker) } \\
\text { Boedijn }\end{array}$} & \multirow{22}{*}{$\begin{array}{l}\text { Pleosporaceae, Dothideomycetes, } \\
\text { Ascomycota } \\
\text { Pleosporaceae, Dothideomycetes, } \\
\text { Ascomycota }\end{array}$} & & & & Mittal et al. 1990 \\
\hline & & seed & Betula & USA & Friedrich et al. 1971, \\
\hline & & & alleghaniensis & & Mittal et al. 1990 \\
\hline & & seeds & Dactylis glomerata & Canada & Conners 1967 \\
\hline & & seeds & $\begin{array}{l}\text { Eucalyptus } \\
\text { citriodora }\end{array}$ & India & $\begin{array}{l}\text { Mittal \& Sharma 1984, } \\
\text { Mittal et al. } 1990\end{array}$ \\
\hline & & seeds & Festuca elatior & Canada & Conners 1967 \\
\hline & & seeds & Linum usitatissimum & Canada & Conners 1967 \\
\hline & & seeds & Picea excelsa & USSR & $\begin{array}{l}\text { Urosevic 1961, } \\
\text { Anderson 1986a }\end{array}$ \\
\hline & & seeds & Pinus sylvestris & USSR & $\begin{array}{l}\text { Urosevic 1961, } \\
\text { Anderson 1986a }\end{array}$ \\
\hline & & seeds & $\begin{array}{l}\text { Platanus } \\
\text { occidentalis }\end{array}$ & USA & $\begin{array}{l}\text { Fakir et al. 1971, Mittal } \\
\text { et al. } 1990\end{array}$ \\
\hline & & seeds & Acacia confusa & Philippines & Agmata 1979, Anderson \\
\hline & & seed & Bauhinia sp. & Thailand & Chalermpongse et al. \\
\hline & & & & & 1984, Mittal et al. 1990 \\
\hline & & seeds & Bombax anceps & Thailand & $\begin{array}{l}\text { Chalermpongse et al. } \\
\text { 1984, Mittal et al. } 1990\end{array}$ \\
\hline & & seeds & Bombax ceiba & Bangladesh & Mittal et al. 1990 \\
\hline & & seeds & Cassia bakeriana & Thailand & $\begin{array}{l}\text { Chalermpongse et al. } \\
\text { 1984, Mittal et al. } 1990\end{array}$ \\
\hline & & seeds & Cassia siamea & Thailand & $\begin{array}{l}\text { Chalermpongse et al. } \\
\text { 1984, Mittal et al. } 1990\end{array}$ \\
\hline & & seeds & $\begin{array}{l}\text { Casuarina } \\
\text { equisetifolia }\end{array}$ & Philippines & $\begin{array}{l}\text { Quiniones 1985, 1987, } \\
\text { Anderson 1986a }\end{array}$ \\
\hline & & seeds & Dalbergia sissoo & India & Naz et al. 2015 \\
\hline & & seeds & Delonix regia & India, Philippines & $\begin{array}{l}\text { Dayan 1986, Mittal et al. } \\
\text { 1990, Sahu et al. } 2003\end{array}$ \\
\hline & & seeds & Eucalyptus grandis & Thailand & $\begin{array}{l}\text { Chalermpongse et al. } \\
\text { 1984, Mittal et al. } 1990\end{array}$ \\
\hline & & seeds & Eucalyptus sp. & India & $\begin{array}{l}\text { Saxena 1985, Mittal et } \\
\text { al. } 1990\end{array}$ \\
\hline
\end{tabular}


Table 2 Continued.

\begin{tabular}{|c|c|c|c|c|c|}
\hline Species & Taxonomy & Substrate & Host & Country & References \\
\hline & & seeds & $\begin{array}{l}\text { Eucalyptus } \\
\text { tereticornis }\end{array}$ & Thailand & $\begin{array}{l}\text { Chalermpongse et al. } \\
\text { 1984, Mittal et al. } 1990\end{array}$ \\
\hline & & seeds & $\begin{array}{l}\text { Lagerstroemia } \\
\text { calyculata }\end{array}$ & Thailand & $\begin{array}{l}\text { Chalermpongse et al. } \\
\text { 1984, Mittal et al. } 1990\end{array}$ \\
\hline & & seeds & $\begin{array}{l}\text { Leucaena } \\
\text { leucocephala }\end{array}$ & $\begin{array}{l}\text { Bangladesh , } \\
\text { Thailand }\end{array}$ & $\begin{array}{l}\text { Chalermpongse et al. } \\
\text { 1984, Mittal et al. 1990, } \\
\text { Islam et al. } 2008\end{array}$ \\
\hline & & seeds & Melia azedarach & Thailand & $\begin{array}{l}\text { Chalermpongse et al. } \\
\text { 1984, Mittal et al. } 1990\end{array}$ \\
\hline & & seeds & $\begin{array}{l}\text { Musanga } \\
\text { cecropioides }\end{array}$ & Philippines & $\begin{array}{l}\text { Agmata 1979, Anderson } \\
\text { 1986a }\end{array}$ \\
\hline & & seeds & Pinus kesiya & Thailand & $\begin{array}{l}\text { Chalermpongse et al. } \\
\text { 1984, Mittal et al. } 1990\end{array}$ \\
\hline & & seeds & Pinus merkusii & Thailand & $\begin{array}{l}\text { Chalermpongse et al. } \\
\text { 1984, Mittal et al. } 1990\end{array}$ \\
\hline & & seeds & Pyrus spp. & India & Sahai \& Otra 1982 \\
\hline & & seeds & Shorea robusta & Thailand & $\begin{array}{l}\text { Chalermpongse et al. } \\
\text { 1984, Mittal et al. } 1990\end{array}$ \\
\hline & & seeds & Stylosanthes humilis & Australia & Nik \& Parbery 1977 \\
\hline & & seeds & $\begin{array}{l}\text { Swietenia } \\
\text { macrophylla }\end{array}$ & Philippines & $\begin{array}{l}\text { Agmata 1979, Anderson } \\
\text { 1986a }\end{array}$ \\
\hline & & seeds & Thuja spp. & India & Sahai \& Otra 1982 \\
\hline & & seeds & Triplaris cumingiana & Philippines & $\begin{array}{l}\text { Quiniones 1985, } \\
\text { Anderson 1986a }\end{array}$ \\
\hline $\begin{array}{l}\text { Curvularia maculans (C.K. } \\
\text { Bancr.) Boedijn }\end{array}$ & $\begin{array}{l}\text { Pleosporaceae, Dothideomycetes, } \\
\text { Ascomycota }\end{array}$ & seeds & Cedrus deodara & India & Mittal 1983 \\
\hline $\begin{array}{l}\text { Curvularia ovoidea (Hiroë) } \\
\text { Munt.-Cvetk. }\end{array}$ & $\begin{array}{l}\text { Pleosporaceae, Dothideomycetes, } \\
\text { Ascomycota }\end{array}$ & seeds & Cosmos sulphureus & $\begin{array}{l}\text { imported to } \\
\text { Taiwan }\end{array}$ & Wu et al. 2006 \\
\hline \multirow[t]{3}{*}{$\begin{array}{l}\text { Curvularia pallescens } \\
\text { Boedijn }\end{array}$} & $\begin{array}{l}\text { Pleosporaceae, Dothideomycetes, } \\
\text { Ascomycota }\end{array}$ & seeds & $\begin{array}{l}\text { Acacia } \\
\text { auriculiformis }\end{array}$ & Philippines & $\begin{array}{l}\text { Quiniones 1985, } \\
\text { Anderson 1986a }\end{array}$ \\
\hline & & seeds & $\begin{array}{l}\text { Anthocephalus } \\
\text { chinensis }\end{array}$ & Philippines & Quiniones 1987 \\
\hline & & seeds & Bombax anceps & Thailand & $\begin{array}{l}\text { Chalermpongse et al. } \\
\text { 1984, Mittal et al. } 1990\end{array}$ \\
\hline
\end{tabular}


Table 2 Continued.

\begin{tabular}{|c|c|c|c|c|c|}
\hline Species & Taxonomy & Substrate & Host & Country & References \\
\hline & & seeds & Cassia floribunda & Thailand & $\begin{array}{l}\text { Chalermpongse et al. } \\
\text { 1984, Mittal et al. } 1990\end{array}$ \\
\hline & & seeds & $\begin{array}{l}\text { Casuarina } \\
\text { equisetifolia }\end{array}$ & India, Philippines & $\begin{array}{l}\text { Quiniones 1985, 1987, } \\
\text { Anderson 1986a, Anju et } \\
\text { al. } 2012\end{array}$ \\
\hline & & seeds & Cedrus deodara & India & Munjal \& Sharma 1975 \\
\hline & & seeds & Eucalyptus alba & Thailand & $\begin{array}{l}\text { Chalermpongse et al. } \\
\text { 1984, Mittal et al. } 1990\end{array}$ \\
\hline & & seeds & Eucalyptus hybrid & India & $\begin{array}{l}\text { Mittal 1986, Mittal et al. } \\
1990\end{array}$ \\
\hline & & seeds & Eucalyptus sp. & India & $\begin{array}{l}\text { Saxena 1985, Mittal et } \\
\text { al. } 1990\end{array}$ \\
\hline & & seeds & Pinus roxburghii & India & $\begin{array}{l}\text { Munjal \& Sharma 1975, } \\
\text { Mittal et al. } 1990\end{array}$ \\
\hline & & seeds & Triplaris cumingiana & Philippines & $\begin{array}{l}\text { Dayan 1986, } \\
\text { Mittal et al. } 1990\end{array}$ \\
\hline $\begin{array}{l}\text { Curvularia prasadii R.L. } \\
\text { Mathur \& B.L. Mathur }\end{array}$ & $\begin{array}{l}\text { Pleosporaceae, Dothideomycetes, } \\
\text { Ascomycota }\end{array}$ & seed & Prunus serrulata & Japan & Watanabe 2010 \\
\hline $\begin{array}{l}\text { Curvularia senegalensis } \\
\text { (Speg.) Subram. }\end{array}$ & $\begin{array}{l}\text { Pleosporaceae, Dothideomycetes, } \\
\text { Ascomycota }\end{array}$ & seed & Pinus thunbergii & Japan & Watanabe 2010 \\
\hline Curvularia spicifera & Pleosporaceae, Dothideomycetes, & seeds & Delonix regia & India & Sahu et al. 2003 \\
\hline (Bainier) Boedijn & Ascomycota & seeds & Setaria glauca & Pakistan & Lodhi \& Naeem 1955 \\
\hline \multirow[t]{9}{*}{ Curvularia spp. } & Pleosporaceae, Dothideomycetes, & pod & Sesbania bispinosa & India & Anita et al. 2009 \\
\hline & Ascomycota & seeds & Albizia lebbeck & India & Natarajan et al. 2003 \\
\hline & & seeds & Cedrus deodara & India & Munjal \& Sharma 1975 \\
\hline & & seeds & Dalbergia sissoides & India & Mohanan et al. 2005 \\
\hline & & seeds & Eucalyptus alba & Thailand & $\begin{array}{l}\text { Chalermpo ngse et al. } \\
\text { 1984, Mittal et al. } 1990\end{array}$ \\
\hline & & seeds & Eucalyptus grandis & $\begin{array}{l}\text { South Africa, } \\
\text { Uruguay }\end{array}$ & $\begin{array}{l}\text { Anderson 1986a, Jimu et } \\
\text { al. } 2016\end{array}$ \\
\hline & & seeds & Eucalyptus maidenii & Uruguay & Anderson 1986a \\
\hline & & seeds & Phyllostachys edulis & China & Shen et al. 2014 \\
\hline & & seeds & $\begin{array}{l}\text { Pinus armandii var. } \\
\text { mastersiana }\end{array}$ & Taiwan & $\begin{array}{l}\text { Jong \& Chen 1966, } \\
\text { Mittal et al. } 1990\end{array}$ \\
\hline
\end{tabular}


Table 2 Continued.

\begin{tabular}{|c|c|c|c|c|c|}
\hline Species & Taxonomy & Substrate & Host & Country & References \\
\hline \multirow{7}{*}{$\begin{array}{l}\text { Curvularia verruculosa } \\
\text { Tandon \& Bilgrami ex M.B. } \\
\text { Ellis }\end{array}$} & & seeds & $\begin{array}{l}\text { Pinus elliottii var. } \\
\text { elliottii }\end{array}$ & Uruguay, USA & $\begin{array}{l}\text { Anderson 1986a, } \\
\text { Fraedrich \& Miller } 1995\end{array}$ \\
\hline & & seeds & Pinus roxburghii & India & $\begin{array}{l}\text { Munjal \& Sharma 1975, } \\
\text { Mittal et al. } 1990\end{array}$ \\
\hline & & seeds & Pinus taeda & USA & $\begin{array}{l}\text { Covington et al. 1982, } \\
\text { Anderson 1986a }\end{array}$ \\
\hline & & seeds & Pinus wallichiana & India & $\begin{array}{l}\text { Munjal \& Sharma 1975, } \\
\text { Mittal et al. } 1990\end{array}$ \\
\hline & & seeds & Tectona grandis & India & Mohanan et al. 2005 \\
\hline & \multirow[t]{2}{*}{$\begin{array}{l}\text { Pleosporaceae, Dothideomycetes, } \\
\text { Ascomycota }\end{array}$} & seeds & Eucalyptus sp. & India & $\begin{array}{l}\text { Saxena 1985, Mittal et } \\
\text { al. } 1990\end{array}$ \\
\hline & & seeds & Pinus caribaea & $\begin{array}{l}\text { Belize [as British } \\
\text { Honduras] }\end{array}$ & Hocking 1968 \\
\hline \multirow{2}{*}{$\begin{array}{l}\text { Cutaneotrichosporon } \\
\text { cutaneum (Beurm., Gougerot } \\
\text { \& Vaucher bis) Xin Zhan } \\
\text { Liu, F.Y. Bai, M. Groenew. } \\
\text { \& Boekhout } \\
\text { [as Trichosporon cutaneum } \\
\text { (Beurm., Gougerot \& } \\
\text { Vaucher bis) M. Ota] }\end{array}$} & \multirow[t]{2}{*}{$\begin{array}{l}\text { Trichosporonaceae, Tremellomycetes, } \\
\text { Basidiomycota }\end{array}$} & seeds & $\begin{array}{l}\text { Rhododendron } \\
\text { smirnowii }\end{array}$ & $\begin{array}{l}\text { Poland or/ } \\
\text { imported to Poland }\end{array}$ & Czeczuga et al. 2009 \\
\hline & & seeds & Valeriana officinalis & $\begin{array}{l}\text { Poland or/ } \\
\text { imported to Poland }\end{array}$ & Czeczuga et al. 2009 \\
\hline $\begin{array}{l}\text { Cyathus ibericus J.C. } \\
\text { Zamora \& Poveda-Molero }\end{array}$ & $\begin{array}{l}\text { Nidulariaceae, Agaricomycetes, } \\
\text { Basidiomycota }\end{array}$ & cones & Pinus halepensis & Spain & Crous et al. 2016 \\
\hline $\begin{array}{l}\text { Cyberlindnera maclurae } \\
\text { (Kurtzman) Minter }{ }^{\#} \\
\text { [as Pichia maclurae } \\
\text { Kurtzman] }\end{array}$ & $\begin{array}{l}\text { Phaffomycetaceae, Saccharomycetes, } \\
\text { Ascomycota }\end{array}$ & pomes & Maclura pomifera & USA & Kurtzman 2000 \\
\hline \multirow{2}{*}{$\begin{array}{l}\text { Cyberlindnera saturnus } \\
\text { (Klöcker) Minter } \\
\text { [as Hansenula saturnus } \\
\text { (Klöcker) Syd. \& P. Syd.] }\end{array}$} & \multirow[t]{2}{*}{$\begin{array}{l}\text { Phaffomycetaceae, Saccharomycetes, } \\
\text { Ascomycota }\end{array}$} & seeds & Bryonia cretica & $\begin{array}{l}\text { Poland or/ } \\
\text { imported to Poland }\end{array}$ & Czeczuga et al. 2009 \\
\hline & & seeds & Humulus lupulus & $\begin{array}{l}\text { Poland or/ } \\
\text { imported to Poland }\end{array}$ & Czeczuga et al. 2009 \\
\hline
\end{tabular}


Table 2 Continued.

\begin{tabular}{|c|c|c|c|c|c|}
\hline Species & Taxonomy & Substrate & Host & Country & References \\
\hline & & seeds & Juniperus communis & $\begin{array}{l}\text { Poland or/ } \\
\text { imported to Poland }\end{array}$ & Czeczuga et al. 2009 \\
\hline & & seeds & Pistacia terebinthus & $\begin{array}{l}\text { Poland or/ } \\
\text { imported to Poland }\end{array}$ & Czeczuga et al. 2009 \\
\hline & & seeds & $\begin{array}{l}\text { Podophyllum } \\
\text { peltatum }\end{array}$ & $\begin{array}{l}\text { Poland or/ } \\
\text { imported to Poland }\end{array}$ & Czeczuga et al. 2009 \\
\hline & & seeds & Rhus copallina & $\begin{array}{l}\text { Poland or/ } \\
\text { imported to Poland }\end{array}$ & Czeczuga et al. 2009 \\
\hline & & seeds & Rhus gueinzii & $\begin{array}{l}\text { Poland or/ } \\
\text { imported to Poland }\end{array}$ & Czeczuga et al. 2009 \\
\hline & & seeds & Sambucus racemosa & $\begin{array}{l}\text { Poland or/ } \\
\text { imported to Poland }\end{array}$ & Czeczuga et al. 2009 \\
\hline $\begin{array}{l}\text { Cycasicola leucaenae } \\
\text { Jayasiri, E.B.G. Jones \& } \\
\text { K.D. Hyde }^{\#}\end{array}$ & $\begin{array}{l}\text { Thyridariaceae, Dothideomycetes, } \\
\text { Ascomycota }\end{array}$ & pod & Leucaena sp. & Thailand & Jayasiri et al. 2019 \\
\hline $\begin{array}{l}\text { Cyclaneusma minus (Butin) } \\
\text { DiCosmo, Peredo \& Minter }\end{array}$ & $\begin{array}{l}\text { Marthamycetaceae, Leotiomycetes, } \\
\text { Ascomycota }\end{array}$ & seeds & Fraxinus excelsior & Sweden & Hayatgheibi 2013 \\
\hline $\begin{array}{l}\text { Cylindroaseptospora } \\
\text { leucaenae Jayasiri, E.B.G. } \\
\text { Jones \& K.D. Hyde }^{\# * *}\end{array}$ & $\begin{array}{l}\text { Didymosphaeriaceae, Dothideomycetes, } \\
\text { Ascomycota }\end{array}$ & pod & Leucaena sp. & Thailand & Jayasiri et al. 2019 \\
\hline $\begin{array}{l}\text { Cylindroaseptospora } \\
\text { siamensis Jayasiri, E.B.G. } \\
\text { Jones \& K.D. Hyde }\end{array}$ & $\begin{array}{l}\text { Didymosphaeriaceae, Dothideomycetes, } \\
\text { Ascomycota }\end{array}$ & pod & Leucaena sp. & Thailand & Jayasiri et al. 2019 \\
\hline Cylindrocephalum spp. & $\begin{array}{l}\text { Incertae sedis, Incertae sedis, } \\
\text { Ascomycota }\end{array}$ & seeds & Acer saccharum & USA & $\begin{array}{l}\text { Friedrich et al. } 1971 \text {, } \\
\text { Mittal et al. } 1990\end{array}$ \\
\hline $\begin{array}{l}\text { Cylindrocephalum stellatum } \\
\text { (Harz) Sacc. }\end{array}$ & $\begin{array}{l}\text { Incertae sedis, Incertae sedis, } \\
\text { Ascomycota }\end{array}$ & seeds & Pinus sylvestris & USSR & $\begin{array}{l}\text { Prisyazhnyuk } 1960 \text {, } \\
\text { Mittal et al. } 1990\end{array}$ \\
\hline $\begin{array}{l}\text { Cylindrochytridium } \\
\text { endobioticum Willoughby }\end{array}$ & $\begin{array}{l}\text { Incertae sedis, Cladochytriomycetes, } \\
\text { Chytridiomycota }\end{array}$ & seeds & Cornus sanguinea & $\begin{array}{l}\text { Poland or/ } \\
\text { imported to Poland }\end{array}$ & Czeczuga et al. 2009 \\
\hline $\begin{array}{l}\text { Cylindrochytridium } \\
\text { johnstonii Karling }\end{array}$ & $\begin{array}{l}\text { Incertae sedis, Cladochytriomycetes, } \\
\text { Chytridiomycota }\end{array}$ & seeds & Cornus capitata & $\begin{array}{l}\text { Poland or/ } \\
\text { imported to Poland }\end{array}$ & Czeczuga et al. 2009 \\
\hline Cylindrocladium spp. & $\begin{array}{l}\text { Nectriaceae, Sordariomycetes, } \\
\text { Ascomycota }\end{array}$ & cones & Pinus densiflora & Japan & Kasai et al. 1995 \\
\hline
\end{tabular}


Table 2 Continued.

\begin{tabular}{|c|c|c|c|c|c|}
\hline Species & Taxonomy & Substrate & Host & Country & References \\
\hline & & pods & Delonix regia & Thailand & Somrithipol et al. 2002b \\
\hline & & seeds & Pinus lambertiana & USA & $\begin{array}{l}\text { Anderson 1986a, } \\
\text { Schubert } 1961\end{array}$ \\
\hline & & seeds & Shorea assamica & Malaysia & $\begin{array}{l}\text { Hong 1981, Mittal et al. } \\
1990\end{array}$ \\
\hline $\begin{array}{l}\text { Cylindrodendrum hubeiense } \\
\text { (W.Y. Zhuang, Y. Nong \& J. } \\
\text { Luo) L. Lombard \& Crous }{ }^{\#} \\
\text { [as Neonectria hubeiensis } \\
\text { W.Y. Zhuang, Y. Nong \& J. } \\
\text { Luo] }\end{array}$ & $\begin{array}{l}\text { Nectriaceae, Sordariomycetes, } \\
\text { Ascomycota }\end{array}$ & fruits & Rhododendron sp. & China & Zhuang et al. 2007 \\
\hline Cylindrodendrum sp. & $\begin{array}{l}\text { Nectriaceae, Sordariomycetes, } \\
\text { Ascomycota }\end{array}$ & seeds & Quercus robur & Turkey & Oskay et al. 2018 \\
\hline $\begin{array}{l}\text { Cylindromyces striatus } \\
\text { Manohar., D.K. Agarwal \& } \\
\text { N.K. Rao }{ }^{\# * *}\end{array}$ & $\begin{array}{l}\text { Incertae sedis, Incertae sedis, } \\
\text { Ascomycota }\end{array}$ & pods & undetermined plant & India & $\begin{array}{l}\text { Manoharachary et al. } \\
2004\end{array}$ \\
\hline $\begin{array}{l}\text { Cylindrosporella alnea } \\
\text { (Pers. ex Lév.) Höhn. }\end{array}$ & $\begin{array}{l}\text { Gnomoniaceae, Sordariomycetes, } \\
\text { Ascomycota }\end{array}$ & seeds & Alnus sp. & Denmark & Anderson 1986a \\
\hline $\begin{array}{l}\text { Cylindrosporium platanoidis } \\
\text { (Allesch.) Died. }\end{array}$ & $\begin{array}{l}\text { Ploettnerulaceae, Leotiomycetes, } \\
\text { Ascomycota }\end{array}$ & seeds & Acer pseudoplatanus & Poland & Krol et al. 2015 \\
\hline Cytosphaera sp. & $\begin{array}{l}\text { Incertae sedis, Incertae sedis, } \\
\text { Ascomycota }\end{array}$ & fruits & Ilex cinerea & Hong Kong & Tang et al. 2003a \\
\hline $\begin{array}{l}\text { Cytospora chrysosperma } \\
\text { (Pers.) Fr. }\end{array}$ & $\begin{array}{l}\text { Cytosporaceae, Sordariomycetes, } \\
\text { Ascomycota }\end{array}$ & seeds, pods & Eucalyptus globulus & Uruguay & Lupo et al. 2001 \\
\hline Cytospora curreyi Sacc. & $\begin{array}{l}\text { Cytosporaceae, Sordariomycetes, } \\
\text { Ascomycota }\end{array}$ & seeds & Larix spp. & UK & $\begin{array}{l}\text { Noble et al. 1958, } \\
\text { Anderson 1986a }\end{array}$ \\
\hline Cytospora intermedia Sacc. & Cytosporaceae, Sordariomycetes, & seeds & Quercus spp. & USSR & Mittal et al. 1990 \\
\hline $\begin{array}{l}\text { Cytospora intermedia Sacc. } \\
\text { [as Valsa intermedia } \\
\text { Nitschke] }\end{array}$ & Ascomycota & seeds & Quercus spp. & USSR & $\begin{array}{l}\text { Urosevic 1962, Mittal et } \\
\text { al. } 1990\end{array}$ \\
\hline Cytospora pinastri Fr. & $\begin{array}{l}\text { Cytosporaceae, Sordariomycetes, } \\
\text { Ascomycota }\end{array}$ & cones, seeds & Pinus albicaulis & Canada & Vujanovic et al. 2000 \\
\hline Cytospora spp. & $\begin{array}{l}\text { Cytosporaceae, Sordariomycetes, } \\
\text { Ascomycota }\end{array}$ & seeds & Acer rubrum & USA & $\begin{array}{l}\text { Friedrich et al. } 1971 \text {, } \\
\text { Mittal et al. } 1990\end{array}$ \\
\hline
\end{tabular}


Table 2 Continued.

\begin{tabular}{|c|c|c|c|c|c|}
\hline Species & Taxonomy & Substrate & Host & Country & References \\
\hline & & seeds & Acer saccharum & USA & $\begin{array}{l}\text { Friedrich et al. } 1971 \text {, } \\
\text { Mittal et al. } 1990\end{array}$ \\
\hline & & seeds & $\begin{array}{l}\text { Platanus } \\
\text { occidentalis }\end{array}$ & USA & $\begin{array}{l}\text { Fakir et al. 1971, Mittal } \\
\text { et al. } 1990\end{array}$ \\
\hline & & seeds & Quercus robur & Turkey & Oskay et al. 2018 \\
\hline \multirow[t]{2}{*}{ Dactylaria spp. } & Calloriaceae, Leotiomycetes, Ascomycota & fruits & Psychotria asiatica & Hong Kong & Tang et al. 2003a \\
\hline & & pods & Delonix regia & Thailand & Somrithipol et al. 2002b \\
\hline $\begin{array}{l}\text { Dactylium dendroides (Bull.) } \\
\text { Fr. }\end{array}$ & Orbiliaceae, Orbiliomycetes, Ascomycota & seed & Linum usitatissimum & Canada & Conners 1967 \\
\hline $\begin{array}{l}\text { Dasyscyphella longistipitata } \\
\text { Hosoya }\end{array}$ & Lachnaceae, Leotiomycetes, Ascomycota & fruit cupules & Fagus crenata & Japan & Fukasawa et al. 2012 \\
\hline $\begin{array}{l}\text { Dasyscyphella longistipitata } \\
\text { Hosoya }^{\#}\end{array}$ & Lachnaceae, Leotiomycetes, Ascomycota & fruit cupule & Fagus sylvatica & Japan & Ono \& Hosoya 2001 \\
\hline $\begin{array}{l}\text { Dasyscyphus fuscescens } \\
\text { (Pers.) Gray }\end{array}$ & $\begin{array}{l}\text { Hyaloscyphaceae, Leotiomycetes, } \\
\text { Ascomycota }\end{array}$ & fruit cupules & Fagus sylvatica & UK & Carré 1964 \\
\hline Dasyscyphus sp. & $\begin{array}{l}\text { Hyaloscyphaceae, Leotiomycetes, } \\
\text { Ascomycota }\end{array}$ & cone & Picea sitchensis & USA & Conners 1967 \\
\hline $\begin{array}{l}\text { Dasyscyphus virgineus } \\
\text { (Batsch) Gray }\end{array}$ & $\begin{array}{l}\text { Hyaloscyphaceae, Leotiomycetes, } \\
\text { Ascomycota }\end{array}$ & fruit cupules & Fagus sylvatica & UK & Carré 1964 \\
\hline $\begin{array}{l}\text { Davidhawksworthia ilicicola } \\
\text { Crous }^{\# * *}\end{array}$ & $\begin{array}{l}\text { Dermateaceae, Leotiomycetes, } \\
\text { Ascomycota }\end{array}$ & fruit & Ilex aquifolium & The Netherlands & $\begin{array}{l}\text { Crous \& Groenewald } \\
2016\end{array}$ \\
\hline Davidiella spp. & $\begin{array}{l}\text { Davidiellaceae, Dothideomycetes, } \\
\text { Ascomycota }\end{array}$ & $\begin{array}{l}\text { seed capsules, } \\
\text { seeds }\end{array}$ & Eucalyptus grandis & South Africa & Jimu et al. 2016 \\
\hline $\begin{array}{l}\text { Delitschia nypae Jayasiri, } \\
\text { E.B.G. Jones \& K.D. Hyde }{ }^{\#}\end{array}$ & $\begin{array}{l}\text { Delitschiaceae, Dothideomycetes, } \\
\text { Ascomycota }\end{array}$ & fruit & Nypa fruticans & Thailand & Jayasiri et al. 2019 \\
\hline $\begin{array}{l}\text { Delonicicola siamense R.H. } \\
\text { Perera, Maharachch. \& K.D. } \\
\text { Hyde }^{\# * *}\end{array}$ & $\begin{array}{l}\text { Delonicicolaceae, Sordariomycetes, } \\
\text { Ascomycota }\end{array}$ & pods & Delonix regia & Thailand & Perera et al. 2017 \\
\hline Dendrophoma sp. & $\begin{array}{l}\text { Chaetosphaeriaceae, Sordariomycetes, } \\
\text { Ascomycota }\end{array}$ & $\begin{array}{l}\text { seeds } \\
\text { seeds } \\
\text { seeds }\end{array}$ & $\begin{array}{l}\text { Cupressus torulosa } \\
\text { Pinus elliottii } \\
\text { Pinus taeda }\end{array}$ & $\begin{array}{l}\text { Uruguay } \\
\text { USA } \\
\text { USA }\end{array}$ & $\begin{array}{l}\text { Anderson 1986a } \\
\text { Mittal et al. } 1990 \\
\text { Anderson 1986a, b }\end{array}$ \\
\hline $\begin{array}{l}\text { Dendrostoma leiphaemia } \\
\text { (Fr.) Senan. \& K.D. Hyde } \\
\text { [as Amphiporthe leiphaemia }\end{array}$ & $\begin{array}{l}\text { Erythrogloeaceae, Sordariomycetes, } \\
\text { Ascomycota }\end{array}$ & seeds & Quercus robur & Germany & Schroder et al. 2004 \\
\hline
\end{tabular}


Table 2 Continued.

\begin{tabular}{|c|c|c|c|c|c|}
\hline Species & Taxonomy & Substrate & Host & Country & References \\
\hline \multicolumn{6}{|l|}{ (Fr.) Butin] } \\
\hline $\begin{array}{l}\text { Dermatosorus schoenoplecti } \\
\text { Vánky \& R.G. Shiva }{ }^{\#}\end{array}$ & $\begin{array}{l}\text { Anthracoideaceae, Ustilaginomycetes, } \\
\text { Basidiomycota }\end{array}$ & seeds & $\begin{array}{l}\text { Schoenoplectus } \\
\text { mucronatus }\end{array}$ & Australia & Vánky \& Shivas 2003 \\
\hline Diaporthales sp. & Sordariomycetes, Ascomycota & seeds & Eucalyptus grandis & South Africa & Jimu et al. 2016 \\
\hline $\begin{array}{l}\text { Diaporthe angelicae (Berk.) } \\
\text { Wehm. }\end{array}$ & $\begin{array}{l}\text { Diaporthaceae, Sordariomycetes, } \\
\text { Ascomycota }\end{array}$ & seeds & $\begin{array}{l}\text { Heracleum } \\
\text { sphondylium }\end{array}$ & France & Gomes et al. 2013 \\
\hline Diaporthe archeri & Diaporthaceae, Sordariomycetes, & fruits & Alocasia odora & Hong Kong & Tang et al. 2003a \\
\hline [as Phomopsis archeri B. & Ascomycota & fruits & Ardisia crenata & Hong Kong & Tang et al. 2003a \\
\hline \multirow[t]{10}{*}{ Sutton] } & & fruits & Bridelia tomentosa & Hong Kong & Tang et al. 2003a \\
\hline & & fruits & Dichroa febrifuga & Hong Kong & Tang et al. 2003a \\
\hline & & fruits & Diplospora dubia & Hong Kong & Tang et al. 2003a \\
\hline & & fruits & Ilex asprella & Hong Kong & Tang et al. 2003a \\
\hline & & fruits & Ilex cinerea & Hong Kong & Tang et al. 2003a \\
\hline & & fruits & Ilex pubescens & Hong Kong & Tang et al. 2003a \\
\hline & & fruits & Litsea rotundifolia & Hong Kong & Tang et al. 2003a \\
\hline & & fruits & $\begin{array}{l}\text { Viburnum } \\
\text { sempervirens }\end{array}$ & Hong Kong & Tang et al. 2003a \\
\hline & & fruits & Wikstroemia nutans & Hong Kong & Tang et al. 2003a \\
\hline & & pods & Eucalyptus globulus & Uruguay & Lupo et al. 2001 \\
\hline Diaporthe arecae (H.C. & Diaporthaceae, Sordariomycetes, & fruits & Areca catechu & India & Gomes et al. 2013 \\
\hline Srivast., Zakia \& Govindar.) & Ascomycota & & & & \\
\hline \multicolumn{6}{|l|}{ R.R. Gomes, Glienke \& } \\
\hline Diaporthe casuarinae [as & Diaporthaceae, Sordariomycetes, & seeds & Casuarina & Australia, USA & Bose 1944, Anderson \\
\hline $\begin{array}{l}\text { Phomopsis casuarinae } \\
\text { (Tassi) Died.] }\end{array}$ & Ascomycota & & equisetifolia & & $\begin{array}{l}\text { 1986a, Bayman et al. } \\
1998\end{array}$ \\
\hline $\begin{array}{l}\text { Diaporthe collariana R.H. } \\
\text { Perera \& K.D. Hyde }\end{array}$ & $\begin{array}{l}\text { Diaporthaceae, Sordariomycetes, } \\
\text { Ascomycota }\end{array}$ & fruits & Magnolia champaca & Thailand & Perera et al. 2018a \\
\hline Diaporthe conorum (Desm.) & Diaporthaceae, Sordariomycetes, & cones, seeds & Pinus mugo ‘Galica’ & Canada & Vujanovic et al. 2000 \\
\hline \multirow{2}{*}{$\begin{array}{l}\text { Niessl [as Phomopsis } \\
\text { conorum (Sacc.) Died.] }\end{array}$} & Ascomycota & cones, seeds & Pinus nigra & Canada & Vujanovic et al. 2000 \\
\hline & & cones, seeds & Pinus sylvestris & Canada & Vujanovic et al. 2000 \\
\hline \multirow[t]{2}{*}{ Diaporthe eres Nitschke } & $\begin{array}{l}\text { Diaporthaceae, Sordariomycetes, } \\
\text { Ascomycota }\end{array}$ & fruits & Fraxinus sp. & The Netherlands & Gomes et al. 2013 \\
\hline & & seeds & Abies nordmanniana & Austria & Talgø et al. 2010 \\
\hline
\end{tabular}


Table 2 Continued.

\begin{tabular}{|c|c|c|c|c|c|}
\hline Species & Taxonomy & Substrate & Host & Country & References \\
\hline \multirow{3}{*}{$\begin{array}{l}\text { Diaporthe heveae } \\
\text { [as Phomopsis heveae } \\
\text { (Petch) Boedijn] }\end{array}$} & \multirow{3}{*}{$\begin{array}{l}\text { Diaporthaceae, Sordariomycetes, } \\
\text { Ascomycota }\end{array}$} & seeds & Fraxinus excelsior & Sweden & Cleary et al. 2013 \\
\hline & & seeds & Hevea brasiliensis & Malaysia & $\begin{array}{l}\text { Mittal et al. 1990, } \\
\text { Srivastava 1956b }\end{array}$ \\
\hline & & seeds & Hevea sp. & Malaysia & $\begin{array}{l}\text { Edwards \& Sutherland } \\
\text { 1979, Mittal et al. } 1990\end{array}$ \\
\hline $\begin{array}{l}\text { Diaporthe hongkongensis } \\
\text { R.R. Gomes, Glienke \& } \\
\text { Crous }^{\#}\end{array}$ & $\begin{array}{l}\text { Diaporthaceae, Sordariomycetes, } \\
\text { Ascomycota }\end{array}$ & fruit & Dichroa febrifuga & Hong Kong & Gomes et al. 2013 \\
\hline $\begin{array}{l}\text { Crous }^{\#} \\
\text { Diaporthe insularis Nitschke }\end{array}$ & $\begin{array}{l}\text { Diaporthaceae, Sordariomycetes, } \\
\text { Ascomycota }\end{array}$ & seeds & Quercus spp. & USSR & $\begin{array}{l}\text { Urosevic 1962, Mittal et } \\
\text { al. } 1990\end{array}$ \\
\hline \multirow{3}{*}{$\begin{array}{l}\text { Diaporthe occulta (Fuckel) } \\
\text { Nitschke [as Phomopsis } \\
\text { occulta Traverso] } \\
\text { Diaporthe pseudotsugae } \\
\text { Dissan., Camporesi \& K.D. } \\
\text { Hyde\# }\end{array}$} & $\begin{array}{l}\text { Diaporthaceae, Sordariomycetes, } \\
\text { Ascomycota }\end{array}$ & seeds & Juniperus virginiana & Denmark & $\begin{array}{l}\text { Neergaard 1958, } \\
\text { Anderson 1986a }\end{array}$ \\
\hline & \multirow[b]{2}{*}{$\begin{array}{l}\text { Diaporthaceae, Sordariomycetes, } \\
\text { Ascomycota }\end{array}$} & seeds & \multirow{2}{*}{$\begin{array}{l}\text { Larix spp. } \\
\text { Pseudotsuga } \\
\text { menziesii }\end{array}$} & \multirow{2}{*}{$\begin{array}{l}\text { Denmark } \\
\text { Italy }\end{array}$} & Anderson 1986a \\
\hline & & cones & & & Dissanayake et al. 2017 \\
\hline Diaporthe quercella & Diaporthaceae, Sordariomycetes, & acorns & Quercus robur & Poland & Jankowiak 2008 \\
\hline $\begin{array}{l}\text { [as Phomopsis quercella } \\
\text { (Sacc. \& Roum.) Died.] }\end{array}$ & Ascomycota & seeds & Quercus spp. & USSR & $\begin{array}{l}\text { Urosevic 1983, Mittal et } \\
\text { al. } 1990\end{array}$ \\
\hline Diaporthe rosae Samarakoon & Diaporthaceae, Sordariomycetes, & fruits & Magnolia champaca & Thailand & Perera et al. 2018c \\
\hline \& K.D. Hyde & Ascomycota & fruits & Senna siamea & Thailand & Perera et al. 2018c \\
\hline \multirow[t]{5}{*}{ Diaporthe spp. } & Diaporthaceae, Sordariomycetes, & cones, seeds & Pinus contorta & Canada & Vujanovic et al. 2000 \\
\hline & Ascomycota & cones, seeds & Pinus mugo 'Galica’ & Canada & Vujanovic et al. 2000 \\
\hline & & fruits & Maesa perlarius & Hong Kong & Gomes et al. 2013 \\
\hline & & seeds & Eucalyptus grandis & South Africa & Jimu et al. 2016 \\
\hline & & seeds & Quercus spp. & USSR & $\begin{array}{l}\text { Urosevic 1983, Mittal et } \\
\text { al. } 1990\end{array}$ \\
\hline \multirow{5}{*}{$\begin{array}{l}\text { Diaporthe spp. } \\
\text { [as Phomopsis] }\end{array}$} & & cones & Pinus densiflora & Japan & Kasai et al. 1995 \\
\hline & & fruit cupules & Fagus crenata & Japan & $\begin{array}{l}\text { Fukasawa et al. 2012, } \\
\text { Tateno et al. } 2015\end{array}$ \\
\hline & & fruits & Dichroa febrifuga & Hong Kong & Tang et al. 2003a \\
\hline & & fruits & Psychotria asiatica & Hong Kong & Tang et al. 2003a \\
\hline & & fruits & Sarcandra glabra & Hong Kong & Tang et al. 2003a \\
\hline
\end{tabular}


Table 2 Continued.

\begin{tabular}{|c|c|c|c|c|c|}
\hline Species & Taxonomy & Substrate & Host & Country & References \\
\hline & & pod & $\begin{array}{l}\text { Colophospermum } \\
\text { mopane }\end{array}$ & Southern Africa & Jordaan et. al 2006 \\
\hline & & seed capsules & Eucalyptus grandis & South Africa & Jimu et al. 2016 \\
\hline & & seeds & $\begin{array}{l}\text { Acacia } \\
\text { auriculiformis }\end{array}$ & Philippines & Quiniones 1987 \\
\hline & & seeds & Acer palmatum & South Korea & $\begin{array}{l}\text { Mathur 1974, Anderson } \\
\text { 1986a }\end{array}$ \\
\hline & & seeds & Acer saccharinum & USA & $\begin{array}{l}\text { Friedrich et al. } 1971 \text {, } \\
\text { Mittal et al. } 1990\end{array}$ \\
\hline & & seeds & Acer saccharum & USA & $\begin{array}{l}\text { Janerette 1979, Mittal et } \\
\text { al. } 1990\end{array}$ \\
\hline & & seeds & $\begin{array}{l}\text { Acrocarpus } \\
\text { fraxinifolius }\end{array}$ & Rwanda & $\begin{array}{l}\text { Mathur 1974, Anderson } \\
\text { 1986a }\end{array}$ \\
\hline & & seeds & Alnus glutinosa & Poland & Krol et al. 2015 \\
\hline & & seeds & Azadirachta indica & India & $\begin{array}{l}\text { Mathur 1974, Mittal et } \\
\text { al. } 1990\end{array}$ \\
\hline & & seeds & $\begin{array}{l}\text { Betula } \\
\text { alleghaniensis }\end{array}$ & USA & $\begin{array}{l}\text { Friedrich et al. } 1971 \text {, } \\
\text { Mittal et al. } 1990\end{array}$ \\
\hline & & seeds & Betula papyrifera & USA & $\begin{array}{l}\text { Friedrich et al. } 1971 \text {, } \\
\text { Mittal et al. } 1990\end{array}$ \\
\hline & & seeds & Cassia siamea & $\begin{array}{l}\text { Philippines, } \\
\text { Thailand }\end{array}$ & $\begin{array}{l}\text { Quiniones 1985, 1987, } \\
\text { Mittal et al. } 1990\end{array}$ \\
\hline & & seeds & Cordia alliodora & Colombia & $\begin{array}{l}\text { Mathur 1974, Anderson } \\
\text { 1986a }\end{array}$ \\
\hline & & seed & $\begin{array}{l}\text { Cryptomeria } \\
\text { japonica }\end{array}$ & Japan & Watanabe 2010 \\
\hline & & seeds & $\begin{array}{l}\text { Eucalyptus } \\
\text { citriodora }\end{array}$ & India & Anderson 1986a \\
\hline & & seeds & Eucalyptus grandis & South Africa & Jimu et al. 2016 \\
\hline & & seeds & Fagus sylvatica & Poland & Krol et al. 2015 \\
\hline & & seeds & Fraxinus americana & USA & $\begin{array}{l}\text { Friedrich et al. } 1971 \text {, } \\
\text { Mittal et al. } 1990\end{array}$ \\
\hline & & seeds & Fraxinus excelsior & Sweden & Hayatgheibi 2013 \\
\hline
\end{tabular}


Table 2 Continued.

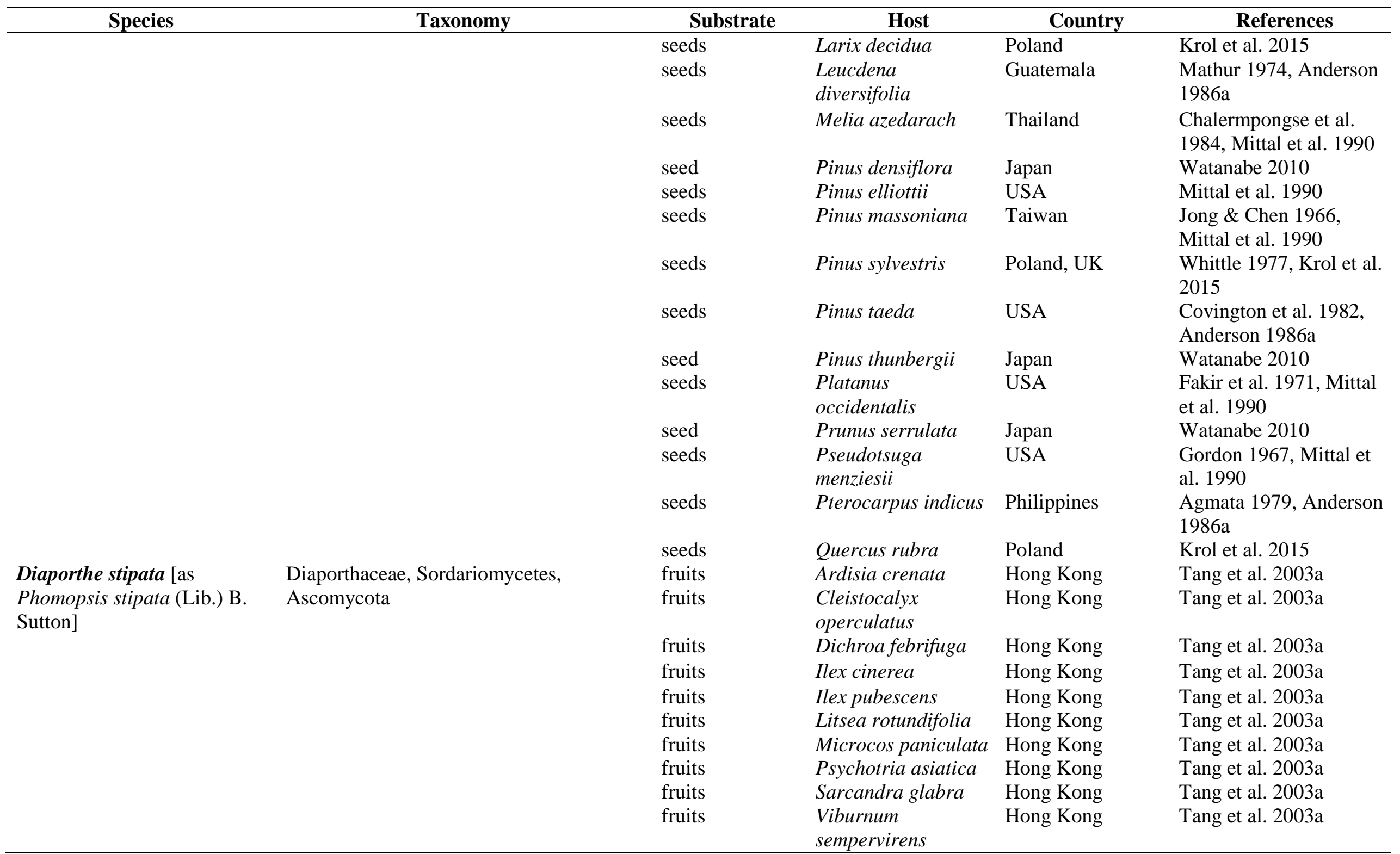


Table 2 Continued.

\begin{tabular}{|c|c|c|c|c|c|}
\hline Species & Taxonomy & Substrate & Host & Country & References \\
\hline $\begin{array}{l}\text { Diaporthe subordinaria } \\
\text { (Desm.) R.R. Gomes, } \\
\text { Glienke \& Crous }\end{array}$ & $\begin{array}{l}\text { Diaporthaceae, Sordariomycetes, } \\
\text { Ascomycota }\end{array}$ & seeds & Plantago lanceolate & New Zealand & Gomes et al. 2013 \\
\hline Diaporthe viticola Nitschke & $\begin{array}{l}\text { Diaporthaceae, Sordariomycetes, } \\
\text { Ascomycota }\end{array}$ & seeds & Fraxinus excelsior & Sweden & Cleary et al. 2013 \\
\hline $\begin{array}{l}\text { Dicoccum asperum (Corda) } \\
\text { Sacc. }\end{array}$ & $\begin{array}{l}\text { Incertae sedis, Incertae sedis, } \\
\text { Ascomycota }\end{array}$ & seeds & Pinus sylvestris & USSR & $\begin{array}{l}\text { Prisyazhnyuk } 1960 \text {, } \\
\text { Mittal et al. } 1990\end{array}$ \\
\hline $\begin{array}{l}\text { Dichotomopilus } \\
\text { dolichotrichus (L.M. Ames) }\end{array}$ & $\begin{array}{l}\text { Chaetomiaceae, Sordariomycetes, } \\
\text { Ascomycota }\end{array}$ & seed & $\begin{array}{l}\text { Cryptomeria } \\
\text { japonica }\end{array}$ & Japan & Watanabe 2010 \\
\hline X.Wei Wang \& Samson [as & & seed & Linum usitatissimum & Canada & Conners 1967 \\
\hline Chaetomium dolichotrichum & & seed & Pinus densiflora & Japan & Watanabe 2010 \\
\hline L.M. Ames] & & seed & Pinus thunbergii & Japan & Watanabe 2010 \\
\hline & & seed & Prunus serrulata & Japan & Watanabe 2010 \\
\hline Dichotomopilus erectus & Chaetomiaceae, Sordariomycetes, & seed & Pinus densiflora & Japan & Watanabe 2010 \\
\hline $\begin{array}{l}\text { (Skolko \& J.W. Groves) } \\
\text { X.Wei Wang \& Samson } \\
\text { [as Chaetomium erectum } \\
\text { Skolko \& J.W. Groves] }\end{array}$ & Ascomycota & seed & Pinus thunbergii & Japan & Watanabe 2010 \\
\hline \multirow{9}{*}{$\begin{array}{l}\text { Dichotomopilus funicola } \\
\text { (Cooke) X.Wei Wang \& } \\
\text { Samson [as Chaetomium } \\
\text { funicola Cooke] }\end{array}$} & Chaetomiaceae, Sordariomycetes, & seeds & Abies nordmanniana & Austria & Talgø et al. 2010 \\
\hline & Ascomycota & seeds & Abies procera & Norway & Talgø et al. 2010 \\
\hline & & seed & Anethum graveolens & Canada & Conners 1967 \\
\hline & & seeds & $\begin{array}{l}\text { Betula } \\
\text { alleghaniensis }\end{array}$ & USA & $\begin{array}{l}\text { Friedrich et al. } 1971 \text {, } \\
\text { Mittal et al. } 1990\end{array}$ \\
\hline & & seed & Eucalyptus globulus & Uruguay & Lupo et al. 2001 \\
\hline & & seed & Fraxinus americana & USA & $\begin{array}{l}\text { Friedrich et al. } 1971 \text {, } \\
\text { Mittal et al. } 1990\end{array}$ \\
\hline & & seeds & Linum usitatissimum & Canada & Conners 1967 \\
\hline & & seeds & Pinus densiflora & Japan & Watanabe 2010 \\
\hline & & seeds & Pinus elliottii & South Africa & Cilliers et al. 1995 \\
\hline $\begin{array}{l}\text { Dichotomopilus indicus } \\
\text { (Corda) X.Wei Wang \& }\end{array}$ & $\begin{array}{l}\text { Chaetomiaceae, Sordariomycetes, } \\
\text { Ascomycota }\end{array}$ & acorns & Quercus robur & Poland & Jankowiak 2008 \\
\hline Samson [as Chaetomium & & seed & Linum usitatissimum & Canada & Conners 1967 \\
\hline indicum Corda] & & seed & Lolium perenne & Scotland & Conners 1967 \\
\hline
\end{tabular}


Table 2 Continued.

\begin{tabular}{|c|c|c|c|c|c|}
\hline Species & Taxonomy & Substrate & Host & Country & References \\
\hline & & seeds & Pinus sylvestris & USSR & $\begin{array}{l}\text { Dolejs 1964, Mittal et al. } \\
1990\end{array}$ \\
\hline $\begin{array}{l}\text { Dichotomopilus reflexus } \\
\text { (Skolko \& J.W. Groves) } \\
\text { X.Wei Wang \& Samson } \\
\text { [as Chaetomium reflexum } \\
\text { Skolko \& J.W. Groves] }\end{array}$ & $\begin{array}{l}\text { Chaetomiaceae, Sordariomycetes, } \\
\text { Ascomycota }\end{array}$ & seed & Pinus densiflora & Japan & Watanabe 2010 \\
\hline $\begin{array}{l}\text { Dictyochaeta fructicola } \\
\text { (M.S. Patil, U.S. Yadav \& } \\
\text { S.D. Patil) Whitton, } \\
\text { McKenzie \& K.D. Hyde } \\
\text { [as Codinaea fruticola M.S. } \\
\text { Patil, U.S. Yadav \& S.D. } \\
\text { Patil] }\end{array}$ & $\begin{array}{l}\text { Chaetosphaeriaceae, Sordariomycetes, } \\
\text { Ascomycota }\end{array}$ & carpus & Butea monosperma & India & Patil et al. 1991 \\
\hline Dictyochaeta sp. & $\begin{array}{l}\text { Chaetosphaeriaceae, Sordariomycetes, } \\
\text { Ascomycota }\end{array}$ & pods & Delonix regia & Thailand & Somrithipol et al. 2002b \\
\hline $\begin{array}{l}\text { Dictyochaetopsis brasiliensis } \\
\text { M. Claduch, Gené, Stchigel } \\
\text { \& Guarro }\end{array}$ & $\begin{array}{l}\text { Chaetosphaeriaceae, Sordariomycetes, } \\
\text { Ascomycota }\end{array}$ & fruit & Papillonaceae plant & Brazil & Calduch et al. 2002 \\
\hline $\begin{array}{l}\text { Dictyocheirospora lithocarpi } \\
\text { Jayasiri, E.B.G. Jones \& } \\
\text { K.D. Hyde }\end{array}$ & $\begin{array}{l}\text { Dictyosporiaceae, Dothideomycetes, } \\
\text { Ascomycota }\end{array}$ & fruit & Lithocarpus sp. & Thailand & Jayasiri et al. 2019 \\
\hline $\begin{array}{l}\text { Dictyocheirospora } \\
\text { nabanheensis Tibpromma \& } \\
\text { K.D. Hyde }\end{array}$ & $\begin{array}{l}\text { Dictyosporiaceae, Dothideomycetes, } \\
\text { Ascomycota }\end{array}$ & pod & Leucaena sp. & Thailand & Jayasiri et al. 2019 \\
\hline Dictyopolyschema & Incertae sedis, Incertae sedis, & seeds & Abies lasiocarpa & Canada, Norway & Talgø et al. 2010 \\
\hline \multirow[t]{2}{*}{ pirozynskii M.B. Ellis } & Ascomycota & seeds & Abies nordmanniana & $\begin{array}{l}\text { Austria, Georgia, } \\
\text { Russia }\end{array}$ & Talgø et al. 2010 \\
\hline & & seeds & Abies procera & Norway & Talgø et al. 2010 \\
\hline $\begin{array}{l}\text { Dictyosporium tetraseriale } \\
\text { Goh, Yanna \& K.D. Hyde }\end{array}$ & $\begin{array}{l}\text { Dictyosporiaceae, Dothideomycetes, } \\
\text { Ascomycota }\end{array}$ & pods & Delonix regia & Thailand & Somrithipol et al. 2002b \\
\hline $\begin{array}{l}\text { Didymella coffeae-arabicae } \\
\text { (Aveskamp, Verkley \& } \\
\text { Gruyter) Qian Chen \& L. Cai }\end{array}$ & $\begin{array}{l}\text { Didymellaceae, Dothideomycetes, } \\
\text { Ascomycota }\end{array}$ & pod & Leucaena sp. & China & Jayasiri et al. 2019 \\
\hline
\end{tabular}


Table 2 Continued.

\begin{tabular}{|c|c|c|c|c|c|}
\hline Species & Taxonomy & Substrate & Host & Country & References \\
\hline $\begin{array}{l}\text { Didymella fabae G.J. Jellis \& } \\
\text { Punith. }\end{array}$ & $\begin{array}{l}\text { Didymellaceae, Dothideomycetes, } \\
\text { Ascomycota }\end{array}$ & seeds & Fraxinus excelsior & Sweden & Hayatgheibi 2013 \\
\hline $\begin{array}{l}\text { Didymella glomerata } \\
\text { (Corda) Qian Chen \& L. Cai }\end{array}$ & $\begin{array}{l}\text { Didymellaceae, Dothideomycetes, } \\
\text { Ascomycota }\end{array}$ & seeds & Quercus robur & Turkey & Oskay et al. 2018 \\
\hline $\begin{array}{l}\text { Didymella glomerata } \\
\text { (Corda) Qian Chen \& L. Cai }\end{array}$ & & seeds & Cedrus deodara & India & $\begin{array}{l}\text { Munjal \& Sharma 1975, } \\
\text { Mittal et al. } 1990\end{array}$ \\
\hline [as Phoma glomerata & & seeds & Larix occidentalis & USA & James et al. 1996 \\
\hline (Corda) Wollenw. \& & & seed & Pinus densiflora & Japan & Watanabe 2010 \\
\hline \multirow[t]{2}{*}{ Hochapfel] } & & seeds & Pinus roxburghii & India & $\begin{array}{l}\text { Munjal \& Sharma 1975, } \\
\text { Mittal et al. } 1990\end{array}$ \\
\hline & & seeds & Pinus wallichiana & India & $\begin{array}{l}\text { Munjal \& Sharma 1975, } \\
\text { Mittal et al. } 1990\end{array}$ \\
\hline $\begin{array}{l}\text { Didymella pomorum } \\
\text { (Thüm.) Qian Chen \& L. Cai } \\
\text { [as Phoma pomorum Thüm.] }\end{array}$ & $\begin{array}{l}\text { Didymellaceae, Dothideomycetes, } \\
\text { Ascomycota }\end{array}$ & seeds & Larix occidentalis & USA & James et al. 1996 \\
\hline $\begin{array}{l}\text { Didymocrea leucaenae } \\
\text { Jayasiri, E.B.G. Jones \& } \\
\text { K.D. Hyde }{ }^{\#}\end{array}$ & $\begin{array}{l}\text { Didymosphaeriaceae, Dothideomycetes, } \\
\text { Ascomycota }\end{array}$ & pod & Leucaena sp. & Thailand & Jayasiri et al. 2019 \\
\hline Didymosphaeria sp. & $\begin{array}{l}\text { Didymosphaeriaceae, Dothideomycetes, } \\
\text { Ascomycota }\end{array}$ & pods & Eucalyptus globulus & Uruguay & Lupo et al. 2001 \\
\hline Dinemasporium strigosum & Chaetosphaeriaceae, Sordariomycetes, & fruits & Ardisia crenata & Hong Kong & Tang et al. 2003a \\
\hline (Pers.) Sacc. & Ascomycota & fruits & Dichroa febrifuga & Hong Kong & Tang et al. 2003a \\
\hline Dioszegia sp. & $\begin{array}{l}\text { Bulleribasidiaceae, Tremellomycetes, } \\
\text { Basidiomycota }\end{array}$ & seeds & Fraxinus excelsior & Sweden & Hayatgheibi 2013 \\
\hline \multirow[t]{2}{*}{ Diplodia conigena Desm. } & $\begin{array}{l}\text { Botryosphaeriaceae, Dothideomycetes, } \\
\text { Ascomycota }\end{array}$ & seeds & Picea abies & USSR & $\begin{array}{l}\text { Prisyazhnyuk } 1960 \text {, } \\
\text { Mittal et al. } 1990\end{array}$ \\
\hline & & seeds & Pinus sylvestris & USSR & $\begin{array}{l}\text { Prisyazhnyuk } 1960 \text {, } \\
\text { Mittal et al. } 1990\end{array}$ \\
\hline Diplodia frumenti Ellis \& & Botryosphaeriaceae, Dothideomycetes, & seed & Pinus densiflora & Japan & Watanabe 2010 \\
\hline Everh. & Ascomycota & seed & Pinus thunbergii & Japan & Watanabe 2010 \\
\hline Diplodia gossypina Cooke & $\begin{array}{l}\text { Botryosphaeriaceae, Dothideomycetes, } \\
\text { Ascomycota }\end{array}$ & seeds & $\begin{array}{l}\text { Pinus elliottii var. } \\
\text { elliottii }\end{array}$ & USA & $\begin{array}{l}\text { Miller \& Bramlett 1979, } \\
\text { Anderson 1986a }\end{array}$ \\
\hline $\begin{array}{l}\text { Diplodia magnoliigena } \\
\text { Jayasiri, E.B.G. Jones \& }\end{array}$ & $\begin{array}{l}\text { Botryosphaeriaceae, Dothideomycetes, } \\
\text { Ascomycota }\end{array}$ & cone & $\begin{array}{l}\text { Magnolia } \\
\text { grandiflora }\end{array}$ & China & Jayasiri et al. 2019 \\
\hline
\end{tabular}


Table 2 Continued.

\begin{tabular}{|c|c|c|c|c|c|}
\hline Species & Taxonomy & Substrate & Host & Country & References \\
\hline \multicolumn{6}{|l|}{ K.D. Hyde } \\
\hline $\begin{array}{l}\text { Diplodia rosulata Gure, } \\
\text { Slippers \& Stenlid }{ }^{\#}\end{array}$ & $\begin{array}{l}\text { Botryosphaeriaceae, Dothideomycetes, } \\
\text { Ascomycota }\end{array}$ & seeds & Prunus africana & Ethiopia & Gure et al. 2005a \\
\hline \multirow[t]{18}{*}{ Diplodia spp. } & Botryosphaeriaceae, Dothideomycetes, & fruits & Dichroa febrifuga & Hong Kong & Tang et al. 2003a \\
\hline & Ascomycota & fruits & Ilex cinerea & Hong Kong & Tang et al. 2003a \\
\hline & & fruits & Litsea rotundifolia & Hong Kong & Tang et al. 2003a \\
\hline & & fruits & $\begin{array}{l}\text { Viburnum } \\
\text { sempervirens }\end{array}$ & Hong Kong & Tang et al. 2003a \\
\hline & & seeds & Acer rubrum & USA & $\begin{array}{l}\text { Friedrich et al. } 1971 \text {, } \\
\text { Mittal et al. } 1990\end{array}$ \\
\hline & & seeds & Acer saccharum & USA & $\begin{array}{l}\text { Friedrich et al. } 1971 \text {, } \\
\text { Mittal et al. } 1990\end{array}$ \\
\hline & & seeds & Fraxinus excelsior & Sweden & Hayatgheibi 2013 \\
\hline & & seeds & $\begin{array}{l}\text { Pinus armandii var. } \\
\text { mastersiana }\end{array}$ & Taiwan & $\begin{array}{l}\text { Jong \& Chen } 1966 \text {, } \\
\text { Mittal et al. } 1990\end{array}$ \\
\hline & & seeds & Pinus elliottii & Taiwan, USA & $\begin{array}{l}\text { Rowan \& Debarr 1974, } \\
\text { Mittal et al. } 1990\end{array}$ \\
\hline & & Seeds & $\begin{array}{l}\text { Pinus elliottii var. } \\
\text { elliottii }\end{array}$ & Georgia & Huang \& Kuhlman 1990 \\
\hline & & seeds & Pinus luchuensis & Taiwan & $\begin{array}{l}\text { Jong \& Chen } 1966 \text {, } \\
\text { Mittal et al. } 1990\end{array}$ \\
\hline & & seeds & Pinus massoniana & Taiwan & $\begin{array}{l}\text { Jong \& Chen } 1966 \text {, } \\
\text { Mittal et al. } 1990\end{array}$ \\
\hline & & seeds & Pinus taeda & USA & Anderson 1986a, b \\
\hline & & seeds & Pinus taiwanensis & Taiwan & $\begin{array}{l}\text { Jong \& Chen 1966, } \\
\text { Mittal et al. } 1990\end{array}$ \\
\hline & & seeds & $\begin{array}{l}\text { Pinus thunbergii } \\
\text { [= Pinus } \\
\text { thunbergiana }]\end{array}$ & Taiwan & $\begin{array}{l}\text { Jong \& Chen } 1966 \text {, } \\
\text { Mittal et al. } 1990\end{array}$ \\
\hline & & seeds & $\begin{array}{l}\text { Platanus } \\
\text { occidentalis }\end{array}$ & USA & $\begin{array}{l}\text { Fakir et al. 1971, Mittal } \\
\text { et al. } 1990\end{array}$ \\
\hline & & seeds & Podocarpus falcatus & Ethiopia & Gure et al. 2005a \\
\hline & & seeds & $\begin{array}{l}\text { Quercus } \\
\text { castaneifolia }\end{array}$ & Iran & Kavosi et al. 2013 \\
\hline
\end{tabular}


Table 2 Continued.

\begin{tabular}{|c|c|c|c|c|c|}
\hline Species & Taxonomy & Substrate & Host & Country & \begin{tabular}{|l|} 
References \\
\end{tabular} \\
\hline & & seed & Sesbania bispinosa & India & Anita et al. 2009 \\
\hline Diplodina sp. & $\begin{array}{l}\text { Gnomoniaceae, Sordariomycetes, } \\
\text { Ascomycota }\end{array}$ & seeds & $\begin{array}{l}\text { Platanus } \\
\text { occidentalis }\end{array}$ & USA & $\begin{array}{l}\text { Fakir et al. 1971, Mittal } \\
\text { et al. } 1990\end{array}$ \\
\hline $\begin{array}{l}\text { Diplodites sweetii Kalgutkar, } \\
\text { Nambudiri \& Tidwell }{ }^{\#}\end{array}$ & Fossil fungus & $\begin{array}{l}\text { permineralized } \\
\text { fruit }\end{array}$ & Viracarpon sp. & India & Kalgutkar et al. 1993 \\
\hline $\begin{array}{l}\text { Discosia querci Jayasiri, } \\
\text { E.B.G. Jones \& K.D. Hyde }\end{array}$ & $\begin{array}{l}\text { Sporocadaceae, Sordariomycetes, } \\
\text { Ascomycota }\end{array}$ & seeds & Quercus sp. & UK & Jayasiri et al. 2018a \\
\hline Discosia sp. & $\begin{array}{l}\text { Sporocadaceae, Sordariomycetes, } \\
\text { Ascomycota }\end{array}$ & seeds & Grevillea robusta & Rwanda & $\begin{array}{l}\text { Mathur 1974, Anderson } \\
\text { 1986a }\end{array}$ \\
\hline $\begin{array}{l}\text { Discotubeufia browneae } \\
\text { Jayasiri, E.B.G. Jones \& } \\
\text { K.D. Hyde }{ }^{\# * *}\end{array}$ & $\begin{array}{l}\text { Tubeufiaceae, Dothideomycetes, } \\
\text { Ascomycota }\end{array}$ & pod & Brownea sp. & Thailand & Jayasiri et al. 2019 \\
\hline $\begin{array}{l}\text { Discula quercina (Cooke) } \\
\text { Sacc. }\end{array}$ & $\begin{array}{l}\text { Gnomoniaceae, Sordariomycetes, } \\
\text { Ascomycota }\end{array}$ & seeds & Quercus robur & Germany & Schroder et al. 2004 \\
\hline \multirow[t]{4}{*}{ Discula spp. } & Gnomoniaceae, Sordariomycetes, & seeds & Acer pseudoplatanus & Poland & Krol et al. 2015 \\
\hline & Ascomycota & seeds & Fagus sylvatica & Poland & Krol et al. 2015 \\
\hline & & seeds & Quercus robur & Poland & Krol et al. 2015 \\
\hline & & seeds & Quercus rubra & Poland & Krol et al. 2015 \\
\hline $\begin{array}{l}\text { Discula umbrinella (Berk. \& } \\
\text { Broome) M. Morelet }\end{array}$ & $\begin{array}{l}\text { Gnomoniaceae, Sordariomycetes, } \\
\text { Ascomycota }\end{array}$ & seeds & Quercus rubra & France & Anderson 1986a \\
\hline Dothidea sp. & $\begin{array}{l}\text { Dothideaceae, Dothideomycetes, } \\
\text { Ascomycota }\end{array}$ & cones, seeds & Pinus spp. & Canada & Vujanovic et al. 2000 \\
\hline $\begin{array}{l}\text { Dothiorella lampangensis } \\
\text { Jayasiri, E.B.G. Jones \& } \\
\text { K.D. Hyde }^{\#}\end{array}$ & $\begin{array}{l}\text { Botryosphaeriaceae, Dothideomycetes, } \\
\text { Ascomycota }\end{array}$ & fruit & Rutaceae plant & Thailand & Jayasiri et al. 2019 \\
\hline $\begin{array}{l}\text { Dothiorella sempervirentis } \\
\text { Abdollahz., Zare \& A.J.L. } \\
\text { Phillips }^{\#}\end{array}$ & $\begin{array}{l}\text { Botryosphaeriaceae, Dothideomycetes, } \\
\text { Ascomycota }\end{array}$ & cones & $\begin{array}{l}\text { Cupressus } \\
\text { sempervirens }\end{array}$ & Iran & $\begin{array}{l}\text { Abdollahzadeh et al. } \\
2014\end{array}$ \\
\hline \multirow[t]{4}{*}{ Dothiorella spp. } & $\begin{array}{l}\text { Botryosphaeriaceae, Dothideomycetes, } \\
\text { Ascomycota }\end{array}$ & seeds & Araucaria excelsa & USA & $\begin{array}{l}\text { Kahn et al. 1965, Mittal } \\
\text { et al. } 1990\end{array}$ \\
\hline & & seeds & Howea forsteriana & North America & Anderson 1986a \\
\hline & & seeds & $\begin{array}{l}\text { Pinus elliottii var. } \\
\text { elliottii }\end{array}$ & Georgia & Huang \& Kuhlman 1990 \\
\hline & & seeds & Podocarpus falcatus & Ethiopia & Gure et al. 2005a \\
\hline
\end{tabular}


Table 2 Continued.

\begin{tabular}{|c|c|c|c|c|c|}
\hline Species & Taxonomy & Substrate & Host & Country & References \\
\hline & & seeds & Pongamia pinnata & India & $\begin{array}{l}\text { Jamaluddin et al. } 1983 \text {, } \\
\text { Mittal et al. } 1990\end{array}$ \\
\hline \multirow[t]{3}{*}{$\begin{array}{l}\text { Drechslera bicolor (Mitra) } \\
\text { Subram. \& B.L. Jain }\end{array}$} & \multirow[t]{3}{*}{$\begin{array}{l}\text { Pleosporaceae, Dothideomycetes, } \\
\text { Ascomycota }\end{array}$} & seed & $\begin{array}{l}\text { Macroptilium } \\
\text { atropurpureum }\end{array}$ & Australia & Nik \& Parbery 1977 \\
\hline & & seeds & Pinus caribaea & Cuba & $\begin{array}{l}\text { Mathur 1974, Anderson } \\
\text { 1986a }\end{array}$ \\
\hline & & seed & Stylosanthes humilis & Australia & Nik \& Parbery 1977 \\
\hline \multirow{3}{*}{$\begin{array}{l}\text { Drechslera dictyoides } \\
\text { (Drechsler) Shoemaker } \\
\text { Drechslera halodes } \\
\text { (Drechsler) Subram. \& B.L. } \\
\text { Jain }\end{array}$} & \multirow{3}{*}{$\begin{array}{l}\text { Pleosporaceae, Dothideomycetes, } \\
\text { Ascomycota } \\
\text { Pleosporaceae, Dothideomycetes, } \\
\text { Ascomycota }\end{array}$} & seed & Festuca elatior & Canada & Conners 1967 \\
\hline & & seeds & Cosmos sulphureus & $\begin{array}{l}\text { imported to } \\
\text { Taiwan }\end{array}$ & Wu et al. 2006 \\
\hline & & seeds & Eucalyptus sp. & India & $\begin{array}{l}\text { Reddy et al. 1982, Mittal } \\
\text { et al. } 1990\end{array}$ \\
\hline \multirow{2}{*}{$\begin{array}{l}\text { Drechslera maydis (Y. Nisik. } \\
\text { \& C. Miyake) Subram. \& } \\
\text { B.L. Jain }\end{array}$} & \multirow[t]{2}{*}{$\begin{array}{l}\text { Pleosporaceae, Dothideomycetes, } \\
\text { Ascomycota }\end{array}$} & seeds & Cosmos sulphureus & $\begin{array}{l}\text { imported to } \\
\text { Taiwan }\end{array}$ & Wu et al. 2006 \\
\hline & & seeds & Pinus insularis & Philippines & $\begin{array}{l}\text { Agmata 1979, Anderson } \\
\text { 1986a }\end{array}$ \\
\hline \multirow{3}{*}{$\begin{array}{l}\text { Drechslera rostrata } \\
\text { (Drechsler) M.J. Richardson } \\
\text { \& E.M. Fraser }\end{array}$} & \multirow[t]{3}{*}{$\begin{array}{l}\text { Pleosporaceae, Dothideomycetes, } \\
\text { Ascomycota }\end{array}$} & seeds & $\begin{array}{l}\text { Chamaecyparis } \\
\text { obtusa }\end{array}$ & South Korea & $\begin{array}{l}\text { Mathur 1974, Anderson } \\
\text { 1986a }\end{array}$ \\
\hline & & seeds & Eucalyptus sp. & India & $\begin{array}{l}\text { Saxena 1985, Mittal et } \\
\text { al. } 1990\end{array}$ \\
\hline & & seeds & Melia azedarach & Thailand & $\begin{array}{l}\text { Chalermpongse et al. } \\
\text { 1984, Mittal et al. } 1990\end{array}$ \\
\hline $\begin{array}{l}\text { Drechslera siccans } \\
\text { (Drechsler) Shoemaker }\end{array}$ & $\begin{array}{l}\text { Pleosporaceae, Dothideomycetes, } \\
\text { Ascomycota }\end{array}$ & seed & Lolium perenne & Canada & Conners 1967 \\
\hline $\begin{array}{l}\text { Drechslera sorokiniana } \\
\text { (Shoemaker) Subram. \& B.L. } \\
\text { Jain }\end{array}$ & $\begin{array}{l}\text { Pleosporaceae, Dothideomycetes, } \\
\text { Ascomycota }\end{array}$ & seeds & Azadirachta indica & India & $\begin{array}{l}\text { Mathur 1974, Mittal et } \\
\text { al. } 1990\end{array}$ \\
\hline \multirow[t]{3}{*}{ Drechslera spp. } & \multirow{3}{*}{$\begin{array}{l}\text { Pleosporaceae, Dothideomycetes, } \\
\text { Ascomycota }\end{array}$} & seeds & Eucalyptus grandis & Uruguay & Anderson 1986a \\
\hline & & seeds & $\begin{array}{l}\text { Jacaranda } \\
\text { mimosifolia }\end{array}$ & India & $\begin{array}{l}\text { Mathur 1974, Anderson } \\
\text { 1986a }\end{array}$ \\
\hline & & seeds & $\begin{array}{l}\text { Pinus elliottii var. } \\
\text { elliottii }\end{array}$ & Uruguay & Anderson 1986a \\
\hline
\end{tabular}


Table 2 Continued.

\begin{tabular}{|c|c|c|c|c|c|}
\hline Species & Taxonomy & Substrate & Host & Country & References \\
\hline & & seeds & Shorea robusta & India & $\begin{array}{l}\text { Mittal \& Sharma 1982b, } \\
\text { Mittal et al. } 1990\end{array}$ \\
\hline & & seeds & Tectona grandis & India & Mohanan et al. 2005 \\
\hline \multirow{2}{*}{$\begin{array}{l}\text { Drechslera tetramera } \\
\text { (McKinney) Subram. \& B.L. } \\
\text { Jain }\end{array}$} & \multirow{2}{*}{$\begin{array}{l}\text { Pleosporaceae, Dothideomycetes, } \\
\text { Ascomycota }\end{array}$} & seeds & Holarrhena & Thailand & Chalermpongse et al. \\
\hline & & seeds & $\begin{array}{l}\text { Leucaena } \\
\text { leucocephala }\end{array}$ & Philippines & $\begin{array}{l}\text { Agmata 1979, Anderson } \\
\text { 1986a }\end{array}$ \\
\hline $\begin{array}{l}\text { Drepanopeziza brunnea } \\
\text { (Ellis \& Everh.) Rossman \& } \\
\text { W.C. Allen [Marssonina } \\
\text { brunnea (Ellis \& Everh.) } \\
\text { Magnus] }\end{array}$ & $\begin{array}{l}\text { Drepanopezizaceae, Leotiomycetes, } \\
\text { Ascomycota }\end{array}$ & seeds & Populus sp. & New Zealand & $\begin{array}{l}\text { Spiers \& Wenilam 1983, } \\
\text { Mittal et al. } 1990\end{array}$ \\
\hline \multirow[t]{2}{*}{ Echinobotryum atrum Corda } & \multirow[t]{2}{*}{$\begin{array}{l}\text { Microascaceae, Sordariomycetes, } \\
\text { Ascomycota }\end{array}$} & seeds & Betula verrucosa & USSR & $\begin{array}{l}\text { Kozlowska 1968, Mittal } \\
\text { et al. } 1990\end{array}$ \\
\hline & & seeds & Quercus pedunculata & USSR & $\begin{array}{l}\text { Kozlowska 1968, Mittal } \\
\text { et al. } 1990\end{array}$ \\
\hline $\begin{array}{l}\text { Endochytrium digitatum } \\
\text { Karling }\end{array}$ & $\begin{array}{l}\text { Endochytriaceae, Chytridiomycetes, } \\
\text { Chytridiomycota }\end{array}$ & seeds & Atropa belladonna & $\begin{array}{l}\text { Poland or/ } \\
\text { imported to Poland }\end{array}$ & Czeczuga et al. 2009 \\
\hline \multirow[t]{2}{*}{$\begin{array}{l}\text { Endochytrium ramosum } \\
\text { Sparrow }\end{array}$} & \multirow[t]{2}{*}{$\begin{array}{l}\text { Endochytriaceae, Chytridiomycetes, } \\
\text { Chytridiomycota }\end{array}$} & seeds & Cornus capitata & $\begin{array}{l}\text { Poland or/ } \\
\text { imported to Poland }\end{array}$ & Czeczuga et al. 2009 \\
\hline & & seeds & Ilex verticillata & $\begin{array}{l}\text { Poland or/ } \\
\text { imported to Poland }\end{array}$ & Czeczuga et al. 2009 \\
\hline $\begin{array}{l}\text { Endoconidioma rosae- } \\
\text { hissaricae Wanas., Gafforov, } \\
\text { E.B.G. Jones \& K.D. Hyde }{ }^{\#}\end{array}$ & $\begin{array}{l}\text { Dothideaceae, Dothideomycetes, } \\
\text { Ascomycota }\end{array}$ & fruits & Rosa canina & Uzbekistan & Wanasinghe et al. 2018 \\
\hline Endophragmia sp. & $\begin{array}{l}\text { Incertae sedis, Incertae sedis, } \\
\text { Ascomycota }\end{array}$ & cones & Pinus densiflora & Japan & Kasai et al. 1995 \\
\hline $\begin{array}{l}\text { Endophragmiella bukkensis } \\
\text { Révay }^{\#}\end{array}$ & $\begin{array}{l}\text { Helminthosphaeriaceae, Sordariomycetes, } \\
\text { Ascomycota }\end{array}$ & cone & Picea abies & Hungary & Révay 1987 \\
\hline $\begin{array}{l}\text { Endophragmiella lignicola } \\
\text { S. Hughes }\end{array}$ & $\begin{array}{l}\text { Helminthosphaeriaceae, Sordariomycetes, } \\
\text { Ascomycota }\end{array}$ & fruit cupules & Fagus sylvatica & UK & Kirk 1986 \\
\hline $\begin{array}{l}\text { Endoraecium kauaianum } \\
\text { Berndt }^{\#}\end{array}$ & $\begin{array}{l}\text { Raveneliaceae, Pucciniomycetes, } \\
\text { Basidiomycota }\end{array}$ & fruits & Acacia koa & USA & Berndt 2011 \\
\hline
\end{tabular}


Table 2 Continued.

\begin{tabular}{|c|c|c|c|c|c|}
\hline Species & Taxonomy & Substrate & Host & Country & References \\
\hline $\begin{array}{l}\text { Entoloma alboproximum } \\
\text { (Largent, Aime \& T.W. } \\
\text { Henkel) Mešić \& Tkalčec\# } \\
\text { [as Nolanea alboproxima } \\
\text { Largent, Aime \& T.W. } \\
\text { Henkel] }\end{array}$ & $\begin{array}{l}\text { Entolomataceae, Agaricomycetes, } \\
\text { Basidiomycota }\end{array}$ & seed husks & Dicymbe sp. & Guyana & Henkel et al. 2014 \\
\hline $\begin{array}{l}\text { Epichlöe canadensis } \\
\text { Charlton \& C.A. Young }\end{array}$ & $\begin{array}{l}\text { Clavicipitaceae, Sordariomycetes, } \\
\text { Ascomycota }\end{array}$ & seeds & Elymus canadensis & USA & Charlton et al. 2012 \\
\hline Epichlöe typhina (Pers.) & Clavicipitaceae, Sordariomycetes, & seed & Festuca arundinacea & USA & Rycyk Jr \& Sharpe 1984 \\
\hline Brockm. & Ascomycota & seed & Festuca rubra & New Zealand & Latch et al. 1984 \\
\hline Epicoccum neglectum & Didymellaceae, Dothideomycetes, & seed & Bromus inermis & Canada & Conners 1967 \\
\hline Desm. & Ascomycota & seed & Festuca rubra & Canada & Conners 1967 \\
\hline & & seed & Lolium perenne & Canada & Conners 1967 \\
\hline & & seed & Phleum pratense & Canada & Conners 1967 \\
\hline & & seed & Poa compressa & Canada & Conners 1967 \\
\hline \multirow[t]{16}{*}{ Epicoccum nigrum Link } & Didymellaceae, Dothideomycetes, & acorns & Quercus robur & Poland & Jankowiak 2008 \\
\hline & Ascomycota & cones, seeds & Pinus nigra & Canada & Vujanovic et al. 2000 \\
\hline & & cones, seeds & Pinus sylvestris & Canada & Vujanovic et al. 2000 \\
\hline & & fruit cupules & Fagus crenata & Japan & Fukasawa et al. 2012 \\
\hline & & seeds & Acer pseudoplatanus & Poland & Krol et al. 2015 \\
\hline & & seed & Agropyron cristatum & Canada & Conners 1967 \\
\hline & & seeds & Alnus glutinosa & Poland & Krol et al. 2015 \\
\hline & & seed & $\begin{array}{l}\text { Apium graveolens } \\
\text { var. dulce }\end{array}$ & USA & Conners 1967 \\
\hline & & seed & $\begin{array}{l}\text { Asparagus officinalis } \\
\text { var. altilis }\end{array}$ & Canada & Conners 1967 \\
\hline & & seeds & Betula pendula & Poland & Krol et al. 2015 \\
\hline & & seed & Dactylis glomerata & Canada & Conners 1967 \\
\hline & & seed & Elymus virginicus & Canada & Conners 1967 \\
\hline & & seeds & Fagus sylvatica & Poland & Krol et al. 2015 \\
\hline & & seed & Festuca elatior & Canada & Conners 1967 \\
\hline & & seeds & Larix decidua & Poland & Krol et al. 2015 \\
\hline & & seed & Linum usitatissimum & Canada & Conners 1967 \\
\hline
\end{tabular}


Table 2 Continued.

\begin{tabular}{|c|c|c|c|c|c|}
\hline Species & Taxonomy & Substrate & Host & Country & References \\
\hline & & seed & $\begin{array}{l}\text { Macroptilium } \\
\text { atropurpureum }\end{array}$ & Australia & Nik \& Parbery 1977 \\
\hline & & seeds & Pinus caribaea & $\begin{array}{l}\text { Belize [as British } \\
\text { Honduras] }\end{array}$ & Hocking 1968 \\
\hline & & seeds & Pinus sylvestris & Poland & Krol et al. 2015 \\
\hline & & seeds & Pinus taeda & USA & $\begin{array}{l}\text { Mason \& van Arsdel } \\
\text { 1978, Anderson 1986a }\end{array}$ \\
\hline & & seeds & $\begin{array}{l}\text { Platanus } \\
\text { occidentalis }\end{array}$ & USA & $\begin{array}{l}\text { Fakir et al. 1971, Mittal } \\
\text { et al. } 1990\end{array}$ \\
\hline & & seeds & Poa annua & Pakistan & Lodhi \& Naeem 1955 \\
\hline & & seeds & Quercus robur & Germany, Poland & $\begin{array}{l}\text { Krol et al. 2015, } \\
\text { Schroder et al. } 2004\end{array}$ \\
\hline & & seed & Setaria viridis & Canada & Conners 1967 \\
\hline & & seed & $\begin{array}{l}\text { Stylosanthes } \\
\text { guianensis }\end{array}$ & Australia & Nik \& Parbery 1977 \\
\hline & & seed & $\begin{array}{l}\text { Trifolium } \\
\text { semipilosum }\end{array}$ & Australia & Nik \& Parbery 1977 \\
\hline $\begin{array}{l}\text { Epicoccum poae Qian Chen, } \\
\text { Crous \& L. Cai" }\end{array}$ & $\begin{array}{l}\text { Didymellaceae, Dothideomycetes, } \\
\text { Ascomycota }\end{array}$ & seeds & Poa annua & USA & Chen et al. 2017 \\
\hline Epicoccum purpurascens & Didymellaceae, Dothideomycetes, & cones & Pinus sylvestris & UK & Whittle 1977 \\
\hline \multirow[t]{9}{*}{ Ehrenb. ex Schltdl. } & Ascomycota & seeds & Abies lasiocarpa & Canada, Norway & Talgø et al. 2010 \\
\hline & & seeds & Abies nordmanniana & Austria, Russia & Talgø et al. 2010 \\
\hline & & seeds & Abies procera & Norway & Talgø et al. 2010 \\
\hline & & seeds & Acer rubrum & USA & $\begin{array}{l}\text { Friedrich et al. } 1971 \text {, } \\
\text { Mittal et al. } 1990\end{array}$ \\
\hline & & seeds & Acer saccharinum & USA & $\begin{array}{l}\text { Friedrich et al. } 1971 \text {, } \\
\text { Mittal et al. } 1990\end{array}$ \\
\hline & & seeds & $\begin{array}{l}\text { Betula } \\
\text { alleghaniensis }\end{array}$ & USA & $\begin{array}{l}\text { Friedrich et al. } 1971 \text {, } \\
\text { Mittal et al. } 1990\end{array}$ \\
\hline & & seeds & Betula papyrifera & USA & $\begin{array}{l}\text { Friedrich et al. } 1971 \text {, } \\
\text { Mittal et al. } 1990\end{array}$ \\
\hline & & seeds & Casuarina spp. & India & Sahai \& Otra 1982 \\
\hline & & seeds & Cedrus deodara & India & Mittal 1983 \\
\hline
\end{tabular}


Table 2 Continued.

\begin{tabular}{|c|c|c|c|c|c|}
\hline Species & Taxonomy & Substrate & Host & Country & References \\
\hline & & seeds, pods & Eucalyptus globulus & Uruguay & Lupo et al. 2001 \\
\hline & & seeds & Fraxinus americana & USA & $\begin{array}{l}\text { Friedrich et al. } 1971 \text {, } \\
\text { Mittal et al. } 1990\end{array}$ \\
\hline & & seeds & Picea glauca & Canada & Mittal \& Wang 1987 \\
\hline & & seed & Pinus densiflora & Japan & Watanabe 2010 \\
\hline & & seeds & Pinus strobus & Canada & Mittal \& Wang 1987 \\
\hline & & seeds & Pinus sylvestris & UK & Whittle 1977 \\
\hline & & seeds & $\begin{array}{l}\text { Pseudotsuga } \\
\text { menziesii }\end{array}$ & USA & $\begin{array}{l}\text { Gordon 1967, Mittal et } \\
\text { al. } 1990\end{array}$ \\
\hline & & seeds & Quercus alba & USA & $\begin{array}{l}\text { Vozzo 1984, Anderson } \\
\text { 1986a }\end{array}$ \\
\hline & & seeds & Quercus falcata & USA & $\begin{array}{l}\text { Vozzo 1984, Anderson } \\
\text { 1986a }\end{array}$ \\
\hline & & seeds & Quercus nigra & USA & $\begin{array}{l}\text { Vozzo 1984, Anderson } \\
\text { 1986a }\end{array}$ \\
\hline & & seeds & Quercus phellos & USA & $\begin{array}{l}\text { Vozzo 1984, Anderson } \\
\text { 1986a }\end{array}$ \\
\hline & & seeds & Thuja spp. & India & Sahai \& Otra 1982 \\
\hline \multirow[t]{6}{*}{ Epicoccum spp. } & $\begin{array}{l}\text { Didymellaceae, Dothideomycetes, } \\
\text { Ascomycota }\end{array}$ & seeds & Acer saccharum & USA & $\begin{array}{l}\text { Janerette 1979, Mittal et } \\
\text { al. } 1990\end{array}$ \\
\hline & & seeds & $\begin{array}{l}\text { Araucaria } \\
\text { heterophylla }\end{array}$ & Egypt & $\begin{array}{l}\text { Kamara et al. } 1981 \text {, } \\
\text { Mittal et al. } 1990\end{array}$ \\
\hline & & seeds & $\begin{array}{l}\text { Cryptomeria } \\
\text { japonica }\end{array}$ & Japan & Watanabe 2010 \\
\hline & & seeds & Fraxinus excelsior & Sweden & Hayatgheibi 2013 \\
\hline & & seed & Picea sitchensis & UK & $\begin{array}{l}\text { Salt 1964, Mittal et al. } \\
1990\end{array}$ \\
\hline & & seeds & $\begin{array}{l}\text { Pinus elliottii var. } \\
\text { elliottii }\end{array}$ & USA & Fraedrich \& Miller 1995 \\
\hline $\begin{array}{l}\text { Epochnium monilioides } \\
\text { Link }\end{array}$ & $\begin{array}{l}\text { Sclerotiniaceae, Leotiomycetes, } \\
\text { Ascomycota }\end{array}$ & seeds & Quercus spp. & USSR & $\begin{array}{l}\text { Urosevic 1962, Mittal et } \\
\text { al. } 1990\end{array}$ \\
\hline Epulorhiza sp. & $\begin{array}{l}\text { Tulasnellaceae, Agaricomycetes, } \\
\text { Basidiomycota }\end{array}$ & seeds & Cypripedium acaule & Canada & Zelmer et al. 1996 \\
\hline
\end{tabular}


Table 2 Continued.

\begin{tabular}{|c|c|c|c|c|c|}
\hline Species & Taxonomy & Substrate & Host & Country & References \\
\hline & & seeds & $\begin{array}{l}\text { Cypripedium } \\
\text { calceolus }\end{array}$ & Canada & Zelmer et al. 1996 \\
\hline & & seeds & $\begin{array}{l}\text { Platanthera } \\
\text { hyperborea }\end{array}$ & Canada & Zelmer et al. 1996 \\
\hline & & seeds & $\begin{array}{l}\text { Platanthera } \\
\text { praeclara }\end{array}$ & Canada & Zelmer et al. 1996 \\
\hline & & seeds & $\begin{array}{l}\text { Spiranthes } \\
\text { magnicamporum }\end{array}$ & Canada & Zelmer et al. 1996 \\
\hline $\begin{array}{l}\text { Eriospora leucostoma Berk. } \\
\text { \& Broome }\end{array}$ & $\begin{array}{l}\text { Stictidaceae, Lecanoromycetes, } \\
\text { Ascomycota }\end{array}$ & fruits & Sarcandra glabra & Hong Kong & Tang et al. 2003a \\
\hline $\begin{array}{l}\text { Ernakulamia krabiensis } \\
\text { Jayasiri, E.B.G. Jones \& } \\
\text { K.D. Hyde }{ }^{\#}\end{array}$ & $\begin{array}{l}\text { Tetraplosphaeriaceae, Dothideomycetes, } \\
\text { Ascomycota }\end{array}$ & pods & Acacia sp. & Thailand & Jayasiri et al. 2019 \\
\hline $\begin{array}{l}\text { Erysiphe diervillae var. } \\
\text { chasanensis V.P. Gelyuta }\end{array}$ & $\begin{array}{l}\text { Erysiphaceae, Leotiomycetes, } \\
\text { Ascomycota }\end{array}$ & fruit & Weigela praecox & Japan & Gelyuta 1990 \\
\hline Erysiphe sp. & $\begin{array}{l}\text { Erysiphaceae, Leotiomycetes, } \\
\text { Ascomycota }\end{array}$ & seeds & Pinus taeda & USA & Anderson 1986a \\
\hline Eudarluca biconica Katum. & $\begin{array}{l}\text { Phaeosphaeriaceae, Dothideomycetes, } \\
\text { Ascomycota }\end{array}$ & seed & Canavalia cathartica & India & Anita \& Sridhar 2009 \\
\hline Eurotium chevalieri L. & Aspergillaceae, Eurotiomycetes, & seed & Prunus serrulata & Japan & Watanabe 2010 \\
\hline Mangin & Ascomycota & pod & Sesbania bispinosa & India & Anita et al. 2009 \\
\hline $\begin{array}{l}\text { Eurotium rubrum Jos. } \\
\text { König, Spieck. \& W. Bremer }\end{array}$ & $\begin{array}{l}\text { Aspergillaceae, Eurotiomycetes, } \\
\text { Ascomycota }\end{array}$ & seeds & $\begin{array}{l}\text { Quercus } \\
\text { castaneifolia }\end{array}$ & Iran & Kavosi et al. 2013 \\
\hline Eurotium spp. & $\begin{array}{l}\text { Aspergillaceae, Eurotiomycetes, } \\
\text { Ascomycota }\end{array}$ & $\begin{array}{l}\text { pods } \\
\text { seeds }\end{array}$ & $\begin{array}{l}\text { Delonix regia } \\
\text { Pinus caribaea }\end{array}$ & $\begin{array}{l}\text { Thailand } \\
\text { Belize [as British } \\
\text { Honduras] }\end{array}$ & $\begin{array}{l}\text { Somrithipol et al. 2002b } \\
\text { Hocking } 1968\end{array}$ \\
\hline $\begin{array}{l}\text { Exobasidium symploci- } \\
\text { japonicae var. carpogenum } \\
\text { Nagao \& S. Ogawa }{ }^{\#}\end{array}$ & $\begin{array}{l}\text { Exobasidiaceae, Exobasidiomycetes, } \\
\text { Basidiomycota }\end{array}$ & fruit & Symplocos lucida & Japan & Nagao et al. 2003 \\
\hline Exophiala spp. & $\begin{array}{l}\text { Herpotrichiellaceae, Eurotiomycetes, } \\
\text { Ascomycota }\end{array}$ & seeds & Eucalyptus grandis & South Africa & Jimu et al. 2016 \\
\hline $\begin{array}{l}\text { Exserohilum rostratum } \\
\text { (Drechsler) K.J. Leonard \& }\end{array}$ & $\begin{array}{l}\text { Pleosporaceae, Dothideomycetes, } \\
\text { Ascomycota }\end{array}$ & seeds & Pinus elliottii & South Africa & Cilliers et al. 1995 \\
\hline
\end{tabular}


Table 2 Continued.

\begin{tabular}{|c|c|c|c|c|c|}
\hline Species & Taxonomy & Substrate & Host & Country & References \\
\hline \multicolumn{6}{|l|}{ Suggs } \\
\hline $\begin{array}{l}\text { Fairmaniella leprosa } \\
\text { (Fairm.) Petr. \& Syd. }\end{array}$ & $\begin{array}{l}\text { Incertae sedis, Incertae sedis, } \\
\text { Ascomycota }\end{array}$ & pods & Eucalyptus globulus & Uruguay & Lupo et al. 2001 \\
\hline Filobasidium wieringae (Á. & Filobasidiaceae, Tremellomycetes, & seeds & Fraxinus excelsior & Sweden & Hayatgheibi 2013 \\
\hline $\begin{array}{l}\text { Fonseca, Scorzetti \& Fell) } \\
\text { Xin Zhan Liu, F.Y. Bai, M. } \\
\text { Groenew. \& Boekhout } \\
\text { [as Cryptococcus wieringae } \\
\text { Á. Fonseca, Scorzetti \& Fell] }\end{array}$ & Basidiomycota & seeds & Shorea talura & Malaysia & Mittal et al. 1990 \\
\hline $\begin{array}{l}\text { Fitzpatrickella operculata } \\
\text { Benny, Samuelson \& Kimbr.\# }\end{array}$ & $\begin{array}{l}\text { Coryneliaceae, Eurotiomycetes, } \\
\text { Ascomycota }\end{array}$ & fruits & Drimys confertifolia & Chile & Benny et al. 1985 \\
\hline $\begin{array}{l}\text { Flabellascoma minimum A. } \\
\text { Hashim., K. Hiray. \& Kaz. } \\
\text { Tanaka }\end{array}$ & $\begin{array}{l}\text { Lophiostomataceae, Dothideomycetes, } \\
\text { Ascomycota }\end{array}$ & pod & $\begin{array}{l}\text { Leucaena } \\
\text { leucocephala }\end{array}$ & Thailand & Jayasiri et al. 2019 \\
\hline Fumago vagans Pers. & $\begin{array}{l}\text { Incertae sedis, Incertae sedis, } \\
\text { Ascomycota }\end{array}$ & cone & Humulus lupulus & Canada & Conners 1967 \\
\hline \multirow{12}{*}{$\begin{array}{l}\text { Fusariella indica R.Y. Roy } \\
\text { \& B. Rai } \\
\text { Fusarium acuminatum Ellis } \\
\text { \& Everh. }\end{array}$} & $\begin{array}{l}\text { Bionectriaceae, Sordariomycetes, } \\
\text { Ascomycota }\end{array}$ & seeds & Dalbergia sissoo & India & $\begin{array}{l}\text { Mittal \& Sharma 1981b, } \\
\text { Mittal et al. } 1990\end{array}$ \\
\hline & Nectriaceae, Sordariomycetes, & cones & Pinus ponderosa & USA & James 1995 \\
\hline & Ascomycota & cones & $\begin{array}{l}\text { Pseudotsuga } \\
\text { menziesii var. glauca }\end{array}$ & USA & James 1995 \\
\hline & & seed & Acer negundo & Canada & Conners 1967 \\
\hline & & seed & Agropyron cristatum & Canada & Conners 1967 \\
\hline & & seed & $\begin{array}{l}\text { Apium graveolens } \\
\text { var. dulce }\end{array}$ & USA & Conners 1967 \\
\hline & & seed & Festuca rubra & Canada & Conners 1967 \\
\hline & & seeds & Larix occidentalis & USA & James et al. 1996 \\
\hline & & seed & Linum usitatissimum & Canada & Conners 1967 \\
\hline & & seeds & Pinus elliottii & Argentina & Lori \& Salerno 2003 \\
\hline & & seeds & Pinus ponderosa & USA & James 1985a, 1995 \\
\hline & & $\begin{array}{l}\text { seeds } \\
\text { seeds }\end{array}$ & $\begin{array}{l}\text { Pinus taeda } \\
\text { Pseudotsuga } \\
\text { menziesii } \\
\end{array}$ & $\begin{array}{l}\text { Argentina } \\
\text { Canada, USA }\end{array}$ & $\begin{array}{l}\text { Lori \& Salerno } 2003 \\
\text { James 1986a, Axelrood } \\
\text { et al. } 1995\end{array}$ \\
\hline
\end{tabular}


Table 2 Continued.

\begin{tabular}{|c|c|c|c|c|c|}
\hline Species & Taxonomy & Substrate & Host & Country & References \\
\hline & & seeds & $\begin{array}{l}\text { Pseudotsuga } \\
\text { menziesii var. glauca }\end{array}$ & USA & James 1995 \\
\hline $\begin{array}{l}\text { Fusarium anthophilum (A. } \\
\text { Braun) Wollenw. }\end{array}$ & $\begin{array}{l}\text { Nectriaceae, Sordariomycetes, } \\
\text { Ascomycota }\end{array}$ & acorns & Quercus robur & Poland & Jankowiak 2008 \\
\hline \multirow{3}{*}{$\begin{array}{l}\text { Fusarium arthrosporioides } \\
\text { Sherb. }\end{array}$} & Nectriaceae, Sordariomycetes, & seed & Lolium perenne & Canada & Conners 1967 \\
\hline & Ascomycota & seeds & Picea excelsa & USSR & $\begin{array}{l}\text { Urosevic 1961, } \\
\text { Anderson 1986a }\end{array}$ \\
\hline & & seeds & Pinus sylvestris & USSR & $\begin{array}{l}\text { Urosevic 1961, } \\
\text { Anderson 1986a }\end{array}$ \\
\hline \multirow{13}{*}{$\begin{array}{l}\text { Fusarium avenaceum (Fr.) } \\
\text { Sacc. [as Gibberella } \\
\text { avenacea R.J. Cook] }\end{array}$} & Nectriaceae, Sordariomycetes, & acorns & Quercus robur & Poland & Jankowiak 2008 \\
\hline & Ascomycota & seeds & Abies sibirica & USSR & $\begin{array}{l}\text { Prisyazhnyuk } 1960 \text {, } \\
\text { Mittal et al. } 1990\end{array}$ \\
\hline & & seeds & Acer pseudoplatanus & Poland & Krol et al. 2015 \\
\hline & & seed & $\begin{array}{l}\text { Asparagus officinalis } \\
\text { var. altilis }\end{array}$ & Canada & Conners 1967 \\
\hline & & seeds & Fraxinus excelsior & Sweden & Cleary et al. 2013 \\
\hline & & seeds & Larix sibirica & USSR & $\begin{array}{l}\text { Prisyazhnyuk } 1960 \text {, } \\
\text { Mittal et al. } 1990\end{array}$ \\
\hline & & seed & Linum usitatissimum & Canada & Conners 1967 \\
\hline & & seed & Lolium perenne & Scotland & Conners 1967 \\
\hline & & seeds & Picea abies & Italy, USSR & $\begin{array}{l}\text { Prisyazhnyuk 1960, } \\
\text { Mittal et al. 1990, Motta } \\
\text { et al. } 1996\end{array}$ \\
\hline & & seeds & Pinus sylvestris & USSR & $\begin{array}{l}\text { Prisyazhnyuk } 1960 \text {, } \\
\text { Mittal et al. } 1990\end{array}$ \\
\hline & & seeds & $\begin{array}{l}\text { Pseudotsuga } \\
\text { menziesii }\end{array}$ & Canada & Axelrood et al. 1995 \\
\hline & & seeds & Quercus robur & Poland & Krol et al. 2015 \\
\hline & & seeds & Quercus spp. & USSR & Mittal et al. 1990 \\
\hline $\begin{array}{l}\text { Fusarium bulbigenum } \\
\text { Cooke \& Massee }\end{array}$ & $\begin{array}{l}\text { Nectriaceae, Sordariomycetes, } \\
\text { Ascomycota }\end{array}$ & seeds & Quercus spp. & USSR & $\begin{array}{l}\text { Urosevic 1962, Mittal et } \\
\text { al. } 1990\end{array}$ \\
\hline $\begin{array}{l}\text { Fusarium bulbigenum var. } \\
\text { blasticola (Rostr.) Wollenw. }\end{array}$ & $\begin{array}{l}\text { Nectriaceae, Sordariomycetes, } \\
\text { Ascomycota }\end{array}$ & seeds & Cedrus deodara & India & $\begin{array}{l}\text { Munjal \& Sharma 1975, } \\
\text { Mittal et al. } 1990\end{array}$ \\
\hline
\end{tabular}


Table 2 Continued.

\begin{tabular}{|c|c|c|c|c|c|}
\hline Species & Taxonomy & Substrate & Host & Country & References \\
\hline & & seeds & Pinus roxburghii & India & $\begin{array}{l}\text { Munjal \& Sharma 1975, } \\
\text { Mittal et al. } 1990\end{array}$ \\
\hline & & seeds & Pinus wallichiana & India & $\begin{array}{l}\text { Munjal \& Sharma 1975, } \\
\text { Mittal et al. } 1990\end{array}$ \\
\hline $\begin{array}{l}\text { Fusarium chlamydosporum } \\
\text { Wollenw. \& Reinking }\end{array}$ & $\begin{array}{l}\text { Nectriaceae, Sordariomycetes, } \\
\text { Ascomycota }\end{array}$ & seeds & $\begin{array}{l}\text { Pinus elliottii var. } \\
\text { elliottii }\end{array}$ & USA & Fraedrich \& Miller 1995 \\
\hline $\begin{array}{l}\text { Fusarium circinatum } \\
\text { Nirenberg \& O'Donnell }\end{array}$ & $\begin{array}{l}\text { Nectriaceae, Sordariomycetes, } \\
\text { Ascomycota }\end{array}$ & seeds & Pinus radiata & USA & Dwinell 1999 \\
\hline $\begin{array}{l}\text { Fusarium caeruleum Lib. ex } \\
\text { Sacc. }\end{array}$ & $\begin{array}{l}\text { Nectriaceae, Sordariomycetes, } \\
\text { Ascomycota }\end{array}$ & seeds & Pinus sylvestris & USSR & $\begin{array}{l}\text { Prisyazhnyuk } 1960 \text {, } \\
\text { Mittal et al. } 1990\end{array}$ \\
\hline $\begin{array}{l}\text { Fusarium compactum } \\
\text { (Wollenw.) Raillo }\end{array}$ & $\begin{array}{l}\text { Nectriaceae, Sordariomycetes, } \\
\text { Ascomycota }\end{array}$ & seeds & $\begin{array}{l}\text { Pinus elliottii var. } \\
\text { elliottii }\end{array}$ & USA & Fraedrich \& Miller 1995 \\
\hline \multirow[t]{8}{*}{$\begin{array}{l}\text { Fusarium culmorum } \\
\text { (Wm.G. Sm.) Sacc. }\end{array}$} & \multirow[t]{8}{*}{$\begin{array}{l}\text { Nectriaceae, Sordariomycetes, } \\
\text { Ascomycota }\end{array}$} & seed & Abies spp. & UK & $\begin{array}{l}\text { Anderson 1986a, Batko } \\
1956\end{array}$ \\
\hline & & seed & Linum usitatissimum & Canada & Conners 1967 \\
\hline & & seed & Lolium perenne & Canada & Conners 1967 \\
\hline & & seed & Medicago sativa & Canada & Conners 1967 \\
\hline & & seeds & Medicago truncatula & Australia & Nik \& Parbery 1977 \\
\hline & & seeds & Picea excelsa & USSR & $\begin{array}{l}\text { Urosevic 1961, } \\
\text { Anderson 1986a }\end{array}$ \\
\hline & & seeds & Pinus sylvestris & USSR & $\begin{array}{l}\text { Urosevic 1961, } \\
\text { Anderson 1986a }\end{array}$ \\
\hline & & seeds & Tectona grandis & - & $\begin{array}{l}\text { Mathur 1974, Anderson } \\
\text { 1986a }\end{array}$ \\
\hline $\begin{array}{l}\text { Fusarium dlaminii Marasas, } \\
\text { P.E. Nelson \& Toussoun }\end{array}$ & $\begin{array}{l}\text { Nectriaceae, Sordariomycetes, } \\
\text { Ascomycota }\end{array}$ & seeds & Picea abies & Italy & Motta et al. 1996 \\
\hline \multirow[t]{4}{*}{$\begin{array}{l}\text { Fusarium equiseti (Corda) } \\
\text { Sacc. }\end{array}$} & \multirow[t]{4}{*}{$\begin{array}{l}\text { Nectriaceae, Sordariomycetes, } \\
\text { Ascomycota }\end{array}$} & seed & $\begin{array}{l}\text { Acrocarpus } \\
\text { fraxinifolius }\end{array}$ & India, Rwanda & $\begin{array}{l}\text { Mathur 1974, Anderson } \\
\text { 1986a }\end{array}$ \\
\hline & & seed & Albizia gummifera & Rwanda & $\begin{array}{l}\text { Mathur 1974, Mittal et } \\
\text { al. } 1990\end{array}$ \\
\hline & & seed & $\begin{array}{l}\text { Apium graveolens } \\
\text { var. dulce }\end{array}$ & Canada & Conners 1967 \\
\hline & & seed & $\begin{array}{l}\text { Asparagus officinalis } \\
\text { var. altilis }\end{array}$ & Canada & Conners 1967 \\
\hline
\end{tabular}


Table 2 Continued.

\begin{tabular}{|c|c|c|c|c|c|}
\hline Species & Taxonomy & Substrate & Host & Country & References \\
\hline & & seed & Azadirachta indica & India & $\begin{array}{l}\text { Mathur 1974, Mittal et } \\
\text { al. } 1990\end{array}$ \\
\hline & & seed & Bombax ceiba & India & $\begin{array}{l}\text { Mathur 1974, Mittal et } \\
\text { al. } 1990\end{array}$ \\
\hline & & seed & Cupressus spp. & Syria & $\begin{array}{l}\text { Mathur 1974, Anderson } \\
\text { 1986a }\end{array}$ \\
\hline & & seed & Delonix regia & Brazil & $\begin{array}{l}\text { Mathur 1974, Anderson } \\
\text { 1986a }\end{array}$ \\
\hline & & seeds & Eucalyptus deglupta & Philippines & $\begin{array}{l}\text { Mathur 1974, Anderson } \\
\text { 1986a }\end{array}$ \\
\hline & & seeds & Eucalyptus sp. & India & $\begin{array}{l}\text { Saxena 1985, Mittal et } \\
\text { al. } 1990\end{array}$ \\
\hline & & seeds & Festuca elatior & Canada & Conners 1967 \\
\hline & & seeds & Festuca rubra & Canada & Conners 1967 \\
\hline & & seeds & Gmelina arborea & India & $\begin{array}{l}\text { Mathur 1974, Anderson } \\
\text { 1986a }\end{array}$ \\
\hline & & seed & Grevillea robusta & Rwanda & $\begin{array}{l}\text { Mathur 1974, Anderson } \\
\text { 1986a }\end{array}$ \\
\hline & & seed & Linum usitatissimum & Canada & Conners 1967 \\
\hline & & seeds & Lolium perenne & Canada & Conners 1967 \\
\hline & & seeds & Panicum mileaceum & Canada & Conners 1967 \\
\hline & & seeds & Phleum pratense & Canada & Conners 1967 \\
\hline & & seeds & Picea abies & Italy, USSR & $\begin{array}{l}\text { Prisyazhnyuk 1960, } \\
\text { Mittal et al. 1990, Motta } \\
\text { et al. } 1996\end{array}$ \\
\hline & & seeds & Pinus caribaea & $\mathrm{UK}$ & $\begin{array}{l}\text { Rees 1982, Mittal et al. } \\
1990\end{array}$ \\
\hline & & seeds & Pinus elliottii & Argentina & Lori \& Salerno 2003 \\
\hline & & seeds & $\begin{array}{l}\text { Pinus elliottii var. } \\
\text { elliottii }\end{array}$ & USA & Fraedrich \& Miller 1995 \\
\hline & & seeds & Pinus merkusii & Zambia & $\begin{array}{l}\text { Mathur 1974, Anderson } \\
\text { 1986a }\end{array}$ \\
\hline & & seeds & Pinus oocarpa & UK & $\begin{array}{l}\text { Rees 1982, Mittal et al. } \\
1990\end{array}$ \\
\hline
\end{tabular}


Table 2 Continued.

\begin{tabular}{|c|c|c|c|c|c|}
\hline Species & Taxonomy & Substrate & Host & Country & References \\
\hline & & seeds & Pinus pseudostrobus & UK & $\begin{array}{l}\text { Rees 1982, Mittal et al. } \\
1990\end{array}$ \\
\hline & & seeds & Pinus roxburghii & India & $\begin{array}{l}\text { Mathur 1974, Anderson } \\
\text { 1986a }\end{array}$ \\
\hline & & seeds & Pinus taeda & Argentina, Georgia & $\begin{array}{l}\text { Huang \& Kuhlman } \\
\text { 1990, Lori \& Salerno } \\
2003\end{array}$ \\
\hline & & seeds & $\begin{array}{l}\text { Portulaca } \\
\text { grandiflora }\end{array}$ & Canada & Conners 1967 \\
\hline & & seeds & Prosopis juliflora & Chile & $\begin{array}{l}\text { Mathur 1974, Mittal et } \\
\text { al. } 1990\end{array}$ \\
\hline & & seeds & Prosopis tamarugo & Chile & $\begin{array}{l}\text { Mathur 1974, Mittal et } \\
\text { al. } 1990\end{array}$ \\
\hline & & seeds & $\begin{array}{l}\text { Robinia } \\
\text { pseudoacacia }\end{array}$ & Hungary & $\begin{array}{l}\text { Hangyal-Balul } 1983 \text {, } \\
\text { Mittal et al. } 1990\end{array}$ \\
\hline & & seeds & Setaria viridis & Canada & Conners 1967 \\
\hline & & seeds & Tectona grandis & - & $\begin{array}{l}\text { Mathur 1974, Anderson } \\
\text { 1986a }\end{array}$ \\
\hline \multirow{2}{*}{$\begin{array}{l}\text { Fusarium graminearum } \\
\text { Schwabe }\end{array}$} & \multirow{2}{*}{$\begin{array}{l}\text { Nectriaceae, Sordariomycetes, } \\
\text { Ascomycota }\end{array}$} & seeds & Acer pseudoplatanus & Poland & Krol et al. 2015 \\
\hline & & seeds & Linum usitatissimum & Canada & Conners 1967 \\
\hline \multirow[t]{3}{*}{$\begin{array}{l}\text { Fusarium heterosporum } \\
\text { Nees \& T. Nees }\end{array}$} & \multirow[t]{3}{*}{$\begin{array}{l}\text { Nectriaceae, Sordariomycetes, } \\
\text { Ascomycota }\end{array}$} & $\begin{array}{l}\text { seeds } \\
\text { seeds }\end{array}$ & $\begin{array}{l}\text { Quercus robur } \\
\text { Picea excelsa }\end{array}$ & $\begin{array}{l}\text { Poland } \\
\text { USSR }\end{array}$ & $\begin{array}{l}\text { Krol et al. } 2015 \\
\text { Urosevic 1961, } \\
\text { Anderson 1986a }\end{array}$ \\
\hline & & seeds & Pinus sylvestris & USSR & $\begin{array}{l}\text { Urosevic 1961, } \\
\text { Anderson 1986a }\end{array}$ \\
\hline & & seeds & Quercus spp. & USSR & $\begin{array}{l}\text { Urosevic 1962, Mittal et } \\
\text { al. } 1990\end{array}$ \\
\hline \multirow{4}{*}{$\begin{array}{l}\text { Fusarium incarnatum } \\
\text { (Desm.) Sacc. } \\
\text { Fusarium lateritium Nees }\end{array}$} & \multirow{4}{*}{$\begin{array}{l}\text { Nectriaceae, Sordariomycetes, } \\
\text { Ascomycota } \\
\text { Nectriaceae, Sordariomycetes, } \\
\text { Ascomycota }\end{array}$} & seeds & Pinus elliottii & Argentina & Lori \& Salerno 2003 \\
\hline & & seeds & Pinus taeda & Argentina & Lori \& Salerno 2003 \\
\hline & & seeds & $\begin{array}{l}\text { Araucaria } \\
\text { heterophylla }\end{array}$ & Egypt & $\begin{array}{l}\text { Kamara et al. } 1981 \text {, } \\
\text { Mittal et al. } 1990\end{array}$ \\
\hline & & seeds & Picea excelsa & USSR & $\begin{array}{l}\text { Urosevic 1961, } \\
\text { Anderson 1986a }\end{array}$ \\
\hline
\end{tabular}


Table 2 Continued.

\begin{tabular}{|c|c|c|c|c|c|}
\hline Species & Taxonomy & Substrate & Host & Country & References \\
\hline & & seeds & Pinus sylvestris & USSR & $\begin{array}{l}\text { Urosevic 1961, } \\
\text { Anderson 1986a }\end{array}$ \\
\hline & & seeds & $\begin{array}{l}\text { Pseudotsuga } \\
\text { menziesii }\end{array}$ & Canada & Axelrood et al. 1995 \\
\hline & & seeds & Quercus spp. & USSR & $\begin{array}{l}\text { Urosevic 1962, Mittal et } \\
\text { al. } 1990\end{array}$ \\
\hline \multirow[t]{14}{*}{$\begin{array}{l}\text { Fusarium moniliforme J. } \\
\text { Sheld. }\end{array}$} & $\begin{array}{l}\text { Nectriaceae, Sordariomycetes, } \\
\text { Ascomycota }\end{array}$ & seeds & Abies grandis & South Korea & $\begin{array}{l}\text { Mathur 1974, Anderson } \\
\text { 1986a }\end{array}$ \\
\hline & & seeds & Abies nordmanniana & South Korea & $\begin{array}{l}\text { Mathur 1974, Anderson } \\
\text { 1986a }\end{array}$ \\
\hline & & seeds & $\begin{array}{l}\text { Acacia } \\
\text { auriculiformis }\end{array}$ & Philippines & Quiniones 1987 \\
\hline & & seeds & Acacia raddiana & Israel & $\begin{array}{l}\text { Mathur 1974, Anderson } \\
\text { 1986a }\end{array}$ \\
\hline & & seeds & Acer sp. & South Korea & $\begin{array}{l}\text { Mathur 1974, Anderson } \\
\text { 1986a }\end{array}$ \\
\hline & & seeds & $\begin{array}{l}\text { Acrocarpus } \\
\text { fraxinifolius }\end{array}$ & India, Rwanda & $\begin{array}{l}\text { Mathur 1974, Anderson } \\
\text { 1986a }\end{array}$ \\
\hline & & seeds & $\begin{array}{l}\text { Adenanthera } \\
\text { microsperma }\end{array}$ & India & $\begin{array}{l}\text { Mathur 1974, Anderson } \\
\text { 1986a }\end{array}$ \\
\hline & & seeds & Adina cordifolia & India & $\begin{array}{l}\text { Mathur 1974, Anderson } \\
\text { 1986a }\end{array}$ \\
\hline & & seed & Albizia falcataria & Philippines & $\begin{array}{l}\text { Mathur 1974, Quiniones } \\
\text { 1987, Anderson 1986a }\end{array}$ \\
\hline & & seed & Albizia julibrissin & Philippines & $\begin{array}{l}\text { Quiniones 1985, 1987, } \\
\text { Mittal et al. } 1990\end{array}$ \\
\hline & & seeds & Albizia procera & Philippines & $\begin{array}{l}\text { Dayan 1986, Mittal et al. } \\
1990\end{array}$ \\
\hline & & seeds & Albizia stipulata & India & $\begin{array}{l}\text { Mathur 1974, Anderson } \\
\text { 1986a }\end{array}$ \\
\hline & & seeds & $\begin{array}{l}\text { Alstonia } \\
\text { macrophylla }\end{array}$ & Philippines & $\begin{array}{l}\text { Quiniones 1985, 1987, } \\
\text { Mittal et al. } 1990\end{array}$ \\
\hline & & seeds & $\begin{array}{l}\text { Anthocephalus } \\
\text { chinensis }\end{array}$ & Philippines & Quiniones 1987 \\
\hline
\end{tabular}


Table 2 Continued.

\begin{tabular}{|c|c|c|c|c|c|}
\hline Species & Taxonomy & Substrate & Host & Country & References \\
\hline & & seeds & Azadirachta indica & India & $\begin{array}{l}\text { Mathur 1974, Mittal et } \\
\text { al. } 1990\end{array}$ \\
\hline & & seeds & Calamus ornatus & Philippines & Quiniones 1987 \\
\hline & & seeds & Cassia bakeriana & Thailand & $\begin{array}{l}\text { Chalermpongse et al. } \\
\text { 1984, Mittal et al. } 1990\end{array}$ \\
\hline & & seeds & Cassia siamea & Philippines & $\begin{array}{l}\text { Quiniones 1985, 1987, } \\
\text { Mittal et al. } 1990\end{array}$ \\
\hline & & seeds & $\begin{array}{l}\text { Casuarina } \\
\text { equisetifolia }\end{array}$ & Philippines & $\begin{array}{l}\text { Quiniones 1985, 1987, } \\
\text { Anderson 1986a }\end{array}$ \\
\hline & & seeds & Cedrela odorata & Colombia & $\begin{array}{l}\text { Mathur 1974, Anderson } \\
\text { 1986a }\end{array}$ \\
\hline & & seeds & Cedrela toona & India & $\begin{array}{l}\text { Mathur 1974, Anderson } \\
\text { 1986a }\end{array}$ \\
\hline & & seeds & Cedrus deodara & India & Mittal 1983 \\
\hline & & seeds & Chukrasia tabularis & India & $\begin{array}{l}\text { Mathur 1974, Anderson } \\
\text { 1986a }\end{array}$ \\
\hline & & seeds & Cordia alliodora & Colombia & $\begin{array}{l}\text { Mathur 1974, Anderson } \\
\text { 1986a }\end{array}$ \\
\hline & & seeds & $\begin{array}{l}\text { Cryptomeria } \\
\text { japonica }\end{array}$ & Japan & Watanabe 2010 \\
\hline & & seeds & Dalbergia sissoo & India, Madagascar & $\begin{array}{l}\text { Mathur 1974, Anderson } \\
\text { 1986a, Kumar } 2014\end{array}$ \\
\hline & & seeds & $\begin{array}{l}\text { Endospermum } \\
\text { peltatum }\end{array}$ & Philippines & $\begin{array}{l}\text { Agmata 1979, Anderson } \\
\text { 1986a }\end{array}$ \\
\hline & & seeds & $\begin{array}{l}\text { Eucalyptus } \\
\text { camaldulensis }\end{array}$ & Thailand & $\begin{array}{l}\text { Chalermpongse et al. } \\
\text { 1984, Mittal et al. } 1990\end{array}$ \\
\hline & & seeds & Eucalyptus grandis & Uruguay & $\begin{array}{l}\text { Mathur 1974, Anderson } \\
\text { 1986a }\end{array}$ \\
\hline & & seeds & Eucalyptus sp. & India & $\begin{array}{l}\text { Saxena 1985, Mittal et } \\
\text { al. } 1990\end{array}$ \\
\hline & & seeds & Ficus benjamina & India & $\begin{array}{l}\text { Mathur 1974, Anderson } \\
\text { 1986a }\end{array}$ \\
\hline & & seeds & Gmelina arborea & India & $\begin{array}{l}\text { Mathur 1974, Anderson } \\
\text { 1986a }\end{array}$ \\
\hline
\end{tabular}


Table 2 Continued.

\begin{tabular}{|c|c|c|c|c|c|}
\hline Species & Taxonomy & Substrate & Host & Country & References \\
\hline & & seeds & Grevillea robusta & Rwanda & $\begin{array}{l}\text { Mathur 1974, Anderson } \\
\text { 1986a }\end{array}$ \\
\hline & & seeds & $\begin{array}{l}\text { Jacaranda } \\
\text { mimosifolia }\end{array}$ & India & $\begin{array}{l}\text { Mathur 1974, Anderson } \\
\text { 1986a }\end{array}$ \\
\hline & & seeds & Juniperus coreana & South Korea & $\begin{array}{l}\text { Mathur 1974, Anderson } \\
\text { 1986a }\end{array}$ \\
\hline & & seeds & Kydia calycina & India & $\begin{array}{l}\text { Mathur 1974, Anderson } \\
\text { 1986a }\end{array}$ \\
\hline & & seeds & $\begin{array}{l}\text { Leucaena } \\
\text { cunningham }\end{array}$ & Malawi & $\begin{array}{l}\text { Mathur 1974, Anderson } \\
\text { 1986a }\end{array}$ \\
\hline & & seeds & $\begin{array}{l}\text { Leucaena } \\
\text { diversifolia }\end{array}$ & Guatemala & $\begin{array}{l}\text { Mathur 1974, Anderson } \\
\text { 1986a }\end{array}$ \\
\hline & & seeds & Leucaena latisiliqua & Philippines & $\begin{array}{l}\text { Mathur 1974, Anderson } \\
\text { 1986a }\end{array}$ \\
\hline & & seeds & $\begin{array}{l}\text { Leucaena } \\
\text { leucocephala }\end{array}$ & $\begin{array}{l}\text { Malaysia, } \\
\text { Philippines }\end{array}$ & $\begin{array}{l}\text { Mathur 1974, Anderson } \\
\text { 1986a }\end{array}$ \\
\hline & & seeds & Leucaena spp. & Philippines & $\begin{array}{l}\text { Mathur 1974, Anderson } \\
\text { 1986a }\end{array}$ \\
\hline & & seeds & Melia spp. & India & Sahai \& Otra 1982 \\
\hline & & seeds & $\begin{array}{l}\text { Mimosa } \\
\text { caesalpiniafolia }\end{array}$ & Brazil & $\begin{array}{l}\text { Mathur 1974, Anderson } \\
\text { 1986a }\end{array}$ \\
\hline & & seeds & Mimosa scabrella & Brazil & $\begin{array}{l}\text { Mathur 1974, Anderson } \\
\text { 1986a }\end{array}$ \\
\hline & & seeds & Picea abies & Italy & Motta et al. 1996 \\
\hline & & seeds & Picea excelsa & USSR & $\begin{array}{l}\text { Urosevic 1961, } \\
\text { Anderson 1986a }\end{array}$ \\
\hline & & seeds & $\begin{array}{l}\text { Piliostigma } \\
\text { malabaricum }\end{array}$ & Philippines & $\begin{array}{l}\text { Dayan 1986, Mittal et al. } \\
1990\end{array}$ \\
\hline & & seeds & Pinus caribaea & $\begin{array}{l}\text { Belize [as British } \\
\text { Honduras],Central } \\
\text { America, } \\
\text { Honduras, } \\
\text { Madagascar }\end{array}$ & $\begin{array}{l}\text { Hocking 1968, Rees } \\
\text { 1982, Anderson 1986a }\end{array}$ \\
\hline
\end{tabular}


Table 2 Continued.

\begin{tabular}{|c|c|c|c|c|c|}
\hline Species & Taxonomy & Substrate & Host & Country & References \\
\hline & & seeds & $\begin{array}{l}\text { Pinus caribaea var. } \\
\text { bahamensis }\end{array}$ & Brazil & $\begin{array}{l}\text { Mathur 1974, Anderson } \\
\text { 1986a }\end{array}$ \\
\hline & & seeds & $\begin{array}{l}\text { Pinus caribaea var. } \\
\text { caribae }\end{array}$ & Brazil & $\begin{array}{l}\text { Mathur 1974, Anderson } \\
\text { 1986a }\end{array}$ \\
\hline & & seeds & $\begin{array}{l}\text { Pinus caribaea var. } \\
\text { hondurensis }\end{array}$ & Brazil & $\begin{array}{l}\text { Mathur 1974, Anderson } \\
\text { 1986a }\end{array}$ \\
\hline & & seeds & Pinus densiflora & Japan & Watanabe 2010 \\
\hline & & seeds & Pinus elliottii & Canada, USA & $\begin{array}{l}\text { Anderson 1986a, Mittal } \\
\text { et al. } 1990\end{array}$ \\
\hline & & seeds & $\begin{array}{l}\text { Pinus elliottii var. } \\
\text { elliottii }\end{array}$ & Brazil, USA & $\begin{array}{l}\text { Matur 1974, Anderson } \\
\text { 1986a, Fraedrich \& } \\
\text { Miller } 1995\end{array}$ \\
\hline & & seeds & Pinus insularis & Philippines & $\begin{array}{l}\text { Agmata 1979, Anderson } \\
\text { 1986a }\end{array}$ \\
\hline & & seeds & Pinus khasya & Brazil, Madagascar & $\begin{array}{l}\text { Mathur 1974, Anderson } \\
\text { 1986a, Mittal et al. } 1990\end{array}$ \\
\hline & & seeds & Pinus merkusii & $\begin{array}{l}\text { Philippines, } \\
\text { Zambia }\end{array}$ & $\begin{array}{l}\text { Agmata 1979, Anderson } \\
\text { 1986a, Mittal et al. } 1990\end{array}$ \\
\hline & & seeds & Pinus occidentalis & Cuba & $\begin{array}{l}\text { Anderson 1986a, } \\
\text { Richardson } 1979\end{array}$ \\
\hline & & seeds & Pinus oocarpa & $\begin{array}{l}\text { Central America, } \\
\text { India, UK }\end{array}$ & $\begin{array}{l}\text { Rees 1982, Anderson } \\
\text { 1986a, Mittal et al. } 1990\end{array}$ \\
\hline & & seeds & Pinus palustris & USA & $\begin{array}{l}\text { Pawuk 1978, Anderson } \\
\text { 1986a }\end{array}$ \\
\hline & & seeds & Pinus pinaster & Italy, Uruguay & $\begin{array}{l}\text { Anderson 1986a, Mittal } \\
\text { et al. } 1990\end{array}$ \\
\hline & & seeds & Pinus pseudostrobus & UK & $\begin{array}{l}\text { Rees 1982, Mittal et al. } \\
1990\end{array}$ \\
\hline & & seeds & Pinus pungens & USA & $\begin{array}{l}\text { Mathur 1974, Anderson } \\
\text { 1986a }\end{array}$ \\
\hline & & seeds & Pinus sylvestris & USSR & $\begin{array}{l}\text { Prisyazhnyuk 1960, } \\
\text { Anderson 1986a }\end{array}$ \\
\hline & & seeds & Pinus taeda & $\begin{array}{l}\text { Brazil, Canada, } \\
\text { USA }\end{array}$ & $\begin{array}{l}\text { Mason \& van Arsdel } \\
\text { 1978, Anderson 1986a }\end{array}$ \\
\hline
\end{tabular}


Table 2 Continued.

\begin{tabular}{|c|c|c|c|c|c|}
\hline Species & Taxonomy & Substrate & \begin{tabular}{|c|} 
Host \\
\end{tabular} & Country & \begin{tabular}{|l|} 
References \\
\end{tabular} \\
\hline & & seeds & Pinus wallichiana & Pakistan & Ashaeer 2000 \\
\hline & & seeds & $\begin{array}{l}\text { Pittosporum } \\
\text { resiniferum }\end{array}$ & Philippines & $\begin{array}{l}\text { Dayan 1986, Mittal et al. } \\
1990\end{array}$ \\
\hline & & seeds & $\begin{array}{l}\text { Platanus } \\
\text { occidentalis }\end{array}$ & USA & $\begin{array}{l}\text { Fakir et al. 1971, Mittal } \\
\text { et al. } 1990\end{array}$ \\
\hline & & seeds & Polyscias nodosa & Philippines & $\begin{array}{l}\text { Agmata 1979, Mittal et } \\
\text { al. } 1990\end{array}$ \\
\hline & & seeds & Prosopis juliflora & Brazil, Chile & $\begin{array}{l}\text { Mathur 1974, Mittal et } \\
\text { al. } 1990\end{array}$ \\
\hline & & seeds & $\begin{array}{l}\text { Pseudotsuga } \\
\text { menziesii }\end{array}$ & Canada & Axelrood et al. 1995 \\
\hline & & seeds & Pterocarpus indicus & Philippines & $\begin{array}{l}\text { Agmata 1979, Anderson } \\
\text { 1986a }\end{array}$ \\
\hline & & seeds & $\begin{array}{l}\text { Pterospermum } \\
\text { acerifolium }\end{array}$ & India & $\begin{array}{l}\text { Mathur 1974, Anderson } \\
\text { 1986a }\end{array}$ \\
\hline & & seeds & Quercus spp. & India & Sahai \& Otra 1982 \\
\hline & & seeds & Tectona grandis & $\begin{array}{l}\text { India, Philippines, } \\
\text { Thailand }\end{array}$ & $\begin{array}{l}\text { Mathur 1974, Anderson } \\
\text { 1986a, Mittal et al. } 1990\end{array}$ \\
\hline & & seeds & Toona calantas & Philippines & Quiniones 1987 \\
\hline & & seeds & Triplaris cumingiana & Philippines & $\begin{array}{l}\text { Quiniones 1985, } \\
\text { Anderson 1986a }\end{array}$ \\
\hline & & seeds & Ulmus davidiana & South Korea & $\begin{array}{l}\text { Mathur 1974, Anderson } \\
\text { 1986a }\end{array}$ \\
\hline $\begin{array}{l}\text { Fusarium moniliforme var. } \\
\text { minus Wollenw. \& Reinking }\end{array}$ & $\begin{array}{l}\text { Nectriaceae, Sordariomycetes, } \\
\text { Ascomycota }\end{array}$ & seeds & Cedrus deodara & India & $\begin{array}{l}\text { Munjal \& Sharma 1975, } \\
\text { Mittal et al. } 1990\end{array}$ \\
\hline $\begin{array}{l}\text { Fusarium moniliforme var. } \\
\text { subglutinans Wollenw. \& }\end{array}$ & $\begin{array}{l}\text { Nectriaceae, Sordariomycetes, } \\
\text { Ascomycota }\end{array}$ & cones, seeds & Pinus taeda & USA & $\begin{array}{l}\text { Barrows-Broaddus \& } \\
\text { Dwinell } 1985\end{array}$ \\
\hline \multirow[t]{3}{*}{ Reinking } & & seeds & Pinus echinata & USA & Mittal et al. 1990 \\
\hline & & seeds & $\begin{array}{l}\text { Pinus elliottii var. } \\
\text { elliottii }\end{array}$ & USA, Philippines & $\begin{array}{l}\text { Miller \& Bramlett 1979, } \\
\text { Anderson 1986a }\end{array}$ \\
\hline & & seeds & Pinus oocarpa & UK & $\begin{array}{l}\text { Rees 1982, Mittal et al. } \\
1990\end{array}$ \\
\hline
\end{tabular}


Table 2 Continued.

\begin{tabular}{|c|c|c|c|c|c|}
\hline Species & Taxonomy & Substrate & Host & Country & References \\
\hline \multirow{20}{*}{$\begin{array}{l}\text { Fusarium nivale Ces. ex } \\
\text { Berl. \& Voglino } \\
\text { Fusarium oxysporum } \\
\text { Schltdl. }\end{array}$} & & seeds & Pinus pinaster & Italy & $\begin{array}{l}\text { Mittal et al. 1990, Motta } \\
1986\end{array}$ \\
\hline & & seeds & Pinus pseudostrobus & UK & $\begin{array}{l}\text { Rees 1982, Mittal et al. } \\
1990\end{array}$ \\
\hline & $\begin{array}{l}\text { Nectriaceae, Sordariomycetes, } \\
\text { Ascomycota }\end{array}$ & seeds & Pinus roxburghii & India & $\begin{array}{l}\text { Munjal \& Sharma 1975, } \\
\text { Mittal et al. } 1990\end{array}$ \\
\hline & Nectriaceae, Sordariomycetes, & cones & Pinus ponderosa & USA & James 1995 \\
\hline & Ascomycota & cones, seeds & $\begin{array}{l}\text { Pseudotsuga } \\
\text { menziesii var. glauca }\end{array}$ & USA & James 1995 \\
\hline & & pod & Canavalia cathartica & India & Anita \& Sridhar 2009 \\
\hline & & pod, seed & Sesbania bispinosa & India & Anita et al. 2009 \\
\hline & & seeds & Abies alba & Poland & Krol et al. 2015 \\
\hline & & seeds & Acer pseudoplatanus & Poland & Krol et al. 2015 \\
\hline & & seeds & $\begin{array}{l}\text { Araucaria } \\
\text { angustifolia }\end{array}$ & Australia & $\begin{array}{l}\text { Kamara et al. } 1981 \text {, } \\
\text { Mittal et al. } 1990\end{array}$ \\
\hline & & seeds & Araucaria bidwillii & Egypt & $\begin{array}{l}\text { Kamara et al. } 1981 \text {, } \\
\text { Mittal et al. } 1990\end{array}$ \\
\hline & & seeds & $\begin{array}{l}\text { Araucaria } \\
\text { cunninghamii }\end{array}$ & Australia & $\begin{array}{l}\text { Kamara et al. } 1981 \text {, } \\
\text { Mittal et al. } 1990\end{array}$ \\
\hline & & seeds & $\begin{array}{l}\text { Araucaria } \\
\text { heterophylla }\end{array}$ & Egypt & $\begin{array}{l}\text { El-Kady et al. } 1986 \text {, } \\
\text { Mittal et al. } 1990\end{array}$ \\
\hline & & seed & $\begin{array}{l}\text { Asparagus officinalis } \\
\text { var. altilis }\end{array}$ & Canada & Conners 1967 \\
\hline & & seeds & Betula pendula & Poland & Krol et al. 2015 \\
\hline & & seeds & Caltha leptosepala & $\begin{array}{l}\text { Poland or/ } \\
\text { imported to Poland }\end{array}$ & Czeczuga et al. 2009 \\
\hline & & seed & Canavalia cathartica & India & Anita \& Sridhar 2009 \\
\hline & & seeds & Crataegus azarolus & $\begin{array}{l}\text { Poland or/ } \\
\text { imported to Poland }\end{array}$ & Czeczuga et al. 2009 \\
\hline & & seed & $\begin{array}{l}\text { Cryptomeria } \\
\text { japonica }\end{array}$ & Japan & Watanabe 2010 \\
\hline & & seeds & $\begin{array}{l}\text { Cupressus } \\
\text { sempervirens }\end{array}$ & Egypt & $\begin{array}{l}\text { Farag et al. 1977, Mittal } \\
\text { et al. } 1990\end{array}$ \\
\hline
\end{tabular}


Table 2 Continued.

\begin{tabular}{|c|c|c|c|c|c|}
\hline Species & Taxonomy & Substrate & Host & Country & References \\
\hline & & seeds & Dalbergia sissoo & India & Kumar 2014 \\
\hline & & seeds & Eucalyptus deglupta & Thailand & $\begin{array}{l}\text { Chalermpongse et al. } \\
\text { 1984, Mittal et al. } 1990\end{array}$ \\
\hline & & seeds & Eucalyptus sp. & India & $\begin{array}{l}\text { Saxena 1985, Mittal et } \\
\text { al. } 1990\end{array}$ \\
\hline & & seed & Hyoscyamus niger & Canada & Conners 1967 \\
\hline & & seeds & Larix decidua & USSR & $\begin{array}{l}\text { Kozlowska 1968, Mittal } \\
\text { et al. } 1990\end{array}$ \\
\hline & & seeds & Larix occidentalis & USA & $\begin{array}{l}\text { James } 1986 b \text {, James et } \\
\text { al. } 1996\end{array}$ \\
\hline & & seeds & Larix sibirica & USSR & $\begin{array}{l}\text { Prisyazhnyuk 1960, } \\
\text { Mittal et al. } 1990\end{array}$ \\
\hline & & seed & Linum usitatissimum & Canada & Conners 1967 \\
\hline & & seeds & Mimosa scabrella & Brazil & $\begin{array}{l}\text { Mathur 1974, Anderson } \\
\text { 1986a }\end{array}$ \\
\hline & & seeds & Picea abies & Italy, Poland & Krol et al. 2015 \\
\hline & & seeds & Picea excelsa & USSR & $\begin{array}{l}\text { Urosevic 1961, } \\
\text { Anderson 1986a }\end{array}$ \\
\hline & & seeds & Picea glauca & Canada, USA & $\begin{array}{l}\text { James 1985b, Mittal \& } \\
\text { Wang 1986, Mittal et al. } \\
1990\end{array}$ \\
\hline & & seeds & Picea pungens & USA & James 1985b \\
\hline & & seeds & Pinus caribaea & Belize [or as & Hocking 1968, Rees \\
\hline & & & & $\begin{array}{l}\text { British Honduras], } \\
\text { Cuba, UK }\end{array}$ & $\begin{array}{l}\text { 1982, Anderson 1986a, } \\
\text { Mittal et al. } 1990\end{array}$ \\
\hline & & seeds & Pinus elliottii & $\begin{array}{l}\text { Argentina, South } \\
\text { Africa }\end{array}$ & $\begin{array}{l}\text { Cilliers et al. 1995, Lori } \\
\text { \& Salerno } 2003\end{array}$ \\
\hline & & seeds & $\begin{array}{l}\text { Pinus elliottii var. } \\
\text { elliottii }\end{array}$ & USA & Fraedrich \& Miller 1995 \\
\hline & & seeds & Pinus lambertiana & USA & Anderson 1986a \\
\hline & & seeds & Pinus oocarpa & UK & $\begin{array}{l}\text { Rees 1982, Mittal et al. } \\
1990\end{array}$ \\
\hline & & seeds & Pinus palustris & USA & $\begin{array}{l}\text { Pawuk 1978, Mittal et al. } \\
1990\end{array}$ \\
\hline
\end{tabular}


Table 2 Continued.

\begin{tabular}{|c|c|c|c|c|c|}
\hline Species & Taxonomy & Substrate & Host & Country & References \\
\hline & & seeds & Pinus ponderosa & USA & $\begin{array}{l}\text { James \& Genz 1982, } \\
\text { Anderson 1986a, } \\
\text { James1986c, 1995 }\end{array}$ \\
\hline & & seeds & Pinus pseudostrobus & UK & $\begin{array}{l}\text { Rees 1982, Mittal et al. } \\
1990\end{array}$ \\
\hline & & seeds & Pinus roxburghii & India & $\begin{array}{l}\text { Mittal \& Sharma 1982c, } \\
\text { Mittal et al. } 1990\end{array}$ \\
\hline & & seeds & Pinus sylvestris & USSR & $\begin{array}{l}\text { Urosevic 1961, } \\
\text { Anderson 1986a }\end{array}$ \\
\hline & & seeds & Pinus taeda & $\begin{array}{l}\text { Argentina, USA, } \\
\text { Uruguay }\end{array}$ & $\begin{array}{l}\text { Mason \& van Arsdel } \\
\text { 1978, Anderson 1986a , } \\
\text { Lori \& Salerno } 2003\end{array}$ \\
\hline & & seed & Prunus serrulata & Japan & Watanabe 2010 \\
\hline & & seeds & $\begin{array}{l}\text { Pseudotsuga } \\
\text { menziesii }\end{array}$ & Canada, USA & $\begin{array}{l}\text { James 1983b, 1984, } \\
\text { 1986a, Axelrood et al. } \\
1995\end{array}$ \\
\hline & & seeds & $\begin{array}{l}\text { Pterospermum } \\
\text { acerifolium }\end{array}$ & India & $\begin{array}{l}\text { Mathur 1974, Anderson } \\
\text { 1986a }\end{array}$ \\
\hline & & seeds & $\begin{array}{l}\text { Quercus } \\
\text { castaneifolia }\end{array}$ & Iran & Kavosi et al. 2013 \\
\hline & & seeds & Quercus pedunculata & USSR & $\begin{array}{l}\text { Kozlowska 1968, Mittal } \\
\text { et al. } 1990\end{array}$ \\
\hline & & seeds & Quercus spp. & USSR & $\begin{array}{l}\text { Urosevic 1964, Mittal et } \\
\text { al. } 1990\end{array}$ \\
\hline & & seeds & $\begin{array}{l}\text { Robinia } \\
\text { pseudoacacia }\end{array}$ & Hungary & $\begin{array}{l}\text { Hangyal-Balul } 1983 \text {, } \\
\text { Mittal et al. } 1990\end{array}$ \\
\hline & & seeds & Shorea materialis & Malaysia & Mittal et al. 1990 \\
\hline & & seeds & Tectona grandis & India & $\begin{array}{l}\text { Mathur 1974, Anderson } \\
\text { 1986a }\end{array}$ \\
\hline $\begin{array}{l}\text { Fusarium oxysporum f. lini } \\
\text { (Bolley) W.C. Snyder \& } \\
\text { H.N. Hansen }\end{array}$ & $\begin{array}{l}\text { Nectriaceae, Sordariomycetes, } \\
\text { Ascomycota }\end{array}$ & seed & Linum usitatissimum & Canada & Conners 1967 \\
\hline $\begin{array}{l}\text { Fusarium oxysporum f. } \\
\text { perniciosum (Hepting) Toole }\end{array}$ & $\begin{array}{l}\text { Nectriaceae, Sordariomycetes, } \\
\text { Ascomycota }\end{array}$ & seeds & Albizia julibrissin & USA & $\begin{array}{l}\text { Quiniones 1985, } \\
\text { Anderson 1986a }\end{array}$ \\
\hline
\end{tabular}


Table 2 Continued.

\begin{tabular}{|c|c|c|c|c|c|}
\hline Species & Taxonomy & Substrate & Host & Country & References \\
\hline $\begin{array}{l}\text { Fusarium oxysporum f.sp. } \\
\text { elaeidis Toovey }\end{array}$ & $\begin{array}{l}\text { Nectriaceae, Sordariomycetes, } \\
\text { Ascomycota }\end{array}$ & seeds & Elaeis guineensis & $\begin{array}{l}\text { Africa, Colombia, } \\
\text { Surinam }\end{array}$ & $\begin{array}{l}\text { Locke \& Colhoun 1973, } \\
\text { Mittal et al. } 1990\end{array}$ \\
\hline $\begin{array}{l}\text { Fusarium oxysporum f.sp. } \\
\text { koae D.E. Gardner }\end{array}$ & $\begin{array}{l}\text { Nectriaceae, Sordariomycetes, } \\
\text { Ascomycota }\end{array}$ & seeds & Acacia spp. & Hawaii & $\begin{array}{l}\text { Anderson 1986a, } \\
\text { Gardner } 1980\end{array}$ \\
\hline $\begin{array}{l}\text { Fusarium oxysporum var. } \\
\text { aurantiacum (Corda) }\end{array}$ & $\begin{array}{l}\text { Nectriaceae, Sordariomycetes, } \\
\text { Ascomycota }\end{array}$ & seeds & Cedrus deodara & India & $\begin{array}{l}\text { Munjal \& Sharma 1975, } \\
\text { Mittal et al. } 1990\end{array}$ \\
\hline \multirow[t]{2}{*}{ Rabenh. } & & seeds & Pinus roxburghii & India & $\begin{array}{l}\text { Munjal \& Sharma } 1975 \text {, } \\
\text { Mittal et al. } 1990\end{array}$ \\
\hline & & seeds & Pinus wallichiana & India & $\begin{array}{l}\text { Munjal \& Sharma } 1975 \text {, } \\
\text { Mittal et al. } 1990\end{array}$ \\
\hline Fusarium poae (Peck) & Nectriaceae, Sordariomycetes, & acorns & Quercus robur & Poland & Jankowiak 2008 \\
\hline \multirow[t]{10}{*}{ Wollenw. } & Ascomycota & seeds & Acer pseudoplatanus & Poland & Krol et al. 2015 \\
\hline & & seed & $\begin{array}{l}\text { Apium graveolens } \\
\text { var. dulce }\end{array}$ & Canada & Conners 1967 \\
\hline & & seeds & Betula pendula & Poland & Krol et al. 2015 \\
\hline & & seeds & Eucalyptus sp. & India & $\begin{array}{l}\text { Saxena 1985, Mittal et } \\
\text { al. } 1990\end{array}$ \\
\hline & & seed & Linum usitatissimum & Canada & Conners 1967 \\
\hline & & seed & Panicum mileaceum & Canada & Conners 1967 \\
\hline & & seeds & Picea abies & Italy & Motta et al. 1996 \\
\hline & & seeds & Pinus sylvestris & UK & Whittle 1977 \\
\hline & & seeds & $\begin{array}{l}\text { Pseudotsuga } \\
\text { menziesii }\end{array}$ & Canada, USA & $\begin{array}{l}\text { Gordon 1967, Mittal et } \\
\text { al. 1990, Axelrood et al. } \\
1995\end{array}$ \\
\hline & & seed & Setaria viridis & Canada & Conners 1967 \\
\hline $\begin{array}{l}\text { Fusarium proliferatum } \\
\text { (Matsush.) Nirenberg ex }\end{array}$ & $\begin{array}{l}\text { Nectriaceae, Sordariomycetes, } \\
\text { Ascomycota }\end{array}$ & seeds & $\begin{array}{l}\text { Dianthus } \\
\text { caryophyllus }\end{array}$ & Taiwan & Li \& Wu 2002 \\
\hline \multirow[t]{4}{*}{ Gerlach \& Nirenberg } & & seeds & Picea abies & Italy & Motta et al. 1996 \\
\hline & & seeds & Pinus elliottii & Argentina & Lori \& Salerno 2003 \\
\hline & & seeds & $\begin{array}{l}\text { Pinus elliottii var. } \\
\text { elliottii }\end{array}$ & USA & Fraedrich \& Miller 1995 \\
\hline & & seeds & Pinus taeda & Argentina & Lori \& Salerno 2003 \\
\hline Fusarium redolens & Nectriaceae, Sordariomycetes, & seeds & Picea excelsa & USSR & Urosevic 1961, \\
\hline Wollenw. & Ascomycota & & & & Anderson 1986a \\
\hline
\end{tabular}


Table 2 Continued.

\begin{tabular}{|c|c|c|c|c|c|}
\hline Species & Taxonomy & Substrate & Host & Country & References \\
\hline & & seeds & Pinus sylvestris & USSR & $\begin{array}{l}\text { Urosevic 1961, } \\
\text { Anderson 1986a }\end{array}$ \\
\hline \multirow{7}{*}{$\begin{array}{l}\text { Fusarium roseum Link [as } \\
\text { Gibberella pulicaris (Kunze) } \\
\text { Sacc.] }\end{array}$} & Nectriaceae, Sordariomycetes, & seeds & Fraxinus excelsior & Sweden & Cleary et al. 2013 \\
\hline & Ascomycota & seeds & Pinus lambertiana & USA & Anderson 1986a \\
\hline & & seed & Pinus palustris & USA & $\begin{array}{l}\text { Pawuk 1978, Mittal et al. } \\
1990\end{array}$ \\
\hline & & seeds & Pinus ponderosa & USA & James 1983a \\
\hline & & seeds & Pinus taeda & USA & $\begin{array}{l}\text { Mason \& van Arsdel } \\
\text { 1978, Anderson 1986a }\end{array}$ \\
\hline & & seeds & $\begin{array}{l}\text { Platanus } \\
\text { occidentalis }\end{array}$ & USA & $\begin{array}{l}\text { Fakir et al. 1971, Mittal } \\
\text { et al. } 1990\end{array}$ \\
\hline & & seeds & Prunus serrulata & Japan & Watanabe 2010 \\
\hline \multirow{7}{*}{$\begin{array}{l}\text { Fusarium sambucinum } \\
\text { Fuckel }\end{array}$} & Nectriaceae, Sordariomycetes, & seed & Hyoscyamus niger & Canada & Conners 1967 \\
\hline & Ascomycota & seeds & Larix decidua & Poland & Krol et al. 2015 \\
\hline & & seeds & Larix occidentalis & USA & $\begin{array}{l}\text { James } 1986 b \text {, James et } \\
\text { al. } 1996\end{array}$ \\
\hline & & seeds & Picea abies & Italy & Motta et al. 1996 \\
\hline & & seeds & Pinus ponderosa & USA & James 1985a \\
\hline & & seeds & Pinus sylvestris & Poland & Krol et al. 2015 \\
\hline & & seeds & $\begin{array}{l}\text { Pseudotsuga } \\
\text { menziesii }\end{array}$ & Canada & Axelrood et al. 1995 \\
\hline $\begin{array}{l}\text { Fusarium sambucinum var. } \\
\text { coeruleum Wollenw. }\end{array}$ & $\begin{array}{l}\text { Nectriaceae, Sordariomycetes, } \\
\text { Ascomycota }\end{array}$ & seed & Agropyron cristatum & Canada & Conners 1967 \\
\hline \multirow[t]{5}{*}{$\begin{array}{l}\text { Fusarium sarcochroum } \\
\text { (Desm.) Sacc. }\end{array}$} & $\begin{array}{l}\text { Nectriaceae, Sordariomycetes, } \\
\text { Ascomycota }\end{array}$ & seeds & Picea excelsa & USSR & $\begin{array}{l}\text { Urosevic 1961, } \\
\text { Anderson 1986a }\end{array}$ \\
\hline & & seeds & Pinus sylvestris & USSR & $\begin{array}{l}\text { Urosevic 1961, } \\
\text { Anderson 1986a }\end{array}$ \\
\hline & & seeds & Abies amabilis & South Korea & $\begin{array}{l}\text { Mathur 1974, Anderson } \\
\text { 1986a }\end{array}$ \\
\hline & & seeds & $\begin{array}{l}\text { Acacia } \\
\text { auriculiformis }\end{array}$ & Philippines & $\begin{array}{l}\text { Quiniones 1985, 1987, } \\
\text { Anderson 1986a }\end{array}$ \\
\hline & & seeds & Acacia modesta & India & $\begin{array}{l}\text { Mathur 1974, Anderson } \\
\text { 1986a }\end{array}$ \\
\hline
\end{tabular}


Table 2 Continued.

\begin{tabular}{|c|c|c|c|c|c|}
\hline Species & Taxonomy & Substrate & Host & Country & References \\
\hline & & seeds & Acer palmatum & South Korea & $\begin{array}{l}\text { Mathur 1974, Anderson } \\
\text { 1986a }\end{array}$ \\
\hline & & seeds & $\begin{array}{l}\text { Acrocarpus } \\
\text { fraxinifolius }\end{array}$ & India, Rwanda & $\begin{array}{l}\text { Mathur 1974, Anderson } \\
\text { 1986a }\end{array}$ \\
\hline & & seeds & $\begin{array}{l}\text { Adenanthera } \\
\text { microsperma }\end{array}$ & India & $\begin{array}{l}\text { Mathur 1974, Anderson } \\
\text { 1986a }\end{array}$ \\
\hline & & seeds & Albizia falcataria & Philippines & $\begin{array}{l}\text { Mathur 1974, Mittal et } \\
\text { al. } 1990\end{array}$ \\
\hline & & seeds & Albizia gummifera & Rwanda & $\begin{array}{l}\text { Mathur 1974, Mittal et } \\
\text { al. } 1990\end{array}$ \\
\hline & & seeds & Albizia julibrissin & Philippines & Quiniones 1987 \\
\hline & & seeds & Albizia procera & Philippines & $\begin{array}{l}\text { Agmata 1979, Quiniones } \\
\text { 1987, Anderson 1986a }\end{array}$ \\
\hline & & seeds & $\begin{array}{l}\text { Alstonia } \\
\text { macrophylla }\end{array}$ & Philippines & $\begin{array}{l}\text { Quiniones 1985, 1987, } \\
\text { Mittal et al. } 1990\end{array}$ \\
\hline & & seeds & Anogeissus pendula & Costa Rica & $\begin{array}{l}\text { Mathur 1974, Mittal et } \\
\text { al. } 1990\end{array}$ \\
\hline & & seeds & $\begin{array}{l}\text { Anthocephalus } \\
\text { chinensis }\end{array}$ & Philippines & Quiniones 1987 \\
\hline & & seeds & Azadirachta indica & India & $\begin{array}{l}\text { Mathur 1974, Mittal et } \\
\text { al. } 1990\end{array}$ \\
\hline & & seeds & Bauhinia sp. & Thailand & $\begin{array}{l}\text { Chalermpongse et al. } \\
\text { 1984, Mittal et al. } 1990\end{array}$ \\
\hline & & seeds & Bombax ceiba & India & $\begin{array}{l}\text { Mathur 1974, Mittal et } \\
\text { al. } 1990\end{array}$ \\
\hline & & seeds & $\begin{array}{l}\text { Bombax } \\
\text { malabaricum }\end{array}$ & Pakistan & Lodhi \& Naeem 1955 \\
\hline & & seeds & Calamus ornatus & Philippines & Quiniones 1987 \\
\hline & & seeds & Carpinus eximia & South Korea & $\begin{array}{l}\text { Mathur 1974, Mittal et } \\
\text { al. } 1990\end{array}$ \\
\hline & & seeds & Cassia acutifolia & Egypt & $\begin{array}{l}\text { Mathur 1974, Mittal et } \\
\text { al. } 1990\end{array}$ \\
\hline & & seeds & Cassia fistula & India & $\begin{array}{l}\text { Mathur 1974, Mittal et } \\
\text { al. } 1990\end{array}$ \\
\hline
\end{tabular}


Table 2 Continued.

\begin{tabular}{|c|c|c|c|c|c|}
\hline Species & Taxonomy & Substrate & Host & Country & References \\
\hline & & seeds & Cassia siamea & Philippines & $\begin{array}{l}\text { Quiniones 1985, 1987, } \\
\text { Mittal et al. } 1990\end{array}$ \\
\hline & & seeds & Casuarina spp. & India & Sahai \& Otra 1982 \\
\hline & & seeds & Cedrela odorata & Colombia & $\begin{array}{l}\text { Mathur 1974, Anderson } \\
\text { 1986a }\end{array}$ \\
\hline & & seeds & Cordia alliodora & Colombia & $\begin{array}{l}\text { Mathur 1974, Anderson } \\
\text { 1986a }\end{array}$ \\
\hline & & seeds & Delonix regia & Brazil, Philippines & $\begin{array}{l}\text { Dayan 1986, Anderson } \\
\text { 1986a, Quiniones } 1987\end{array}$ \\
\hline & & seeds & $\begin{array}{l}\text { Eucalyptus } \\
\text { camaldulensis }\end{array}$ & Egypt & $\begin{array}{l}\text { Mathur 1974, Anderson } \\
\text { 1986a }\end{array}$ \\
\hline & & seeds & Eucalyptus maidenii & Uruguay & Anderson 1986a \\
\hline & & seeds & Eucalyptus sp. & India & $\begin{array}{l}\text { Saxena 1985, Mittal et } \\
\text { al. } 1990\end{array}$ \\
\hline & & seeds & Ficus benjamina & India & $\begin{array}{l}\text { Mathur 1974, Anderson } \\
\text { 1986a }\end{array}$ \\
\hline & & seeds & Gmelina arborea & India & $\begin{array}{l}\text { Mathur 1974, Anderson } \\
\text { 1986a }\end{array}$ \\
\hline & & seeds & Gmelina moluccana & Solomon Islands & $\begin{array}{l}\text { Mathur 1974, Anderson } \\
\text { 1986a }\end{array}$ \\
\hline & & seeds & Grevillea robusta & Rwanda & $\begin{array}{l}\text { Mathur 1974, Anderson } \\
\text { 1986a }\end{array}$ \\
\hline & & seeds & Kydia calycina & India & $\begin{array}{l}\text { Mathur 1974, Anderson } \\
\text { 1986a }\end{array}$ \\
\hline & & seeds & $\begin{array}{l}\text { Leucaena } \\
\text { leucocephala }\end{array}$ & India, Philippines & $\begin{array}{l}\text { Singh et al. 1983, } \\
\text { Anderson 1986a, } \\
\text { Quiniones 1987, Mittal } \\
\text { et al. } 1990\end{array}$ \\
\hline & & seeds & Leucaena spp. & Philippines & $\begin{array}{l}\text { Mathur 1974, Anderson } \\
\text { 1986a }\end{array}$ \\
\hline & & seeds & Melaleuca spp. & India & Anderson 1986a \\
\hline & & seeds & Melia spp. & India & Sahai \& Otra 1982 \\
\hline & & seeds & $\begin{array}{l}\text { Mimosa } \\
\text { caesalpiniafolia }\end{array}$ & Brazil & $\begin{array}{l}\text { Mathur 1974, Anderson } \\
\text { 1986a }\end{array}$ \\
\hline
\end{tabular}


Table 2 Continued.

\begin{tabular}{|c|c|c|c|c|c|}
\hline Species & Taxonomy & Substrate & Host & Country & References \\
\hline & & seeds & Mimosa scabrella & Brazil & $\begin{array}{l}\text { Mathur 1974, Anderson } \\
\text { 1986a }\end{array}$ \\
\hline & & seeds & Picea excelsa & USSR & $\begin{array}{l}\text { Urosevic 1961, } \\
\text { Anderson 1986a }\end{array}$ \\
\hline & & seeds & $\begin{array}{l}\text { Piliostigma } \\
\text { malabaricum }\end{array}$ & Philippines & $\begin{array}{l}\text { Quiniones 1985, 1987, } \\
\text { Anderson 1986a }\end{array}$ \\
\hline & & seeds & Pinus caribaea & Cuba, UK & $\begin{array}{l}\text { Rees 1982, Anderson } \\
\text { 1986a, Mittal et al. } 1990\end{array}$ \\
\hline & & seeds & $\begin{array}{l}\text { Pinus caribaea var. } \\
\text { hondurensis }\end{array}$ & Brazil & $\begin{array}{l}\text { Mathur 1974, Anderson } \\
\text { 1986a }\end{array}$ \\
\hline & & seeds & Pinus elliottii & Argentina, USA & $\begin{array}{l}\text { Mathur 1974, Anderson } \\
\text { 1986a, Lori \& Salerno } \\
2003\end{array}$ \\
\hline & & seeds & $\begin{array}{l}\text { Pinus elliottii var. } \\
\text { elliottii }\end{array}$ & $\begin{array}{l}\text { Georgia, Uruguay, } \\
\text { USA }\end{array}$ & $\begin{array}{l}\text { Anderson 1986a, Huang } \\
\text { \& Kuhlman 1990, } \\
\text { Fraedrich \& Miller } 1995\end{array}$ \\
\hline & & seeds & Pinus kesiya & Philippines & $\begin{array}{l}\text { Quiniones 1985, } \\
\text { Anderson 1986a }\end{array}$ \\
\hline & & seeds & Pinus khasya & Madagascar & $\begin{array}{l}\text { Mathur 1974, Anderson } \\
\text { 1986a }\end{array}$ \\
\hline & & seeds & Pinus merkusii & Philippines & $\begin{array}{l}\text { Agmata 1979, Anderson } \\
\text { 1986a }\end{array}$ \\
\hline & & seeds & Pinus oocarpa & UK & $\begin{array}{l}\text { Rees 1982, Mittal et al. } \\
1990\end{array}$ \\
\hline & & seeds & Pinus patula & Madagascar & $\begin{array}{l}\text { Anderson 1986a, Gibson } \\
1957\end{array}$ \\
\hline & & seeds & Pinus pinaster & Uruguay & Anderson 1986a \\
\hline & & seeds & Pinus pseudostrobus & UK & $\begin{array}{l}\text { Rees 1982, Mittal et al. } \\
1990\end{array}$ \\
\hline & & seeds & Pinus roxburghii & India & $\begin{array}{l}\text { Mittal \& Sharma 1982c, } \\
\text { Mittal et al. } 1990\end{array}$ \\
\hline & & seeds & Pinus sylvestris & USSR & $\begin{array}{l}\text { Urosevic 1961, } \\
\text { Anderson 1986a }\end{array}$ \\
\hline
\end{tabular}


Table 2 Continued.

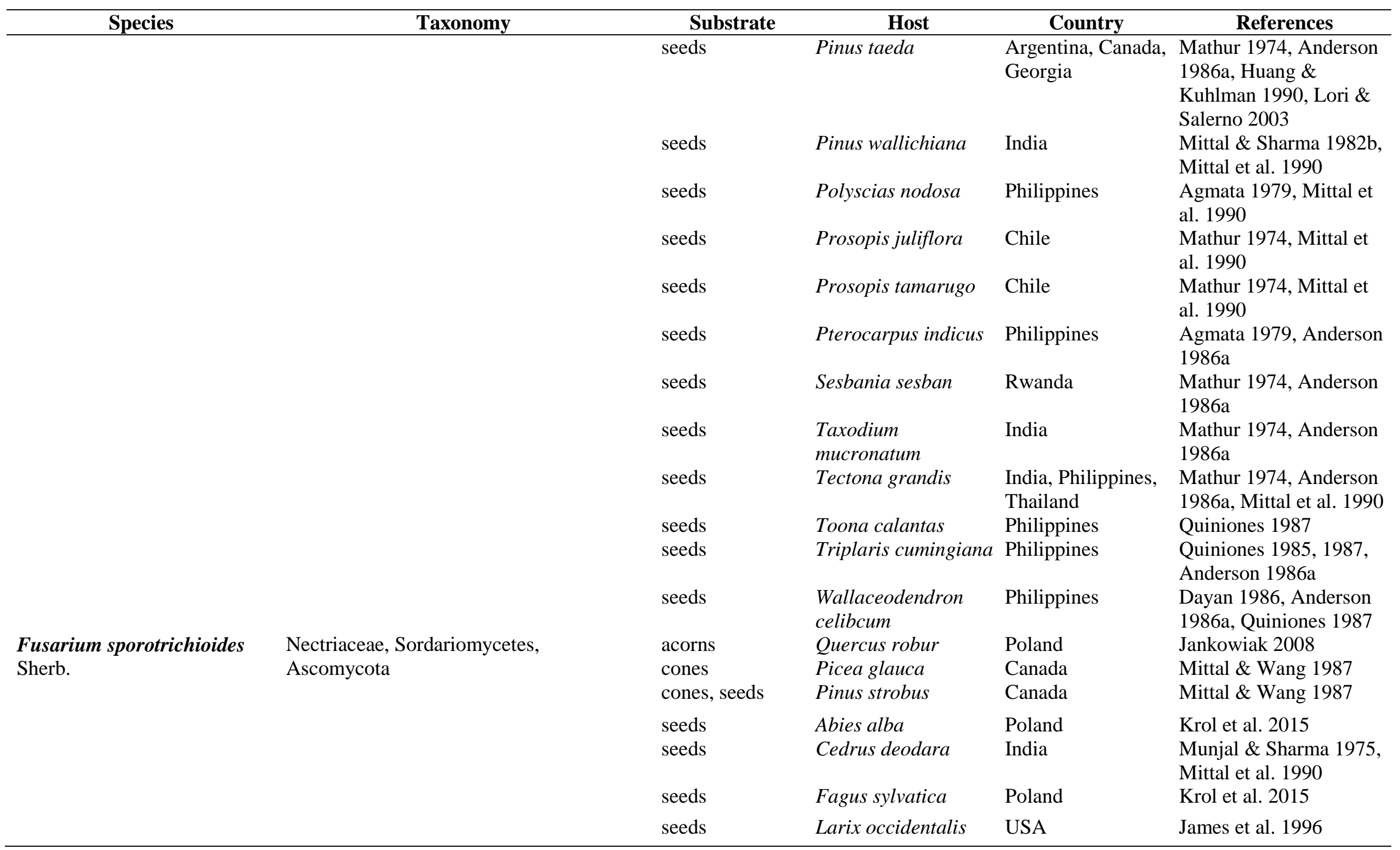


Table 2 Continued.

\begin{tabular}{|c|c|c|c|c|c|}
\hline Species & Taxonomy & Substrate & Host & Country & References \\
\hline & & seeds & Picea abies & USSR & $\begin{array}{l}\text { Prisyazhnyuk } 1960 \text {, } \\
\text { Mittal et al. } 1990\end{array}$ \\
\hline & & seeds & Picea excelsa & USSR & $\begin{array}{l}\text { Urosevic 1961, } \\
\text { Anderson 1986a }\end{array}$ \\
\hline & & seeds & Picea glauca & Canada & Mittal \& Wang 1987 \\
\hline & & seeds & Pinus ponderosa & USA & James 1985a \\
\hline & & seeds & Pinus roxburghii & India & $\begin{array}{l}\text { Munjal \& Sharma } 1975 \text {, } \\
\text { Mittal et al. } 1990\end{array}$ \\
\hline & & seeds & Pinus sylvestris & USSR & $\begin{array}{l}\text { Prisyazhnyuk 1960, } \\
\text { Anderson 1986a }\end{array}$ \\
\hline & & seeds & Pinus wallichiana & India & $\begin{array}{l}\text { Munjal \& Sharma } 1975 \text {, } \\
\text { Mittal et al. } 1990\end{array}$ \\
\hline & & seeds & Quercus robur & Poland & Krol et al. 2015 \\
\hline & & seeds & $\begin{array}{l}\text { Robinia } \\
\text { pseudoacacia }\end{array}$ & Hungary & $\begin{array}{l}\text { Hangyal-Balul } 1983 \text {, } \\
\text { Mittal et al. } 1990\end{array}$ \\
\hline \multirow[t]{15}{*}{ Fusarium spp. } & Nectriaceae, Sordariomycetes, & cones, seeds & Pinus nigra & Canada & Vujanovic et al. 2000 \\
\hline & Ascomycota & cones, seeds & $\begin{array}{l}\text { Pinus nigra ssp. } \\
\text { nigra }\end{array}$ & Canada & Vujanovic et al. 2000 \\
\hline & & cones, seeds & $\begin{array}{l}\text { Pinus sylvestris } \\
\text { 'Argentea- } \\
\text { Compacta' }\end{array}$ & Canada & Vujanovic et al. 2000 \\
\hline & & cones, seeds & Pinus uncinata & Canada & Vujanovic et al. 2000 \\
\hline & & fruits & Alocasia odora & Hong Kong & Tang et al. 2003a \\
\hline & & fruits & Ardisia punctata & Hong Kong & Tang et al. 2003a \\
\hline & & fruits & Avicennia marina & India & Mehdi \& Saifullah 2000 \\
\hline & & fruits & Diplospora dubia & Hong Kong & Tang et al. 2003a \\
\hline & & fruits & Ilex asprella & Hong Kong & Tang et al. 2003a \\
\hline & & fruits & Ilex cinerea & Hong Kong & Tang et al. 2003a \\
\hline & & fruits & Ilex pubescens & Hong Kong & Tang et al. 2003a \\
\hline & & fruits & Microcos paniculata & Hong Kong & Tang et al. 2003a \\
\hline & & fruits & Psychotria asiatica & Hong Kong & Tang et al. 2003a \\
\hline & & fruits & $\begin{array}{l}\text { Rhodomyrtus } \\
\text { tomentosa }\end{array}$ & Hong Kong & Tang et al. 2003a \\
\hline & & seed capsules & Eucalyptus grandis & South Africa & Jimu et al. 2016 \\
\hline
\end{tabular}


Table 2 Continued.

\begin{tabular}{|c|c|c|c|c|c|}
\hline Species & Taxonomy & Substrate & Host & Country & References \\
\hline & & seeds & Abies alba & Poland & Krol et al. 2015 \\
\hline & & seeds & Abies nordmanniana & Austria, Georgia & Talgø et al. 2010 \\
\hline & & seeds & Abies procera & Norway & Talgø et al. 2010 \\
\hline & & seeds & Acer pseudoplatanus & Poland & Krol et al. 2015 \\
\hline & & seeds & Acer rubrum & USA & $\begin{array}{l}\text { Friedrich et al. } 1971 \text {, } \\
\text { Mittal et al. } 1990\end{array}$ \\
\hline & & seeds & Acer saccharinum & USA & $\begin{array}{l}\text { Friedrich et al. } 1971 \text {, } \\
\text { Mittal et al. } 1990\end{array}$ \\
\hline & & seeds & Albizia lebbeck & India & $\begin{array}{l}\text { Natarajan et al. 2003, } \\
\text { Mohanan et al. } 2005\end{array}$ \\
\hline & & seeds & Alnus glutinosa & Poland & Krol et al. 2015 \\
\hline & & seeds & $\begin{array}{l}\text { Araucaria } \\
\text { angustifolia }\end{array}$ & Australia & $\begin{array}{l}\text { Kamara et al. } 1981 \text {, } \\
\text { Mittal et al. } 1990\end{array}$ \\
\hline & & seeds & $\begin{array}{l}\text { Araucaria } \\
\text { cunninghamii }\end{array}$ & Australia & $\begin{array}{l}\text { Kamara et al. } 1981 \text {, } \\
\text { Mittal et al. } 1990\end{array}$ \\
\hline & & seeds & $\begin{array}{l}\text { Araucaria } \\
\text { heterophylla }\end{array}$ & Egypt & $\begin{array}{l}\text { Kamara et al. } 1981 \text {, } \\
\text { Mittal et al. } 1990\end{array}$ \\
\hline & & seeds & Azadirachta indica & India & $\begin{array}{l}\text { Mathur 1974, Mittal et } \\
\text { al. } 1990\end{array}$ \\
\hline & & seeds & $\begin{array}{l}\text { Betula } \\
\text { alleghaniensis }\end{array}$ & USA & $\begin{array}{l}\text { Friedrich et al. } 1971 \text {, } \\
\text { Mittal et al. } 1990\end{array}$ \\
\hline & & seeds & Betula papyrifera & USA & $\begin{array}{l}\text { Friedrich et al. } 1971 \text {, } \\
\text { Mittal et al. } 1990\end{array}$ \\
\hline & & seeds & Betula pendula & Poland & Krol et al. 2015 \\
\hline & & seeds & Bombax ceiba & India & Mittal et al. 1990 \\
\hline & & seeds & $\begin{array}{l}\text { Cupressus } \\
\text { sempervirens }\end{array}$ & Egypt & $\begin{array}{l}\text { Farag et al. 1977, Mittal } \\
\text { et al. } 1990\end{array}$ \\
\hline & & seeds & Dalbergia sissoides & India & Mohanan et al. 2005 \\
\hline & & seeds & Dalbergia sissoo & India & $\begin{array}{l}\text { Mittal \& Sharma 1981b, } \\
\text { Mittal et al. } 1990\end{array}$ \\
\hline & & seeds & $\begin{array}{l}\text { Dianthus } \\
\text { caryophyllus }\end{array}$ & Taiwan & Li \& Wu 2002 \\
\hline & & seeds & Dianthus spp. & Taiwan & Li \& Wu 2002 \\
\hline
\end{tabular}


Table 2 Continued.

\begin{tabular}{|c|c|c|c|c|c|}
\hline Species & Taxonomy & Substrate & Host & Country & References \\
\hline & & seeds & $\begin{array}{l}\text { Eucalyptus } \\
\text { citriodora }\end{array}$ & India & $\begin{array}{l}\text { Mittal \& Sharma 1984, } \\
\text { Mittal et al. } 1990\end{array}$ \\
\hline & & seeds & Eucalyptus globulus & Portugal & $\begin{array}{l}\text { Mathur 1974, Anderson } \\
\text { 1986a }\end{array}$ \\
\hline & & seeds & Eucalyptus grandis & South Africa & Jimu et al. 2016 \\
\hline & & seeds & Eucalyptus hybrid & India & $\begin{array}{l}\text { Mittal 1986, Mittal et al. } \\
1990\end{array}$ \\
\hline & & seeds & Fagus sylvatica & Hungary, Poland & $\begin{array}{l}\text { Mittal et al. 1990, Krol } \\
\text { et al. } 2015\end{array}$ \\
\hline & & seeds & Fraxinus americana & USA & $\begin{array}{l}\text { Friedrich et al. } 1971 \text {, } \\
\text { Mittal et al. } 1990\end{array}$ \\
\hline & & seeds & Fraxinus excelsior & Sweden & $\begin{array}{l}\text { Cleary et al. 2013, } \\
\text { Hayatgheibi } 2013\end{array}$ \\
\hline & & seeds & Larix decidua & Poland & Krol et al. 2015 \\
\hline & & seeds & $\begin{array}{l}\text { Leucaena } \\
\text { leucocephala }\end{array}$ & Bangladesh & Islam et al. 2008 \\
\hline & & seeds & Maesopsis eminii & Malaysia & Mittal et al. 1990 \\
\hline & & seeds & Phyllostachys edulis & China & Shen et al. 2014 \\
\hline & & seeds & Picea abies & Poland & Krol et al. 2015 \\
\hline & & seeds & Picea sitchensis & UK & $\begin{array}{l}\text { Salt 1964, Mittal et al. } \\
1990\end{array}$ \\
\hline & & seeds & $\begin{array}{l}\text { Pinus armandii var. } \\
\text { mastersiana }\end{array}$ & Taiwan & $\begin{array}{l}\text { Jong \& Chen } 1966 \text {, } \\
\text { Mittal et al. } 1990\end{array}$ \\
\hline & & seeds & Pinus elliottii & Taiwan, USA & Mittal et al. 1990 \\
\hline & & seeds & $\begin{array}{l}\text { Pinus elliottii var. } \\
\text { elliottii }\end{array}$ & USA & Fraedrich \& Miller 1995 \\
\hline & & seeds & Pinus luchuensis & Taiwan & $\begin{array}{l}\text { Jong \& Chen } 1966 \text {, } \\
\text { Mittal et al. } 1990\end{array}$ \\
\hline & & seeds & Pinus massoniana & Taiwan & $\begin{array}{l}\text { Jong \& Chen 1966, } \\
\text { Mittal et al. } 1990\end{array}$ \\
\hline & & seeds & Pinus nigra & Hungary & $\begin{array}{l}\text { Hangyal 1973, Mittal et } \\
\text { al. } 1990\end{array}$ \\
\hline & & seeds & Pinus sylvestris & Hungary, Poland & $\begin{array}{l}\text { Hangyal 1973, Mittal et } \\
\text { al. 1990, Krol et al. } 2015\end{array}$ \\
\hline
\end{tabular}


Table 2 Continued.

\begin{tabular}{|c|c|c|c|c|c|}
\hline Species & Taxonomy & Substrate & Host & Country & References \\
\hline & & seeds & Pinus taeda & USA & $\begin{array}{l}\text { Covington et al. } 1982 \text {, } \\
\text { Mittal et al. } 1990\end{array}$ \\
\hline & & seeds & Pinus taiwanensis & Taiwan & $\begin{array}{l}\text { Jong \& Chen } 1966 \text {, } \\
\text { Mittal et al. } 1990\end{array}$ \\
\hline & & seeds & $\begin{array}{l}\text { Pinus thunbergii } \\
\text { [= Pinus } \\
\text { thunbergiana }]\end{array}$ & Taiwan & $\begin{array}{l}\text { Jong \& Chen } 1966 \text {, } \\
\text { Mittal et al. } 1990\end{array}$ \\
\hline & & seed & $\begin{array}{l}\text { Portulaca } \\
\text { grandiflora }\end{array}$ & Canada & Conners 1967 \\
\hline & & seeds & $\begin{array}{l}\text { Pseudotsuga } \\
\text { menziesii }\end{array}$ & Canada & $\begin{array}{l}\text { Salisbury 1955, Mittal et } \\
\text { al. } 1990\end{array}$ \\
\hline & & seeds & Quercus robur & $\begin{array}{l}\text { Germany, Poland, } \\
\text { Turkey }\end{array}$ & $\begin{array}{l}\text { Schroder et al. 2004, } \\
\text { Krol et al. 2015, Oskay } \\
\text { et al. } 2018\end{array}$ \\
\hline & & seeds & Quercus rubra & Poland & Krol et al. 2015 \\
\hline & & seeds & Quercus spp. & USSR & $\begin{array}{l}\text { Urosevic 1983, Mittal et } \\
\text { al. } 1990\end{array}$ \\
\hline & & seeds & Shorea assamica & Malaysia & $\begin{array}{l}\text { Hong 1981, Mittal et al. } \\
1990\end{array}$ \\
\hline & & seeds & Shorea robusta & India & $\begin{array}{l}\text { Mittal \& Sharma 1982b, } \\
\text { Mittal et al. } 1990\end{array}$ \\
\hline & & seeds & Shorea talura & Malaysia & Mittal et al. 1990 \\
\hline & & seeds & Tectona grandis & India & Mohanan et al. 2005 \\
\hline $\begin{array}{l}\text { Fusarium stilboides } \\
\text { Wollenw. }\end{array}$ & $\begin{array}{l}\text { Nectriaceae, Sordariomycetes, } \\
\text { Ascomycota }\end{array}$ & acorns & Quercus robur & Poland & Jankowiak 2008 \\
\hline $\begin{array}{l}\text { Fusarium subglutinans } \\
\text { (Wollenw. \& Reinking) P.E. } \\
\text { Nelson, Toussoun \& Marasas }\end{array}$ & $\begin{array}{l}\text { Nectriaceae, Sordariomycetes, } \\
\text { Ascomycota }\end{array}$ & seeds & $\begin{array}{l}\text { Pinus elliottii var. } \\
\text { elliottii }\end{array}$ & USA & Fraedrich \& Miller 1995 \\
\hline $\begin{array}{l}\text { Fusarium subglutinans f.sp. } \\
\text { pini J.C. Correll, T.R. } \\
\text { Gordon, McCain, J.W. Fox, } \\
\text { Koehler, D.L. Wood \& M.E. } \\
\text { Schultz }\end{array}$ & $\begin{array}{l}\text { Nectriaceae, Sordariomycetes, } \\
\text { Ascomycota }\end{array}$ & $\begin{array}{l}\text { cones } \\
\text { seeds }\end{array}$ & $\begin{array}{l}\text { Pinus radiata } \\
\text { Pinus radiata }\end{array}$ & $\begin{array}{l}\text { USA } \\
\text { USA }\end{array}$ & $\begin{array}{l}\text { Dwinell } 1998 \\
\text { Dwinell 1998, Storer et } \\
\text { al. } 1998\end{array}$ \\
\hline
\end{tabular}


Table 2 Continued.

\begin{tabular}{|c|c|c|c|c|c|}
\hline \multirow{2}{*}{$\begin{array}{l}\text { Species } \\
\text { Fusarium torulosum (Berk. } \\
\text { \& M.A. Curtis) Nirenberg }\end{array}$} & \multirow{2}{*}{$\begin{array}{l}\text { Taxonomy } \\
\text { Nectriaceae, Sordariomycetes, } \\
\text { Ascomycota }\end{array}$} & \multirow{2}{*}{$\begin{array}{l}\text { Substrate } \\
\text { acorns }\end{array}$} & \multirow{2}{*}{$\begin{array}{c}\text { Host } \\
\text { Quercus robur }\end{array}$} & \multirow{2}{*}{$\begin{array}{l}\text { Country } \\
\text { Poland }\end{array}$} & \multirow{2}{*}{$\begin{array}{r}\text { References } \\
\text { Jankowiak } 2008\end{array}$} \\
\hline & & & & & \\
\hline \multirow{5}{*}{$\begin{array}{l}\text { Fusarium tricinctum } \\
\text { (Corda) Sacc. }\end{array}$} & \multirow{5}{*}{$\begin{array}{l}\text { Nectriaceae, Sordariomycetes, } \\
\text { Ascomycota }\end{array}$} & seeds & Picea abies & Italy & Motta et al. 1996 \\
\hline & & seeds & Picea glauca & USA & James 1985b \\
\hline & & seeds & Picea pungens & USA & James $1985 b$ \\
\hline & & seeds & Pinus palustris & USA & $\begin{array}{l}\text { Pawuk 1978, Mittal et al. } \\
1990\end{array}$ \\
\hline & & seeds & Pinus taeda & USA & $\begin{array}{l}\text { Mason \& van Arsdel } \\
\text { 1978, Anderson 1986a }\end{array}$ \\
\hline \multirow{4}{*}{$\begin{array}{l}\text { Fusarium verticillioides } \\
\text { (Sacc.) Nirenberg }\end{array}$} & \multirow{4}{*}{$\begin{array}{l}\text { Nectriaceae, Sordariomycetes, } \\
\text { Ascomycota }\end{array}$} & seeds & Alnus glutinosa & Poland & Krol et al. 2015 \\
\hline & & seeds & Larix decidua & Poland & Krol et al. 2015 \\
\hline & & seeds & Pinus elliottii & Argentina & Lori \& Salerno 2003 \\
\hline & & seeds & Pinus taeda & Argentina & Lori \& Salerno 2003 \\
\hline $\begin{array}{l}\text { Fusella olivacea (Corda) } \\
\text { Sacc. }\end{array}$ & $\begin{array}{l}\text { Incertae sedis, Incertae sedis, } \\
\text { Ascomycota }\end{array}$ & seeds & Quercus spp. & USSR & $\begin{array}{l}\text { Urosevic 1962, Mittal et } \\
\text { al. } 1990\end{array}$ \\
\hline \multirow[t]{7}{*}{ Fusicoccum spp. } & \multirow{7}{*}{$\begin{array}{l}\text { Botryosphaeriaceae, Dothideomycetes, } \\
\text { Ascomycota }\end{array}$} & cones, seeds & Pinus flexilis & Canada & Vujanovic et al. 2000 \\
\hline & & cones, seeds & Pinus mugo 'Galica' & Canada & Vujanovic et al. 2000 \\
\hline & & seeds & $\begin{array}{l}\text { Eucalyptus } \\
\text { citriodora }\end{array}$ & India & Anderson 1986a \\
\hline & & seeds & Pinus elliottii & USA & $\begin{array}{l}\text { Rowan \& Debarr 1974, } \\
\text { Mittal et al. } 1990\end{array}$ \\
\hline & & seeds & $\begin{array}{l}\text { Pinus elliottii var. } \\
\text { elliottii }\end{array}$ & USA & Fraedrich \& Miller 1995 \\
\hline & & seeds & Pinus massoniana & Taiwan & $\begin{array}{l}\text { Jong \& Chen } 1966 \text {, } \\
\text { Mittal et al. } 1990\end{array}$ \\
\hline & & seeds & Tectona grandis & India & Anderson 1986a \\
\hline \multirow{2}{*}{$\begin{array}{l}\text { Fusicolla acetilerea (Tubaki, } \\
\text { C. Booth \& T. Harada) } \\
\text { Gräfenhan \& Seifert } \\
\text { [as Fusarium merismoides } \\
\text { var. acetilereum Tubaki, C. } \\
\text { Booth \& T. Harada] }\end{array}$} & \multirow[t]{2}{*}{$\begin{array}{l}\text { Nectriaceae, Sordariomycetes, } \\
\text { Ascomycota }\end{array}$} & seeds & Pinus oocarpa & UK & $\begin{array}{l}\text { Rees 1982, Mittal et al. } \\
1990\end{array}$ \\
\hline & & seeds & Pinus pseudostrobus & UK & $\begin{array}{l}\text { Rees 1982, Mittal et al. } \\
1990\end{array}$ \\
\hline $\begin{array}{l}\text { Fusicolla merismoides } \\
\text { (Corda) Gräfenhan [as }\end{array}$ & $\begin{array}{l}\text { Nectriaceae, Sordariomycetes, } \\
\text { Ascomycota }\end{array}$ & seeds & Pinus caribaea & $\mathrm{UK}$ & $\begin{array}{l}\text { Rees 1982, Mittal et al. } \\
1990\end{array}$ \\
\hline
\end{tabular}


Table 2 Continued.

\begin{tabular}{|c|c|c|c|c|c|}
\hline Species & Taxonomy & Substrate & Host & Country & References \\
\hline $\begin{array}{l}\text { Fusarium merismoides } \\
\text { Corda] }\end{array}$ & & seeds & Quercus spp. & USSR & Mittal et al. 1990 \\
\hline Fusidium spp. & $\begin{array}{l}\text { Nectriaceae, Sordariomycetes, } \\
\text { Ascomycota }\end{array}$ & $\begin{array}{l}\text { acorns } \\
\text { cones } \\
\text { seeds }\end{array}$ & $\begin{array}{l}\text { Quercus robur } \\
\text { Pinus densiflora } \\
\text { Acer saccharum }\end{array}$ & $\begin{array}{l}\text { Poland } \\
\text { Japan } \\
\text { USA }\end{array}$ & $\begin{array}{l}\text { Jankowiak } 2008 \\
\text { Kasai et al. } 1995 \\
\text { Friedrich et al. } 1971 \text {, } \\
\text { Mittal et al. } 1990\end{array}$ \\
\hline Gaeumannomyces sp. & $\begin{array}{l}\text { Magnaporthaceae, Sordariomycetes, } \\
\text { Ascomycota }\end{array}$ & $\begin{array}{l}\text { fruits } \\
\text { fruits } \\
\text { fruits }\end{array}$ & $\begin{array}{l}\text { Dichroa febrifuga } \\
\text { Psychotria asiatica } \\
\text { Sarcandra glabra }\end{array}$ & $\begin{array}{l}\text { Hong Kong } \\
\text { Hong Kong } \\
\text { Hong Kong }\end{array}$ & $\begin{array}{l}\text { Tang et al. 2003a } \\
\text { Tang et al. 2003a } \\
\text { Tang et al. 2003a }\end{array}$ \\
\hline \multirow[t]{8}{*}{$\begin{array}{l}\text { Genicularia spp. } \\
\text { Geniculodendron pyriforme } \\
\text { G.A. Salt }\end{array}$} & $\begin{array}{l}\text { Orbiliaceae, Orbiliomycetes, Ascomycota } \\
\text { Caloscyphaceae, Pezizomycetes, } \\
\text { Ascomycota }\end{array}$ & $\begin{array}{l}\text { seeds } \\
\text { seeds }\end{array}$ & $\begin{array}{l}\text { Abies procera } \\
\text { Abies concolor } \\
\text { 'glauca' }\end{array}$ & $\begin{array}{l}\text { Norway } \\
\text { USA }\end{array}$ & $\begin{array}{l}\text { Talgø et al. } 2010 \\
\text { Schröder et al. } 2002\end{array}$ \\
\hline & & seeds & Abies concolor & $\begin{array}{l}\text { Germany } \\
\text { (imported seeds } \\
\text { from USA and } \\
\text { Canada) }\end{array}$ & Schröder et al. 2002 \\
\hline & & seeds & Abies grandis & $\begin{array}{l}\text { Germany } \\
\text { (imported seeds } \\
\text { from USA and } \\
\text { Canada), USA }\end{array}$ & Schröder et al. 2002 \\
\hline & & seeds & Larix occidentalis & USA & James et al. 1996 \\
\hline & & seeds & Picea engelmannii & $\begin{array}{l}\text { Germany } \\
\text { (imported seeds } \\
\text { from USA and } \\
\text { Canada) }\end{array}$ & Schröder et al. 2002 \\
\hline & & seeds & Picea sitchensis & $\begin{array}{l}\text { Canada, Germany } \\
\text { (imported seeds } \\
\text { from USA and } \\
\text { Canada) }\end{array}$ & $\begin{array}{l}\text { Sutherland \& Woods } \\
\text { 1978, Schröder et al. } \\
2002\end{array}$ \\
\hline & & seeds & Picea spp. & Canada & $\begin{array}{l}\text { Woods et al. 1982, } \\
\text { Anderson 1986a }\end{array}$ \\
\hline & & seeds & Pinus resinosa & Canada & $\begin{array}{l}\text { Epners 1964, Mittal et } \\
\text { al. } 1990\end{array}$ \\
\hline
\end{tabular}


Table 2 Continued.

\begin{tabular}{|c|c|c|c|c|c|}
\hline Species & Taxonomy & Substrate & Host & Country & References \\
\hline & & seeds & Pinus spp. & UK & $\begin{array}{l}\text { Salt 1974, Mittal et al. } \\
1990\end{array}$ \\
\hline & & seeds & Pinus sylvestris & Canada & $\begin{array}{l}\text { Epners 1964, Mittal et } \\
\text { al. } 1990\end{array}$ \\
\hline \multirow[t]{2}{*}{ Geniculosporium spp. } & Xylariaceae, Sordariomycetes, & cones & Pinus densiflora & Japan & Kasai et al. 1995 \\
\hline & Ascomycota & fruit cupules & Fagus crenata & Japan & $\begin{array}{l}\text { Fukasawa et al. 2012, } \\
\text { Tateno et al. } 2015\end{array}$ \\
\hline Geomyces sp. & $\begin{array}{l}\text { Thelebolaceae, Leotiomycetes, } \\
\text { Ascomycota }\end{array}$ & seed & Pinus monticola & USA & $\begin{array}{l}\text { Ganley \& Newcombe } \\
2006\end{array}$ \\
\hline Geopyxis sp. & Tarzettaceae, Pezizomycetes, Ascomycota & seed & Pinus monticola & USA & $\begin{array}{l}\text { Ganley \& Newcombe } \\
2006\end{array}$ \\
\hline \multirow[t]{10}{*}{ Geotrichum spp. } & $\begin{array}{l}\text { Dipodascaceae, Saccharomycetes, } \\
\text { Ascomycota }\end{array}$ & fruits & $\begin{array}{l}\text { Parahancornia } \\
\text { amapa }\end{array}$ & Brazil & Morais et al. 1995 \\
\hline & & seeds & Acer rubrum & USA & $\begin{array}{l}\text { Friedrich et al. } 1971 \text {, } \\
\text { Mittal et al. } 1990\end{array}$ \\
\hline & & seeds & Acer saccharinum & USA & $\begin{array}{l}\text { Friedrich et al. } 1971 \text {, } \\
\text { Mittal et al. } 1990\end{array}$ \\
\hline & & seeds & Albizia lebbeck & India & Natarajan et al. 2003 \\
\hline & & seed & Cercis chinensis & Japan & Watanabe 2010 \\
\hline & & seeds & Dalbergia sissoo & India & Naz et al. 2015 \\
\hline & & seeds & Fraxinus americana & USA & $\begin{array}{l}\text { Friedrich et al. } 1971 \text {, } \\
\text { Mittal et al. } 1990\end{array}$ \\
\hline & & seeds & Pinus elliottii & USA & Mittal et al. 1990 \\
\hline & & seeds & Pinus taeda & USA & $\begin{array}{l}\text { Mason \& van Arsdel } \\
\text { 1978, Anderson 1986a }\end{array}$ \\
\hline & & seed & Prunus serrulata & Japan & Watanabe 2010 \\
\hline $\begin{array}{l}\text { Gibbera sphyrospermi G.J. } \\
\text { Samuel, M.E. Barr \& C.T. } \\
\text { Rogers }^{\#}\end{array}$ & $\begin{array}{l}\text { Incertae sedis, Dothideomycetes, } \\
\text { Ascomycota }\end{array}$ & fruit & $\begin{array}{l}\text { Sphyrospermum } \\
\text { cordifolium }\end{array}$ & Panama & Samuels et al. 1988 \\
\hline \multirow[t]{2}{*}{ Gilmaniella spp. } & $\begin{array}{l}\text { Incertae sedis, Incertae sedis, } \\
\text { Ascomycota }\end{array}$ & seeds & Cassia bakeriana & Thailand & $\begin{array}{l}\text { Chalermpongse et al. } \\
\text { 1984, Mittal et al. } 1990\end{array}$ \\
\hline & & seeds & Pinus taeda & USA & Anderson 1986a, b \\
\hline $\begin{array}{l}\text { Glaxoa pellucida P.F. } \\
\text { Cannon }{ }^{\# * *}\end{array}$ & $\begin{array}{l}\text { Incertae sedis, Dothideomycetes, } \\
\text { Ascomycota }\end{array}$ & cones & $\begin{array}{l}\text { Cupressus } \\
\text { semipervirens }\end{array}$ & UK & Cannon 1997 \\
\hline
\end{tabular}


Table 2 Continued.

\begin{tabular}{|c|c|c|c|c|c|}
\hline Species & Taxonomy & Substrate & Host & Country & References \\
\hline $\begin{array}{l}\text { Gliocephalotrichum } \\
\text { bulbilium J.J. Ellis \& } \\
\text { Hesselt. }\end{array}$ & $\begin{array}{l}\text { Nectriaceae, Sordariomycetes, } \\
\text { Ascomycota }\end{array}$ & cones & Pinus densiflora & Japan & Kasai et al. 1995 \\
\hline Gliocephalotrichum sp. & $\begin{array}{l}\text { Nectriaceae, Sordariomycetes, } \\
\text { Ascomycota }\end{array}$ & seeds & $\begin{array}{l}\text { Eucalyptus } \\
\text { citriodora }\end{array}$ & India & Anderson 1986a \\
\hline \multirow[t]{6}{*}{ Gliocladium roseum Bainier } & \multirow[t]{6}{*}{$\begin{array}{l}\text { Bionectriaceae, Sordariomycetes, } \\
\text { Ascomycota }\end{array}$} & seed & Picea excelsa & USSR & $\begin{array}{l}\text { Urosevic 1961, } \\
\text { Anderson 1986a }\end{array}$ \\
\hline & & seeds & Picea sitchensis & UK & $\begin{array}{l}\text { Salt 1964, Mittal et al. } \\
1990\end{array}$ \\
\hline & & seeds & Pinus densiflora & Japan & Watanabe 2010 \\
\hline & & seeds & Pinus elliottii & South Africa & Cilliers et al. 1995 \\
\hline & & seeds & Pinus sylvestris & USSR & $\begin{array}{l}\text { Urosevic 1961, } \\
\text { Anderson 1986a }\end{array}$ \\
\hline & & seeds & Pinus wallichiana & India & Mittal \& Sharma 1982b \\
\hline Gliocladium solani (Harting) & Bionectriaceae, Sordariomycetes, & fruits & Alocasia odora & Hong Kong & Tang et al. 2003a \\
\hline \multirow[t]{5}{*}{ Petch } & \multirow[t]{5}{*}{ Ascomycota } & fruits & Diplospora dubia & Hong Kong & Tang et al. 2003a \\
\hline & & fruits & Ilex asprella & Hong Kong & Tang et al. 2003a \\
\hline & & fruits & Ilex cinerea & Hong Kong & Tang et al. 2003a \\
\hline & & fruits & Psychotria asiatica & Hong Kong & Tang et al. 2003a \\
\hline & & fruits & $\begin{array}{l}\text { Viburnum } \\
\text { sempervirens }\end{array}$ & Hong Kong & Tang et al. 2003a \\
\hline \multirow[t]{6}{*}{ Gliocladium spp. } & \multirow{6}{*}{$\begin{array}{l}\text { Hypocreaceae, Sordariomycetes, } \\
\text { Ascomycota }\end{array}$} & cones & Pinus densiflora & Japan & Kasai et al. 1995 \\
\hline & & seed & Gentiana sp. & Japan & Watanabe 2010 \\
\hline & & seeds & $\begin{array}{l}\text { Pinus elliottii var. } \\
\text { elliottii }\end{array}$ & Uruguay & Anderson 1986a \\
\hline & & seeds & Pinus ponderosa & USA & $\begin{array}{l}\text { James \& Genz 1982, } \\
\text { Anderson 1986a }\end{array}$ \\
\hline & & seeds & Pinus taeda & USA & Anderson 1986a, b \\
\hline & & seeds & $\begin{array}{l}\text { Pseudotsuga } \\
\text { menziesii }\end{array}$ & Canada & $\begin{array}{l}\text { Bloomberg 1969, Mittal } \\
\text { et al. } 1990\end{array}$ \\
\hline $\begin{array}{l}\text { Gliocladium verticillioides } \\
\text { (G.A. Newton) Pidoplitschka }\end{array}$ & $\begin{array}{l}\text { Hypocreaceae, Sordariomycetes, } \\
\text { Ascomycota }\end{array}$ & seeds & Quercus spp. & USSR & $\begin{array}{l}\text { Urosevic 1962, Mittal et } \\
\text { al. } 1990\end{array}$ \\
\hline $\begin{array}{l}\text { Gliomastix roseogrisea (S.B. } \\
\text { Saksena) Summerb. }\end{array}$ & $\begin{array}{l}\text { Bionectriaceae, Sordariomycetes, } \\
\text { Ascomycota }\end{array}$ & seeds & Cedrus deodara & India & Mittal 1983 \\
\hline
\end{tabular}


Table 2 Continued.

\begin{tabular}{|c|c|c|c|c|c|}
\hline $\begin{array}{c}\text { Species } \\
\end{array}$ & Taxonomy & Substrate & Host & Country & References \\
\hline \multicolumn{6}{|l|}{$\begin{array}{l}\text { [as Cephalosporium } \\
\text { roseogriseum S.B. Saksena] }\end{array}$} \\
\hline $\begin{array}{l}\text { Gliocladiopsis tenuis } \\
\text { (Bugnic.) Crous \& M.J. } \\
\text { Wingf. [as Cylindrocarpon } \\
\text { tenue Bugnic.] }\end{array}$ & $\begin{array}{l}\text { Nectriaceae, Sordariomycetes, } \\
\text { Ascomycota }\end{array}$ & cones & $\begin{array}{l}\text { Pseudotsuga } \\
\text { menziesii var. glauca }\end{array}$ & USA & James 1995 \\
\hline $\begin{array}{l}\text { Gloeosporium acericola } \\
\text { Allesch. }\end{array}$ & $\begin{array}{l}\text { Dermateaceae, Leotiomycetes, } \\
\text { Ascomycota }\end{array}$ & seeds & Acer sp. & USSR & Anderson 1986a \\
\hline $\begin{array}{l}\text { Gloeosporium bolleyi R. } \\
\text { Sprague }\end{array}$ & $\begin{array}{l}\text { Dermateaceae, Leotiomycetes, } \\
\text { Ascomycota }\end{array}$ & seed & Setaria viridis & Canada & Conners 1967 \\
\hline \multirow[t]{5}{*}{ Gloeosporium spp. } & $\begin{array}{l}\text { Dermateaceae, Leotiomycetes, } \\
\text { Ascomycota }\end{array}$ & seeds & Acer saccharinum & USA & $\begin{array}{l}\text { Friedrich et al. } 1971 \text {, } \\
\text { Mittal et al. } 1990\end{array}$ \\
\hline & & seeds & $\begin{array}{l}\text { Liriodendron } \\
\text { tulipifera }\end{array}$ & USA & Anderson 1986a \\
\hline & & seeds & $\begin{array}{l}\text { Pinus armandii var. } \\
\text { mastersiana }\end{array}$ & Taiwan & $\begin{array}{l}\text { Jong \& Chen } 1966 \text {, } \\
\text { Mittal et al. } 1990\end{array}$ \\
\hline & & seeds & Pinus massoniana & Taiwan & $\begin{array}{l}\text { Jong \& Chen } 1966 \text {, } \\
\text { Mittal et al. } 1990\end{array}$ \\
\hline & & seeds & $\begin{array}{l}\text { Platanus } \\
\text { occidentalis }\end{array}$ & USA & $\begin{array}{l}\text { Fakir et al. 1971, Mittal } \\
\text { et al. } 1990\end{array}$ \\
\hline \multirow[t]{2}{*}{$\begin{array}{l}\text { Gloeosporium ulmicola } \\
\text { Miles }\end{array}$} & $\begin{array}{l}\text { Dermateaceae, Leotiomycetes, } \\
\text { Ascomycota }\end{array}$ & seeds & Ulmus pumila & Romania & $\begin{array}{l}\text { Georgescu \& Petrescu } \\
\text { 1954, Mittal et al. } 1990\end{array}$ \\
\hline & & seeds & Ulmus spp. & Romania & $\begin{array}{l}\text { Richardson 1979, } \\
\text { Anderson 1986a }\end{array}$ \\
\hline $\begin{array}{l}\text { Gloeotinia temulenta (Prill. } \\
\text { \& Delacr.) M. Wilson, Noble } \\
\text { \& E.G. Gray }\end{array}$ & Helotiaceae, Leotiomycetes, Ascomycota & seed & Lolium perenne & Scotland & Conners 1967 \\
\hline $\begin{array}{l}\text { Gloniopsis fluctiformis } \\
\text { Jayasiri, E.B.G. Jones \& } \\
\text { K.D. Hyde }^{\#}\end{array}$ & $\begin{array}{l}\text { Hysteriaceae, Dothideomycetes, } \\
\text { Ascomycota }\end{array}$ & fruit & $\begin{array}{l}\text { Combretaceae } \\
\text { plant }\end{array}$ & Thailand & Jayasiri et al. 2019 \\
\hline $\begin{array}{l}\text { Gloniopsis leucaenae } \\
\text { Jayasiri, E.B.G. Jones \& } \\
\text { K.D. Hyde }{ }\end{array}$ & $\begin{array}{l}\text { Hysteriaceae, Dothideomycetes, } \\
\text { Ascomycota }\end{array}$ & pod & Leucaena sp. & Thailand & Jayasiri et al. 2019 \\
\hline
\end{tabular}


Table 2 Continued.

\begin{tabular}{|c|c|c|c|c|c|}
\hline Species & Taxonomy & Substrate & Host & Country & References \\
\hline Gnomonia quercina Kleb. & $\begin{array}{l}\text { Gnomoniaceae, Sordariomycetes, } \\
\text { Ascomycota }\end{array}$ & seeds & Quercus spp. & USSR & $\begin{array}{l}\text { Urosevic 1962, Mittal et } \\
\text { al. } 1990\end{array}$ \\
\hline $\begin{array}{l}\text { Gnomoniopsis fructicola (G. } \\
\text { Arnaud) Sogonov [as } \\
\text { Gnomonia fructicola (G. } \\
\text { Arnaud) Fall] }\end{array}$ & $\begin{array}{l}\text { Gnomoniaceae, Sordariomycetes, } \\
\text { Ascomycota }\end{array}$ & fruits & Litsea rotundifolia & Hong Kong & Tang et al. 2003a \\
\hline Gonapodya bohemica Cejp & $\begin{array}{l}\text { Gonapodyaceae, Monoblepharidomycetes, } \\
\text { Chytridiomycota }\end{array}$ & seeds & Sambucus racemosa & $\begin{array}{l}\text { Poland or/ } \\
\text { imported to Poland }\end{array}$ & Czeczuga et al. 2009 \\
\hline \multirow[t]{8}{*}{$\begin{array}{l}\text { Gonapodya polymorpha } \\
\text { Thaxt. }\end{array}$} & $\begin{array}{l}\text { Gonapodyaceae, Monoblepharidomycetes, } \\
\text { Chytridiomycota }\end{array}$ & seeds & Calluna vulgaris & $\begin{array}{l}\text { Poland or/ } \\
\text { imported to Poland }\end{array}$ & Czeczuga et al. 2009 \\
\hline & & seeds & Cornus capitata & $\begin{array}{l}\text { Poland or/ } \\
\text { imported to Poland }\end{array}$ & Czeczuga et al. 2009 \\
\hline & & seeds & Crataegus azarolus & $\begin{array}{l}\text { Poland or/ } \\
\text { imported to Poland }\end{array}$ & Czeczuga et al. 2009 \\
\hline & & seeds & $\begin{array}{l}\text { Lonicera } \\
\text { periclymenum }\end{array}$ & $\begin{array}{l}\text { Poland or/ } \\
\text { imported to Poland }\end{array}$ & Czeczuga et al. 2009 \\
\hline & & seeds & Lotus uliginosa & $\begin{array}{l}\text { Poland or/ } \\
\text { imported to Poland }\end{array}$ & Czeczuga et al. 2009 \\
\hline & & seeds & Lycium chinense & $\begin{array}{l}\text { Poland or/ } \\
\text { imported to Poland }\end{array}$ & Czeczuga et al. 2009 \\
\hline & & seeds & $\begin{array}{l}\text { Prunella grandiflora } \\
\text { ssp. pyrenaia }\end{array}$ & $\begin{array}{l}\text { Poland or/ } \\
\text { imported to Poland }\end{array}$ & Czeczuga et al. 2009 \\
\hline & & seeds & Rosa mollis & $\begin{array}{l}\text { Poland or/ } \\
\text { imported to Poland }\end{array}$ & Czeczuga et al. 2009 \\
\hline \multirow[t]{5}{*}{$\begin{array}{l}\text { Gonapodya prolifera } \\
\text { (Cornu) A. Fisch. }\end{array}$} & $\begin{array}{l}\text { Gonapodyaceae, Monoblepharidomycetes, } \\
\text { Chytridiomycota }\end{array}$ & seeds & Borago officinalis & $\begin{array}{l}\text { Poland or/ } \\
\text { imported to Poland }\end{array}$ & Czeczuga et al. 2009 \\
\hline & & seeds & Ginkgo biloba & $\begin{array}{l}\text { Poland or/ } \\
\text { imported to Poland }\end{array}$ & Czeczuga et al. 2009 \\
\hline & & seeds & Humulus lupulus & $\begin{array}{l}\text { Poland or/ } \\
\text { imported to Poland }\end{array}$ & Czeczuga et al. 2009 \\
\hline & & seeds & Humulus scandens & $\begin{array}{l}\text { Poland or/ } \\
\text { imported to Poland }\end{array}$ & Czeczuga et al. 2009 \\
\hline & & seeds & Rhus gueinzii & $\begin{array}{l}\text { Poland or/ } \\
\text { imported to Poland }\end{array}$ & Czeczuga et al. 2009 \\
\hline
\end{tabular}


Table 2 Continued.

\begin{tabular}{|c|c|c|c|c|c|}
\hline Species & Taxonomy & Substrate & Host & Country & References \\
\hline \multirow{6}{*}{$\begin{array}{l}\text { Gonapodya siliquiformis } \\
\text { (Reinsch) Thaxt. } \\
\text { [as 'siliquaeformis'] }\end{array}$} & \multirow[t]{6}{*}{$\begin{array}{l}\text { Gonapodyaceae, Monoblepharidomycetes, } \\
\text { Chytridiomycota }\end{array}$} & seeds & Bryonia alba & $\begin{array}{l}\text { Poland or/ } \\
\text { imported to Poland }\end{array}$ & Czeczuga et al. 2009 \\
\hline & & seeds & Daphne gnidium & $\begin{array}{l}\text { Poland or/ } \\
\text { imported to Poland }\end{array}$ & Czeczuga et al. 2009 \\
\hline & & seeds & Daphne mezereum & $\begin{array}{l}\text { Poland or/ } \\
\text { imported to Poland }\end{array}$ & Czeczuga et al. 2009 \\
\hline & & seeds & Hyoscyamus niger & $\begin{array}{l}\text { Poland or/ } \\
\text { imported to Poland }\end{array}$ & Czeczuga et al. 2009 \\
\hline & & seeds & Sambucus ebulus & $\begin{array}{l}\text { Poland or/ } \\
\text { imported to Poland }\end{array}$ & Czeczuga et al. 2009 \\
\hline & & seeds & Sorbus torminalis & $\begin{array}{l}\text { Poland or/ } \\
\text { imported to Poland }\end{array}$ & Czeczuga et al. 2009 \\
\hline Gonatobotrys flavus Bonord & $\begin{array}{l}\text { Ceratostomataceae, Sordariomycetes, } \\
\text { Ascomycota }\end{array}$ & seeds & Quercus spp. & USSR & Mittal et al. 1990 \\
\hline Gonatobotryum spp. & $\begin{array}{l}\text { Incertae sedis, Incertae sedis, } \\
\text { Ascomycota }\end{array}$ & $\begin{array}{l}\text { seeds } \\
\text { seeds }\end{array}$ & $\begin{array}{l}\text { Pinus taeda } \\
\text { Tectona grandis }\end{array}$ & $\begin{array}{l}\text { USA } \\
\text { India }\end{array}$ & $\begin{array}{l}\text { Anderson 1986a, b } \\
\text { Anderson 1986a }\end{array}$ \\
\hline $\begin{array}{l}\text { Gonytrichum macrocladum } \\
\text { (Sacc.) S. Hughes }\end{array}$ & $\begin{array}{l}\text { Chaetosphaeriaceae, Sordariomycetes, } \\
\text { Ascomycota }\end{array}$ & pods & Delonix regia & Thailand & Somrithipol et al. 2002b \\
\hline $\begin{array}{l}\text { Graminopassalora graminis } \\
\text { (Fuckel) U. Braun, C. } \\
\text { Nakash., Videira \& Crous } \\
\text { [as Passalora graminis } \\
\text { (Fuckel) Höhn.] }\end{array}$ & $\begin{array}{l}\text { Mycosphaerellaceae, Dothideomycetes, } \\
\text { Ascomycota }\end{array}$ & seed & Phleum pratense & Canada & Conners 1967 \\
\hline $\begin{array}{l}\text { Graphium rigidum (Pers.) } \\
\text { Sacc. }\end{array}$ & $\begin{array}{l}\text { Graphiaceae, Sordariomycetes, } \\
\text { Ascomycota }\end{array}$ & fruit & Apeiba sp. & Panama & Morris \& Finley 1956 \\
\hline Graphium sp. & $\begin{array}{l}\text { Graphiaceae, Sordariomycetes, } \\
\text { Ascomycota }\end{array}$ & seeds & $\begin{array}{l}\text { Pinus armandii var. } \\
\text { mastersiana }\end{array}$ & Taiwan & $\begin{array}{l}\text { Jong \& Chen } 1966 \text {, } \\
\text { Mittal et al. } 1990\end{array}$ \\
\hline \multirow{5}{*}{$\begin{array}{l}\text { Guignardia cocogena } \\
\text { (Cooke) Punith. }\end{array}$} & \multirow{5}{*}{$\begin{array}{l}\text { Phyllostictaceae, Dothideomycetes, } \\
\text { Ascomycota }\end{array}$} & fruits & Ardisia punctata & Hong Kong & Tang et al. 2003a \\
\hline & & fruits & Ilex pubescens & Hong Kong & Tang et al. 2003a \\
\hline & & fruits & Microcos paniculata & Hong Kong & Tang et al. 2003a \\
\hline & & fruits & Psychotria asiatica & Hong Kong & Tang et al. 2003a \\
\hline & & fruits & $\begin{array}{l}\text { Viburnum } \\
\text { sempervirens }\end{array}$ & Hong Kong & Tang et al. 2003a \\
\hline
\end{tabular}


Table 2 Continued.

\begin{tabular}{|c|c|c|c|c|c|}
\hline Species & Taxonomy & Substrate & Host & Country & References \\
\hline $\begin{array}{l}\text { Guignardia dioscoreae } \\
\text { Sawada ex Katum. }\end{array}$ & $\begin{array}{l}\text { Phyllostictaceae, Dothideomycetes, } \\
\text { Ascomycota }\end{array}$ & fruits & Psychotria asiatica & Hong Kong & Tang et al. 2003a \\
\hline Guignardia musae Racib. & $\begin{array}{l}\text { Phyllostictaceae, Dothideomycetes, } \\
\text { Ascomycota }\end{array}$ & fruits & Diplospora dubia & Hong Kong & Tang et al. 2003a \\
\hline $\begin{array}{l}\text { Guignardia robiniae S. Ito \& } \\
\text { Kobayasi }\end{array}$ & $\begin{array}{l}\text { Phyllostictaceae, Dothideomycetes, } \\
\text { Ascomycota }\end{array}$ & seeds & $\begin{array}{l}\text { Robinia } \\
\text { pseudoacacia }\end{array}$ & Japan & $\begin{array}{l}\text { Sato \& Shoji 1960, } \\
\text { Anderson } 1986 a\end{array}$ \\
\hline $\begin{array}{l}\text { Gymnoascus bourquelotii } \\
\text { Boud. }\end{array}$ & $\begin{array}{l}\text { Gymnoascaceae, Eurotiomycetes, } \\
\text { Ascomycota }\end{array}$ & seeds & Gossypium sp. & Pakistan & Lodhi \& Naeem 1955 \\
\hline $\begin{array}{l}\text { Gymnopus asetosus Antonín, } \\
\text { Ryoo \& } \mathrm{Ka}^{\#}\end{array}$ & $\begin{array}{l}\text { Omphalotaceae, Agaricomycetes, } \\
\text { Basidiomycota }\end{array}$ & cone & undetermined plant & South Korea & Antonín et al. 2014 \\
\hline $\begin{array}{l}\text { Gymnosporangium } \\
\text { clavariiforme (Wulfen) DC. }\end{array}$ & $\begin{array}{l}\text { Pucciniaceae, Pucciniomycetes, } \\
\text { Basidiomycota }\end{array}$ & fruit & Amelanchier sp. & Canada & Conners 1967 \\
\hline $\begin{array}{l}\text { Gymnosporangium clavipes } \\
\text { Cooke \& Peck }\end{array}$ & $\begin{array}{l}\text { Pucciniaceae, Pucciniomycetes, } \\
\text { Basidiomycota }\end{array}$ & fruit & Amelanchier sp. & Canada & Conners 1967 \\
\hline $\begin{array}{l}\text { Gymnosporangium } \\
\text { inconspicuum F. Kern }\end{array}$ & $\begin{array}{l}\text { Pucciniaceae, Pucciniomycetes, } \\
\text { Basidiomycota }\end{array}$ & fruit & Amelanchier florida & Canada & Conners 1967 \\
\hline $\begin{array}{l}\text { Hanseniaspora } \\
\text { guilliermondii Pijper }\end{array}$ & $\begin{array}{l}\text { Saccharomycodaceae, Saccharomycetes, } \\
\text { Ascomycota }\end{array}$ & fruits & $\begin{array}{l}\text { Parahancornia } \\
\text { amapa }\end{array}$ & Brazil & Morais et al. 1995 \\
\hline $\begin{array}{l}\text { Hanseniaspora meyeri } \\
\text { Cadez, Poot, Raspor \& M.T. } \\
\text { Sm. }^{\text {I }}\end{array}$ & $\begin{array}{l}\text { Saccharomycodaceae, Saccharomycetes, } \\
\text { Ascomycota }\end{array}$ & fruit & Sapindus sp. & USA & Cadez et al. 2003 \\
\hline $\begin{array}{l}\text { Hanseniaspora occidentalis } \\
\text { M.T. Sm. }\end{array}$ & $\begin{array}{l}\text { Saccharomycodaceae, Saccharomycetes, } \\
\text { Ascomycota }\end{array}$ & fruits & $\begin{array}{l}\text { Parahancornia } \\
\text { amapa }\end{array}$ & Brazil & Morais et al. 1995 \\
\hline Hansfordia spp. & $\begin{array}{l}\text { Hansfordiaceae, Sordariomycetes, } \\
\text { Ascomycota }\end{array}$ & $\begin{array}{l}\text { seeds } \\
\text { seeds }\end{array}$ & $\begin{array}{l}\text { Pinus taeda } \\
\text { Tectona grandis }\end{array}$ & $\begin{array}{l}\text { USA } \\
\text { India }\end{array}$ & $\begin{array}{l}\text { Anderson 1986a, b } \\
\text { Anderson 1986a }\end{array}$ \\
\hline $\begin{array}{l}\text { Hapalophragmiopsis } \\
\text { ponderosa (Syd., P. Syd. \& } \\
\text { E.J. Butler) Thirum. } \\
\text { [as 'ponderosum'] }\end{array}$ & $\begin{array}{l}\text { Raveneliaceae, Pucciniomycetes, } \\
\text { Basidiomycota }\end{array}$ & seeds & Acacia spp. & India & $\begin{array}{l}\text { Malia \& Tattar 1978, } \\
\text { Anderson 1986a }\end{array}$ \\
\hline Helicoma guttulatum Y.Z. & Tubeufiaceae, Dothideomycetes, & fruits & Lithocarpus sp. & Thailand & Jayasiri et al. 2019 \\
\hline Lu, Boonmee \& K.D. Hyde & Ascomycota & fruits & undetermined plant & Thailand & Jayasiri et al. 2019 \\
\hline $\begin{array}{l}\text { Helicomyces candidus } \\
\text { (Preuss) Sacc. }\end{array}$ & $\begin{array}{l}\text { Tubeufiaceae, Dothideomycetes, } \\
\text { Ascomycota }\end{array}$ & seeds & Pinus sylvestris & USSR & $\begin{array}{l}\text { Prisyazhnyuk } 1960 \text {, } \\
\text { Mittal et al. } 1990\end{array}$ \\
\hline
\end{tabular}


Table 2 Continued.

\begin{tabular}{|c|c|c|c|c|c|}
\hline Species & Taxonomy & Substrate & Host & Country & References \\
\hline $\begin{array}{l}\text { Helicomyces elegans } \\
\text { Morgan }\end{array}$ & $\begin{array}{l}\text { Tubeufiaceae, Dothideomycetes, } \\
\text { Ascomycota }\end{array}$ & seeds & Pinus sylvestris & USSR & $\begin{array}{l}\text { Prisyazhnyuk 1960, } \\
\text { Mittal et al. } 1990\end{array}$ \\
\hline Helicomyces roseus Link & $\begin{array}{l}\text { Tubeufiaceae, Dothideomycetes, } \\
\text { Ascomycota }\end{array}$ & pods & Delonix regia & Thailand & Somrithipol et al. 2002b \\
\hline $\begin{array}{l}\text { Helicosporium } \\
\text { melghatianum Dharkar, } \\
\text { Subhedar, Hande \& } \\
\text { Shahezad }^{\#}\end{array}$ & $\begin{array}{l}\text { Tubeufiaceae, Dothideomycetes, } \\
\text { Ascomycota }\end{array}$ & pods & Pongamia pinnata & India & Dharkar et al. 2010 \\
\hline $\begin{array}{l}\text { Helicosporium pulvinatum } \\
\text { (Nees \& T. Nees) Pers. }\end{array}$ & $\begin{array}{l}\text { Tubeufiaceae, Dothideomycetes, } \\
\text { Ascomycota }\end{array}$ & seeds & Quercus spp. & USSR & $\begin{array}{l}\text { Urosevic 1962, Mittal et } \\
\text { al. } 1990\end{array}$ \\
\hline Helicosporium sp. & $\begin{array}{l}\text { Tubeufiaceae, Dothideomycetes, } \\
\text { Ascomycota }\end{array}$ & pods & Delonix regia & Thailand & Somrithipol et al. 2002b \\
\hline $\begin{array}{l}\text { Helminthosporium linicola } \\
\text { Kletsh. }\end{array}$ & $\begin{array}{l}\text { Massarinaceae, Dothideomycetes, } \\
\text { Ascomycota }\end{array}$ & seed & Linum usitatissimum & Canada & Conners 1967 \\
\hline \multirow[t]{2}{*}{$\begin{array}{l}\text { Helminthosporium } \\
\text { rostratum Drechsler }\end{array}$} & \multirow[t]{2}{*}{$\begin{array}{l}\text { Massarinaceae, Dothideomycetes, } \\
\text { Ascomycota }\end{array}$} & seeds & Picea excelsa & USSR & $\begin{array}{l}\text { Urosevic 1961, } \\
\text { Anderson 1986a }\end{array}$ \\
\hline & & seeds & Pinus sylvestris & USSR & $\begin{array}{l}\text { Urosevic 1961, } \\
\text { Anderson 1986a }\end{array}$ \\
\hline Helminthosporium sativum & Massarinaceae, Dothideomycetes, & seeds & Bombax ceiba & Pakistan & Lodhi \& Naeem 1955 \\
\hline \multirow[t]{2}{*}{ Pammel, C.M. King \& Bakke } & \multirow[t]{2}{*}{ Ascomycota } & seeds & Picea excelsa & USSR & $\begin{array}{l}\text { Urosevic 1961, } \\
\text { Anderson 1986a }\end{array}$ \\
\hline & & seeds & Pinus sylvestris & USSR & $\begin{array}{l}\text { Urosevic 1961, } \\
\text { Anderson 1986a }\end{array}$ \\
\hline \multirow[t]{5}{*}{ Helminthosporium spp. } & \multirow[t]{5}{*}{$\begin{array}{l}\text { Massarinaceae, Dothideomycetes, } \\
\text { Ascomycota }\end{array}$} & seeds & Acer saccharinum & USA & $\begin{array}{l}\text { Friedrich et al. } 1971 \text {, } \\
\text { Mittal et al. } 1990\end{array}$ \\
\hline & & seeds & Acer saccharum & USA & $\begin{array}{l}\text { Friedrich et al. } 1971 \text {, } \\
\text { Mittal et al. } 1990\end{array}$ \\
\hline & & seeds & Araucaria bidwillii & Egypt & $\begin{array}{l}\text { Kamara et al. } 1981 \text {, } \\
\text { Mittal et al. } 1990\end{array}$ \\
\hline & & seeds & $\begin{array}{l}\text { Araucaria } \\
\text { cunninghamii }\end{array}$ & Australia & $\begin{array}{l}\text { Kamara et al. } 1981 \text {, } \\
\text { Mittal et al. } 1990\end{array}$ \\
\hline & & seeds & $\begin{array}{l}\text { Betula } \\
\text { alleghaniensis }\end{array}$ & USA & $\begin{array}{l}\text { Friedrich et al. } 1971 \text {, } \\
\text { Mittal et al. } 1990\end{array}$ \\
\hline
\end{tabular}


Table 2 Continued.

\begin{tabular}{|c|c|c|c|c|c|}
\hline Species & Taxonomy & Substrate & Host & Country & References \\
\hline & & seeds & Cedrus deodara & India & $\begin{array}{l}\text { Munjal \& Sharma 1975, } \\
\text { Mittal et al. } 1990\end{array}$ \\
\hline & & seeds & Dalbergia sissoo & India & $\begin{array}{l}\text { Mittal \& Sharma 1981b, } \\
\text { Mittal et al. 1990, Naz et } \\
\text { al. } 2015\end{array}$ \\
\hline & & seeds & Fraxinus americana & USA & $\begin{array}{l}\text { Friedrich et al. } 1971 \text {, } \\
\text { Mittal et al. } 1990\end{array}$ \\
\hline & & seeds & Pinus caribaea & $\begin{array}{l}\text { Belize [as British } \\
\text { Honduras] }\end{array}$ & $\begin{array}{l}\text { Hocking 1968, Mittal et } \\
\text { al. } 1990\end{array}$ \\
\hline & & seeds & Pinus elliottii & USA & $\begin{array}{l}\text { Rowan \& Debarr 1974, } \\
\text { Mittal et al. } 1990\end{array}$ \\
\hline & & seeds & Pinus roxburghii & India & $\begin{array}{l}\text { Munjal \& Sharma 1975, } \\
\text { Mittal et al. } 1990\end{array}$ \\
\hline & & seeds & Pinus taeda & USA & $\begin{array}{l}\text { Covington et al. 1982, } \\
\text { Anderson 1986a }\end{array}$ \\
\hline & & seeds & Pinus wallichiana & India & $\begin{array}{l}\text { Munjal \& Sharma 1975, } \\
\text { Mittal et al. } 1990\end{array}$ \\
\hline $\begin{array}{l}\text { Helminthosporium } \\
\text { spiciferum (Bainier) Nicot }\end{array}$ & $\begin{array}{l}\text { Massarinaceae, Dothideomycetes, } \\
\text { Ascomycota }\end{array}$ & seeds & $\begin{array}{l}\text { Platanus } \\
\text { occidentalis }\end{array}$ & USA & $\begin{array}{l}\text { Fakir et al. 1971, Mittal } \\
\text { et al. } 1990\end{array}$ \\
\hline $\begin{array}{l}\text { Helminthosporium } \\
\text { tetramerum McKinney } \\
\text { [as 'tetramer'] }\end{array}$ & $\begin{array}{l}\text { Massarinaceae, Dothideomycetes, } \\
\text { Ascomycota }\end{array}$ & seeds & Eucalyptus sp. & India & $\begin{array}{l}\text { Saxena 1985, Mittal et } \\
\text { al. } 1990\end{array}$ \\
\hline Helotiales sp. & Leotiomycetes, Ascomycota & seeds & Fraxinus excelsior & Sweden & Hayatgheibi 2013 \\
\hline $\begin{array}{l}\text { Helotium fagineum (Pers.) } \\
\text { Fr. }\end{array}$ & Helotiaceae, Leotiomycetes, Ascomycota & fruit cupules & Fagus sylvatica & UK & Carré 1964 \\
\hline $\begin{array}{l}\text { Helotium strobilinum (Fr.) } \\
\text { Fuckel }\end{array}$ & Helotiaceae, Leotiomycetes, Ascomycota & seeds & Larix sibirica & USSR & $\begin{array}{l}\text { Prisyazhnyuk } 1960 \text {, } \\
\text { Mittal et al. } 1990\end{array}$ \\
\hline \multirow[t]{2}{*}{ Helotium virgultorum Fr. } & Helotiaceae, Leotiomycetes, Ascomycota & seeds & Picea abies & USSR & $\begin{array}{l}\text { Barrows-Broaddus \& } \\
\text { Dwinell 1985, Mittal et } \\
\text { al. } 1990\end{array}$ \\
\hline & & seeds & Pinus sylvestris & USSR & $\begin{array}{l}\text { Prisyazhnyuk } 1960 \text {, } \\
\text { Mittal et al. } 1990\end{array}$ \\
\hline Hendersonia strobilina Curr. & $\begin{array}{l}\text { Phaeosphaeriaceae, Dothideomycetes, } \\
\text { Ascomycota }\end{array}$ & seeds & Pinus sylvestris & USSR & $\begin{array}{l}\text { Prisyazhnyuk } 1960 \text {, } \\
\text { Mittal et al. } 1990\end{array}$ \\
\hline
\end{tabular}


Table 2 Continued.

\begin{tabular}{|c|c|c|c|c|c|}
\hline Species & Taxonomy & Substrate & Host & Country & References \\
\hline $\begin{array}{l}\text { Hermatomyces sphaericus } \\
\text { (Sacc.) S. Hughes }\end{array}$ & $\begin{array}{l}\text { Hermatomycetaceae, Dothideomycetes, } \\
\text { Ascomycota }\end{array}$ & pod & Entada phaseoloides & Thailand & Jayasiri et al. 2019 \\
\hline Herpotrichia juniperi (Sacc.) & Melanommataceae, Dothideomycetes, & cones, seeds & Pinus contorta & Canada & Vujanovic et al. 2000 \\
\hline \multirow[t]{7}{*}{ Petr. } & Ascomycota & cones, seeds & Pinus densiflora & Canada & Vujanovic et al. 2000 \\
\hline & & cones, seeds & Pinus mugo 'Galica’ & Canada & Vujanovic et al. 2000 \\
\hline & & cones, seeds & Pinus ponderosa & Canada & Vujanovic et al. 2000 \\
\hline & & cones, seeds & Pinus rigida & Canada & Vujanovic et al. 2000 \\
\hline & & cones, seeds & $\begin{array}{l}\text { Pinus sylvestris } \\
\text { 'Argentea- } \\
\text { Compacta' }\end{array}$ & Canada & Vujanovic et al. 2000 \\
\hline & & cones, seeds & $\begin{array}{l}\text { Pinus sylvestris } \\
\text { 'Beuvronensis' }\end{array}$ & Canada & Vujanovic et al. 2000 \\
\hline & & cones, seeds & Pinus tabulaeformis & Canada & Vujanovic et al. 2000 \\
\hline $\begin{array}{l}\text { Heterobasidion annosum } \\
\text { (Fr.) Bref. }\end{array}$ & $\begin{array}{l}\text { Bondarzewiaceae, Agaricomycetes, } \\
\text { Basidiomycota }\end{array}$ & seeds & Abies spp. & North America & $\begin{array}{l}\text { Batko 1959, Anderson } \\
\text { 1986a }\end{array}$ \\
\hline $\begin{array}{l}\text { Heteroconium decorosum } \\
\text { R.F. Castaňeda, Saikawa \& } \\
\text { Guaro }^{\#}\end{array}$ & $\begin{array}{l}\text { Antennulariellaceae, Dothideomycetes, } \\
\text { Ascomycota }\end{array}$ & pod & Samanea saman & Cuba & $\begin{array}{l}\text { Castañeda-Ruíz et al. } \\
1999\end{array}$ \\
\hline $\begin{array}{l}\text { Hirsutella uncinata Seifert } \\
\text { \& H. Boulay }\end{array}$ & $\begin{array}{l}\text { Ophiocordycipitaceae, Sordariomycetes, } \\
\text { Ascomycota }\end{array}$ & follicles & Hakea sp. & Australia & Seifert \& Boulay 2004 \\
\hline $\begin{array}{l}\text { Hormiscium antiquum } \\
\text { (Corda) Sacc. }\end{array}$ & $\begin{array}{l}\text { Torulaceae, Dothideomycetes, } \\
\text { Ascomycota }\end{array}$ & seeds & Pinus sylvestris & USSR & $\begin{array}{l}\text { Prisyazhnyuk } 1960 \text {, } \\
\text { Mittal et al. } 1990\end{array}$ \\
\hline \multirow[t]{3}{*}{$\begin{array}{l}\text { Hormiscium stilbosporum } \\
\text { (Corda) Sacc. }\end{array}$} & $\begin{array}{l}\text { Torulaceae, Dothideomycetes, } \\
\text { Ascomycota }\end{array}$ & seeds & Abies sibirica & USSR & $\begin{array}{l}\text { Prisyazhnyuk } 1960 \text {, } \\
\text { Mittal et al. } 1990\end{array}$ \\
\hline & & seeds & Picea abies & USSR & $\begin{array}{l}\text { Prisyazhnyuk } 1960 \text {, } \\
\text { Mittal et al. } 1990\end{array}$ \\
\hline & & seeds & Pinus sylvestris & USSR & $\begin{array}{l}\text { Prisyazhnyuk } 1960 \text {, } \\
\text { Mittal et al. } 1990\end{array}$ \\
\hline \multirow[t]{3}{*}{$\begin{array}{l}\text { Hormodendrum sp. } \\
\text { [as 'Hormodendron'] }\end{array}$} & $\begin{array}{l}\text { Cladosporiaceae, Dothideomycetes, } \\
\text { Ascomycota }\end{array}$ & seeds & Acer rubrum & USA & $\begin{array}{l}\text { Friedrich et al. } 1971 \text {, } \\
\text { Mittal et al. } 1990\end{array}$ \\
\hline & & seeds & Acer saccharinum & USA & $\begin{array}{l}\text { Friedrich et al. } 1971 \text {, } \\
\text { Mittal et al. } 1990\end{array}$ \\
\hline & & seeds & Acer saccharum & USA & $\begin{array}{l}\text { Friedrich et al. } 1971 \text {, } \\
\text { Mittal et al. } 1990\end{array}$ \\
\hline
\end{tabular}


Table 2 Continued.

\begin{tabular}{|c|c|c|c|c|c|}
\hline Species & Taxonomy & Substrate & Host & Country & References \\
\hline & & seeds & $\begin{array}{l}\text { Betula } \\
\text { alleghaniensis }\end{array}$ & USA & $\begin{array}{l}\text { Friedrich et al. } 1971 \text {, } \\
\text { Mittal et al. } 1990\end{array}$ \\
\hline & & seeds & Betula papyrifera & USA & $\begin{array}{l}\text { Friedrich et al. } 1971 \text {, } \\
\text { Mittal et al. } 1990\end{array}$ \\
\hline & & seeds & Fraxinus americana & USA & $\begin{array}{l}\text { Friedrich et al. } 1971 \text {, } \\
\text { Mittal et al. } 1990\end{array}$ \\
\hline & & seeds & $\begin{array}{l}\text { Pseudotsuga } \\
\text { menziesii }\end{array}$ & Canada, USA & $\begin{array}{l}\text { Salisbury 1955, Mittal et } \\
\text { al. } 1990\end{array}$ \\
\hline \multirow[t]{4}{*}{ Hormonema spp. } & Incertae sedis, Dothideomycetes, & pods & Eucalyptus globulus & Uruguay & Lupo et al. 2001 \\
\hline & Ascomycota & seed capsules & Eucalyptus grandis & South Africa & Jimu et al. 2016 \\
\hline & & seeds & Eucalyptus grandis & South Africa & Jimu et al. 2016 \\
\hline & & seed & Pinus monticola & USA & $\begin{array}{l}\text { Ganley \& Newcombe } \\
2006\end{array}$ \\
\hline Humicola fuscoatra Traaen & $\begin{array}{l}\text { Chaetomiaceae, Sordariomycetes, } \\
\text { Ascomycota }\end{array}$ & seed & Prunus serrulata & Japan & Watanabe 2010 \\
\hline \multirow{3}{*}{$\begin{array}{l}\text { Humicola homopilata } \\
\text { (Omvik) X. Wei Wang \& } \\
\text { Houbraken [as Chaetomium } \\
\text { homopilatum Omvik] }\end{array}$} & $\begin{array}{l}\text { Chaetomiaceae, Sordariomycetes, } \\
\text { Ascomycota }\end{array}$ & seeds & $\begin{array}{l}\text { Eucalyptus } \\
\text { citriodora }\end{array}$ & India & $\begin{array}{l}\text { Mittal \& Sharma 1984, } \\
\text { Mittal et al. } 1990\end{array}$ \\
\hline & & seeds & Pinus roxburghii & India & $\begin{array}{l}\text { Mittal \& Sharma 1982c, } \\
\text { Mittal et al. } 1990\end{array}$ \\
\hline & & seeds & Pinus wallichiana & India & $\begin{array}{l}\text { Mittal \& Sharma 1982b, } \\
\text { Mittal et al. } 1990\end{array}$ \\
\hline \multirow[t]{4}{*}{ Humicola sp. } & Chaetomiaceae, Sordariomycetes, & seeds & Picea jezoensis & Japan & Cheng \& Igarashi 1987 \\
\hline & Ascomycota & seeds & Picea sitchensis & UK & $\begin{array}{l}\text { Salt 1964, Mittal et al. } \\
1990\end{array}$ \\
\hline & & seeds & Pinus taeda & USA & Anderson 1986a, b \\
\hline & & seeds & Tectona grandis & India & Anderson 1986a \\
\hline $\begin{array}{l}\text { Hyalocephalotrichum } \\
\text { indicum Nagaraju, Kunwar, } \\
\text { Sureshk. \& Manohar. }^{\text {\& }}\end{array}$ & $\begin{array}{l}\text { Incertae sedis, Incertae sedis, } \\
\text { Ascomycota }\end{array}$ & fruit litter & $\begin{array}{l}\text { Acacia } \\
\text { auriculiformis }\end{array}$ & India & Nagaraju et al. 2011c \\
\hline Hyalodendron sp. & $\begin{array}{l}\text { Trichosporonaceae, Tremellomycetes, } \\
\text { Basidiomycota }\end{array}$ & seeds & Pinus taeda & USA & $\begin{array}{l}\text { Mason \& van Arsdel } \\
\text { 1978, Anderson 1986a }\end{array}$ \\
\hline $\begin{array}{l}\text { Hyaloscypha cupularum } \\
\text { Svrček }^{\#}\end{array}$ & $\begin{array}{l}\text { Hyaloscyphaceae, Leotiomycetes, } \\
\text { Ascomycota }\end{array}$ & cupule & Quercus sp. & $\begin{array}{l}\text { former } \\
\text { Czechoslovakia }\end{array}$ & Svrcek 1987 \\
\hline
\end{tabular}


Table 2 Continued.

\begin{tabular}{|c|c|c|c|c|c|}
\hline Species & Taxonomy & Substrate & Host & Country & References \\
\hline $\begin{array}{l}\text { Hyaloscypha leuconica } \\
\text { (Cooke ex Stev.) Nannf. }\end{array}$ & $\begin{array}{l}\text { Hyaloscyphaceae, Leotiomycetes, } \\
\text { Ascomycota }\end{array}$ & fruit cupules & Fagus sylvatica & UK & Carré 1964 \\
\hline $\begin{array}{l}\text { Hyaloscypha strobilicola } \\
\text { var. parvispora Huhtinen }{ }^{\#}\end{array}$ & $\begin{array}{l}\text { Hyaloscyphaceae, Leotiomycetes, } \\
\text { Ascomycota }\end{array}$ & cone scales & Pinus sabiniana & USA & Huhtinen 1990 \\
\hline $\begin{array}{l}\text { Hyaloscypha } \\
\text { sulphureopilosa Svrček }{ }^{\#}\end{array}$ & $\begin{array}{l}\text { Hyaloscyphaceae, Leotiomycetes, } \\
\text { Ascomycota }\end{array}$ & cones & Picea abies & $\begin{array}{l}\text { former } \\
\text { Czechoslovakia }\end{array}$ & Svrcek 1986 \\
\hline Hydnum auriscalpium L. & $\begin{array}{l}\text { Hydnaceae, Agaricomycetes, } \\
\text { Basidiomycota }\end{array}$ & seeds & Pinus sylvestris & USSR & $\begin{array}{l}\text { Prisyazhnyuk } 1960 \text {, } \\
\text { Mittal et al. } 1990\end{array}$ \\
\hline $\begin{array}{l}\text { Hymenoscyphus cassiae } \\
\text { M.P. Sharma\# }\end{array}$ & Helotiaceae, Leotiomycetes, Ascomycota & pods & Cassia sp. & India & Sharma 1991 \\
\hline $\begin{array}{l}\text { Hymenoscyphus fraxineus } \\
\text { (T. Kowalski) Baral, Queloz } \\
\text { \& Hosoya [as Chalara } \\
\text { fraxinea T. Kowalski] }\end{array}$ & Helotiaceae, Leotiomycetes, Ascomycota & seeds & Fraxinus excelsior & Sweden & $\begin{array}{l}\text { Cleary et al. 2013, } \\
\text { Hayatgheibi 2013, } \\
\text { Marčiulyniené et al. } \\
2017\end{array}$ \\
\hline $\begin{array}{l}\text { Hymenoscyphus seminis- } \\
\text { alni Baral, B. Grauw. \& M. } \\
\text { Eckel }^{\#}\end{array}$ & Helotiaceae, Leotiomycetes, Ascomycota & seeds & Alnus glutinosa & Germany & Baral 1996 \\
\hline $\begin{array}{l}\text { Hymenoscyphus splendens } \\
\text { Abdullah, Descals \& J. } \\
\text { Webster }^{\#}\end{array}$ & Helotiaceae, Leotiomycetes, Ascomycota & fruit cupules & Fagus sylvatica & UK & Abdullah et al. 1981 \\
\hline $\begin{array}{l}\text { Hyphopichia buzzinii L.R. } \\
\text { Ribeiro, A.R.O. Santos, N. } \\
\text { Jacques, Grondin, Casareg., } \\
\text { C.A. Lara, Lachance \& C.A. } \\
\text { Rosa\# }\end{array}$ & $\begin{array}{l}\text { Incertae sedis, Saccharomycetes, } \\
\text { Ascomycota }\end{array}$ & fruit & Clidemia sp. & Guiana & Ribeiro et al. 2017 \\
\hline Hypocreales sp. & Sordariomycetes, Ascomycota & seeds & Fraxinus excelsior & Sweden & Cleary et al. 2013 \\
\hline \multirow[t]{3}{*}{$\begin{array}{l}\text { Hypoderma conigenum } \\
\text { (Pers.) DC. }\end{array}$} & $\begin{array}{l}\text { Rhytismataceae, Leotiomycetes, } \\
\text { Ascomycota }\end{array}$ & seeds & Larix sibirica & USSR & $\begin{array}{l}\text { Prisyazhnyuk 1960, } \\
\text { Mittal et al. } 1990\end{array}$ \\
\hline & & seeds & Picea abies & USSR & $\begin{array}{l}\text { Prisyazhnyuk } 1960 \text {, } \\
\text { Mittal et al. } 1990\end{array}$ \\
\hline & & seeds & Pinus sylvestris & USSR & $\begin{array}{l}\text { Prisyazhnyuk } 1960 \text {, } \\
\text { Mittal et al. } 1990\end{array}$ \\
\hline $\begin{array}{l}\text { Hypoxylon hinnuleum (G. } \\
\text { Sm.) Kuhnert \& Sir }\end{array}$ & $\begin{array}{l}\text { Hypoxylaceae, Sordariomycetes, } \\
\text { Ascomycota }\end{array}$ & seeds & $\begin{array}{l}\text { Platanus } \\
\text { occidentalis }\end{array}$ & USA & $\begin{array}{l}\text { Fakir et al. 1971, Mittal } \\
\text { et al. } 1990\end{array}$ \\
\hline
\end{tabular}


Table 2 Continued.

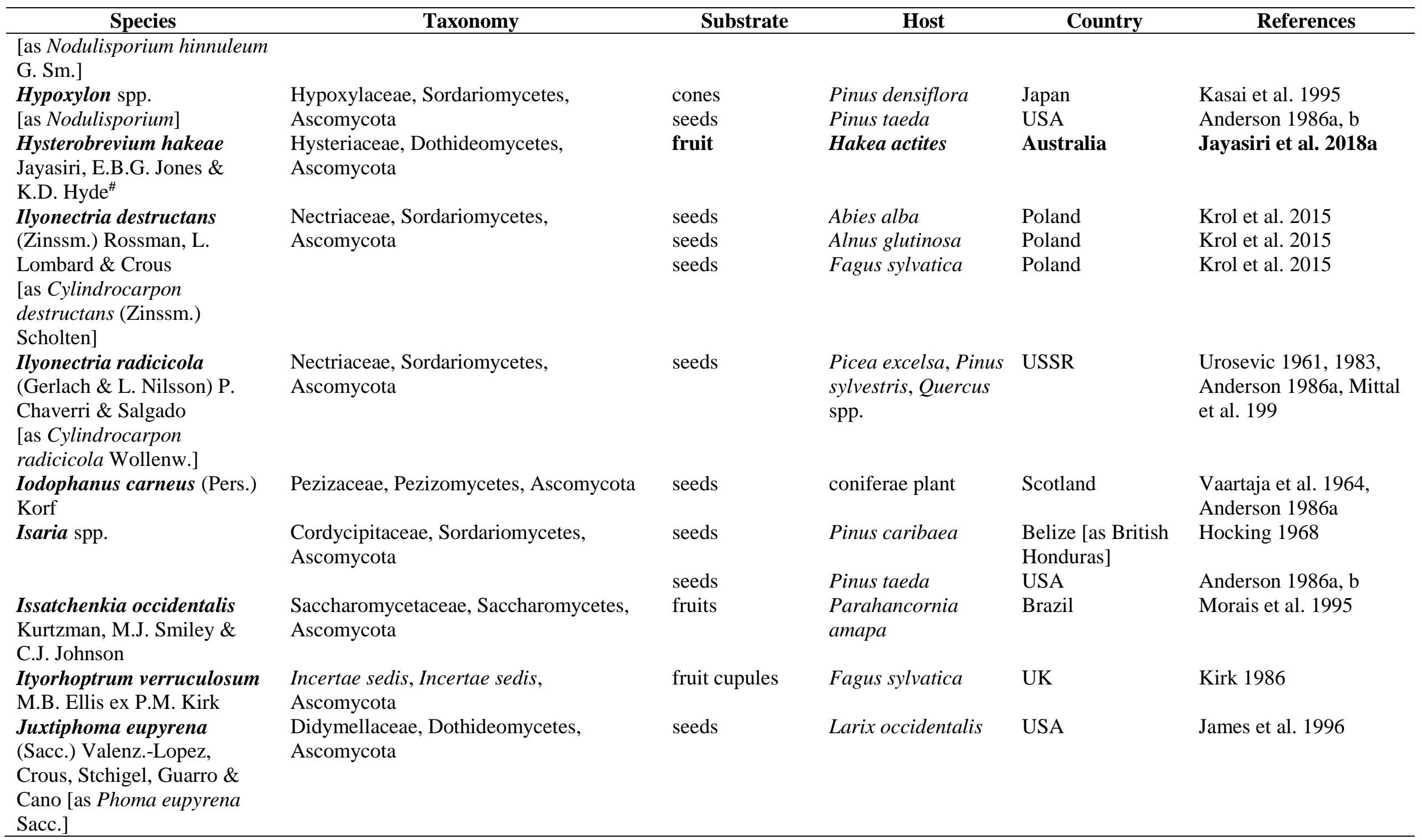


Table 2 Continued.

\begin{tabular}{|c|c|c|c|c|c|}
\hline Species & Taxonomy & Substrate & Host & Country & References \\
\hline Kallichroma tethys (Kohlm. & Bionectriaceae, Sordariomycetes, & seed & Canavalia cathartica & India & Anita \& Sridhar 2009 \\
\hline $\begin{array}{l}\text { \& E. Kohlm.) Kohlm. \& } \\
\text { Volkm.-Kohlm. }\end{array}$ & Ascomycota & pod & Sesbania bispinosa & India & Anita et al. 2009 \\
\hline $\begin{array}{l}\text { Rhizophlyctis hyalina } \\
\text { (Karling) Sparrow Karlingia } \\
\text { hyalina Karling }\end{array}$ & $\begin{array}{l}\text { Rhizophlyctidaceae, Chytridiomycetes, } \\
\text { Chytridiomycota }\end{array}$ & seeds & Trifolium pratense & $\begin{array}{l}\text { Poland or/ } \\
\text { imported to Poland }\end{array}$ & Czeczuga et al. 2009 \\
\hline Karlingia polonica Hassan & $\begin{array}{l}\text { Rhizophlyctidaceae, Chytridiomycetes, } \\
\text { Chytridiomycota }\end{array}$ & seeds & $\begin{array}{l}\text { Armeria maritima } \\
\text { ssp. maritima }\end{array}$ & $\begin{array}{l}\text { Poland or/ } \\
\text { imported to Poland }\end{array}$ & Czeczuga et al. 2009 \\
\hline $\begin{array}{l}\text { Karlingia rosea (de Bary \& } \\
\text { Woronin) A.E. Johanson }\end{array}$ & $\begin{array}{l}\text { Rhizophlyctidaceae, Chytridiomycetes, } \\
\text { Chytridiomycota }\end{array}$ & seeds & Vicia sativa & Poland & Kiziewicz 2005 \\
\hline Karlingia spinosa Karling & $\begin{array}{l}\text { Rhizophlyctidaceae, Chytridiomycetes, } \\
\text { Chytridiomycota }\end{array}$ & seeds & Ficus pumila & $\begin{array}{l}\text { Poland or/ } \\
\text { imported to Poland }\end{array}$ & Czeczuga et al. 2009 \\
\hline $\begin{array}{l}\text { Karlingiella elongata } \\
\text { (Karling) G.H. Jerônimo, }\end{array}$ & $\begin{array}{l}\text { Incertae sedis, Chytridiomycetes, } \\
\text { Chytridiomycota }\end{array}$ & seeds & Cicuta virosa & $\begin{array}{l}\text { Poland or/ } \\
\text { imported to Poland }\end{array}$ & Czeczuga et al. 2009 \\
\hline $\begin{array}{l}\text { A.L. Jesus \& Pires-Zottar. } \\
\text { [as Nowakowskiella elongata } \\
\text { Karling] }\end{array}$ & & seeds & Cuscuta europaea & $\begin{array}{l}\text { Poland or/ } \\
\text { imported to Poland }\end{array}$ & Czeczuga et al. 2009 \\
\hline $\begin{array}{l}\text { Karlingiomyces lobatus } \\
\text { (Karling) Sparrow }\end{array}$ & $\begin{array}{l}\text { Polychytriaceae, Polychytriomycetes, } \\
\text { Chytridiomycota }\end{array}$ & seeds & Ginkgo biloba & $\begin{array}{l}\text { Poland or/ } \\
\text { imported to Poland }\end{array}$ & Czeczuga et al. 2009 \\
\hline Khuskia oryzae H.J. Huds. & $\begin{array}{l}\text { Incertae sedis, Sordariomycetes, } \\
\text { Ascomycota }\end{array}$ & seeds & Delonix regia & India & Sahu et al. 2003 \\
\hline $\begin{array}{l}\text { Kionocephala catenulata } \\
\text { M.B. Ellis ex P.M. Kirk }{ }^{\# * *}\end{array}$ & $\begin{array}{l}\text { Incertae sedis, Incertae sedis, } \\
\text { Ascomycota }\end{array}$ & fruit cupule & Fagus sylvatica & UK & Kirk 1986 \\
\hline $\begin{array}{l}\text { Kloeckera africana } \\
\text { (Klöcker) Janke }\end{array}$ & $\begin{array}{l}\text { Saccharomycetaceae, Saccharomycetes, } \\
\text { Ascomycota }\end{array}$ & fruits & $\begin{array}{l}\text { Parahancornia } \\
\text { amapa }\end{array}$ & Brazil & Morais et al. 1995 \\
\hline $\begin{array}{l}\text { Kloeckera apiculata (Reess) } \\
\text { Janke }\end{array}$ & $\begin{array}{l}\text { Saccharomycetaceae, Saccharomycetes, } \\
\text { Ascomycota }\end{array}$ & fruits & $\begin{array}{l}\text { Parahancornia } \\
\text { amapa }\end{array}$ & Brazil & Morais et al. 1995 \\
\hline $\begin{array}{l}\text { Kuraishia borneana } \\
\text { Kurtzman, Kurtzman \& } \\
\text { Robnett }^{\#}\end{array}$ & $\begin{array}{l}\text { Pichiaceae, Saccharomycetes, } \\
\text { Ascomycota }\end{array}$ & fruit waste & undetermined plant & Brunei & $\begin{array}{l}\text { Kurtzman \& Robnett } \\
2014\end{array}$ \\
\hline $\begin{array}{l}\text { Lacellina graminicola (Berk. } \\
\text { \& Broome) Petch }\end{array}$ & $\begin{array}{l}\text { Incertae sedis, Incertae sedis, } \\
\text { Ascomycota }\end{array}$ & seeds & Pinus ponderosa & USA & $\begin{array}{l}\text { James \& Genz 1982, } \\
\text { Anderson 1986a }\end{array}$ \\
\hline $\begin{array}{l}\text { Lachnum tengii W.Y } \\
\text { Zhuang\# }^{\#}\end{array}$ & Lachnaceae, Leotiomycetes, Ascomycota & nuts & undetermined plant & China & Zhuang 2002 \\
\hline
\end{tabular}


Table 2 Continued.

\begin{tabular}{|c|c|c|c|c|c|}
\hline Species & Taxonomy & Substrate & Host & Country & References \\
\hline $\begin{array}{l}\text { Laetinaevia colobanthi I.J. } \\
\text { Gamundi \& H.A. Soubedi }\end{array}$ & Calloriaceae, Leotiomycetes, Ascomycota & fruit & $\begin{array}{l}\text { Colobanthus } \\
\text { quitensis }\end{array}$ & Antarctica & $\begin{array}{l}\text { Gamundí \& Spinedi } \\
1988\end{array}$ \\
\hline Lanceispora sp. & $\begin{array}{l}\text { Incertae sedis, Sordariomycetes, } \\
\text { Ascomycota }\end{array}$ & pods & Delonix regia & Thailand & Somrithipol et al. 2002b \\
\hline Lasiodiplodia sp. & $\begin{array}{l}\text { Botryosphaeriaceae, Dothideomycetes, } \\
\text { Ascomycota }\end{array}$ & seeds & Eucalyptus grandis & South Africa & Jimu et al. 2016 \\
\hline $\begin{array}{l}\text { Lasiodiplodia avicenniarum } \\
\text { Jayasiri, E.B.G. Jones \& } \\
\text { K.D. Hyde }^{\#}\end{array}$ & $\begin{array}{l}\text { Botryosphaeriaceae, Dothideomycetes, } \\
\text { Ascomycota }\end{array}$ & fruit & Avicennia marina & Thailand & Jayasiri et al. 2019 \\
\hline Lasiodiplodia & Botryosphaeriaceae, Dothideomycetes, & pod & Afzelia xylocarpa & Thailand & Jayasiri et al. 2019 \\
\hline $\begin{array}{l}\text { pseudotheobromae A.J.L. } \\
\text { Phillips, A. Alves \& Crous }\end{array}$ & Ascomycota & pod & Quercus sp. & Thailand & Jayasiri et al. 2019 \\
\hline $\begin{array}{l}\text { Lasiodiplodia swieteniae } \\
\text { Jayasiri, E.B.G. Jones \& } \\
\text { K.D. Hyde }\end{array}$ & $\begin{array}{l}\text { Botryosphaeriaceae, Dothideomycetes, } \\
\text { Ascomycota }\end{array}$ & fruit & Swietenia sp. & Thailand & Jayasiri et al. 2019 \\
\hline \multirow{8}{*}{$\begin{array}{l}\text { Lasiodiplodia theobromae } \\
\text { (Pat.) Griffon \& Maubl. }\end{array}$} & Botryosphaeriaceae, Dothideomycetes, & fruit & undetermined plant & Guinea & Phillips et al. 2013 \\
\hline & Ascomycota & fruit, pod & undetermined plant & Thailand & Jayasiri et al. 2019 \\
\hline & & pod & Acacia sp. & Thailand & Jayasiri et al. 2019 \\
\hline & & pod & $\begin{array}{l}\text { Calophyllum } \\
\text { inophyllum }\end{array}$ & Thailand & Jayasiri et al. 2019 \\
\hline & & seeds & Pinus caribaea & Central America & Rees 1988 \\
\hline & & seeds & Pinus elliottii & $\begin{array}{l}\text { Brazil, South } \\
\text { Africa }\end{array}$ & $\begin{array}{l}\text { Cilliers et al. 1995, } \\
\text { Maciel et al. } 2015\end{array}$ \\
\hline & & seeds & $\begin{array}{l}\text { Pinus elliottii var. } \\
\text { elliottii }\end{array}$ & USA & Fraedrich \& Miller 1995 \\
\hline & & seeds & Pinus taeda & Brazil & Maciel et al. 2015 \\
\hline $\begin{array}{l}\text { Lauriomyces ellipticus } \\
\text { Somrith. \& E.B.G. Jones }\end{array}$ & $\begin{array}{l}\text { Lauriomycetaceae, Leotiomycetes, } \\
\text { Ascomycota }\end{array}$ & fruit & undetermined plant & Thailand & $\begin{array}{l}\text { Somrithipol \& Jones } \\
2007\end{array}$ \\
\hline $\begin{array}{l}\text { Lauriomyces sakaeratensis } \\
\text { Somrith., Kosol \& E.B.G. } \\
\text { Jones }^{\#}\end{array}$ & $\begin{array}{l}\text { Lauriomycetaceae, Leotiomycetes, } \\
\text { Ascomycota }\end{array}$ & fruits & $\begin{array}{l}\text { Dipterocarpus } \\
\text { costatus }\end{array}$ & Thailand & Somrithipol et al. 2006 \\
\hline $\begin{array}{l}\text { Lecanora shangrilaensis } \\
\text { Z.T. Zhao \& L. Lü̈ }\end{array}$ & $\begin{array}{l}\text { Lecanoraceae, Lecanoromycetes, } \\
\text { Ascomycota }\end{array}$ & cones & Pinus sp. & China & Lü \& Zhao 2017 \\
\hline
\end{tabular}


Table 2 Continued.

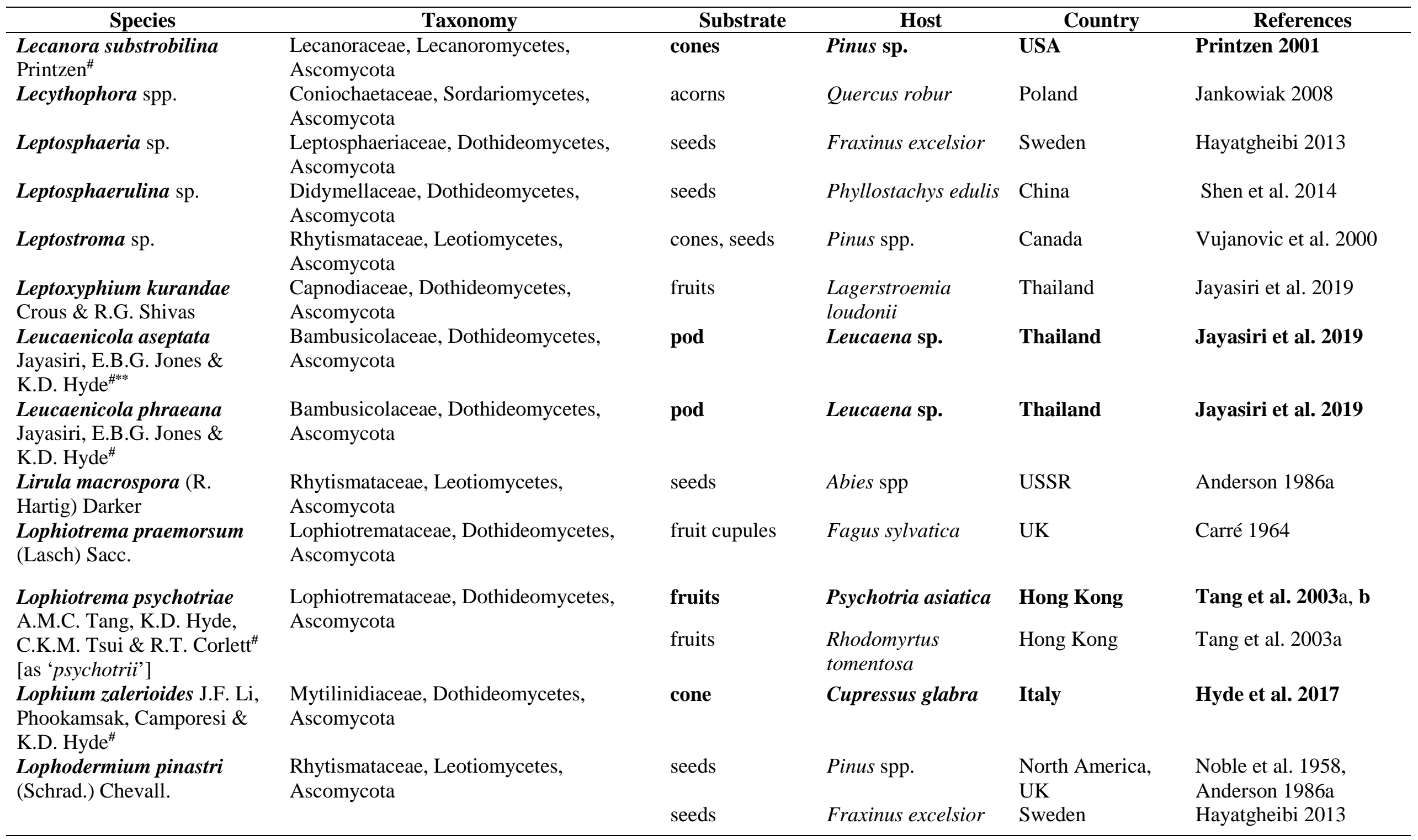


Table 2 Continued.

\begin{tabular}{|c|c|c|c|c|c|}
\hline Species & Taxonomy & Substrate & Host & Country & References \\
\hline $\begin{array}{l}\text { Lophodermium seditiosum } \\
\text { Minter, Staley \& Millar }\end{array}$ & $\begin{array}{l}\text { Rhytismataceae, Leotiomycetes, } \\
\text { Ascomycota }\end{array}$ & seeds & Pinus sylvestris & Germany & Bentele et al. 2014 \\
\hline \multirow[t]{11}{*}{$\begin{array}{l}\text { Macrochytrium botrydioides } \\
\text { Minden }\end{array}$} & $\begin{array}{l}\text { Incertae sedis, Chytridiomycetes, } \\
\text { Chytridiomycota }\end{array}$ & seeds & $\begin{array}{l}\text { Armeria } \\
\text { transmontana }\end{array}$ & $\begin{array}{l}\text { Poland or/ } \\
\text { imported to Poland }\end{array}$ & Czeczuga et al. 2009 \\
\hline & & seeds & Asparagus officinalis & $\begin{array}{l}\text { Poland or/ } \\
\text { imported to Poland }\end{array}$ & Czeczuga et al. 2009 \\
\hline & & seeds & Atropa belladonna & $\begin{array}{l}\text { Poland or/ } \\
\text { imported to Poland }\end{array}$ & Czeczuga et al. 2009 \\
\hline & & seeds & Atropa caucasica & $\begin{array}{l}\text { Poland or/ } \\
\text { imported to Poland }\end{array}$ & Czeczuga et al. 2009 \\
\hline & & seeds & Atropa komarovii & $\begin{array}{l}\text { Poland or/ } \\
\text { imported to Poland }\end{array}$ & Czeczuga et al. 2009 \\
\hline & & seeds & Bryonia dioica & $\begin{array}{l}\text { Poland or/ } \\
\text { imported to Poland }\end{array}$ & Czeczuga et al. 2009 \\
\hline & & seeds & Celtis australis & $\begin{array}{l}\text { Poland or/ } \\
\text { imported to Poland }\end{array}$ & Czeczuga et al. 2009 \\
\hline & & seeds & Cornus capitata & $\begin{array}{l}\text { Poland or/ } \\
\text { imported to Poland }\end{array}$ & Czeczuga et al. 2009 \\
\hline & & seeds & Crataegus azarolus & $\begin{array}{l}\text { Poland or/ } \\
\text { imported to Poland }\end{array}$ & Czeczuga et al. 2009 \\
\hline & & seeds & $\begin{array}{l}\text { Podophyllum } \\
\text { peltatum }\end{array}$ & $\begin{array}{l}\text { Poland or/ } \\
\text { imported to Poland }\end{array}$ & Czeczuga et al. 2009 \\
\hline & & seeds & Sorbus torminalis & $\begin{array}{l}\text { Poland or/ } \\
\text { imported to Poland }\end{array}$ & Czeczuga et al. 2009 \\
\hline Macrophoma fraxini Delacr. & $\begin{array}{l}\text { Botryosphaeriaceae, Dothideomycetes, } \\
\text { Ascomycota }\end{array}$ & seeds & Fraxinus sp. & $\begin{array}{l}\text { former } \\
\text { Czechoslovakia }\end{array}$ & Anderson 1986a \\
\hline $\begin{array}{l}\text { Macrophoma nitens Berl. \& } \\
\text { Voglino }\end{array}$ & $\begin{array}{l}\text { Botryosphaeriaceae, Dothideomycetes, } \\
\text { Ascomycota }\end{array}$ & seeds & Quercus spp. & USSR & $\begin{array}{l}\text { Urosevic 1959, Mittal et } \\
\text { al. } 1990\end{array}$ \\
\hline \multirow[t]{5}{*}{ Macrophoma spp. } & Botryosphaeriaceae, Dothideomycetes, & fruits & Ardisia qinquegona & Hong Kong & Tang et al. 2003a \\
\hline & Ascomycota & fruits & $\begin{array}{l}\text { Cleistocalyx } \\
\text { operculatus }\end{array}$ & Hong Kong & Tang et al. 2003a \\
\hline & & fruits & Litsea rotundifolia & Hong Kong & Tang et al. 2003a \\
\hline & & fruits & Microcos paniculata & Hong Kong & Tang et al. 2003a \\
\hline & & fruits & Psychotria asiatica & Hong Kong & Tang et al. 2003a \\
\hline
\end{tabular}


Table 2 Continued.

\begin{tabular}{|c|c|c|c|c|c|}
\hline Species & Taxonomy & Substrate & Host & Country & References \\
\hline & & fruits & $\begin{array}{l}\text { Rhodomyrtus } \\
\text { tomentosa }\end{array}$ & Hong Kong & Tang et al. 2003a \\
\hline & & seeds & $\begin{array}{l}\text { Eucalyptus } \\
\text { camaldulensis }\end{array}$ & Thailand & $\begin{array}{l}\text { Chalermpongse et al. } \\
\text { 1984, Mittal et al. } 1990\end{array}$ \\
\hline & & seeds & $\begin{array}{l}\text { Eucalyptus } \\
\text { citriodora }\end{array}$ & India & Anderson 1986a \\
\hline \multirow[t]{14}{*}{$\begin{array}{l}\text { Macrophomina phaseolina } \\
\text { (Tassi) Goid. }\end{array}$} & $\begin{array}{l}\text { Botryosphaeriaceae, Dothideomycetes, } \\
\text { Ascomycota }\end{array}$ & seeds & Albizia stipulata & India & $\begin{array}{l}\text { Mathur 1974, Anderson } \\
\text { 1986a }\end{array}$ \\
\hline & & seeds & Azadirachta indica & India & $\begin{array}{l}\text { Mathur 1974, Mittal et } \\
\text { al. } 1990\end{array}$ \\
\hline & & seeds & Cassia siamea & Philippines & $\begin{array}{l}\text { Dayan 1986, Mittal et al. } \\
1990\end{array}$ \\
\hline & & seeds & $\begin{array}{l}\text { Casuarina } \\
\text { equisetifolia }\end{array}$ & Philippines & $\begin{array}{l}\text { Quiniones 1985, } \\
\text { Anderson 1986a }\end{array}$ \\
\hline & & seeds & Cedrela odorata & Colombia & $\begin{array}{l}\text { Mathur 1974, Anderson } \\
\text { 1986a }\end{array}$ \\
\hline & & seeds & Eucalyptus sp. & India & $\begin{array}{l}\text { Saxena 1985, Mittal et } \\
\text { al. } 1990\end{array}$ \\
\hline & & seeds & $\begin{array}{l}\text { Leucdena } \\
\text { diversifolia }\end{array}$ & Guatemala & $\begin{array}{l}\text { Mathur 1974, Anderson } \\
\text { 1986a }\end{array}$ \\
\hline & & seeds & $\begin{array}{l}\text { Musanga } \\
\text { cecropioides }\end{array}$ & Philippines & $\begin{array}{l}\text { Agmata 1979, Anderson } \\
\text { 1986a }\end{array}$ \\
\hline & & seeds & Pinus caribaea & $\begin{array}{l}\text { Central America, } \\
\text { Madagascar, UK }\end{array}$ & $\begin{array}{l}\text { Rees 1982, Anderson } \\
\text { 1986a, Mittal et al. } 1990\end{array}$ \\
\hline & & seeds & Pinus insularis & Philippines & $\begin{array}{l}\text { Agmata 1979, Anderson } \\
\text { 1986a }\end{array}$ \\
\hline & & seeds & Pinus merkusii & Philippines & $\begin{array}{l}\text { Agmata 1979, Anderson } \\
\text { 1986a }\end{array}$ \\
\hline & & seeds & Pterocarpus indicus & Philippines & $\begin{array}{l}\text { Agmata 1979, Anderson } \\
\text { 1986a }\end{array}$ \\
\hline & & seeds & $\begin{array}{l}\text { Swietenia } \\
\text { macrophylla }\end{array}$ & Philippines & $\begin{array}{l}\text { Mathur 1974, Anderson } \\
\text { 1986a }\end{array}$ \\
\hline & & seeds & $\begin{array}{l}\text { Tabebuia } \\
\text { heptaphylla }\end{array}$ & Brazil & $\begin{array}{l}\text { Mathur 1974, Anderson } \\
\text { 1986a }\end{array}$ \\
\hline
\end{tabular}


Table 2 Continued.

\begin{tabular}{|c|c|c|c|c|c|}
\hline Species & Taxonomy & Substrate & Host & Country & References \\
\hline $\begin{array}{l}\text { Macrophomina phaseolina } \\
\text { (Tassi) Goid. } \\
\text { [as Macrophomina phaseoli } \\
\text { (Maubl.) S.F. Ashby] }\end{array}$ & & seeds & Triplaris cumingiana & Philippines & $\begin{array}{l}\text { Quiniones 1985, } \\
\text { Anderson 1986a }\end{array}$ \\
\hline $\begin{array}{l}\text { Macrophomina phaseolina } \\
\text { (Tassi) Goid. }\end{array}$ & & seeds & $\begin{array}{l}\text { Acacia } \\
\text { auriculiformis }\end{array}$ & Philippines & Quiniones 1987 \\
\hline $\begin{array}{l}\text { [as Macrophoma phaseoli } \\
\text { Maubl.] }\end{array}$ & & seeds & $\begin{array}{l}\text { Casuarina } \\
\text { equisetifolia }\end{array}$ & Philippines & Quiniones 1987 \\
\hline \multirow{9}{*}{ Macrophomina spp. } & \multirow{9}{*}{$\begin{array}{l}\text { Botryosphaeriaceae, Dothideomycetes, } \\
\text { Ascomycota }\end{array}$} & seeds & Gmelina arborea & Philippines & Quiniones 1987 \\
\hline & & seeds & Toona calantas & Philippines & Quiniones 1987 \\
\hline & & seeds & Chukrasia tabularis & India & $\begin{array}{l}\text { Mathur 1974, Anderson } \\
\text { 1986a }\end{array}$ \\
\hline & & seeds & Cupressus arizonica & Uruguay & Anderson 1986a \\
\hline & & seeds & Eucalyptus grandis & South Africa & Jimu et al. 2016 \\
\hline & & seeds & Leucaena spp. & Philippines & $\begin{array}{l}\text { Mathur 1974, Anderson } \\
\text { 1986a }\end{array}$ \\
\hline & & seeds & Shorea acuminata & Malaysia & Mittal et al. 1990 \\
\hline & & seeds & Shorea talura & Malaysia & Mittal et al. 1990 \\
\hline & & seeds & Tectona grandis & - & $\begin{array}{l}\text { Mathur 1974, Anderson } \\
\text { 1986a }\end{array}$ \\
\hline $\begin{array}{l}\text { Malassezia globosa Midgley, } \\
\text { E. Guého \& J. Guillot }\end{array}$ & $\begin{array}{l}\text { Malasseziaceae, Malasseziomycetes, } \\
\text { Basidiomycota }\end{array}$ & seeds & Fraxinus excelsior & Sweden & Hayatgheibi 2013 \\
\hline Malassezia sp. & $\begin{array}{l}\text { Malasseziaceae, Malasseziomycetes, } \\
\text { Basidiomycota }\end{array}$ & seeds & Fraxinus excelsior & Sweden & Hayatgheibi 2013 \\
\hline $\begin{array}{l}\text { Marasmius cryptocystidiatus } \\
\text { Corner }^{\#}\end{array}$ & $\begin{array}{l}\text { Marasmiaceae, Agaricomycetes, } \\
\text { Basidiomycota }\end{array}$ & fruit husks & Willughbeia sp. & Singapore & Corner 1996 \\
\hline $\begin{array}{l}\text { Marasmius fagi (Kalamees) } \\
\text { Kalamees }^{\#} \\
\text { [as Collybia fagi Kalamees] }\end{array}$ & $\begin{array}{l}\text { Marasmiaceae, Agaricomycetes, } \\
\text { Basidiomycota }\end{array}$ & fruit & Fagus sp. & Russia & Vaasma et al. 1986 \\
\hline $\begin{array}{l}\text { Marasmius micromerus } \\
\text { Corner }^{\#}\end{array}$ & $\begin{array}{l}\text { Marasmiaceae, Agaricomycetes, } \\
\text { Basidiomycota }\end{array}$ & fruits & Polyalthia sp. & Malaysia & Corner 1996 \\
\hline Massarina spp. & $\begin{array}{l}\text { Massarinaceae, Dothideomycetes, } \\
\text { Ascomycota }\end{array}$ & cones, seeds & Pinus spp. & Canada & Vujanovic et al. 2000 \\
\hline
\end{tabular}


Table 2 Continued.

\begin{tabular}{|c|c|c|c|c|c|}
\hline Species & Taxonomy & Substrate & Host & Country & References \\
\hline & & fruits & Ilex pubescens & Hong Kong & Tang et al. 2003a \\
\hline & & fruits & Microcos paniculata & Hong Kong & Tang et al. 2003a \\
\hline & & fruits & Psychotria asiatica & Hong Kong & Tang et al. 2003a \\
\hline & & fruits & $\begin{array}{l}\text { Viburnum } \\
\text { sempervirens }\end{array}$ & Hong Kong & Tang et al. 2003a \\
\hline Melampsora abietis- & Melampsoraceae, Pucciniomycetes, & cone & Tsuga canadensis & Canada & Conners 1967 \\
\hline Ludw. & & & & & \\
\hline $\begin{array}{l}\text { Melanconium apiocarpum } \\
\text { Link }\end{array}$ & \multirow[t]{2}{*}{$\begin{array}{l}\text { Melanconidaceae, Sordariomycetes, } \\
\text { Ascomycota }\end{array}$} & seeds & Pinus sylvestris & USSR & $\begin{array}{l}\text { Mittal et al. } 1990 \text {, } \\
\text { Urosevic } 1961\end{array}$ \\
\hline $\begin{array}{l}\text { Melanconium apiocarpum } \\
\text { Link [as 'apiocarpon'] }\end{array}$ & & seeds & Picea excelsa & USSR & $\begin{array}{l}\text { Urosevic 1961, } \\
\text { Anderson 1986a }\end{array}$ \\
\hline Melanconium bicolor Nees & $\begin{array}{l}\text { Melanconidaceae, Sordariomycetes, } \\
\text { Ascomycota }\end{array}$ & seeds & $\begin{array}{l}\text { Picea excelsa, Pinus } \\
\text { sylvestris }\end{array}$ & USSR & $\begin{array}{l}\text { Urosevic 1961, } \\
\text { Anderson 1986a }\end{array}$ \\
\hline $\begin{array}{l}\text { Melanospora papillata } \\
\text { Hotson }\end{array}$ & $\begin{array}{l}\text { Ceratostomataceae, Sordariomycetes, } \\
\text { Ascomycota }\end{array}$ & seed & $\begin{array}{l}\text { Linum usitatissimum, } \\
\text { Lolium perenne }\end{array}$ & Canada & Conners 1967 \\
\hline Melanospora spp. & Ceratostomataceae, Sordariomycetes, & seeds & Pinus taeda & USA & Anderson 1986a, b \\
\hline \multirow{3}{*}{$\begin{array}{l}\text { Melanospora spp. } \\
\text { [as Gonatobotrys] }\end{array}$} & \multirow[t]{3}{*}{ Ascomycota } & seed & Prunus serrulata & Japan & Watanabe 2010 \\
\hline & & seeds & Pinus sylvestris & USSR & $\begin{array}{l}\text { Dolejs 1964, } \\
\text { Mittal et al. } 1990\end{array}$ \\
\hline & & seeds & Pinus taeda & USA & Anderson 1986a, b \\
\hline \multirow{6}{*}{$\begin{array}{l}\text { Melanospora simplex } \\
\text { (Corda) D. Hawksw. } \\
\text { [as Gonatobotrys simplex } \\
\text { Corda] } \\
\text { Melanospora zamiae Corda }\end{array}$} & \multirow{2}{*}{$\begin{array}{l}\text { Ceratostomataceae, Sordariomycetes, } \\
\text { Ascomycota }\end{array}$} & seed & Agropyron cristatum & Canada & Conners 1967 \\
\hline & & seed & Linum usitatissimum & Canada & Conners 1967 \\
\hline & \multirow[t]{4}{*}{$\begin{array}{l}\text { Ceratostomataceae, Sordariomycetes, } \\
\text { Ascomycota }\end{array}$} & seeds & Abies spp. & UK & $\begin{array}{l}\text { Batko 1959, Anderson } \\
\text { 1986a }\end{array}$ \\
\hline & & seed & Gossypium sp. & Pakistan & Lodhi \& Naeem 1955 \\
\hline & & seed & Linum usitatissimum & Canada & Conners 1967 \\
\hline & & seeds & Lolium perenne & Canada & Conners 1967 \\
\hline \multirow{2}{*}{$\begin{array}{l}\text { Memnoniella echinata } \\
\text { (Rivolta) Galloway }\end{array}$} & \multirow{2}{*}{$\begin{array}{l}\text { Stachybotryaceae, Sordariomycetes, } \\
\text { Ascomycota }\end{array}$} & pods & Delonix regia & Thailand & Somrithipol et al. 2002b \\
\hline & & seeds & Cassia fistula & India & $\begin{array}{l}\text { Mittal \& Sharma 1981a, } \\
\text { Mittal et al. } 1990\end{array}$ \\
\hline
\end{tabular}


Table 2 Continued.

\begin{tabular}{|c|c|c|c|c|c|}
\hline Species & Taxonomy & Substrate & Host & Country & References \\
\hline & & seeds & Cassia siamea & Thailand & $\begin{array}{l}\text { Chalermpongse et al. } \\
\text { 1984, Mittal et al. } 1990\end{array}$ \\
\hline & & seeds & Cedrus deodara & India & $\begin{array}{l}\text { Munjal \& Sharma 1975, } \\
\text { Mittal et al. } 1990\end{array}$ \\
\hline & & seeds & Dalbergia sissoo & India & $\begin{array}{l}\text { Mittal \& Sharma 1981b, } \\
\text { Mittal et al. } 1990\end{array}$ \\
\hline & & seeds & Eucalyptus hybrid & India & $\begin{array}{l}\text { Mittal 1986, Mittal et al. } \\
1990\end{array}$ \\
\hline & & seeds & Eucalyptus sp. & India & $\begin{array}{l}\text { Reddy et al. 1982, Mittal } \\
\text { et al. } 1990\end{array}$ \\
\hline & & seeds & Pinus merkusii & Thailand & $\begin{array}{l}\text { Chalermpongse et al. } \\
\text { 1984, Mittal et al. } 1990\end{array}$ \\
\hline & & seeds & Pinus roxburghii & India & $\begin{array}{l}\text { Mittal \& Sharma 1982c, } \\
\text { Mittal et al. } 1990\end{array}$ \\
\hline & & seeds & Pinus wallichiana & India & $\begin{array}{l}\text { Munjal \& Sharma 1975, } \\
\text { Mittal et al. } 1990\end{array}$ \\
\hline Memnoniella spp. & $\begin{array}{l}\text { Stachybotryaceae, Sordariomycetes, } \\
\text { Ascomycota }\end{array}$ & seeds & Tectona grandis & India & Anderson 1986a \\
\hline $\begin{array}{l}\text { Meniscoideisporites cretacea } \\
\text { K. Watan., H. Nishida \& } \\
\text { Tak. Kobay }{ }^{\# * *}\end{array}$ & Fossil fungus & cone & $\begin{array}{l}\text { Cycadeoidella } \\
\text { japonica }\end{array}$ & Japan & Watanabe et al. 1999 \\
\hline Menisporopsis sp. & $\begin{array}{l}\text { Chaetosphaeriaceae, Sordariomycetes, } \\
\text { Ascomycota }\end{array}$ & fruits & $\begin{array}{l}\text { Rhodomyrtus } \\
\text { tomentosa }\end{array}$ & Hong Kong & Tang et al. 2003a \\
\hline Metarhizium sp. & $\begin{array}{l}\text { Clavicipitaceae, Sordariomycetes, } \\
\text { Ascomycota }\end{array}$ & seeds & Pinus taeda & USA & Anderson 1986a, b \\
\hline $\begin{array}{l}\text { Metschnikowia laotica } \\
\text { Sipiczki\# }\end{array}$ & $\begin{array}{l}\text { Metschnikowiaceae, Saccharomycetes, } \\
\text { Ascomycota }\end{array}$ & fruit & undetermined plant & Laos & Sipiczki 2014 \\
\hline $\begin{array}{l}\text { Meyerozyma guilliermondii } \\
\text { (Wick.) Kurtzman \& M. } \\
\text { Suzuki [as Candida } \\
\text { guilliermondii (Castell.) } \\
\text { Langeron \& Guerra] }\end{array}$ & $\begin{array}{l}\text { Debaryomycetaceae, Saccharomycetes, } \\
\text { Ascomycota }\end{array}$ & fruits & $\begin{array}{l}\text { Parahancornia } \\
\text { amapa }\end{array}$ & Brazil & Morais et al. 1995 \\
\hline Micraspis strobilina Dennis & $\begin{array}{l}\text { Micraspidaceae, Leotiomycetes, } \\
\text { Ascomycota }\end{array}$ & cones & Pinus sylvestris & UK & Dennis 1971 \\
\hline
\end{tabular}


Table 2 Continued.

\begin{tabular}{|c|c|c|c|c|c|}
\hline Species & Taxonomy & Substrate & Host & Country & References \\
\hline $\begin{array}{l}\text { Microascus longirostris } \\
\text { Zukal }\end{array}$ & $\begin{array}{l}\text { Microascaceae, Sordariomycetes, } \\
\text { Ascomycota }\end{array}$ & seed & $\begin{array}{l}\text { Cryptomeria } \\
\text { japonica }\end{array}$ & Japan & Watanabe 2010 \\
\hline $\begin{array}{l}\text { Microbotryum perfoliatae } \\
\text { Vánky }{ }^{\#}\end{array}$ & $\begin{array}{l}\text { Microbotryaceae, Microbotryomycetes, } \\
\text { Basidiomycota }\end{array}$ & seeds & Claytonia perfoliata & USA & Vánky 1998 \\
\hline $\begin{array}{l}\text { Microbotryum silybum } \\
\text { Vánky \& Berner }^{\#}\end{array}$ & $\begin{array}{l}\text { Microbotryaceae, Microbotryomycetes, } \\
\text { Basidiomycota }\end{array}$ & seed & Silybum marianum & Greece & Vánky \& Berner 2003 \\
\hline $\begin{array}{l}\text { Microdochium sp. [as } \\
\text { Monographella] }\end{array}$ & $\begin{array}{l}\text { Microdochiaceae, Sordariomycetes, } \\
\text { Ascomycota }\end{array}$ & seeds & Phyllostachys edulis & China & Shen et al. 2014 \\
\hline $\begin{array}{l}\text { Microsphaeropsis globulosa } \\
\text { (Sousa da Câmara) B. Sutton }\end{array}$ & $\begin{array}{l}\text { Didymosphaeriaceae, Dothideomycetes, } \\
\text { Ascomycota }\end{array}$ & fruits & $\begin{array}{l}\text { Cleistocalyx } \\
\text { operculatus }\end{array}$ & Hong Kong & Tang et al. 2003a \\
\hline $\begin{array}{l}\text { Microthecium sepedonioides } \\
\text { (Preuss) Y. Marín, Stchigel, } \\
\text { Guarro \& Cano } \\
\text { [as Papulaspora } \\
\text { sepedonioides Preuss] }\end{array}$ & $\begin{array}{l}\text { Ceratostomataceae, Sordariomycetes, } \\
\text { Ascomycota }\end{array}$ & seeds & Quercus spp. & USSR & $\begin{array}{l}\text { Urosevic 1962, Mittal et } \\
\text { al. } 1990\end{array}$ \\
\hline $\begin{array}{l}\text { Millerozyma acaciae (Van } \\
\text { der Walt) Kurtzman \& M. } \\
\text { Suzuki [as Pichia acaciae } \\
\text { Van der Walt] }\end{array}$ & $\begin{array}{l}\text { Debaryomycetaceae, Saccharomycetes, } \\
\text { Ascomycota }\end{array}$ & fruits & $\begin{array}{l}\text { Parahancornia } \\
\text { amapa }\end{array}$ & Brazil & Morais et al. 1995 \\
\hline $\begin{array}{l}\text { Mollisia ligni (Desm.) P. } \\
\text { Karst. }\end{array}$ & Mollisiaceae, Leotiomycetes, Ascomycota & fruit cupules & Fagus sylvatica & UK & Carré 1964 \\
\hline Mollisia sp. & Mollisiaceae, Leotiomycetes, Ascomycota & cones, seeds & Pinus spp. & Canada & Vujanovic et al. 2000 \\
\hline Monacrosporium sp. & Orbiliaceae, Orbiliomycetes, Ascomycota & cones & Pinus densiflora & Japan & Kasai et al. 1995 \\
\hline \multirow[t]{3}{*}{$\begin{array}{l}\text { Monilia sitophila (Mont.) } \\
\text { Sacc. }\end{array}$} & $\begin{array}{l}\text { Sclerotiniaceae, Leotiomycetes, } \\
\text { Ascomycota }\end{array}$ & seeds & Larix sibirica & USSR & $\begin{array}{l}\text { Prisyazhnyuk 1960, } \\
\text { Mittal et al. } 1990\end{array}$ \\
\hline & & seeds & Picea abies & USSR & $\begin{array}{l}\text { Prisyazhnyuk } 1960 \text {, } \\
\text { Mittal et al. } 1990\end{array}$ \\
\hline & & seeds & Quercus spp. & USSR & $\begin{array}{l}\text { Urosevic 1962, Mittal et } \\
\text { al. } 1990\end{array}$ \\
\hline Monilia sitophila (Mont.) & & seeds & Abies sibirica & USSR & Mittal et al. 1990, \\
\hline Sacc. [as sitophyla] & & seeds & Pinus sylvestris & USSR & $\begin{array}{l}\text { Prisyazhnyuk 1960, } \\
\text { Mittal et al. } 1990\end{array}$ \\
\hline Monilia sp. & $\begin{array}{l}\text { Sclerotiniaceae, Leotiomycetes, } \\
\text { Ascomycota }\end{array}$ & seeds & Alnus sibirica & South Korea & $\begin{array}{l}\text { Mathur 1974, Mittal et } \\
\text { al. } 1990\end{array}$ \\
\hline
\end{tabular}


Table 2 Continued.

\begin{tabular}{|c|c|c|c|c|c|}
\hline Species & Taxonomy & Substrate & Host & Country & References \\
\hline & & seeds & Cassia bakeriana & Thailand & $\begin{array}{l}\text { Chalermpongse et al. } \\
\text { 1984, Mittal et al. } 1990\end{array}$ \\
\hline & & seeds & $\begin{array}{l}\text { Chamaecyparis } \\
\text { obtusa }\end{array}$ & South Korea & $\begin{array}{l}\text { Mathur 1974, Anderson } \\
\text { 1986a }\end{array}$ \\
\hline & & seeds & $\begin{array}{l}\text { Pinus elliottii var. } \\
\text { elliottii }\end{array}$ & Georgia & Huang \& Kuhlman 1990 \\
\hline & & seeds & Pinus taeda & USA & Anderson 1986a, b \\
\hline & & seeds & Samanea saman & Thailand & $\begin{array}{l}\text { Chalermpongse et al. } \\
\text { 1984, Mittal et al. } 1990\end{array}$ \\
\hline $\begin{array}{l}\text { Monilinia amelanchieris } \\
\text { (J.M. Reade) Honey }\end{array}$ & $\begin{array}{l}\text { Sclerotiniaceae, Leotiomycetes, } \\
\text { Ascomycota }\end{array}$ & fruit & Amelanchier sp. & Canada & Conners 1967 \\
\hline $\begin{array}{l}\text { Monilinia azaleae Honey } \\
\text { [as Monilia azalea L.R. } \\
\text { Batra] }\end{array}$ & $\begin{array}{l}\text { Sclerotiniaceae, Leotiomycetes, } \\
\text { Ascomycota }\end{array}$ & fruits & $\begin{array}{l}\text { Rhododendron } \\
\text { roseum }\end{array}$ & USA & Batra 1991 \\
\hline $\begin{array}{l}\text { Monilinia fructicola (G. } \\
\text { Winter) Honey }\end{array}$ & $\begin{array}{l}\text { Sclerotiniaceae, Leotiomycetes, } \\
\text { Ascomycota }\end{array}$ & fruits & Psychotria asiatica & Hong Kong & Tang et al. 2003a \\
\hline 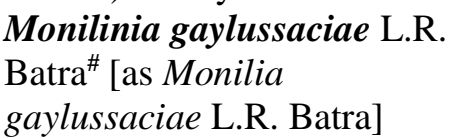 & $\begin{array}{l}\text { Sclerotiniaceae, Leotiomycetes, } \\
\text { Ascomycota }\end{array}$ & fruits & Gaylussacia baccata & USA & Batra 1988 \\
\hline $\begin{array}{l}\text { Monilinia jezoensis Yuk. } \\
\text { Takah., T. Sano \& Y. } \\
\text { Harada\# [as Monilia jezoensis } \\
\text { Yuk. Takah., Ichihashi \& Y. } \\
\text { Harada] }\end{array}$ & $\begin{array}{l}\text { Sclerotiniaceae, Leotiomycetes, } \\
\text { Ascomycota }\end{array}$ & fruits & $\begin{array}{l}\text { Rhododendron } \\
\text { kaempferi }\end{array}$ & Japan & Takahashi et al. 2005 \\
\hline $\begin{array}{l}\text { Monilinia ssiori Y. Harada, } \\
\text { Ma. Saaki \& T. Sano }\end{array}$ & $\begin{array}{l}\text { Sclerotiniaceae, Leotiomycetes, } \\
\text { Ascomycota }\end{array}$ & fruits & Prunus ssiori & Japan & Harada et al. 2005 \\
\hline $\begin{array}{l}\text { Monochaetia ilicina (Sacc.) } \\
\text { Nag Raj }\end{array}$ & $\begin{array}{l}\text { Sporocadaceae, Sordariomycetes, } \\
\text { Ascomycota }\end{array}$ & fruits & Ardisia crenata & Hong Kong & Tang et al. 2003a \\
\hline $\begin{array}{l}\text { Monochaetia karstenii } \\
\text { (Sacc. \& P. Syd.) B. Sutton }\end{array}$ & $\begin{array}{l}\text { Sporocadaceae, Sordariomycetes, } \\
\text { Ascomycota }\end{array}$ & fruits & Ilex cinerea & Hong Kong & Tang et al. 2003a \\
\hline Monochaetia sp. & $\begin{array}{l}\text { Sporocadaceae, Sordariomycetes, } \\
\text { Ascomycota }\end{array}$ & cones & Picea glauca & Canada & Mittal \& Wang 1987 \\
\hline Monocillium spp. & $\begin{array}{l}\text { Niessliaceae, Sordariomycetes, } \\
\text { Ascomycota }\end{array}$ & seeds & $\begin{array}{l}\text { Eucalyptus } \\
\text { citriodora }\end{array}$ & India & Anderson 1986a \\
\hline
\end{tabular}


Table 2 Continued.

\begin{tabular}{|c|c|c|c|c|c|}
\hline Species & Taxonomy & Substrate & Host & Country & References \\
\hline & & seeds & Eucalyptus globulus & India & Anderson 1986a \\
\hline & & seeds & Eucalyptus grandis & India & Anderson 1986a \\
\hline & & seeds & $\begin{array}{l}\text { Eucalyptus } \\
\text { tereticornis }\end{array}$ & India & Anderson 1986a \\
\hline & & seeds & Pinus taeda & USA & Anderson 1986a, b \\
\hline & & seeds & Tectona grandis & India & Anderson 1986a \\
\hline Monotospora sp. & $\begin{array}{l}\text { Incertae sedis, Incertae sedis, } \\
\text { Ascomycota }\end{array}$ & seeds & Pinus taeda & USA & Anderson 1986a, b \\
\hline Monotosporella sp. & $\begin{array}{l}\text { Pleurotheciaceae, Sordariomycetes, } \\
\text { Ascomycota }\end{array}$ & pods & Delonix regia & Thailand & Somrithipol et al. 2002b \\
\hline $\begin{array}{l}\text { Mortierella candelabrum } \\
\text { Tiegh. \& G. Le Monn. }\end{array}$ & $\begin{array}{l}\text { Mortierellaceae, Mortierellomycetes, } \\
\text { Mucoromycota }\end{array}$ & seeds & Crataegus azarolus & $\begin{array}{l}\text { Poland or/ } \\
\text { imported to Poland }\end{array}$ & Czeczuga et al. 2009 \\
\hline $\begin{array}{l}\text { Mortierella hyalina (Harz) } \\
\text { W. Gams }\end{array}$ & $\begin{array}{l}\text { Mortierellaceae, Mortierellomycetes, } \\
\text { Mucoromycota }\end{array}$ & cones & Pinus densiflora & Japan & Kasai et al. 1995 \\
\hline Mucor adventitius Oudem. & $\begin{array}{l}\text { Mucoraceae, Mucoromycetes, } \\
\text { Mucoromycota }\end{array}$ & seeds & Shorea robusta & India & $\begin{array}{l}\text { Mittal \& Sharma 1982b, } \\
\text { Mittal et al. } 1990\end{array}$ \\
\hline \multirow[t]{2}{*}{ Mucor circinelloides Tiegh. } & $\begin{array}{l}\text { Mucoraceae, Mucoromycetes, } \\
\text { Mucoromycota }\end{array}$ & seed & $\begin{array}{l}\text { Cryptomeria } \\
\text { japonica }\end{array}$ & Japan & Watanabe 2010 \\
\hline & & seed & Prunus serrulata & Japan & Watanabe 2010 \\
\hline \multirow[t]{2}{*}{ Mucor erectus Bainier } & $\begin{array}{l}\text { Mucoraceae, Mucoromycetes, } \\
\text { Mucoromycota }\end{array}$ & seeds & Crataegus azarolus & $\begin{array}{l}\text { Poland or/ } \\
\text { imported to Poland }\end{array}$ & Czeczuga et al. 2009 \\
\hline & & seeds & Sorbus torminalis & $\begin{array}{l}\text { Poland or/ } \\
\text { imported to Poland }\end{array}$ & Czeczuga et al. 2009 \\
\hline \multirow[t]{5}{*}{ Mucor globosus Schreb. } & $\begin{array}{l}\text { Mucoraceae, Mucoromycetes, } \\
\text { Mucoromycota }\end{array}$ & seeds & Betula verrucosa & USSR & $\begin{array}{l}\text { Kozlowska 1968, Mittal } \\
\text { et al. } 1990\end{array}$ \\
\hline & & seeds & Cedrus deodara & India & $\begin{array}{l}\text { Munjal \& Sharma 1975, } \\
\text { Mittal et al. } 1990\end{array}$ \\
\hline & & seeds & Pinus roxburghii & India & $\begin{array}{l}\text { Munjal \& Sharma 1975, } \\
\text { Mittal et al. } 1990\end{array}$ \\
\hline & & seeds & Quercus pedunculata & USSR & $\begin{array}{l}\text { Kozlowska 1968, Mittal } \\
\text { et al. } 1990\end{array}$ \\
\hline & & seeds & Quercus spp. & USSR & $\begin{array}{l}\text { Urosevic 1959, Mittal et } \\
\text { al. } 1990\end{array}$ \\
\hline
\end{tabular}


Table 2 Continued.

\begin{tabular}{|c|c|c|c|c|c|}
\hline Species & Taxonomy & Substrate & Host & Country & References \\
\hline $\begin{array}{l}\text { Mucor globosus Schreb. } \\
\text { [as 'globosum'] }\end{array}$ & & seeds & Pinus wallichiana & India & $\begin{array}{l}\text { Munjal \& Sharma 1975, } \\
\text { Mittal et al. } 1990\end{array}$ \\
\hline \multirow[t]{14}{*}{ Mucor hiemalis Wehmer } & $\begin{array}{l}\text { Mucoraceae, Mucoromycetes, } \\
\text { Mucoromycota }\end{array}$ & cones & $\begin{array}{l}\text { Asparagus officinalis } \\
\text { var. altilis }\end{array}$ & Canada & Conners 1967 \\
\hline & & seed & Abies alba & Poland & Krol et al. 2015 \\
\hline & & seed & Acer pseudoplatanus & Poland & Krol et al. 2015 \\
\hline & & seeds & Betula pendula & Poland & Krol et al. 2015 \\
\hline & & seeds & Cedrus deodara & India & $\begin{array}{l}\text { Munjal \& Sharma 1975, } \\
\text { Mittal et al. } 1990\end{array}$ \\
\hline & & seeds & Eucalyptus sp. & India & $\begin{array}{l}\text { Saxena 1985, Mittal et } \\
\text { al. } 1990\end{array}$ \\
\hline & & seeds & Larix decidua & Poland & Krol et al. 2015 \\
\hline & & seeds & Linum usitatissimum & Canada & Conners 1967 \\
\hline & & seeds & Picea glauca & Canada & Mittal \& Wang 1987 \\
\hline & & seeds & Pinus caribaea & $\begin{array}{l}\text { Belize [as British } \\
\text { Honduras] }\end{array}$ & $\begin{array}{l}\text { Hocking 1968, Mittal et } \\
\text { al. } 1990\end{array}$ \\
\hline & & seeds & Pinus patula & Kenya & $\begin{array}{l}\text { Gibson 1957, Mittal et } \\
\text { al. } 1990\end{array}$ \\
\hline & & seeds & Pinus roxburghii & India & $\begin{array}{l}\text { Munjal \& Sharma 1975, } \\
\text { Mittal et al. } 1990\end{array}$ \\
\hline & & seeds & Pinus strobus & Canada & Mittal \& Wang 1987 \\
\hline & & seeds & Pinus wallichiana & India & $\begin{array}{l}\text { Munjal \& Sharma 1975, } \\
\text { Mittal et al. } 1990\end{array}$ \\
\hline \multirow[t]{2}{*}{ Mucor microsporus Bonord. } & Mucoraceae, Mucoromycetes, & pod & Canavalia cathartica & India & Anita \& Sridhar 2009 \\
\hline & Mucoromycota & seed & Sesbania bispinosa & India & Anita et al. 2009 \\
\hline \multirow[t]{4}{*}{ Mucor mucedo L. } & $\begin{array}{l}\text { Mucoraceae, Mucoromycetes, } \\
\text { Mucoromycota }\end{array}$ & seeds & Abies sibirica & USSR & $\begin{array}{l}\text { Prisyazhnyuk } 1960 \text {, } \\
\text { Mittal et al. } 1990\end{array}$ \\
\hline & & seeds & Larix sibirica & USSR & $\begin{array}{l}\text { Prisyazhnyuk } 1960 \text {, } \\
\text { Mittal et al. } 1990\end{array}$ \\
\hline & & seeds & Picea abies & USSR & $\begin{array}{l}\text { Prisyazhnyuk } 1960 \text {, } \\
\text { Mittal et al. } 1990\end{array}$ \\
\hline & & seeds & Pinus ponderosa & USSR & $\begin{array}{l}\text { James \& Genz 1982, } \\
\text { Anderson 1986a }\end{array}$ \\
\hline
\end{tabular}


Table 2 Continued.

\begin{tabular}{|c|c|c|c|c|c|}
\hline Species & Taxonomy & Substrate & Host & Country & References \\
\hline & & seeds & Pinus sylvestris & USSR & $\begin{array}{l}\text { Dolejs 1964, } \\
\text { Prisyazhnyuk 1960, } \\
\text { Mittal et al. 1990, }\end{array}$ \\
\hline Mucor piriformis A. Fisch. & $\begin{array}{l}\text { Mucoraceae, Mucoromycetes, } \\
\text { Mucoromycota }\end{array}$ & seeds & Rosa mollis & $\begin{array}{l}\text { Poland or/ } \\
\text { imported to Poland }\end{array}$ & Czeczuga et al. 2009 \\
\hline \multirow[t]{3}{*}{$\begin{array}{l}\text { Mucor piriformis A. Fisch. } \\
\text { [as Mucor alboater Naumov] }\end{array}$} & & seeds & Abies sibirica & USSR & $\begin{array}{l}\text { Prisyazhnyuk 1960, } \\
\text { Mittal et al. } 1990\end{array}$ \\
\hline & & seeds & Picea abies & USSR & $\begin{array}{l}\text { Prisyazhnyuk } 1960 \text {, } \\
\text { Mittal et al. } 1990\end{array}$ \\
\hline & & seeds & Pinus sylvestris & USSR & $\begin{array}{l}\text { Prisyazhnyuk } 1960 \text {, } \\
\text { Mittal et al. } 1990\end{array}$ \\
\hline \multirow[t]{6}{*}{ Mucor plumbeus Bonord. } & $\begin{array}{l}\text { Mucoraceae, Mucoromycetes, } \\
\text { Mucoromycota }\end{array}$ & pod & Sesbania bispinosa & India & Anita et al. 2009 \\
\hline & & seeds & Abies sibirica & USSR & $\begin{array}{l}\text { Prisyazhnyuk 1960, } \\
\text { Anderson 1986a }\end{array}$ \\
\hline & & seeds & Picea abies & USSR & $\begin{array}{l}\text { Prisyazhnyuk } 1960 \text {, } \\
\text { Mittal et al. } 1990\end{array}$ \\
\hline & & seeds & Pinus densiflora & Japan & Watanabe 2010 \\
\hline & & seeds & Picea excelsa & USSR & $\begin{array}{l}\text { Urosevic 1961, } \\
\text { Anderson 1986a }\end{array}$ \\
\hline & & seeds & Pinus sylvestris & USSR & $\begin{array}{l}\text { Prisyazhnyuk 1960, } \\
\text { Anderson 1986a }\end{array}$ \\
\hline \multirow[t]{5}{*}{ Mucor racemosus Bull. } & $\begin{array}{l}\text { Mucoraceae, Mucoromycetes, } \\
\text { Mucoromycota }\end{array}$ & seeds & Abies sibirica & USSR & $\begin{array}{l}\text { Prisyazhnyuk } 1960 \text {, } \\
\text { Mittal et al. } 1990\end{array}$ \\
\hline & & seeds & Casuarina spp. & India & Sahai \& Otra 1982 \\
\hline & & seeds & Larix sibirica & USSR & $\begin{array}{l}\text { Prisyazhnyuk } 1960 \text {, } \\
\text { Mittal et al. } 1990\end{array}$ \\
\hline & & seeds & Picea abies & USSR & $\begin{array}{l}\text { Prisyazhnyuk } 1960 \text {, } \\
\text { Mittal et al. } 1990\end{array}$ \\
\hline & & seeds & Picea excelsa & USSR & $\begin{array}{l}\text { Urosevic 1961, } \\
\text { Anderson 1986a }\end{array}$ \\
\hline
\end{tabular}


Table 2 Continued.

\begin{tabular}{|c|c|c|c|c|c|}
\hline Species & Taxonomy & Substrate & Host & Country & References \\
\hline & & seeds & Pinus sylvestris & UK, USSR & $\begin{array}{l}\text { Prisyazhnyuk 1960, } \\
\text { Urosevic 1961, Whittle } \\
\text { 1977, Anderson 1986a }\end{array}$ \\
\hline & & seeds & $\begin{array}{l}\text { Pseudotsuga } \\
\text { menziesii }\end{array}$ & USA & $\begin{array}{l}\text { Gordon 1967, Mittal et } \\
\text { al. } 1990\end{array}$ \\
\hline & & seeds & Pyrus spp. & India & Sahai \& Otra 1982 \\
\hline & & seeds & Quercus spp. & India & Sahai \& Otra 1982 \\
\hline & & seeds & Sorbus torminalis & $\begin{array}{l}\text { Poland or/ } \\
\text { imported to Poland }\end{array}$ & Czeczuga et al. 2009 \\
\hline Mucor silvaticus Hagem & $\begin{array}{l}\text { Mucoraceae, Mucoromycetes, } \\
\text { Mucoromycota }\end{array}$ & seeds & Rosa mollis & $\begin{array}{l}\text { Poland or/ } \\
\text { imported to Poland }\end{array}$ & Czeczuga et al. 2009 \\
\hline \multirow[t]{16}{*}{ Mucor spp. } & $\begin{array}{l}\text { Mucoraceae, Mucoromycetes, } \\
\text { Mucoromycota }\end{array}$ & acorns & Quercus robur & Poland & Jankowiak 2008 \\
\hline & & cones & Pinus densiflora & Japan & Kasai et al. 1995 \\
\hline & & cones & Pinus sylvestris & UK & Whittle 1977 \\
\hline & & seeds & Abies nordmanniana & Georgia, Russia & Talgø et al. 2010 \\
\hline & & seeds & Abies procera & Norway & Talgø et al. 2010 \\
\hline & & seeds & Acer saccharum & USA & $\begin{array}{l}\text { Janerette 1979, Mittal et } \\
\text { al. } 1990\end{array}$ \\
\hline & & seeds & Albizia lebbeck & India & Natarajan et al. 2003 \\
\hline & & seeds & Abies nordmanniana & Georgia, Russia & Talgø et al. 2010 \\
\hline & & seeds & Bombax anceps & Thailand & $\begin{array}{l}\text { Chalermpongse et al. } \\
\text { 1984, Mittal et al. } 1990\end{array}$ \\
\hline & & seeds & Bombax ceiba & India & Mittal et al. 1990 \\
\hline & & seeds & Cassia fistula & India & $\begin{array}{l}\text { Mittal \& Sharma 1981a, } \\
\text { Mittal et al. } 1990\end{array}$ \\
\hline & & seeds & Cedrus deodara & India & $\begin{array}{l}\text { Mittal 1983, Mittal et al. } \\
1990\end{array}$ \\
\hline & & seeds & $\begin{array}{l}\text { Dalbergia } \\
\text { cochinchinensis }\end{array}$ & Thailand & $\begin{array}{l}\text { Chalermpongse et al. } \\
\text { 1984, Mittal et al. } 1990\end{array}$ \\
\hline & & seeds & Eucalyptus globulus & India & Anderson 1986a \\
\hline & & seeds & Eucalyptus grandis & India & Anderson 1986a \\
\hline & & seeds & Eucalyptus hybrid & India & $\begin{array}{l}\text { Mittal 1986, Mittal et al. } \\
1990\end{array}$ \\
\hline
\end{tabular}


Table 2 Continued.

\begin{tabular}{|c|c|c|c|c|c|}
\hline Species & Taxonomy & Substrate & Host & Country & References \\
\hline & & seeds & $\begin{array}{l}\text { Eucalyptus } \\
\text { tereticornis }\end{array}$ & India & Anderson 1986a \\
\hline & & seeds & Fraxinus americana & USA & $\begin{array}{l}\text { Friedrich et al. } 1971 \text {, } \\
\text { Mittal et al. } 1990\end{array}$ \\
\hline & & seeds & Larix occidentalis & USA & James et al. 1996 \\
\hline & & seeds & $\begin{array}{l}\text { Macroptilium } \\
\text { atropurpureum }\end{array}$ & Australia & Nik \& Parbery 1977 \\
\hline & & seeds & Picea engelmannii & USA & $\begin{array}{l}\text { Fuller \& Hildebrand } \\
\text { 1985, Mittal et al. } 1990\end{array}$ \\
\hline & & seeds & Picea sitchensis & UK & $\begin{array}{l}\text { Salt 1964, Mittal et al. } \\
1990\end{array}$ \\
\hline & & seeds & $\begin{array}{l}\text { Pinus armandii var. } \\
\text { mastersiana }\end{array}$ & Taiwan & $\begin{array}{l}\text { Jong \& Chen } 1966 \text {, } \\
\text { Mittal et al. } 1990\end{array}$ \\
\hline & & seeds & Pinus caribaea & $\begin{array}{l}\text { Belize [as British } \\
\text { Honduras] }\end{array}$ & $\begin{array}{l}\text { Hocking 1968, Mittal et } \\
\text { al. } 1990\end{array}$ \\
\hline & & seeds & Pinus contorta & USA & $\begin{array}{l}\text { Fuller \& Hildebrand } \\
\text { 1985, Mittal et al. } 1990\end{array}$ \\
\hline & & seeds & $\begin{array}{l}\text { Pinus elliottii var. } \\
\text { elliottii }\end{array}$ & Georgia, USA & $\begin{array}{l}\text { Huang \& Kuhlman } \\
\text { 1990, Fraedrich \& Miller } \\
1995\end{array}$ \\
\hline & & seeds & Pinus lambertiana & USA & $\begin{array}{l}\text { Schubert 1961, } \\
\text { Anderson 1986a }\end{array}$ \\
\hline & & seeds & Pinus nigra & Hungary & $\begin{array}{l}\text { Hangyal 1973, Mittal et } \\
\text { al. } 1990\end{array}$ \\
\hline & & seeds & Pinus patula & East Africa & $\begin{array}{l}\text { Gibson 1957, Mittal et } \\
\text { al. } 1990\end{array}$ \\
\hline & & seeds & Pinus ponderosa & USA & $\begin{array}{l}\text { Fuller \& Hildebrand } \\
\text { 1985, Mittal et al. } 1990\end{array}$ \\
\hline & & seeds & Pinus sylvestris & $\begin{array}{l}\text { Hungary, Poland, } \\
\text { UK }\end{array}$ & $\begin{array}{l}\text { Hangyal 1973, Whittle } \\
\text { 1977, Mittal et al. } 1990\end{array}$ \\
\hline & & seeds & Pinus wallichiana & India & $\begin{array}{l}\text { Mittal \& Sharma 1982b, } \\
\text { Mittal et al. } 1990\end{array}$ \\
\hline & & seeds & $\begin{array}{l}\text { Pseudotsuga } \\
\text { menziesii }\end{array}$ & Canada, USA & $\begin{array}{l}\text { Anderson 1986a, James } \\
1984\end{array}$ \\
\hline
\end{tabular}


Table 2 Continued.

\begin{tabular}{|c|c|c|c|c|c|}
\hline Species & Taxonomy & Substrate & Host & Country & References \\
\hline & & seeds & Quercus robur & Germany & Schroder et al. 2004 \\
\hline & & seeds & Quercus spp. & USSR & $\begin{array}{l}\text { Urosevic 1962, Mittal et } \\
\text { al. } 1990\end{array}$ \\
\hline & & seeds & $\begin{array}{l}\text { Swietenia } \\
\text { macrophylla }\end{array}$ & Thailand & $\begin{array}{l}\text { Chalermpongse et al. } \\
\text { 1984, Mittal et al. } 1990\end{array}$ \\
\hline & & seeds & Tectona grandis & India & $\begin{array}{l}\text { Anderson 1986a, } \\
\text { Mohanan et al. } 2005\end{array}$ \\
\hline Mucor spinosus Schrank & $\begin{array}{l}\text { Mucoraceae, Mucoromycetes, } \\
\text { Mucoromycota }\end{array}$ & seed & Linum usitatissimum & Canada & Conners 1967 \\
\hline Mucor strictus Hagem & $\begin{array}{l}\text { Mucoraceae, Mucoromycetes, } \\
\text { Mucoromycota }\end{array}$ & seeds & Sorbus torminalis & $\begin{array}{l}\text { Poland or/ } \\
\text { imported to Poland }\end{array}$ & Czeczuga et al. 2009 \\
\hline $\begin{array}{l}\text { Mundkurella japonica } \\
\text { Denchev \& Kakish." }\end{array}$ & $\begin{array}{l}\text { Urocystidaceae, Ustilaginomycetes, } \\
\text { Basidiomycota }\end{array}$ & fruits & Kalopanax pictus & Japan & $\begin{array}{l}\text { Denchev \& Kakishima } \\
2007\end{array}$ \\
\hline $\begin{array}{l}\text { Muyocopron dipterocarpi } \\
\text { Mapook, Boonmee \& K.D. } \\
\text { Hyde }\end{array}$ & $\begin{array}{l}\text { Muyocopronaceae, Dothideomycetes, } \\
\text { Ascomycota }\end{array}$ & pod & Delonix regia & Thailand & Jayasiri et al. 2019 \\
\hline Muyocopron lithocarpi & Muyocopronaceae, Dothideomycetes, & pod & Cercis chinensis & China, Thailand & Jayasiri et al. 2019 \\
\hline $\begin{array}{l}\text { Mapook, Boonmee \& K.D. } \\
\text { Hyde }\end{array}$ & Ascomycota & pod & Peltophorum sp. & China, Thailand & Jayasiri et al. 2019 \\
\hline 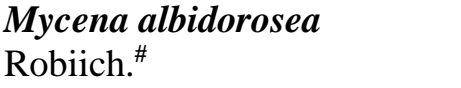 & $\begin{array}{l}\text { Mycenaceae, Agaricomycetes, } \\
\text { Basidiomycota }\end{array}$ & cones & Picea abies & Italy & Robich 2003 \\
\hline $\begin{array}{l}\text { Mycena cupressincola } \\
\text { Robich }^{\#}\end{array}$ & $\begin{array}{l}\text { Mycenaceae, Agaricomycetes, } \\
\text { Basidiomycota }\end{array}$ & cones & Cupressus sp. & Italy & Robich 2016 \\
\hline $\begin{array}{l}\text { Mycena deeptha Aravind. \& } \\
\text { Manim.\# }\end{array}$ & $\begin{array}{l}\text { Mycenaceae, Agaricomycetes, } \\
\text { Basidiomycota }\end{array}$ & fruits & Vateria indica & India & $\begin{array}{l}\text { Aravindakshan et al. } \\
2012\end{array}$ \\
\hline Mycena depilata R. Singer & $\begin{array}{l}\text { Mycenaceae, Agaricomycetes, } \\
\text { Basidiomycota }\end{array}$ & fruit & $\begin{array}{l}\text { dicotyledonous } \\
\text { plant }\end{array}$ & Brazil & Singer 1989 \\
\hline $\begin{array}{l}\text { Mycena detrusa Mass Geest } \\
\text { \& E Horak }{ }^{\#}\end{array}$ & $\begin{array}{l}\text { Mycenaceae, Agaricomycetes, } \\
\text { Basidiomycota }\end{array}$ & cupule & Lithocarpus sp. & $\begin{array}{l}\text { Papua New } \\
\text { Guinea }\end{array}$ & $\begin{array}{l}\text { Geesteranus \& Horak } \\
1995\end{array}$ \\
\hline $\begin{array}{l}\text { Mycena extenuata Mass } \\
\text { Geest. \& de Meijer }{ }^{\#}\end{array}$ & $\begin{array}{l}\text { Mycenaceae, Agaricomycetes, } \\
\text { Basidiomycota }\end{array}$ & fruit & Bignoniaceae plant & Brazil & $\begin{array}{l}\text { Geesteranus \& de } \\
\text { Meijer } 1998\end{array}$ \\
\hline Mycena mirata (Peck) Sacc & $\begin{array}{l}\text { Mycenaceae, Agaricomycetes, } \\
\text { Basidiomycota }\end{array}$ & fruit cupules & Fagus sylvatica & UK & Carré 1964 \\
\hline
\end{tabular}


Table 2 Continued.

\begin{tabular}{|c|c|c|c|c|c|}
\hline Species & Taxonomy & Substrate & Host & Country & References \\
\hline $\begin{array}{l}\text { Mycena plumipes (Kalchbr.) } \\
\text { P.A. Moreau }{ }^{\#} \text { [as Agaricus } \\
\text { plumipes Kalchbr.] }\end{array}$ & $\begin{array}{l}\text { Mycenaceae, Agaricomycetes, } \\
\text { Basidiomycota }\end{array}$ & cones & Pinus sp. & Hungary & Moreau 2003 \\
\hline $\begin{array}{l}\text { Mycocalicium } \\
\text { hyaloparvicellulum } \\
\text { Daranagama \& K.D. Hyde }\end{array}$ & $\begin{array}{l}\text { Mycocaliciaceae, Eurotiomycetes, } \\
\text { Ascomycota }\end{array}$ & cone & Pinus halepensis & Italy & Ariyawansa et al. 2015 \\
\hline $\begin{array}{l}\text { Mycocalicium llimonae } \\
\text { Hladun \& D. Muňiz }\end{array}$ & $\begin{array}{l}\text { Mycocaliciaceae, Eurotiomycetes, } \\
\text { Ascomycota }\end{array}$ & cones & Pinus halepensis & Spain & Muñiz \& Hladun 2007 \\
\hline Mycogone sp. & $\begin{array}{l}\text { Hypocreaceae, Sordariomycetes, } \\
\text { Ascomycota }\end{array}$ & seeds & Nothofagus sp. & UK & Anderson 1986a \\
\hline Mycosphaerella sp. & $\begin{array}{l}\text { Mycosphaerellaceae, Dothideomycetes, } \\
\text { Ascomycota }\end{array}$ & seeds & Eucalyptus grandis & South Africa & Jimu et al. 2016 \\
\hline Mycosphaerellaceae sp. & $\begin{array}{l}\text { Mycosphaerellaceae, Dothideomycetes, } \\
\text { Ascomycota }\end{array}$ & seed capsules & Eucalyptus grandis & South Africa & Jimu et al. 2016 \\
\hline $\begin{array}{l}\text { Mycovellosiella fulva } \\
\text { (Cooke) Arx }\end{array}$ & $\begin{array}{l}\text { Mycosphaerellaceae, Dothideomycetes, } \\
\text { Ascomycota }\end{array}$ & fruit & $\begin{array}{l}\text { Artocarpus } \\
\text { communis }\end{array}$ & Nigeria & Amusa et al. 2002 \\
\hline $\begin{array}{l}\text { Myrothecium mucunae R.F. } \\
\text { Castaneda \& W.B. Kendr" }\end{array}$ & $\begin{array}{l}\text { Stachybotriaceae, Sordariomycetes, } \\
\text { Ascomycota }\end{array}$ & seed & Mucuna urens & Cuba & $\begin{array}{l}\text { Castañeda-Ruíz \& } \\
\text { Kendrick } 1991\end{array}$ \\
\hline \multirow[t]{2}{*}{ Myrothecium spp. } & $\begin{array}{l}\text { Stachybotriaceae, Sordariomycetes, } \\
\text { Ascomycota }\end{array}$ & $\begin{array}{l}\text { pods } \\
\text { pod } \\
\text { seeds }\end{array}$ & $\begin{array}{l}\text { Delonix regia } \\
\text { Sesbania bispinosa } \\
\text { Tectona grandis }\end{array}$ & $\begin{array}{l}\text { Thailand } \\
\text { India } \\
\text { - }\end{array}$ & $\begin{array}{l}\text { Somrithipol et al. 2002b } \\
\text { Anita et al. } 2009 \\
\text { Mathur 1974, Anderson } \\
\text { 1986a }\end{array}$ \\
\hline & & seeds & Tilia americana & USA & $\begin{array}{l}\text { Mathur 1974, Anderson } \\
\text { 1986a }\end{array}$ \\
\hline $\begin{array}{l}\text { Mytilinidion didymospora } \\
\text { Jayasiri, Camporesi \& K.D. } \\
\text { Hyde }^{\#}\end{array}$ & $\begin{array}{l}\text { Mytilinidiaceae, Dothideomycetes, } \\
\text { Ascomycota }\end{array}$ & cones & Cupressus glabra & Italy & Jayasiri et al. 2018a \\
\hline $\begin{array}{l}\text { Mytilinidion scolecosporum } \\
\text { M.L. Lohman }\end{array}$ & $\begin{array}{l}\text { Mytilinidiaceae, Dothideomycetes, } \\
\text { Ascomycota }\end{array}$ & cones, seeds & Pinus spp. & Canada & Vujanovic et al. 2000 \\
\hline $\begin{array}{l}\text { Naemacyclus fimbriatus } \\
\text { (Schwein.) DiCosmo, Peredo } \\
\text { \& Minter }\end{array}$ & $\begin{array}{l}\text { Marthamycetaceae, Leotiomycetes, } \\
\text { Ascomycota }\end{array}$ & cones, seeds & Pinus spp. & Canada & Vujanovic et al. 2000 \\
\hline Naemacyclus minor Butin & $\begin{array}{l}\text { Marthamycetaceae, Leotiomycetes, } \\
\text { Ascomycota }\end{array}$ & seeds & Fraxinus excelsior & Sweden & Hayatgheibi 2013 \\
\hline
\end{tabular}


Table 2 Continued.

\begin{tabular}{|c|c|c|c|c|c|}
\hline Species & Taxonomy & Substrate & Host & Country & References \\
\hline $\begin{array}{l}\text { Nakazawaea ernobii (Lodder } \\
\text { \& Kreger-van Rij) Kurtzman } \\
\text { \& Robnett } \\
\text { [as Candida karawaiewii } \\
\text { Yarrow \& S.A. Mey.] }\end{array}$ & $\begin{array}{l}\text { Incertae sedis, Saccharomycetes, } \\
\text { Ascomycota }\end{array}$ & fruits & $\begin{array}{l}\text { Parahancornia } \\
\text { amapa }\end{array}$ & Brazil & Morais et al. 1995 \\
\hline $\begin{array}{l}\text { Naranus cryptomeriae Ts. } \\
\text { Watan..** }\end{array}$ & $\begin{array}{l}\text { Incertae sedis, Incertae sedis, } \\
\text { Ascomycota }\end{array}$ & seeds & $\begin{array}{l}\text { Cryptomeria } \\
\text { japonica }\end{array}$ & Japan & Watanabe 1995 \\
\hline $\begin{array}{l}\text { Nawawia filiformis } \\
\text { (Nawawi) Marvanová }\end{array}$ & $\begin{array}{l}\text { Chaetosphaeriaceae, Sordariomycetes, } \\
\text { Ascomycota }\end{array}$ & pods & Delonix regia & Thailand & Somrithipol et al. 2002b \\
\hline Nectria gigantospora Zimm. & $\begin{array}{l}\text { Nectriaceae, Sordariomycetes, } \\
\text { Ascomycota }\end{array}$ & fruits & $\begin{array}{l}\text { Didymopanax } \\
\text { morototoni }\end{array}$ & Panama & $\begin{array}{l}\text { Samuels \& Dumon } \\
\text { 1982, Piepenbring } 2006\end{array}$ \\
\hline $\begin{array}{l}\text { Nectria haematococca Berk. } \\
\text { \& Broome }\end{array}$ & $\begin{array}{l}\text { Nectriaceae, Sordariomycetes, } \\
\text { Ascomycota }\end{array}$ & fruit & palm tree & Panama & Samuels \& Dumon 1982 \\
\hline $\begin{array}{l}\text { Nectria radicicola Gerlach \& } \\
\text { L. Nilsson }\end{array}$ & $\begin{array}{l}\text { Nectriaceae, Sordariomycetes, } \\
\text { Ascomycota }\end{array}$ & fruit & $\begin{array}{l}\text { Balanophoraceae } \\
\text { plant }\end{array}$ & Peru & Samuels \& Dumon 1982 \\
\hline Nectria spp. & Nectriaceae, Sordariomycetes, & $\begin{array}{l}\text { fruit } \\
\text { pods }\end{array}$ & $\begin{array}{l}\text { undetermined plant } \\
\text { Delonix regia }\end{array}$ & $\begin{array}{l}\text { Panama } \\
\text { Thailand }\end{array}$ & $\begin{array}{l}\text { Samuels \& Dumon } 1982 \\
\text { Somrithipol et al. 2002b }\end{array}$ \\
\hline & Ascomycota & fruits & Diplospora dubia & Hong Kong & Tang et al. 2003a \\
\hline & & fruits & Psychotria asiatica & Hong Kong & Tang et al. 2003a \\
\hline & & fruits & Sarcandra glabra & Hong Kong & Tang et al. 2003a \\
\hline Nectriaceae sp. & $\begin{array}{l}\text { Nectriaceae, Sordariomycetes, } \\
\text { Ascomycota }\end{array}$ & seeds & Quercus robur & Turkey & Oskay et al. 2018 \\
\hline \multirow{6}{*}{$\begin{array}{l}\text { Neocosmospora solani } \\
\text { (Mart.) L. Lombard \& Crous } \\
\text { [as Fusarium solani (Mart.) } \\
\text { Sacc.] }\end{array}$} & $\begin{array}{l}\text { Nectriaceae, Sordariomycetes, } \\
\text { Ascomycota }\end{array}$ & seeds & $\begin{array}{l}\text { Acacia } \\
\text { auriculiformis }\end{array}$ & Philippines & Quiniones 1987 \\
\hline & & seeds & Agathis dammara & Philippines & $\begin{array}{l}\text { Quiniones 1985, Mittal } \\
\text { et al. } 1990\end{array}$ \\
\hline & & seeds & Albizia lebbeck & Philippines & $\begin{array}{l}\text { Quiniones 1985, 1987, } \\
\text { Mittal et al. } 1990\end{array}$ \\
\hline & & seeds & Albizia procera & Philippines & $\begin{array}{l}\text { Quiniones 1985, 1987, } \\
\text { Mittal et al. } 1990\end{array}$ \\
\hline & & seeds & Albizia stipulata & India & $\begin{array}{l}\text { Mathur 1974, Anderson } \\
\text { 1986a }\end{array}$ \\
\hline & & seeds & Alnus glutinosa & Poland & Krol et al. 2015 \\
\hline
\end{tabular}


Table 2 Continued.

\begin{tabular}{|c|c|c|c|c|c|}
\hline Species & Taxonomy & Substrate & Host & Country & References \\
\hline & & seeds & $\begin{array}{l}\text { Alstonia } \\
\text { macrophylla }\end{array}$ & Philippines & $\begin{array}{l}\text { Quiniones 1985, 1987, } \\
\text { Mittal et al. } 1990\end{array}$ \\
\hline & & seeds & $\begin{array}{l}\text { Araucaria } \\
\text { angustifolia }\end{array}$ & Australia & $\begin{array}{l}\text { Kamara et al. } 1981 \text {, } \\
\text { Mittal et al. } 1990\end{array}$ \\
\hline & & seeds & Araucaria bidwillii & Egypt & $\begin{array}{l}\text { Kamara et al. } 1981 \text {, } \\
\text { Mittal et al. } 1990\end{array}$ \\
\hline & & seeds & $\begin{array}{l}\text { Araucaria } \\
\text { cunninghamii }\end{array}$ & Australia & $\begin{array}{l}\text { Kamara et al. } 1981 \text {, } \\
\text { Mittal et al. } 1990\end{array}$ \\
\hline & & seeds & $\begin{array}{l}\text { Araucaria } \\
\text { heterophylla }\end{array}$ & Egypt & $\begin{array}{l}\text { El-Kady et al. } 1986 \text {, } \\
\text { Mittal et al. } 1990\end{array}$ \\
\hline & & seeds & Azadirachta indica & India & $\begin{array}{l}\text { Mathur 1974, Mittal et } \\
\text { al. } 1990\end{array}$ \\
\hline & & seeds & Bombax ceiba & India & $\begin{array}{l}\text { Mathur 1974, Mittal et } \\
\text { al. } 1990\end{array}$ \\
\hline & & seed & Bromus inermis & Canada & Conners 1967 \\
\hline & & seeds & Calamus ornatus & Philippines & $\begin{array}{l}\text { Quiniones 1985, 1987, } \\
\text { Mittal et al. } 1990\end{array}$ \\
\hline & & seeds & Cassia siamea & Philippines & $\begin{array}{l}\text { Quiniones 1985, 1987, } \\
\text { Mittal et al. } 1990\end{array}$ \\
\hline & & seeds & $\begin{array}{l}\text { Casuarina } \\
\text { equisetifolia }\end{array}$ & Philippines & Quiniones 1987 \\
\hline & & seeds & Cedrela odorata & Colombia & $\begin{array}{l}\text { Mathur 1974, Anderson } \\
\text { 1986a }\end{array}$ \\
\hline & & seeds & $\begin{array}{l}\text { Chamaecyparis } \\
\text { obtusa }\end{array}$ & South Korea & $\begin{array}{l}\text { Mathur 1974, Anderson } \\
\text { 1986a }\end{array}$ \\
\hline & & seeds & Dalbergia sissoo & India, Madagascar & $\begin{array}{l}\text { Mathur 1974, Anderson } \\
\text { 1986a, Kumar } 2014\end{array}$ \\
\hline & & seeds & $\begin{array}{l}\text { Endospermum } \\
\text { peltatum }\end{array}$ & Philippines & $\begin{array}{l}\text { Agmata 1979, Anderson } \\
\text { 1986a }\end{array}$ \\
\hline & & seeds & $\begin{array}{l}\text { Eucalyptus } \\
\text { citriodora }\end{array}$ & India & $\begin{array}{l}\text { Mittal \& Sharma 1984, } \\
\text { Mittal et al. } 1990\end{array}$ \\
\hline & & seeds & Fagus sylvatica & Poland & Krol et al. 2015 \\
\hline
\end{tabular}


Table 2 Continued.

\begin{tabular}{|c|c|c|c|c|c|}
\hline Species & Taxonomy & Substrate & Host & Country & References \\
\hline & & seeds & Gmelina arborea & India, Philippines & $\begin{array}{l}\text { Mathur 1974, Anderson } \\
\text { 1986a, Quiniones } 1987\end{array}$ \\
\hline & & seeds & Grevillea robusta & Rwanda & $\begin{array}{l}\text { Mathur 1974, } \\
\text { Anderson 1986a }\end{array}$ \\
\hline & & seeds & Larix decidua & Poland & Krol et al. 2015 \\
\hline & & seeds & $\begin{array}{l}\text { Leucaena } \\
\text { leucocephala }\end{array}$ & Philippines & $\begin{array}{l}\text { Quiniones 1985, 1987, } \\
\text { Mittal et al. } 1990\end{array}$ \\
\hline & & seeds & Leucaena spp. & Philippines & $\begin{array}{l}\text { Mathur 1974, Anderson } \\
\text { 1986a }\end{array}$ \\
\hline & & seeds & Picea abies & USSR & $\begin{array}{l}\text { Prisyazhnyuk } 1960 \text {, } \\
\text { Mittal et al. } 1990\end{array}$ \\
\hline & & seeds & Picea excelsa & USSR & $\begin{array}{l}\text { Urosevic 1961, } \\
\text { Anderson 1986a }\end{array}$ \\
\hline & & seeds & Picea glauca & USA & James 1985b \\
\hline & & seeds & Picea pungens & USA & James 1985b \\
\hline & & seeds & $\begin{array}{l}\text { Piliostigma } \\
\text { malabaricum }\end{array}$ & Philippines & $\begin{array}{l}\text { Dayan 1986, Mittal et al. } \\
1990\end{array}$ \\
\hline & & seeds & Pinus caribaea & Cuba, UK & $\begin{array}{l}\text { Rees 1982, Anderson } \\
\text { 1986a, Mittal et al. } 1990\end{array}$ \\
\hline & & seeds & $\begin{array}{l}\text { Pinus caribaea var. } \\
\text { caribae }\end{array}$ & Brazil & $\begin{array}{l}\text { Mathur 1974, Anderson } \\
\text { 1986a }\end{array}$ \\
\hline & & seeds & Pinus elliottii & Argentina, USA & $\begin{array}{l}\text { Rowan \& Debarr 1974, } \\
\text { Anderson } 1986 a \text {, Lori \& } \\
\text { Salerno } 2003\end{array}$ \\
\hline & & seeds & $\begin{array}{l}\text { Pinus elliottii var. } \\
\text { elliottii }\end{array}$ & Brazil & $\begin{array}{l}\text { Mathur 1974, Anderson } \\
\text { 1986a }\end{array}$ \\
\hline & & seeds & Pinus merkusii & Philippines & $\begin{array}{l}\text { Agmata 1979, Anderson } \\
\text { 1986a }\end{array}$ \\
\hline & & seeds & Pinus oocarpa & Central America & $\begin{array}{l}\text { Rees 1982, Anderson } \\
\text { 1986a }\end{array}$ \\
\hline & & seeds & Pinus palustris & USA & $\begin{array}{l}\text { Pawuk 1978, Mittal et al. } \\
1990\end{array}$ \\
\hline & & seeds & Pinus ponderosa & USA & James \& Genz 1982 \\
\hline
\end{tabular}


Table 2 Continued.

\begin{tabular}{|c|c|c|c|c|c|}
\hline Species & Taxonomy & Substrate & Host & Country & References \\
\hline & & seeds & Pinus pseudostrobus & UK & $\begin{array}{l}\text { Rees 1982, Mittal et al. } \\
1990\end{array}$ \\
\hline & & seeds & Pinus sylvestris & USSR & $\begin{array}{l}\text { Prisyazhnyuk 1960, } \\
\text { Anderson 1986a }\end{array}$ \\
\hline & & seeds & Pinus taeda & Argentina, USA & $\begin{array}{l}\text { Mathur 1974, Anderson } \\
\text { 1986a, Lori \& Salerno } \\
2003\end{array}$ \\
\hline & & seeds & $\begin{array}{l}\text { Pittosporum } \\
\text { resiniferum }\end{array}$ & Philippines & $\begin{array}{l}\text { Quiniones 1985, } \\
\text { Anderson 1986a, } 1987\end{array}$ \\
\hline & & seeds & Prosopis juliflora & Chile & $\begin{array}{l}\text { Mathur 1974, Mittal et } \\
\text { al. } 1990\end{array}$ \\
\hline & & seed & Prunus serrulata & Japan & Watanabe 2010 \\
\hline & & seeds & $\begin{array}{l}\text { Pseudotsuga } \\
\text { menziesii }\end{array}$ & USA & James 1983b, 1984 \\
\hline & & seeds & Pterocarpus indicus & Philippines & $\begin{array}{l}\text { Agmata 1979, Anderson } \\
\text { 1986a, Quiniones } 1987\end{array}$ \\
\hline & & seeds & $\begin{array}{l}\text { Pterospermum } \\
\text { acerifolium }\end{array}$ & India & $\begin{array}{l}\text { Mathur 1974, Anderson } \\
\text { 1986a }\end{array}$ \\
\hline & & seeds & Quercus alba & USA & $\begin{array}{l}\text { Vozzo 1984, Anderson } \\
\text { 1986a }\end{array}$ \\
\hline & & seeds & Quercus falcata & USA & $\begin{array}{l}\text { Vozzo 1984, Anderson } \\
\text { 1986a }\end{array}$ \\
\hline & & seeds & Quercus nigra & USA & $\begin{array}{l}\text { Vozzo 1984, Anderson } \\
\text { 1986a }\end{array}$ \\
\hline & & seeds & Quercus phellos & USA & $\begin{array}{l}\text { Vozzo 1984, Anderson } \\
\text { 1986a }\end{array}$ \\
\hline & & seeds & Quercus spp. & USSR & $\begin{array}{l}\text { Urosevic 1962, Mittal et } \\
\text { al. } 1990\end{array}$ \\
\hline & & seeds & $\begin{array}{l}\text { Swietenia } \\
\text { macrophylla }\end{array}$ & Philippines & $\begin{array}{l}\text { Agmata 1979, Anderson } \\
\text { 1986a }\end{array}$ \\
\hline & & seeds & $\begin{array}{l}\text { Taxodium } \\
\text { mucronatum }\end{array}$ & India & $\begin{array}{l}\text { Mathur 1974, Anderson } \\
\text { 1986a }\end{array}$ \\
\hline & & seeds & Tectona grandis & Philippines & $\begin{array}{l}\text { Mathur 1974, Anderson } \\
\text { 1986a }\end{array}$ \\
\hline
\end{tabular}


Table 2 Continued.

\begin{tabular}{|c|c|c|c|c|c|}
\hline Species & Taxonomy & Substrate & Host & Country & References \\
\hline & & seeds & Toona calantas & Philippines & Quiniones 1987 \\
\hline & & seeds & Triplaris cumingiana & Philippines & Quiniones 1985, \\
\hline & & & & & Anderson 1986a, 1987 \\
\hline & & seeds & Ulmus davidiana & South Korea & $\begin{array}{l}\text { Mathur 1974, Anderson } \\
\text { 1986a }\end{array}$ \\
\hline & & seeds & $\begin{array}{l}\text { Wallaceodendron } \\
\text { celibcum }\end{array}$ & Philippines & $\begin{array}{l}\text { Quiniones 1985, 1987, } \\
\text { Anderson 1986a }\end{array}$ \\
\hline $\begin{array}{l}\text { Neodeightonia planchoniae } \\
\text { Jayasiri \& K.D. Hyde }\end{array}$ & $\begin{array}{l}\text { Botryosphaeriaceae, Dothideomycetes, } \\
\text { Ascomycota }\end{array}$ & fruit & Planchonia sp. & Thailand & Jayasiri et al. 2019 \\
\hline $\begin{array}{l}\text { Neofusicoccum } \\
\text { eucalyptorum (Crous, H. Sm. } \\
\text { ter \& M.J. Wingf.) Crous, } \\
\text { Slippers \& A.J.L. Phillips }\end{array}$ & $\begin{array}{l}\text { Botryosphaeriaceae, Dothideomycetes, } \\
\text { Ascomycota }\end{array}$ & $\begin{array}{l}\text { seeds, seed } \\
\text { capsules }\end{array}$ & Eucalyptus grandis & South Africa & Jimu et al. 2016 \\
\hline $\begin{array}{l}\text { Neofusicoccum mangiferae } \\
\text { (Syd. \& P. Syd.) Crous, } \\
\text { Slippers \& A.J.L. Phillips } \\
\text { [as Fusicoccum eucalypti } \\
\text { Sousa da Câmara] }\end{array}$ & $\begin{array}{l}\text { Botryosphaeriaceae, Dothideomycetes, } \\
\text { Ascomycota }\end{array}$ & pods & Eucalyptus globulus & Uruguay & Lupo et al. 2001 \\
\hline $\begin{array}{l}\text { Neofusicoccum parvum } \\
\text { (Pennycook \& Samuels) }\end{array}$ & $\begin{array}{l}\text { Botryosphaeriaceae, Dothideomycetes, } \\
\text { Ascomycota }\end{array}$ & fruit & $\begin{array}{l}\text { Magnolia } \\
\text { grandiflora }\end{array}$ & China & Jayasiri et al. 2019 \\
\hline Crous, Slippers \& A.J.L. & & pod & Cercis chinensis & China & Jayasiri et al. 2019 \\
\hline $\begin{array}{l}\text { Phillips } \\
\text { [as Botryosphaeria parva } \\
\text { Pennycook \& Samuels] }\end{array}$ & & seeds & Podocarpus falcatus & Ethiopia & Gure et al. 2005a \\
\hline $\begin{array}{l}\text { Neofusicoccum ribis } \\
\text { (Slippers, Crous \& M.J. } \\
\text { Wingf.) Crous, Slippers \& } \\
\text { A.J.L. Phillips } \\
\text { [as Botryosphaeria ribis } \\
\text { Grossenb. \& Duggar] }\end{array}$ & $\begin{array}{l}\text { Botryosphaeriaceae, Dothideomycetes, } \\
\text { Ascomycota }\end{array}$ & seeds & Pinus caribaea & UK & Mittal et al. 1990 \\
\hline $\begin{array}{l}\text { Neohelicosporium } \\
\text { fusisporum Jayasiri \& K.D. } \\
\text { Hyde }^{\#}\end{array}$ & $\begin{array}{l}\text { Tubeufiaceae, Dothideomycetes, } \\
\text { Ascomycota }\end{array}$ & fruit & Malvaceae plant & Thailand & Jayasiri et al. 2017b \\
\hline
\end{tabular}


Table 2 Continued.

\begin{tabular}{|c|c|c|c|c|c|}
\hline Species & Taxonomy & Substrate & Host & Country & References \\
\hline $\begin{array}{l}\text { Neohelicosporium } \\
\text { hyalosporum Y.Z. Lu, J.C. } \\
\text { Kang \& K.D. Hyde }\end{array}$ & $\begin{array}{l}\text { Tubeufiaceae, Dothideomycetes, } \\
\text { Ascomycota }\end{array}$ & pod & Delonix regia & Thailand & Jayasiri et al. 2019 \\
\hline 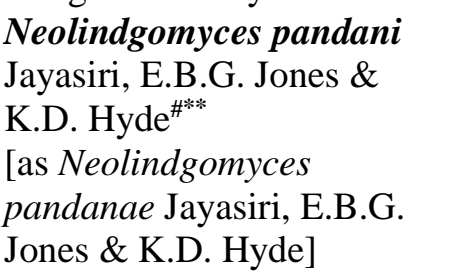 & $\begin{array}{l}\text { Lindgomycetaceae, Dothideomycetes, } \\
\text { Ascomycota }\end{array}$ & fruit & Pandanus sp. & Thailand & Jayasiri et al. 2019 \\
\hline \multirow{4}{*}{$\begin{array}{l}\text { Neonectria didymum } \\
\text { [as Cylindrocarpon didymum } \\
\text { (Harting) Wollenw.] }\end{array}$} & \multirow{4}{*}{$\begin{array}{l}\text { Nectriaceae, Sordariomycetes, } \\
\text { Ascomycota }\end{array}$} & cones & Pinus ponderosa & USA & James 1995 \\
\hline & & cones & $\begin{array}{l}\text { Pseudotsuga } \\
\text { menziesii var. glauca }\end{array}$ & USA & James 1995 \\
\hline & & seeds & Pinus elliottii & South Africa & Cilliers et al. 1995 \\
\hline & & seeds & $\begin{array}{l}\text { Pseudotsuga } \\
\text { menziesii var. glauca }\end{array}$ & USA & James 1995 \\
\hline $\begin{array}{l}\text { Neonectria ramulariae } \\
\text { Wollenw. }\end{array}$ & $\begin{array}{l}\text { Nectriaceae, Sordariomycetes, } \\
\text { Ascomycota }\end{array}$ & seeds & Abies koreana & Korea & Cho et al. 2007 \\
\hline \multirow{5}{*}{$\begin{array}{l}\text { Neonectria spp. } \\
\text { [as Cylindrocarpon] }\end{array}$} & \multirow{5}{*}{$\begin{array}{l}\text { Nectriaceae, Sordariomycetes, } \\
\text { Ascomycota }\end{array}$} & seeds & Abies nordmanniana & Georgia & Talgø et al. 2010 \\
\hline & & seeds & Eucalyptus grandis & South Africa & Jimu et al. 2016 \\
\hline & & seeds & Picea jezoensis & Japan & Cheng \& Igarashi 1987 \\
\hline & & seeds & Picea sitchensis & UK & $\begin{array}{l}\text { Salt 1964, Mittal et al. } \\
1990\end{array}$ \\
\hline & & seeds & Pinus caribaea & $\begin{array}{l}\text { Belize [as British } \\
\text { Honduras] }\end{array}$ & Hocking 1968 \\
\hline $\begin{array}{l}\text { Neopestalotiopsis clavispora } \\
\text { (G.F. Atk.) Maharachch., } \\
\text { K.D. Hyde \& Crous [as } \\
\text { Pestalotia clavispora G.F. } \\
\text { Atk.] }\end{array}$ & $\begin{array}{l}\text { Sporocadaceae, Sordariomycetes, } \\
\text { Ascomycota }\end{array}$ & acorns & Quercus robur & Poland & Jankowiak 2008 \\
\hline $\begin{array}{l}\text { Neopestalotiopsis foedans } \\
\text { (Sacc. \& Ellis) Maharachch., } \\
\text { K.D. Hyde \& Crous } \\
\text { [as Pestalotiopsis foedans } \\
\text { (Sacc. \& Ellis) Steyaert] }\end{array}$ & $\begin{array}{l}\text { Sporocadaceae, Sordariomycetes, } \\
\text { Ascomycota }\end{array}$ & seeds & Pinus oocarpa & Central America & $\begin{array}{l}\text { Mathur 1974, Anderson } \\
\text { 1986a }\end{array}$ \\
\hline
\end{tabular}


Table 2 Continued.

\begin{tabular}{|c|c|c|c|c|c|}
\hline Species & Taxonomy & Substrate & Host & Country & References \\
\hline $\begin{array}{l}\text { Neopyrenochaeta cercidis } \\
\text { Jayasiri, E.B.G. Jones \& } \\
\text { K.D. Hyde }{ }^{\#}\end{array}$ & $\begin{array}{l}\text { Neopyrenochaetaceae, Dothideomycetes, } \\
\text { Ascomycota }\end{array}$ & pod & Cercis chinensis & China & Jayasiri et al. 2019 \\
\hline $\begin{array}{l}\text { Neoroussoella entadae } \\
\text { Jayasiri, E.B.G. Jones \& } \\
\text { K.D. Hyde }\end{array}$ & $\begin{array}{l}\text { Roussoellaceae, Dothideomycetes, } \\
\text { Ascomycota }\end{array}$ & pod & Leucaena sp. & Thailand & Jayasiri et al. 2019 \\
\hline $\begin{array}{l}\text { Neoroussoella leucaenae } \\
\text { Jayasiri, E.B.G. Jones \& } \\
\text { K.D. Hyde }^{\#}\end{array}$ & $\begin{array}{l}\text { Roussoellaceae, Dothideomycetes, } \\
\text { Ascomycota }\end{array}$ & pod & Leucaena sp. & Thailand & Jayasiri et al. 2019 \\
\hline $\begin{array}{l}\text { Neosartorya delicata H.Z. } \\
\text { Kong }^{\#}\end{array}$ & $\begin{array}{l}\text { Aspergillaceae, Eurotiomycetes, } \\
\text { Ascomycota }\end{array}$ & fruit & undetermined plant & China & Kong 1997 \\
\hline $\begin{array}{l}\text { Neoscytalidium dimidiatum } \\
\text { (Penz.) Crous \& Slippers } \\
\text { [as Hendersonula toruloidea } \\
\text { Nattrass] }\end{array}$ & $\begin{array}{l}\text { Botryosphaeriaceae, Dothideomycetes, } \\
\text { Ascomycota }\end{array}$ & fruits & Ilex pubescens & Hong Kong & Tang et al. 2003a \\
\hline $\begin{array}{l}\text { Neosetophoma lunariae } \\
\text { Crous \& R.K. Schumach.\# }\end{array}$ & $\begin{array}{l}\text { Phaeosphaeriaceae, Dothideomycetes, } \\
\text { Ascomycota }\end{array}$ & seeds & Lunaria annua & Germany & $\begin{array}{l}\text { Hernandez-Restrepo et } \\
\text { al. } 2016\end{array}$ \\
\hline $\begin{array}{l}\text { Neotyphodium huerfanum } \\
\text { (J.F. White, G.T. Cole \& } \\
\text { Morgan-Jones) Glenn, C.W. } \\
\text { Bacon \& Hanlin }{ }^{\#} \\
\text { [as Acremonium huerfanum } \\
\text { J.F. White, G.T. Cole \& } \\
\text { Morgan-Jones] }\end{array}$ & $\begin{array}{l}\text { Clavicipitaceae, Sordariomycetes, } \\
\text { Ascomycota }\end{array}$ & seeds & Festuca arizonica & USA & White et al. 1987 \\
\hline $\begin{array}{l}\text { Neurospora cerealis } \\
\text { (Dowding) Dania García, } \\
\text { Stchigel \& Guarro }\end{array}$ & $\begin{array}{l}\text { Sordariaceae, Sordariomycetes, } \\
\text { Ascomycota }\end{array}$ & seed & Linum usitatissimum & Canada & Conners 1967 \\
\hline $\begin{array}{l}\text { [as Gelasinospora cerealis } \\
\text { Dowding] }\end{array}$ & & seed & Stylosanthes humilis & Australia & Nik \& Parbery 1977 \\
\hline Neurospora sp. & $\begin{array}{l}\text { Sordariaceae, Sordariomycetes, } \\
\text { Ascomycota }\end{array}$ & seeds & Fraxinus excelsior & Sweden & Hayatgheibi 2013 \\
\hline
\end{tabular}


Table 2 Continued.

\begin{tabular}{|c|c|c|c|c|c|}
\hline Species & Taxonomy & Substrate & Host & Country & References \\
\hline $\begin{array}{l}\text { Neurospora tetraspora Dania } \\
\text { García, Stchigel \& Guarro } \\
\text { [as Gelasinospora } \\
\text { tetrasperma Dowding] }\end{array}$ & $\begin{array}{l}\text { Sordariaceae, Sordariomycetes, } \\
\text { Ascomycota }\end{array}$ & seed & Festuca rubra & Canada & Conners 1967 \\
\hline $\begin{array}{l}\text { Niesslia pusilla (Speg. \& } \\
\text { Roum.) G. Winter }\end{array}$ & $\begin{array}{l}\text { Niessliaceae, Sordariomycetes, } \\
\text { Ascomycota }\end{array}$ & fruit cupules & Fagus sylvatica & UK & Carré 1964 \\
\hline Nigrospora gossypii Jacz. & $\begin{array}{l}\text { Apiosporaceae, Sordariomycetes, } \\
\text { Ascomycota }\end{array}$ & seeds & $\begin{array}{l}\text { Quercus } \\
\text { castaneifolia }\end{array}$ & Iran & Kavosi et al. 2013 \\
\hline Nigrospora oryzae (Berk. \& & Apiosporaceae, Sordariomycetes, & seed & Bromus inermis & Canada & Conners 1967 \\
\hline \multirow[t]{6}{*}{ Broome) Petch } & Ascomycota & seed & Cercis chinensis & Japan & Watanabe 2010 \\
\hline & & seeds & Eucalyptus globulus & Uruguay & Lupo et al. 2001 \\
\hline & & seed & Festuca elatior & Canada & Conners 1967 \\
\hline & & seed & Pinus thunbergii & Japan & Watanabe 2010 \\
\hline & & seed & Prunus serrulata & Japan & Watanabe 2010 \\
\hline & & seeds & Quercus spp. & USSR & Mittal et al. 1990 \\
\hline Nigrospora sacchari (Speg.) & Apiosporaceae, Sordariomycetes, & pods & Eucalyptus globulus & Uruguay & Lupo et al. 2001 \\
\hline E.W. Mason & Ascomycota & seed & Pinus thunbergii & Japan & Watanabe 2010 \\
\hline Nigrospora sphaerica & Apiosporaceae, Sordariomycetes, & pods & Eucalyptus globulus & Uruguay & Lupo et al. 2001 \\
\hline \multirow[t]{6}{*}{ (Sacc.) E.W. Mason } & Ascomycota & seed & Agropyron cristatum & Canada & Conners 1967 \\
\hline & & seed & Elymus virginicus & Canada & Conners 1967 \\
\hline & & seeds & Eucalyptus globulus & Uruguay & Lupo et al. 2001 \\
\hline & & seeds & Helichrysum sp. & Pakistan & Lodhi \& Naeem 1955 \\
\hline & & seed & Poa compressa & Canada & Conners 1967 \\
\hline & & seed & Setaria viridis & Canada & Conners 1967 \\
\hline \multirow[t]{5}{*}{ Nigrospora spp. } & Apiosporaceae, Sordariomycetes, & fruit cupules & Fagus crenata & Japan & Fukasawa et al. 2012 \\
\hline & Ascomycota & pod & Canavalia cathartica & India & Anita \& Sridhar 2009 \\
\hline & & $\begin{array}{l}\text { seeds, seed } \\
\text { capsules }\end{array}$ & Eucalyptus grandis & South Africa & Jimu et al. 2016 \\
\hline & & seeds & Cupressus spp. & Syria & $\begin{array}{l}\text { Mathur 1974, Anderson } \\
\text { 1986a }\end{array}$ \\
\hline & & seeds & $\begin{array}{l}\text { Pinus elliottii var. } \\
\text { elliottii }\end{array}$ & Georgia, USA & $\begin{array}{l}\text { Huang \& Kuhlman } \\
\text { 1990, Fraedrich \& Miller } \\
1995\end{array}$ \\
\hline
\end{tabular}


Table 2 Continued.

\begin{tabular}{|c|c|c|c|c|c|}
\hline Species & Taxonomy & Substrate & Host & Country & References \\
\hline & & seeds & Pinus taeda & USA & $\begin{array}{l}\text { Covington et al. 1982, } \\
\text { Anderson 1986a }\end{array}$ \\
\hline & & seeds & $\begin{array}{l}\text { Platanus } \\
\text { occidentalis }\end{array}$ & USA & $\begin{array}{l}\text { Fakir et al. 1971, Mittal } \\
\text { et al. } 1990\end{array}$ \\
\hline & & seeds & Pterocarpus indicus & Philippines & $\begin{array}{l}\text { Agmata 1979, Anderson } \\
\text { 1986a }\end{array}$ \\
\hline & & seed & Sesbania bispinosa & India & Anita et al. 2009 \\
\hline & & seeds & $\begin{array}{l}\text { Swietenia } \\
\text { macrophylla }\end{array}$ & Philippines & $\begin{array}{l}\text { Agmata 1979, Anderson } \\
\text { 1986a }\end{array}$ \\
\hline $\begin{array}{l}\text { Nothophoma quercina (Syd. } \\
\text { \& P. Syd.) Qian Chen \& L. } \\
\text { Cai }\end{array}$ & $\begin{array}{l}\text { Didymellaceae, Dothideomycetes, } \\
\text { Ascomycota }\end{array}$ & cone & Keteleeria fortune & China & Jayasiri et al. 2019 \\
\hline \multirow[t]{10}{*}{$\begin{array}{l}\text { Nowakowskiella elegans } \\
\text { (Nowak.) J. Schröt. }\end{array}$} & $\begin{array}{l}\text { Nowakowskiellaceae, Chytridiomycetes, } \\
\text { Chytridiomycota }\end{array}$ & seeds & $\begin{array}{l}\text { Armeria maritima } \\
\text { ssp. maritima }\end{array}$ & $\begin{array}{l}\text { Poland or/ } \\
\text { imported to Poland }\end{array}$ & Czeczuga et al. 2009 \\
\hline & & seeds & $\begin{array}{l}\text { Armeria } \\
\text { transmontana }\end{array}$ & $\begin{array}{l}\text { Poland or/ } \\
\text { imported to Poland }\end{array}$ & Czeczuga et al. 2009 \\
\hline & & seeds & Atropa belladonna & $\begin{array}{l}\text { Poland or/ } \\
\text { imported to Poland }\end{array}$ & Czeczuga et al. 2009 \\
\hline & & seeds & Cicuta virosa & $\begin{array}{l}\text { Poland or/ } \\
\text { imported to Poland }\end{array}$ & Czeczuga et al. 2009 \\
\hline & & seeds & Cornus capitata & $\begin{array}{l}\text { Poland or/ } \\
\text { imported to Poland }\end{array}$ & Czeczuga et al. 2009 \\
\hline & & seeds & Cuscuta europaea & $\begin{array}{l}\text { Poland or/ } \\
\text { imported to Poland }\end{array}$ & Czeczuga et al. 2009 \\
\hline & & seeds & Ficus pumila & $\begin{array}{l}\text { Poland or/ } \\
\text { imported to Poland }\end{array}$ & Czeczuga et al. 2009 \\
\hline & & seeds & Lotus uliginosa & $\begin{array}{l}\text { Poland or/ } \\
\text { imported to Poland }\end{array}$ & Czeczuga et al. 2009 \\
\hline & & seeds & $\begin{array}{l}\text { Prunella grandiflora } \\
\text { ssp. pyrenaia }\end{array}$ & $\begin{array}{l}\text { Poland or/ } \\
\text { imported to Poland }\end{array}$ & Czeczuga et al. 2009 \\
\hline & & seeds & Sambucus ebulus & $\begin{array}{l}\text { Poland or/ } \\
\text { imported to Poland }\end{array}$ & Czeczuga et al. 2009 \\
\hline
\end{tabular}


Table 2 Continued.

\begin{tabular}{|c|c|c|c|c|c|}
\hline Species & Taxonomy & Substrate & Host & Country & References \\
\hline & & seeds & Ulex europaeus & $\begin{array}{l}\text { Poland or/ } \\
\text { imported to Poland }\end{array}$ & Czeczuga et al. 2009 \\
\hline & & seeds & Vicia sativa & Poland & Kiziewicz 2005 \\
\hline $\begin{array}{l}\text { Nowakowskiella } \\
\text { hemisphaerospora Shanor }\end{array}$ & $\begin{array}{l}\text { Nowakowskiellaceae, Chytridiomycetes, } \\
\text { Chytridiomycota }\end{array}$ & seeds & $\begin{array}{l}\text { Armeria maritima } \\
\text { ssp. maritima }\end{array}$ & $\begin{array}{l}\text { Poland or/ } \\
\text { imported to Poland }\end{array}$ & Czeczuga et al. 2009 \\
\hline $\begin{array}{l}\text { Nowakowskiella macrospora } \\
\text { Karling }\end{array}$ & $\begin{array}{l}\text { Nowakowskiellaceae, Chytridiomycetes, } \\
\text { Chytridiomycota }\end{array}$ & seeds & Ficus pumila & $\begin{array}{l}\text { Poland or/ } \\
\text { imported to Poland }\end{array}$ & Czeczuga et al. 2009 \\
\hline $\begin{array}{l}\text { Nowakowskiella profusa } \\
\text { Karling }\end{array}$ & $\begin{array}{l}\text { Nowakowskiellaceae, Chytridiomycetes, } \\
\text { Chytridiomycota }\end{array}$ & seeds & Ficus pumila & $\begin{array}{l}\text { Poland or/ } \\
\text { imported to Poland }\end{array}$ & Czeczuga et al. 2009 \\
\hline \multirow[t]{2}{*}{$\begin{array}{l}\text { Nowakowskiella ramosa E.J. } \\
\text { Butler }\end{array}$} & $\begin{array}{l}\text { Nowakowskiellaceae, Chytridiomycetes, } \\
\text { Chytridiomycota }\end{array}$ & seeds & Crataegus azarolus & $\begin{array}{l}\text { Poland or/ } \\
\text { imported to Poland }\end{array}$ & Czeczuga et al. 2009 \\
\hline & & seeds & Ulex europaeus & $\begin{array}{l}\text { Poland or/ } \\
\text { imported to Poland }\end{array}$ & Czeczuga et al. 2009 \\
\hline $\begin{array}{l}\text { Ochroconis ailanthi Jayasiri, } \\
\text { E.B.G. Jones \& K.D. Hyde }{ }^{\#}\end{array}$ & $\begin{array}{l}\text { Sympoventuriaceae, Dothideomycetes, } \\
\text { Ascomycota }\end{array}$ & pod & Ailanthus sp. & Thailand & Jayasiri et al. 2019 \\
\hline \multirow[t]{4}{*}{$\begin{array}{l}\text { Oedocephalum } \\
\text { glomerulosum (Bull.) Sacc. }\end{array}$} & $\begin{array}{l}\text { Incertae sedis, Pezizomycetes, } \\
\text { Ascomycota }\end{array}$ & seeds & Cedrus deodara & India & $\begin{array}{l}\text { Munjal \& Sharma 1975, } \\
\text { Mittal et al. } 1990\end{array}$ \\
\hline & & seeds & Pinus roxburghii & India & $\begin{array}{l}\text { Munjal \& Sharma 1975, } \\
\text { Mittal et al. } 1990\end{array}$ \\
\hline & & seeds & Pinus sylvestris & Poland & $\begin{array}{l}\text { Garbowski 1936, Mittal } \\
\text { et al. } 1990\end{array}$ \\
\hline & & seeds & Quercus spp. & USSR & Mittal et al. 1990 \\
\hline Oedocephalum lineatum & Incertae sedis, Pezizomycetes, & seeds & Cedrus deodara & India & Munjal \& Sharma 1975 \\
\hline B.K. Bakshi & Ascomycota & seeds & Pinus roxburghii & India & $\begin{array}{l}\text { Mittal \& Sharma 1982c, } \\
\text { Mittal et al. } 1990\end{array}$ \\
\hline \multirow[t]{3}{*}{ Oedocephalum spp. } & $\begin{array}{l}\text { Incertae sedis, Pezizomycetes, } \\
\text { Ascomycota }\end{array}$ & seeds & $\begin{array}{l}\text { Pinus elliottii var. } \\
\text { elliottii }\end{array}$ & Uruguay & Anderson 1986a \\
\hline & & seeds & Pinus wallichiana & India & $\begin{array}{l}\text { Munjal \& Sharma 1975, } \\
\text { Mittal et al. } 1990\end{array}$ \\
\hline & & seeds & Tectona grandis & India & Anderson 1986a \\
\hline Oidiodendron spp. & $\begin{array}{l}\text { Myxotrichaceae, Leotiomycetes, } \\
\text { Ascomycota }\end{array}$ & seed & Prunus serrulata & Japan & Watanabe 2010 \\
\hline $\begin{array}{l}\text { Oidiodendron cereale } \\
\text { (Thüm.) G.L. Barron }\end{array}$ & $\begin{array}{l}\text { Myxotrichaceae, Leotiomycetes, } \\
\text { Ascomycota }\end{array}$ & seed & Pinus densiflora & Japan & Watanabe 2010 \\
\hline
\end{tabular}


Table 2 Continued.

\begin{tabular}{|c|c|c|c|c|c|}
\hline Species & Taxonomy & Substrate & Host & Country & References \\
\hline & & seed & Pinus thunbergii & Japan & Watanabe 2010 \\
\hline \multirow{3}{*}{ Oidiodendron flavum Svilv. } & \multirow{3}{*}{$\begin{array}{l}\text { Myxotrichaceae, Leotiomycetes, } \\
\text { Ascomycota }\end{array}$} & seed & Pinus densiflora & Japan & Watanabe 2010 \\
\hline & & seed & Pinus thunbergii & Japan & Watanabe 2010 \\
\hline & & seed & Prunus serrulata & Japan & Watanabe 2010 \\
\hline $\begin{array}{l}\text { Oidiodendron griseum } \\
\text { Robak }\end{array}$ & $\begin{array}{l}\text { Myxotrichaceae, Leotiomycetes, } \\
\text { Ascomycota }\end{array}$ & seeds & Quercus spp. & USSR & $\begin{array}{l}\text { Urosevic 1962, Mittal et } \\
\text { al. } 1990\end{array}$ \\
\hline Oidium sp. & $\begin{array}{l}\text { Erysiphaceae, Leotiomycetes, } \\
\text { Ascomycota }\end{array}$ & seeds & Pinus taeda & USA & Anderson 1986a, b \\
\hline $\begin{array}{l}\text { Olpidium gregarium } \\
\text { (Nowak.) J. Schröt. }\end{array}$ & $\begin{array}{l}\text { Olpidiaceae, Chytridiomycetes, } \\
\text { Chytridiomycota }\end{array}$ & seeds & Calla palustris & $\begin{array}{l}\text { Poland or/ } \\
\text { imported to Poland }\end{array}$ & Czeczuga et al. 2009 \\
\hline Olpitrichum sp. & $\begin{array}{l}\text { Incertae sedis, Incertae sedis, } \\
\text { Ascomycota }\end{array}$ & seeds & Pinus taeda & USA & Anderson 1986a, b \\
\hline $\begin{array}{l}\text { Ombrophila strobilina (Fr.) } \\
\text { P. Karst. }\end{array}$ & $\begin{array}{l}\text { Gelatinodiscaceae, Leotiomycetes, } \\
\text { Ascomycota }\end{array}$ & seeds & Picea abies & USSR & $\begin{array}{l}\text { Prisyazhnyuk 1960, } \\
\text { Mittal et al. } 1990\end{array}$ \\
\hline $\begin{array}{l}\text { Oospora glauca (Preuss) } \\
\text { Sacc. }\end{array}$ & $\begin{array}{l}\text { Erysiphaceae, Leotiomycetes, } \\
\text { Ascomycota }\end{array}$ & seeds & Quercus spp. & USSR & $\begin{array}{l}\text { Urosevic 1962, Mittal et } \\
\text { al. } 1990\end{array}$ \\
\hline \multirow[t]{4}{*}{$\begin{array}{l}\text { Oospora lactis (Fresen.) } \\
\text { Sacc. }\end{array}$} & $\begin{array}{l}\text { Erysiphaceae, Leotiomycetes, } \\
\text { Ascomycota }\end{array}$ & seed & $\begin{array}{l}\text { Apium graveolens } \\
\text { var. dulce }\end{array}$ & USA & Conners 1967 \\
\hline & & seed & $\begin{array}{l}\text { Asparagus officinalis } \\
\text { var. altilis }\end{array}$ & Canada & Conners 1967 \\
\hline & & seed & Linum usitatissimum & Canada & Conners 1967 \\
\hline & & seed & Phleum pratense & Canada & Conners 1967 \\
\hline \multirow[t]{2}{*}{ Oospora verticillioides Sacc. } & $\begin{array}{l}\text { Erysiphaceae, Leotiomycetes, } \\
\text { Ascomycota }\end{array}$ & seeds & Picea excelsa & USSR & $\begin{array}{l}\text { Urosevic 1961, } \\
\text { Anderson 1986a }\end{array}$ \\
\hline & & seeds & Pinus sylvestris & USSR & $\begin{array}{l}\text { Urosevic 1961, } \\
\text { Anderson 1986a }\end{array}$ \\
\hline $\begin{array}{l}\text { Ophiostoma conicola Marm. } \\
\text { \& Butin }{ }^{\#}\end{array}$ & $\begin{array}{l}\text { Ophiostomataceae, Sordariomycetes, } \\
\text { Ascomycota }\end{array}$ & cones & Pinus cembroides & Mexico & $\begin{array}{l}\text { Marmolejo \& Butin } \\
1990\end{array}$ \\
\hline \multirow[t]{3}{*}{ Ophiostoma spp. } & $\begin{array}{l}\text { Ophiostomataceae, Sordariomycetes, } \\
\text { Ascomycota }\end{array}$ & seeds & Picea excelsa & USSR & $\begin{array}{l}\text { Urosevic 1961, } \\
\text { Anderson 1986a }\end{array}$ \\
\hline & & seeds & Pinus sylvestris & USSR & $\begin{array}{l}\text { Urosevic 1961, } \\
\text { Anderson 1986a }\end{array}$ \\
\hline & & seeds & Quercus spp. & USSR & $\begin{array}{l}\text { Urosevic 1962, 1983, } \\
\text { Mittal et al. } 1990\end{array}$ \\
\hline
\end{tabular}


Table 2 Continued.

\begin{tabular}{|c|c|c|c|c|c|}
\hline Species & Taxonomy & Substrate & Host & Country & References \\
\hline $\begin{array}{l}\text { Ophiostoma valachicum } \\
\text { Georgescu, Teodoru \& Badea }\end{array}$ & $\begin{array}{l}\text { Ophiostomataceae, Sordariomycetes, } \\
\text { Ascomycota }\end{array}$ & seeds & Quercus spp. & USSR & $\begin{array}{l}\text { Urosevic 1964, Mittal et } \\
\text { al. } 1990\end{array}$ \\
\hline \multirow{2}{*}{$\begin{array}{l}\text { Ovatospora brasiliensis (Bat. } \\
\text { \& Pontual) X.Wei Wang \& } \\
\text { Samson [as Chaetomium } \\
\text { brasiliense Bat. \& Pontual] }\end{array}$} & $\begin{array}{l}\text { Chaetomiaceae, Sordariomycetes, } \\
\text { Ascomycota }\end{array}$ & seed & Desmodium intortum & Australia & Nik \& Parbery 1977 \\
\hline & & seed & $\begin{array}{l}\text { Stylosanthes } \\
\text { guianensis }\end{array}$ & Australia & Nik \& Parbery 1977 \\
\hline $\begin{array}{l}\text { Paecilomyces divaricatus } \\
\text { (Thom) Samson, Houbraken } \\
\text { \& Frisvad[as Spicaria } \\
\text { divaricata (Thom) J.C. } \\
\text { Gilman \& E.V. Abbott] }\end{array}$ & $\begin{array}{l}\text { Thermoascaceae, Eurotiomycetes, } \\
\text { Ascomycota }\end{array}$ & seeds & Pinus sylvestris & USSR & $\begin{array}{l}\text { Dolejs 1964, Mittal et al. } \\
1990\end{array}$ \\
\hline $\begin{array}{l}\text { Paecilomyces puntonii } \\
\text { (Vuill.) Nann. }\end{array}$ & $\begin{array}{l}\text { Thermoascaceae, Eurotiomycetes, } \\
\text { Ascomycota }\end{array}$ & seed & $\begin{array}{l}\text { Cryptomeria } \\
\text { japonica }\end{array}$ & Japan & Watanabe 2010 \\
\hline \multirow[t]{2}{*}{$\begin{array}{l}\text { Paecilomyces roseolus G. } \\
\text { Sm. }\end{array}$} & $\begin{array}{l}\text { Thermoascaceae, Eurotiomycetes, } \\
\text { Ascomycota }\end{array}$ & seed & $\begin{array}{l}\text { Cryptomeria } \\
\text { japonica }\end{array}$ & Japan & Watanabe 2010 \\
\hline & & seed & Pinus densiflora & Japan & Watanabe 2010 \\
\hline \multirow[t]{11}{*}{ Paecilomyces spp. } & $\begin{array}{l}\text { Thermoascaceae, Eurotiomycetes, } \\
\text { Ascomycota }\end{array}$ & seeds & Acer saccharum & USA & $\begin{array}{l}\text { Friedrich et al. } 1971 \text {, } \\
\text { Mittal et al. } 1990\end{array}$ \\
\hline & & seeds & Albizia lebbeck & India & Mohanan et al. 2005 \\
\hline & & seeds & Dalbergia sissoides & India & Mohanan et al. 2005 \\
\hline & & seeds & Delonix regia & India & Sahu et al. 2003 \\
\hline & & seeds & Eucalyptus alba & Thailand & $\begin{array}{l}\text { Chalermpongse et al. } \\
\text { 1984, Mittal et al. } 1990\end{array}$ \\
\hline & & seeds & $\begin{array}{l}\text { Eucalyptus } \\
\text { citriodora }\end{array}$ & India & Anderson 1986a \\
\hline & & seeds & Eucalyptus deglupta & Thailand & $\begin{array}{l}\text { Chalermpongse et al. } \\
\text { 1984, Mittal et al. } 1990\end{array}$ \\
\hline & & seeds & Eucalyptus globulus & India & Anderson 1986a \\
\hline & & seeds & Linum usitatissimum & Canada & Conners 1967 \\
\hline & & seeds & Picea excelsa & USSR & $\begin{array}{l}\text { Urosevic 1961, } \\
\text { Anderson 1986a }\end{array}$ \\
\hline & & seeds & Picea glauca & Canada & Mittal \& Wang 1987 \\
\hline
\end{tabular}


Table 2 Continued.

\begin{tabular}{|c|c|c|c|c|c|}
\hline Species & Taxonomy & Substrate & Host & Country & References \\
\hline & & seeds & Pinus contorta & USA & $\begin{array}{l}\text { Fuller \& Hildebrand } \\
\text { 1985, Mittal et al. } 1990\end{array}$ \\
\hline \multirow[t]{6}{*}{ Paecilomyces variotii Bainier } & \multirow[t]{6}{*}{$\begin{array}{l}\text { Thermoascaceae, Eurotiomycetes, } \\
\text { Ascomycota }\end{array}$} & seed & Pinus merkusii & Thailand & $\begin{array}{l}\text { Chalermpongse et al. } \\
\text { 1984, Mittal et al. } 1990\end{array}$ \\
\hline & & seeds & Pinus sylvestris & USSR & $\begin{array}{l}\text { Urosevic 1961, } \\
\text { Anderson 1986a }\end{array}$ \\
\hline & & seeds & Pyrus spp. & India & Sahai \& Otra 1982 \\
\hline & & seeds & Quercus spp. & USSR & $\begin{array}{l}\text { Urosevic 1962, Mittal et } \\
\text { al. } 1990\end{array}$ \\
\hline & & seeds & Tectona grandis & India & Mohanan et al. 2005 \\
\hline & & seeds & Tectona grandis & India & Anderson 1986a \\
\hline $\begin{array}{l}\text { Palaeodiplodites yezoensis } \\
\text { K. Watan., H. Nishida \& } \\
\text { Tak. Kobay }^{\# * *}\end{array}$ & Fossil fungus & cone & $\begin{array}{l}\text { Cycadeoidella } \\
\text { japonica }\end{array}$ & Japan & Watanabe et al. 1999 \\
\hline $\begin{array}{l}\text { Palaeopericonia fritzschei } \\
\text { C.G. Ibáñez \& Zamuner }{ }^{\# * *}\end{array}$ & Fossil fungus & fossilised cones & Araucaria mirabilis & USA & $\begin{array}{l}\text { Ibáñez \& Zamuner } \\
1996\end{array}$ \\
\hline $\begin{array}{l}\text { Papiliotrema laurentii } \\
\text { (Kuff.) Xin Zhan Liu, F.Y. } \\
\text { Bai, M. Groenew. \& } \\
\text { Boekhout [as Cryptococcus } \\
\text { laurentii (Kuff.) C.E. } \\
\text { Skinner] }\end{array}$ & $\begin{array}{l}\text { Rhynchogastremaceae, Tremellomycetes, } \\
\text { Basidiomycota }\end{array}$ & seeds & Fraxinus excelsior & Sweden & Hayatgheibi 2013 \\
\hline \multirow{8}{*}{$\begin{array}{l}\text { Papularia arundinis (Corda) } \\
\text { Fr. }\end{array}$} & \multirow{8}{*}{$\begin{array}{l}\text { Apiosporaceae, Sordariomycetes, } \\
\text { Ascomycota }\end{array}$} & seed & Acer sp. & China & Conners 1967 \\
\hline & & seed & Agropyron cristatum & Canada & Conners 1967 \\
\hline & & seed & Elymus virginicus & Canada & Conners 1967 \\
\hline & & seed & Festuca rubra & Canada & Conners 1967 \\
\hline & & seed & Linum usitatissimum & Canada & Conners 1967 \\
\hline & & seed & Phleum pratense & Canada & Conners 1967 \\
\hline & & seed & Poa compressa & Canada & Conners 1967 \\
\hline & & seed & Setaria viridis & Canada & Conners 1967 \\
\hline $\begin{array}{l}\text { Papularia sphaerosperma } \\
\text { (Pers.) Höhn. }\end{array}$ & $\begin{array}{l}\text { Apiosporaceae, Sordariomycetes, } \\
\text { Ascomycota }\end{array}$ & seed & Lolium perenne & Canada & Conners 1967 \\
\hline Papulaspora spp. & $\begin{array}{l}\text { Incertae sedis, Sordariomycetes, } \\
\text { Ascomycota }\end{array}$ & acorns & Quercus robur & Poland & Jankowiak 2008 \\
\hline
\end{tabular}


Table 2 Continued.

\begin{tabular}{|c|c|c|c|c|c|}
\hline Species & Taxonomy & Substrate & Host & Country & References \\
\hline & & seeds & Araucaria bidwillii & Egypt & $\begin{array}{l}\text { Kamara et al. } 1981 \text {, } \\
\text { Mittal et al. } 1990\end{array}$ \\
\hline & & seeds & Manilkara bidentata & USA & Bayman et al. 1998 \\
\hline & & seeds & Picea glauca & Canada & Mittal \& Wang 1987 \\
\hline & & seeds & Picea jezoensis & Japan & Cheng \& Igarashi 1987 \\
\hline & & seeds & Pinus taeda & USA & Anderson 1986a, b \\
\hline & & seeds & $\begin{array}{l}\text { Pseudotsuga } \\
\text { menziesii }\end{array}$ & USA & $\begin{array}{l}\text { Gordon 1967, Mittal et } \\
\text { al. } 1990\end{array}$ \\
\hline & & seeds & Quercus spp. & USSR & $\begin{array}{l}\text { Urosevic 1959, Mittal et } \\
\text { al. } 1990\end{array}$ \\
\hline $\begin{array}{l}\text { Paraconiothyrium } \\
\text { archidendri Verkley, Göker } \\
\text { \& Stielow } \\
\text { [as Austropleospora } \\
\text { archidendri (Verkley, Göker } \\
\text { \& Stielow) Ariyaw. \& K.D. } \\
\text { Hyde] }\end{array}$ & $\begin{array}{l}\text { Didymosphaeriaceae, Dothideomycetes, } \\
\text { Ascomycota }\end{array}$ & pod & Leucaena sp. & Thailand & Jayasiri et al. 2019 \\
\hline Paraconiothyrium fuckelii & Didymosphaeriaceae, Dothideomycetes, & seeds & Acer pseudoplatanus & Poland & Krol et al. 2015 \\
\hline (Sacc.) Verkley \& Gruyter & Ascomycota & seeds & Cercis chinensis & Japan & Watanabe 2010 \\
\hline [as Coniothyrium fuckelii & & seeds & Fagus sylvatica & Poland & Krol et al. 2015 \\
\hline Sacc.] & & seeds & Fraxinus excelsior & Sweden & Hayatgheibi 2013 \\
\hline & & seeds & Picea abies & Poland & Krol et al. 2015 \\
\hline & & seed & Pinus sylvestris & Poland & Krol et al. 2015 \\
\hline $\begin{array}{l}\text { Parascedosporium } \\
\text { putredinis (Corda) Lackner } \\
\text { \& de Hoog }\end{array}$ & $\begin{array}{l}\text { Microascaceae, Sordariomycetes, } \\
\text { Ascomycota }\end{array}$ & pod & Delonix regia & Thailand & Perera et al. 2018b \\
\hline \multirow{3}{*}{$\begin{array}{l}\text { Paramyrothecium roridum } \\
\text { (Tode) L. Lombard \& Crous } \\
\text { [as Myrothecium roridum } \\
\text { Tode] }\end{array}$} & $\begin{array}{l}\text { Stachybotryaceae, Sordariomycetes, } \\
\text { Ascomycota }\end{array}$ & seeds & $\begin{array}{l}\text { Acrocarpus } \\
\text { fraxinifolius }\end{array}$ & Rwanda & $\begin{array}{l}\text { Mathur 1974, Anderson } \\
\text { 1986a }\end{array}$ \\
\hline & & seeds & Azadirachta indica & India & $\begin{array}{l}\text { Mathur 1974, Mittal et } \\
\text { al. } 1990\end{array}$ \\
\hline & & seeds & Eucalyptus grandis & Uruguay & $\begin{array}{l}\text { Mathur 1974, Anderson } \\
\text { 1986a }\end{array}$ \\
\hline
\end{tabular}


Table 2 Continued.

\begin{tabular}{|c|c|c|c|c|c|}
\hline Species & Taxonomy & Substrate & Host & Country & \begin{tabular}{|l|} 
References \\
\end{tabular} \\
\hline & & seeds & Eucalyptus sp. & India & $\begin{array}{l}\text { Saxena 1985, Mittal et } \\
\text { al. } 1990\end{array}$ \\
\hline & & seeds & Pinus taeda & USA & $\begin{array}{l}\text { Mathur 1974, Anderson } \\
\text { 1986a }\end{array}$ \\
\hline Passalora sp. & $\begin{array}{l}\text { Mycosphaerellaceae, Dothideomycetes, } \\
\text { Ascomycota }\end{array}$ & seeds & Quercus spp. & USSR & $\begin{array}{l}\text { Urosevic 1962, Mittal et } \\
\text { al. } 1990\end{array}$ \\
\hline $\begin{array}{l}\text { Penicilliopsis africana } \\
\text { Samson \& Seifert } \#\end{array}$ & $\begin{array}{l}\text { Aspergillaceae, Eurotiomycetes, } \\
\text { Ascomycota }\end{array}$ & seed & undetermined plant & Africa & Samson \& Seifert 1986 \\
\hline $\begin{array}{l}\text { Penicilliopsis indica K. } \\
\text { Swapna \& Nagaveni }^{\#}\end{array}$ & $\begin{array}{l}\text { Aspergillaceae, Eurotiomycetes, } \\
\text { Ascomycota }\end{array}$ & seeds & $\begin{array}{l}\text { Dysoxylum } \\
\text { malabaricum }\end{array}$ & India & Priya \& Nagveni 2012 \\
\hline \multirow[t]{5}{*}{ Penicillium albicans Bainier } & Aspergillaceae, Eurotiomycetes, & seeds & Cedrus deodara & India & Mittal 1983 \\
\hline & Ascomycota & seeds & $\begin{array}{l}\text { Eucalyptus } \\
\text { citriodora }\end{array}$ & India & $\begin{array}{l}\text { Mittal \& Sharma 1984, } \\
\text { Mittal et al. } 1990\end{array}$ \\
\hline & & seeds & Eucalyptus hybrid & India & $\begin{array}{l}\text { Mittal 1986, Mittal et al. } \\
1990\end{array}$ \\
\hline & & seeds & Pinus wallichiana & India & $\begin{array}{l}\text { Mittal \& Sharma 1982b, } \\
\text { Mittal et al. } 1990\end{array}$ \\
\hline & & seeds & Shorea robusta & India & $\begin{array}{l}\text { Mittal \& Sharma 1982b, } \\
\text { Mittal et al. } 1990\end{array}$ \\
\hline \multirow{3}{*}{$\begin{array}{l}\text { Penicillium arenarium } \\
\text { Shaposhn. \& Manteifel }\end{array}$} & Aspergillaceae, Eurotiomycetes, & seeds & Picea excelsa & USSR & Urosevic 1961, \\
\hline & Ascomycota & & & & Anderson 1986a \\
\hline & & seeds & Pinus sylvestris & USSR & $\begin{array}{l}\text { Urosevic 1961, } \\
\text { Anderson 1986a }\end{array}$ \\
\hline \multirow[t]{4}{*}{$\begin{array}{l}\text { Penicillium aurantiogriseum } \\
\text { Dierckx }\end{array}$} & $\begin{array}{l}\text { Aspergillaceae, Eurotiomycetes, } \\
\text { Ascomycota }\end{array}$ & cones, seeds & Pinus strobus & Canada & Mittal \& Wang 1987 \\
\hline & & seeds & $\begin{array}{l}\text { Casuarina } \\
\text { equisetifolia }\end{array}$ & India & Anju et al. 2012 \\
\hline & & seeds & Delonix regia & India & Sahu et al. 2003 \\
\hline & & seeds & Picea glauca & Canada & Mittal \& Wang 1987 \\
\hline $\begin{array}{l}\text { Penicillium brefeldianum } \\
\text { B.O. Dodge }\end{array}$ & $\begin{array}{l}\text { Aspergillaceae, Eurotiomycetes, } \\
\text { Ascomycota }\end{array}$ & seeds & $\begin{array}{l}\text { Eucalyptus } \\
\text { citriodora }\end{array}$ & India & $\begin{array}{l}\text { Mittal \& Sharma 1984, } \\
\text { Mittal et al. } 1990\end{array}$ \\
\hline $\begin{array}{l}\text { Penicillium brevicompactum } \\
\text { Dierckx }\end{array}$ & $\begin{array}{l}\text { Aspergillaceae, Eurotiomycetes, } \\
\text { Ascomycota }\end{array}$ & seeds & Pinus roxburghii & India & $\begin{array}{l}\text { Mittal \& Sharma 1982c, } \\
\text { Mittal et al. } 1990\end{array}$ \\
\hline
\end{tabular}


Table 2 Continued.

\begin{tabular}{|c|c|c|c|c|c|}
\hline Species & Taxonomy & Substrate & Host & Country & References \\
\hline & & seeds & $\begin{array}{l}\text { Platanus } \\
\text { occidentalis }\end{array}$ & USA & $\begin{array}{l}\text { Fakir et al. 1971, Mittal } \\
\text { et al. } 1990\end{array}$ \\
\hline $\begin{array}{l}\text { Penicillium arenicola } \\
\text { Chalab. [as Penicillium }\end{array}$ & $\begin{array}{l}\text { Aspergillaceae, Eurotiomycetes, } \\
\text { Ascomycota }\end{array}$ & seeds & Cassia fistula & India & $\begin{array}{l}\text { Mittal \& Sharma 1981a, } \\
\text { Mittal et al. } 1990\end{array}$ \\
\hline \multirow[t]{5}{*}{ canadense G. Sm.] } & & seeds & Cedrus deodara & India & Mittal 1983 \\
\hline & & seeds & $\begin{array}{l}\text { Eucalyptus } \\
\text { citriodora }\end{array}$ & India & $\begin{array}{l}\text { Mittal \& Sharma 1984, } \\
\text { Mittal et al. } 1990\end{array}$ \\
\hline & & seeds & Pinus roxburghii & India & $\begin{array}{l}\text { Mittal \& Sharma 1982c, } \\
\text { Mittal et al. } 1990\end{array}$ \\
\hline & & seeds & Pinus wallichiana & India, Pakistan & $\begin{array}{l}\text { Mittal \& Sharma 1982b, } \\
\text { Mittal et al. } 1990 \text {, } \\
\text { Ashaeer } 2000\end{array}$ \\
\hline & & seeds & Shorea robusta & India & $\begin{array}{l}\text { Mittal \& Sharma 1982b, } \\
\text { Mittal et al. } 1990\end{array}$ \\
\hline $\begin{array}{l}\text { Penicillium arenicola } \\
\text { Chalab. = Penicillium } \\
\text { canadense G. Sm. } \\
\text { [as 'canadicum'] }\end{array}$ & & seeds & Pinus sylvestris & USSR & $\begin{array}{l}\text { Prisyazhnyuk 1960, } \\
\text { Mittal et al. } 1990\end{array}$ \\
\hline Penicillium candidum Link & $\begin{array}{l}\text { Aspergillaceae, Eurotiomycetes, } \\
\text { Ascomycota }\end{array}$ & seeds & Picea abies & USSR & $\begin{array}{l}\text { Prisyazhnyuk } 1960 \text {, } \\
\text { Mittal et al. } 1990\end{array}$ \\
\hline $\begin{array}{l}\text { Penicillium capsulatum } \\
\text { Raper \& Fennell }\end{array}$ & $\begin{array}{l}\text { Aspergillaceae, Eurotiomycetes, } \\
\text { Ascomycota }\end{array}$ & seeds & $\begin{array}{l}\text { Casuarina } \\
\text { equisetifolia }\end{array}$ & India & Anju et al. 2012 \\
\hline $\begin{array}{l}\text { Penicillium cataractarum } \\
\text { Visagie, Malloch \& Seifert }{ }^{\#}\end{array}$ & $\begin{array}{l}\text { Aspergillaceae, Eurotiomycetes, } \\
\text { Ascomycota }\end{array}$ & nuts & Carya cordiformis & Canada & Visagie et al. 2016 \\
\hline Penicillium citrinum Thom & $\begin{array}{l}\text { Aspergillaceae, Eurotiomycetes, } \\
\text { Ascomycota }\end{array}$ & seeds & Dalbergia sissoo & India & Naz et al. 2015 \\
\hline \multirow[t]{5}{*}{$\begin{array}{l}\text { Penicillium chrysogenum } \\
\text { Thom }\end{array}$} & $\begin{array}{l}\text { Aspergillaceae, Eurotiomycetes, } \\
\text { Ascomycota }\end{array}$ & pod & Canavalia cathartica & India & Anita \& Sridhar 2009 \\
\hline & & pod, seed & Sesbania bispinosa & India & Anita et al. 2009 \\
\hline & & seeds & Alnus glutinosa & Poland & Krol et al. 2015 \\
\hline & & seed & Canavalia cathartica & India & Anita \& Sridhar 2009 \\
\hline & & seeds & $\begin{array}{l}\text { Casuarina } \\
\text { equisetifolia }\end{array}$ & India & Anju et al. 2012 \\
\hline
\end{tabular}


Table 2 Continued.

\begin{tabular}{|c|c|c|c|c|c|}
\hline Species & Taxonomy & Substrate & Host & Country & References \\
\hline & & seeds & Eucalyptus sp. & India & $\begin{array}{l}\text { Saxena 1985, Mittal et } \\
\text { al. } 1990\end{array}$ \\
\hline & & seeds & Larix occidentalis & USA & James et al. 1996 \\
\hline & & seeds & Melia spp. & India & Sahai \& Otra 1982 \\
\hline & & seeds & Picea excelsa & USSR & $\begin{array}{l}\text { Urosevic 1961, } \\
\text { Anderson 1986a }\end{array}$ \\
\hline & & seeds & Pinus ponderosa & USA & $\begin{array}{l}\text { James \& Genz 1982, } \\
\text { Anderson 1986a }\end{array}$ \\
\hline & & seeds & Pinus sylvestris & USSR & $\begin{array}{l}\text { Anderson 1986a, } \\
\text { Urosevic } 1961\end{array}$ \\
\hline & & seeds & $\begin{array}{l}\text { Pseudotsuga } \\
\text { menziesii }\end{array}$ & USA & $\begin{array}{l}\text { James 1984, Anderson } \\
\text { 1986a }\end{array}$ \\
\hline & & seeds & Quercus robur & Poland & Krol et al. 2015 \\
\hline & & seeds & Quercus rubra & Poland & Krol et al. 2015 \\
\hline & & seeds & Sapium spp. & India & Sahai \& Otra 1982 \\
\hline & & seeds & Thuja spp. & India & Sahai \& Otra 1982 \\
\hline \multirow[t]{3}{*}{$\begin{array}{l}\text { Penicillium chrysogenum } \\
\text { Thom } \\
\text { [as } P \text {. notatum Westling] }\end{array}$} & & seeds & Pinus caribaea & $\begin{array}{l}\text { Belize [as British } \\
\text { Honduras], } \\
\text { Thailand }\end{array}$ & $\begin{array}{l}\text { Hocking 1968, Mittal et } \\
\text { al. } 1990\end{array}$ \\
\hline & & seeds & Pinus roxburghii & India & $\begin{array}{l}\text { Mittal \& Sharma 1982c, } \\
\text { Mittal et al. } 1990\end{array}$ \\
\hline & & seeds & Pinus wallichiana & India & $\begin{array}{l}\text { Mittal \& Sharma 1982b, } \\
\text { Mittal et al. } 1990\end{array}$ \\
\hline $\begin{array}{l}\text { Penicillium chrysogenum } \\
\text { var. chrysogenum Thom }\end{array}$ & $\begin{array}{l}\text { Aspergillaceae, Eurotiomycetes, } \\
\text { Ascomycota }\end{array}$ & seeds & Delonix regia & India & Sahu et al. 2003 \\
\hline \multirow[t]{5}{*}{ Penicillium citrinum Thom } & $\begin{array}{l}\text { Aspergillaceae, Eurotiomycetes, } \\
\text { Ascomycota }\end{array}$ & pod, seed & Canavalia cathartica & India & Anita \& Sridhar 2009 \\
\hline & & seeds & Canavalia maritima & India & Seena \& Sridhar 2004 \\
\hline & & seeds & $\begin{array}{l}\text { Casuarina } \\
\text { equisetifolia }\end{array}$ & India & Anju et al. 2012 \\
\hline & & seeds & $\begin{array}{l}\text { Cupressus } \\
\text { sempervirens }\end{array}$ & Egypt & $\begin{array}{l}\text { Farag et al. 1977, Mittal } \\
\text { et al. } 1990\end{array}$ \\
\hline & & seeds & Eucalyptus sp. & India & $\begin{array}{l}\text { Reddy et al. 1982, Mittal } \\
\text { et al. } 1990\end{array}$ \\
\hline
\end{tabular}


Table 2 Continued.

\begin{tabular}{|c|c|c|c|c|c|}
\hline Species & Taxonomy & Substrate & Host & Country & References \\
\hline & & seeds & Larix occidentalis & USA & James et al. 1996 \\
\hline & & seeds & Melia spp. & India & Sahai \& Otra 1982 \\
\hline & & seeds & Pyrus spp. & India & Sahai \& Otra 1982 \\
\hline & & seeds & Thuja spp. & India & Sahai \& Otra 1982 \\
\hline $\begin{array}{l}\text { Penicillium coccotrypicola } \\
\text { Holdom, Y.P. Tan \& R.G. } \\
\text { Shivas }^{\#}\end{array}$ & $\begin{array}{l}\text { Aspergillaceae, Eurotiomycetes, } \\
\text { Ascomycota }\end{array}$ & seeds & $\begin{array}{l}\text { Archontophoenix } \\
\text { cunninghamiana }\end{array}$ & Australia & Crous et al. 2014 \\
\hline \multirow{3}{*}{$\begin{array}{l}\text { Penicillium crustaceum (L.) } \\
\text { Fr. }\end{array}$} & \multirow{3}{*}{$\begin{array}{l}\text { Aspergillaceae, Eurotiomycetes, } \\
\text { Ascomycota }\end{array}$} & seeds & Picea excelsa & USSR & Urosevic 1961, \\
\hline & & & & & Anderson 1986a \\
\hline & & seeds & Pinus sylvestris & USSR & $\begin{array}{l}\text { Garbowski 1936, } \\
\text { Anderson 1986a }\end{array}$ \\
\hline \multirow{13}{*}{$\begin{array}{l}\text { Penicillium cyclopium } \\
\text { Westling }\end{array}$} & \multirow{13}{*}{$\begin{array}{l}\text { Aspergillaceae, Eurotiomycetes, } \\
\text { Ascomycota }\end{array}$} & seeds & Casuarina spp. & India & Sahai \& Otra 1982 \\
\hline & & seeds & $\begin{array}{l}\text { Cupressus } \\
\text { sempervirens }\end{array}$ & Egypt & $\begin{array}{l}\text { Farag et al. 1977, Mittal } \\
\text { et al. } 1990\end{array}$ \\
\hline & & seed & $\begin{array}{l}\text { Desmodium } \\
\text { uncinatum }\end{array}$ & Australia & Nik \& Parbery 1977 \\
\hline & & seed & Linum usitatissimum & Canada & Conners 1967 \\
\hline & & seed & Lotononis angolensis & Australia & Nik \& Parbery 1977 \\
\hline & & seed & $\begin{array}{l}\text { Macroptilium } \\
\text { atropurpureum }\end{array}$ & Australia & Nik \& Parbery 1977 \\
\hline & & seeds & Pinus sylvestris & UK & Whittle 1977 \\
\hline & & seeds & Pyrus spp. & India & Sahai \& Otra 1982 \\
\hline & & seeds & Quercus spp. & India & Sahai \& Otra 1982 \\
\hline & & seed & Stylosanthes gracilis & Australia & Nik \& Parbery 1977 \\
\hline & & seed & $\begin{array}{l}\text { Trifolium } \\
\text { burchellianum }\end{array}$ & Australia & Nik \& Parbery 1977 \\
\hline & & seed & Trifolium repens & Australia & Nik \& Parbery 1977 \\
\hline & & seed & $\begin{array}{l}\text { Trifolium } \\
\text { semipilosum }\end{array}$ & Australia & Nik \& Parbery 1977 \\
\hline $\begin{array}{l}\text { Penicillium decumbens } \\
\text { Thom }\end{array}$ & $\begin{array}{l}\text { Aspergillaceae, Eurotiomycetes, } \\
\text { Ascomycota }\end{array}$ & seeds & $\begin{array}{l}\text { Eucalyptus } \\
\text { citriodora }\end{array}$ & India & $\begin{array}{l}\text { Mittal \& Sharma 1984, } \\
\text { Mittal et al. } 1990\end{array}$ \\
\hline
\end{tabular}


Table 2 Continued.

\begin{tabular}{|c|c|c|c|c|c|}
\hline Species & Taxonomy & Substrate & Host & Country & References \\
\hline & & seed & $\begin{array}{l}\text { Macroptilium } \\
\text { lathyroides }\end{array}$ & Australia & Nik \& Parbery 1977 \\
\hline & & seeds & Pinus roxburghii & India & $\begin{array}{l}\text { Mittal \& Sharma 1982c, } \\
\text { Mittal et al. } 1990\end{array}$ \\
\hline & & seed & Stylosanthes gracilis & Australia & Nik \& Parbery 1977 \\
\hline & & seed & $\begin{array}{l}\text { Stylosanthes } \\
\text { guianensis }\end{array}$ & Australia & Nik \& Parbery 1977 \\
\hline \multirow{5}{*}{$\begin{array}{l}\text { Penicillium digitatum (Pers.) } \\
\text { Sacc. }\end{array}$} & Aspergillaceae, Eurotiomycetes, & fruits & Ardisia crenata & Hong Kong & Tang et al. 2003a \\
\hline & Ascomycota & fruits & $\begin{array}{l}\text { Cleistocalyx } \\
\text { operculatus }\end{array}$ & Hong Kong & Tang et al. 2003a \\
\hline & & fruits & Ilex cinerea & Hong Kong & Tang et al. 2003a \\
\hline & & fruits & Psychotria asiatica & Hong Kong & Tang et al. 2003a \\
\hline & & fruits & $\begin{array}{l}\text { Rhodomyrtus } \\
\text { tomentosa }\end{array}$ & Hong Kong & Tang et al. 2003a \\
\hline \multirow[t]{3}{*}{$\begin{array}{l}\text { Penicillium divergens } \\
\text { Bainier \& Sartory }\end{array}$} & $\begin{array}{l}\text { Aspergillaceae, Eurotiomycetes, } \\
\text { Ascomycota }\end{array}$ & seeds & Picea excelsa & USSR & $\begin{array}{l}\text { Urosevic 1961, } \\
\text { Anderson 1986a }\end{array}$ \\
\hline & & seeds & Pinus sylvestris & USSR, Poland & $\begin{array}{l}\text { Urosevic 1961, } \\
\text { Anderson 1986a, Mittal } \\
\text { et al. } 1990\end{array}$ \\
\hline & & seeds & Quercus spp. & USSR & $\begin{array}{l}\text { Urosevic 1959, Mittal et } \\
\text { al. } 1990\end{array}$ \\
\hline $\begin{array}{l}\text { Penicillium } \\
\text { ellipsoideosporum L. Wang } \\
\text { \& H.Z. Kong\# }\end{array}$ & $\begin{array}{l}\text { Aspergillaceae, Eurotiales, } \\
\text { Eurotiomycetes }\end{array}$ & seeds & Ficus microcarpa & China & Wang \& Kong 2000 \\
\hline \multirow[t]{6}{*}{ Penicillium expansum Link } & $\begin{array}{l}\text { Aspergillaceae, Eurotiales, } \\
\text { Eurotiomycetes }\end{array}$ & seeds & Abies alba & Poland & Krol et al. 2015 \\
\hline & & seeds & Abies sibirica & USSR & $\begin{array}{l}\text { Prisyazhnyuk } 1960 \text {, } \\
\text { Mittal et al. } 1990\end{array}$ \\
\hline & & seeds & Alnus glutinosa & Poland & Krol et al. 2015 \\
\hline & & seeds & Betula verrucosa & USSR & $\begin{array}{l}\text { Kozlowska 1968, Mittal } \\
\text { et al. } 1990\end{array}$ \\
\hline & & seeds & $\begin{array}{l}\text { Eucalyptus } \\
\text { citriodora }\end{array}$ & India & $\begin{array}{l}\text { Mittal \& Sharma } 1984, \\
\text { Mittal et al. } 1990\end{array}$ \\
\hline & & seeds & Larix occidentalis & USA & James et al. 1996 \\
\hline
\end{tabular}


Table 2 Continued.

\begin{tabular}{|c|c|c|c|c|c|}
\hline Species & Taxonomy & Substrate & Host & Country & References \\
\hline & & seeds & Larix sibirica & USSR & $\begin{array}{l}\text { Prisyazhnyuk } 1960 \text {, } \\
\text { Mittal et al. } 1990\end{array}$ \\
\hline & & seeds & Picea abies & USSR & $\begin{array}{l}\text { Prisyazhnyuk } 1960 \text {, } \\
\text { Mittal et al. } 1990\end{array}$ \\
\hline & & seeds & Pinus ponderosa & USA & $\begin{array}{l}\text { James \& Genz 1982, } \\
\text { Anderson 1986a }\end{array}$ \\
\hline & & seeds & Pinus sylvestris & Poland, USSR & $\begin{array}{l}\text { Prisyazhnyuk 1960, } \\
\text { Mittal et al. 1990, Krol } \\
\text { et al. } 2015\end{array}$ \\
\hline & & seeds & Pinus wallichiana & India & $\begin{array}{l}\text { Mittal \& Sharma 1982b, } \\
\text { Mittal et al. } 1990\end{array}$ \\
\hline & & seeds & Quercus pedunculata & USSR & $\begin{array}{l}\text { Kozlowska 1968, Mittal } \\
\text { et al. } 1990\end{array}$ \\
\hline & & seeds & Quercus robur & Poland & Krol et al. 2015 \\
\hline & & seeds & Quercus rubra & Poland & Krol et al. 2015 \\
\hline & & seeds & Quercus spp. & USSR & $\begin{array}{l}\text { Urosevic 1959, Mittal et } \\
\text { al. } 1990\end{array}$ \\
\hline Penicillium frequentans & Aspergillaceae, Eurotiales, & seed & Casuarina spp. & UK & Whittle 1977 \\
\hline \multirow[t]{8}{*}{ Westling } & Eurotiomycetes & seed & Cupressus spp & UK & Whittle 1977 \\
\hline & & seed & Eucalyptus sp. & India & $\begin{array}{l}\text { Saxena 1985, Mittal et } \\
\text { al. } 1990\end{array}$ \\
\hline & & seeds & Lotononis angolensis & Australia & Nik \& Parbery 1977 \\
\hline & & seeds & Melia spp. & India & Sahai \& Otra 1982 \\
\hline & & seeds & Pinus sylvestris & UK & Whittle 1977 \\
\hline & & seeds & Shorea robusta & India & $\begin{array}{l}\text { Mittal \& Sharma 1982b, } \\
\text { Mittal et al. } 1990\end{array}$ \\
\hline & & seeds & Stylosanthes humilis & Australia & Nik \& Parbery 1977 \\
\hline & & seeds & Teramnus uncinatus & Australia & Nik \& Parbery 1977 \\
\hline Penicillium fuscum (Sopp) & Aspergillaceae, Eurotiales, & seeds & Larix occidentalis & USA & James et al. 1996 \\
\hline Raper \& Thom & Eurotiomycetes & seeds & Pinus ponderosa & USA & $\begin{array}{l}\text { James \& Genz 1982, } \\
\text { Anderson 1986a }\end{array}$ \\
\hline $\begin{array}{l}\text { Penicillium glabrum } \\
\text { (Wehmer) Westling }\end{array}$ & $\begin{array}{l}\text { Aspergillaceae, Eurotiales, } \\
\text { Eurotiomycetes }\end{array}$ & pod, seed & Canavalia cathartica & India & Anita \& Sridhar 2009 \\
\hline
\end{tabular}


Table 2 Continued.

\begin{tabular}{|c|c|c|c|c|c|}
\hline Species & Taxonomy & Substrate & Host & Country & References \\
\hline & & seeds & Dalbergia sissoo & India & Kumar 2014 \\
\hline & & $\begin{array}{l}\text { seeds } \\
\text { seeds } \\
\text { seeds }\end{array}$ & $\begin{array}{l}\text { Larix occidentalis } \\
\text { Pinus elliottii } \\
\text { Pinus ponderosa }\end{array}$ & $\begin{array}{l}\text { USA } \\
\text { South Africa } \\
\text { USA }\end{array}$ & $\begin{array}{l}\text { James et al. } 1996 \\
\text { Cilliers et al. } 1995 \\
\text { James \& Genz 1982, } \\
\text { Anderson 1986a }\end{array}$ \\
\hline $\begin{array}{l}\text { Penicillium glabrum } \\
\text { (Wehmer) Westling } \\
\text { [as 'glabra'] }\end{array}$ & & fruits & Ardisia crenata & Hong Kong & Tang et al. 2003a \\
\hline \multirow{2}{*}{$\begin{array}{l}\text { Penicillium glaucoalbidum } \\
\text { (Desm.) Houbraken \& } \\
\text { Samson [as Thysanophora } \\
\text { penicillioides (Roum.) W.B. } \\
\text { Kendr.] }\end{array}$} & \multirow[t]{2}{*}{$\begin{array}{l}\text { Aspergillaceae, Eurotiomycetes, } \\
\text { Ascomycota }\end{array}$} & cones, seed & Pinus densiflora & Japan & $\begin{array}{l}\text { Kasai et al. 1995, } \\
\text { Watanabe } 2010\end{array}$ \\
\hline & & seeds & Picea abies & Finland & $\begin{array}{l}\text { Tillman-Sutela et al. } \\
2004\end{array}$ \\
\hline Penicillium glaucum Link & $\begin{array}{l}\text { Aspergillaceae, Eurotiomycetes, } \\
\text { Ascomycota }\end{array}$ & seeds & Pinus sylvestris & USSR & $\begin{array}{l}\text { Dolejs 1964, Mittal et al. } \\
1990\end{array}$ \\
\hline \multirow[t]{7}{*}{$\begin{array}{l}\text { Penicillium granulatum } \\
\text { Bainier }\end{array}$} & \multirow[t]{7}{*}{$\begin{array}{l}\text { Aspergillaceae, Eurotiomycetes, } \\
\text { Ascomycota }\end{array}$} & seeds & Betula verrucosa & USSR & $\begin{array}{l}\text { Kozlowska 1968, Mittal } \\
\text { et al. } 1990\end{array}$ \\
\hline & & seeds & Cedrus deodara & India & Mittal 1983 \\
\hline & & seeds & Cupressus spp. & India & Sahai \& Otra 1982 \\
\hline & & seeds & Larix decidua & USSR & $\begin{array}{l}\text { Kozlowska 1968, Mittal } \\
\text { et al. } 1990\end{array}$ \\
\hline & & seeds & Quercus pedunculata & USSR & $\begin{array}{l}\text { Kozlowska 1968, Mittal } \\
\text { et al. } 1990\end{array}$ \\
\hline & & seeds & Quercus spp. & India & Sahai \& Otra 1982 \\
\hline & & seeds & Sapium spp. & India & Sahai \& Otra 1982 \\
\hline \multirow{4}{*}{$\begin{array}{l}\text { Penicillium implicatum } \\
\text { Biourge }\end{array}$} & \multirow{4}{*}{$\begin{array}{l}\text { Aspergillaceae, Eurotiomycetes, } \\
\text { Ascomycota }\end{array}$} & seeds & Pyrus spp. & India & Sahai \& Otra 1982 \\
\hline & & seeds & $\begin{array}{l}\text { Quercus } \\
\text { castaneifolia }\end{array}$ & Iran & Kavosi et al. 2013 \\
\hline & & seeds & Quercus spp. & India & Sahai \& Otra 1982 \\
\hline & & seeds & Sapium spp. & India & Sahai \& Otra 1982 \\
\hline $\begin{array}{l}\text { Penicillium italicum } \\
\text { Wehmer }\end{array}$ & $\begin{array}{l}\text { Aspergillaceae, Eurotiomycetes, } \\
\text { Ascomycota }\end{array}$ & pod & Canavalia cathartica & India & Anita \& Sridhar 2009 \\
\hline
\end{tabular}


Table 2 Continued.

\begin{tabular}{|c|c|c|c|c|c|}
\hline Species & Taxonomy & Substrate & Host & Country & References \\
\hline & & pod & Sesbania bispinosa & India & Anita et al. 2009 \\
\hline & & seeds & Dalbergia sissoo & India & $\begin{array}{l}\text { Mittal \& Sharma 1981b, } \\
\text { Mittal et al. } 1990\end{array}$ \\
\hline & & seeds & $\begin{array}{l}\text { Pseudotsuga } \\
\text { menziesii }\end{array}$ & USA & $\begin{array}{l}\text { James 1984, Anderson } \\
\text { 1986a }\end{array}$ \\
\hline $\begin{array}{l}\text { Penicillium janthinellum } \\
\text { Biourge }\end{array}$ & $\begin{array}{l}\text { Aspergillaceae, Eurotiomycetes, } \\
\text { Ascomycota }\end{array}$ & seeds & Pinus sylvestris & UK & Whittle 1977 \\
\hline \multirow[t]{11}{*}{$\begin{array}{l}\text { Penicillium Ianosum } \\
\text { Westling }\end{array}$} & $\begin{array}{l}\text { Aspergillaceae, Eurotiomycetes, } \\
\text { Ascomycota }\end{array}$ & seed & Lotononis angolensis & Australia & Nik \& Parbery 1977 \\
\hline & & seed & $\begin{array}{l}\text { Macroptilium } \\
\text { atropurpureum }\end{array}$ & Australia & Nik \& Parbery 1977 \\
\hline & & seed & Medicago sativa & Australia & Nik \& Parbery 1977 \\
\hline & & seed & $\begin{array}{l}\text { Medicago } \\
\text { tribuloides }\end{array}$ & Australia & Nik \& Parbery 1977 \\
\hline & & seed & Medicago truncatula & Australia & Nik \& Parbery 1977 \\
\hline & & seed & $\begin{array}{l}\text { Stylosanthes } \\
\text { guianensis }\end{array}$ & Australia & Nik \& Parbery 1977 \\
\hline & & seed & Stylosanthes humilis & Australia & Nik \& Parbery 1977 \\
\hline & & seed & Teramnus uncinatus & Australia & Nik \& Parbery 1977 \\
\hline & & seed & $\begin{array}{l}\text { Trifolium } \\
\text { burchellianum }\end{array}$ & Australia & Nik \& Parbery 1977 \\
\hline & & seed & Trifolium repens & Australia & Nik \& Parbery 1977 \\
\hline & & seed & $\begin{array}{l}\text { Trifolium } \\
\text { semipilosum }\end{array}$ & Australia & Nik \& Parbery 1977 \\
\hline $\begin{array}{l}\text { Penicillium lapidosum Raper } \\
\text { \& Fennell }\end{array}$ & $\begin{array}{l}\text { Aspergillaceae, Eurotiomycetes, } \\
\text { Ascomycota }\end{array}$ & seeds & Pinus caribaea & $\begin{array}{l}\text { Belize [as British } \\
\text { Honduras] }\end{array}$ & Hocking 1968 \\
\hline $\begin{array}{l}\text { Penicillium luteoviride } \\
\text { Biourge }\end{array}$ & $\begin{array}{l}\text { Aspergillaceae, Eurotiomycetes, } \\
\text { Ascomycota }\end{array}$ & seeds & Quercus spp. & USSR & $\begin{array}{l}\text { Urosevic 1959, Mittal et } \\
\text { al. } 1990\end{array}$ \\
\hline Penicillium martensii & Aspergillaceae, Eurotiomycetes, & seeds & Cupressus spp. & India & Sahai \& Otra 1982 \\
\hline Biourge & Ascomycota & seeds & Melia spp. & India & Sahai \& Otra 1982 \\
\hline & & seeds & Thuja spp. & India & Sahai \& Otra 1982 \\
\hline Penicillium multicolor & Aspergillaceae, Eurotiomycetes, & seeds & Casuarina spp. & India & Sahai \& Otra 1982 \\
\hline Grig.-Man. \& Porad. & Ascomycota & seeds & Pyrus spp. & India & Sahai \& Otra 1982 \\
\hline
\end{tabular}


Table 2 Continued.

\begin{tabular}{|c|c|c|c|c|c|}
\hline Species & Taxonomy & Substrate & Host & Country & References \\
\hline & & seeds & Thuja spp. & India & Sahai \& Otra 1982 \\
\hline \multirow[t]{6}{*}{$\begin{array}{l}\text { Penicillium nigricans K.W. } \\
\text { Zaleski }\end{array}$} & $\begin{array}{l}\text { Aspergillaceae, Eurotiomycetes, } \\
\text { Ascomycota }\end{array}$ & seeds & Casuarina spp. & India & Sahai \& Otra 1982 \\
\hline & & seeds & Pinus taeda & Georgia & Huang \& Kuhlman 1990 \\
\hline & & seeds & Pyrus spp. & India & Sahai \& Otra 1982 \\
\hline & & seeds & Quercus spp. & India & Sahai \& Otra 1982 \\
\hline & & seeds & Sapium spp & India & Sahai \& Otra 1982 \\
\hline & & seeds & Thuja spp. & India & Sahai \& Otra 1982 \\
\hline $\begin{array}{l}\text { Penicillium nucicola } \\
\text { Visagie, Malloch \& Seifert }{ }^{\#}\end{array}$ & $\begin{array}{l}\text { Aspergillaceae, Eurotiales, } \\
\text { Eurotiomycetes }\end{array}$ & nuts & Carya ovata & Canada & Visagie et al. 2016 \\
\hline \multirow[t]{2}{*}{$\begin{array}{l}\text { Penicillium oxalicum Currie } \\
\text { \& Thom }\end{array}$} & $\begin{array}{l}\text { Aspergillaceae, Eurotiales, } \\
\text { Eurotiomycetes }\end{array}$ & seeds & Larix occidentalis & USA & James et al. 1996 \\
\hline & & seeds & Pinus ponderosa & USA & $\begin{array}{l}\text { James \& Genz 1982, } \\
\text { Anderson 1986a }\end{array}$ \\
\hline Penicillium patulum Bainier & $\begin{array}{l}\text { Aspergillaceae, Eurotiales, } \\
\text { Eurotiomycetes }\end{array}$ & seeds & Larix occidentalis & USA & James et al. 1996 \\
\hline $\begin{array}{l}\text { Penicillium restrictum J.C. } \\
\text { Gilman \& E.V. Abbott }\end{array}$ & $\begin{array}{l}\text { Aspergillaceae, Eurotiales, } \\
\text { Eurotiomycetes }\end{array}$ & seeds & Cedrus deodara & India & Mittal 1983 \\
\hline \multirow[t]{2}{*}{ Penicillium roqueforti Thom } & $\begin{array}{l}\text { Aspergillaceae, Eurotiales, } \\
\text { Eurotiomycetes }\end{array}$ & seeds & Picea excelsa & USSR & $\begin{array}{l}\text { Urosevic 1961, } \\
\text { Anderson 1986a }\end{array}$ \\
\hline & & seeds & Pinus sylvestris & USSR & $\begin{array}{l}\text { Urosevic 1961, } \\
\text { Anderson 1986a }\end{array}$ \\
\hline \multirow[t]{6}{*}{ Penicillium spp. } & $\begin{array}{l}\text { Aspergillaceae, Eurotiomycetes, } \\
\text { Ascomycota }\end{array}$ & acorns, seeds & Quercus robur & Poland & $\begin{array}{l}\text { Jankowiak 2008, Krol et } \\
\text { al. } 2015\end{array}$ \\
\hline & & cones, seed & Pinus densiflora & Japan & $\begin{array}{l}\text { Kasai et al. 1995, } \\
\text { Watanabe } 2010\end{array}$ \\
\hline & & cones, seeds & Pinus ponderosa & USA & $\begin{array}{l}\text { Fuller \& Hildebrand } \\
\text { 1985, James 1995, Mittal } \\
\text { et al. } 1990\end{array}$ \\
\hline & & cones, seeds & Pinus spp. & Canada & Vujanovic et al. 2000 \\
\hline & & cones, seeds & Pinus sylvestris & UK & Whittle 1977 \\
\hline & & cones, seeds & $\begin{array}{l}\text { Pseudotsuga } \\
\text { menziesii var. glauca }\end{array}$ & USA & James 1995 \\
\hline
\end{tabular}


Table 2 Continued.

\begin{tabular}{|c|c|c|c|c|c|}
\hline Species & Taxonomy & Substrate & Host & Country & References \\
\hline & & fruit cupules & Fagus crenata & Japan & Fukasawa et al. 2012 \\
\hline & & fruit & $\begin{array}{l}\text { Artocarpus } \\
\text { communis }\end{array}$ & Nigeria & Amusa et al. 2002 \\
\hline & & fruits & Avicennia marina & India & Mehdi \& Saifullah 2000 \\
\hline & & fruits & Bridelia tomentosa & Hong Kong & Tang et al. 2003a \\
\hline & & fruits & Diplospora dubia & Hong Kong & Tang et al. 2003a \\
\hline & & fruits & Sarcandra glabra & Hong Kong & Tang et al. 2003a \\
\hline & & pod, seed & Canavalia cathartica & India & Anita \& Sridhar 2009 \\
\hline & & pods & Delonix regia & $\begin{array}{l}\text { Thailand, } \\
\text { Philippines }\end{array}$ & $\begin{array}{l}\text { Dayan 1986, Mittal et al. } \\
\text { 1990, Somrithipol et al. } \\
\text { 2002b }\end{array}$ \\
\hline & & seeds & Abies alba & Poland & Krol et al. 2015 \\
\hline & & seeds & Abies lasiocarpa & Canada, Norway & Talgø et al. 2010 \\
\hline & & seeds & Abies nordmanniana & $\begin{array}{l}\text { Austria, Georgia, } \\
\text { Russia }\end{array}$ & Talgø et al. 2010 \\
\hline & & seeds & Abies procera & Norway & Talgø et al. 2010 \\
\hline & & seeds & $\begin{array}{l}\text { Acacia } \\
\text { auriculiformis }\end{array}$ & $\begin{array}{l}\text { Philippines, } \\
\text { Thailand }\end{array}$ & $\begin{array}{l}\text { Chalermpongse et al. } \\
\text { 1984, Quiniones 1987, } \\
\text { Mittal et al. } 1990\end{array}$ \\
\hline & & seeds & Acacia confusa & Philippines & $\begin{array}{l}\text { Agmata 1979, } \\
\text { Anderson 1986a }\end{array}$ \\
\hline & & seeds & Acer rubrum & USA & $\begin{array}{l}\text { Friedrich et al. } 1971 \text {, } \\
\text { Mittal et al. } 1990\end{array}$ \\
\hline & & seeds & Acer saccharinum & USA & $\begin{array}{l}\text { Friedrich et al. } 1971 \text {, } \\
\text { Mittal et al. } 1990\end{array}$ \\
\hline & & seeds & Acer saccharum & USA & $\begin{array}{l}\text { Fuller \& Hildebrand } \\
\text { 1985, Mittal et al. } 1990\end{array}$ \\
\hline & & seeds & Albizia falcataria & Philippines & $\begin{array}{l}\text { Dayan 1986, Quiniones } \\
\text { 1987, Mittal et al. } 1990\end{array}$ \\
\hline & & seeds & Albizia julibrissin & Philippines & $\begin{array}{l}\text { Quiniones 1985, 1987, } \\
\text { Mittal et al. } 1990\end{array}$ \\
\hline & & seeds & Albizia lebbeck & India, Philippines & $\begin{array}{l}\text { Agmata 1979, Anderson } \\
\text { 1986a, Quiniones 1987, } \\
\text { Natarajan et al. 2003, }\end{array}$ \\
\hline
\end{tabular}


Table 2 Continued.

\begin{tabular}{|c|c|c|c|c|c|}
\hline Species & Taxonomy & Substrate & Host & Country & References \\
\hline & & seeds & Albizia procera & Philippines & $\begin{array}{l}\text { Mohanan et al. } 2005 \\
\text { Agmata 1979, Anderson } \\
\text { 1986a, Quiniones } 1987\end{array}$ \\
\hline & & seeds & Alnus glutinosa & Poland & Krol et al. 2015 \\
\hline & & seeds & $\begin{array}{l}\text { Alstonia } \\
\text { macrophylla }\end{array}$ & Philippines & $\begin{array}{l}\text { Quiniones 1985, 1987, } \\
\text { Mittal et al. } 1990\end{array}$ \\
\hline & & seeds & $\begin{array}{l}\text { Anthocephalus } \\
\text { chinensis }\end{array}$ & Philippines & $\begin{array}{l}\text { Agmata 1979, Quiniones } \\
\text { 1987, Mittal et al. } 1990\end{array}$ \\
\hline & & seeds & $\begin{array}{l}\text { Antidesma } \\
\text { ghaesembilla }\end{array}$ & Philippines & $\begin{array}{l}\text { Dayan 1986, Mittal et al. } \\
1990\end{array}$ \\
\hline & & seeds & $\begin{array}{l}\text { Araucaria } \\
\text { angustifolia }\end{array}$ & Australia & $\begin{array}{l}\text { Kamara et al. } 1981 \text {, } \\
\text { Mittal et al. } 1990\end{array}$ \\
\hline & & seeds & $\begin{array}{l}\text { Araucaria } \\
\text { cunninghamii }\end{array}$ & Australia & $\begin{array}{l}\text { Kamara et al. } 1981 \text {, } \\
\text { Mittal et al. } 1990\end{array}$ \\
\hline & & seeds & $\begin{array}{l}\text { Araucaria } \\
\text { heterophylla }\end{array}$ & Egypt & $\begin{array}{l}\text { Kamara et al. } 1981 \text {, } \\
\text { Mittal et al. } 1990\end{array}$ \\
\hline & & seeds & Bauhinia acuminata & Philippines & $\begin{array}{l}\text { Quiniones 1985, 1987, } \\
\text { Mittal et al. } 1990\end{array}$ \\
\hline & & seeds & Bauhinia sp. & Thailand & $\begin{array}{l}\text { Chalermpongse et al. } \\
\text { 1984, Mittal et al. } 1990\end{array}$ \\
\hline & & seeds & $\begin{array}{l}\text { Betula } \\
\text { alleghaniensis }\end{array}$ & USA & $\begin{array}{l}\text { Smoot \& Segall 1963, } \\
\text { Mittal et al. } 1990\end{array}$ \\
\hline & & seeds & Betula papyrifera & USA & $\begin{array}{l}\text { Friedrich et al. } 1971 \text {, } \\
\text { Mittal et al. } 1990\end{array}$ \\
\hline & & seeds & Betula pendula & Finland, Poland & $\begin{array}{l}\text { Lilja 1979, Mittal et al. } \\
\text { 1990, Krol et al. } 2015\end{array}$ \\
\hline & & seeds & Bombax anceps & Thailand & $\begin{array}{l}\text { Chalermpongse et al. } \\
\text { 1984, Mittal et al. } 1990\end{array}$ \\
\hline & & seeds & Bombax ceiba & Bangladesh, India & Mittal et al. 1990 \\
\hline & & seeds & Cassia bakeriana & Thailand & $\begin{array}{l}\text { Chalermpongse et al. } \\
\text { 1984, Mittal et al. } 1990\end{array}$ \\
\hline & & seeds & Cassia fistula & India, Thailand & $\begin{array}{l}\text { Mittal \& Sharma 1981a, } \\
\text { Mittal et al. } 1990\end{array}$ \\
\hline
\end{tabular}


Table 2 Continued.

\begin{tabular}{|c|c|c|c|c|c|}
\hline Species & Taxonomy & Substrate & Host & Country & References \\
\hline & & seeds & Cassia siamea & $\begin{array}{l}\text { Philippines, } \\
\text { Thailand }\end{array}$ & $\begin{array}{l}\text { Quiniones 1985, 1987, } \\
\text { Mittal et al. } 1990\end{array}$ \\
\hline & & seeds & $\begin{array}{l}\text { Casuarina } \\
\text { equisetifolia }\end{array}$ & Philippines, USA & $\begin{array}{l}\text { Quiniones 1985, 1987, } \\
\text { Anderson 1986a , } \\
\text { Bayman et al. } 1998\end{array}$ \\
\hline & & seeds & Cedrus deodara & India & $\begin{array}{l}\text { Munjal \& Sharma 1975, } \\
\text { Mittal et al. } 1990\end{array}$ \\
\hline & & seed & $\begin{array}{l}\text { Cryptomeria } \\
\text { japonica }\end{array}$ & Japan & Watanabe 2010 \\
\hline & & seeds & Dalbergia sissoides & India & Mohanan et al. 2005 \\
\hline & & seed & $\begin{array}{l}\text { Desmodium } \\
\text { uncinatum }\end{array}$ & Australia & Nik \& Parbery 1977 \\
\hline & & seeds & $\begin{array}{l}\text { Dianthus } \\
\text { caryophyllus }\end{array}$ & Taiwan & Li \& Wu 2002 \\
\hline & & seeds & Dianthus spp. & Taiwan & Li \& Wu 2002 \\
\hline & & seeds & $\begin{array}{l}\text { Eucalyptus } \\
\text { citriodora }\end{array}$ & India & Anderson 1986a \\
\hline & & seeds & Eucalyptus deglupta & Philippines & $\begin{array}{l}\text { Agmata 1979, Anderson } \\
\text { 1986a }\end{array}$ \\
\hline & & seeds & Eucalyptus globulus & India & Anderson 1986a \\
\hline & & seeds & Eucalyptus grandis & India, South Africa & $\begin{array}{l}\text { Anderson 1986a, Jimu et } \\
\text { al. } 2016\end{array}$ \\
\hline & & seeds & Eucalyptus maidenii & Uruguay & Anderson 1986a \\
\hline & & seeds & $\begin{array}{l}\text { Eucalyptus } \\
\text { tereticornis }\end{array}$ & India & Anderson 1986a \\
\hline & & seeds & Fagus sylvatica & Poland & Krol et al. 2015 \\
\hline & & seeds & Fraxinus americana & USA & $\begin{array}{l}\text { Friedrich et al. } 1971 \text {, } \\
\text { Mittal et al. } 1990\end{array}$ \\
\hline & & seeds & Fraxinus excelsior & Sweden & Hayatgheibi 2013 \\
\hline & & seeds & Gliricidia sepium & Philippines & $\begin{array}{l}\text { Dayan 1986, Mittal et al. } \\
1990\end{array}$ \\
\hline & & seeds & Gmelina arborea & $\begin{array}{l}\text { Philippines, } \\
\text { Thailand }\end{array}$ & $\begin{array}{l}\text { Quiniones 1985, } \\
\text { Anderson 1986a, Mittal } \\
\text { et al. } 1990\end{array}$ \\
\hline
\end{tabular}


Table 2 Continued.

\begin{tabular}{|c|c|c|c|c|c|}
\hline Species & Taxonomy & Substrate & Host & Country & References \\
\hline & & seeds & $\begin{array}{l}\text { Lagerstroemia } \\
\text { calyculata }\end{array}$ & Thailand & $\begin{array}{l}\text { Chalermpongse et al. } \\
\text { 1984, Mittal et al. } 1990\end{array}$ \\
\hline & & seeds & $\begin{array}{l}\text { Lagerstroemia } \\
\text { speciosa }\end{array}$ & Philippines & $\begin{array}{l}\text { Quiniones 1985, } \\
\text { Anderson 1986a }\end{array}$ \\
\hline & & seeds & $\begin{array}{l}\text { Leucaena } \\
\text { leucocephala }\end{array}$ & $\begin{array}{l}\text { Bangladesh, } \\
\text { Philippines, } \\
\text { Thailand }\end{array}$ & $\begin{array}{l}\text { Chalermpongse et al. } \\
\text { 1984, Anderson 1986a, } \\
\text { Quiniones 1987, Mittal } \\
\text { et al. 1990, Islam et al. } \\
2008\end{array}$ \\
\hline & & seeds & Manilkara bidentata & USA & Bayman et al. 1998 \\
\hline & & seed & Medicago truncatula & Australia & Nik \& Parbery 1977 \\
\hline & & seeds & Melia azedarach & Thailand & $\begin{array}{l}\text { Chalermpongse et al. } \\
\text { 1984, Mittal et al. } 1990\end{array}$ \\
\hline & & seeds & $\begin{array}{l}\text { Musanga } \\
\text { cecropioides }\end{array}$ & Philippines & $\begin{array}{l}\text { Agmata 1979, Anderson } \\
\text { 1986a }\end{array}$ \\
\hline & & seeds & Parkia roxburghii & Philippines & $\begin{array}{l}\text { Dayan 1986, Anderson } \\
\text { 1986a, Quiniones } 1987\end{array}$ \\
\hline & & seeds & Phyllostachys edulis & China & Shen et al. 2014 \\
\hline & & seeds & Picea abies & Poland & Krol et al. 2015 \\
\hline & & seeds & Picea engelmannii & USA & $\begin{array}{l}\text { Fuller \& Hildebrand } \\
\text { 1985, Mittal et al. } 1990\end{array}$ \\
\hline & & seeds & Picea jezoensis & Japan & Cheng \& Igarashi 1987 \\
\hline & & seeds & Picea sitchensis & UK & $\begin{array}{l}\text { Salt 1964, Mittal et al. } \\
1990\end{array}$ \\
\hline & & seeds & $\begin{array}{l}\text { Piliostigma } \\
\text { malabaricum }\end{array}$ & Philippines & $\begin{array}{l}\text { Quiniones 1985, } \\
\text { Anderson 1986a, } 1987\end{array}$ \\
\hline & & seeds & Pinus caribaea & Belize [or as & Hocking 1968, \\
\hline & & & & British Honduras] & $\begin{array}{l}\text { Chalermpongse et al. } \\
\text { 1984, Mittal et al. } 1990\end{array}$ \\
\hline & & seeds & Pinus contorta & USA & $\begin{array}{l}\text { Fuller \& Hildebrand } \\
\text { 1985, Mittal et al. } 1990\end{array}$ \\
\hline & & seeds & Pinus elliottii & Taiwan, USA & $\begin{array}{l}\text { Rowan \& Debarr 1974, } \\
\text { Mittal et al. } 1990\end{array}$ \\
\hline
\end{tabular}


Table 2 Continued.

\begin{tabular}{|c|c|c|c|c|c|}
\hline Species & Taxonomy & Substrate & Host & Country & References \\
\hline & & seeds & $\begin{array}{l}\text { Pinus elliottii var. } \\
\text { elliottii }\end{array}$ & Uruguay, USA & $\begin{array}{l}\text { Anderson 1986a, } \\
\text { Fraedrich \& Miller } 1995\end{array}$ \\
\hline & & seeds & Pinus kesiya & $\begin{array}{l}\text { Philippines, } \\
\text { Thailand }\end{array}$ & $\begin{array}{l}\text { Quiniones 1985, } \\
\text { Anderson 1986a, Mittal } \\
\text { et al. } 1990\end{array}$ \\
\hline & & seeds & Pinus lambertiana & USA & $\begin{array}{l}\text { Anderson 1986a, } \\
\text { Schubert } 1961\end{array}$ \\
\hline & & seeds & Pinus merkusii & Philippines & $\begin{array}{l}\text { Agmata 1979, Anderson } \\
\text { 1986a }\end{array}$ \\
\hline & & seeds & Pinus nigra & Hungary & $\begin{array}{l}\text { Hangyal 1973, Mittal et } \\
\text { al. } 1990\end{array}$ \\
\hline & & seeds & Pinus roxburghii & India & $\begin{array}{l}\text { Munjal \& Sharma 1975, } \\
\text { Mittal et al. } 1990\end{array}$ \\
\hline & & seeds & Pinus sylvestris & Hungary, Poland & $\begin{array}{l}\text { Hangyal 1973, Mittal et } \\
\text { al. 1990, Krol et al. } 2015\end{array}$ \\
\hline & & seeds & Pinus taeda & USA & $\begin{array}{l}\text { Mason \& van Arsdel } \\
\text { 1978, Anderson 1986a }\end{array}$ \\
\hline & & seeds & Pinus wallichiana & India & $\begin{array}{l}\text { Munjal \& Sharma 1975, } \\
\text { Mittal et al. } 1990\end{array}$ \\
\hline & & seeds & $\begin{array}{l}\text { Pittosporum } \\
\text { resiniferum }\end{array}$ & Philippines & $\begin{array}{l}\text { Dayan 1986, Mittal et al. } \\
1990\end{array}$ \\
\hline & & seeds & $\begin{array}{l}\text { Platanus } \\
\text { occidentalis }\end{array}$ & USA & $\begin{array}{l}\text { Fakir et al. 1971, Mittal } \\
\text { et al. } 1990\end{array}$ \\
\hline & & seeds & $\begin{array}{l}\text { Pseudotsuga } \\
\text { menziesii }\end{array}$ & Canada, USA & $\begin{array}{l}\text { Salisbury 1955, Mittal et } \\
\text { al. } 1990\end{array}$ \\
\hline & & seeds & Quercus robur & Germany & Schroder et al. 2004 \\
\hline & & seeds & Quercus rubra & Poland & Krol et al. 2015 \\
\hline & & seeds & Quercus spp. & USSR & Mittal et al. 1990 \\
\hline & & seeds & Samanea saman & $\begin{array}{l}\text { Philippines, } \\
\text { Thailand }\end{array}$ & $\begin{array}{l}\text { Quiniones 1985, 1987, } \\
\text { Anderson 1986a, Mittal } \\
\text { et al. } 1990\end{array}$ \\
\hline & & seeds & $\begin{array}{l}\text { Sequoia } \\
\text { sempervirens }\end{array}$ & USA & $\begin{array}{l}\text { Davidson 1970, Mittal et } \\
\text { al. } 1990\end{array}$ \\
\hline
\end{tabular}


Table 2 Continued.

\begin{tabular}{|c|c|c|c|c|c|}
\hline Species & Taxonomy & Substrate & Host & Country & References \\
\hline & & seeds & Serialbizzia acle & Philippines & $\begin{array}{l}\text { Quiniones 1985, } \\
\text { Anderson 1986a, } 1987\end{array}$ \\
\hline & & seeds & Shorea assamica & Malaysia & $\begin{array}{l}\text { Hong 1981, Mittal et al. } \\
1990\end{array}$ \\
\hline & & seeds & Shorea robusta & Thailand & $\begin{array}{l}\text { Chalermpongse et al. } \\
\text { 1984, Mittal et al. } 1990\end{array}$ \\
\hline & & seed & Stylosanthes humilis & Australia & Nik \& Parbery 1977 \\
\hline & & seeds & $\begin{array}{l}\text { Swietenia } \\
\text { macrophylla }\end{array}$ & Philippines & $\begin{array}{l}\text { Dayan 1986, Mittal et al. } \\
1990\end{array}$ \\
\hline & & seeds & Tectona grandis & India & $\begin{array}{l}\text { Anderson 1986a, } \\
\text { Mohanan et al. } 2005\end{array}$ \\
\hline & & seeds & Triplaris cumingiana & Philippines & $\begin{array}{l}\text { Quiniones 1985, } \\
\text { Anderson 1986a }\end{array}$ \\
\hline & & seeds & Vitex parviflora & Philippines & $\begin{array}{l}\text { Agmata 1979, Anderson } \\
\text { 1986a }\end{array}$ \\
\hline & & seeds & $\begin{array}{l}\text { Wallaceodendron } \\
\text { celibcum }\end{array}$ & Philippines & $\begin{array}{l}\text { Dayan 1986, Mittal et al. } \\
1990\end{array}$ \\
\hline & & seeds & $\begin{array}{l}\text { Xylia xylocarpa var. } \\
\text { kerrii }\end{array}$ & Thailand & $\begin{array}{l}\text { Chalermpongse et al. } \\
\text { 1984, Mittal et al. } 1990\end{array}$ \\
\hline \multirow[t]{2}{*}{$\begin{array}{l}\text { Penicillium steckii K.W. } \\
\text { Zaleski }\end{array}$} & $\begin{array}{l}\text { Aspergillaceae, Eurotiomycetes, } \\
\text { Ascomycota }\end{array}$ & seeds & Casuarina spp. & India & Sahai \& Otra 1982 \\
\hline & & seeds & Quercus spp. & India & Sahai \& Otra 1982 \\
\hline Penicillium thomii Maire & $\begin{array}{l}\text { Aspergillaceae, Eurotiomycetes, } \\
\text { Ascomycota }\end{array}$ & $\begin{array}{l}\text { seeds } \\
\text { cones }\end{array}$ & $\begin{array}{l}\text { Sapium spp. } \\
\text { Picea glauca }\end{array}$ & $\begin{array}{l}\text { India } \\
\text { Canada }\end{array}$ & $\begin{array}{l}\text { Sahai \& Otra } 1982 \\
\text { Mittal \& Wang } 1987\end{array}$ \\
\hline Penicillium urticae Bainier & $\begin{array}{l}\text { Aspergillaceae, Eurotiomycetes, } \\
\text { Ascomycota }\end{array}$ & $\begin{array}{l}\text { cones, seeds } \\
\text { seeds }\end{array}$ & $\begin{array}{l}\text { Pinus strobus } \\
\text { Cupressus spp. }\end{array}$ & $\begin{array}{l}\text { Canada } \\
\text { India }\end{array}$ & $\begin{array}{l}\text { Mittal \& Wang } 1987 \\
\text { Sahai \& Otra } 1982\end{array}$ \\
\hline \multirow[t]{2}{*}{$\begin{array}{l}\text { Penicillium viridicatum } \\
\text { Westling }\end{array}$} & $\begin{array}{l}\text { Aspergillaceae, Eurotiomycetes, } \\
\text { Ascomycota }\end{array}$ & $\begin{array}{l}\text { seeds } \\
\text { seeds }\end{array}$ & $\begin{array}{l}\text { Pyrus spp. } \\
\text { Larix occidentalis }\end{array}$ & $\begin{array}{l}\text { India } \\
\text { USA }\end{array}$ & $\begin{array}{l}\text { Sahai \& Otra } 1982 \\
\text { James et al. } 1996\end{array}$ \\
\hline & & seeds & Pinus ponderosa & USA & $\begin{array}{l}\text { James \& Genz 1982, } \\
\text { Anderson 1986a }\end{array}$ \\
\hline
\end{tabular}


Table 2 Continued.

\begin{tabular}{|c|c|c|c|c|c|}
\hline Species & Taxonomy & Substrate & Host & Country & References \\
\hline $\begin{array}{l}\text { Penicillium vulpinum } \\
\text { (Cooke \& Massee) Seifert \& } \\
\text { Samson [as Penicillium } \\
\text { claviforme Bainier ] }\end{array}$ & $\begin{array}{l}\text { Aspergillaceae, Eurotiomycetes, } \\
\text { Ascomycota }\end{array}$ & seeds & Pinus ponderosa & USA & $\begin{array}{l}\text { James \& Genz 1982, } \\
\text { Anderson 1986a }\end{array}$ \\
\hline Peniophora sp. & $\begin{array}{l}\text { Peniophoraceae, Agaricomycetes, } \\
\text { Basidiomycota }\end{array}$ & seeds & Quercus robur & Turkey & Oskay et al. 2018 \\
\hline \multirow[t]{3}{*}{ Periconia byssoides Pers. } & $\begin{array}{l}\text { Periconiaceae, Dothideomycetes, } \\
\text { Ascomycota }\end{array}$ & fruits & $\begin{array}{l}\text { Magnolia } \\
\text { grandiflora }\end{array}$ & Thailand & Jayasiri et al. 2019 \\
\hline & & pod & Peltophorum sp. & Thailand & Jayasiri et al. 2019 \\
\hline & & seed & Acer sp. & Canada & Conners 1967 \\
\hline \multirow{2}{*}{$\begin{array}{l}\text { Periconia byssoides Pers. } \\
\text { [as Periconia pycnospora } \\
\text { Fresen.] }\end{array}$} & & seed & Agropyron cristatum & China & Conners 1967 \\
\hline & & seed & $\begin{array}{l}\text { Cryptomeria } \\
\text { japonica }\end{array}$ & Japan & Watanabe 2010 \\
\hline $\begin{array}{l}\text { Periconia circinata (L. } \\
\text { Mangin) Sacc. \& D. Sacc. }\end{array}$ & $\begin{array}{l}\text { Periconiaceae, Dothideomycetes, } \\
\text { Ascomycota }\end{array}$ & seed & Linum usitatissimum & Canada & Conners 1967 \\
\hline $\begin{array}{l}\text { Periconia delonicis Jayasiri, } \\
\text { E.B.G. Jones \& K.D. Hyd }{ }^{\#}\end{array}$ & $\begin{array}{l}\text { Periconiaceae, Dothideomycetes, } \\
\text { Ascomycota }\end{array}$ & pod & Delonix regia & Thailand & Jayasiri et al. 2019 \\
\hline \multirow[t]{4}{*}{ Periconia spp. } & $\begin{array}{l}\text { Periconiaceae, Dothideomycetes, } \\
\text { Ascomycota }\end{array}$ & pod & Sesbania bispinosa & India & Anita et al. 2009 \\
\hline & & seeds & Eucalyptus sp. & India & $\begin{array}{l}\text { Saxena 1985, Mittal et } \\
\text { al. } 1990\end{array}$ \\
\hline & & seeds & Pinus roxburghii & India & $\begin{array}{l}\text { Mittal \& Sharma 1982c, } \\
\text { Mittal et al. } 1990\end{array}$ \\
\hline & & seeds & Tectona grandis & India & Anderson 1986a \\
\hline \multirow{2}{*}{$\begin{array}{l}\text { Peridermium conorum- } \\
\text { piceae (Reess) Arthur \& F. } \\
\text { Kern }\end{array}$} & $\begin{array}{l}\text { Cronartiaceae, Pucciniomycetes, } \\
\text { Basidiomycota }\end{array}$ & cones & Picea canadensis & USA & Arthur \& Kern 1906 \\
\hline & & cones & Picea engelmannii & USA & Arthur \& Kern 1906 \\
\hline
\end{tabular}


Table 2 Continued.

\begin{tabular}{|c|c|c|c|c|c|}
\hline Species & Taxonomy & Substrate & Host & Country & References \\
\hline & & cones & Picea excelsa & USA & Arthur \& Kern 1906 \\
\hline & & cones & Picea Mariana & USA & Arthur \& Kern 1906 \\
\hline & & cones & Picea rubra & USA & Arthur \& Kern 1906 \\
\hline Perisporium vulgare Corda & $\begin{array}{l}\text { Incertae sedis, Incertae sedis, } \\
\text { Ascomycota }\end{array}$ & seeds & Pinus wallichiana & India & $\begin{array}{l}\text { Munjal \& Sharma 1975, } \\
\text { Mittal et al. } 1990\end{array}$ \\
\hline \multirow[t]{2}{*}{$\begin{array}{l}\text { Pestalotiopsis adusta (Ellis } \\
\text { \& Everh.) Steyaert }\end{array}$} & $\begin{array}{l}\text { Sporocadaceae, Sordariomycetes, } \\
\text { Ascomycota }\end{array}$ & fruits & $\begin{array}{l}\text { Diploclisia } \\
\text { glaucescens }\end{array}$ & Hong Kong & $\begin{array}{l}\text { Maharachchikumbura et } \\
\text { al. } 2014\end{array}$ \\
\hline & & $\begin{array}{l}\text { fruits } \\
\text { fruits }\end{array}$ & $\begin{array}{l}\text { Ilex asprella } \\
\text { Ilex pubescens }\end{array}$ & $\begin{array}{l}\text { Hong Kong } \\
\text { Hong Kong }\end{array}$ & $\begin{array}{l}\text { Tang et al. 2003a } \\
\text { Tang et al. 2003a }\end{array}$ \\
\hline $\begin{array}{l}\text { Pestalotiopsis diploclisiae } \\
\text { Maharachch., K.D. Hyde \& } \\
\text { Crous }^{\#}\end{array}$ & $\begin{array}{l}\text { Sporocadaceae, Sordariomycetes, } \\
\text { Ascomycota }\end{array}$ & fruit & Litsea rotundifolia & Hong Kong & Tang et al. 2003a \\
\hline $\begin{array}{l}\text { Pestalotiopsis espaillatii } \\
\text { (Cif. \& Gonz. Frag.) Satya }\end{array}$ & $\begin{array}{l}\text { Sporocadaceae, Sordariomycetes, } \\
\text { Ascomycota }\end{array}$ & fruits & Ilex pubescens & Hong Kong & Tang et al. 2003a \\
\hline $\begin{array}{l}\text { Pestalotiopsis flavidula } \\
\text { (Tassi) Y.X. Chen } \\
\text { [as Pestalotia flavidula } \\
\text { Tassi] }\end{array}$ & $\begin{array}{l}\text { Sporocadaceae, Sordariomycetes, } \\
\text { Ascomycota }\end{array}$ & seeds & $\begin{array}{l}\text { Pinus elliottii var. } \\
\text { elliottii }\end{array}$ & Georgia & Huang \& Kuhlman 1990 \\
\hline \multirow[t]{8}{*}{$\begin{array}{l}\text { Pestalotiopsis funerea } \\
\text { (Desm.) Steyaert }\end{array}$} & $\begin{array}{l}\text { Sporocadaceae, Sordariomycetes, } \\
\text { Ascomycota }\end{array}$ & cones, seeds & Pinus koraiensis & Canada & Vujanovic et al. 2000 \\
\hline & & cones, seeds & Pinus mugo 'Hesse' & Canada & Vujanovic et al. 2000 \\
\hline & & cones, seeds & $\begin{array}{l}\text { Pinus mugo var. } \\
\text { pumilio }\end{array}$ & Canada & Vujanovic et al. 2000 \\
\hline & & seeds & $\begin{array}{l}\text { Chamaecyparis } \\
\text { lawsoniana }\end{array}$ & France, Italy & $\begin{array}{l}\text { Motta \& Saponaro 1983, } \\
\text { Mittal et al. } 1990\end{array}$ \\
\hline & & seeds & $\begin{array}{l}\text { Cupressus } \\
\text { abramsiana }\end{array}$ & France, Italy & $\begin{array}{l}\text { Saponaro \& Motta 1984, } \\
\text { Mittal et al. } 1990\end{array}$ \\
\hline & & seeds & Cupressus arizonica & France, Italy & $\begin{array}{l}\text { Saponaro \& Motta 1984, } \\
\text { Mittal et al. } 1990\end{array}$ \\
\hline & & seeds & Cupressus funebris & Uruguay & Anderson 1986a \\
\hline & & seeds & Cupressus glabra & France, Italy & $\begin{array}{l}\text { Saponaro \& Motta 1984, } \\
\text { Mittal et al. } 1990\end{array}$ \\
\hline
\end{tabular}


Table 2 Continued.

\begin{tabular}{|c|c|c|c|c|c|}
\hline Species & Taxonomy & Substrate & Host & Country & References \\
\hline & & seeds & $\begin{array}{l}\text { Cupressus } \\
\text { goveniana }\end{array}$ & France, Italy & $\begin{array}{l}\text { Saponaro \& Motta 1984, } \\
\text { Mittal et al. } 1990\end{array}$ \\
\hline & & seeds & Cupressus lusitanica & France, Italy & $\begin{array}{l}\text { Saponaro \& Motta 1984, } \\
\text { Mittal et al. } 1990\end{array}$ \\
\hline & & seeds & $\begin{array}{l}\text { Cupressus lusitanica } \\
\text { var. benthamii }\end{array}$ & France, Italy & $\begin{array}{l}\text { Saponaro \& Motta 1984, } \\
\text { Mittal et al. } 1990\end{array}$ \\
\hline & & seeds & $\begin{array}{l}\text { Cupressus } \\
\text { sempervirens }\end{array}$ & France, Italy & $\begin{array}{l}\text { Motta \& Saponaro 1983, } \\
\text { Mittal et al. } 1990\end{array}$ \\
\hline & & seeds & Cupressus torulosa & France, Italy & $\begin{array}{l}\text { Saponaro \& Motta 1984, } \\
\text { Mittal et al. } 1990\end{array}$ \\
\hline & & seeds & Eucalyptus alba & India & $\begin{array}{l}\text { Mathur 1974, Anderson } \\
\text { 1986a }\end{array}$ \\
\hline & & seeds & Eucalyptus grandis & Uruguay & Anderson 1986a \\
\hline & & seeds & $\begin{array}{l}\text { Libocedrus } \\
\text { decurrens }\end{array}$ & UK & Anderson 1986a \\
\hline & & seeds & Pinus spp. & UK & Anderson 1986a \\
\hline & & seeds & $\begin{array}{l}\text { Pseudotsuga } \\
\text { menziesii }\end{array}$ & $\mathrm{UK}$ & Anderson 1986a \\
\hline & & seeds & Thuja orientalis & Italy, France & $\begin{array}{l}\text { Motta \& Saponaro 1983, } \\
\text { Mittal et al. } 1990\end{array}$ \\
\hline $\begin{array}{l}\text { Pestalotiopsis glandicola } \\
\text { (Castagne) Steyaert } \\
\text { [as Pestalotia castagnei } \\
\text { Desm.] }\end{array}$ & $\begin{array}{l}\text { Sporocadaceae, Sordariomycetes, } \\
\text { Ascomycota }\end{array}$ & seeds & Quercus spp. & USSR & Mittal et al. 1990 \\
\hline \multirow{3}{*}{$\begin{array}{l}\text { Pestalotiopsis glandicola } \\
\text { (Castagne) Steyaert } \\
\text { [as Pestalotia glandicola } \\
\text { (Castagne) Guba] }\end{array}$} & & seeds & Picea excelsa & USSR & $\begin{array}{l}\text { Urosevic 1961, } \\
\text { Anderson 1986a }\end{array}$ \\
\hline & & seeds & Pinus sylvestris & USSR & $\begin{array}{l}\text { Urosevic 1961, } \\
\text { Anderson 1986a }\end{array}$ \\
\hline & & seeds & Quercus spp. & USSR & $\begin{array}{l}\text { Urosevic 1959, Mittal et } \\
\text { al. } 1990\end{array}$ \\
\hline
\end{tabular}


Table 2 Continued.

\begin{tabular}{|c|c|c|c|c|c|}
\hline Species & Taxonomy & Substrate & Host & Country & References \\
\hline $\begin{array}{l}\text { Pestalotiopsis gracilis } \\
\text { (Kleb.) Steyaert }\end{array}$ & $\begin{array}{l}\text { Sporocadaceae, Sordariomycetes, } \\
\text { Ascomycota }\end{array}$ & fruits & Litsea rotundifolia & Hong Kong & Tang et al. 2003a \\
\hline \multirow{2}{*}{$\begin{array}{l}\text { Pestalotiopsis guepinii } \\
\text { (Desm.) Steyaert } \\
\text { [as 'guepini’] }\end{array}$} & $\begin{array}{l}\text { Sporocadaceae, Sordariomycetes, } \\
\text { Ascomycota }\end{array}$ & seeds & $\begin{array}{l}\text { Cupressus } \\
\text { macrocarpa }\end{array}$ & Uruguay & Anderson 1986a \\
\hline & & $\begin{array}{l}\text { seeds } \\
\text { seeds }\end{array}$ & $\begin{array}{l}\text { Pinus elliottii } \\
\text { Pinus elliottii var. } \\
\text { elliottii }\end{array}$ & $\begin{array}{l}\text { South Africa } \\
\text { Uruguay }\end{array}$ & $\begin{array}{l}\text { Cilliers et al. } 1995 \\
\text { Anderson } 1986 a\end{array}$ \\
\hline $\begin{array}{l}\text { Pestalotiopsis mangiferae } \\
\text { (Henn.) Steyaert }\end{array}$ & $\begin{array}{l}\text { Sporocadaceae, Sordariomycetes, } \\
\text { Ascomycota }\end{array}$ & seeds & Eucalyptus sp. & India & $\begin{array}{l}\text { Reddy et al. 1982, Mittal } \\
\text { et al. } 1990\end{array}$ \\
\hline $\begin{array}{l}\text { Pestalotiopsis neglecta } \\
\text { (Thüm.) Steyaert }\end{array}$ & $\begin{array}{l}\text { Sporocadaceae, Sordariomycetes, } \\
\text { Ascomycota }\end{array}$ & fruits & Diplospora dubia & Hong Kong & Tang et al. 2003a \\
\hline $\begin{array}{l}\text { Pestalotiopsis phoenicis } \\
\text { (Vize) Y.X. Chen }\end{array}$ & $\begin{array}{l}\text { Sporocadaceae, Sordariomycetes, } \\
\text { Ascomycota }\end{array}$ & fruits & $\begin{array}{l}\text { Cleistocalyx } \\
\text { operculatus }\end{array}$ & Hong Kong & Tang et al. 2003a \\
\hline $\begin{array}{l}\text { Pestalotiopsis shoreae Yu } \\
\text { Song, Tangthir., K.D. Hyde } \\
\text { \& Y. Wang\# }\end{array}$ & $\begin{array}{l}\text { Sporocadaceae, Sordariomycetes, } \\
\text { Ascomycota }\end{array}$ & $\begin{array}{l}\text { fruits } \\
\text { seeds }\end{array}$ & $\begin{array}{l}\text { Psychotria asiatica } \\
\text { Shorea obtuse }\end{array}$ & $\begin{array}{l}\text { Hong Kong } \\
\text { Thailand }\end{array}$ & $\begin{array}{l}\text { Tang et al. 2003a } \\
\text { Song et al. } 2014\end{array}$ \\
\hline \multirow[t]{9}{*}{ Pestalotiopsis spp. } & $\begin{array}{l}\text { Sporocadaceae, Sordariomycetes, } \\
\text { Ascomycota }\end{array}$ & cones & Pinus densiflora & Japan & Kasai et al. 1995 \\
\hline & & fruits & $\begin{array}{l}\text { Cleistocalyx } \\
\text { operculatus }\end{array}$ & Hong Kong & Tang et al. 2003a \\
\hline & & fruits & Microcos paniculata & Hong Kong & Tang et al. 2003a \\
\hline & & seeds & Bauhinia purpurea & India & $\begin{array}{l}\text { Mathur 1974, Mittal et } \\
\text { al. } 1990\end{array}$ \\
\hline & & seeds & Bauhinia sp. & Thailand & $\begin{array}{l}\text { Chalermpongse et al. } \\
\text { 1984, Mittal et al. } 1990\end{array}$ \\
\hline & & seeds & Bauhinia variegata & India & $\begin{array}{l}\text { Mathur 1974, Mittal et } \\
\text { al. } 1990\end{array}$ \\
\hline & & seeds & Cassia bakeriana & Thailand & $\begin{array}{l}\text { Chalermpongse et al. } \\
\text { 1984, Mittal et al. } 1990\end{array}$ \\
\hline & & seeds & $\begin{array}{l}\text { Casuarina } \\
\text { equisetifolia }\end{array}$ & Mauritius & $\begin{array}{l}\text { Bose 1944, Anderson } \\
\text { 1986a }\end{array}$ \\
\hline & & seeds & Chamaecyparis sp. & Japan & Anderson 1986a \\
\hline
\end{tabular}


Table 2 Continued.

\begin{tabular}{|c|c|c|c|c|c|}
\hline Species & Taxonomy & Substrate & Host & Country & References \\
\hline & & seeds & Eucalyptus grandis & South Africa & Jimu et al. 2016 \\
\hline & & seeds & Eucalyptus maidenii & Uruguay & Anderson 1986a \\
\hline & & seeds & Phyllostachys edulis & China & Shen et al. 2014 \\
\hline & & seeds & Pinus massoniana & Taiwan & $\begin{array}{l}\text { Jong \& Chen } 1966 \text {, } \\
\text { Mittal et al. } 1990\end{array}$ \\
\hline & & seeds & Quercus spp. & Scotland & Anderson 1986a \\
\hline $\begin{array}{l}\text { Pestalotiopsis spp. } \\
\text { [as Pestalosphaeria] }\end{array}$ & & seed & Sesbania bispinosa & India & Anita et al. 2009 \\
\hline $\begin{array}{l}\text { Pestalotiopsis versicolor } \\
\text { (Speg.) Steyaert }\end{array}$ & $\begin{array}{l}\text { Sporocadaceae, Sordariomycetes, } \\
\text { Ascomycota }\end{array}$ & fruits & $\begin{array}{l}\text { Viburnum } \\
\text { sempervirens }\end{array}$ & Hong Kong & Tang et al. 2003a \\
\hline Peyronellaea sp. & $\begin{array}{l}\text { Didymellaceae, Dothideomycetes, } \\
\text { Ascomycota }\end{array}$ & seeds & $\begin{array}{l}\text { Platanus } \\
\text { occidentalis }\end{array}$ & USA & $\begin{array}{l}\text { Fakir et al. 1971, Mittal } \\
\text { et al. } 1990\end{array}$ \\
\hline $\begin{array}{l}\text { Pezicula cinnamomea (DC.) } \\
\text { Sacc. }\end{array}$ & $\begin{array}{l}\text { Dermateaceae, Leotiomycetes, } \\
\text { Ascomycota }\end{array}$ & acorns & Quercus robur & Poland & Jankowiak 2008 \\
\hline $\begin{array}{l}\text { Pezicula eucalypti Korf \& } \\
\text { Iturr }^{\#}\end{array}$ & $\begin{array}{l}\text { Dermateaceae, Leotiomycetes, } \\
\text { Ascomycota }\end{array}$ & capsule & Eucalyptus sp. & Spain & Iturriaga \& Korf 1997 \\
\hline Peziza sciophila Medardi ${ }^{\#}$ & Pezizaceae, Pezizomycetes, Ascomycota & cones & Picea sp. & Italy & Medardi 2007 \\
\hline $\begin{array}{l}\text { Phaeoisaria clematidis } \\
\text { (Fuckel) S. Hughes }\end{array}$ & $\begin{array}{l}\text { Pleurotheciaceae, Sordariomycetes, } \\
\text { Ascomycota }\end{array}$ & pods & Delonix regia & Thailand & Somrithipol et al. 2002b \\
\hline $\begin{array}{l}\text { Phaeomarasmius } \\
\text { laccarioides Har. Takah.\# }\end{array}$ & $\begin{array}{l}\text { Tubariaceae, Agaricomycetes, } \\
\text { Basidiomycota }\end{array}$ & $\begin{array}{l}\text { fruits } \\
\text { fruit }\end{array}$ & $\begin{array}{l}\text { Microcos paniculata } \\
\text { Liquidambar } \\
\text { styraciflua }\end{array}$ & $\begin{array}{l}\text { Hong Kong } \\
\text { Japan }\end{array}$ & $\begin{array}{l}\text { Tang et al. 2003a } \\
\text { Takahashi } 2001\end{array}$ \\
\hline $\begin{array}{l}\text { Phaeosphaeria lunariae } \\
\text { Crous \& R.K. Schumach." }\end{array}$ & $\begin{array}{l}\text { Phaeosphaeriaceae, Dothideomycetes, } \\
\text { Ascomycota }\end{array}$ & pods & Lunaria annua & Germany & $\begin{array}{l}\text { Hernandez-Restrepo et } \\
\text { al. } 2016\end{array}$ \\
\hline $\begin{array}{l}\text { Phaeosphaeria sinensis } \\
\text { Jayasiri, E.B.G. Jones \& } \\
\text { K.D. Hyde }\end{array}$ & $\begin{array}{l}\text { Phaeosphaeriaceae, Dothideomycetes, } \\
\text { Ascomycota }\end{array}$ & pod & Wisteria sp. & China & Jayasiri et al. 2019 \\
\hline $\begin{array}{l}\text { Phialophora atrovirens } \\
\text { (J.F.H. Beyma) Schol- }\end{array}$ & $\begin{array}{l}\text { Herpotrichiellaceae, Eurotiomycetes, } \\
\text { Ascomycota }\end{array}$ & seed & $\begin{array}{l}\text { Cryptomeria } \\
\text { japonica }\end{array}$ & Japan & Watanabe 2010 \\
\hline Schwarz & & seed & Pinus thunbergii & Japan & Watanabe 2010 \\
\hline Phialophora spp. & $\begin{array}{l}\text { Herpotrichiellaceae, Eurotiomycetes, } \\
\text { Ascomycota }\end{array}$ & acorns & Quercus robur & Poland & Jankowiak 2008 \\
\hline
\end{tabular}


Table 2 Continued.

\begin{tabular}{|c|c|c|c|c|c|}
\hline Species & Taxonomy & Substrate & Host & Country & References \\
\hline & & seed & $\begin{array}{l}\text { Cryptomeria } \\
\text { japonica }\end{array}$ & Japan & Watanabe 2010 \\
\hline \multirow[t]{2}{*}{$\begin{array}{l}\text { Phlyctochytrium aureliae } \\
\text { Ajello }\end{array}$} & $\begin{array}{l}\text { Phlyctochytriaceae, Chytridiomycetes, } \\
\text { Chytridiomycota }\end{array}$ & seeds & Arum korolkowii & $\begin{array}{l}\text { Poland or/ } \\
\text { imported to Poland }\end{array}$ & Czeczuga et al. 2009 \\
\hline & & seeds & $\begin{array}{l}\text { Zantedeschia albo- } \\
\text { maculata }\end{array}$ & $\begin{array}{l}\text { Poland or/ } \\
\text { imported to Poland }\end{array}$ & Czeczuga et al. 2009 \\
\hline Phoma anceps Sacc. & $\begin{array}{l}\text { Didymellaceae, Dothideomycetes, } \\
\text { Ascomycota }\end{array}$ & seed & Medicago sativa & Canada & Conners 1967 \\
\hline Phoma araucariae Traverso & $\begin{array}{l}\text { Didymellaceae, Dothideomycetes, } \\
\text { Ascomycota }\end{array}$ & seeds & Araucaria excelsa & USA & Mittal et al. 1990 \\
\hline Phoma herbarum Westend. & $\begin{array}{l}\text { Didymellaceae, Dothideomycetes, } \\
\text { Ascomycota }\end{array}$ & seeds & Larix occidentalis & USA & James et al. 1996 \\
\hline \multirow[t]{3}{*}{$\begin{array}{l}\text { Phoma hibernica Grimes, } \\
\text { M. O'Connor \& Cummins }\end{array}$} & $\begin{array}{l}\text { Didymellaceae, Dothideomycetes, } \\
\text { Ascomycota }\end{array}$ & seeds & Cedrus deodara & India & $\begin{array}{l}\text { Munjal \& Sharma 1975, } \\
\text { Mittal et al. } 1990\end{array}$ \\
\hline & & seeds & Pinus roxburghii & India & $\begin{array}{l}\text { Munjal \& Sharma 1975, } \\
\text { Mittal et al. } 1990\end{array}$ \\
\hline & & seeds & Pinus wallichiana & India & $\begin{array}{l}\text { Munjal \& Sharma } 1975 \text {, } \\
\text { Mittal et al. } 1990\end{array}$ \\
\hline Phoma lineolata Desm. & $\begin{array}{l}\text { Didymellaceae, Dothideomycetes, } \\
\text { Ascomycota }\end{array}$ & seeds & Larix spp. & $\begin{array}{l}\text { Denmark, UK, } \\
\text { USA }\end{array}$ & $\begin{array}{l}\text { Noble et al. 1958, } \\
\text { Anderson 1986a }\end{array}$ \\
\hline \multirow[t]{10}{*}{ Phoma spp. } & $\begin{array}{l}\text { Didymellaceae, Dothideomycetes, } \\
\text { Ascomycota }\end{array}$ & acorns & Quercus robur & Poland & Jankowiak 2008 \\
\hline & & cones, seeds & Pinus sylvestris & Canada & Vujanovic et al. 2000 \\
\hline & & cones, seeds & $\begin{array}{l}\text { Pinus sylvestris } \\
\text { 'Watereri' }\end{array}$ & Canada & Vujanovic et al. 2000 \\
\hline & & fruits & Alocasia odora & Hong Kong & Tang et al. 2003a \\
\hline & & fruits & Ardisia crenata & Hong Kong & Tang et al. 2003a \\
\hline & & fruits & Bridelia tomentosa & Hong Kong & Tang et al. 2003a \\
\hline & & fruits & $\begin{array}{l}\text { Cleistocalyx } \\
\text { operculatus }\end{array}$ & Hong Kong & Tang et al. 2003a \\
\hline & & fruits & Dichroa febrifuga & Hong Kong & Tang et al. 2003a \\
\hline & & fruits & Ilex asprella & Hong Kong & Tang et al. 2003a \\
\hline & & fruits & Ilex cinerea & Hong Kong & Tang et al. 2003a \\
\hline
\end{tabular}


Table 2 Continued.

\begin{tabular}{|c|c|c|c|c|c|}
\hline Species & Taxonomy & Substrate & Host & Country & References \\
\hline & & fruits & Ilex pubescens & Hong Kong & Tang et al. 2003a \\
\hline & & fruits & Litsea rotundifolia & Hong Kong & Tang et al. 2003a \\
\hline & & fruits & Psychotria asiatica & Hong Kong & Tang et al. 2003a \\
\hline & & fruits & $\begin{array}{l}\text { Viburnum } \\
\text { sempervirens }\end{array}$ & Hong Kong & Tang et al. 2003a \\
\hline & & pod & $\begin{array}{l}\text { Colophospermum } \\
\text { mopane }\end{array}$ & Southern Africa & Jordaan et al. 2006 \\
\hline & & pods & Delonix regia & Thailand & Somrithipol et al. 2002b \\
\hline & & seed & Larix sp. & Japan & Watanabe 2010 \\
\hline & & seed & Prunus serrulata & Japan & Watanabe 2010 \\
\hline & & seeds & Abies alba & Poland & Krol et al. 2015 \\
\hline & & seeds & Abies lasiocarpa & Canada, Norway & Talgø et al. 2010 \\
\hline & & seeds & Abies nordmanniana & $\begin{array}{l}\text { Austria, Georgia, } \\
\text { Russia }\end{array}$ & Talgø et al. 2010 \\
\hline & & seeds & Abies procera & Norway & Talgø et al. 2010 \\
\hline & & seeds & $\begin{array}{l}\text { Acacia } \\
\text { auriculiformis }\end{array}$ & Philippines & $\begin{array}{l}\text { Mathur 1974, Anderson } \\
\text { 1986a, Quiniones } 1987\end{array}$ \\
\hline & & seeds & Acacia confusa & Philippines & $\begin{array}{l}\text { Agmata 1979, Anderson } \\
\text { 1986a }\end{array}$ \\
\hline & & seeds & Acacia modesta & India & $\begin{array}{l}\text { Mathur 1974, Anderson } \\
\text { 1986a }\end{array}$ \\
\hline & & seeds & Acacia raddiana & Israel & $\begin{array}{l}\text { Mathur 1974, Anderson } \\
\text { 1986a }\end{array}$ \\
\hline & & seeds & Acacia spp. & Egypt & $\begin{array}{l}\text { Mathur 1974, Anderson } \\
\text { 1986a }\end{array}$ \\
\hline & & seeds & Acer palmatum & South Korea & $\begin{array}{l}\text { Mathur 1974, Anderson } \\
\text { 1986a }\end{array}$ \\
\hline & & seeds & Acer pseudoplatanus & Poland & Krol et al. 2015 \\
\hline & & seeds & $\begin{array}{l}\text { Acrocarpus } \\
\text { fraxinifolius }\end{array}$ & India, Rwanda & $\begin{array}{l}\text { Mathur 1974, Anderson } \\
\text { 1986a }\end{array}$ \\
\hline & & seeds & $\begin{array}{l}\text { Adenanthera } \\
\text { microsperma }\end{array}$ & India & $\begin{array}{l}\text { Mathur 1974, Anderson } \\
\text { 1986a }\end{array}$ \\
\hline
\end{tabular}


Table 2 Continued.

\begin{tabular}{|c|c|c|c|c|c|}
\hline Species & Taxonomy & Substrate & Host & Country & References \\
\hline & & seeds & Adina cordifolia & India & $\begin{array}{l}\text { Mathur 1974, Anderson } \\
\text { 1986a }\end{array}$ \\
\hline & & seeds & Agathis robusta & Philippines , UK & $\begin{array}{l}\text { Quiniones 1985, 1987, } \\
\text { Anderson 1986a }\end{array}$ \\
\hline & & seeds & Albizia falcataria & Philippines & $\begin{array}{l}\text { Mathur 1974, Mittal et } \\
\text { al. } 1990\end{array}$ \\
\hline & & seeds & Albizia lebbeck & Philippines & Quiniones 1987 \\
\hline & & seeds & Albizia stipulata & India & $\begin{array}{l}\text { Mathur 1974, Anderson } \\
\text { 1986a }\end{array}$ \\
\hline & & seeds & Alnus glutinosa & Poland & Krol et al. 2015 \\
\hline & & seeds & Alnus sibirica & South Korea & $\begin{array}{l}\text { Mathur 1974, Mittal et } \\
\text { al. } 1990\end{array}$ \\
\hline & & seeds & $\begin{array}{l}\text { Alstonia } \\
\text { macrophylla }\end{array}$ & Philippines & Quiniones 1987 \\
\hline & & seeds & Anogeissus pendula & Costa Rica & $\begin{array}{l}\text { Mathur 1974, Mittal et } \\
\text { al. } 1990\end{array}$ \\
\hline & & seeds & $\begin{array}{l}\text { Anthocephalus } \\
\text { cadamba }\end{array}$ & India & $\begin{array}{l}\text { Mathur 1974, Mittal et } \\
\text { al. } 1990\end{array}$ \\
\hline & & seeds & Azadirachta indica & India & $\begin{array}{l}\text { Mathur 1974, Mittal et } \\
\text { al. } 1990\end{array}$ \\
\hline & & seeds & Bauhinia sp. & Thailand & $\begin{array}{l}\text { Chalermpongse et al. } \\
\text { 1984, Mittal et al. } 1990\end{array}$ \\
\hline & & seeds & Bauhinia variegata & India & $\begin{array}{l}\text { Mathur 1974, Mittal et } \\
\text { al. } 1990\end{array}$ \\
\hline & & seeds & Betula pendula & Poland & Krol et al. 2015 \\
\hline & & seeds & Bombax ceiba & India & $\begin{array}{l}\text { Mathur 1974, Mittal et } \\
\text { al. } 1990\end{array}$ \\
\hline & & seeds & $\begin{array}{l}\text { Callistephus } \\
\text { chinensis }\end{array}$ & China & $\begin{array}{l}\text { Gloyer 1931, Crosier \& } \\
\text { Heit } 1948\end{array}$ \\
\hline & & seeds & Cassia fistula & Thailand & $\begin{array}{l}\text { Chalermpongse et al. } \\
\text { 1984, Mittal et al. } 1990\end{array}$ \\
\hline & & seeds & Cassia siamea & Philippines & $\begin{array}{l}\text { Quiniones 1985, Mittal } \\
\text { et al. } 1990\end{array}$ \\
\hline
\end{tabular}


Table 2 Continued.

\begin{tabular}{|c|c|c|c|c|c|}
\hline Species & Taxonomy & Substrate & Host & Country & References \\
\hline & & seeds & $\begin{array}{l}\text { Casuarina } \\
\text { equisetifolia }\end{array}$ & Philippines & $\begin{array}{l}\text { Mathur 1974, Anderson } \\
\text { 1986a }\end{array}$ \\
\hline & & seeds & Cedrela odorata & Colombia & $\begin{array}{l}\text { Mathur 1974, Anderson } \\
\text { 1986a }\end{array}$ \\
\hline & & seeds & Cedrela serrata & India & $\begin{array}{l}\text { Mathur 1974, Anderson } \\
\text { 1986a }\end{array}$ \\
\hline & & seeds & Cedrela serrulata & Rwanda & $\begin{array}{l}\text { Mathur 1974, Anderson } \\
\text { 1986a }\end{array}$ \\
\hline & & seeds & Chukrasia tabularis & India & $\begin{array}{l}\text { Mathur 1974, Anderson } \\
\text { 1986a }\end{array}$ \\
\hline & & seeds & Cordia alliodora & Colombia & $\begin{array}{l}\text { Mathur 1974, Anderson } \\
\text { 1986a }\end{array}$ \\
\hline & & seeds & $\begin{array}{l}\text { Cryptomeria } \\
\text { japonica }\end{array}$ & Madagascar & $\begin{array}{l}\text { Mathur 1974, Anderson } \\
\text { 1986a }\end{array}$ \\
\hline & & seeds & $\begin{array}{l}\text { Cupressus } \\
\text { cashmeriana }\end{array}$ & India & $\begin{array}{l}\text { Mathur 1974, Anderson } \\
\text { 1986a }\end{array}$ \\
\hline & & seeds & Cupressus lusitanica & India & $\begin{array}{l}\text { Mathur 1974, Anderson } \\
\text { 1986a }\end{array}$ \\
\hline & & seeds & Cupressus spp. & Syria & $\begin{array}{l}\text { Mathur 1974, Anderson } \\
\text { 1986a }\end{array}$ \\
\hline & & seeds & Dalbergia sissoo & India & $\begin{array}{l}\text { Mittal \& Sharma 1981b, } \\
\text { Mittal et al. } 1990\end{array}$ \\
\hline & & seeds & Delonix regia & India, Brazil & $\begin{array}{l}\text { Mathur 1974, Anderson } \\
\text { 1986a }\end{array}$ \\
\hline & & seeds & $\begin{array}{l}\text { Dianthus } \\
\text { caryophyllus }\end{array}$ & Taiwan & Li \& Wu 2002 \\
\hline & & seeds & Dianthus spp. & Taiwan & Li \& Wu 2002 \\
\hline & & seeds & Eucalyptus alba & India & $\begin{array}{l}\text { Mathur 1974, Anderson } \\
\text { 1986a }\end{array}$ \\
\hline & & seeds & $\begin{array}{l}\text { Eucalyptus } \\
\text { camaldulensis }\end{array}$ & India & $\begin{array}{l}\text { Mathur 1974, Anderson } \\
\text { 1986a }\end{array}$ \\
\hline & & seeds & Eucalyptus grandis & India, South Africa & $\begin{array}{l}\text { Mathur 1974, Anderson } \\
\text { 1986a, Jimu et al. } 2016\end{array}$ \\
\hline
\end{tabular}


Table 2 Continued.

\begin{tabular}{|c|c|c|c|c|c|}
\hline Species & Taxonomy & Substrate & Host & Country & References \\
\hline & & seeds & Eucalyptus sp. & India & $\begin{array}{l}\text { Reddy et al. 1982, Mittal } \\
\text { et al. } 1990\end{array}$ \\
\hline & & seeds & $\begin{array}{l}\text { Eucalyptus } \\
\text { tereticornis }\end{array}$ & India & $\begin{array}{l}\text { Mathur 1974, Anderson } \\
\text { 1986a }\end{array}$ \\
\hline & & seeds & Eucommia ulmoides & USA & $\begin{array}{l}\text { Mathur 1974, Anderson } \\
\text { 1986a }\end{array}$ \\
\hline & & seeds & Fraxinus excelsior & Sweden & $\begin{array}{l}\text { Cleary et al. 2013, } \\
\text { Hayatgheibi } 2013\end{array}$ \\
\hline & & seeds & Gliricidia sepium & Philippines & Quiniones 1987 \\
\hline & & seeds & Gmelina arborea & India, Philippines & $\begin{array}{l}\text { Mathur 1974, Anderson } \\
\text { 1986a, Quiniones } 1987\end{array}$ \\
\hline & & seeds & Hovenia dulcis & India & $\begin{array}{l}\text { Mathur 1974, Anderson } \\
\text { 1986a }\end{array}$ \\
\hline & & seeds & $\begin{array}{l}\text { Jacaranda } \\
\text { mimosifolia }\end{array}$ & India & $\begin{array}{l}\text { Mathur 1974, Anderson } \\
\text { 1986a }\end{array}$ \\
\hline & & seeds & Kydia calycina & India & $\begin{array}{l}\text { Mathur 1974, Anderson } \\
\text { 1986a }\end{array}$ \\
\hline & & seeds & $\begin{array}{l}\text { Lagerstroemia } \\
\text { speciosa }\end{array}$ & India, Philippines & $\begin{array}{l}\text { Mathur 1974, Anderson } \\
\text { 1986a, Quiniones } 1987\end{array}$ \\
\hline & & seeds & $\begin{array}{l}\text { Leucaena } \\
\text { leucocephala }\end{array}$ & Philippines & $\begin{array}{l}\text { Quiniones 1985, 1987, } \\
\text { Mittal et al. } 1990\end{array}$ \\
\hline & & Seeds & $\begin{array}{l}\text { Leucaena } \\
\text { leucocephala var. } \\
\text { Cunningham }\end{array}$ & Cuba & $\begin{array}{l}\text { Mathur 1974, Anderson } \\
\text { 1986a }\end{array}$ \\
\hline & & seeds & Leucaena spp. & Philippines & $\begin{array}{l}\text { Mathur 1974, Anderson } \\
\text { 1986a }\end{array}$ \\
\hline & & seeds & $\begin{array}{l}\text { Leucdena } \\
\text { diversifolia }\end{array}$ & Guatemala & $\begin{array}{l}\text { Mathur 1974, Anderson } \\
\text { 1986a }\end{array}$ \\
\hline & & seeds & Maesopsis eminii & Malaysia & Mittal et al. 1990 \\
\hline & & seeds & $\begin{array}{l}\text { Mimosa } \\
\text { caesalpiniafolia }\end{array}$ & Brazil & $\begin{array}{l}\text { Mathur 1974, Anderson } \\
\text { 1986a }\end{array}$ \\
\hline & & seeds & $\begin{array}{l}\text { Ougeinia } \\
\text { dalbergioides }\end{array}$ & - & $\begin{array}{l}\text { Mathur 1974, Anderson } \\
\text { 1986a }\end{array}$ \\
\hline
\end{tabular}


Table 2 Continued.

\begin{tabular}{|c|c|c|c|c|c|}
\hline Species & Taxonomy & Substrate & Host & Country & References \\
\hline & & seeds & Parkia roxburghii & Philippines & Quiniones 1987 \\
\hline & & seeds & Phyllostachys edulis & China & Shen et al. 2014 \\
\hline & & seeds & Picea abies & Italy & Motta et al. 1996 \\
\hline & & seeds & $\begin{array}{l}\text { Pinus armandii var. } \\
\text { mastersiana }\end{array}$ & Taiwan & $\begin{array}{l}\text { Jong \& Chen } 1966 \text {, } \\
\text { Mittal et al. } 1990\end{array}$ \\
\hline & & seeds & Pinus caribaea & $\begin{array}{l}\text { Belize [as British } \\
\text { Honduras] }\end{array}$ & Hocking 1968 \\
\hline & & seeds & $\begin{array}{l}\text { Pinus caribaea var. } \\
\text { hondurensis }\end{array}$ & India & $\begin{array}{l}\text { Mathur 1974, Anderson } \\
\text { 1986a }\end{array}$ \\
\hline & & seeds & Pinus insularis & Philippines & $\begin{array}{l}\text { Agmata 1979, Anderson } \\
\text { 1986a }\end{array}$ \\
\hline & & seeds & Pinus kesiya & Philippines & Quiniones 1987 \\
\hline & & seeds & Pinus khasya & $\begin{array}{l}\text { Philippines, } \\
\text { Zambia }\end{array}$ & $\begin{array}{l}\text { Mathur 1974, Anderson } \\
\text { 1986a, Mittal et al. } 1990\end{array}$ \\
\hline & & seeds & Pinus merkusii & Zambia & $\begin{array}{l}\text { Chalermpongse et al. } \\
\text { 1984, Anderson 1986a }\end{array}$ \\
\hline & & seeds & Pinus oocarpa & Central America & $\begin{array}{l}\text { Mathur 1974, Anderson } \\
\text { 1986a }\end{array}$ \\
\hline & & seeds & Pinus ponderosa & USA & $\begin{array}{l}\text { James \& Genz 1982, } \\
\text { Anderson 1986a }\end{array}$ \\
\hline & & seeds & Pinus spp. & Japan & $\begin{array}{l}\text { Homechin et al. 1986, } \\
\text { Anderson 1986a }\end{array}$ \\
\hline & & seeds & Pinus sylvestris & Poland & Krol et al. 2015 \\
\hline & & seeds & $\begin{array}{l}\text { Platanus } \\
\text { occidentalis }\end{array}$ & USA & $\begin{array}{l}\text { Fakir et al. 1971, Mittal } \\
\text { et al. } 1990\end{array}$ \\
\hline & & seeds & Polyscias nodosa & Philippines & $\begin{array}{l}\text { Agmata 1979, Mittal et } \\
\text { al. } 1990\end{array}$ \\
\hline & & seeds & Pongamia pinnata & India & $\begin{array}{l}\text { Jamaluddin et al. 1983, } \\
\text { Mittal et al. } 1990\end{array}$ \\
\hline & & seeds & $\begin{array}{l}\text { Pseudotsuga } \\
\text { menziesii }\end{array}$ & USA & $\begin{array}{l}\text { Gordon 1967, Mittal et } \\
\text { al. } 1990\end{array}$ \\
\hline & & seeds & Pterocarpus indicus & Philippines & $\begin{array}{l}\text { Agmata 1979, Anderson } \\
\text { 1986a }\end{array}$ \\
\hline
\end{tabular}


Table 2 Continued.

\begin{tabular}{|c|c|c|c|c|c|}
\hline Species & Taxonomy & Substrate & Host & Country & References \\
\hline & & seeds & $\begin{array}{l}\text { Pterospermum } \\
\text { acerifolium }\end{array}$ & India & $\begin{array}{l}\text { Mathur 1974, Anderson } \\
\text { 1986a }\end{array}$ \\
\hline & & seeds & Quercus robur & Germany & Schroder et al. 2004 \\
\hline & & seeds & Samanea saman & $\begin{array}{l}\text { Philippines , } \\
\text { Thailand }\end{array}$ & $\begin{array}{l}\text { Chalermpongse et al. } \\
\text { 1984, Quiniones 1987, } \\
\text { Mittal et al. } 1990\end{array}$ \\
\hline & & seeds & $\begin{array}{l}\text { Sequoia } \\
\text { sempervirens }\end{array}$ & USA & $\begin{array}{l}\text { Davidson 1970, Mittal et } \\
\text { al. } 1990\end{array}$ \\
\hline & & seeds & Serialbizia acle & Philippines & Quiniones 1987 \\
\hline & & seeds & Sesbania sesban & Rwanda & $\begin{array}{l}\text { Mathur 1974, Anderson } \\
\text { 1986a }\end{array}$ \\
\hline & & seeds & Shorea materialis & Malaysia & Mittal et al. 1990 \\
\hline & & seeds & Shorea robusta & Thailand & $\begin{array}{l}\text { Chalermpongse et al. } \\
\text { 1984, Mittal et al. } 1990\end{array}$ \\
\hline & & seeds & $\begin{array}{l}\text { Swietenia } \\
\text { macrophylla }\end{array}$ & Philippines & $\begin{array}{l}\text { Agmata 1979, Anderson } \\
\text { 1986a, Quiniones } 1987\end{array}$ \\
\hline & & seeds & $\begin{array}{l}\text { Taxodium } \\
\text { mucronatum }\end{array}$ & India & $\begin{array}{l}\text { Mathur 1974, Anderson } \\
\text { 1986a }\end{array}$ \\
\hline & & seeds & Tectona grandis & India, Philippines & $\begin{array}{l}\text { Mathur 1974, Mittal et } \\
\text { al. 1990, Mohanan et al. } \\
2005\end{array}$ \\
\hline & & seeds & Toona calantas & Philippines & Quiniones 1987 \\
\hline & & seeds & Pinus sylvestris & Poland & Krol et al. 2015 \\
\hline & & seeds & $\begin{array}{l}\text { Wallaceodendron } \\
\text { celibcum }\end{array}$ & Philippines & Quiniones 1987 \\
\hline Phoma strobiligena Desm. & $\begin{array}{l}\text { Didymellaceae, Dothideomycetes, } \\
\text { Ascomycota }\end{array}$ & seeds & Abies sibirica & USSR & $\begin{array}{l}\text { Prisyazhnyuk 1960, } \\
\text { Mittal et al. } 1990\end{array}$ \\
\hline \multirow[t]{2}{*}{$\begin{array}{l}\text { Phragmotrichum chailletii } \\
\text { Kunze }^{\#}\end{array}$} & $\begin{array}{l}\text { Melanommataceae, Dothideomycetes, } \\
\text { Ascomycota }\end{array}$ & cones & Abies sp. & Switzerland & Schmidt \& Kunze 1823 \\
\hline & & seeds & Pinus sylvestris & USSR & $\begin{array}{l}\text { Prisyazhnyuk } 1960 \text {, } \\
\text { Mittal et al. } 1990\end{array}$ \\
\hline $\begin{array}{l}\text { Phyllosticta aucubae- } \\
\text { japonicae N. Zhou \& L. Cai\# }\end{array}$ & $\begin{array}{l}\text { Phyllostictaceae, Dothideomycetes, } \\
\text { Ascomycota }\end{array}$ & fruit & Aucuba japonica & Japan & $\begin{array}{l}\text { Hernandez-Restrepo et } \\
\text { al. } 2016\end{array}$ \\
\hline
\end{tabular}


Table 2 Continued.

\begin{tabular}{|c|c|c|c|c|c|}
\hline Species & Taxonomy & Substrate & Host & Country & References \\
\hline Phyllosticta cocoicola (Bat.) & Phyllostictaceae, Dothideomycetes, & fruits & Diplospora dubia & Hong Kong & Tang et al. 2003a \\
\hline \multirow[t]{2}{*}{ Sivan. } & Ascomycota & fruits & Ilex cinerea & Hong Kong & Tang et al. 2003a \\
\hline & & fruits & Microcos paniculata & Hong Kong & Tang et al. 2003a \\
\hline $\begin{array}{l}\text { Phyllosticta osteospora var. } \\
\text { samaricola D. Sacc. }\end{array}$ & $\begin{array}{l}\text { Phyllostictaceae, Dothideomycetes, } \\
\text { Ascomycota }\end{array}$ & seeds & Fraxinus sp. & USSR & Anderson 1986a \\
\hline $\begin{array}{l}\text { Phyllosticta platanoides f. } \\
\text { negundinis Sacc. }\end{array}$ & $\begin{array}{l}\text { Phyllostictaceae, Dothideomycetes, } \\
\text { Ascomycota }\end{array}$ & seeds & Acer sp. & USSR & Anderson 1986a \\
\hline \multirow[t]{3}{*}{ Phyllosticta spp. } & $\begin{array}{l}\text { Phyllostictaceae, Dothideomycetes, } \\
\text { Ascomycota }\end{array}$ & seeds & Acer sp. & - & $\begin{array}{l}\text { Noble et al. 1958, } \\
\text { Anderson 1986a }\end{array}$ \\
\hline & & seeds & Alnus glutinosa & Poland & Krol et al. 2015 \\
\hline & & seeds & Betula pendula & Poland & Krol et al. 2015 \\
\hline $\begin{array}{l}\text { Physalidiella elegans (Luppi } \\
\text { Mosca) Rulamort } \\
\text { [as Physalidium elegans } \\
\text { Luppi Mosca] }\end{array}$ & $\begin{array}{l}\text { Incertae sedis, Incertae sedis, } \\
\text { Ascomycota }\end{array}$ & pods & Delonix regia & Thailand & Somrithipol et al. 2002b \\
\hline \multirow[t]{4}{*}{$\begin{array}{l}\text { Physalospora obtusa } \\
\text { (Schwein.) Cooke }\end{array}$} & $\begin{array}{l}\text { Didymellaceae, Dothideomycetes, } \\
\text { Ascomycota }\end{array}$ & seeds & Acer rubrum & USA & $\begin{array}{l}\text { Friedrich et al. } 1971 \text {, } \\
\text { Mittal et al. } 1990\end{array}$ \\
\hline & & seeds & Acer saccharinum & USA & $\begin{array}{l}\text { Friedrich et al. } 1971 \text {, } \\
\text { Mittal et al. } 1990\end{array}$ \\
\hline & & seeds & Acer saccharum & USA & $\begin{array}{l}\text { Friedrich et al. } 1971 \text {, } \\
\text { Mittal et al. } 1990\end{array}$ \\
\hline & & seeds & Fraxinus americana & USA & $\begin{array}{l}\text { Friedrich et al. } 1971 \text {, } \\
\text { Mittal et al. } 1990\end{array}$ \\
\hline $\begin{array}{l}\text { Physocladia obscura } \\
\text { (Sparrow) Sparrow }\end{array}$ & $\begin{array}{l}\text { Chytriomycetaceae, Chytridiomycetes, } \\
\text { Chytridiomycota }\end{array}$ & seeds & $\begin{array}{l}\text { Zantedeschia } \\
\text { aethiopica }\end{array}$ & $\begin{array}{l}\text { Poland or/ } \\
\text { imported to Poland }\end{array}$ & Czeczuga et al. 2009 \\
\hline
\end{tabular}


Table 2 Continued.

\begin{tabular}{|c|c|c|c|c|c|}
\hline Species & Taxonomy & Substrate & Host & Country & References \\
\hline $\begin{array}{l}\text { Pichia kluyveri var. } \\
\text { kluyveri Bedford ex } \\
\text { Kudryavtsev }\end{array}$ & $\begin{array}{l}\text { Pichiaceae, Saccharomycetes, } \\
\text { Ascomycota }\end{array}$ & fruits & $\begin{array}{l}\text { Parahancornia } \\
\text { amapa }\end{array}$ & Brazil & Morais et al. 1995 \\
\hline Pichia kluyveri-like & Saccharomycetes, Ascomycota & fruits & $\begin{array}{l}\text { Parahancornia } \\
\text { amapa }\end{array}$ & Brazil & Morais et al. 1995 \\
\hline Pichia membranifaciens-like & Saccharomycetes, Ascomycota & fruits & $\begin{array}{l}\text { Parahancornia } \\
\text { amapa }\end{array}$ & Brazil & Morais et al. 1995 \\
\hline Pichia muscicola-like & Saccharomycetes, Ascomycota & fruits & $\begin{array}{l}\text { Parahancornia } \\
\text { amapa }\end{array}$ & Brazil & Morais et al. 1995 \\
\hline Pichia sargentensis-like & Saccharomycetes, Ascomycota & fruits & $\begin{array}{l}\text { Parahancornia } \\
\text { amapa }\end{array}$ & Brazil & Morais et al. 1995 \\
\hline $\begin{array}{l}\text { Pilaira anomala (Ces.) J. } \\
\text { Schröt. }\end{array}$ & $\begin{array}{l}\text { Mucoraceae, Mucoromycetes, } \\
\text { Mucoromycota }\end{array}$ & seeds & Rosa mollis & $\begin{array}{l}\text { Poland or/ } \\
\text { imported to Poland }\end{array}$ & Czeczuga et al. 2009 \\
\hline Pilobolus sp. & $\begin{array}{l}\text { Pilobolaceae, Mucoromycetes, } \\
\text { Mucoromycota }\end{array}$ & fruits & Avicennia marina & India & Mehdi \& Saifullah 2000 \\
\hline $\begin{array}{l}\text { Pilocintractia adriana } \\
\text { Vánky }^{\#}\end{array}$ & $\begin{array}{l}\text { Anthracoideaceae, Ustilaginomycetes, } \\
\text { Basidiomycota }\end{array}$ & nutlets & $\begin{array}{l}\text { Fimbristylis } \\
\text { miliacea }\end{array}$ & India & Vánky 2006 \\
\hline Pilophora agaricina Wallr. & $\begin{array}{l}\text { Rhizopodaceae, Mucoromycetes, } \\
\text { Mucoromycota }\end{array}$ & pod & Canavalia cathartica & India & Anita \& Sridhar 2009 \\
\hline $\begin{array}{l}\text { Piptocephalis freseniana de } \\
\text { Bary }\end{array}$ & $\begin{array}{l}\text { Piptocephalidaceae, Zoopagomycetes, } \\
\text { Mucoromycota }\end{array}$ & seeds & Quercus spp. & USSR & $\begin{array}{l}\text { Urosevic 1959, Mittal et } \\
\text { al. } 1990\end{array}$ \\
\hline $\begin{array}{l}\text { Pithomyces alabamensis } \\
\text { (Matsush.) P.M. Kirk }\end{array}$ & $\begin{array}{l}\text { Astrosphaeriellaceae, Dothideomycetes, } \\
\text { Ascomycota }\end{array}$ & pods & Delonix regia & Thailand & Somrithipol et al. 2002b \\
\hline $\begin{array}{l}\text { Pithomyces cynodontis M.B. } \\
\text { Ellis }\end{array}$ & $\begin{array}{l}\text { Astrosphaeriellaceae, Dothideomycetes, } \\
\text { Ascomycota }\end{array}$ & seeds & $\begin{array}{l}\text { Casuarina spp., } \\
\text { Thuja spp. }\end{array}$ & India & Sahai \& Otra 1982 \\
\hline Pithomyces spp. & $\begin{array}{l}\text { Astrosphaeriellaceae, Dothideomycetes, } \\
\text { Ascomycota }\end{array}$ & seeds & Tectona grandis & India & Anderson 1986a \\
\hline $\begin{array}{l}\text { Pithyella hispida Rativ. \& R } \\
\text { Galán }{ }^{\#}\end{array}$ & Helotiaceae, Leotiomycetes, Ascomycota & fruit & Quercus agrifolia & USA & Galán et al. 1994 \\
\hline Pleiochaeta sp. & $\begin{array}{l}\text { Dothidotthiaceae, Dothideomycetes, } \\
\text { Ascomycota }\end{array}$ & seeds & $\begin{array}{l}\text { Araucaria } \\
\text { angustifolia }\end{array}$ & Australia & $\begin{array}{l}\text { Kamara et al. } 1981 \text {, } \\
\text { Mittal et al. } 1990\end{array}$ \\
\hline $\begin{array}{l}\text { Pleogibberella calamia } \\
\text { (Cooke) Berl. \& Voglino }\end{array}$ & $\begin{array}{l}\text { Nectriaceae, Sordariomycetes, } \\
\text { Ascomycota }\end{array}$ & fruit & $\begin{array}{l}\text { Calamus } \\
\text { fasciculatus }\end{array}$ & India & Cooke 1884 \\
\hline
\end{tabular}


Table 2 Continued.

\begin{tabular}{|c|c|c|c|c|c|}
\hline Species & Taxonomy & Substrate & Host & Country & References \\
\hline \multicolumn{6}{|l|}{$\begin{array}{l}\text { [as Gibberella calamia } \\
\text { Cooke] }\end{array}$} \\
\hline $\begin{array}{l}\text { Pleohelicoon fagi Jayasiri, } \\
\text { E.B.G. Jones \& K.D. } \\
\text { HydeK }{ }^{\# *}\end{array}$ & $\begin{array}{l}\text { Pleomonodictydaceae, Dothideomycetes, } \\
\text { Ascomycota }\end{array}$ & cupule & Fagus sylvatica & UK & Jayasiri et al. 2019 \\
\hline Pleospora infectoria Fuckel & $\begin{array}{l}\text { Pleosporaceae, Dothideomycetes, } \\
\text { Ascomycota }\end{array}$ & seeds & Eucalyptus sp. & India & $\begin{array}{l}\text { Saxena 1985, Mittal et } \\
\text { al. } 1990\end{array}$ \\
\hline Pleospora laricina Rehm & $\begin{array}{l}\text { Pleosporaceae, Dothideomycetes, } \\
\text { Ascomycota }\end{array}$ & cones & Pinus mugo 'Hesse' & Canada & Vujanovic et al. 2000 \\
\hline \multirow[t]{2}{*}{ Pleospora spp. } & $\begin{array}{l}\text { Pleosporaceae, Dothideomycetes, } \\
\text { Ascomycota }\end{array}$ & cones, seeds & Pinus ponderosa & Canada & Vujanovic et al. 2000 \\
\hline & & seeds & Fraxinus americana & USA & $\begin{array}{l}\text { Friedrich et al. } 1971 \text {, } \\
\text { Mittal et al. } 1990\end{array}$ \\
\hline Pleosporales spp. & Dothideomycetes, Ascomycota & $\begin{array}{l}\text { seeds } \\
\text { seeds }\end{array}$ & $\begin{array}{l}\text { Eucalyptus grandis } \\
\text { Fraxinus excelsior }\end{array}$ & $\begin{array}{l}\text { South Africa } \\
\text { Sweden }\end{array}$ & $\begin{array}{l}\text { Jimu et al. } 2016 \\
\text { Cleary et al. } 2013\end{array}$ \\
\hline $\begin{array}{l}\text { Pleurophoma italica } \\
\text { Tibpromma, Camporesi \& } \\
\text { K.D. Hyde }{ }^{\#}\end{array}$ & $\begin{array}{l}\text { Lentitheciaceae, Sordariomycetes, } \\
\text { Ascomycota }\end{array}$ & cone & Pinus nigra & Italy & Tibpromma et al. 2017 \\
\hline Pleurophragmium sp. & $\begin{array}{l}\text { Incertae sedis, Sordariomycetes, } \\
\text { Ascomycota }\end{array}$ & fruits & Microcos paniculata & Hong Kong & Tang et al. 2003a \\
\hline $\begin{array}{l}\text { Podospora bulbillosa (W. } \\
\text { Gams \& Mouch.) X. Wei } \\
\text { Wang \& Houbraken } \\
\text { [as Cladorrhinum } \\
\text { bulbillosum W. Gams \& } \\
\text { Mouch.] }\end{array}$ & $\begin{array}{l}\text { Podosporaceae, Sordariomycetes, } \\
\text { Ascomycota }\end{array}$ & seed & Prunus serrulata & Japan & Watanabe 2010 \\
\hline $\begin{array}{l}\text { Polydesmia turbinata Raitv. } \\
\text { \& R. Galán\# }\end{array}$ & $\begin{array}{l}\text { Amorphothecaceae, Leotiomycetes, } \\
\text { Ascomycota }\end{array}$ & fruit capsule & Eucalyptus globulus & Spain & Raitviír \& Galán 1995 \\
\hline \multirow[t]{3}{*}{$\begin{array}{l}\text { Polyphagus euglenae (Bail) } \\
\text { Nowak. }\end{array}$} & $\begin{array}{l}\text { Polyphagaceae, Chytridiomycetes, } \\
\text { Chytridiomycota }\end{array}$ & seeds & Atropa belladonna & $\begin{array}{l}\text { Poland or/ } \\
\text { imported to Poland }\end{array}$ & Czeczuga et al. 2009 \\
\hline & & seeds & Atropa caucasica & $\begin{array}{l}\text { Poland or/ } \\
\text { imported to Poland }\end{array}$ & Czeczuga et al. 2009 \\
\hline & & seeds & Atropa pallidiflora & $\begin{array}{l}\text { Poland or/ } \\
\text { imported to Poland }\end{array}$ & Czeczuga et al. 2009 \\
\hline
\end{tabular}


Table 2 Continued.

\begin{tabular}{|c|c|c|c|c|c|}
\hline Species & Taxonomy & Substrate & Host & Country & References \\
\hline & & seeds & Hyoscyamus albus & $\begin{array}{l}\text { Poland or/ } \\
\text { imported to Poland }\end{array}$ & Czeczuga et al. 2009 \\
\hline & & seeds & Paris quadrifolia & $\begin{array}{l}\text { Poland or/ } \\
\text { imported to Poland }\end{array}$ & Czeczuga et al. 2009 \\
\hline & & seeds & $\begin{array}{l}\text { Podophyllum } \\
\text { peltatum }\end{array}$ & $\begin{array}{l}\text { Poland or/ } \\
\text { imported to Poland }\end{array}$ & Czeczuga et al. 2009 \\
\hline Polyspora lini Laff. & $\begin{array}{l}\text { Saccotheciaceae, Dothideomycetes, } \\
\text { Ascomycota }\end{array}$ & seed & Linum usitatissimum & Canada & Conners 1967 \\
\hline $\begin{array}{l}\text { Propolis faginea (Schrad.) P. } \\
\text { Karst. }\end{array}$ & $\begin{array}{l}\text { Marthamycetaceae, Leotiomycetes, } \\
\text { Ascomycota }\end{array}$ & seeds & Larix sibirica & USSR & $\begin{array}{l}\text { Prisyazhnyuk } 1960 \text {, } \\
\text { Mittal et al. } 1990\end{array}$ \\
\hline Propolis occulta Chlebická\# & $\begin{array}{l}\text { Marthamycetaceae, Leotiomycetes, } \\
\text { Ascomycota }\end{array}$ & cones & Pinus sylvestris & Czech Republic & Chlebická 2014 \\
\hline $\begin{array}{l}\text { Propolis rhodoleuca } \\
\text { (Sommerf.) Fr. }\end{array}$ & $\begin{array}{l}\text { Marthamycetaceae, Leotiomycetes, } \\
\text { Ascomycota }\end{array}$ & seeds & Pinus sylvestris & USSR & $\begin{array}{l}\text { Dolejs 1964, Mittal et al. } \\
1990\end{array}$ \\
\hline \multirow{2}{*}{$\begin{array}{l}\text { Protrudomyces lateralis (A. } \\
\text { Braun) Letcher } \\
\text { [as Rhizophydium laterale } \\
\text { (A. Braun) Rabenh.] }\end{array}$} & $\begin{array}{l}\text { Protrudomycetaceae, } \\
\text { Rhizophydiomycetes, Chytridiomycota }\end{array}$ & seeds & Hyoscyamus niger & $\begin{array}{l}\text { Poland or/ } \\
\text { imported to Poland }\end{array}$ & Czeczuga et al. 2009 \\
\hline & & seeds & Ilex aquifolium & $\begin{array}{l}\text { Poland or/ } \\
\text { imported to Poland }\end{array}$ & Czeczuga et al. 2009 \\
\hline $\begin{array}{l}\text { Prosopidicola mexicana } \\
\text { Crous \& C.L. Lennox }{ }^{\# * *}\end{array}$ & $\begin{array}{l}\text { Prosopidicolaceae, Sordariomycetes, } \\
\text { Ascomycota }\end{array}$ & pods & Prosopis glandulosa & USA & Lennox et al. 2004 \\
\hline $\begin{array}{l}\text { Pseudoanthostomella pini- } \\
\text { nigrae Daranag., Camporesi } \\
\text { \& K.D. Hyde }{ }^{\#}\end{array}$ & $\begin{array}{l}\text { Incertae sedis, Sordariomycetes, } \\
\text { Ascomycota }\end{array}$ & cones & Pinus nigra & Italy & Daranagama et al. 2016 \\
\hline $\begin{array}{l}\text { Pseudoberkleasmium } \\
\text { acaciae Jayasiri, E.B.G. } \\
\text { Jones \& K.D. Hyde }\end{array}$ & $\begin{array}{l}\text { Pseudoberkleasmiaceae, } \\
\text { Dothideomycetes, Ascomycota }\end{array}$ & pod & Acacia sp. & Thailand & Jayasiri et al. 2019 \\
\hline $\begin{array}{l}\text { Pseudoboubovia benkertii } \\
\text { (B. Perić) U. Lindem., M. } \\
\text { Vega, B. Perić \& R. Tena } \\
\text { [as Kotlabaea benkertii B. } \\
\text { Perić] }\end{array}$ & $\begin{array}{l}\text { Pulvinulaceae, Pezizomycetes, } \\
\text { Ascomycota }\end{array}$ & cones & $\begin{array}{l}\text { Cupressus } \\
\text { sempervirens, Pinus } \\
\text { halepensis }\end{array}$ & Montenegro & Lindemann et al. 2015 \\
\hline
\end{tabular}


Table 2 Continued.

\begin{tabular}{|c|c|c|c|c|c|}
\hline Species & Taxonomy & Substrate & Host & Country & References \\
\hline $\begin{array}{l}\text { Pseudocamarosporium } \\
\text { piceae Wijayaw., Camporesi } \\
\text { \& K.D. Hyde }{ }^{\#}\end{array}$ & $\begin{array}{l}\text { Montagnulaceae, Dothideomycetes, } \\
\text { Ascomycota }\end{array}$ & cones & Picea excelsa & Italy & $\begin{array}{l}\text { Wijayawardene et al. } \\
2014\end{array}$ \\
\hline $\begin{array}{l}\text { Pseudocercospora } \\
\text { mapelanensis J.A. Osorio \& } \\
\text { Jol. Roux }{ }^{\#}\end{array}$ & $\begin{array}{l}\text { Mycosphaerellaceae, Dothideomycetes, } \\
\text { Ascomycota }\end{array}$ & fruits & $\begin{array}{l}\text { Barringtonia } \\
\text { racemosa }\end{array}$ & South Africa & Osorio et al. 2015 \\
\hline $\begin{array}{l}\text { Pseudocercospora ulei } \\
\text { (Henn.) B.T. Hora \& } \\
\text { Mizubuti [as Microcyclus } \\
\text { ulei (Henn.) Arx] }\end{array}$ & $\begin{array}{l}\text { Mycosphaerellaceae, Dothideomycetes, } \\
\text { Ascomycota }\end{array}$ & seeds & Hevea brasiliensis & $\begin{array}{l}\text { South and Central } \\
\text { America, } \\
\text { Caribbean }\end{array}$ & Mittal et al. 1990 \\
\hline $\begin{array}{l}\text { Pseudochaetosphaeronema } \\
\text { siamensis Jayasiri, E.B.G. } \\
\text { Jones \& K.D. Hyde" }\end{array}$ & $\begin{array}{l}\text { Macrodiplodiopsidaceae, } \\
\text { Dothideomycetes, Ascomycota }\end{array}$ & pod & Tamarindus sp. & Thailand & Jayasiri et al. 2019 \\
\hline $\begin{array}{l}\text { Pseudocoleophoma } \\
\text { bauhiniae Jayasiri, E.B.G. } \\
\text { Jones \& K.D. Hyde\# }\end{array}$ & $\begin{array}{l}\text { Dictyosporiaceae, Dothideomycetes, } \\
\text { Ascomycota }\end{array}$ & pod & Bauhinia sp. & Thailand & Jayasiri et al. 2019 \\
\hline $\begin{array}{l}\text { Pseudodictyosporium } \\
\text { wauense Matsush. }\end{array}$ & $\begin{array}{l}\text { Dictyosporiaceae, Dothideomycetes, } \\
\text { Ascomycota }\end{array}$ & fruit cupule & Fagus sylvatica & UK & Jayasiri et al. 2019 \\
\hline $\begin{array}{l}\text { Pseudofusicoccum } \\
\text { calophylli Jayasiri, E.B.G. } \\
\text { Jones \& K.D. Hyde }\end{array}$ & $\begin{array}{l}\text { Phyllostictaceae, Dothideomycetes, } \\
\text { Ascomycota }\end{array}$ & fruit & $\begin{array}{l}\text { Calophyllum } \\
\text { inophyllum }\end{array}$ & Thailand & Jayasiri et al. 2019 \\
\hline $\begin{array}{l}\text { Pseudohalonectria fagicola } \\
\text { R.H. Perera, E.B.G. Jones \& } \\
\text { K.D. Hyde }{ }^{\#}\end{array}$ & $\begin{array}{l}\text { Pseudohalonectriaceae, Sordariomycetes, } \\
\text { Ascomycota }\end{array}$ & cupule & Fagus sylvatica & UK & Perera et al. 2016a \\
\hline $\begin{array}{l}\text { Pseudohalonectria } \\
\text { hampshirensis R.H. Perera, } \\
\text { E.B.G. Jones \& K.D. Hyde }\end{array}$ & $\begin{array}{l}\text { Pseudohalonectriaceae, Sordariomycetes, } \\
\text { Ascomycota }\end{array}$ & cupule & Fagus sylvatica & UK & Perera et al. 2016a \\
\hline $\begin{array}{l}\text { Pseudohelicomyces } \\
\text { aquaticus Y.Z. Lu, Boonmee } \\
\text { \& K.D. Hyde }\end{array}$ & $\begin{array}{l}\text { Tubeufiaceae, Dothideomycetes, } \\
\text { Ascomycota }\end{array}$ & pod & Tamarindus indica & Thailand & Jayasiri et al. 2019 \\
\hline $\begin{array}{l}\text { Pseudohelicomyces } \\
\text { menglunicus J.F. Li, } \\
\text { Rungtiwa Phookamsak \& }\end{array}$ & $\begin{array}{l}\text { Tubeufiaceae, Dothideomycetes, } \\
\text { Ascomycota }\end{array}$ & seed & undetermined plant & China & Phookamsak et al. 2019 \\
\hline
\end{tabular}


Table 2 Continued.

\begin{tabular}{|c|c|c|c|c|c|}
\hline Species & Taxonomy & Substrate & Host & Country & References \\
\hline $\begin{array}{l}\text { Pseudohelicomyces quercus } \\
\text { Jayasiri, E.B.G. Jones \& } \\
\text { K.D. Hyde }{ }^{\#}\end{array}$ & $\begin{array}{l}\text { Tubeufiaceae, Dothideomycetes, } \\
\text { Ascomycota }\end{array}$ & cupule & Quercus sp. & Thailand & Jayasiri et al. 2019 \\
\hline $\begin{array}{l}\text { Pseudohelicomyces talbotii } \\
\text { (Goos) Y.Z. Lu \& K.D. Hyde }\end{array}$ & $\begin{array}{l}\text { Tubeufiaceae, Dothideomycetes, } \\
\text { Ascomycota }\end{array}$ & fruits & Meliaceae plant & Thailand & Jayasiri et al. 2019 \\
\hline \multirow{4}{*}{$\begin{array}{l}\text { Pseudopithomyces } \\
\text { chartarum (Berk. \& M.A. } \\
\text { Curtis) Jun F. Li, Ariyaw. \& } \\
\text { K.D. Hyde [as Sporidesmium } \\
\text { bakeri Syd. \& P. Syd.] }\end{array}$} & $\begin{array}{l}\text { Didymosphaeriaceae, Dothideomycetes, } \\
\text { Ascomycota }\end{array}$ & fruits & $\begin{array}{l}\text { Magnolia } \\
\text { grandiflora }\end{array}$ & Thailand & Jayasiri et al. 2019 \\
\hline & & pod & Bauhinia sp. & Thailand & Jayasiri et al. 2019 \\
\hline & & pod & Leucaena sp. & Thailand & Jayasiri et al. 2019 \\
\hline & & $\begin{array}{l}\text { pod } \\
\text { seed }\end{array}$ & $\begin{array}{l}\text { Radermachera sinica } \\
\text { Pinus thunbergii }\end{array}$ & $\begin{array}{l}\text { Thailand } \\
\text { Japan }\end{array}$ & $\begin{array}{l}\text { Jayasiri et al. } 2019 \\
\text { Watanabe } 2010\end{array}$ \\
\hline $\begin{array}{l}\text { Pseudopithomyces } \\
\text { chartarum (Berk. \& M.A. } \\
\text { Curtis) Jun F. Li, Ariyaw. \& } \\
\text { K.D. Hyde [as Pithomyces } \\
\text { chartarum (Berk. \& M.A. } \\
\text { Curtis) M.B. Ellis] }\end{array}$ & & $\begin{array}{l}\text { seed } \\
\text { seed }\end{array}$ & $\begin{array}{l}\text { Pinus thunbergii } \\
\text { Stylosanthes } \\
\text { guianensis }\end{array}$ & $\begin{array}{l}\text { Japan } \\
\text { Australia }\end{array}$ & $\begin{array}{l}\text { Watanabe } 2010 \\
\text { Nik \& Parbery } 1977\end{array}$ \\
\hline $\begin{array}{l}\text { Pseudopithomyces entadae } \\
\text { Jayasiri, E.B.G. Jones \& } \\
\text { K.D. Hyde }^{\#}\end{array}$ & $\begin{array}{l}\text { Didymosphaeriaceae, Dothideomycetes, } \\
\text { Ascomycota }\end{array}$ & pod & Entada phaseoloides & Thailand & Jayasiri et al. 2019 \\
\hline $\begin{array}{l}\text { Pseudopithomyces maydicus } \\
\text { (Sacc.) Jun F. Li, Ariyaw. \& }\end{array}$ & $\begin{array}{l}\text { Didymellaceae, Dothideomycetes, } \\
\text { Ascomycota }\end{array}$ & seeds & Eucalyptus sp. & India & $\begin{array}{l}\text { Reddy et al. 1982, Mittal } \\
\text { et al. } 1990\end{array}$ \\
\hline $\begin{array}{l}\text { K.D. Hyde [as Pithomyces } \\
\text { maydicus (Sacc.) M.B. Ellis] }\end{array}$ & & seed & Pinus thunbergii & Japan & Watanabe 2010 \\
\hline \multirow{5}{*}{$\begin{array}{l}\text { Pseudothielavia terricola } \\
\text { (J.C. Gilman \& E.V. Abbott) } \\
\text { X. WeiWang \& Houbraken } \\
\text { [as Thielavia terricola (J.C. } \\
\text { Gilman \& E.V. Abbott) C.W. } \\
\text { Emmons] }\end{array}$} & $\begin{array}{l}\text { Chaetomiaceae, Sordariomycetes, } \\
\text { Ascomycota }\end{array}$ & seed & $\begin{array}{l}\text { Cryptomeria } \\
\text { japonica }\end{array}$ & Japan & Watanabe 2010 \\
\hline & & seed & Pinus densiflora & Japan & Watanabe 2010 \\
\hline & & seeds & Pinus roxburghii & India & $\begin{array}{l}\text { Munjal \& Sharma 1975, } \\
\text { Mittal et al. } 1990\end{array}$ \\
\hline & & seeds & Pinus wallichiana & India & $\begin{array}{l}\text { Munjal \& Sharma } 1975 \text {, } \\
\text { Mittal et al. } 1990\end{array}$ \\
\hline & & seeds & Shorea robusta & India & $\begin{array}{l}\text { Mittal \& Sharma 1982b, } \\
\text { Mittal et al. } 1990\end{array}$ \\
\hline
\end{tabular}


Table 2 Continued.

\begin{tabular}{|c|c|c|c|c|c|}
\hline Species & Taxonomy & Substrate & Host & Country & References \\
\hline $\begin{array}{l}\text { Puccinia salihae Krigbag \& } \\
\text { aime }^{\#}\end{array}$ & $\begin{array}{l}\text { Pucciniaceae, Pucciniomycetes, } \\
\text { Basidiomycota }\end{array}$ & fruit & Thymelaea aucheri & Turkey & Kirbag et al. 2011 \\
\hline $\begin{array}{l}\text { Pucciniastrum areolatum } \\
\text { (Fr.) G.H. Otth }\end{array}$ & $\begin{array}{l}\text { Pucciniastraceae, Pucciniomycetes, } \\
\text { Basidiomycota }\end{array}$ & seeds & Picea spp. & Germany & Anderson 1986a \\
\hline $\begin{array}{l}\text { Pucciniastrum epilobii } \\
\text { (Pers.) G.H. Otth }\end{array}$ & $\begin{array}{l}\text { Pucciniastraceae, Pucciniomycetes, } \\
\text { Basidiomycota }\end{array}$ & cone & Abies lasiocarpa & Canada & Conners 1967 \\
\hline \multirow[t]{4}{*}{$\begin{array}{l}\text { Pullularia pullulans (de } \\
\text { Bary \& Löwenthal) Berkhout }\end{array}$} & $\begin{array}{l}\text { Saccotheciaceae, Dothideomycetes, } \\
\text { Ascomycota }\end{array}$ & seeds & Abies sibirica & USSR & $\begin{array}{l}\text { Prisyazhnyuk } 1960 \text {, } \\
\text { Mittal et al. } 1990\end{array}$ \\
\hline & & seeds & Larix sibirica & USSR & $\begin{array}{l}\text { Prisyazhnyuk } 1960 \text {, } \\
\text { Mittal et al. } 1990\end{array}$ \\
\hline & & seeds & Picea abies & USSR & $\begin{array}{l}\text { Prisyazhnyuk } 1960 \text {, } \\
\text { Mittal et al. } 1990\end{array}$ \\
\hline & & seeds & Quercus spp. & USSR & $\begin{array}{l}\text { Urosevic 1959, Mittal et } \\
\text { al. } 1990\end{array}$ \\
\hline \multirow[t]{2}{*}{ Pullularia spp. } & $\begin{array}{l}\text { Saccotheciaceae, Dothideomycetes, } \\
\text { Ascomycota }\end{array}$ & seeds & Picea excelsa & USSR & $\begin{array}{l}\text { Urosevic 1961, } \\
\text { Anderson 1986a }\end{array}$ \\
\hline & & seeds & Pinus sylvestris & USSR & $\begin{array}{l}\text { Prisyazhnyuk 1960, } \\
\text { Anderson 1986a }\end{array}$ \\
\hline $\begin{array}{l}\text { Purpurepithecium } \\
\text { murisporum Jayasiri \& K.D. } \\
\text { Hyde }^{\#}\end{array}$ & $\begin{array}{l}\text { Gloniaceae, Dothideomycetes, } \\
\text { Ascomycota }\end{array}$ & cone & Pinus sp. & Italy & Jayasiri et al. 2017a \\
\hline $\begin{array}{l}\text { Pyrenochaeta globosa Ts. } \\
\text { Watanabe }^{\#}\end{array}$ & $\begin{array}{l}\text { Incertae sedis, Dothideomycetes, } \\
\text { Ascomycota }\end{array}$ & seeds & Pinus thunbergii & Japan & Watanabe 1992 \\
\hline \multirow[t]{3}{*}{ Pyrenochaeta spp. } & $\begin{array}{l}\text { Incertae sedis, Dothideomycetes, } \\
\text { Ascomycota }\end{array}$ & seeds & Pinus ponderosa & USA & $\begin{array}{l}\text { James \& Genz 1982, } \\
\text { Anderson 1986a }\end{array}$ \\
\hline & & seeds & Pinus roxburghii & India & $\begin{array}{l}\text { Mittal \& Sharma 1982c, } \\
\text { Mittal et al. } 1990\end{array}$ \\
\hline & & seeds & Quercus robur & Germany & Schroder et al. 2004 \\
\hline $\begin{array}{l}\text { Pyrenophora dematioidea } \\
\text { (Bubák \& Wróbl.) Rossman } \\
\text { \& K.D. Hyde [as Drechslera } \\
\text { dematioidea (Bubák \& } \\
\text { Wróbl.) Scharif] }\end{array}$ & $\begin{array}{l}\text { Pleosporaceae, Dothideomycetes, } \\
\text { Ascomycota }\end{array}$ & seeds & Quercus robur & Poland & Krol et al. 2015 \\
\hline
\end{tabular}


Table 2 Continued.

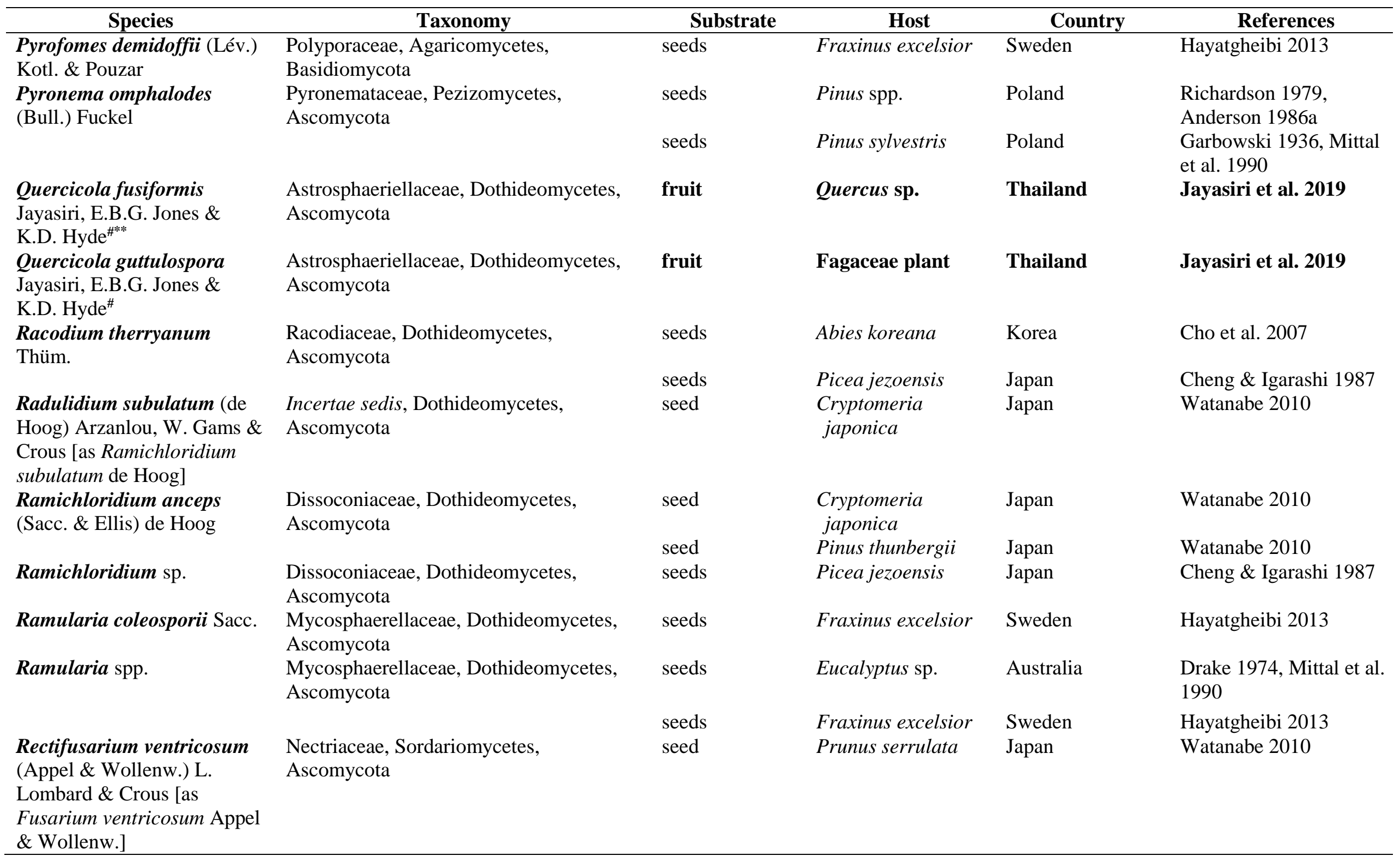


Table 2 Continued.

\begin{tabular}{|c|c|c|c|c|c|}
\hline Species & Taxonomy & Substrate & Host & Country & References \\
\hline $\begin{array}{l}\text { Remotididymella bauhiniae } \\
\text { Jayasiri, E.B.G. Jones \& } \\
\text { K.D. Hyde }{ }^{\#^{* *}}\end{array}$ & $\begin{array}{l}\text { Didymellaceae, Dothideomycetes, } \\
\text { Ascomycota }\end{array}$ & pod & Bauhinia sp. & Thailand & Jayasiri et al. 2019 \\
\hline $\begin{array}{l}\text { Repetoblastiella olivacea } \\
\text { R.F. Castañeda, Minter \& M. } \\
\text { Stadler }{ }^{\# * *}\end{array}$ & $\begin{array}{l}\text { Incertae sedis, Incertae sedis, } \\
\text { Ascomycota }\end{array}$ & nuts & $\begin{array}{l}\text { Couroupita } \\
\text { guianensis }\end{array}$ & Cuba & $\begin{array}{l}\text { Castañeda-Ruíz et al. } \\
2010\end{array}$ \\
\hline $\begin{array}{l}\text { Resinicium bicolor (Alb. \& } \\
\text { Schwein.) Parmasto }\end{array}$ & $\begin{array}{l}\text { Rickenellaceae. Agaricomycetes, } \\
\text { Basidiomycota }\end{array}$ & seeds & Fraxinus excelsior & Sweden & Hayatgheibi 2013 \\
\hline Restilago capensis Vánky ${ }^{\# * *}$ & $\begin{array}{l}\text { Incertae sedis, Incertae sedis, } \\
\text { Basidiomycota }\end{array}$ & nutlets & $\begin{array}{l}\text { Ischyrolepis } \\
\text { capensis }\end{array}$ & South Africa & Vánky 2008a \\
\hline $\begin{array}{l}\text { Restiosporium anarthriae } \\
\text { Vánky }^{\#}\end{array}$ & $\begin{array}{l}\text { Websdaneaceae, Ustilaginomycetes, } \\
\text { Basidiomycota }\end{array}$ & nutlets & Anarthria laevis & Australia & Vánky \& Shivas 2006 \\
\hline $\begin{array}{l}\text { Restiosporium apodasmiae } \\
\text { Vánky\# }\end{array}$ & $\begin{array}{l}\text { Websdaneaceae, Ustilaginomycetes, } \\
\text { Basidiomycota }\end{array}$ & nutlets & $\begin{array}{l}\text { Apodasmia } \\
\text { eremophila }\end{array}$ & Australia & Vánky \& Shivas 2006 \\
\hline $\begin{array}{l}\text { Restiosporium baloskionis } \\
\text { Vánky \& R.G. Shivas }{ }^{\#}\end{array}$ & $\begin{array}{l}\text { Websdaneaceae, Ustilaginomycetes, } \\
\text { Basidiomycota }\end{array}$ & nutlets & $\begin{array}{l}\text { Baloskion } \\
\text { tetraphyllum }\end{array}$ & Australia & Vánky \& Shivas 2003 \\
\hline $\begin{array}{l}\text { Restiosporium chaetanthi } \\
\text { Vánky }^{\#}\end{array}$ & $\begin{array}{l}\text { Websdaneaceae, Ustilaginomycetes, } \\
\text { Basidiomycota }\end{array}$ & nutlets & $\begin{array}{l}\text { Chaetanthus } \\
\text { aristatus }\end{array}$ & Australia & Vánky \& Shivas 2006 \\
\hline $\begin{array}{l}\text { Restiosporium dapsilanthi } \\
\text { Vanky }^{\#}\end{array}$ & $\begin{array}{l}\text { Websdaneaceae, Ustilaginomycetes, } \\
\text { Basidiomycota }\end{array}$ & nutlets & Dapsilanthus elatior & Australia & Vánky 2003 \\
\hline $\begin{array}{l}\text { Restiosporium desmocladi } \\
\text { Vánky\# }\end{array}$ & $\begin{array}{l}\text { Websdaneaceae, Ustilaginomycetes, } \\
\text { Basidiomycota }\end{array}$ & nutlets & $\begin{array}{l}\text { Desmocladus } \\
\text { elongatus }\end{array}$ & Australia & Vánky \& Shivas 2006 \\
\hline $\begin{array}{l}\text { Restiosporium eurychordae } \\
\text { Vánky\# }\end{array}$ & $\begin{array}{l}\text { Websdaneaceae, Ustilaginomycetes, } \\
\text { Basidiomycota }\end{array}$ & nutlets & $\begin{array}{l}\text { Eurychorda } \\
\text { complanata }\end{array}$ & Australia & Vánky \& Shivas 2006 \\
\hline $\begin{array}{l}\text { Restiosporium flexuosum } \\
\text { Vánky }^{\#}\end{array}$ & $\begin{array}{l}\text { Websdaneaceae, Ustilaginomycetes, } \\
\text { Basidiomycota }\end{array}$ & nutlets & $\begin{array}{l}\text { Desmocladus } \\
\text { flexuosus }\end{array}$ & Australia & Vánky \& Shivas 2006 \\
\hline $\begin{array}{l}\text { Restiosporium guringaliae } \\
\text { Vánky \& R.G. Shivas" }\end{array}$ & $\begin{array}{l}\text { Websdaneaceae, Ustilaginomycetes, } \\
\text { Basidiomycota }\end{array}$ & nutlets & $\begin{array}{l}\text { Guringalia } \\
\text { dimorpha }\end{array}$ & Australia & Vánky 2007 \\
\hline $\begin{array}{l}\text { Restiosporium hypolaenae } \\
\text { Vánky }{ }^{\#}\end{array}$ & $\begin{array}{l}\text { Websdaneaceae, Ustilaginomycetes, } \\
\text { Basidiomycota }\end{array}$ & nutlets & $\begin{array}{l}\text { Hypolaena } \\
\text { fastigiata }\end{array}$ & Australia & Vánky \& Shivas 2006 \\
\hline $\begin{array}{l}\text { Restiosporium lepyrodiae } \\
\text { Vánky" }\end{array}$ & $\begin{array}{l}\text { Websdaneaceae, Ustilaginomycetes, } \\
\text { Basidiomycota }\end{array}$ & nutlets & Lepyrodia scariosa & Australia & Vánky \& Shivas 2006 \\
\hline
\end{tabular}


Table 2 Continued.

\begin{tabular}{|c|c|c|c|c|c|}
\hline Species & Taxonomy & Substrate & Host & Country & References \\
\hline $\begin{array}{l}\text { Restiosporium pallentis } \\
\text { Vánky\& R.G. Shiva }\end{array}$ & $\begin{array}{l}\text { Websdaneaceae, Ustilaginomycetes, } \\
\text { Basidiomycota }\end{array}$ & nutlets & Baloskion pallens & Australia & Vánky \& Shivas 2006 \\
\hline Restiosporium patei Vánky\# & $\begin{array}{l}\text { Websdaneaceae, Ustilaginomycetes, } \\
\text { Basidiomycota }\end{array}$ & nutlets & Lepidobolus densus & Australia & Vánky \& Shivas 2006 \\
\hline $\begin{array}{l}\text { Restiosporium proliferum } \\
\text { Vánky\# }\end{array}$ & $\begin{array}{l}\text { Websdaneaceae, Ustilaginomycetes, } \\
\text { Basidiomycota }\end{array}$ & nutlets & Anarthria prolifera & Australia & Vánky \& Shivas 2006 \\
\hline $\begin{array}{l}\text { Restiosporium spathacei } \\
\text { Vánky. R.G. Shivas \& C. } \\
\text { Vánky\# }\end{array}$ & $\begin{array}{l}\text { Websdaneaceae, Ustilaginomycetes, } \\
\text { Basidiomycota }\end{array}$ & capsules & $\begin{array}{l}\text { Dapsilanthus } \\
\text { spathaceus }\end{array}$ & Australia & Vánky \& Shivas 2006 \\
\hline $\begin{array}{l}\text { Restiosporium sphacelatum } \\
\text { Vánky }\end{array}$ & $\begin{array}{l}\text { Websdaneaceae, Ustilaginomycetes, } \\
\text { Basidiomycota }\end{array}$ & nutlets & $\begin{array}{l}\text { Chordifex } \\
\text { sphacelatus }\end{array}$ & Australia & Vánky \& Shivas 2006 \\
\hline Rhinocladiella spp. & $\begin{array}{l}\text { Herpotrichiellaceae, Eurotiomycetes, } \\
\text { Ascomycota }\end{array}$ & seeds & Picea jezoensis & Japan & Cheng \& Igarashi 1987 \\
\hline Rhinotrichum repens Preuss & $\begin{array}{l}\text { Incertae sedis, Incertae sedis, } \\
\text { Ascomycota }\end{array}$ & seeds & Pinus sylvestris & USSR & $\begin{array}{l}\text { Dolejs 1964, Mittal et al. } \\
1990\end{array}$ \\
\hline Rhizidium lignicola Lindau & $\begin{array}{l}\text { Chytriomycetaceae, Chytridiomycetes, } \\
\text { Chytridiomycota }\end{array}$ & seeds & $\begin{array}{l}\text { Polygonum } \\
\text { amphibium }\end{array}$ & $\begin{array}{l}\text { Poland or/ } \\
\text { imported to Poland }\end{array}$ & Czeczuga et al. 2009 \\
\hline $\begin{array}{l}\text { Rhizidium nowakowskii } \\
\text { Karling }\end{array}$ & $\begin{array}{l}\text { Chytriomycetaceae, Chytridiomycetes, } \\
\text { Chytridiomycota }\end{array}$ & seeds & Hyoscyamus albus & $\begin{array}{l}\text { Poland or/ } \\
\text { imported to Poland }\end{array}$ & Czeczuga et al. 2009 \\
\hline $\begin{array}{l}\text { Rhizidium richmondense } \\
\text { Willoughby }\end{array}$ & $\begin{array}{l}\text { Chytriomycetaceae, Chytridiomycetes, } \\
\text { Chytridiomycota }\end{array}$ & seeds & Atropa caucasica & $\begin{array}{l}\text { Poland or/ } \\
\text { imported to Poland }\end{array}$ & Czeczuga et al. 2009 \\
\hline \multirow[t]{6}{*}{$\begin{array}{l}\text { Rhizoctonia solani J.G. } \\
\text { Kühn }\end{array}$} & $\begin{array}{l}\text { Ceratobasidiaceae, Agaricomycetes, } \\
\text { Basidiomycota }\end{array}$ & seeds & Abies balsamea & USA & Mittal et al. 1990 \\
\hline & & seeds & Abies fraseri & USA & Mittal et al. 1990 \\
\hline & & seeds & Abies spp & USA & $\begin{array}{l}\text { Heit \& Natti } 1969 \text {, } \\
\text { Mittal et al. } 1990\end{array}$ \\
\hline & & seeds & $\begin{array}{l}\text { Araucaria } \\
\text { angustifolia }\end{array}$ & Australia & $\begin{array}{l}\text { Kamara et al. } 1981 \text {, } \\
\text { Mittal et al. } 1990\end{array}$ \\
\hline & & seeds & Araucaria bidwillii & Egypt & $\begin{array}{l}\text { Kamara et al. } 1981 \text {, } \\
\text { Mittal et al. } 1990\end{array}$ \\
\hline & & seeds & $\begin{array}{l}\text { Araucaria } \\
\text { cunninghamii }\end{array}$ & Australia & $\begin{array}{l}\text { Kamara et al. } 1981 \text {, } \\
\text { Mittal et al. } 1990\end{array}$ \\
\hline
\end{tabular}


Table 2 Continued.

\begin{tabular}{|c|c|c|c|c|c|}
\hline Species & Taxonomy & Substrate & Host & Country & References \\
\hline & & seeds & $\begin{array}{l}\text { Araucaria } \\
\text { heterophylla }\end{array}$ & Egypt & $\begin{array}{l}\text { Kamara et al. } 1981 \text {, } \\
\text { Mittal et al. } 1990\end{array}$ \\
\hline & & seeds & $\begin{array}{l}\text { Callistephus } \\
\text { chinensis }\end{array}$ & China & $\begin{array}{l}\text { Gloyer } 1931 \text {, Crosier \& } \\
\text { Heit } 1948\end{array}$ \\
\hline & & seeds & Fagus sylvatica & France, Poland & $\begin{array}{l}\text { Richardson 1983, } \\
\text { Anderson 1986a, Krol et } \\
\text { al. } 2015\end{array}$ \\
\hline & & seeds & Leucaena spp. & Philippines & $\begin{array}{l}\text { Mathur 1974, Anderson } \\
\text { 1986a }\end{array}$ \\
\hline & & seed & Linum usitatissimum & Canada & Conners 1967 \\
\hline & & seeds & Picea sitchensis & UK & $\begin{array}{l}\text { Salt 1964, Mittal et al. } \\
1990\end{array}$ \\
\hline & & seeds & Pinus nigra & Italy & $\begin{array}{l}\text { Turchetti 1982, Mittal et } \\
\text { al. } 1990\end{array}$ \\
\hline & & seed & Prunus serrulata & Japan & Watanabe 2010 \\
\hline & & seeds & Ulmus americana & USA & Mittal et al. 1990 \\
\hline & & seeds & Ulmus spp. & Denmark & $\begin{array}{l}\text { Richardson 1979, } \\
\text { Anderson 1986a }\end{array}$ \\
\hline \multirow[t]{6}{*}{ Rhizoctonia spp. } & \multirow{6}{*}{$\begin{array}{l}\text { Ceratobasidiaceae, Agaricomycetes, } \\
\text { Basidiomycota }\end{array}$} & acorns & Quercus robur & Poland & Jankowiak 2008 \\
\hline & & seeds & Cedrus deodara & India & Mittal 1983 \\
\hline & & seed & Cercis chinensis & Japan & Watanabe 2010 \\
\hline & & seeds & $\begin{array}{l}\text { Cupressus } \\
\text { sempervirens }\end{array}$ & Egypt & $\begin{array}{l}\text { Farag et al. 1977, Mittal } \\
\text { et al. } 1990\end{array}$ \\
\hline & & seeds & Pinus elliottii & Taiwan & $\begin{array}{l}\text { Rowan \& Debarr 1974, } \\
\text { Mittal et al. } 1990\end{array}$ \\
\hline & & seed & Prunus serrulata & Japan & Watanabe 2010 \\
\hline Rhizomarasmius setosus & \multirow{2}{*}{$\begin{array}{l}\text { Physalacriaceae, Agaricomycetes, } \\
\text { Basidiomycota }\end{array}$} & fruit cupules & Fagus sylvatica & UK & Carré 1964 \\
\hline $\begin{array}{l}\text { (Sowerby) Antonín \& A. } \\
\text { Urb. [as Marasmius recubans } \\
\text { Quél. as 'reenbans'] }\end{array}$ & & seeds & Picea abies & USSR & $\begin{array}{l}\text { Prisyazhnyuk } 1960 \text {, } \\
\text { Mittal et al. } 1990\end{array}$ \\
\hline $\begin{array}{l}\text { Rhizomucor pusillus (Lindt) } \\
\text { Schipper [as Mucor pusillus } \\
\text { Lindt] }\end{array}$ & $\begin{array}{l}\text { Lichtheimiaceae, Mucoromycetes, } \\
\text { Mucoromycota }\end{array}$ & seeds & $\begin{array}{l}\text { Cupressus spp., } \\
\text { Sapium spp. }\end{array}$ & India & Sahai \& Otra 1982 \\
\hline
\end{tabular}


Table 2 Continued.

\begin{tabular}{|c|c|c|c|c|c|}
\hline Species & Taxonomy & Substrate & Host & Country & References \\
\hline \multirow[t]{15}{*}{$\begin{array}{l}\text { Rhizophlyctis rosea (de Bary } \\
\text { \& Woronin) A. Fisch. }\end{array}$} & $\begin{array}{l}\text { Rhizophlyctidaceae, Chytridiomycetes, } \\
\text { Chytridiomycota }\end{array}$ & seeds & $\begin{array}{l}\text { Armeria maritima } \\
\text { ssp. maritima }\end{array}$ & $\begin{array}{l}\text { Poland or/ } \\
\text { imported to Poland }\end{array}$ & Czeczuga et al. 2009 \\
\hline & & seeds & Atropa belladonna & $\begin{array}{l}\text { Poland or/ } \\
\text { imported to Poland }\end{array}$ & Czeczuga et al. 2009 \\
\hline & & seeds & Atropa caucasica & $\begin{array}{l}\text { Poland or/ } \\
\text { imported to Poland }\end{array}$ & Czeczuga et al. 2009 \\
\hline & & seeds & Atropa komarovii & $\begin{array}{l}\text { Poland or/ } \\
\text { imported to Poland }\end{array}$ & Czeczuga et al. 2009 \\
\hline & & seeds & Atropa pallidiflora & $\begin{array}{l}\text { Poland or/ } \\
\text { imported to Poland }\end{array}$ & Czeczuga et al. 2009 \\
\hline & & seeds & Cicuta virosa & $\begin{array}{l}\text { Poland or/ } \\
\text { imported to Poland }\end{array}$ & Czeczuga et al. 2009 \\
\hline & & seeds & Cornus australis & $\begin{array}{l}\text { Poland or/ } \\
\text { imported to Poland }\end{array}$ & Czeczuga et al. 2009 \\
\hline & & seeds & Cuscuta europaea & $\begin{array}{l}\text { Poland or/ } \\
\text { imported to Poland }\end{array}$ & Czeczuga et al. 2009 \\
\hline & & seeds & Ginkgo biloba & $\begin{array}{l}\text { Poland or/ } \\
\text { imported to Poland }\end{array}$ & Czeczuga et al. 2009 \\
\hline & & seeds & Hyoscyamus albus & $\begin{array}{l}\text { Poland or/ } \\
\text { imported to Poland }\end{array}$ & Czeczuga et al. 2009 \\
\hline & & seeds & Hyoscyamus aureus & $\begin{array}{l}\text { Poland or/ } \\
\text { imported to Poland }\end{array}$ & Czeczuga et al. 2009 \\
\hline & & seeds & Lotus uliginosa & $\begin{array}{l}\text { Poland or/ } \\
\text { imported to Poland }\end{array}$ & Czeczuga et al. 2009 \\
\hline & & seeds & $\begin{array}{l}\text { Prunella grandiflora } \\
\text { ssp. pyrenaia }\end{array}$ & $\begin{array}{l}\text { Poland or/ } \\
\text { imported to Poland }\end{array}$ & Czeczuga et al. 2009 \\
\hline & & seeds & Rosa mollis & $\begin{array}{l}\text { Poland or/ } \\
\text { imported to Poland }\end{array}$ & Czeczuga et al. 2009 \\
\hline & & seeds & Ulex europaeus & $\begin{array}{l}\text { Poland or/ } \\
\text { imported to Poland }\end{array}$ & Czeczuga et al. 2009 \\
\hline $\begin{array}{l}\text { Rhizophydium ampullaceum } \\
\text { (A. Braun) A. Fisch. }\end{array}$ & $\begin{array}{l}\text { Rhizophydiaceae, Rhizophydiomycetes, } \\
\text { Chytridiomycota }\end{array}$ & seeds & Atropa belladonna & $\begin{array}{l}\text { Poland or/ } \\
\text { imported to Poland }\end{array}$ & Czeczuga et al. 2009 \\
\hline
\end{tabular}


Table 2 Continued.

\begin{tabular}{|c|c|c|c|c|c|}
\hline Species & Taxonomy & Substrate & Host & Country & References \\
\hline & & seeds & Bryonia cretica & $\begin{array}{l}\text { Poland or/ } \\
\text { imported to Poland }\end{array}$ & Czeczuga et al. 2009 \\
\hline & & seeds & $\begin{array}{l}\text { Polygonum } \\
\text { amphibium }\end{array}$ & $\begin{array}{l}\text { Poland or/ } \\
\text { imported to Poland }\end{array}$ & Czeczuga et al. 2009 \\
\hline \multirow[t]{2}{*}{$\begin{array}{l}\text { Rhizophydium carpophilum } \\
\text { Zopf }\end{array}$} & $\begin{array}{l}\text { Rhizophydiaceae, Rhizophydiomycetes, } \\
\text { Chytridiomycota }\end{array}$ & seeds & $\begin{array}{l}\text { Lonicera } \\
\text { periclymenum }\end{array}$ & $\begin{array}{l}\text { Poland or/ } \\
\text { imported to Poland }\end{array}$ & Czeczuga et al. 2009 \\
\hline & & seeds & $\begin{array}{l}\text { Rhododendron } \\
\text { insigne }\end{array}$ & $\begin{array}{l}\text { Poland or/ } \\
\text { imported to Poland }\end{array}$ & Czeczuga et al. 2009 \\
\hline \multirow{4}{*}{$\begin{array}{l}\text { Rhizophydium coronum } \\
\text { A.M. Hanson } \\
\text { Rhizophydium globosum (A. } \\
\text { Braun) Rabenh. }\end{array}$} & $\begin{array}{l}\text { Rhizophydiaceae, Rhizophydiomycetes, } \\
\text { Chytridiomycota }\end{array}$ & seeds & Bryonia cretica & $\begin{array}{l}\text { Poland or/ } \\
\text { imported to Poland }\end{array}$ & Czeczuga et al. 2009 \\
\hline & $\begin{array}{l}\text { Rhizophydiaceae, Rhizophydiomycetes, } \\
\text { Chytridiomycota }\end{array}$ & seeds & $\begin{array}{l}\text { Hyoscyamus } \\
\text { gyoerffyi }\end{array}$ & $\begin{array}{l}\text { Poland or/ } \\
\text { imported to Poland }\end{array}$ & Czeczuga et al. 2009 \\
\hline & & seeds & Hyoscyamus muticus & $\begin{array}{l}\text { Poland or/ } \\
\text { imported to Poland }\end{array}$ & Czeczuga et al. 2009 \\
\hline & & seeds & Sambucus ebulus & $\begin{array}{l}\text { Poland or/ } \\
\text { imported to Poland }\end{array}$ & Czeczuga et al. 2009 \\
\hline \multirow{8}{*}{$\begin{array}{l}\text { Rhizophydium olla H.E. } \\
\text { Petersen } \\
\text { Rhizopus arrhizus A. Fisch. }\end{array}$} & $\begin{array}{l}\text { Rhizophydiaceae, Rhizophydiomycetes, } \\
\text { Chytridiomycota }\end{array}$ & seeds & Paris quadrifolia & $\begin{array}{l}\text { Poland or/ } \\
\text { imported to Poland }\end{array}$ & Czeczuga et al. 2009 \\
\hline & $\begin{array}{l}\text { Rhizopodaceae, Mucoromycetes, } \\
\text { Mucoromycota }\end{array}$ & seeds & Cedrus deodara & India & $\begin{array}{l}\text { Munjal \& Sharma 1975, } \\
\text { Mittal et al. } 1990\end{array}$ \\
\hline & & seeds & Crataegus azarolus & $\begin{array}{l}\text { Poland or/ } \\
\text { imported to Poland }\end{array}$ & Czeczuga et al. 2009 \\
\hline & & seeds & Eucalyptus sp. & India & $\begin{array}{l}\text { Saxena 1985, Mittal et } \\
\text { al. } 1990\end{array}$ \\
\hline & & seeds & Picea excelsa & USSR & $\begin{array}{l}\text { Urosevic 1961, } \\
\text { Anderson 1986a }\end{array}$ \\
\hline & & seeds & Pinus patula & Kenya & $\begin{array}{l}\text { Gibson 1957, Mittal et } \\
\text { al. } 1990\end{array}$ \\
\hline & & seeds & Pinus ponderosa & USA & $\begin{array}{l}\text { James \& Genz 1982, } \\
\text { Anderson 1986a }\end{array}$ \\
\hline & & seeds & Pinus roxburghii & India & $\begin{array}{l}\text { Mittal \& Sharma 1982c, } \\
\text { Mittal et al. } 1990\end{array}$ \\
\hline
\end{tabular}


Table 2 Continued.

\begin{tabular}{|c|c|c|c|c|c|}
\hline Species & Taxonomy & Substrate & Host & Country & References \\
\hline & & seeds & Pinus sylvestris & Poland, USSR & $\begin{array}{l}\text { Garbowski 1936, } \\
\text { Anderson 1986a }\end{array}$ \\
\hline & & seeds & Pinus taeda & USA & $\begin{array}{l}\text { Mason \& van Arsdel } \\
\text { 1978, Mittal et al. } 1990\end{array}$ \\
\hline & & seeds & Pinus wallichiana & India & $\begin{array}{l}\text { Munjal \& Sharma 1975, } \\
\text { Mittal et al. } 1990\end{array}$ \\
\hline & & seeds & $\begin{array}{l}\text { Pseudotsuga } \\
\text { menziesii }\end{array}$ & USA & $\begin{array}{l}\text { Gordon 1967, Mittal et } \\
\text { al. } 1990\end{array}$ \\
\hline \multirow[t]{2}{*}{ Rhizopus betivorus Nevod } & $\begin{array}{l}\text { Rhizopodaceae, Mucoromycetes, } \\
\text { Mucoromycota }\end{array}$ & seeds & Betula verrucosa & USSR & $\begin{array}{l}\text { Kozlowska 1968, Mittal } \\
\text { et al. } 1990\end{array}$ \\
\hline & & seeds & Quercus pedunculata & USSR & $\begin{array}{l}\text { Kozlowska 1968, Mittal } \\
\text { et al. } 1990\end{array}$ \\
\hline $\begin{array}{l}\text { Rhizopus cohnii Berl. \& De } \\
\text { Toni }\end{array}$ & $\begin{array}{l}\text { Rhizopodaceae, Mucoromycetes, } \\
\text { Mucoromycota }\end{array}$ & seeds & Pinus roxburghii & India & $\begin{array}{l}\text { Mittal \& Sharma 1982c, } \\
\text { Mittal et al. } 1990\end{array}$ \\
\hline \multirow[t]{14}{*}{ Rhizopus nigricans Ehrenb. } & $\begin{array}{l}\text { Rhizopodaceae, Mucoromycetes, } \\
\text { Mucoromycota }\end{array}$ & cones & Picea glauca & Canada & Mittal \& Wang 1987 \\
\hline & & cones, seeds & Pinus strobus & Canada & Mittal \& Wang 1987 \\
\hline & & Seeds & Abies alba & Poland & Krol et al. 2015 \\
\hline & & seeds & Abies sibirica & USSR & $\begin{array}{l}\text { Prisyazhnyuk 1960, } \\
\text { Mittal et al. } 1990\end{array}$ \\
\hline & & seeds & Betula pendula & Poland & Krol et al. 2015 \\
\hline & & seeds & $\begin{array}{l}\text { Casuarina } \\
\text { equisetifolia }\end{array}$ & India & Anju et al. 2012 \\
\hline & & seeds & Casuarina spp. & India & Sahai \& Otra 1982 \\
\hline & & seeds & Cedrus deodara & India & Mittal 1983 \\
\hline & & seeds & Dalbergia sissoo & India & Kumar 2014 \\
\hline & & seeds & Fagus sylvatica & Poland & Krol et al. 2015 \\
\hline & & seeds & Larix sibirica & USSR & $\begin{array}{l}\text { Prisyazhnyuk } 1960 \text {, } \\
\text { Mittal et al. } 1990\end{array}$ \\
\hline & & seeds & Picea glauca & Canada & Mittal \& Wang 1987 \\
\hline & & seeds & Pinus nigra & Hungary & $\begin{array}{l}\text { Hangyal 1973, Mittal et } \\
\text { al. } 1990\end{array}$ \\
\hline & & seeds & Pinus sylvestris & $\begin{array}{l}\text { Hungary, Poland, } \\
\text { UK, USSR }\end{array}$ & $\begin{array}{l}\text { Hangyal 1973, Whittle } \\
\text { 1977, Mittal et al. 1990, }\end{array}$ \\
\hline
\end{tabular}


Table 2 Continued.

\begin{tabular}{|c|c|c|c|c|c|}
\hline Species & Taxonomy & Substrate & Host & Country & References \\
\hline & & & & & Krol et al. 2015 \\
\hline & & seeds & Pinus wallichiana & Pakistan & Ashaeer 2000 \\
\hline & & seeds & Quercus rubra & Poland & Krol et al. 2015 \\
\hline & & seeds & Quercus spp. & USSR & $\begin{array}{l}\text { Urosevic 1959, Mittal et } \\
\text { al. } 1990\end{array}$ \\
\hline & & seeds & Shorea robusta & Thailand & $\begin{array}{l}\text { Mittal \& Sharma 1982b, } \\
\text { Mittal et al. } 1990\end{array}$ \\
\hline Rhizopus nodosus Namysł. & $\begin{array}{l}\text { Rhizopodaceae, Mucoromycetes, } \\
\text { Mucoromycota }\end{array}$ & seeds & Crataegus azarolus & $\begin{array}{l}\text { Poland or/ } \\
\text { imported to Poland }\end{array}$ & Czeczuga et al. 2009 \\
\hline \multirow{12}{*}{$\begin{array}{l}\text { Rhizopus oryzae Went \& } \\
\text { Prins. Geerl. }\end{array}$} & Rhizopodaceae, Mucoromycetes, & seed & Agropyron cristatum & Canada & Conners 1967 \\
\hline & & seed & Larix sp. & Japan & Watanabe 2010 \\
\hline & & seed & Prunus serrulata & Japan & Watanabe 2010 \\
\hline & & seeds & Albizia lebbeck & India & $\begin{array}{l}\text { Mittal \& Sharma 1982a, } \\
\text { Mittal et al. } 1990\end{array}$ \\
\hline & & seeds & Cassia fistula & India & $\begin{array}{l}\text { Mittal \& Sharma 1981a, } \\
\text { Mittal et al. } 1990\end{array}$ \\
\hline & & seeds & Cedrus deodara & India & Mittal 1983 \\
\hline & & seeds & Dalbergia sissoo & India & $\begin{array}{l}\text { Mittal \& Sharma 1981b, } \\
\text { Mittal et al. } 1990\end{array}$ \\
\hline & & seeds & $\begin{array}{l}\text { Eucalyptus } \\
\text { citriodora }\end{array}$ & India & $\begin{array}{l}\text { Mittal \& Sharma 1984, } \\
\text { Mittal et al. } 1990\end{array}$ \\
\hline & & seeds & Eucalyptus hybrid & India & $\begin{array}{l}\text { Mittal 1986, Mittal et al. } \\
1990\end{array}$ \\
\hline & & seeds & Pinus roxburghii & India & $\begin{array}{l}\text { Mittal \& Sharma 1982c, } \\
\text { Mittal et al. } 1990\end{array}$ \\
\hline & & seeds & Pinus wallichiana & India, Pakistan & $\begin{array}{l}\text { Mittal \& Sharma 1982b, } \\
\text { Mittal et al. 1990, } \\
\text { Ashaeer } 2000\end{array}$ \\
\hline & & seeds & Shorea robusta & India & $\begin{array}{l}\text { Mittal \& Sharma 1982b, } \\
\text { Mittal et al. } 1990\end{array}$ \\
\hline \multirow[t]{2}{*}{ Rhizopus spp. } & $\begin{array}{l}\text { Rhizopodaceae, Mucoromycetes, } \\
\text { Mucoromycota }\end{array}$ & fruits & Avicennia marina & India & Mehdi \& Saifullah 2000 \\
\hline & & pods & Delonix regia & Thailand & Somrithipol et al. 2002b \\
\hline
\end{tabular}


Table 2 Continued.

\begin{tabular}{|c|c|c|c|c|c|}
\hline Species & Taxonomy & Substrate & Host & Country & References \\
\hline & & seeds & Abies lasiocarpa & Canada & Talgø et al. 2010 \\
\hline & & seeds & Abies nordmanniana & Georgia & Talgø et al. 2010 \\
\hline & & seeds & Abies procera & Norway & Talgø et al. 2010 \\
\hline & & seeds & Acacia confusa & Philippines & $\begin{array}{l}\text { Agmata 1979, Anderson } \\
\text { 1986a }\end{array}$ \\
\hline & & seeds & Acer saccharum & USA & $\begin{array}{l}\text { Fuller \& Hildebrand } \\
\text { 1985, Mittal et al. } 1990\end{array}$ \\
\hline & & seeds & Albizia falcataria & Philippines & $\begin{array}{l}\text { Dayan 1986, Mittal et al. } \\
1990\end{array}$ \\
\hline & & seeds & Albizia lebbeck & India & Mohanan et al. 2005 \\
\hline & & seeds & $\begin{array}{l}\text { Araucaria } \\
\text { angustifolia }\end{array}$ & Australia & $\begin{array}{l}\text { Kamara et al. } 1981 \text {, } \\
\text { Mittal et al. } 1990\end{array}$ \\
\hline & & seeds & $\begin{array}{l}\text { Araucaria } \\
\text { cunninghamii }\end{array}$ & Australia & $\begin{array}{l}\text { Kamara et al. } 1981 \text {, } \\
\text { Mittal et al. } 1990\end{array}$ \\
\hline & & seeds & $\begin{array}{l}\text { Betula } \\
\text { alleghaniensis }\end{array}$ & USA & $\begin{array}{l}\text { Friedrich et al. } 1971 \text {, } \\
\text { Mittal et al. } 1990\end{array}$ \\
\hline & & seeds & Betula papyrifera & USA & $\begin{array}{l}\text { Friedrich et al. } 1971 \text {, } \\
\text { Mittal et al. } 1990\end{array}$ \\
\hline & & seeds & Bombax ceiba & Bangladesh, India & Mittal et al. 1990 \\
\hline & & seed & Canavalia cathartica & India & Anita \& Sridhar 2009 \\
\hline & & seeds & Cassia fistula & Thailand & $\begin{array}{l}\text { Chalermpongse et al. } \\
\text { 1984, Mittal et al. } 1990\end{array}$ \\
\hline & & seeds & $\begin{array}{l}\text { Dianthus } \\
\text { caryophyllus }\end{array}$ & Taiwan & Li \& Wu 2002 \\
\hline & & seeds & Dianthus spp. & Taiwan & Li \& Wu 2002 \\
\hline & & seeds & Eucalyptus deglupta & Thailand & $\begin{array}{l}\text { Chalermpongse et al. } \\
\text { 1984, Mittal et al. } 1990\end{array}$ \\
\hline & & seeds & Eucalyptus grandis & Uruguay & Anderson 1986a \\
\hline & & seeds & Fraxinus americana & USA & $\begin{array}{l}\text { Friedrich et al. } 1971 \text {, } \\
\text { Mittal et al. } 1990\end{array}$ \\
\hline & & seeds & Gmelina arborea & Thailand & $\begin{array}{l}\text { Chalermpongse et al. } \\
\text { 1984, Mittal et al. } 1990\end{array}$ \\
\hline & & seeds & Larix occidentalis & USA & James et al. 1996 \\
\hline
\end{tabular}


Table 2 Continued.

\begin{tabular}{|c|c|c|c|c|c|}
\hline Species & Taxonomy & Substrate & Host & Country & References \\
\hline & & seeds & $\begin{array}{l}\text { Leucaena } \\
\text { leucocephala }\end{array}$ & $\begin{array}{l}\text { Bangladesh, } \\
\text { Thailand }\end{array}$ & $\begin{array}{l}\text { Chalermpongse et al. } \\
\text { 1984, Mittal et al. 1990, } \\
\text { Islam et al. } 2008\end{array}$ \\
\hline & & seeds & Picea engelmannii & USA & $\begin{array}{l}\text { Fuller \& Hildebrand } \\
\text { 1985, Mittal et al. } 1990\end{array}$ \\
\hline & & seeds & Picea jezoensis & Japan & Cheng \& Igarashi 1987 \\
\hline & & seeds & Picea sitchensis & UK & $\begin{array}{l}\text { Salt 1964, Mittal et al. } \\
1990\end{array}$ \\
\hline & & seeds & Pinus contorta & USA & $\begin{array}{l}\text { Fuller \& Hildebrand } \\
\text { 1985, Mittal et al. } 1990\end{array}$ \\
\hline & & seeds & $\begin{array}{l}\text { Pinus elliottii var. } \\
\text { elliottii }\end{array}$ & $\begin{array}{l}\text { Georgia, Uruguay, } \\
\text { USA }\end{array}$ & $\begin{array}{l}\text { Anderson 1986a, Huang } \\
\text { \& Kuhlman 1990, } \\
\text { Fraedrich \& Miller } 1995\end{array}$ \\
\hline & & seeds & Pinus lambertiana & USA & $\begin{array}{l}\text { Anderson 1986a, } \\
\text { Schubert } 1961\end{array}$ \\
\hline & & seeds & Pinus massoniana & Taiwan & $\begin{array}{l}\text { Jong \& Chen } 1966 \text {, } \\
\text { Mittal et al. } 1990\end{array}$ \\
\hline & & seeds & Pinus patula & East Africa & $\begin{array}{l}\text { Gibson 1957, Mittal et } \\
\text { al. } 1990\end{array}$ \\
\hline & & seeds & Pinus ponderosa & USA & $\begin{array}{l}\text { Fuller \& Hildebrand } \\
\text { 1985, Mittal et al. } 1990\end{array}$ \\
\hline & & seeds & Pinus taeda & Georgia, USA & $\begin{array}{l}\text { Covington et al. 1982, } \\
\text { Anderson 1986a, Huang } \\
\text { \& Kuhlman } 1990\end{array}$ \\
\hline & & seeds & Pinus taiwanensis & Taiwan & $\begin{array}{l}\text { Jong \& Chen } 1966 \text {, } \\
\text { Mittal et al. } 1990\end{array}$ \\
\hline & & seeds & $\begin{array}{l}\text { Pinus thunbergii } \\
\text { [= Pinus } \\
\text { thunbergiana }]\end{array}$ & Taiwan & $\begin{array}{l}\text { Jong \& Chen } 1966 \text {, } \\
\text { Mittal et al. } 1990\end{array}$ \\
\hline & & seeds & $\begin{array}{l}\text { Pseudotsuga } \\
\text { menziesii }\end{array}$ & Canada, USA & $\begin{array}{l}\text { Anderson 1986a, } \\
\text { Bloomberg 1966, James } \\
1984\end{array}$ \\
\hline & & seeds & Quercus spp. & USSR & $\begin{array}{l}\text { Urosevic 1962, Mittal et } \\
\text { al. } 1990\end{array}$ \\
\hline
\end{tabular}


Table 2 Continued.

\begin{tabular}{|c|c|c|c|c|c|}
\hline Species & Taxonomy & Substrate & Host & Country & References \\
\hline & & seeds & $\begin{array}{l}\text { Swietenia } \\
\text { macrophylla }\end{array}$ & Philippines & $\begin{array}{l}\text { Dayan 1986, Mittal et al. } \\
1990\end{array}$ \\
\hline \multirow[t]{5}{*}{$\begin{array}{l}\text { Rhizopus stolonifer } \\
\text { (Ehrenb.) Vuill. }\end{array}$} & $\begin{array}{l}\text { Rhizopodaceae, Mucoromycetes, } \\
\text { Mucoromycota }\end{array}$ & fruit & $\begin{array}{l}\text { Artocarpus } \\
\text { communis }\end{array}$ & Nigeria & Amusa et al. 2002 \\
\hline & & seeds & Picea abies & USSR & $\begin{array}{l}\text { Prisyazhnyuk } 1960 \text {, } \\
\text { Mittal et al. } 1990\end{array}$ \\
\hline & & seeds & $\begin{array}{l}\text { Pseudotsuga } \\
\text { menziesii }\end{array}$ & USA & $\begin{array}{l}\text { Gordon 1967, Mittal et } \\
\text { al. } 1990\end{array}$ \\
\hline & & seed & $\begin{array}{l}\text { Stylosanthes } \\
\text { guianensis }\end{array}$ & Australia & Nik \& Parbery 1977 \\
\hline & & seed & Stylosanthes humilis & Australia & Nik \& Parbery 1977 \\
\hline \multirow[t]{2}{*}{ Rhizopus tonkinensis Vuill. } & $\begin{array}{l}\text { Rhizopodaceae, Mucoromycetes, } \\
\text { Mucoromycota }\end{array}$ & seed & Linum usitatissimum & Canada & Conners 1967 \\
\hline & & seed & Phleum pratense & Canada & Conners 1967 \\
\hline Rhodotorula sp. & $\begin{array}{l}\text { Sporidiobolaceae, Microbotryomycetes, } \\
\text { Basidiomycota }\end{array}$ & seeds & Fraxinus excelsior & Sweden & Hayatgheibi 2013 \\
\hline $\begin{array}{l}\text { Rhytidhysteron rufulum } \\
\text { (Spreng.) Speg. }\end{array}$ & $\begin{array}{l}\text { Hysteriaceae, Dothideomycetes, } \\
\text { Ascomycota }\end{array}$ & fruit & Swietenia mahagoni & Thailand & Jayasiri et al. 2019 \\
\hline \multirow[t]{4}{*}{$\begin{array}{l}\text { Rosellinia limonispora Ellis } \\
\text { \& Everh. [as 'limoniiformis'] }\end{array}$} & $\begin{array}{l}\text { Xylariaceae, Sordariomycetes, } \\
\text { Ascomycota }\end{array}$ & seed & Agropyron cristatum & Canada & Conners 1967 \\
\hline & & seed & Bromus inermis & Canada & Conners 1967 \\
\hline & & seed & Festuca rubra & Canada & Conners 1967 \\
\hline & & seed & Linum usitatissimum & Canada & Conners 1967 \\
\hline $\begin{array}{l}\text { Rosellinia obliquata var. } \\
\text { americana (Sommerf.) Sacc. }\end{array}$ & $\begin{array}{l}\text { Xylariaceae, Sordariomycetes, } \\
\text { Ascomycota }\end{array}$ & cone & Pinus ponderosa & Canada & Conners 1967 \\
\hline $\begin{array}{l}\text { Saccharomycopsis amapae } \\
\text { (P.B. Morais, C.A. Rosa, } \\
\text { S.A. Mey., Mend.-Hagler \& } \\
\text { Hagler) Casarég. \& N. } \\
\text { Jacques" [as Candida amapae } \\
\text { P.B. Morais, C.A. Rosa, S.A. } \\
\text { Mey., Mend.-Hagler \& } \\
\text { Hagler] }\end{array}$ & $\begin{array}{l}\text { Saccharomycopsidaceae, } \\
\text { Saccharomycetes, Ascomycota }\end{array}$ & fruit & $\begin{array}{l}\text { Parahancornia } \\
\text { amapa }\end{array}$ & Brazil & Morais et al. 1995 \\
\hline
\end{tabular}


Table 2 Continued.

\begin{tabular}{|c|c|c|c|c|c|}
\hline Species & Taxonomy & Substrate & Host & Country & References \\
\hline Sarcinomyces sp. & $\begin{array}{l}\text { Incertae sedis, Eurotiomycetes, } \\
\text { Ascomycota }\end{array}$ & seeds & Pinus monticola & USA & $\begin{array}{l}\text { Ganley \& Newcombe } \\
2006\end{array}$ \\
\hline $\begin{array}{l}\text { Sarocladium kiliense (Grütz) } \\
\text { Summerb. [as Acremonium } \\
\text { kiliense Grütz] }\end{array}$ & $\begin{array}{l}\text { Sarocladiaceae, Sordariomycetes, } \\
\text { Ascomycota }\end{array}$ & acorns & Quercus robur & Poland & Jankowiak 2008 \\
\hline \multirow{3}{*}{$\begin{array}{l}\text { Sarocladium strictum (W. } \\
\text { Gams) Summerb. } \\
\text { [as Acremonium strictum W. } \\
\text { Gams] }\end{array}$} & $\begin{array}{l}\text { Sarocladiaceae, Sordariomycetes, } \\
\text { Ascomycota }\end{array}$ & seeds & Eucalyptus globulus & Uruguay & Lupo et al. 2001 \\
\hline & & $\begin{array}{l}\text { seeds } \\
\text { seeds }\end{array}$ & $\begin{array}{l}\text { Eucalyptus sp. } \\
\text { Fraxinus excelsior }\end{array}$ & $\begin{array}{l}\text { India } \\
\text { Sweden }\end{array}$ & $\begin{array}{l}\text { Mittal et al. } 1990 \\
\text { Reddy et al. 1982, } \\
\text { Hayatgheibi } 2013\end{array}$ \\
\hline & & seeds & Quercus robur & Poland & Jankowiak 2008 \\
\hline \multirow{6}{*}{$\begin{array}{l}\text { Sarocladium strictum (W. } \\
\text { Gams) Summerb. } \\
\text { [as Cephalosporium } \\
\text { acremonium Corda] }\end{array}$} & & seeds & Melia spp. & India & Sahai \& Otra 1982 \\
\hline & & seeds & Picea excelsa & USSR & $\begin{array}{l}\text { Urosevic 1961, } \\
\text { Anderson 1986a }\end{array}$ \\
\hline & & seeds & Pinus kesiya & Thailand & $\begin{array}{l}\text { Chalermpongse et al. } \\
\text { 1984, Mittal et al. } 1990\end{array}$ \\
\hline & & seeds & Pinus sylvestris & USSR & $\begin{array}{l}\text { Urosevic 1961, } \\
\text { Anderson 1986a }\end{array}$ \\
\hline & & seeds & Quercus spp. & USSR & $\begin{array}{l}\text { Urosevic 1962, Mittal et } \\
\text { al. } 1990\end{array}$ \\
\hline & & seeds & Sapium spp. & India & Sahai \& Otra 1982 \\
\hline $\begin{array}{l}\text { Sarocladium zeae (W. Gams } \\
\text { \& D.R. Sumner) Summerb. } \\
\text { [as Acremonium zeae W. } \\
\text { Gams \& D.R. Sumner] }\end{array}$ & $\begin{array}{l}\text { Sarocladiaceae, Sordariomycetes, } \\
\text { Ascomycota }\end{array}$ & acorns & Quercus robur & Poland & Jankowiak 2008 \\
\hline $\begin{array}{l}\text { Saturnispora diversa (Ohara, } \\
\text { Nonom. \& Yunome ex van } \\
\text { Uden \& Buckley) Kurtzman }\end{array}$ & $\begin{array}{l}\text { Pichiaceae, Saccharomycetes, } \\
\text { Ascomycota }\end{array}$ & fruits & $\begin{array}{l}\text { Parahancornia } \\
\text { amapa }\end{array}$ & Brazil & Morais et al. 1995 \\
\hline
\end{tabular}


Table 2 Continued.

\begin{tabular}{|c|c|c|c|c|c|}
\hline Species & Taxonomy & Substrate & Host & Country & References \\
\hline \multicolumn{6}{|l|}{$\begin{array}{l}\text { [as Candida diversa Ohara, } \\
\text { Nonom. \& Yunome ex van } \\
\text { Uden \& Buckley] }\end{array}$} \\
\hline $\begin{array}{l}\text { Stauronematopsis sojae } \\
\text { (Uecker \& Kulik) Abbas, B. } \\
\text { Sutton \& Ghaffar } \\
\text { [as Pseudorobillarda sojae } \\
\text { Uecker \& Kulik] }\end{array}$ & $\begin{array}{l}\text { Incertae sedis, Incertae sedis, } \\
\text { Ascomycota }\end{array}$ & pods & Delonix regia & Thailand & Somrithipol et al. 2002b \\
\hline $\begin{array}{l}\text { Schizophyllum alneum (L.) } \\
\text { J. Schröt. }\end{array}$ & $\begin{array}{l}\text { Schizophyllaceae, Agaricomycetes, } \\
\text { Basidiomycota }\end{array}$ & $\begin{array}{l}\text { fruits } \\
\text { seeds }\end{array}$ & $\begin{array}{l}\text { Ilex cinerea } \\
\text { Quercus spp. }\end{array}$ & $\begin{array}{l}\text { Hong Kong } \\
\text { USSR }\end{array}$ & $\begin{array}{l}\text { Tang et al. 2003a } \\
\text { Mittal et al. } 1990\end{array}$ \\
\hline \multirow[t]{7}{*}{ Schizophyllum commune Fr. } & $\begin{array}{l}\text { Schizophyllaceae, Agaricomycetes, } \\
\text { Basidiomycota }\end{array}$ & $\begin{array}{l}\text { seeds } \\
\text { seeds }\end{array}$ & $\begin{array}{l}\text { Cassia fistula } \\
\text { Cassia javanica }[= \\
\text { Cassia nodosa }]\end{array}$ & $\begin{array}{l}\text { India } \\
\text { India }\end{array}$ & $\begin{array}{l}\text { Goswami \& Ojha } 2004 \\
\text { Goswami \& Ojha } 2004\end{array}$ \\
\hline & & seeds & Elaeis guineensis & Malaysia & $\begin{array}{l}\text { Turner 1981, Mittal et al. } \\
1990\end{array}$ \\
\hline & & seeds & $\begin{array}{l}\text { Pinus elliottii var. } \\
\text { elliottii }\end{array}$ & USA & Fraedrich \& Miller 1995 \\
\hline & & seeds & $\begin{array}{l}\text { Pseudotsuga } \\
\text { menziesii }\end{array}$ & USA & $\begin{array}{l}\text { Rediske \& Shea 1965, } \\
\text { Anderson 1986a }\end{array}$ \\
\hline & & seeds & Quercus spp. & USSR & $\begin{array}{l}\text { Urosevic 1962, Mittal et } \\
\text { al. } 1990\end{array}$ \\
\hline & & seeds & Shorea assamica & Malaysia & $\begin{array}{l}\text { Hong 1981, Mittal et al. } \\
1990\end{array}$ \\
\hline & & seeds & Shorea robusta & India & Goswami \& Ojha 2004 \\
\hline $\begin{array}{l}\text { Schizothecium curvisporum } \\
\text { (Cain) N. Lundq. } \\
\text { [as Sordaria curvispora } \\
\text { Cain] }\end{array}$ & $\begin{array}{l}\text { Lasiosphaeriaceae, Sordariomycetes, } \\
\text { Ascomycota }\end{array}$ & seed & $\begin{array}{l}\text { Apium graveolens } \\
\text { var. dulce }\end{array}$ & USA & Conners 1967 \\
\hline $\begin{array}{l}\text { Schwanniomyces etchellsii } \\
\text { (Kreger-van Rij) M. Suzuki } \\
\text { \& Kurtzman [as Pichia } \\
\text { etchellsii Kreger-van Rij] }\end{array}$ & $\begin{array}{l}\text { Debaryomycetaceae, Saccharomycetes, } \\
\text { Ascomycota }\end{array}$ & fruits & $\begin{array}{l}\text { Parahancornia } \\
\text { amapa }\end{array}$ & Brazil & Morais et al. 1995 \\
\hline
\end{tabular}


Table 2 Continued.

\begin{tabular}{|c|c|c|c|c|c|}
\hline Species & Taxonomy & Substrate & Host & Country & References \\
\hline $\begin{array}{l}\text { Schwanniomyces vanrijiae } \\
\text { (Van der Walt \& } \\
\text { Tscheuschner) M. Suzuki \& } \\
\text { Kurtzman } \\
\text { [as Debaryomyces vanrijiae } \\
\text { var. yarrowii (Santa María \& } \\
\text { C. García) Kreger-van Rij] }\end{array}$ & $\begin{array}{l}\text { Debaryomycetaceae, Saccharomycetes, } \\
\text { Ascomycota }\end{array}$ & fruits & $\begin{array}{l}\text { Parahancornia } \\
\text { amapa }\end{array}$ & Brazil & Morais et al. 1995 \\
\hline Sclerophoma spp. & $\begin{array}{l}\text { Dothioraceae, Dothideomycetes, } \\
\text { Ascomycota }\end{array}$ & $\begin{array}{l}\text { seeds } \\
\text { seeds } \\
\text { seeds }\end{array}$ & $\begin{array}{l}\text { Abies lasiocarpa } \\
\text { Abies nordmanniana } \\
\text { Abies procera }\end{array}$ & $\begin{array}{l}\text { Canada, Norway } \\
\text { Russia } \\
\text { Norway }\end{array}$ & $\begin{array}{l}\text { Talgø et al. } 2010 \\
\text { Talgø et al. } 2010 \\
\text { Talgø et al. } 2010\end{array}$ \\
\hline \multirow[t]{5}{*}{$\begin{array}{l}\text { Sclerophoma pithyophila } \\
\text { (Corda) Höhn. }\end{array}$} & $\begin{array}{l}\text { Dothioraceae, Dothideomycetes, } \\
\text { Ascomycota }\end{array}$ & cones & Pinus sylvestris & UK & Whittle 1977 \\
\hline & & cones, seeds & $\begin{array}{l}\text { Pinus } \times \text { schwerinii } \\
(P . \text { strobus } \times P . \\
\text { wallichiana })\end{array}$ & Canada & Vujanovic et al. 2000 \\
\hline & & cones, seeds & $\begin{array}{l}\text { Pinus nigra ssp. } \\
\text { laricio }\end{array}$ & Canada & Vujanovic et al. 2000 \\
\hline & & pods & Eucalyptus globulus & Uruguay & Lupo et al. 2001 \\
\hline & & seeds & Pinus sylvestris & UK & Whittle 1977 \\
\hline Sclerotinia betulae Woronin & $\begin{array}{l}\text { Sclerotiniaceae, Leotiomycetes, } \\
\text { Ascomycota }\end{array}$ & seed & Betula papyrifera & Canada & Conners 1967 \\
\hline Sclerotinia libertiana Fuckel & $\begin{array}{l}\text { Sclerotiniaceae, Leotiomycetes, } \\
\text { Ascomycota }\end{array}$ & seeds & Quercus spp. & USSR & Mittal et al. 1990 \\
\hline $\begin{array}{l}\text { Sclerotinia pseudotuberosa } \\
\text { (Rehm) Rehm }\end{array}$ & $\begin{array}{l}\text { Sclerotiniaceae, Leotiomycetes, } \\
\text { Ascomycota }\end{array}$ & seeds & Quercus spp. & USSR & $\begin{array}{l}\text { Urosevic 1959, Mittal et } \\
\text { al. } 1990\end{array}$ \\
\hline $\begin{array}{l}\text { Sclerotinia sclerotiorum } \\
\text { (Lib.) de Bary }\end{array}$ & $\begin{array}{l}\text { Sclerotiniaceae, Leotiomycetes, } \\
\text { Ascomycota }\end{array}$ & seeds & Quercus spp. & USSR & $\begin{array}{l}\text { Urosevic 1962, Mittal et } \\
\text { al. } 1990\end{array}$ \\
\hline \multirow[t]{4}{*}{ Sclerotium spp. } & $\begin{array}{l}\text { Sclerotiniaceae, Leotiomycetes, } \\
\text { Ascomycota }\end{array}$ & seeds & Abies spp. & - & $\begin{array}{l}\text { Ono 1974, Anderson } \\
\text { 1986a }\end{array}$ \\
\hline & & seeds & $\begin{array}{l}\text { Araucaria } \\
\text { cunninghamii }\end{array}$ & Australia & $\begin{array}{l}\text { Kamara et al. } 1981 \text {, } \\
\text { Mittal et al. } 1990\end{array}$ \\
\hline & & seeds & Prunus serrulata & Japan & Watanabe 2010 \\
\hline & & seed & $\begin{array}{l}\text { Pseudotsuga } \\
\text { menziesii }\end{array}$ & Canada & $\begin{array}{l}\text { Salisbury 1955, Mittal et } \\
\text { al. } 1990\end{array}$ \\
\hline
\end{tabular}


Table 2 Continued.

\begin{tabular}{|c|c|c|c|c|c|}
\hline Species & Taxonomy & Substrate & Host & Country & References \\
\hline \multirow{3}{*}{$\begin{array}{l}\text { Scolecobasidium } \\
\text { constrictum E.V. Abbott }\end{array}$} & \multirow{3}{*}{$\begin{array}{l}\text { Incertae sedis, Dothideomycetes, } \\
\text { Ascomycota }\end{array}$} & seed & Cercis chinensis & Japan & Watanabe 2010 \\
\hline & & seed & $\begin{array}{l}\text { Cryptomeria } \\
\text { japonica }\end{array}$ & Japan & Watanabe 2010 \\
\hline & & seed & Pinus densiflora & Japan & Watanabe 2010 \\
\hline $\begin{array}{l}\text { Scolecobasidium humicola } \\
\text { G.L. Barron \& L.V. Busch }\end{array}$ & $\begin{array}{l}\text { Incertae sedis, Dothideomycetes, } \\
\text { Ascomycota }\end{array}$ & seed & Prunus serrulata & Japan & Watanabe 2010 \\
\hline \multirow{3}{*}{$\begin{array}{l}\text { Scopulariopsis brevicaulis } \\
\text { Bainier }\end{array}$} & \multirow{3}{*}{$\begin{array}{l}\text { Microascaceae, Sordariomycetes, } \\
\text { Ascomycota }\end{array}$} & seed & Pinus densiflora & Japan & Watanabe 2010 \\
\hline & & seeds & Pinus sylvestris & USSR & $\begin{array}{l}\text { Prisyazhnyuk } 1960 \text {, } \\
\text { Mittal et al. } 1990\end{array}$ \\
\hline & & seeds & Quercus spp. & USSR & $\begin{array}{l}\text { Urosevic 1959, Mittal et } \\
\text { al. } 1990\end{array}$ \\
\hline $\begin{array}{l}\text { Scopulariopsis canadensis } \\
\text { F.J. Morton \& G. Sm. }\end{array}$ & $\begin{array}{l}\text { Microascaceae, Sordariomycetes, } \\
\text { Ascomycota }\end{array}$ & seed & Pinus densiflora & Japan & Watanabe 2010 \\
\hline $\begin{array}{l}\text { Scutellinia pseudovitreola } \\
\text { W.Y. Zhuang \& Zhu L. } \\
\text { Yang }\end{array}$ & $\begin{array}{l}\text { Pyronemataceae, Pezizomycetes, } \\
\text { Ascomycota }\end{array}$ & fruits & Platanus sp. & China & Zhuang 2013 \\
\hline $\begin{array}{l}\text { Scytalidium lignicola } \\
\text { Pesante }\end{array}$ & $\begin{array}{l}\text { Hyaloscyphaceae, Leotiomycetes, } \\
\text { Ascomycota }\end{array}$ & pod & Sesbania bispinosa & India & Anita et al. 2009 \\
\hline \multirow[t]{2}{*}{ Scytalidium spp. } & \multirow[t]{2}{*}{$\begin{array}{l}\text { Hyaloscyphaceae, Leotiomycetes, } \\
\text { Ascomycota }\end{array}$} & fruits & $\begin{array}{l}\text { Cleistocalyx } \\
\text { operculatus }\end{array}$ & Hong Kong & Tang et al. 2003a \\
\hline & & fruits & $\begin{array}{l}\text { Viburnum } \\
\text { sempervirens }\end{array}$ & Hong Kong & Tang et al. 2003a \\
\hline $\begin{array}{l}\text { Scytinostroma } \\
\text { mediterraneense Boidin \& } \\
\text { Lanq. }\end{array}$ & $\begin{array}{l}\text { Peniophoraceae, Agaricomycetes, } \\
\text { Basidiomycota }\end{array}$ & cones & Pinus halepensis & France & $\begin{array}{l}\text { Boidin \& Lanquetin } \\
1987\end{array}$ \\
\hline Sebacina sp. & $\begin{array}{l}\text { Sebacinaceae, Agaricomycetes, } \\
\text { Basidiomycota }\end{array}$ & seeds & Phyllostachys edulis & China & Shen et al. 2014 \\
\hline \multirow[t]{3}{*}{$\begin{array}{l}\text { Seimatosporium caninum } \\
\text { (Brunaud) B. Sutton }\end{array}$} & $\begin{array}{l}\text { Sporocadaceae, Sordariomycetes, } \\
\text { Ascomycota }\end{array}$ & fruits & Ardisia crenata & Hong Kong & Tang et al. 2003a \\
\hline & & fruits & Ardisia punctata & Hong Kong & Tang et al. 2003a \\
\hline & & fruits & $\begin{array}{l}\text { Cleistocalyx } \\
\text { operculatus }\end{array}$ & Hong Kong & Tang et al. 2003a \\
\hline
\end{tabular}


Table 2 Continued.

\begin{tabular}{|c|c|c|c|c|c|}
\hline Species & Taxonomy & Substrate & Host & Country & References \\
\hline \multirow[t]{3}{*}{ Seimatosporium spp. } & \multirow{3}{*}{$\begin{array}{l}\text { Sporocadaceae, Sordariomycetes, } \\
\text { Ascomycota }\end{array}$} & fruits & Ilex cinerea & Hong Kong & Tang et al. 2003a \\
\hline & & fruits & Microcos paniculata & Hong Kong & Tang et al. 2003a \\
\hline & & fruits & $\begin{array}{l}\text { Viburnum } \\
\text { sempervirens }\end{array}$ & Hong Kong & Tang et al. 2003a \\
\hline \multirow{12}{*}{$\begin{array}{l}\text { Seiridium cardinale (W.W. } \\
\text { Wagener) B. Sutton \& I.A.S. } \\
\text { Gibson }\end{array}$} & \multirow[t]{12}{*}{$\begin{array}{l}\text { Sporocadaceae, Sordariomycetes, } \\
\text { Ascomycota }\end{array}$} & seeds & $\begin{array}{l}\text { Chamaecyparis } \\
\text { lawsoniana }\end{array}$ & France, Italy & $\begin{array}{l}\text { Motta \& Saponaro 1983, } \\
\text { Mittal et al. } 1990\end{array}$ \\
\hline & & seeds & $\begin{array}{l}\text { Cupressus } \\
\text { abramsiana }\end{array}$ & France, Italy & $\begin{array}{l}\text { Saponaro \& Motta 1984, } \\
\text { Mittal et al. } 1990\end{array}$ \\
\hline & & seeds & Cupressus arizonica & France, Italy & $\begin{array}{l}\text { Saponaro \& Motta 1984, } \\
\text { Mittal et al. } 1990\end{array}$ \\
\hline & & seeds & Cupressus glabra & France, Italy & $\begin{array}{l}\text { Saponaro \& Motta 1984, } \\
\text { Mittal et al. } 1990\end{array}$ \\
\hline & & seeds & $\begin{array}{l}\text { Cupressus } \\
\text { goveniana }\end{array}$ & France, Italy & $\begin{array}{l}\text { Saponaro \& Motta 1984, } \\
\text { Mittal et al. } 1990\end{array}$ \\
\hline & & seeds & Cupressus lusitanica & France, Italy & $\begin{array}{l}\text { Saponaro \& Motta 1984, } \\
\text { Mittal et al. } 1990\end{array}$ \\
\hline & & seeds & $\begin{array}{l}\text { Cupressus lusitanica } \\
\text { var. benthamii }\end{array}$ & France, Italy & $\begin{array}{l}\text { Saponaro \& Motta 1984, } \\
\text { Mittal et al. } 1990\end{array}$ \\
\hline & & seeds & $\begin{array}{l}\text { Cupressus } \\
\text { macrocarpa }\end{array}$ & Italy & $\begin{array}{l}\text { Motta 1984, Mittal et al. } \\
1990\end{array}$ \\
\hline & & seeds & $\begin{array}{l}\text { Cupressus } \\
\text { sempervirens }\end{array}$ & France, Italy & $\begin{array}{l}\text { Motta 1984, Mittal et al. } \\
1990\end{array}$ \\
\hline & & seeds & Cupressus spp. & Italy & Mittal et al. 1990 \\
\hline & & seeds & Cupressus torulosa & France, Italy & $\begin{array}{l}\text { Saponaro \& Motta 1984, } \\
\text { Mittal et al. } 1990\end{array}$ \\
\hline & & seeds & Thuja orientalis & Italy, France & $\begin{array}{l}\text { Motta 1984, Mittal et al. } \\
1990\end{array}$ \\
\hline $\begin{array}{l}\text { Seiridium herteri } \\
=\text { Pestalotia herteri Petr. [as } \\
\text { 'hertigii’] }\end{array}$ & $\begin{array}{l}\text { Sporocadaceae, Sordariomycetes, } \\
\text { Ascomycota }\end{array}$ & seeds & Quercus pedunculata & USSR & $\begin{array}{l}\text { Kozlowska 1968, Mittal } \\
\text { et al. } 1990\end{array}$ \\
\hline $\begin{array}{l}\text { 'Seiridium kawakamii' } \\
=\text { Pestalotia kawakamii } \\
\text { Sawada }\end{array}$ & $\begin{array}{l}\text { Sporocadaceae, Sordariomycetes, } \\
\text { Ascomycota }\end{array}$ & fruits & Ilex cinerea & Hong Kong & Tang et al. 2003a \\
\hline
\end{tabular}


Table 2 Continued.

\begin{tabular}{|c|c|c|c|c|c|}
\hline Species & Taxonomy & Substrate & Host & Country & References \\
\hline \multicolumn{6}{|l|}{$\begin{array}{l}\text { [as 'Pestalotiopsis } \\
\text { kawakamii'] }\end{array}$} \\
\hline $\begin{array}{l}\text { 'Seiridium parrotiae' } \\
\text { [as Pestalotia parrotiae T.M. } \\
\text { Achundov] }\end{array}$ & $\begin{array}{l}\text { Sporocadaceae, Sordariomycetes, } \\
\text { Ascomycota }\end{array}$ & seeds & Parrotia persica & USSR & $\begin{array}{l}\text { Akhundov \& Papusha } \\
\text { 1981, Mittal et al. } 1990\end{array}$ \\
\hline \multirow[t]{14}{*}{$\begin{array}{l}\text { Seiridium spp. } \\
\text { [as Pestalotia] }\end{array}$} & $\begin{array}{l}\text { Sporocadaceae, Sordariomycetes, } \\
\text { Ascomycota }\end{array}$ & seeds & Acer palmatum & South Korea & $\begin{array}{l}\text { Mathur 1974, Anderson } \\
\text { 1986a }\end{array}$ \\
\hline & & seeds & $\begin{array}{l}\text { Adenanthera } \\
\text { microsperma }\end{array}$ & India & $\begin{array}{l}\text { Mathur 1974, Anderson } \\
\text { 1986a }\end{array}$ \\
\hline & & seeds & Albizia falcataria & Philippines & $\begin{array}{l}\text { Mathur 1974, Mittal et } \\
\text { al. } 1990\end{array}$ \\
\hline & & seeds & Albizia procera & Philippines & $\begin{array}{l}\text { Quiniones 1985, } \\
\text { Anderson 1986a }\end{array}$ \\
\hline & & seeds & $\begin{array}{l}\text { Araucaria } \\
\text { cunninghamii }\end{array}$ & Australia & $\begin{array}{l}\text { Kamara et al. } 1981 \text {, } \\
\text { Mittal et al. } 1990\end{array}$ \\
\hline & & seeds & Araucaria excelsa & USA & $\begin{array}{l}\text { Kahn et al. 1965, Mittal } \\
\text { et al. } 1990\end{array}$ \\
\hline & & seeds & $\begin{array}{l}\text { Callistemon } \\
\text { viminalis }\end{array}$ & India & $\begin{array}{l}\text { Mathur 1974, Mittal et } \\
\text { al. } 1990\end{array}$ \\
\hline & & seeds & $\begin{array}{l}\text { Casuarina } \\
\text { equisetifolia }\end{array}$ & Philippines, USA & $\begin{array}{l}\text { Mathur 1974, Mittal et } \\
\text { al. } 1990 \text {, Bayman et al. } \\
1998\end{array}$ \\
\hline & & seeds & Eucalyptus deglupta & Philippines & $\begin{array}{l}\text { Mathur 1974, Anderson } \\
\text { 1986a }\end{array}$ \\
\hline & & seeds & Grevillea robusta & Rwanda & $\begin{array}{l}\text { Mathur 1974, Anderson } \\
\text { 1986a }\end{array}$ \\
\hline & & seeds & Larix occidentalis & USA & James et al. 1996 \\
\hline & & seeds & $\begin{array}{l}\text { Leucaena } \\
\text { leucocephala }\end{array}$ & Philippines & $\begin{array}{l}\text { Mathur 1974, Anderson } \\
\text { 1986a }\end{array}$ \\
\hline & & seeds & Manilkara bidentata & USA & Bayman et al. 1998 \\
\hline & & seeds & $\begin{array}{l}\text { Mimosa } \\
\text { caesalpiniafolia }\end{array}$ & Brazil & $\begin{array}{l}\text { Mathur 1974, Anderson } \\
\text { 1986a }\end{array}$ \\
\hline
\end{tabular}


Table 2 Continued.

\begin{tabular}{|c|c|c|c|c|c|}
\hline Species & Taxonomy & Substrate & Host & Country & References \\
\hline & & seeds & $\begin{array}{l}\text { Pinus armandii var. } \\
\text { mastersiana }\end{array}$ & Taiwan & $\begin{array}{l}\text { Jong \& Chen } 1966 \text {, } \\
\text { Mittal et al. } 1990\end{array}$ \\
\hline & & seeds & Pinus caribaea & Madagascar & $\begin{array}{l}\text { Mathur 1974, Anderson } \\
\text { 1986a }\end{array}$ \\
\hline & & seed & Pinus densiflora & Japan & Watanabe 2010 \\
\hline & & seeds & Pinus elliottii & USA & $\begin{array}{l}\text { Rowan \& Debarr 1974, } \\
\text { Anderson 1986a }\end{array}$ \\
\hline & & seeds & $\begin{array}{l}\text { Pinus elliottii var. } \\
\text { elliottii }\end{array}$ & USA & Fraedrich \& Miller 1995 \\
\hline & & seeds & Pinus insularis & Philippines & $\begin{array}{l}\text { Agmata 1979, Anderson } \\
\text { 1986a }\end{array}$ \\
\hline & & seeds & Pinus khasya & $\begin{array}{l}\text { Zambia, } \\
\text { Madagascar }\end{array}$ & $\begin{array}{l}\text { Mathur } 1974 \text { Mittal et al. } \\
1990\end{array}$ \\
\hline & & seeds & Pinus massoniana & Taiwan & $\begin{array}{l}\text { Jong \& Chen } 1966 \text {, } \\
\text { Mittal et al. } 1990\end{array}$ \\
\hline & & seeds & Pinus merkusii & Zambia & $\begin{array}{l}\text { Mathur 1974, Anderson } \\
\text { 1986a }\end{array}$ \\
\hline & & seeds & Pinus oocarpa & Zambia & $\begin{array}{l}\text { Mathur 1974, Anderson } \\
\text { 1986a }\end{array}$ \\
\hline & & seeds & Pinus patula & Madagascar & $\begin{array}{l}\text { Gibson 1957, Anderson } \\
\text { 1986a }\end{array}$ \\
\hline & & seeds & Pinus roxburghii & India & $\begin{array}{l}\text { Munjal \& Sharma 1975, } \\
\text { Mittal et al. } 1990\end{array}$ \\
\hline & & seeds & Pinus taeda & $\begin{array}{l}\text { Georgia, USA, } \\
\text { Uruguay }\end{array}$ & $\begin{array}{l}\text { Mason \& van Arsdel } \\
\text { 1978, Huang \& } \\
\text { Kuhlman 1990, Mittal et } \\
\text { al. } 1990\end{array}$ \\
\hline & & seeds & $\begin{array}{l}\text { Pinus thunbergii }[= \\
\text { Pinus thunbergiana] }\end{array}$ & Japan, Taiwan & $\begin{array}{l}\text { Jong \& Chen } 1966 \text {, } \\
\text { Mittal et al. } 1990 \text {, } \\
\text { Watanabe } 2010\end{array}$ \\
\hline & & seeds & Pinus taiwanensis & Taiwan & $\begin{array}{l}\text { Jong \& Chen } 1966 \text {, } \\
\text { Mittal et al. } 1990\end{array}$ \\
\hline & & seeds & Pinus wallichiana & India & $\begin{array}{l}\text { Munjal \& Sharma 1975, } \\
\text { Mittal et al. } 1990\end{array}$ \\
\hline
\end{tabular}


Table 2 Continued.

\begin{tabular}{|c|c|c|c|c|c|}
\hline Species & Taxonomy & Substrate & Host & Country & References \\
\hline & & seeds & $\begin{array}{l}\text { Platanus } \\
\text { occidentalis }\end{array}$ & USA & $\begin{array}{l}\text { Fakir et al. 1971, Mittal } \\
\text { et al. } 1990\end{array}$ \\
\hline & & seeds & Prosopis juliflora & Brazil & $\begin{array}{l}\text { Mathur 1974, Mittal et } \\
\text { al. } 1990\end{array}$ \\
\hline & & seed & Prunus serrulata & Japan & Watanabe 2010 \\
\hline & & seeds & Pterocarpus indicus & Philippines & $\begin{array}{l}\text { Agmata 1979, Anderson } \\
\text { 1986a }\end{array}$ \\
\hline & & seeds & $\begin{array}{l}\text { Pterospermum } \\
\text { acerifolium }\end{array}$ & India & $\begin{array}{l}\text { Mathur 1974, Anderson } \\
\text { 1986a }\end{array}$ \\
\hline & & seeds & $\begin{array}{l}\text { Sequoia } \\
\text { sempervirens }\end{array}$ & USA & $\begin{array}{l}\text { Davidson 1970, Mittal et } \\
\text { al. } 1990\end{array}$ \\
\hline & & seeds & $\begin{array}{l}\text { Swietenia } \\
\text { macrophylla }\end{array}$ & Philippines & $\begin{array}{l}\text { Agmata 1979, Anderson } \\
\text { 1986a }\end{array}$ \\
\hline & & seeds & $\begin{array}{l}\text { Taxodium } \\
\text { mucronatum }\end{array}$ & India & $\begin{array}{l}\text { Mathur 1974, Anderson } \\
\text { 1986a }\end{array}$ \\
\hline & & seeds & Tectona grandis & India & $\begin{array}{l}\text { Anderson 1986a, } \\
\text { Mohanan et al. } 2005\end{array}$ \\
\hline & & seeds & $\begin{array}{l}\text { Terminalia } \\
\text { myriocarpa }\end{array}$ & India & $\begin{array}{l}\text { Mathur 1974, Anderson } \\
\text { 1986a }\end{array}$ \\
\hline & & seeds & Thuja spp. & Spain & Anderson 1986a \\
\hline & & seeds & Triplaris cumingiana & Philippines & $\begin{array}{l}\text { Quiniones 1985, } \\
\text { Anderson 1986a }\end{array}$ \\
\hline & & seeds & Ulmus davidiana & South Korea & $\begin{array}{l}\text { Mathur 1974, Anderson } \\
\text { 1986a }\end{array}$ \\
\hline \multirow{3}{*}{$\begin{array}{l}\text { Seiridium quercina } \\
\text { [as Pestalotia quercina } \\
\text { Guba] }\end{array}$} & $\begin{array}{l}\text { Sporocadaceae, Sordariomycetes, } \\
\text { Ascomycota }\end{array}$ & seeds & Picea excelsa & USSR & $\begin{array}{l}\text { Urosevic 1961, } \\
\text { Anderson 1986a }\end{array}$ \\
\hline & & seeds & Pinus sylvestris & USSR & $\begin{array}{l}\text { Urosevic 1961, } \\
\text { Anderson 1986a }\end{array}$ \\
\hline & & seeds & Quercus spp. & USSR & $\begin{array}{l}\text { Urosevic 1962, Mittal et } \\
\text { al. } 1990\end{array}$ \\
\hline $\begin{array}{l}\text { Selenophoma bromigena } \\
\text { (Sacc.) R. Sprague \& Aar.G. } \\
\text { Johnson }\end{array}$ & $\begin{array}{l}\text { Saccotheciaceae, Dothideomycetes, } \\
\text { Ascomycota }\end{array}$ & seed & Agropyron cristatum & Canada & Conners 1967 \\
\hline
\end{tabular}


Table 2 Continued.

\begin{tabular}{|c|c|c|c|c|c|}
\hline Species & Taxonomy & Substrate & Host & Country & References \\
\hline & & seed & Bromus inermis & Canada & Conners 1967 \\
\hline Selenophoma donacis & Saccotheciaceae, Dothideomycetes, & seed & Agropyron cristatum & Canada & Conners 1967 \\
\hline $\begin{array}{l}\text { (Pass.) R. Sprague \& Aar.G. } \\
\text { Johnson }\end{array}$ & Ascomycota & seed & Festuca elatior & Canada & Conners 1967 \\
\hline $\begin{array}{l}\text { Selenophoma obtusa R. } \\
\text { Sprague \& Aar.G. Johnson }\end{array}$ & $\begin{array}{l}\text { Saccotheciaceae, Dothideomycetes, } \\
\text { Ascomycota }\end{array}$ & seed & Bromus inermis & Canada & Conners 1967 \\
\hline $\begin{array}{l}\text { Selenosporella curvispora G. } \\
\text { Arnaud ex MacGarvie }\end{array}$ & $\begin{array}{l}\text { Incertae sedis, Sordariomycetes, } \\
\text { Ascomycota }\end{array}$ & cones & Pinus densiflora & Japan & Kasai et al. 1995 \\
\hline Selenosporella sp. & $\begin{array}{l}\text { Incertae sedis, Sordariomycetes, } \\
\text { Ascomycota }\end{array}$ & pods & Delonix regia & Thailand & Somrithipol et al. 2002b \\
\hline $\begin{array}{l}\text { Sepedonium chrysospermum } \\
\text { (Bull.) Fr. }\end{array}$ & $\begin{array}{l}\text { Hypocreaceae, Sordariomycetes, } \\
\text { Ascomycota }\end{array}$ & seeds & Quercus spp. & USSR & $\begin{array}{l}\text { Urosevic 1959, Mittal et } \\
\text { al. } 1990\end{array}$ \\
\hline $\begin{array}{l}\text { Sepedonium sp. } \\
\text { [as 'Sepedonicum'] }\end{array}$ & $\begin{array}{l}\text { Hypocreaceae, Sordariomycetes, } \\
\text { Ascomycota }\end{array}$ & seeds & $\begin{array}{l}\text { Pseudotsuga } \\
\text { menziesii }\end{array}$ & USA & $\begin{array}{l}\text { Gordon 1967, Mittal et } \\
\text { al. } 1990\end{array}$ \\
\hline $\begin{array}{l}\text { Septobasidium sabal-minor } \\
\text { Couch ex L.D. Gómez \& } \\
\text { Henk }^{\#}\end{array}$ & $\begin{array}{l}\text { Septobasidiaceae, Pucciniomycetes, } \\
\text { Basidiomycota }\end{array}$ & fruits & Sabal minor & USA & Gómez \& Henk 2004 \\
\hline \multirow[t]{6}{*}{$\begin{array}{l}\text { Septochytrium variabile } \\
\text { Berdan }\end{array}$} & $\begin{array}{l}\text { Septochytriaceae, Chytridiomycetes, } \\
\text { Chytridiomycota }\end{array}$ & seeds & Bryonia cretica & $\begin{array}{l}\text { Poland or/ } \\
\text { imported to Poland }\end{array}$ & Czeczuga et al. 2009 \\
\hline & & seeds & Crataegus azarolus & $\begin{array}{l}\text { Poland or/ } \\
\text { imported to Poland }\end{array}$ & Czeczuga et al. 2009 \\
\hline & & seeds & $\begin{array}{l}\text { Hyoscyamus } \\
\text { gyoerffyi }\end{array}$ & $\begin{array}{l}\text { Poland or/ } \\
\text { imported to Poland }\end{array}$ & Czeczuga et al. 2009 \\
\hline & & seeds & Juniperus oxycedrus & $\begin{array}{l}\text { Poland or/ } \\
\text { imported to Poland }\end{array}$ & Czeczuga et al. 2009 \\
\hline & & seeds & Vicia sativa & Poland & Kiziewicz 2005 \\
\hline & & seeds & $\begin{array}{l}\text { Zantedeschia albo- } \\
\text { maculata }\end{array}$ & $\begin{array}{l}\text { Poland or/ } \\
\text { imported to Poland }\end{array}$ & Czeczuga et al. 2009 \\
\hline \multirow[t]{3}{*}{ Septoria spp. } & $\begin{array}{l}\text { Mycosphaerellaceae, Dothideomycetes, } \\
\text { Ascomycota }\end{array}$ & cones & Pinus ayacahuite & Canada & Vujanovic et al. 2000 \\
\hline & & fruits & Dichroa febrifuga & Hong Kong & Tang et al. 2003a \\
\hline & & seeds & Acer palmatum & South Korea & $\begin{array}{l}\text { Mathur 1974, Anderson } \\
\text { 1986a }\end{array}$ \\
\hline
\end{tabular}


Table 2 Continued.

\begin{tabular}{|c|c|c|c|c|c|}
\hline Species & Taxonomy & Substrate & Host & Country & References \\
\hline & & seeds & Fraxinus excelsior & Sweden & $\begin{array}{l}\text { Cleary et al. 2013, } \\
\text { Hayatgheibi } 2013\end{array}$ \\
\hline & & seeds & $\begin{array}{l}\text { Mimosa } \\
\text { caesalpiniafolia }\end{array}$ & Brazil & $\begin{array}{l}\text { Mathur 1974, Anderson } \\
\text { 1986a }\end{array}$ \\
\hline Shiraia spp. & $\begin{array}{l}\text { Shiraiaceae, Dothideomycetes, } \\
\text { Ascomycota }\end{array}$ & seeds & Phyllostachys edulis & China & Shen et al. 2014 \\
\hline $\begin{array}{l}\text { Simocybe panamensis R. } \\
\text { Singer }{ }^{\#}\end{array}$ & $\begin{array}{l}\text { Crepidotaceae, Agaricomycetes, } \\
\text { Basidiomycota }\end{array}$ & fruit & undetermined plant & Panama & Singer 1989 \\
\hline Simplicillium sp. & $\begin{array}{l}\text { Cordycipitaceae, Sordariomycetes, } \\
\text { Ascomycota }\end{array}$ & seeds & Phyllostachys edulis & China & Shen et al. 2014 \\
\hline \multirow[t]{4}{*}{$\begin{array}{l}\text { Sirococcus conigenus (Pers.) } \\
\text { P.F. Cannon \& Minter }\end{array}$} & $\begin{array}{l}\text { Gnomoniaceae, Sordariomycetes, } \\
\text { Ascomycota }\end{array}$ & cones, seeds & $\begin{array}{l}\text { Pinus nigra ssp. } \\
\text { laricio }\end{array}$ & Canada & Vujanovic et al. 2000 \\
\hline & & cones, seeds & Pinus ponderosa & Canada & Vujanovic et al. 2000 \\
\hline & & seeds & Abies procera & Norway & Talgø et al. 2010 \\
\hline & & seeds & Picea abies & Italy & Motta et al. 1996 \\
\hline \multirow{7}{*}{$\begin{array}{l}\text { Sirococcus conigenus (Pers.) } \\
\text { P.F. Cannon \& Minter [as S. } \\
\text { strobilinus Preuss] }\end{array}$} & & cones & Pinus abies & Germany & Preuss 1855 \\
\hline & & seeds & Picea abies & Canada & $\begin{array}{l}\text { Sutherland et al. } 1981 \text {, } \\
\text { Mittal et al. } 1990\end{array}$ \\
\hline & & seeds & Picea engelmannii & Canada, USA & $\begin{array}{l}\text { Sutherland et al. 1981, } \\
\text { Anderson 1986a }\end{array}$ \\
\hline & & seeds & Picea glauca & Canada & $\begin{array}{l}\text { Sutherland et al. 1981, } \\
\text { Mittal et al. } 1990\end{array}$ \\
\hline & & seeds & $\begin{array}{l}\text { Picea glauca } \times P \text {. } \\
\text { engelmannii }\end{array}$ & Canada & $\begin{array}{l}\text { Sutherland et al. 1981, } \\
\text { Mittal et al. } 1990\end{array}$ \\
\hline & & seeds & Picea sitchensis & Canada & $\begin{array}{l}\text { Salt 1964, Mittal et al. } \\
1990\end{array}$ \\
\hline & & seeds & Picea spp. & Canada & $\begin{array}{l}\text { Sutherland et al. 1981, } \\
\text { Anderson 1986a }\end{array}$ \\
\hline $\begin{array}{l}\text { Sirococcus piceicola } \\
\text { Rosssman, Castl., DF Farr \& } \\
\text { Stanosz }^{\#}\end{array}$ & $\begin{array}{l}\text { Gnomoniaceae, Sordariomycetes, } \\
\text { Ascomycota }\end{array}$ & cone scales & Picea sitchensis & Canada & Rossman et al. 2008 \\
\hline $\begin{array}{l}\text { Soloacrosporiella acaciae } \\
\text { Crous \& M.J. Wingf." }{ }^{* *}\end{array}$ & $\begin{array}{l}\text { Incertae sedis, Dothideomycetes, } \\
\text { Ascomycota }\end{array}$ & pods & Acacia mangium & Malaysia & Crous et al. 2015a \\
\hline
\end{tabular}


Table 2 Continued.

\begin{tabular}{|c|c|c|c|c|c|}
\hline Species & Taxonomy & Substrate & Host & Country & References \\
\hline Sordaria alcina N. Lundq. & $\begin{array}{l}\text { Sordariaceae, Sordariomycetes, } \\
\text { Ascomycota }\end{array}$ & seeds & Abies lasiocarpa & Norway & Talgø et al. 2010 \\
\hline \multirow[t]{12}{*}{$\begin{array}{l}\text { Sordaria fimicola (Roberge } \\
\text { ex Desm.) Ces. \& De Not. }\end{array}$} & $\begin{array}{l}\text { Sordariaceae, Sordariomycetes, } \\
\text { Ascomycota }\end{array}$ & seeds & Acer saccharinum & USA & $\begin{array}{l}\text { Friedrich et al. } 1971 \text {, } \\
\text { Mittal et al. } 1990\end{array}$ \\
\hline & & seed & $\begin{array}{l}\text { Apium graveolens } \\
\text { var. dulce }\end{array}$ & Canada & Conners 1967 \\
\hline & & seed & Bromus sp. & Canada & Conners 1967 \\
\hline & & seed & Festuca rubra & Canada & Conners 1967 \\
\hline & & seeds & Fraxinus americana & USA & $\begin{array}{l}\text { Friedrich et al. } 1971 \text {, } \\
\text { Mittal et al. } 1990\end{array}$ \\
\hline & & seed & Linum usitatissimum & Canada & Conners 1967 \\
\hline & & seeds & Picea abies & USSR & $\begin{array}{l}\text { Prisyazhnyuk 1960, } \\
\text { Mittal et al. } 1990\end{array}$ \\
\hline & & seeds & Pinus roxburghii & India & $\begin{array}{l}\text { Munjal \& Sharma 1975, } \\
\text { Mittal et al. } 1990\end{array}$ \\
\hline & & seeds & Pinus sylvestris & USSR & $\begin{array}{l}\text { Dolejs 1964, Mittal et al. } \\
1990\end{array}$ \\
\hline & & seeds & Pinus wallichiana & India & $\begin{array}{l}\text { Munjal \& Sharma 1975, } \\
\text { Mittal et al. } 1990\end{array}$ \\
\hline & & seeds & $\begin{array}{l}\text { Platanus } \\
\text { occidentalis }\end{array}$ & USA & $\begin{array}{l}\text { Fakir et al. 1971, Mittal } \\
\text { et al. } 1990\end{array}$ \\
\hline & & seed & Prunus serrulata & Japan & Watanabe 2010 \\
\hline $\begin{array}{l}\text { Sordaria nodulifera } \\
\text { TsuneoWatanabe }^{\#}\end{array}$ & $\begin{array}{l}\text { Sordariaceae, Sordariomycetes, } \\
\text { Ascomycota }\end{array}$ & seeds & Prunus jamasakura & Japan & Watanabe 1989 \\
\hline $\begin{array}{l}\text { Sordaria tamaensis Ts. } \\
\text { Watan }{ }^{\#}\end{array}$ & $\begin{array}{l}\text { Sordariaceae, Sordariomycetes, } \\
\text { Ascomycota }\end{array}$ & seeds & Prunus jamasakura & Japan & Watanabe 1989 \\
\hline $\begin{array}{l}\text { Spegazzinia flabellata S.M. } \\
\text { Leão \& Gusmão }\end{array}$ & $\begin{array}{l}\text { Didymosphaeriaceae, Dothideomycetes, } \\
\text { Ascomycota }\end{array}$ & fruit & undetermined plant & Brazil & $\begin{array}{l}\text { Leão-Ferreira \& } \\
\text { Gusmão } 2010\end{array}$ \\
\hline $\begin{array}{l}\text { Spegazzinia radermacherae } \\
\text { Jayasiri, E.B.G. Jones \& } \\
\text { K.D. Hyde }^{\#}\end{array}$ & $\begin{array}{l}\text { Didymosphaeriaceae, Dothideomycetes, } \\
\text { Ascomycota }\end{array}$ & pod & $\begin{array}{l}\text { Radermachera } \\
\text { sinica }\end{array}$ & Thailand & Jayasiri et al. 2019 \\
\hline $\begin{array}{l}\text { Spegazzinia tessarthra } \\
\text { (Berk. \& M.A. Curtis) Sacc. }\end{array}$ & $\begin{array}{l}\text { Didymosphaeriaceae, Dothideomycetes, } \\
\text { Ascomycota }\end{array}$ & seed & Cercis chinensis & Japan & Watanabe 2010 \\
\hline
\end{tabular}


Table 2 Continued.

\begin{tabular}{|c|c|c|c|c|c|}
\hline Species & Taxonomy & Substrate & Host & Country & References \\
\hline Sphaeronaema sp. & $\begin{array}{l}\text { Incertae sedis, Incertae sedis, } \\
\text { Ascomycota, }\end{array}$ & seeds & Pinus taeda & USA & Anderson 1986a, b \\
\hline Sphaeropsis sapinea (Fr.) & Botryosphaeriaceae, Dothideomycetes, & cones & Pinus nigra & Sweden & Oliva et al. 2013 \\
\hline \multirow[t]{12}{*}{ Dyko \& B. Sutton } & Ascomycota & cones & Pinus patula & South Africa & Smith et al. 1996 \\
\hline & & cones & Pinus radiate & South Africa & Smith et al. 1996 \\
\hline & & cones & Pinus sylvestris & Sweden & Oliva et al. 2013 \\
\hline & & cones, seeds & $\begin{array}{l}\text { Pinus albicaulis } \\
\text { Engelm. }\end{array}$ & Canada & Vujanovic et al. 2000 \\
\hline & & cones, seeds & Pinus resinosa & Canada & Vujanovic et al. 2000 \\
\hline & & cones, seeds & Pinus sylvestris & Canada & Vujanovic et al. 2000 \\
\hline & & seeds & Fraxinus excelsior & Sweden & Hayatgheibi 2013 \\
\hline & & seeds & Pinus caribaea & Australia & Rees \& Webber 1988 \\
\hline & & seeds & $\begin{array}{l}\text { Pinus devoniana }[= \\
\text { Pinus michoacana }]\end{array}$ & Zambia & Rees \& Webber 1988 \\
\hline & & seeds & $\begin{array}{l}\text { Pinus elliottii var. } \\
\text { elliottii }\end{array}$ & USA & Fraedrich \& Miller 1995 \\
\hline & & seeds & Pinus oocarpa & Honduras, Zambia & Rees \& Webber 1988 \\
\hline & & seeds & Pinus sylvestris & Poland & Krol et al. 2015 \\
\hline Sphaeropsis sapinea (Fr.) & & seeds & Pinus ponderosa & USA & James \& Genz 1982, \\
\hline Dyko \& B. Sutton & & & & & Anderson 1986a \\
\hline [as Diplodia pinea (Desm.) J. & & seeds & Pinus spp. & UK, USA & Anderson 1986a \\
\hline \multirow[t]{2}{*}{ Kickx f.] } & & seeds & Pinus taeda & Uruguay & Anderson 1986a \\
\hline & & seeds & Pinus wallichiana & Pakistan & Ashaeer 2000 \\
\hline Sphaeropsis sapinea (Fr.) & & cones & Pinus nigra & The Netherlands & Phillips et al. 2013 \\
\hline Dyko \& B. Sutton & & cone & Pinus sp. & China & Jayasiri et al. 2019 \\
\hline [Diplodia sapinea (Fr.) & & cones, seeds & Pinus nigra ssp. & France & Decourcelle et al. 2015 \\
\hline \multirow[t]{2}{*}{ Fuckel] } & & & laricio & & \\
\hline & & cones & Pinus sylvestris & France & Decourcelle et al. 2015 \\
\hline Sphaeropsis sapinea (Fr.) & & seeds & Pinus oocarpa & UK & Rees 1982, Mittal et al. \\
\hline Dyko \& B. Sutton & & & & & 1990 \\
\hline $\begin{array}{l}\text { [as Macrophoma sapinea } \\
\text { (Fr.) Petr.] }\end{array}$ & & seeds & Pinus pseudostrobus & UK & $\begin{array}{l}\text { Rees 1982, Mittal et al. } \\
1990\end{array}$ \\
\hline Sphaeropsis spp. & $\begin{array}{l}\text { Botryosphaeriaceae, Dothideomycetes, } \\
\text { Ascomycota }\end{array}$ & seeds & $\begin{array}{l}\text { Pinus armandii var. } \\
\text { mastersiana }\end{array}$ & Taiwan & $\begin{array}{l}\text { Jong \& Chen } 1966 \text {, } \\
\text { Mittal et al. } 1990\end{array}$ \\
\hline
\end{tabular}


Table 2 Continued.

\begin{tabular}{|c|c|c|c|c|c|}
\hline Species & Taxonomy & Substrate & Host & Country & $\begin{array}{l}\text { References } \\
\end{array}$ \\
\hline & & seeds & Pinus elliottii & Taiwan, USA & $\begin{array}{l}\text { Rowan \& Debarr 1974, } \\
\text { Mittal et al. } 1990\end{array}$ \\
\hline & & seeds & $\begin{array}{l}\text { Pinus elliottii var. } \\
\text { elliottii }\end{array}$ & Georgia & Huang \& Kuhlman 1990 \\
\hline & & seeds & Pinus massoniana & Taiwan & $\begin{array}{l}\text { Jong \& Chen } 1966 \text {, } \\
\text { Mittal et al. } 1990\end{array}$ \\
\hline & & seeds & Pinus taeda & Georgia, USA & $\begin{array}{l}\text { Covington et al. 1982, } \\
\text { Huang \& Kuhlman } \\
\text { 1990, Mittal et al. } 1990\end{array}$ \\
\hline & & seeds & Pinus taiwanensis & Taiwan & $\begin{array}{l}\text { Jong \& Chen } 1966 \text {, } \\
\text { Mittal et al. } 1990\end{array}$ \\
\hline & & seeds & $\begin{array}{l}\text { Pinus thunbergii } \\
\text { [= Pinus } \\
\text { thunbergiana }]\end{array}$ & Taiwan & $\begin{array}{l}\text { Jong \& Chen } 1966 \text {, } \\
\text { Mittal et al. } 1990\end{array}$ \\
\hline $\begin{array}{l}\text { Sphaerostilbella } \\
\text { penicillioides (Corda) }\end{array}$ & $\begin{array}{l}\text { Hypocreaceae, Sordariomycetes, } \\
\text { Ascomycota }\end{array}$ & seeds & $\begin{array}{l}\text { Eucalyptus } \\
\text { citriodora }\end{array}$ & India & $\begin{array}{l}\text { Mittal \& Sharma 1984, } \\
\text { Mittal et al. } 1990\end{array}$ \\
\hline $\begin{array}{l}\text { Rossman, L. Lombard \& } \\
\text { Crous [as Gliocladium } \\
\text { penicillioides Corda] }\end{array}$ & & seeds & Quercus spp. & USSR & $\begin{array}{l}\text { Urosevic 1962, Mittal et } \\
\text { al. } 1990\end{array}$ \\
\hline $\begin{array}{l}\text { Sphaerulina mimosae- } \\
\text { pigrae H.C. Evans \& G. } \\
\text { Carrión\# }{ }^{\#}\end{array}$ & $\begin{array}{l}\text { Mycosphaerellaceae, Dothideomycetes, } \\
\text { Ascomycota }\end{array}$ & pods & Mimosa pigra & Australia & Evans et al. 1993 \\
\hline \multirow[t]{4}{*}{$\begin{array}{l}\text { Spicaria elegans (Corda) } \\
\text { Harz }\end{array}$} & $\begin{array}{l}\text { Incertae sedis, Incertae sedis, } \\
\text { Ascomycota }\end{array}$ & seeds & Abies sibirica & USSR & $\begin{array}{l}\text { Prisyazhnyuk 1960, } \\
\text { Mittal et al. } 1990\end{array}$ \\
\hline & & seeds & Larix sibirica & USSR & $\begin{array}{l}\text { Prisyazhnyuk } 1960 \text {, } \\
\text { Mittal et al. } 1990\end{array}$ \\
\hline & & seeds & Picea abies & USSR & $\begin{array}{l}\text { Prisyazhnyuk } 1960 \text {, } \\
\text { Mittal et al. } 1990\end{array}$ \\
\hline & & seeds & Pinus sylvestris & USSR & $\begin{array}{l}\text { Dolejs 1964, Mittal et al. } \\
1990\end{array}$ \\
\hline $\begin{array}{l}\text { Spicaria simplicissima } \\
\text { Oudem. }\end{array}$ & $\begin{array}{l}\text { Incertae sedis, Incertae sedis, } \\
\text { Ascomycota }\end{array}$ & seeds & Albizia lebbeck & India & $\begin{array}{l}\text { Mittal \& Sharma 1982a, } \\
\text { Mittal et al. } 1990\end{array}$ \\
\hline
\end{tabular}


Table 2 Continued.

\begin{tabular}{|c|c|c|c|c|c|}
\hline Species & Taxonomy & Substrate & Host & Country & References \\
\hline Spicaria sp. & $\begin{array}{l}\text { Incertae sedis, Incertae sedis, } \\
\text { Ascomycota }\end{array}$ & seeds & $\begin{array}{l}\text { Pseudotsuga } \\
\text { menziesii }\end{array}$ & Canada & $\begin{array}{l}\text { Salisbury 1955, Mittal et } \\
\text { al. } 1990\end{array}$ \\
\hline Spondylocladiella sp. & $\begin{array}{l}\text { Incertae sedis, Incertae sedis, } \\
\text { Ascomycota }\end{array}$ & seeds & Pinus caribaea & $\begin{array}{l}\text { Belize [as British } \\
\text { Honduras] }\end{array}$ & Hocking 1968 \\
\hline Spondylocladium sp. & $\begin{array}{l}\text { Incertae sedis, Incertae sedis, } \\
\text { Ascomycota }\end{array}$ & seeds & Pinus taeda & USA & Anderson 1986a, b \\
\hline $\begin{array}{l}\text { Sporidesmium britannicum } \\
\text { B. Sutton }{ }^{\#}\end{array}$ & $\begin{array}{l}\text { Sporidesmiaceae, Sordariomycetes, } \\
\text { Ascomycota }\end{array}$ & cupule & Fagus sylvatica & UK & Minter 1986 \\
\hline $\begin{array}{l}\text { Sporobolomyces roseus } \\
\text { Kluyver \& C.B. Niel }\end{array}$ & $\begin{array}{l}\text { Sporidiobolaceae, Microbotryomycetes, } \\
\text { Basidiomycota }\end{array}$ & seeds & Fraxinus excelsior & Sweden & Hayatgheibi 2013 \\
\hline Sporobolomyces spp. & $\begin{array}{l}\text { Sporidiobolaceae, Microbotryomycetes, } \\
\text { Basidiomycota }\end{array}$ & $\begin{array}{l}\text { seeds } \\
\text { seed } \\
\text { seeds }\end{array}$ & $\begin{array}{l}\text { Eucalyptus grandis } \\
\text { Fraxinus excelsior } \\
\text { Pinus thunbergii }\end{array}$ & $\begin{array}{l}\text { South Africa } \\
\text { Sweden } \\
\text { Japan }\end{array}$ & $\begin{array}{l}\text { Jimu et al. } 2016 \\
\text { Hayatgheibi } 2013 \\
\text { Watanabe } 2010\end{array}$ \\
\hline $\begin{array}{l}\text { Sporoschisma hemipsilum } \\
\text { (Berk. \& Broome) Zelski, } \\
\text { A.N. Mill. \& Shearer } \\
\text { [as Melanochaeta hemipsila } \\
\text { (Berk. \& Broome) E. Müll., } \\
\text { Harr \& Sulmont] }\end{array}$ & $\begin{array}{l}\text { Chaetosphaeriaceae, Sordariomycetes, } \\
\text { Ascomycota }\end{array}$ & pods & Delonix regia & Thailand & Somrithipol et al. 2002b \\
\hline $\begin{array}{l}\text { Sporoschisma hemipsilum } \\
\text { (Berk. \& Broome) Zelski, } \\
\text { A.N. Mill. \& Shearer [as } \\
\text { Sporoschisma saccardoi } \\
\text { E.W. Mason \& S. Hughes] }\end{array}$ & $\begin{array}{l}\text { Chaetosphaeriaceae, Sordariomycetes, } \\
\text { Ascomycota }\end{array}$ & pods & Delonix regia & Thailand & Somrithipol et al. 2002b \\
\hline $\begin{array}{l}\text { Sporoschisma } \\
\text { nigroseptatum D. Rao \& } \\
\text { P.Rag. Rao }\end{array}$ & $\begin{array}{l}\text { Chaetosphaeriaceae, Sordariomycetes, } \\
\text { Ascomycota }\end{array}$ & pods & Delonix regia & Thailand & Somrithipol et al. 2002b \\
\hline Sporoschismopsis sp. & $\begin{array}{l}\text { Reticulascaceae, Sordariomycetes, } \\
\text { Ascomycota }\end{array}$ & pods & Delonix regia & Thailand & Somrithipol et al. 2002b \\
\hline $\begin{array}{l}\text { Sporothrix dimorphospora } \\
\text { (Roxon \& S.C. Jong) Madrid, } \\
\text { Gené, Cano \& Guarro }\end{array}$ & $\begin{array}{l}\text { Ophiostomataceae, Sordariomycetes, } \\
\text { Ascomycota }\end{array}$ & seed & Pinus thunbergii & Japan & Watanabe 2010 \\
\hline
\end{tabular}


Table 2 Continued.

\begin{tabular}{|c|c|c|c|c|c|}
\hline Species & Taxonomy & Substrate & Host & Country & References \\
\hline \multicolumn{6}{|l|}{$\begin{array}{l}\text { [as Humicola dimorphospora } \\
\text { Roxon \& S.C. Jong] }\end{array}$} \\
\hline \multirow[t]{2}{*}{ Sporothrix spp. } & Ophiostomataceae, Sordariomycetes, & seeds & Pinus taeda & USA & Anderson 1986a, b \\
\hline & Ascomycota & seeds & Tectona grandis & India & Anderson 1986a \\
\hline $\begin{array}{l}\text { Sporothrix stenoceras } \\
\text { (Robak) Z.W. de Beer, T.A. } \\
\text { Duong \& M.J. Wingf. } \\
\text { [as Ophiostoma stenoceras } \\
\text { (Robak) Nannf.] }\end{array}$ & $\begin{array}{l}\text { Ophiostomataceae, Sordariomycetes, } \\
\text { Ascomycota }\end{array}$ & seeds & Pinus elliottii & South Africa & Cilliers et al. 1995 \\
\hline Sporotrichum roseum Link & $\begin{array}{l}\text { Fomitopsidaceae, Agaricomycetes, } \\
\text { Basidiomycota }\end{array}$ & seeds & Quercus spp. & USSR & $\begin{array}{l}\text { Urosevic 1959, Mittal et } \\
\text { al. } 1990\end{array}$ \\
\hline \multirow[t]{3}{*}{ Sporotrichum spp. } & $\begin{array}{l}\text { Fomitopsidaceae, Agaricomycetes, } \\
\text { Basidiomycota }\end{array}$ & seed & Betula papyrifera & USA & $\begin{array}{l}\text { Friedrich et al. } 1971 \text {, } \\
\text { Mittal et al. } 1990\end{array}$ \\
\hline & & seeds & Pinus taeda & USA & Anderson 1986a, b \\
\hline & & seeds & Pinus thunbergii & Japan & Watanabe 2010 \\
\hline \multirow[t]{3}{*}{$\begin{array}{l}\text { Stachybotrys alternans } \\
\text { Bonord. }\end{array}$} & $\begin{array}{l}\text { Stachybotryaceae, Sordariomycetes, } \\
\text { Ascomycota }\end{array}$ & seeds & Betula verrucosa & USSR & $\begin{array}{l}\text { Kozlowska 1968, Mittal } \\
\text { et al. } 1990\end{array}$ \\
\hline & & seeds & Larix decidua & USSR & $\begin{array}{l}\text { Kozlowska 1968, Mittal } \\
\text { et al. } 1990\end{array}$ \\
\hline & & seeds & Pinus roxburghii & India & $\begin{array}{l}\text { Mittal \& Sharma 1982c, } \\
\text { Mittal et al. } 1990\end{array}$ \\
\hline \multirow[t]{2}{*}{$\begin{array}{l}\text { Stachybotrys chartarum } \\
\text { (Ehrenb.) S. Hughes }\end{array}$} & $\begin{array}{l}\text { Stachybotryaceae, Sordariomycetes, } \\
\text { Ascomycota }\end{array}$ & seed & Eucalyptus sp. & India & $\begin{array}{l}\text { Reddy et al. 1982, Mittal } \\
\text { et al. } 1990\end{array}$ \\
\hline & & seeds & Linum usitatissimum & Canada & Conners 1967 \\
\hline $\begin{array}{l}\text { Stachybotrys chartarum } \\
\text { (Ehrenb.) S. Hughes }\end{array}$ & & seeds & Cedrus deodara & India & $\begin{array}{l}\text { Munjal \& Sharma 1975, } \\
\text { Mittal et al. } 1990\end{array}$ \\
\hline \multirow[t]{3}{*}{$\begin{array}{l}\text { = Stachybotrys atrus Corda } \\
\text { [as 'atra'] }\end{array}$} & & seeds & Eucalyptus sp. & India & $\begin{array}{l}\text { Saxena 1985, Mittal et } \\
\text { al. } 1990\end{array}$ \\
\hline & & seeds & Pinus roxburghii & India & $\begin{array}{l}\text { Munjal \& Sharma 1975, } \\
\text { Mittal et al. } 1990\end{array}$ \\
\hline & & seeds & Pinus wallichiana & India & $\begin{array}{l}\text { Munjal \& Sharma 1975, } \\
\text { Mittal et al. } 1990\end{array}$ \\
\hline $\begin{array}{l}\text { Stachybotrys chartarum } \\
\text { (Ehrenb.) S. Hughes }\end{array}$ & & seeds & Pinus sylvestris & Poland, USSR & $\begin{array}{l}\text { Garbowski 1936, Mittal } \\
\text { et al. } 1990\end{array}$ \\
\hline
\end{tabular}


Table 2 Continued.

\begin{tabular}{|c|c|c|c|c|c|}
\hline Species & Taxonomy & Substrate & Host & Country & References \\
\hline \multicolumn{6}{|l|}{$=$ Stachybotrys lobulatus } \\
\hline \multicolumn{6}{|l|}{ Berk. [as 'lobulata'] } \\
\hline Stachybotrys parvisporus S. & Stachybotryaceae, Sordariomycetes, & fruits & Ardisia punctata & Hong Kong & Tang et al. 2003a \\
\hline Hughes [as 'parvispora'] & Ascomycota & fruits & Ilex cinerea & Hong Kong & Tang et al. 2003a \\
\hline & & seeds & Cedrus deodara & India & Mittal 1983 \\
\hline $\begin{array}{l}\text { Stachybotrys phaeophialis L. } \\
\text { Lombard \& Crous }{ }^{\#}\end{array}$ & $\begin{array}{l}\text { Stachybotryaceae, Sordariomycetes, } \\
\text { Ascomycota }\end{array}$ & seed & undetermined plant & China & Lombard et al. 2016 \\
\hline \multirow[t]{7}{*}{ Stachybotrys spp. } & $\begin{array}{l}\text { Stachybotryaceae, Sordariomycetes, } \\
\text { Ascomycota }\end{array}$ & seeds & $\begin{array}{l}\text { Callistephus } \\
\text { chinensis }\end{array}$ & $\begin{array}{l}\text { imported to } \\
\text { Taiwan }\end{array}$ & Wu et al. 2006 \\
\hline & & seeds & Cosmos sulphureus & $\begin{array}{l}\text { imported to } \\
\text { Taiwan }\end{array}$ & Wu et al. 2006 \\
\hline & & seeds & Dahlia $\times$ hybrida & $\begin{array}{l}\text { imported to } \\
\text { Taiwan }\end{array}$ & Wu et al. 2006 \\
\hline & & seeds & $\begin{array}{l}\text { Dianthus } \\
\text { caryophyllus }\end{array}$ & Taiwan & Li \& Wu 2002 \\
\hline & & seeds & Dianthus spp. & Taiwan & Li \& Wu 2002 \\
\hline & & seeds & Eucalyptus globulus & India & Anderson 1986a \\
\hline & & seeds & Primula $\times$ polyantha & $\begin{array}{l}\text { imported to } \\
\text { Taiwan }\end{array}$ & Wu et al. 2006 \\
\hline $\begin{array}{l}\text { Stagonospora arenaria } \\
\text { (Sacc.) Sacc. }\end{array}$ & $\begin{array}{l}\text { Massarinaceae, Dothideomycetes, } \\
\text { Ascomycota }\end{array}$ & seed & Agropyron cristatum & Canada & Conners 1967 \\
\hline Stagonospora spp. & $\begin{array}{l}\text { Massarinaceae, Dothideomycetes, } \\
\text { Ascomycota }\end{array}$ & seeds & Phragmites australis & Germany & Ernst et al. 2003 \\
\hline $\begin{array}{l}\text { Stagonosporopsis pini } \\
\text { Jayasiri, E.B.G. Jones \& } \\
\text { K.D. Hyde }^{\#}\end{array}$ & $\begin{array}{l}\text { Didymellaceae, Dothideomycetes, } \\
\text { Ascomycota }\end{array}$ & cone & Pinus sp. & China & Jayasiri et al. 2019 \\
\hline Staphylotrichum sp. & $\begin{array}{l}\text { Chaetomiaceae, Sordariomycetes, } \\
\text { Ascomycota }\end{array}$ & seeds & Pinus taeda & USA & Anderson 1986a, b \\
\hline \multirow{3}{*}{$\begin{array}{l}\text { Stemphylium botryosum } \\
\text { Wallr. }\end{array}$} & Pleosporaceae, Dothideomycetes, & seed & Anethum graveolens & Canada & Conners 1967 \\
\hline & Ascomycota & seed & $\begin{array}{l}\text { Apium graveolens } \\
\text { var. dulce }\end{array}$ & Canada & Conners 1967 \\
\hline & & seed & $\begin{array}{l}\text { Asparagus officinalis } \\
\text { var. altilis }\end{array}$ & Canada & Conners 1967 \\
\hline
\end{tabular}


Table 2 Continued.

\begin{tabular}{|c|c|c|c|c|c|}
\hline Species & Taxonomy & Substrate & Host & Country & References \\
\hline & & seeds & $\begin{array}{l}\text { Casuarina } \\
\text { equisetifolia }\end{array}$ & Philippines & $\begin{array}{l}\text { Mathur 1974, Anderson } \\
\text { 1986a }\end{array}$ \\
\hline & & seeds & Cedrus deodara & India & $\begin{array}{l}\text { Munjal \& Sharma 1975, } \\
\text { Mittal et al. } 1990\end{array}$ \\
\hline & & seed & Festuca elatior & Canada & Conners 1967 \\
\hline & & seed & Festuca rubra & Canada & Conners 1967 \\
\hline & & seed & Linum usitatissimum & Canada & Conners 1967 \\
\hline & & seeds & Pinus roxburghii & India & $\begin{array}{l}\text { Munjal \& Sharma 1975, } \\
\text { Mittal et al. } 1990\end{array}$ \\
\hline & & seeds & Pinus wallichiana & India & $\begin{array}{l}\text { Munjal \& Sharma 1975, } \\
\text { Mittal et al. } 1990\end{array}$ \\
\hline & & seed & Salvia officinalis & Canada & Conners 1967 \\
\hline & & seed & Setaria viridis & Canada & Conners 1967 \\
\hline \multirow[t]{4}{*}{ Stemphylium ilicis Tengwall } & $\begin{array}{l}\text { Pleosporaceae, Dothideomycetes, } \\
\text { Ascomycota }\end{array}$ & seeds & Picea excelsa & USSR & $\begin{array}{l}\text { Urosevic 1961, } \\
\text { Anderson 1986a }\end{array}$ \\
\hline & & seeds & Pinus caribaea & $\begin{array}{l}\text { Belize [as British } \\
\text { Honduras] }\end{array}$ & Hocking 1968 \\
\hline & & seeds & Pinus sylvestris & USSR & $\begin{array}{l}\text { Urosevic 1961, } \\
\text { Anderson 1986a }\end{array}$ \\
\hline & & seeds & Quercus spp. & USSR & $\begin{array}{l}\text { Urosevic 1962, Mittal et } \\
\text { al. } 1990\end{array}$ \\
\hline $\begin{array}{l}\text { Stemphylium parasiticum } \\
\text { (Thüm.) J.A. Elliott }\end{array}$ & $\begin{array}{l}\text { Pleosporaceae, Dothideomycetes, } \\
\text { Ascomycota }\end{array}$ & seeds & $\begin{array}{l}\text { Callistephus } \\
\text { chinensis }\end{array}$ & China & $\begin{array}{l}\text { Gloyer 1931, Crosier \& } \\
\text { Heit } 1948\end{array}$ \\
\hline $\begin{array}{l}\text { Stemphylium paxianum } \\
\text { (Szabó) Lindau }\end{array}$ & $\begin{array}{l}\text { Pleosporaceae, Dothideomycetes, } \\
\text { Ascomycota }\end{array}$ & seeds & Pinus sylvestris & USSR & $\begin{array}{l}\text { Dolejs 1964, Mittal et al. } \\
1990\end{array}$ \\
\hline \multirow{3}{*}{$\begin{array}{l}\text { Stemphylium pyriforme } \\
\text { Bonord. } \\
\text { [as 'piriforme'] }\end{array}$} & $\begin{array}{l}\text { Pleosporaceae, Dothideomycetes, } \\
\text { Ascomycota }\end{array}$ & seeds & Picea excelsa & USSR & $\begin{array}{l}\text { Urosevic 1961, } \\
\text { Anderson 1986a }\end{array}$ \\
\hline & & seeds & Pinus sylvestris & USSR & $\begin{array}{l}\text { Urosevic 1961, } \\
\text { Anderson 1986a }\end{array}$ \\
\hline & & seeds & Quercus spp. & USSR & $\begin{array}{l}\text { Urosevic 1962, Mittal et } \\
\text { al. } 1990\end{array}$ \\
\hline
\end{tabular}


Table 2 Continued.

\begin{tabular}{|c|c|c|c|c|c|}
\hline Species & Taxonomy & Substrate & Host & Country & References \\
\hline $\begin{array}{l}\text { Stemphylium radicinum } \\
\text { (Meier, Drechsler \& E.D. } \\
\text { Eddy) Neerg. }\end{array}$ & $\begin{array}{l}\text { Pleosporaceae, Dothideomycetes, } \\
\text { Ascomycota }\end{array}$ & seeds & Pinus insularis & Philippines & $\begin{array}{l}\text { Agmata 1979, Anderson } \\
\text { 1986a }\end{array}$ \\
\hline \multirow[t]{5}{*}{ Stemphylium spp. } & \multirow{5}{*}{$\begin{array}{l}\text { Pleosporaceae, Dothideomycetes, } \\
\text { Ascomycota }\end{array}$} & seeds & Fraxinus excelsior & Sweden & Hayatgheibi 2013 \\
\hline & & seeds & Picea sitchensis & UK & $\begin{array}{l}\text { Salt 1964, Mittal et al. } \\
1990\end{array}$ \\
\hline & & seeds & Pinus caribaea & Belize & $\begin{array}{l}\text { Hocking 1968, Mittal et } \\
\text { al. } 1990\end{array}$ \\
\hline & & seeds & Pinus massoniana & Taiwan & $\begin{array}{l}\text { Jong \& Chen } 1966 \text {, } \\
\text { Mittal et al. } 1990\end{array}$ \\
\hline & & seeds & $\begin{array}{l}\text { Platanus } \\
\text { occidentalis }\end{array}$ & USA & $\begin{array}{l}\text { Fakir et al. 1971, Mittal } \\
\text { et al. } 1990\end{array}$ \\
\hline \multirow[t]{6}{*}{$\begin{array}{l}\text { Stemphylium vesicarium } \\
\text { (Wallr.) E.G. Simmons }\end{array}$} & \multirow[t]{6}{*}{$\begin{array}{l}\text { Pleosporaceae, Dothideomycetes, } \\
\text { Ascomycota }\end{array}$} & seeds & $\begin{array}{l}\text { Callistephus } \\
\text { chinensis }\end{array}$ & $\begin{array}{l}\text { imported to } \\
\text { Taiwan }\end{array}$ & Wu et al. 2006 \\
\hline & & seeds & Cosmos sulphureus & $\begin{array}{l}\text { imported to } \\
\text { Taiwan }\end{array}$ & Wu et al. 2006 \\
\hline & & seeds & $\begin{array}{l}\text { Cupressus } \\
\text { sempervirens }\end{array}$ & Egypt & $\begin{array}{l}\text { Farag et al. 1977, Mittal } \\
\text { et al. } 1990\end{array}$ \\
\hline & & seeds & $\begin{array}{l}\text { Dianthus } \\
\text { caryophyllus }\end{array}$ & Taiwan & Li \& Wu 2002 \\
\hline & & seeds & Dianthus spp. & Taiwan & Li \& Wu 2002 \\
\hline & & seeds & Tagetes patula & $\begin{array}{l}\text { imported to } \\
\text { Taiwan }\end{array}$ & Wu et al. 2006 \\
\hline $\begin{array}{l}\text { Stereum hirsutum (Willd.) } \\
\text { Pers. [as 'Sterum'] }\end{array}$ & $\begin{array}{l}\text { Stereaceae, Agaricomycetes, } \\
\text { Basidiomycota }\end{array}$ & seeds & Quercus spp. & USSR & $\begin{array}{l}\text { Urosevic 1962, Mittal et } \\
\text { al. } 1990\end{array}$ \\
\hline \multirow[t]{5}{*}{ Sterile mycelia } & \multirow[t]{5}{*}{ Fungi } & pods & Delonix regia & Thailand & Somrithipol et al. 2002b \\
\hline & & seeds & Abies koreana & Korea & Cho et al. 2007 \\
\hline & & seeds & Eucalyptus globulus & India & $\begin{array}{l}\text { Sharma \& Mohanan } \\
\text { 1980, Mittal et al. } 1990\end{array}$ \\
\hline & & seeds & Eucalyptus hybrid & India & $\begin{array}{l}\text { Mittal 1986, Mittal et al. } \\
1990\end{array}$ \\
\hline & & seeds & Picea engelmannii & USA & $\begin{array}{l}\text { Fuller \& Hildebrand } \\
\text { 1985, Mittal et al. } 1990\end{array}$ \\
\hline
\end{tabular}


Table 2 Continued.

\begin{tabular}{|c|c|c|c|c|c|}
\hline Species & Taxonomy & Substrate & Host & Country & References \\
\hline & & seeds & Picea sitchensis & UK & $\begin{array}{l}\text { Salt 1964, Mittal et al. } \\
1990\end{array}$ \\
\hline & & seeds & $\begin{array}{l}\text { Pinus armandii var. } \\
\text { mastersiana }\end{array}$ & Taiwan & $\begin{array}{l}\text { Jong \& Chen 1966, } \\
\text { Mittal et al. } 1990\end{array}$ \\
\hline & & seeds & Pinus caribaea & $\begin{array}{l}\text { Belize [as British } \\
\text { Honduras] }\end{array}$ & $\begin{array}{l}\text { Hocking 1968, Mittal et } \\
\text { al. } 1990\end{array}$ \\
\hline & & seeds & Pinus contorta & USA & $\begin{array}{l}\text { Fuller \& Hildebrand } \\
\text { 1985, Mittal et al. } 1990\end{array}$ \\
\hline & & seeds & Pinus elliottii & $\begin{array}{l}\text { South Africa, } \\
\text { Taiwan, USA }\end{array}$ & $\begin{array}{l}\text { Rowan \& Debarr 1974, } \\
\text { Mittal et al. 1990, } \\
\text { Cilliers et al. 1995 }\end{array}$ \\
\hline & & seeds & Pinus insularis & Philippines & $\begin{array}{l}\text { Agmata 1979, Anderson } \\
\text { 1986a }\end{array}$ \\
\hline & & seeds & Pinus luchuensis & Taiwan & $\begin{array}{l}\text { Jong \& Chen } 1966 \text {, } \\
\text { Mittal et al. } 1990\end{array}$ \\
\hline & & seeds & Pinus massoniana & Taiwan & $\begin{array}{l}\text { Jong \& Chen } 1966 \text {, } \\
\text { Mittal et al. } 1990\end{array}$ \\
\hline & & seeds & Pinus nigra & Hungary & $\begin{array}{l}\text { Hangyal 1973, Mittal et } \\
\text { al. } 1990\end{array}$ \\
\hline & & seeds & Pinus ponderosa & USA & $\begin{array}{l}\text { Fuller \& Hildebrand } \\
\text { 1985, Mittal et al. } 1990\end{array}$ \\
\hline & & seeds & Pinus taiwanensis & Taiwan & $\begin{array}{l}\text { Jong \& Chen } 1966 \text {, } \\
\text { Mittal et al. } 1990\end{array}$ \\
\hline & & seeds & $\begin{array}{l}\text { Pinus thunbergii } \\
\text { [= Pinus } \\
\text { thunbergiana }]\end{array}$ & Taiwan & $\begin{array}{l}\text { Jong \& Chen } 1966 \text {, } \\
\text { Mittal et al. } 1990\end{array}$ \\
\hline & & seeds & Pinus wallichiana & India & $\begin{array}{l}\text { Munjal \& Sharma 1975, } \\
\text { Mittal et al. } 1990\end{array}$ \\
\hline & & seeds & Quercus spp. & India & Sahai \& Otra 1982 \\
\hline & & seeds & Sapium spp. & India & Sahai \& Otra 1982 \\
\hline & & seeds & $\begin{array}{l}\text { Swietenia } \\
\text { macrophylla }\end{array}$ & Philippines & $\begin{array}{l}\text { Agmata 1979, Mittal et } \\
\text { al. } 1990\end{array}$ \\
\hline & & seeds & Tectona grandis & India & $\begin{array}{l}\text { Sharma \& Mohanan } \\
\text { 1980, Mittal et al. } 1990\end{array}$ \\
\hline
\end{tabular}


Table 2 Continued.

\begin{tabular}{|c|c|c|c|c|c|}
\hline \multirow{2}{*}{$\begin{array}{c}\text { Species } \\
\text { Stictis fimbriata Schwein. }\end{array}$} & Taxonomy & Substrate & Host & Country & References \\
\hline & $\begin{array}{l}\text { Stictidaceae, Lecanoromycetes, } \\
\text { Ascomycota }\end{array}$ & seeds & Pinus sylvestris & USSR & $\begin{array}{l}\text { Dolejs 1964, Mittal et al. } \\
1990\end{array}$ \\
\hline \multirow{3}{*}{$\begin{array}{l}\text { Stilbum nanum (Ehrenb.) } \\
\text { Spreng. } \\
\text { [as 'Stilbella nanum'] }\end{array}$} & \multirow[t]{3}{*}{$\begin{array}{l}\text { Chionosphaeraceae, } \\
\text { Agaricostilbomycetes, Basidiomycota }\end{array}$} & seeds & Cedrus deodara & India & $\begin{array}{l}\text { Munjal \& Sharma 1975, } \\
\text { Mittal et al. } 1990\end{array}$ \\
\hline & & seeds & Pinus roxburghii & India & $\begin{array}{l}\text { Munjal \& Sharma 1975, } \\
\text { Mittal et al. } 1990\end{array}$ \\
\hline & & seeds & Pinus wallichiana & India & $\begin{array}{l}\text { Munjal \& Sharma 1975, } \\
\text { Mittal et al. } 1990\end{array}$ \\
\hline $\begin{array}{l}\text { Stomiopeltis phyllanthi } \\
\text { Jayasiri, E.B.G. Jones \& } \\
\text { K.D. Hyde }^{\#}\end{array}$ & $\begin{array}{l}\text { Micropeltidaceae, Dothideomycetes, } \\
\text { Ascomycota }\end{array}$ & fruit & Phyllanthus emblica & China & Jayasiri et al. 2019 \\
\hline $\begin{array}{l}\text { Stomiopeltis sinensis } \\
\text { Jayasiri, E.B.G. Jones \& } \\
\text { K.D. Hyde }^{\#}\end{array}$ & $\begin{array}{l}\text { Micropeltidaceae, Dothideomycetes, } \\
\text { Ascomycota }\end{array}$ & fruit & Harpephyllum sp. & China & Jayasiri et al. 2019 \\
\hline $\begin{array}{l}\text { Strobiloscypha keliae N.S. } \\
\text { Weber \& Denison }^{\#^{* *}}\end{array}$ & $\begin{array}{l}\text { Strobiloscyphaceae, Pezizomycetes, } \\
\text { Ascomycota }\end{array}$ & cones & $\begin{array}{l}\text { Chamaecyparis } \\
\text { lawsoniana }\end{array}$ & USA & Weber \& Denison 1995 \\
\hline $\begin{array}{l}\text { Strobilurus diminutivus } \\
\text { Desjardin }\end{array}$ & $\begin{array}{l}\text { Physalacriaceae, Agaricomycetes, } \\
\text { Basidiomycota }\end{array}$ & cone scales & Pinus sp. & USA & Desjardin 2000 \\
\hline $\begin{array}{l}\text { Strobilurus esculentus } \\
\text { (Wulfen) Singer }\end{array}$ & $\begin{array}{l}\text { Physalacriaceae, Agaricomycetes, } \\
\text { Basidiomycota }\end{array}$ & cones & Picea smithiana & India & Goswami \& Ojha 2004 \\
\hline $\begin{array}{l}\text { Strobilurus stephanocystis } \\
\text { (Kühner \& Romagn. ex } \\
\text { Hora) Singer }\end{array}$ & $\begin{array}{l}\text { Physalacriaceae, Agaricomycetes, } \\
\text { Basidiomycota }\end{array}$ & cones & Pinus sp. & India & Goswami \& Ojha 2004 \\
\hline \multirow[t]{2}{*}{ Stysanus medius Sacc. } & \multirow[t]{2}{*}{$\begin{array}{l}\text { Microascaceae, Sordariomycetes, } \\
\text { Ascomycota }\end{array}$} & seeds & Pinus roxburghii & India & $\begin{array}{l}\text { Munjal \& Sharma 1975, } \\
\text { Mittal et al. } 1990\end{array}$ \\
\hline & & seeds & Pinus sylvestris & Poland & $\begin{array}{l}\text { Garbowski 1936, Mittal } \\
\text { et al. } 1990\end{array}$ \\
\hline Stysanus microsporus Sacc. & $\begin{array}{l}\text { Microascaceae, Sordariomycetes, } \\
\text { Ascomycota }\end{array}$ & seeds & Quercus spp. & USSR & $\begin{array}{l}\text { Urosevic 1959, Mittal et } \\
\text { al. } 1990\end{array}$ \\
\hline $\begin{array}{l}\text { Stysanus stemonitis (Pers.) } \\
\text { Corda }\end{array}$ & $\begin{array}{l}\text { Microascaceae, Sordariomycetes, } \\
\text { Ascomycota }\end{array}$ & seeds & Quercus spp. & USSR & $\begin{array}{l}\text { Urosevic 1959, Mittal et } \\
\text { al. } 1990\end{array}$ \\
\hline
\end{tabular}


Table 2 Continued.

\begin{tabular}{|c|c|c|c|c|c|}
\hline Species & Taxonomy & Substrate & Host & Country & References \\
\hline \multirow[t]{2}{*}{$\begin{array}{l}\text { Stysanus stemonitis (Pers.) } \\
\text { Corda [as 'stemonites'] }\end{array}$} & & seeds & Betula verrucosa & USSR & $\begin{array}{l}\text { Kozlowska 1968, Mittal } \\
\text { et al. } 1990\end{array}$ \\
\hline & & seeds & Quercus pedunculata & USSR & $\begin{array}{l}\text { Kozlowska 1968, Mittal } \\
\text { et al. } 1990\end{array}$ \\
\hline Sydowia spp. & $\begin{array}{l}\text { Dothioraceae, Dothideomycetes, } \\
\text { Ascomycota }\end{array}$ & seeds & Eucalyptus grandis & South Africa & Jimu et al. 2016 \\
\hline \multirow[t]{5}{*}{$\begin{array}{l}\text { Sydowia polyspora (Bref. \& } \\
\text { Tavel) E. Müll. }\end{array}$} & $\begin{array}{l}\text { Dothioraceae, Dothideomycetes, } \\
\text { Ascomycota }\end{array}$ & seeds & Abies koreana & Korea & $\begin{array}{l}\text { Cho et al. 2005, Cho et } \\
\text { al. } 2007\end{array}$ \\
\hline & & seeds & Abies lasiocarpa & Canada, Norway & Talgø et al. 2010 \\
\hline & & seeds & Abies nordmanniana & $\begin{array}{l}\text { Austria, Georgia, } \\
\text { Russia }\end{array}$ & Talgø et al. 2010 \\
\hline & & seeds & Abies procera & Norway & Talgø et al. 2010 \\
\hline & & seeds & Fraxinus excelsior & Sweden & Hayatgheibi 2013 \\
\hline $\begin{array}{l}\text { Symmetrospora coprosmae } \\
\text { (Hamam. \& Nakase) Q.M. } \\
\text { Wang, F.Y. Bai, M. } \\
\text { Groenew. \& Boekhout }^{\#} \\
\text { [as Bullera coprosmae } \\
\text { Hamam. \& Nakase] }\end{array}$ & $\begin{array}{l}\text { Symmetrosporaceae, } \\
\text { Cystobasidiomycetes, Basidiomycota }\end{array}$ & fruit & Coprosma tenuifolia & New Zealand & $\begin{array}{l}\text { Hamamoto \& Nakase } \\
1996\end{array}$ \\
\hline $\begin{array}{l}\text { Symphyosira clematidis } \\
\text { Baral }^{\#}\end{array}$ & Helotiaceae, Leotiomycetes, Ascomycota & fruit & Clematis vitalba & Germany & Weber 1992 \\
\hline $\begin{array}{l}\text { Symphyosirinia chaerophylli } \\
\text { M. Svrček }{ }^{\#}\end{array}$ & Helotiaceae, Leotiomycetes, Ascomycota & seeds & $\begin{array}{l}\text { Chaerophyllum } \\
\text { hirsutum }\end{array}$ & $\begin{array}{l}\text { former } \\
\text { Czechoslovakia }\end{array}$ & Svrcek 1989 \\
\hline $\begin{array}{l}\text { Sympodiella goidanichii } \\
\text { (Rambelli) Crous \& Hern.- } \\
\text { Restr.\# [as Ceratosporella } \\
\text { goidanichii Rambelli] }\end{array}$ & $\begin{array}{l}\text { Incertae sedis, Incertae sedis, } \\
\text { Ascomycota }\end{array}$ & cupule & Fagus sylvatica & Italy & Crous et al. 2019 \\
\hline $\begin{array}{l}\text { Syncephalastrum cinereum } \\
\text { Bainier }\end{array}$ & $\begin{array}{l}\text { Syncephalastraceae, Mucoromycetes, } \\
\text { Mucoromycota }\end{array}$ & seeds & Pinus sylvestris & USSR & $\begin{array}{l}\text { Prisyazhnyuk } 1960 \text {, } \\
\text { Mittal et al. } 1990\end{array}$ \\
\hline \multirow{2}{*}{$\begin{array}{l}\text { Syncephalastrum } \\
\text { racemosum Cohn ex J. } \\
\text { Schröt. }\end{array}$} & $\begin{array}{l}\text { Syncephalastraceae, Mucoromycetes, } \\
\text { Mucoromycota }\end{array}$ & seeds & $\begin{array}{l}\text { Acacia } \\
\text { auriculiformis }\end{array}$ & Thailand & $\begin{array}{l}\text { Chalermpongse et al. } \\
\text { 1984, Mittal et al. } 1990\end{array}$ \\
\hline & & seeds & Cassia fistula & Thailand & $\begin{array}{l}\text { Chalermpongse et al. } \\
\text { 1984, Mittal et al. } 1990\end{array}$ \\
\hline
\end{tabular}


Table 2 Continued.

\begin{tabular}{|c|c|c|c|c|c|}
\hline Species & Taxonomy & Substrate & Host & Country & References \\
\hline & & seed & Cercis chinensis & Japan & Watanabe 2010 \\
\hline & & seeds & Eucalyptus alba & Thailand & $\begin{array}{l}\text { Chalermpongse et al. } \\
\text { 1984, Mittal et al. } 1990\end{array}$ \\
\hline & & seeds & Gmelina arborea & Thailand & $\begin{array}{l}\text { Chalermpongse et al. } \\
\text { 1984, Mittal et al. } 1990\end{array}$ \\
\hline & & seeds & Pinus merkusii & Thailand & $\begin{array}{l}\text { Chalermpongse et al. } \\
\text { 1984, Mittal et al. } 1990\end{array}$ \\
\hline & & seeds & Pinus taeda & USA & $\begin{array}{l}\text { Mason \& van Arsdel } \\
\text { 1978, Anderson 1986a }\end{array}$ \\
\hline & & seeds & Samanea saman & Thailand & $\begin{array}{l}\text { Chalermpongse et al. } \\
\text { 1984, Mittal et al. } 1990\end{array}$ \\
\hline & & seeds & Shorea robusta & Thailand & $\begin{array}{l}\text { Chalermpongse et al. } \\
\text { 1984, Mittal et al. } 1990\end{array}$ \\
\hline \multirow[t]{2}{*}{ Syncephalastrum sp. } & $\begin{array}{l}\text { Syncephalastraceae, Mucoromycetes, } \\
\text { Mucoromycota }\end{array}$ & seeds & $\begin{array}{l}\text { Pseudotsuga } \\
\text { menziesii }\end{array}$ & USA & $\begin{array}{l}\text { Gordon 1967, Mittal et } \\
\text { al. } 1990\end{array}$ \\
\hline & & seeds & Tectona grandis & India & Anderson 1986a \\
\hline $\begin{array}{l}\text { Tainosphaeria crassiparies } \\
\text { F.A. Fernández \& Hundorf }{ }^{* *}\end{array}$ & $\begin{array}{l}\text { Chaetosphaeriaceae, Sordariomycetes, } \\
\text { Ascomycota }\end{array}$ & pod & Hymenaea sp. & USA & $\begin{array}{l}\text { Fernández \& } \\
\text { Huhndorf } 2005\end{array}$ \\
\hline $\begin{array}{l}\text { Tainosphaeria siamensis } \\
\text { Jiao Yang, K.D. Hyde \& Jian } \\
\text { K. Liu }\end{array}$ & $\begin{array}{l}\text { Chaetosphaeriaceae, Sordariomycetes, } \\
\text { Ascomycota }\end{array}$ & seeds & Eucalyptus sp. & India & $\begin{array}{l}\text { Saxena 1985, Mittal et } \\
\text { al. } 1990\end{array}$ \\
\hline $\begin{array}{l}\text { Talaromyces aculeatus } \\
\text { (Raper \& Fennell) Samson, } \\
\text { N. Yilmaz, Frisvad \& Seifert } \\
\text { [as Penicillium aculeatum } \\
\text { Raper \& Fennell] }\end{array}$ & $\begin{array}{l}\text { Trichocomaceae, Eurotiomycetes, } \\
\text { Ascomycota }\end{array}$ & seeds & Pinus taeda & Georgia & Huang \& Kuhlman 1990 \\
\hline $\begin{array}{l}\text { Talaromyces coalescens } \\
\text { (Quintan.) Samson, N. } \\
\text { Yilmaz \& Frisvad } \\
\text { [as Penicillium coalescens } \\
\text { Quintan.] }\end{array}$ & $\begin{array}{l}\text { Trichocomaceae, Eurotiomycetes, } \\
\text { Ascomycota }\end{array}$ & cones & Pinus densiflora & Japan & Kasai et al. 1995 \\
\hline $\begin{array}{l}\text { Talaromyces diversus (Raper } \\
\text { \& Fennell) Samson, N. } \\
\text { Yilmaz \& Frisvad }\end{array}$ & $\begin{array}{l}\text { Trichocomaceae, Eurotiomycetes, } \\
\text { Ascomycota }\end{array}$ & seeds & Pinus sylvestris & UK & Whittle 1977 \\
\hline
\end{tabular}


Table 2 Continued.

\begin{tabular}{|c|c|c|c|c|c|}
\hline Species & Taxonomy & Substrate & Host & Country & References \\
\hline $\begin{array}{l}\text { [as Penicillium diversum } \\
\text { Raper \& Fennell] }\end{array}$ & & & & & \\
\hline $\begin{array}{l}\text { Talaromyces funiculosus } \\
\text { (Thom) Samson, N. Yilmaz, } \\
\text { Frisvad \& Seifert } \\
\text { [as Penicillium funiculosum } \\
\text { Thom] }\end{array}$ & $\begin{array}{l}\text { Trichocomaceae, Eurotiomycetes, } \\
\text { Ascomycota }\end{array}$ & seeds & Quercus spp. & USSR & $\begin{array}{l}\text { Urosevic 1959, Mittal et } \\
\text { al. } 1990\end{array}$ \\
\hline $\begin{array}{l}\text { Talaromyces islandicus } \\
\text { (Sopp) Samson, N. Yilmaz, } \\
\text { Frisvad \& Seifert } \\
\text { = Penicillium islandicum } \\
\text { Sopp [as 'islandium’] }\end{array}$ & $\begin{array}{l}\text { Trichocomaceae, Eurotiomycetes, } \\
\text { Ascomycota }\end{array}$ & seeds & Quercus spp. & India & Sahai \& Otra 1982 \\
\hline $\begin{array}{l}\text { Talaromyces palmae } \\
\text { (Samson, Stolk \& Frisvad) } \\
\text { Samson, N. Yilmaz, Frisvad } \\
\text { \& Seifert }{ }^{\#} \text { [as Penicillium } \\
\text { palmae Samson, Stolk \& } \\
\text { Frisvad] }\end{array}$ & $\begin{array}{l}\text { Trichocomaceae, Eurotiomycetes, } \\
\text { Ascomycota }\end{array}$ & seeds & $\begin{array}{l}\text { Chrysalidocarpus } \\
\text { lutescen }\end{array}$ & The Netherlands & Samson et al. 1989 \\
\hline $\begin{array}{l}\text { Talaromyces purpureogenus } \\
\text { Samson, N. Yilmaz, } \\
\text { Houbraken, Spierenb., } \\
\text { Seifert, Peterson, Varga \& } \\
\text { Frisvad [as Penicillium } \\
\text { purpureogenum Stoll] }\end{array}$ & $\begin{array}{l}\text { Trichocomaceae, Eurotiomycetes, } \\
\text { Ascomycota }\end{array}$ & seeds & Pinus sylvestris & Poland & Krol et al. 2015 \\
\hline $\begin{array}{l}\text { Talaromyces ruber (Stoll) N. } \\
\text { Yilmaz, Houbraken, Frisvad }\end{array}$ & $\begin{array}{l}\text { Trichocomaceae, Eurotiomycetes, } \\
\text { Ascomycota }\end{array}$ & seeds & $\begin{array}{l}\text { Eucalyptus } \\
\text { citriodora }\end{array}$ & India & $\begin{array}{l}\text { Mittal \& Sharma 1984, } \\
\text { Mittal et al. } 1990\end{array}$ \\
\hline \& Samson [as Penicillium & & seeds & Larix occidentalis & USA & James et al. 1996 \\
\hline rubrum Stoll] & & seeds & Pinus roxburghii & India & $\begin{array}{l}\text { Mittal \& Sharma 1982c, } \\
\text { Mittal et al. } 1990\end{array}$ \\
\hline $\begin{array}{l}\text { Talaromyces ruber (Stoll) N. } \\
\text { Yilmaz, Houbraken, Frisvad }\end{array}$ & & seed & $\begin{array}{l}\text { Macroptilium } \\
\text { atropurpureum }\end{array}$ & Australia & Nik \& Parbery 1977 \\
\hline $\begin{array}{l}\text { \& Samson } \\
=\text { Penicillium rubrum Stoll }\end{array}$ & & seed & $\begin{array}{l}\text { Macroptilium } \\
\text { lathyroides }\end{array}$ & Australia & Nik \& Parbery 1977 \\
\hline [as 'ruber'] & & seed & Trifolium repens & Australia & Nik \& Parbery 1977 \\
\hline
\end{tabular}


Table 2 Continued.

\begin{tabular}{|c|c|c|c|c|c|}
\hline Species & Taxonomy & Substrate & Host & Country & References \\
\hline $\begin{array}{l}\text { Talaromyces rugulosus } \\
\text { (Thom) Samson, N. Yilmaz, } \\
\text { Frisvad \& Seifert } \\
\text { [as Penicillium rugulosum } \\
\text { Thom] }\end{array}$ & $\begin{array}{l}\text { Trichocomaceae, Eurotiomycetes, } \\
\text { Ascomycota }\end{array}$ & seeds & Pinus sylvestris & UK & Whittle 1977 \\
\hline $\begin{array}{l}\text { Talaromyces variabilis } \\
\text { (Sopp) Samson, N. Yilmaz, }\end{array}$ & $\begin{array}{l}\text { Trichocomaceae, Eurotiomycetes, } \\
\text { Ascomycota }\end{array}$ & seeds & $\begin{array}{l}\text { Eucalyptus } \\
\text { citriodora }\end{array}$ & India & $\begin{array}{l}\text { Mittal \& Sharma 1984, } \\
\text { Mittal et al. } 1990\end{array}$ \\
\hline $\begin{array}{l}\text { Frisvad \& Seifert } \\
\text { [as Penicillium variabile }\end{array}$ & & seeds & Picea glauca & Canada & $\begin{array}{l}\text { Mittal \& Wang } 1986 \text {, } \\
\text { Mittal et al. } 1990\end{array}$ \\
\hline Sopp] & & seeds & Pinus sylvestris & UK & Whittle 1977 \\
\hline $\begin{array}{l}\text { Talaromyces verruculosus } \\
\text { (Peyronel) Samson, N. } \\
\text { Yilmaz, Frisvad \& Seifert } \\
\text { [as Penicillium verruculosum } \\
\text { Peyronel] }\end{array}$ & $\begin{array}{l}\text { Trichocomaceae, Eurotiomycetes, } \\
\text { Ascomycota }\end{array}$ & seed & Medicago sativa & Canada & Conners 1967 \\
\hline $\begin{array}{l}\text { Talaromyces wortmannii } \\
\text { (Klöcker) C.R. Benj } \\
\text { [as Penicillium wortmannii } \\
\text { Klöcker] }\end{array}$ & $\begin{array}{l}\text { Trichocomaceae, Eurotiomycetes, } \\
\text { Ascomycota }\end{array}$ & seeds & Cedrus deodara & India & Mittal 1983 \\
\hline $\begin{array}{l}\text { Taphrina alni-incanae (J.G. } \\
\text { Kühn) Magnus }\end{array}$ & $\begin{array}{l}\text { Taphrinaceae, Taphrinomycetes, } \\
\text { Ascomycota }\end{array}$ & seeds & Alnus sp. & Poland & Anderson 1986a \\
\hline Taphrina sp. & $\begin{array}{l}\text { Taphrinaceae, Taphrinomycetes, } \\
\text { Ascomycota }\end{array}$ & seeds & Fraxinus excelsior & Sweden & Hayatgheibi 2013 \\
\hline $\begin{array}{l}\text { Teichospora thailandica } \\
\text { (Thambug. \& K.D. Hyde) } \\
\text { Jaklitsch \& Voglmayr } \\
\text { [as Ramusculicola } \\
\text { thailandica Thambug. \& } \\
\text { K.D. Hyde] }\end{array}$ & $\begin{array}{l}\text { Teichosporaceae, Dothideomycetes, } \\
\text { Ascomycota }\end{array}$ & pod & Acacia sp. & Thailand & Jayasiri et al. 2019 \\
\hline $\begin{array}{l}\text { Teratosphaeria zuluensis } \\
\text { (M.J. Wingf., Crous \& T.A. } \\
\text { Cout.) M.J. Wingf. \& Crous }\end{array}$ & $\begin{array}{l}\text { Teratosphaeriaceae, Dothideomycetes, } \\
\text { Ascomycota }\end{array}$ & $\begin{array}{l}\text { seed capsules, } \\
\text { seeds }\end{array}$ & Eucalyptus grandis & South Africa & Jimu et al. 2016 \\
\hline Tetracoccosporium sp. & $\begin{array}{l}\text { Incertae sedis, Incertae sedis, } \\
\text { Ascomycota }\end{array}$ & seeds & Pinus taeda & USA & Anderson 1986a, b \\
\hline
\end{tabular}


Table 2 Continued.

\begin{tabular}{|c|c|c|c|c|c|}
\hline Species & Taxonomy & Substrate & Host & Country & References \\
\hline \multirow[t]{4}{*}{ Thamnidium elegans Link } & \multirow[t]{4}{*}{$\begin{array}{l}\text { Mucoraceae, Mucoromycetes, } \\
\text { Mucoromycota }\end{array}$} & seeds & Abies sibirica & USSR & $\begin{array}{l}\text { Prisyazhnyuk 1960, } \\
\text { Mittal et al. } 1990\end{array}$ \\
\hline & & seeds & Larix sibirica & USSR & $\begin{array}{l}\text { Prisyazhnyuk 1960, } \\
\text { Mittal et al. } 1990\end{array}$ \\
\hline & & seeds & Picea abies & USSR & $\begin{array}{l}\text { Prisyazhnyuk 1960, } \\
\text { Mittal et al. } 1990\end{array}$ \\
\hline & & seeds & Pinus sylvestris & USSR & $\begin{array}{l}\text { Dolejs 1964, Mittal et al. } \\
1990\end{array}$ \\
\hline $\begin{array}{l}\text { Thamnostylum lucknowense } \\
\text { (J.N. Rai, J.P. Tewari \& } \\
\text { Mukerji) Arx \& H.P. } \\
\text { Upadhyay }\end{array}$ & $\begin{array}{l}\text { Lichtheimiaceae, Mucoromycetes, } \\
\text { Mucoromycota }\end{array}$ & seeds & Eucalyptus hybrid & India & $\begin{array}{l}\text { Mittal 1986, Mittal et al. } \\
1990\end{array}$ \\
\hline $\begin{array}{l}\text { Thecaphora hosackiae } \\
\text { Vánky" }\end{array}$ & $\begin{array}{l}\text { Glomosporiaceae, Ustilaginomycetes, } \\
\text { Basidiomycota }\end{array}$ & pods & Hosackia parviflora & USA & Vánky 2008b \\
\hline $\begin{array}{l}\text { Thecaphora maireanae R.G. } \\
\text { Shivas \& Vánky }{ }^{\#}\end{array}$ & $\begin{array}{l}\text { Glomosporiaceae, Ustilaginomycetes, } \\
\text { Basidiomycota }\end{array}$ & fruits & $\begin{array}{l}\text { Maireana } \\
\text { pentagona }\end{array}$ & Australia & Vánky \& Shivas 2003 \\
\hline $\begin{array}{l}\text { Thecaphora oberwinkleri K. } \\
\text { Vánky\# }\end{array}$ & $\begin{array}{l}\text { Glomosporiaceae, Ustilaginomycetes, } \\
\text { Basidiomycota }\end{array}$ & seeds & $\begin{array}{l}\text { Androsace } \\
\text { fedtschenkoi }\end{array}$ & China & Vánky 1988 \\
\hline $\begin{array}{l}\text { Thecaphora oxytropis S.R. } \\
\text { Wang\# }\end{array}$ & $\begin{array}{l}\text { Glomosporiaceae, Ustilaginomycetes, } \\
\text { Basidiomycota }\end{array}$ & seeds & $\begin{array}{l}\text { Oxytropis } \\
\text { ochrocephala }\end{array}$ & China & Wang \& Zeng 2006 \\
\hline $\begin{array}{l}\text { Thecaphora pakistanica } \\
\text { Vánky, S.H. Iqbal \& Khalid }{ }^{\#}\end{array}$ & $\begin{array}{l}\text { Glomosporiaceae, Ustilaginomycetes, } \\
\text { Basidiomycota }\end{array}$ & capsules & $\begin{array}{l}\text { Androsace } \\
\text { rotundifolia }\end{array}$ & Pakistan & Vánky et al. 2007 \\
\hline Thecaphora ulicis Vánky\# & $\begin{array}{l}\text { Glomosporiaceae, Ustilaginomycetes, } \\
\text { Basidiomycota }\end{array}$ & seeds & Ulex minor & UK & Vánky 2008b \\
\hline $\begin{array}{l}\text { Thekopsora padi (Kunze \& } \\
\text { J.C. Schmidt) Kleb. } \\
\text { [as 'Thecospora padi'] }\end{array}$ & $\begin{array}{l}\text { Pucciniastraceae, Pucciniomycetes, } \\
\text { Basidiomycota }\end{array}$ & seeds & Picea abies & USSR & $\begin{array}{l}\text { Prisyazhnyuk 1960, } \\
\text { Mittal et al. } 1990\end{array}$ \\
\hline \multirow[t]{3}{*}{$\begin{array}{l}\text { Thekopsora areolata (Fr.) } \\
\text { Magnus }\end{array}$} & \multirow[t]{3}{*}{$\begin{array}{l}\text { Pucciniastraceae, Pucciniomycetes, } \\
\text { Basidiomycota }\end{array}$} & cones & Picea abies & Finland & $\begin{array}{l}\text { Kaitera 2013, Kaitera et } \\
\text { al. 2009, } 2014\end{array}$ \\
\hline & & cones & Picea engelmannii & Finland & Kaitera et al. 2014 \\
\hline & & cones & Picea glauca & Finland & Kaitera et al. 2014 \\
\hline
\end{tabular}


Table 2 Continued.

\begin{tabular}{|c|c|c|c|c|c|}
\hline Species & Taxonomy & Substrate & Host & Country & References \\
\hline Thielaviopsis sp. & $\begin{array}{l}\text { Ceratocystidaceae, Sordariomycetes, } \\
\text { Ascomycota }\end{array}$ & seeds & Picea jezoensis & Japan & Cheng \& Igarashi 1987 \\
\hline Thielavia spp. & $\begin{array}{l}\text { Chaetomiaceae, Sordariomycetes, } \\
\text { Ascomycota }\end{array}$ & seeds & Bombax ceiba & India & Mittal et al. 1990 \\
\hline $\begin{array}{l}\text { Thozetella cristata Piroz. \& } \\
\text { Hodges }\end{array}$ & $\begin{array}{l}\text { Chaetosphaeriaceae, Sordariomycetes, } \\
\text { Ascomycota }\end{array}$ & cones & Pinus densiflora & Japan & Kasai et al. 1995 \\
\hline $\begin{array}{l}\text { Thozetella fabacearum R.H. } \\
\text { Perera \& K.D. Hyde }\end{array}$ & $\begin{array}{l}\text { Chaetosphaeriaceae, Sordariomycetes, } \\
\text { Ascomycota }\end{array}$ & pod & Fabaceae plant & Thailand & Perera et al. 2016b \\
\hline $\begin{array}{l}\text { Thozetella lithocarpi R.H. } \\
\text { Perera \& K.D. Hyde }\end{array}$ & $\begin{array}{l}\text { Chaetosphaeriaceae, Sordariomycetes, } \\
\text { Ascomycota }\end{array}$ & fruit & Lithocarpus sp. & Thailand & Phookamsak et al. 2019 \\
\hline $\begin{array}{l}\text { Thozetella nivea (Berk.) } \\
\text { Kuntze }\end{array}$ & $\begin{array}{l}\text { Chaetosphaeriaceae, Sordariomycetes, } \\
\text { Ascomycota }\end{array}$ & pods & Delonix regia & Thailand & Somrithipol et al. 2002b \\
\hline $\begin{array}{l}\text { Tilletia brachypodii- } \\
\text { mexicani Vánky\# }\end{array}$ & $\begin{array}{l}\text { Tilletiaceae, Exobasidiomycetes, } \\
\text { Basidiomycota }\end{array}$ & seeds & $\begin{array}{l}\text { Brachypodium } \\
\text { mexicanum }\end{array}$ & Venezuela & Vánky 1995 \\
\hline Tilletia colombiana Vánky" & $\begin{array}{l}\text { Tilletiaceae, Exobasidiomycetes, } \\
\text { Basidiomycota }\end{array}$ & seeds & $\begin{array}{l}\text { Brachypodium } \\
\text { mexicanum }\end{array}$ & Colombia & Vánky 1995 \\
\hline $\begin{array}{l}\text { Tilletia laguri G.M. Zhang, } \\
\text { G.X. Lin\& J.R Deng }\end{array}$ & $\begin{array}{l}\text { Tilletiaceae, Exobasidiomycetes, } \\
\text { Basidiomycota }\end{array}$ & seeds & Lagurus ovatus & Italy & Zhang et al. 1995 \\
\hline $\begin{array}{l}\text { Tilletia walkeri Castl. \& } \\
\text { Carris" }^{\#}\end{array}$ & $\begin{array}{l}\text { Tilletiaceae, Exobasidiomycetes, } \\
\text { Basidiomycota }\end{array}$ & seeds & $\begin{array}{l}\text { Lolium multiflorum } \\
\text { [= Festuca perennis }]\end{array}$ & USA & $\begin{array}{l}\text { Castlebury \& Carris } \\
1999\end{array}$ \\
\hline $\begin{array}{l}\text { Tomentella afrostuposa } \\
\text { Yorou }^{\#}\end{array}$ & $\begin{array}{l}\text { Thelephoraceae, Agaricomycetes, } \\
\text { Basidiomycota }\end{array}$ & pods & Afzelia africana & Guinea & Yorou et al. 2011 \\
\hline \multirow[t]{3}{*}{ Torula convoluta Harz } & $\begin{array}{l}\text { Torulaceae, Dothideomycetes, } \\
\text { Ascomycota }\end{array}$ & seeds & Picea excelsa & USSR & $\begin{array}{l}\text { Urosevic 1961, } \\
\text { Anderson 1986a }\end{array}$ \\
\hline & & seeds & Pinus sylvestris & USSR & $\begin{array}{l}\text { Urosevic 1961, } \\
\text { Anderson 1986a }\end{array}$ \\
\hline & & seeds & Quercus spp. & USSR & $\begin{array}{l}\text { Urosevic 1962, Mittal et } \\
\text { al. } 1990\end{array}$ \\
\hline Torula fici Crous & $\begin{array}{l}\text { Torulaceae, Dothideomycetes, } \\
\text { Ascomycota }\end{array}$ & fruit & Garcinia sp. & Thailand & Jayasiri et al. 2019 \\
\hline $\begin{array}{l}\text { Torula herbarum (Pers.) } \\
\text { Link }\end{array}$ & $\begin{array}{l}\text { Torulaceae, Dothideomycetes, } \\
\text { Ascomycota }\end{array}$ & seed & $\begin{array}{l}\text { Cryptomeria } \\
\text { japonica }\end{array}$ & Japan & Watanabe 2010 \\
\hline
\end{tabular}


Table 2 Continued.

\begin{tabular}{|c|c|c|c|c|c|}
\hline Species & Taxonomy & Substrate & Host & Country & References \\
\hline & & seeds & Pinus sylvestris & USSR & $\begin{array}{l}\text { Dolejs 1964, Mittal et al. } \\
1990\end{array}$ \\
\hline \multirow[t]{4}{*}{ Torula spp. } & Torulaceae, Dothideomycetes, & acorns & Quercus robur & Poland & Jankowiak 2008 \\
\hline & Ascomycota & seed & Cercis chinensis & Japan & Watanabe 2010 \\
\hline & & seeds & Pinus taeda & USA & $\begin{array}{l}\text { Covington et al. 1982, } \\
\text { Anderson 1986a }\end{array}$ \\
\hline & & seeds & Tectona grandis & India & Anderson 1986a \\
\hline Torulomyces lagena Delitsch & $\begin{array}{l}\text { Aspergillaceae, Eurotiomycetes, } \\
\text { Ascomycota, }\end{array}$ & seed & $\begin{array}{l}\text { Cryptomeria } \\
\text { japonica }\end{array}$ & Japan & Watanabe 2010 \\
\hline Tremellales & Tremellomycetes, Basidiomycota & seeds & Eucalyptus grandis & South Africa & Jimu et al. 2016 \\
\hline Trichaegum sp. & $\begin{array}{l}\text { Incertae sedis, Incertae sedis, } \\
\text { Ascomycota }\end{array}$ & seeds & Pinus taeda & USA & Anderson 1986a, b \\
\hline $\begin{array}{l}\text { Trichocladium griseum } \\
\text { (Traaen) X. Wei Wang \& }\end{array}$ & $\begin{array}{l}\text { Chaetomiaceae, Sordariomycetes, } \\
\text { Ascomycota }\end{array}$ & seeds & $\begin{array}{l}\text { Casuarina } \\
\text { equisetifolia }\end{array}$ & India & Anju et al. 2012 \\
\hline $\begin{array}{l}\text { Houbraken [as Humicola } \\
\text { grisea Traaen] }\end{array}$ & & seeds & Dalbergia sissoo & India & $\begin{array}{l}\text { Mittal \& Sharma 1981b, } \\
\text { Mittal et al. } 1990\end{array}$ \\
\hline $\begin{array}{l}\text { Trichoderma asperellum } \\
\text { Samuels, Lieckf. \& } \\
\text { Nirenberg }\end{array}$ & $\begin{array}{l}\text { Hypocreaceae, Sordariomycetes, } \\
\text { Ascomycota }\end{array}$ & seeds & Abies koreana & Korea & Cho et al. 2007 \\
\hline $\begin{array}{l}\text { 'Trichoderma conispora' } \\
\text { [as Hypocrea conispora Y. } \\
\text { Doi \& G.J. Samuels]" }\end{array}$ & $\begin{array}{l}\text { Hypocreaceae, Sordariomycetes, } \\
\text { Ascomycota }\end{array}$ & fruit & undetermined plant & Indonesia & Samuels et al. 1990 \\
\hline $\begin{array}{l}\text { Trichoderma deliquescens } \\
\text { (Sopp) Jaklitsch } \\
\text { [as Gliocladium deliquescens } \\
\text { Sopp] }\end{array}$ & $\begin{array}{l}\text { Hypocreaceae, Sordariomycetes, } \\
\text { Ascomycota }\end{array}$ & seeds & Sapium spp. & India & Sahai \& Otra 1982 \\
\hline \multirow[t]{2}{*}{$\begin{array}{l}\text { Trichoderma hamatum } \\
\text { (Bonord.) Bainier }\end{array}$} & $\begin{array}{l}\text { Hypocreaceae, Sordariomycetes, } \\
\text { Ascomycota }\end{array}$ & pod & Sesbania bispinosa & India & Anita et al. 2009 \\
\hline & & seed & Prunus serrulata & Japan & Watanabe 2010 \\
\hline \multirow[t]{4}{*}{$\begin{array}{l}\text { Trichoderma harzianum } \\
\text { Rifai }\end{array}$} & $\begin{array}{l}\text { Hypocreaceae, Sordariomycetes, } \\
\text { Ascomycota }\end{array}$ & cones & Pinus densiflora & Japan & Kasai et al. 1995 \\
\hline & & pod & Sesbania bispinosa & India & Anita et al. 2009 \\
\hline & & seed & Pinus densiflora & Japan & Watanabe 2010 \\
\hline & & seeds & Pinus elliottii & South Africa & Cilliers et al. 1995 \\
\hline
\end{tabular}


Table 2 Continued.

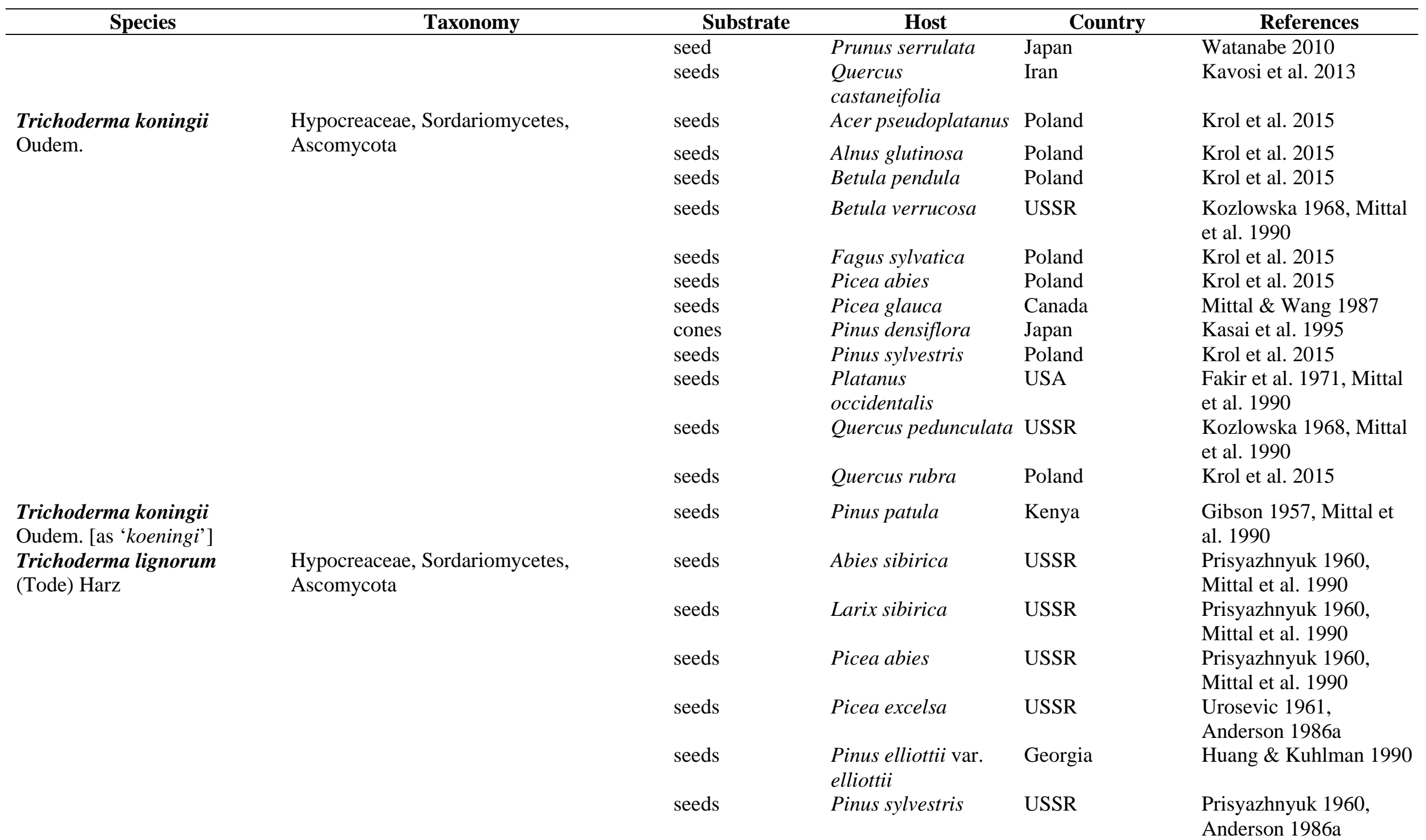


Table 2 Continued.

\begin{tabular}{|c|c|c|c|c|c|}
\hline Species & Taxonomy & Substrate & Host & Country & References \\
\hline & & seeds & Quercus spp. & USSR & $\begin{array}{l}\text { Urosevic 1959, Mittal et } \\
\text { al. } 1990\end{array}$ \\
\hline \multirow[t]{20}{*}{ Trichoderma spp. } & Hypocreaceae, Sordariomycetes, & cones & Pinus ponderosa & USA & James 1995 \\
\hline & Ascomycota & cones, seeds & Pinus spp. & Canada & Vujanovic et al. 2000 \\
\hline & & cones, seeds & $\begin{array}{l}\text { Pseudotsuga } \\
\text { menziesii var. glauca }\end{array}$ & USA & James 1995 \\
\hline & & fruit cupules & Fagus crenata & Japan & Fukasawa et al. 2012 \\
\hline & & fruits & Avicennia marina & India & Mehdi \& Saifullah 2000 \\
\hline & & pods & Delonix regia & Thailand & Somrithipol et al. 2002b \\
\hline & & seeds & Abies lasiocarpa & Canada, Norway & Talgø et al. 2010 \\
\hline & & seeds & Abies nordmanniana & $\begin{array}{l}\text { Austria, Georgia, } \\
\text { Russia }\end{array}$ & Talgø et al. 2010 \\
\hline & & seeds & Abies procera & Norway & Talgø et al. 2010 \\
\hline & & seeds & Albizia lebbeck & India & Mohanan et al. 2005 \\
\hline & & seeds & Dalbergia sissoides & India & Mohanan et al. 2005 \\
\hline & & seeds & $\begin{array}{l}\text { Dianthus } \\
\text { caryophyllus }\end{array}$ & Taiwan & Li \& Wu 2002 \\
\hline & & seeds & Larix occidentalis & USA & James et al. 1996 \\
\hline & & seeds & Picea engelmannii & USA & $\begin{array}{l}\text { Fuller \& Hildebrand } \\
\text { 1985, Mittal et al. } 1990\end{array}$ \\
\hline & & seeds & Picea sitchensis & UK & $\begin{array}{l}\text { Salt 1964, Mittal et al. } \\
1990\end{array}$ \\
\hline & & seeds & Pinus caribaea & $\begin{array}{l}\text { Belize [as British } \\
\text { Honduras] }\end{array}$ & $\begin{array}{l}\text { Hocking 1968, Mittal et } \\
\text { al. } 1990\end{array}$ \\
\hline & & seeds & Pinus contorta & USA & $\begin{array}{l}\text { Fuller \& Hildebrand } \\
\text { 1985, Mittal et al. } 1990\end{array}$ \\
\hline & & seeds & Pinus elliottii & South Africa, USA & $\begin{array}{l}\text { Mittal et al. } 1990 \text {, } \\
\text { Cilliers et al. } 1995\end{array}$ \\
\hline & & seeds & $\begin{array}{l}\text { Pinus elliottii var. } \\
\text { elliottii }\end{array}$ & USA & Fraedrich \& Miller 1995 \\
\hline & & seeds & Pinus nigra & Hungary & $\begin{array}{l}\text { Friedrich et al. } 1971 \text {, } \\
\text { Mittal et al. } 1990\end{array}$ \\
\hline
\end{tabular}


Table 2 Continued.

\begin{tabular}{|c|c|c|c|c|c|}
\hline Species & Taxonomy & Substrate & Host & Country & References \\
\hline \multirow{21}{*}{ Trichoderma viride Pers. } & \multirow{21}{*}{$\begin{array}{l}\text { Hypocreaceae, Sordariomycetes, } \\
\text { Ascomycota }\end{array}$} & seeds & Pinus oocarpa & Honduras & Mittal et al. 1990 \\
\hline & & seeds & Pinus patula & East Africa & $\begin{array}{l}\text { Gibson 1957, Mittal et } \\
\text { al. } 1990\end{array}$ \\
\hline & & seeds & Pinus ponderosa & USA & $\begin{array}{l}\text { Fuller \& Hildebrand } \\
\text { 1985, Mittal et al. 1990, } \\
\text { Cilliers et al. } 1995\end{array}$ \\
\hline & & seeds & Pinus sylvestris & Hungary & $\begin{array}{l}\text { Garbowski 1936, Mittal } \\
\text { et al. } 1990\end{array}$ \\
\hline & & seeds & Pinus taeda & USA & $\begin{array}{l}\text { Covington et al. 1982, } \\
\text { Anderson 1986a }\end{array}$ \\
\hline & & seeds & $\begin{array}{l}\text { Pseudotsuga } \\
\text { menziesii }\end{array}$ & Canada & $\begin{array}{l}\text { Salisbury 1955, Mittal et } \\
\text { al. } 1990\end{array}$ \\
\hline & & seeds & Tectona grandis & India & Mohanan et al. 2005 \\
\hline & & acorns & Quercus robur & Poland & Jankowiak 2008 \\
\hline & & cones & Pinus ponderosa & USA & James 1983a \\
\hline & & cones & Pinus sylvestris & UK & Whittle 1977 \\
\hline & & seeds & Acer rubrum & USA & $\begin{array}{l}\text { Friedrich et al. } 1971 \text {, } \\
\text { Mittal et al. } 1990\end{array}$ \\
\hline & & seeds & Acer saccharinum & USA & $\begin{array}{l}\text { Friedrich et al. } 1971 \text {, } \\
\text { Mittal et al. } 1990\end{array}$ \\
\hline & & seeds & Acer saccharum & USA & $\begin{array}{l}\text { Friedrich et al. } 1971 \text {, } \\
\text { Mittal et al. } 1990\end{array}$ \\
\hline & & seed & Agropyron cristatum & Canada & Conners 1967 \\
\hline & & seeds & Betula pendula & Finland & $\begin{array}{l}\text { Lilja 1979, Mittal et al. } \\
1990\end{array}$ \\
\hline & & seeds & Cedrus deodara & India & $\begin{array}{l}\text { Munjal \& Sharma 1975, } \\
\text { Mittal et al. } 1990\end{array}$ \\
\hline & & seeds & Dalbergia sissoo & India & Kumar 2014 \\
\hline & & seeds & $\begin{array}{l}\text { Eucalyptus } \\
\text { citriodora }\end{array}$ & India & $\begin{array}{l}\text { Mittal \& Sharma 1984, } \\
\text { Mittal et al. } 1990\end{array}$ \\
\hline & & seeds & Eucalyptus hybrid & India & $\begin{array}{l}\text { Mittal 1986, Mittal et al. } \\
1990\end{array}$ \\
\hline & & seeds & Eucalyptus maidenii & Uruguay & Anderson 1986a \\
\hline & & seed & Festuca elatior & Canada & Conners 1967 \\
\hline
\end{tabular}


Table 2 Continued.

\begin{tabular}{|c|c|c|c|c|c|}
\hline Species & Taxonomy & Substrate & Host & Country & References \\
\hline & & seeds & Fraxinus americana & USA & $\begin{array}{l}\text { Friedrich et al. } 1971 \text {, } \\
\text { Mittal et al. } 1990\end{array}$ \\
\hline & & seed & Linum usitatissimum & Canada & Conners 1967 \\
\hline & & seed & Medicago sativa & Canada & Conners 1967 \\
\hline & & seed & Phleum pratense & Canada & Conners 1967 \\
\hline & & seeds & Picea glauca & Canada & Mittal \& Wang 1987 \\
\hline & & seeds & $\begin{array}{l}\text { Pinus elliottii var. } \\
\text { elliottii }\end{array}$ & Uruguay & Anderson 1986a \\
\hline & & seeds & Pinus ponderosa & USA & $\begin{array}{l}\text { James \& Genz 1982, } \\
\text { Anderson 1986a }\end{array}$ \\
\hline & & seeds & Pinus roxburghii & India & $\begin{array}{l}\text { Munjal \& Sharma } 1975 \text {, } \\
\text { Mittal et al. } 1990\end{array}$ \\
\hline & & seeds & Pinus strobus & Canada & Mittal \& Wang 1987 \\
\hline & & seeds & Pinus sylvestris & UK & Whittle 1977 \\
\hline & & seeds & Pinus wallichiana & India & $\begin{array}{l}\text { Munjal \& Sharma } 1975 \text {, } \\
\text { Mittal et al. } 1990\end{array}$ \\
\hline & & seeds & $\begin{array}{l}\text { Pseudotsuga } \\
\text { menziesii }\end{array}$ & Canada, USA & $\begin{array}{l}\text { Bloomberg 1966, } \\
\text { Anderson 1986a, James } \\
1984\end{array}$ \\
\hline & & seeds & Quercus spp. & India & Sahai \& Otra 1982 \\
\hline & & seed & Setaria viridis & Canada & Conners 1967 \\
\hline & & seeds & Thuja spp. & India & Sahai \& Otra 1982 \\
\hline $\begin{array}{l}\text { Trichophaea abundans (P. } \\
\text { Karst.) Boud. } \\
\text { [as Patella abundans (P. } \\
\text { Karst.) Seaver] }\end{array}$ & $\begin{array}{l}\text { Pyronemataceae, Pezizomycetes, } \\
\text { Ascomycota }\end{array}$ & seed & Linum usitatissimum & Canada & Conners 1967 \\
\hline Trichosporon sp. & $\begin{array}{l}\text { Trichosporonaceae, Tremellomycetes, } \\
\text { Basidiomycota }\end{array}$ & seeds & Eucalyptus grandis & South Africa & Jimu et al. 2016 \\
\hline $\begin{array}{l}\text { Trichosporum cerealis } \\
\text { (Thüm.) Sacc. }\end{array}$ & $\begin{array}{l}\text { Piedraiaceae, Dothideomycetes, } \\
\text { Ascomycota }\end{array}$ & seeds & Quercus spp. & USSR & Mittal et al. 1990 \\
\hline $\begin{array}{l}\text { Trichosporum olivatrum } \\
\text { Sacc. }\end{array}$ & $\begin{array}{l}\text { Piedraiaceae, Dothideomycetes, } \\
\text { Ascomycota }\end{array}$ & seeds & Quercus spp. & USSR & $\begin{array}{l}\text { Urosevic 1962, Mittal et } \\
\text { al. } 1990\end{array}$ \\
\hline Trichothecium roseum & Myrotheciomycetaceae, Sordariomycetes, & cones, seeds & Pinus leucodermis & Canada & Vujanovic et al. 2000 \\
\hline (Pers.) Link & Ascomycota & cones, seeds & Pinus mugo & Canada & Vujanovic et al. 2000 \\
\hline
\end{tabular}


Table 2 Continued.

\begin{tabular}{|c|c|c|c|c|c|}
\hline Species & Taxonomy & Substrate & Host & Country & References \\
\hline & & cones, seeds & Pinus mugo 'Hesse' & Canada & Vujanovic et al. 2000 \\
\hline & & cones, seeds & $\begin{array}{l}\text { Pinus mugo var. } \\
\text { pumilio }\end{array}$ & Canada & Vujanovic et al. 2000 \\
\hline & & cones, seeds & Pinus nigra & Canada & Vujanovic et al. 2000 \\
\hline & & cones, seeds & $\begin{array}{l}\text { Pinus nigra ssp. } \\
\text { laricio }\end{array}$ & Canada & Vujanovic et al. 2000 \\
\hline & & cones, seeds & $\begin{array}{l}\text { Pinus nigra ssp. } \\
\text { nigra }\end{array}$ & Canada & Vujanovic et al. 2000 \\
\hline & & cones, seeds & Pinus strobus & Canada & Mittal \& Wang 1987 \\
\hline & & cones, seeds & $\begin{array}{l}\text { Pinus sylvestris } \\
\text { 'Euro-Asia' }\end{array}$ & Canada & Vujanovic et al. 2000 \\
\hline & & cones, seeds & Pinus uncinata & Canada & Vujanovic et al. 2000 \\
\hline & & seeds & Abies alba & Poland & Krol et al. 2015 \\
\hline & & seed & Abies balsamea & Canada & Conners 1967 \\
\hline & & seeds & Abies lasiocarpa & Canada & Talgø et al. 2010 \\
\hline & & seeds & Abies nordmanniana & Georgia, Russia & Talgø et al. 2010 \\
\hline & & seeds & Abies procera & Norway & Talgø et al. 2010 \\
\hline & & seeds & Acer pseudoplatanus & Poland & Krol et al. 2015 \\
\hline & & seeds & $\begin{array}{l}\text { Betula } \\
\text { alleghaniensis }\end{array}$ & USA & $\begin{array}{l}\text { Friedrich et al. } 1971 \text {, } \\
\text { Mittal et al. } 1990\end{array}$ \\
\hline & & seeds & Betula pendula & Finland, Poland & $\begin{array}{l}\text { Lilja 1979, Mittal et al. } \\
\text { 1990, Krol et al. } 2015\end{array}$ \\
\hline & & seeds & Cedrus deodara & Uruguay & $\begin{array}{l}\text { Manoharachary et al. } \\
\text { 1978, Anderson 1986a }\end{array}$ \\
\hline & & seeds & Dalbergia sissoo & India & Kumar 2014 \\
\hline & & seeds & Eucalyptus sp. & India & $\begin{array}{l}\text { Saxena 1985, Mittal et } \\
\text { al. } 1990\end{array}$ \\
\hline & & seeds & Fagus sylvatica & Poland & Krol et al. 2015 \\
\hline & & seeds & Larix occidentalis & USA & James et al. 1996 \\
\hline & & seeds & Picea abies & Poland & Krol et al. 2015 \\
\hline & & seeds & Picea excelsa & USSR & $\begin{array}{l}\text { Urosevic 1961, } \\
\text { Anderson 1986a }\end{array}$ \\
\hline
\end{tabular}


Table 2 Continued.

\begin{tabular}{|c|c|c|c|c|c|}
\hline Species & Taxonomy & Substrate & Host & Country & References \\
\hline & & seeds & Picea glauca & Canada & Mittal \& Wang 1987 \\
\hline & & seeds & $\begin{array}{l}\text { Pinus elliottii var. } \\
\text { elliottii }\end{array}$ & Uruguay & Anderson 1986a \\
\hline & & seeds & Pinus nigra & Hungary & $\begin{array}{l}\text { Friedrich et al. } 1971 \text {, } \\
\text { Mittal et al. } 1990\end{array}$ \\
\hline & & seeds & Pinus ponderosa & USA & $\begin{array}{l}\text { James \& Genz 1982, } \\
\text { Anderson 1986a }\end{array}$ \\
\hline & & seeds & Pinus sylvestris & $\begin{array}{l}\text { Hungary, Poland, } \\
\text { USSR }\end{array}$ & $\begin{array}{l}\text { Hangyal 1973, Anderson } \\
\text { 1986a }\end{array}$ \\
\hline & & seeds & Pinus taeda & Uruguay & Anderson 1986a \\
\hline & & seed & Poa compressa & Canada & Conners 1967 \\
\hline & & seeds & $\begin{array}{l}\text { Pseudotsuga } \\
\text { menziesii }\end{array}$ & USA & $\begin{array}{l}\text { Gordon 1967, Mittal et } \\
\text { al. } 1990\end{array}$ \\
\hline & & seeds & $\begin{array}{l}\text { Quercus } \\
\text { castaneifolia }\end{array}$ & Iran & Kavosi et al. 2013 \\
\hline & & seeds & Quercus robur & Germany & Schroder et al. 2004 \\
\hline & & seeds & Quercus spp. & India, USSR & $\begin{array}{l}\text { Urosevic 1959, Sahai \& } \\
\text { Otra 1982, Mittal et al. } \\
1990\end{array}$ \\
\hline & & seed & Setaria viridis & Canada & Conners 1967 \\
\hline & & seeds & Thuja spp. & India & Sahai \& Otra 1982 \\
\hline \multirow[t]{5}{*}{ Trichothecium spp. } & $\begin{array}{l}\text { Myrotheciomycetaceae, Sordariomycetes, } \\
\text { Ascomycota }\end{array}$ & seeds & Araucaria bidwillii & Egypt & $\begin{array}{l}\text { Kamara et al. } 1981 \text {, } \\
\text { Mittal et al. } 1990\end{array}$ \\
\hline & & seeds & Pinus patula & East Africa & $\begin{array}{l}\text { Gibson 1957, Mittal et } \\
\text { al. } 1990\end{array}$ \\
\hline & & seeds & Pinus taeda & USA & $\begin{array}{l}\text { Covington et al. 1982, } \\
\text { Anderson } 1986 \mathrm{a}\end{array}$ \\
\hline & & seeds & $\begin{array}{l}\text { Pseudotsuga } \\
\text { menziesii }\end{array}$ & Canada & $\begin{array}{l}\text { Bloomberg 1969, Mittal } \\
\text { et al. } 1990\end{array}$ \\
\hline & & seeds & Tectona grandis & India & Anderson 1986a \\
\hline $\begin{array}{l}\text { Tripospermum myrti (Lind) } \\
\text { S. Hughes }\end{array}$ & $\begin{array}{l}\text { Neodevriesiaceae, Dothideomycetes, } \\
\text { Ascomycota }\end{array}$ & seed & Pinus thunbergii & Japan & Watanabe 2010 \\
\hline Tripospermum sp. & $\begin{array}{l}\text { Neodevriesiaceae, Dothideomycetes, } \\
\text { Ascomycota }\end{array}$ & seed & Pinus densiflora & Japan & Watanabe 2010 \\
\hline
\end{tabular}


Table 2 Continued.

\begin{tabular}{|c|c|c|c|c|c|}
\hline Species & Taxonomy & Substrate & Host & Country & References \\
\hline \multirow[t]{2}{*}{ Tritirachium spp. } & \multirow{2}{*}{$\begin{array}{l}\text { Tritirachiaceae, Tritirachiomycetes, } \\
\text { Basidiomycota }\end{array}$} & seeds & Picea jezoensis & Japan & Cheng \& Igarashi 1987 \\
\hline & & seeds & Pinus taeda & Uruguay & Anderson 1986a, b \\
\hline \multirow{3}{*}{$\begin{array}{l}\text { Truncatella angustata } \\
\text { (Pers.) S. Hughes }\end{array}$} & \multirow{4}{*}{$\begin{array}{l}\text { Sporocadaceae, Sordariomycetes, } \\
\text { Ascomycota }\end{array}$} & seeds & Larix decidua & Poland & Krol et al. 2015 \\
\hline & & seeds & Picea abies & Poland & Krol et al. 2015 \\
\hline & & seeds & Pinus sylvestris & Poland & Krol et al. 2015 \\
\hline \multirow{10}{*}{$\begin{array}{l}\text { Truncatella angustata } \\
\text { (Pers.) S. Hughes } \\
\text { [as Pestalotia truncata Lév.] } \\
\text { Truncatella hartigii (Tubeuf) } \\
\text { Steyaert }\end{array}$} & & seeds & Quercus spp. & USSR & $\begin{array}{l}\text { Urosevic 1962, Mittal et } \\
\text { al. } 1990\end{array}$ \\
\hline & \multirow{9}{*}{$\begin{array}{l}\text { Sporocadaceae, Sordariomycetes, } \\
\text { Ascomycota }\end{array}$} & cones, seeds & Pinus contorta & Canada & Vujanovic et al. 2000 \\
\hline & & cones, seeds & Pinus densiflora & Canada & Vujanovic et al. 2000 \\
\hline & & cones, seeds & $\begin{array}{l}\text { Pinus sylvestris } \\
\text { 'Argentea- } \\
\text { Compacta' }\end{array}$ & Canada & Vujanovic et al. 2000 \\
\hline & & cones, seeds & $\begin{array}{l}\text { Pinus sylvestris } \\
\text { 'Beuvronensis' }\end{array}$ & Canada & Vujanovic et al. 2000 \\
\hline & & cones, seeds & $\begin{array}{l}\text { Pinus sylvestris } \\
\text { 'Fastigiata' }\end{array}$ & Canada & Vujanovic et al. 2000 \\
\hline & & cones, seeds & $\begin{array}{l}\text { Pinus sylvestris } \\
\text { 'Watereri' }\end{array}$ & Canada & Vujanovic et al. 2000 \\
\hline & & cones, seeds & Pinus tabulaeformis & Canada & Vujanovic et al. 2000 \\
\hline & & seeds & Abies spp & UK & $\begin{array}{l}\text { Batko 1956, Anderson } \\
\text { 1986a }\end{array}$ \\
\hline & & seeds & Nothofagus sp. & UK & Anderson 1986a \\
\hline \multirow{7}{*}{$\begin{array}{l}\text { Truncatella laurocerasi } \\
\text { (Westend.) Steyaert } \\
\text { Tubaria keralensis K.P.D. } \\
\text { Latha \& Manim.\# } \\
\text { Tubercularia spp. }\end{array}$} & \multirow{7}{*}{$\begin{array}{l}\text { Sporocadaceae, Sordariomycetes, } \\
\text { Ascomycota } \\
\text { Tubariaceae, Agaricomycetes, } \\
\text { Basidiomycota } \\
\text { Icmadophilaceae, Lecanoromycetes, } \\
\text { Ascomycota }\end{array}$} & seeds & Pyrus spp. & Romania & Richardson 1979 \\
\hline & & fruits & Casuarina sp. & India & Latha et al. 2016 \\
\hline & & cones, seeds & $\begin{array}{l}\text { Pinus } \times \text { schwerinii } \\
(P . \text { strobus } \times P . \\
\text { wallichiana })\end{array}$ & Canada & Vujanovic et al. 2000 \\
\hline & & cones, seeds & Pinus ayacahuite & Canada & Vujanovic et al. 2000 \\
\hline & & cones, seeds & Pinus parviflora & Canada & Vujanovic et al. 2000 \\
\hline & & cones, seeds & Pinus peuce & Canada & Vujanovic et al. 2000 \\
\hline & & cones, seeds & Pinus ponderosa & Canada & Vujanovic et al. 2000 \\
\hline
\end{tabular}


Table 2 Continued.

\begin{tabular}{|c|c|c|c|c|c|}
\hline Species & Taxonomy & Substrate & Host & Country & References \\
\hline $\begin{array}{l}\text { Tubeufia dictyospora Y.Z. } \\
\text { Lu, Boonmee \& K.D. Hyde }\end{array}$ & $\begin{array}{l}\text { Tubeufiaceae, Dothideomycetes, } \\
\text { Ascomycota }\end{array}$ & pod & Delonix regia & Thailand & Jayasiri et al. 2019 \\
\hline $\begin{array}{l}\text { Tubeufia entadae Jayasiri, } \\
\text { E.B.G. Jones \& K.D. Hyde }\end{array}$ & $\begin{array}{l}\text { Tubeufiaceae, Dothideomycetes, } \\
\text { Ascomycota }\end{array}$ & pod & Entada phaseoloides & Thailand & Jayasiri et al. 2019 \\
\hline \multirow[t]{2}{*}{ Typhula peronata (Pers.) Fr. } & $\begin{array}{l}\text { Typhulaceae, Agaricomycetes, } \\
\text { Basidiomycota }\end{array}$ & seeds & Picea abies & USSR & $\begin{array}{l}\text { Prisyazhnyuk } 1960 \text {, } \\
\text { Mittal et al. } 1990\end{array}$ \\
\hline & & seeds & Pinus sylvestris & USSR & $\begin{array}{l}\text { Prisyazhnyuk } 1960 \text {, } \\
\text { Mittal et al. } 1990\end{array}$ \\
\hline \multirow{2}{*}{$\begin{array}{l}\text { Umbelopsis isabellina } \\
\text { (Oudem.) W. Gams } \\
\text { [as Mortierella isabellina } \\
\text { Oudem.] }\end{array}$} & $\begin{array}{l}\text { Umbelopsidaceae, Incertae sedis, } \\
\text { Mucoromycota }\end{array}$ & cones & Pinus densiflora & Japan & Kasai et al. 1995 \\
\hline & & $\begin{array}{l}\text { seed } \\
\text { seeds }\end{array}$ & $\begin{array}{l}\text { Pinus thunbergii } \\
\text { Pinus wallichiana }\end{array}$ & $\begin{array}{l}\text { Japan } \\
\text { India }\end{array}$ & $\begin{array}{l}\text { Watanabe } 2010 \\
\text { Mittal \& Sharma 1982b, } \\
\text { Mittal et al. } 1990\end{array}$ \\
\hline $\begin{array}{l}\text { Umbelopsis ramanniana } \\
\text { (Möller) W. Gams } \\
\text { [as Mortierella ramanniana } \\
\text { (Möller) Linnem.] }\end{array}$ & $\begin{array}{l}\text { Umbelopsidaceae, Incertae sedis, } \\
\text { Mucoromycota }\end{array}$ & cones & Pinus densiflora & Japan & Kasai et al. 1995 \\
\hline \multirow[t]{3}{*}{$\begin{array}{l}\text { Umbelopsis ramanniana } \\
\text { (Möller) W. Gams } \\
\text { [as Mucor ramannianus } \\
\text { Möller] }\end{array}$} & & seeds & Picea excelsa & USSR & $\begin{array}{l}\text { Urosevic 1961, } \\
\text { Anderson 1986a }\end{array}$ \\
\hline & & seeds & Pinus caribaea & $\begin{array}{l}\text { Belize [as British } \\
\text { Honduras] }\end{array}$ & $\begin{array}{l}\text { Hocking 1968, Mittal et } \\
\text { al. } 1990\end{array}$ \\
\hline & & seeds & Pinus sylvestris & USSR & $\begin{array}{l}\text { Urosevic 1961, } \\
\text { Anderson 1986a }\end{array}$ \\
\hline Umbelopsis sp. & $\begin{array}{l}\text { Umbelopsidaceae, Incertae sedis, } \\
\text { Mucoromycota }\end{array}$ & seeds & Pinus taeda & USA & Anderson 1986a, b \\
\hline Undetermined Ascomycete & - & seeds & Fraxinus excelsior & Sweden & Hayatgheibi 2013 \\
\hline Undetermined Basidiomycete & - & seeds & Fraxinus excelsior & Sweden & Hayatgheibi 2013 \\
\hline Undetermined fungi & - & $\begin{array}{l}\text { seed capsules } \\
\text { seeds } \\
\text { seeds }\end{array}$ & $\begin{array}{l}\text { Eucalyptus grandis } \\
\text { Calypso bulbosa } \\
\text { Eucalyptus grandis }\end{array}$ & $\begin{array}{l}\text { South Africa } \\
\text { Canada } \\
\text { South Africa }\end{array}$ & $\begin{array}{l}\text { Jimu et al. } 2016 \\
\text { Zelmer et al. } 1996 \\
\text { Jimu et al. } 2016\end{array}$ \\
\hline
\end{tabular}


Table 2 Continued.

\begin{tabular}{|c|c|c|c|c|c|}
\hline Species & Taxonomy & Substrate & Host & Country & References \\
\hline & & seeds & Phyllostachys edulis & China & Shen et al. 2014 \\
\hline & & seeds & $\begin{array}{l}\text { Platanthera } \\
\text { hyperborea }\end{array}$ & Canada & Zelmer et al. 1996 \\
\hline $\begin{array}{l}\text { Urceolella pinicola Raitv. \& } \\
\text { R. Galán }{ }^{\#}\end{array}$ & Calloriaceae, Leotiomycetes, Ascomycota & cone & Pinus halepensis & Spain & Raitviír \& Galán 1993 \\
\hline $\begin{array}{l}\text { Urceolella triseptata Raitv. } \\
\text { \& R. Galán\# }\end{array}$ & Calloriaceae, Leotiomycetes, Ascomycota & cupule & $\begin{array}{l}\text { Quercus } \\
\text { rotundifolia }\end{array}$ & Spain & Raitviír \& Galán 1993 \\
\hline Urocystis aurea Vánky & $\begin{array}{l}\text { Urocystidaceae, Ustilaginomycetes, } \\
\text { Basidiomycota }\end{array}$ & capsules & Hypoxis aurea & India & Vánky 2004 \\
\hline $\begin{array}{l}\text { Uromycladium } \\
\text { naracoortense Berndt [as } \\
\text { 'naracoortensis'] }]^{\#}\end{array}$ & $\begin{array}{l}\text { Pileolariaceae, Pucciniomycetes, } \\
\text { Basidiomycota }\end{array}$ & pods & Acacia cf. iteaphylla & Australia & Berndt 2010 \\
\hline $\begin{array}{l}\text { Ustilago echinochloae L. } \\
\text { Guo \& Y.-c. Wang }\end{array}$ & $\begin{array}{l}\text { Ustilaginaceae, Ustilaginomycetes, } \\
\text { Basidiomycota }\end{array}$ & seeds & $\begin{array}{l}\text { Echinochloa crus- } \\
\text { galli }\end{array}$ & China & Vánky \& Guo 1987 \\
\hline $\begin{array}{l}\text { Ustilago hordei (Pers.) } \\
\text { Lagerh. }\end{array}$ & $\begin{array}{l}\text { Ustilaginaceae, Ustilaginomycetes, } \\
\text { Basidiomycota }\end{array}$ & seed & Agropyron cristatum & Canada & Conners 1967 \\
\hline $\begin{array}{l}\text { Vaginatispora nypae } \\
\text { Jayasiri, E.B.G. Jones \& } \\
\text { K.D. Hyde }^{\#}\end{array}$ & $\begin{array}{l}\text { Lophiostomataceae, Dothideomycetes, } \\
\text { Ascomycota }\end{array}$ & fruit & Nypa sp. & Thailand & Jayasiri et al. 2019 \\
\hline $\begin{array}{l}\text { Valsa pini (Alb. \& Schwein.) } \\
\text { Fr. }\end{array}$ & Valsaceae, Sordariomycetes, Ascomycota & cones, seeds & Pinus flexilis & Canada & Vujanovic et al. 2000 \\
\hline $\begin{array}{l}\text { Valsa spp. } \\
\text { Vargamyces aquaticus } \\
\text { (Dudka) Tóth }\end{array}$ & $\begin{array}{l}\text { Valsaceae, Sordariomycetes, Ascomycota } \\
\text { Amniculicolaceae, Dothideomycetes, } \\
\text { Ascomycota }\end{array}$ & $\begin{array}{l}\text { cones, seeds } \\
\text { seeds } \\
\text { fruit cupule }\end{array}$ & $\begin{array}{l}\text { Pinus peuce } \\
\text { Eucalyptus grandis } \\
\text { Fagus sylvatica }\end{array}$ & $\begin{array}{l}\text { Canada } \\
\text { South Africa } \\
\text { UK }\end{array}$ & $\begin{array}{l}\text { Vujanovic et al. } 2000 \\
\text { Jimu et al. } 2016 \\
\text { Jayasiri et al. } 2019\end{array}$ \\
\hline $\begin{array}{l}\text { Verruculina enalia (Kohlm.) } \\
\text { Kohlm. \& Volkm.-Kohlm. }\end{array}$ & $\begin{array}{l}\text { Testudinaceae, Dothideomycetes, } \\
\text { Ascomycota }\end{array}$ & fruit & Pandanus sp. & Thailand & Jayasiri et al. 2019 \\
\hline \multirow[t]{3}{*}{$\begin{array}{l}\text { Verticillium albo-atrum } \\
\text { Reinke \& Berthold }\end{array}$} & $\begin{array}{l}\text { Plectosphaerellaceae, Sordariomycetes, } \\
\text { Ascomycota }\end{array}$ & seed & $\begin{array}{l}\text { Apium graveolens } \\
\text { var. dulce }\end{array}$ & USA & Conners 1967 \\
\hline & & seeds & Eucalyptus sp. & India & $\begin{array}{l}\text { Lee \& Ahmad } 1982 \text {, } \\
\text { Mittal et al. } 1990\end{array}$ \\
\hline & & seeds & Picea excelsa & USSR & $\begin{array}{l}\text { Urosevic 1961, } \\
\text { Anderson 1986a }\end{array}$ \\
\hline
\end{tabular}


Table 2 Continued.

\begin{tabular}{|c|c|c|c|c|c|}
\hline Species & Taxonomy & Substrate & Host & Country & References \\
\hline & & seeds & Pinus sylvestris & USSR & $\begin{array}{l}\text { Dolejs 1964, Anderson } \\
\text { 1986a }\end{array}$ \\
\hline \multirow{3}{*}{$\begin{array}{l}\text { Verticillium epimyces Berk. } \\
\text { \& Broome } \\
\text { Verticillium glaucum } \\
\text { Bonord. }\end{array}$} & $\begin{array}{l}\text { Plectosphaerellaceae, Sordariomycetes, } \\
\text { Ascomycota }\end{array}$ & seeds & Quercus spp. & USSR & $\begin{array}{l}\text { Urosevic 1962, Mittal et } \\
\text { al. } 1990\end{array}$ \\
\hline & $\begin{array}{l}\text { Plectosphaerellaceae, Sordariomycetes, } \\
\text { Ascomycota }\end{array}$ & seeds & Picea abies & USSR & $\begin{array}{l}\text { Prisyazhnyuk } 1960 \text {, } \\
\text { Mittal et al. } 1990\end{array}$ \\
\hline & & seeds & Pinus sylvestris & USSR & $\begin{array}{l}\text { Prisyazhnyuk } 1960 \text {, } \\
\text { Mittal et al. } 1990\end{array}$ \\
\hline \multirow[t]{15}{*}{ Verticillium spp. } & Plectosphaerellaceae, Sordariomycetes, & pods & Delonix regia & Thailand & Somrithipol et al. 2002b \\
\hline & Ascomycota & seeds & Acer campestre & South Korea & $\begin{array}{l}\text { Mathur 1974, Anderson } \\
\text { 1986a }\end{array}$ \\
\hline & & seeds & Azadirachta indica & India & $\begin{array}{l}\text { Mathur 1974, Mittal et } \\
\text { al. } 1990\end{array}$ \\
\hline & & seeds & $\begin{array}{l}\text { Betula } \\
\text { alleghaniensis }\end{array}$ & USA & $\begin{array}{l}\text { Friedrich et al. } 1971 \text {, } \\
\text { Mittal et al. } 1990\end{array}$ \\
\hline & & seeds & Dalbergia sissoides & India & Mohanan et al. 2005 \\
\hline & & seeds & Eucalyptus grandis & Uruguay & Anderson 1986a \\
\hline & & seeds & Fagus spp. & $\begin{array}{l}\text { former } \\
\text { Czechoslovakia }\end{array}$ & $\begin{array}{l}\text { Urosevic 1964, Mittal et } \\
\text { al. } 1990\end{array}$ \\
\hline & & seeds & Ficus benjamina & India & $\begin{array}{l}\text { Mathur 1974, Anderson } \\
\text { 1986a }\end{array}$ \\
\hline & & seeds & Picea abies & UK & Mittal et al. 1990 \\
\hline & & seeds & Picea sitchensis & UK & $\begin{array}{l}\text { Salt 1964, Mittal et al. } \\
1990\end{array}$ \\
\hline & & seeds & Picea spp. & UK & Anderson 1986a \\
\hline & & seeds & $\begin{array}{l}\text { Pinus armandii var. } \\
\text { mastersiana }\end{array}$ & Taiwan & $\begin{array}{l}\text { Jong \& Chen } 1966 \text {, } \\
\text { Mittal et al. } 1990\end{array}$ \\
\hline & & seeds & Pinus elliottii & Taiwan, USA & $\begin{array}{l}\text { Rowan \& Debarr 1974, } \\
\text { Anderson 1986a, Mittal } \\
\text { et al. } 1990\end{array}$ \\
\hline & & seeds & $\begin{array}{l}\text { Pinus elliottii var. } \\
\text { elliottii }\end{array}$ & Uruguay & Anderson 1986a \\
\hline & & seeds & Pinus massoniana & Taiwan & $\begin{array}{l}\text { Jong \& Chen } 1966 \text {, } \\
\text { Mittal et al. } 1990\end{array}$ \\
\hline
\end{tabular}


Table 2 Continued.

\begin{tabular}{|c|c|c|c|c|c|}
\hline Species & Taxonomy & Substrate & Host & Country & References \\
\hline & & seeds & Pinus ponderosa & USA & $\begin{array}{l}\text { James \& Genz 1982, } \\
\text { Anderson 1986a }\end{array}$ \\
\hline & & seeds & Pinus taeda & USA & Anderson 1986a, b \\
\hline & & seeds & Pinus taiwanensis & Taiwan & $\begin{array}{l}\text { Jong \& Chen } 1966 \text {, } \\
\text { Mittal et al. } 1990\end{array}$ \\
\hline & & seeds & $\begin{array}{l}\text { Pseudotsuga } \\
\text { menziesii }\end{array}$ & Canada, UK, USA & $\begin{array}{l}\text { Gordon 1967, Anderson } \\
\text { 1986a, Mittal et al. } 1990\end{array}$ \\
\hline & & seeds & Pterocarpus indicus & Philippines & $\begin{array}{l}\text { Mathur 1974, Anderson } \\
\text { 1986a }\end{array}$ \\
\hline & & seeds & Quercus robur & Germany & Schroder et al. 2004 \\
\hline & & seeds & Quercus spp. & USSR & $\begin{array}{l}\text { Urosevic 1983, Mittal et } \\
\text { al. } 1990\end{array}$ \\
\hline & & seeds & Tectona grandis & India & $\begin{array}{l}\text { Mathur 1974, Anderson } \\
\text { 1986a, Mohanan et al. } \\
2005\end{array}$ \\
\hline & & seeds & Tsuga heterophylla & UK & Anderson 1986a \\
\hline $\begin{array}{l}\text { Vishniacozyma carnescens } \\
\text { (Verona \& Luchetti) Xin } \\
\text { Zhan Liu, F.Y. Bai, M. } \\
\text { Groenew. \& Boekhout } \\
\text { [as Cryptococcus carnescens } \\
\text { (Verona \& Luchetti) M. } \\
\text { Takash., Sugita, Shinoda \& } \\
\text { Nakase] }\end{array}$ & $\begin{array}{l}\text { Bulleribasidiaceae, Tremellomycetes, } \\
\text { Basidiomycota }\end{array}$ & seeds & Fraxinus excelsior & Sweden & Cleary et al. 2013 \\
\hline $\begin{array}{l}\text { Vishniacozyma victoriae } \\
\text { (M.J. Montes, Belloch, } \\
\text { Galiana, M.D. García, C. } \\
\text { Andrés, S. Ferrer, Torr.- } \\
\text { Rodr. \& J. Guinea) Xin Zhan } \\
\text { Liu, F.Y. Bai, M. Groenew. } \\
\text { \& Boekhout } \\
\text { [as Cryptococcus victoriae } \\
\text { M.J. Montes, Belloch, } \\
\text { Galiana, M.D. García, C. }\end{array}$ & $\begin{array}{l}\text { Bulleribasidiaceae, Tremellomycetes, } \\
\text { Basidiomycota }\end{array}$ & seeds & Fraxinus excelsior & Sweden & Hayatgheibi 2013 \\
\hline
\end{tabular}


Table 2 Continued.

\begin{tabular}{|c|c|c|c|c|c|}
\hline Species & Taxonomy & Substrate & Host & Country & References \\
\hline \multicolumn{6}{|l|}{$\begin{array}{l}\text { Andrés, S. Ferrer, Torr.- } \\
\text { Rodr. \& J. Guinea] }\end{array}$} \\
\hline $\begin{array}{l}\text { Volutella ciliata (Alb. \& } \\
\text { Schwein.) Fr. }\end{array}$ & $\begin{array}{l}\text { Nectriaceae, Sordariomycetes, } \\
\text { Ascomycota }\end{array}$ & cone & Abies balsamea & Canada & Conners 1967 \\
\hline Volutella sp. & $\begin{array}{l}\text { Nectriaceae, Sordariomycetes, } \\
\text { Ascomycota }\end{array}$ & pods & Delonix regia & Thailand & Somrithipol et al. 2002b \\
\hline $\begin{array}{l}\text { Vuilleminia comedens } \\
\text { (Nees) Maire }\end{array}$ & $\begin{array}{l}\text { Vuilleminiaceae, Bartheletiomycetes, } \\
\text { Basidiomycota }\end{array}$ & seeds & Quercus spp. & USSR & Mittal et al. 1990 \\
\hline \multirow[t]{7}{*}{ White, sterile fungi } & - & seeds & Acer rubrum & USA & $\begin{array}{l}\text { Friedrich et al. } 1971 \text {, } \\
\text { Mittal et al. } 1990\end{array}$ \\
\hline & & seeds & Acer saccharinum & USA & $\begin{array}{l}\text { Friedrich et al. } 1971 \text {, } \\
\text { Mittal et al. } 1990\end{array}$ \\
\hline & & seeds & Acer saccharum & USA & $\begin{array}{l}\text { Friedrich 1969, Mittal et } \\
\text { al. } 1990\end{array}$ \\
\hline & & seeds & Albizia lebbeck & India & Mohanan et al. 2005 \\
\hline & & seeds & Cedrus deodara & India & $\begin{array}{l}\text { Munjal \& Sharma 1975, } \\
\text { Mittal et al. } 1990\end{array}$ \\
\hline & & seeds & Dalbergia sissoo & India & Naz et al. 2015 \\
\hline & & seeds & Fraxinus americana & USA & $\begin{array}{l}\text { Friedrich et al. } 1971 \text {, } \\
\text { Mittal et al. } 1990\end{array}$ \\
\hline $\begin{array}{l}\text { Wickerhamiella fructicola } \\
\text { Pagnocca, C.A. Rosa, Dayo- } \\
\text { Owoy. \& A. Rodrigues }{ }^{\#}\end{array}$ & $\begin{array}{l}\text { Trichomonascaceae, Saccharomycetes, } \\
\text { Ascomycota }\end{array}$ & $\begin{array}{l}\text { seeds } \\
\text { fruits }\end{array}$ & $\begin{array}{l}\text { Tectona grandis } \\
\text { Hedychium } \\
\text { coronarium }\end{array}$ & $\begin{array}{l}\text { India } \\
\text { Brazil }\end{array}$ & $\begin{array}{l}\text { Mohanan et al. } 2005 \\
\text { Dayo-Owoyemi et al. } \\
\mathbf{2 0 1 4}\end{array}$ \\
\hline $\begin{array}{l}\text { Wickerhamiella sergipiensis } \\
\text { (R.C. Trindade, M.A. } \\
\text { Resende, Lachance \& C.A. } \\
\text { Rosa) C. Vega \& Lachance }{ }^{\#} \\
\text { [as Candida sergipensis R.C. } \\
\text { Trindade, M.A. Resende, } \\
\text { Lachance \& C.A. Rosa] }\end{array}$ & $\begin{array}{l}\text { Trichomonascaceae, Saccharomycetes, } \\
\text { Ascomycota }\end{array}$ & $\begin{array}{l}\text { frozen fruit } \\
\text { pulp }\end{array}$ & Hancornia speciosa & Brazil & Trindade et al. 2004 \\
\hline $\begin{array}{l}\text { Wickerhamiella versatilis } \\
\text { (Etchells \& T.A. Bell) de } \\
\text { Vega \& Lachance }\end{array}$ & $\begin{array}{l}\text { Trichomonascaceae, Saccharomycetes, } \\
\text { Ascomycota }\end{array}$ & fruits & $\begin{array}{l}\text { Parahancornia } \\
\text { amapa }\end{array}$ & Brazil & Morais et al. 1995 \\
\hline
\end{tabular}


Table 2 Continued.

\begin{tabular}{|c|c|c|c|c|c|}
\hline Species & Taxonomy & Substrate & Host & Country & References \\
\hline $\begin{array}{l}\text { [as Candida versatilis } \\
\text { (Etchells \& T.A. Bell) S.A. } \\
\text { Mey. \& Yarrow] }\end{array}$ & & & & & \\
\hline $\begin{array}{l}\text { Wickerhamomyces } \\
\text { anomalus (E.C. Hansen) } \\
\text { Kurtzman, Robnett \& Bas.- } \\
\text { Powers } \\
\text { [as Hansenula anomala (E.C. } \\
\text { Hansen) Syd. \& P. Syd.] }\end{array}$ & $\begin{array}{l}\text { Phaffomycetaceae, Saccharomycetes, } \\
\text { Ascomycota }\end{array}$ & seeds & Atropa komarovii & $\begin{array}{l}\text { Poland or/ } \\
\text { imported to Poland }\end{array}$ & Czeczuga et al. 2009 \\
\hline $\begin{array}{l}\text { Wickerhamomyces pijperi } \\
\text { (Van der Walt \& } \\
\text { Tscheuschner) Kurtzman, } \\
\text { Robnett \& Bas.-Powers } \\
\text { [as Pichia pijperi Van der } \\
\text { Walt \& Tscheuschner] }\end{array}$ & $\begin{array}{l}\text { Phaffomycetaceae, Saccharomycetes, } \\
\text { Ascomycota }\end{array}$ & fruits & $\begin{array}{l}\text { Parahancornia } \\
\text { amapa }\end{array}$ & Brazil & Morais et al. 1995 \\
\hline $\begin{array}{l}\text { Wiesneriomyces javanicus } \\
\text { Koord. }\end{array}$ & $\begin{array}{l}\text { Wiesneriomycetaceae Incertae sedis, } \\
\text { Ascomycota }\end{array}$ & fruit & Scheelea zonensis & Panama & Goos \& Pirozynski 1975 \\
\hline $\begin{array}{l}\text { Xenocamarosporium } \\
\text { acaciae Crous \& M.J. Wingf. }\end{array}$ & $\begin{array}{l}\text { Didymosphaeriaceae, Dothideomycetes, } \\
\text { Ascomycota }\end{array}$ & pod & Leucaena sp. & Thailand & Jayasiri et al. 2019 \\
\hline $\begin{array}{l}\text { Xylaria aethiopica J. Fourn., } \\
\text { Y.M. Ju, H.M. Hsieh \& U. } \\
\text { Lindem.\# }\end{array}$ & $\begin{array}{l}\text { Xylariaceae, Sordariomycetes, } \\
\text { Ascomycota }\end{array}$ & pods & Millettia ferruginea & Ethiopia & Fournier et al. 2018 \\
\hline $\begin{array}{l}\text { Xylaria alata F. San Martín } \\
\text { \& J.D. Rogers }{ }^{\#}\end{array}$ & $\begin{array}{l}\text { Xylariaceae, Sordariomycetes, } \\
\text { Ascomycota }\end{array}$ & fruit & undetermined plant & Mexico & $\begin{array}{l}\text { San Martín González } \\
\text { \& Rogers } 1989\end{array}$ \\
\hline Xylaria arbuscula Sacc & $\begin{array}{l}\text { Xylariaceae, Sordariomycetes, } \\
\text { Ascomycota }\end{array}$ & fruits & Quercus sp. & Mexico & $\begin{array}{l}\text { San Martín González \& } \\
\text { Rogers } 1989\end{array}$ \\
\hline Xylaria aristata Mont. & $\begin{array}{l}\text { Xylariaceae, Sordariomycetes, } \\
\text { Ascomycota }\end{array}$ & pod & Guazuma ulmifolia & Mexico & $\begin{array}{l}\text { San Martín González \& } \\
\text { Rogers } 1989\end{array}$ \\
\hline $\begin{array}{l}\text { Xylaria beilschmiediae G. } \\
\text { Huang \& L. Guo }\end{array}$ & $\begin{array}{l}\text { Xylariaceae, Sordariomycetes, } \\
\text { Ascomycota }\end{array}$ & fruit & $\begin{array}{l}\text { Beilschmiedia } \\
\text { percoriacea }\end{array}$ & China & Huang et al. 2014a \\
\hline $\begin{array}{l}\text { Xylaria byttneriae G. Huang, } \\
\text { L. Guo \& Na Liü\# }\end{array}$ & $\begin{array}{l}\text { Xylariaceae, Sordariomycetes, } \\
\text { Ascomycota }\end{array}$ & $\begin{array}{l}\text { fruits } \\
\text { fruits }\end{array}$ & $\begin{array}{l}\text { Byttneria integrifolia } \\
\text { Byttneria pilosa }\end{array}$ & $\begin{array}{l}\text { China } \\
\text { China }\end{array}$ & $\begin{array}{l}\text { Huang et al. 2014b } \\
\text { Huang et al. 2014b }\end{array}$ \\
\hline
\end{tabular}


Table 2 Continued.

\begin{tabular}{|c|c|c|c|c|c|}
\hline Species & Taxonomy & Substrate & Host & Country & References \\
\hline $\begin{array}{l}\text { Xylaria carpophila (Pers.) } \\
\text { Fr. }\end{array}$ & $\begin{array}{l}\text { Xylariaceae, Sordariomycetes, } \\
\text { Ascomycota }\end{array}$ & fruit cupules & Fagus crenata & Japan & Fukasawa et al. 2012 \\
\hline $\begin{array}{l}\text { Xylaria duranii F. San } \\
\text { Martín \& Vanoye }{ }^{\#}\end{array}$ & $\begin{array}{l}\text { Xylariaceae, Sordariomycetes, } \\
\text { Ascomycota }\end{array}$ & fruit remaining & $\begin{array}{l}\text { Quercus } \\
\text { polymoroha }\end{array}$ & Mexico & San Martín et al. 2001 \\
\hline $\begin{array}{l}\text { Xylaria euphorbiicola } \\
\text { Rehm }^{\#}\end{array}$ & $\begin{array}{l}\text { Xylariaceae, Sordariomycetes, } \\
\text { Ascomycota }\end{array}$ & fruits & Euphorbia sp. & Brazil & Rehm 1901 \\
\hline $\begin{array}{l}\text { Xylaria guazumae González } \\
\text { \& Rogers }{ }^{\#}\end{array}$ & $\begin{array}{l}\text { Xylariaceae, Sordariomycetes, } \\
\text { Ascomycota }\end{array}$ & fruits & Guazuma ulmifolia & Mexico & $\begin{array}{l}\text { San Martín González } \\
\text { \& Rogers } 1989\end{array}$ \\
\hline $\begin{array}{l}\text { Xylaria heloidea Penz. \& } \\
\text { Sacc.\# }\end{array}$ & $\begin{array}{l}\text { Xylariaceae, Sordariomycetes, } \\
\text { Ascomycota }\end{array}$ & fruits & Legume plant & Indonesia & $\begin{array}{l}\text { Penzig \& Saccardo } \\
1897\end{array}$ \\
\hline $\begin{array}{l}\text { Xylaria himalayensis Narula } \\
\text { \& Rawla\# }\end{array}$ & $\begin{array}{l}\text { Xylariaceae, Sordariomycetes, } \\
\text { Ascomycota }\end{array}$ & seeds & $\begin{array}{l}\text { angiospermous } \\
\text { plant }\end{array}$ & India & Narula et al. 1984 \\
\hline $\begin{array}{l}\text { Xylaria jaliscoensis F. San } \\
\text { Martín, J.D. Rogers \& Y.M. } \\
\text { Ju}^{\#}\end{array}$ & $\begin{array}{l}\text { Xylariaceae, Sordariomycetes, } \\
\text { Ascomycota }\end{array}$ & fruits & Magnolia iltisiana & Mexico & Rogers et al. 2002 \\
\hline $\begin{array}{l}\text { Xylaria karyophthora } \\
\text { Husbands, Urbina \& Aime }\end{array}$ & $\begin{array}{l}\text { Xylariaceae, Sordariomycetes, } \\
\text { Ascomycota }\end{array}$ & seeds & $\begin{array}{l}\text { Chlorocardium } \\
\text { rodiei }\end{array}$ & Guyana & Husbands et al. 2018 \\
\hline $\begin{array}{l}\text { Xylaria liquidambaris J.D. } \\
\text { Rogers, Y.M. Ju \& F. San } \\
\text { Martín [as 'liquidambar'] }\end{array}$ & $\begin{array}{l}\text { Xylariaceae, Sordariomycetes, } \\
\text { Ascomycota }\end{array}$ & fruits & $\begin{array}{l}\text { Liquidambar } \\
\text { styraciflua }\end{array}$ & $\begin{array}{l}\text { USA, Mexico, } \\
\text { China }\end{array}$ & $\begin{array}{l}\text { Rogers 1979a, San } \\
\text { Martín González \& } \\
\text { Rogers 1989, Rogers et } \\
\text { al. } 200\end{array}$ \\
\hline $\begin{array}{l}\text { Xylaria magnoliae J.D. } \\
\text { Rogers }^{\#}\end{array}$ & $\begin{array}{l}\text { Xylariaceae, Sordariomycetes, } \\
\text { Ascomycota }\end{array}$ & fruits & $\begin{array}{l}\text { Magnolia } \\
\text { grandiflora }\end{array}$ & USA & Rogers 1979a \\
\hline & & $\begin{array}{l}\text { fruits } \\
\text { fruits }\end{array}$ & $\begin{array}{l}\text { Magnolia } \\
\text { schiedeana } \\
\text { Magnolia sp. }\end{array}$ & $\begin{array}{l}\text { Mexico } \\
\text { USA }\end{array}$ & $\begin{array}{l}\text { San Martín González \& } \\
\text { Rogers } 1989 \\
\text { Rogers 1979a }\end{array}$ \\
\hline $\begin{array}{l}\text { Xylaria magnoliae var. } \\
\text { microspora J.D. Rogers, }\end{array}$ & $\begin{array}{l}\text { Xylariaceae, Sordariomycetes, } \\
\text { Ascomycota }\end{array}$ & fruits & Talauma ovata & Brazil & $\begin{array}{l}\text { Trierveiler-Pereira et al. } \\
2009\end{array}$ \\
\hline Y.M. Ju \& Whalley & & fruit & Magnolia sp. & Thailand & Rogers et al. 2002 \\
\hline $\begin{array}{l}\text { Xylaria oxyacanthae Tul. \& } \\
\text { C. Tul. }\end{array}$ & $\begin{array}{l}\text { Xylariaceae, Sordariomycetes, } \\
\text { Ascomycota }\end{array}$ & seeds & $\begin{array}{l}\text { Crataegus } \\
\text { monogyna }\end{array}$ & USA, Mexico & $\begin{array}{l}\text { Rogers et al. 2008, San } \\
\text { Martín González \& } \\
\text { Rogers } 1989 \\
\end{array}$ \\
\hline
\end{tabular}


Table 2 Continued.

\begin{tabular}{|c|c|c|c|c|c|}
\hline Species & Taxonomy & Substrate & Host & Country & References \\
\hline & & pod & Fabaceae plant & Mexico & $\begin{array}{l}\text { San Martín González \& } \\
\text { Rogers } 1989\end{array}$ \\
\hline & & seeds & Vitaceae plant & USA, Mexico & $\begin{array}{l}\text { Rogers et al. 2008, San } \\
\text { Martín González \& } \\
\text { Rogers } 1989\end{array}$ \\
\hline $\begin{array}{l}\text { Xylaria persicaria } \\
\text { (Schwein.) Berk. \& M.A. }\end{array}$ & $\begin{array}{l}\text { Xylariaceae, Sordariomycetes, } \\
\text { Ascomycota }\end{array}$ & fruits & $\begin{array}{l}\text { Liquidambar } \\
\text { formosana }\end{array}$ & Korea & Han \& Shin 2007 \\
\hline Curtis & & fruits & $\begin{array}{l}\text { Liquidambar } \\
\text { styraciflua }\end{array}$ & Korea & Han \& Shin 2007 \\
\hline Xylaria rhizocola (Mont.) & Xylariaceae, Sordariomycetes, & seed & Palm & Brazil & Ju et al. 2018 \\
\hline Mont. & Ascomycota & seeds & undetermined plant & $\begin{array}{l}\text { France [as French } \\
\text { Guiana] }\end{array}$ & Ju et al. 2018 \\
\hline $\begin{array}{l}\text { Xylaria rossmaniae Y.M. Ju, } \\
\text { J.D. Rogers \& H.M. Hsieh } \\
\text { [as 'rossmanae']” }\end{array}$ & $\begin{array}{l}\text { Xylariaceae, Sordariomycetes, } \\
\text { Ascomycota }\end{array}$ & pod & Elizabetha sp. & Venezuela & Ju et al. 2018 \\
\hline \multirow[t]{5}{*}{ Xylaria spp. } & $\begin{array}{l}\text { Xylariaceae, Sordariomycetes, } \\
\text { Ascomycota }\end{array}$ & $\begin{array}{l}\text { cones } \\
\text { fruit cupules }\end{array}$ & $\begin{array}{l}\text { Pinus densiflora } \\
\text { Fagus crenata }\end{array}$ & $\begin{array}{l}\text { Japan } \\
\text { Japan }\end{array}$ & $\begin{array}{l}\text { Kasai et al. } 1995 \\
\text { Fukasawa et al. } 2012 \text {, } \\
\text { Tateno et al. } 2015\end{array}$ \\
\hline & & seeds & $\begin{array}{l}\text { Casuarina } \\
\text { equisetifolia }\end{array}$ & USA & Bayman et al. 1998 \\
\hline & & seeds & Eucalyptus globulus & Uruguay & Lupo et al. 2001 \\
\hline & & seeds & Phyllostachys edulis & China & Shen et al. 2014 \\
\hline & & seeds & $\begin{array}{l}\text { Platanus } \\
\text { occidentalis }\end{array}$ & USA & $\begin{array}{l}\text { Fakir et al. 1971, Mittal } \\
\text { et al. } 1990\end{array}$ \\
\hline $\begin{array}{l}\text { Xylaria terminaliae- } \\
\text { bellericae A. Pande \& } \\
\text { Waing." }\end{array}$ & $\begin{array}{l}\text { Xylariaceae, Sordariomycetes, } \\
\text { Ascomycota }\end{array}$ & fruits & Terminalia bellerica & India & $\begin{array}{l}\text { Pande \& Waingankar } \\
2004\end{array}$ \\
\hline $\begin{array}{l}\text { Xylaria terminaliae- } \\
\text { crenulatae A. Pande \& } \\
\text { Waing. }\end{array}$ & $\begin{array}{l}\text { Xylariaceae, Sordariomycetes, } \\
\text { Ascomycota }\end{array}$ & fruits & $\begin{array}{l}\text { Terminalia } \\
\text { crenulata }\end{array}$ & India & $\begin{array}{l}\text { Pande \& Waingankar } \\
2004\end{array}$ \\
\hline $\begin{array}{l}\text { Xylaria vivantii Y.M. Ju, } \\
\text { J.D. Rogers, J. Fourn. \& }\end{array}$ & $\begin{array}{l}\text { Xylariaceae, Sordariomycetes, } \\
\text { Ascomycota }\end{array}$ & fruits & $\begin{array}{l}\text { Magnolia } \\
\text { dodecapetala }\end{array}$ & French West Indies & Ju et al. 2018 \\
\hline H.M. Hsieh & & fruits & Magnolia sp. & Brazil & Ju et al. 2018 \\
\hline
\end{tabular}


Table 2 Continued.

\begin{tabular}{|c|c|c|c|c|c|}
\hline Species & Taxonomy & Substrate & Host & Country & References \\
\hline $\begin{array}{l}\text { Xylaria vivantii Y.-M. Ju, J. } \\
\text { D. Rogers, J. Fournier \& H.- } \\
\text { M. Hsieh\# }\end{array}$ & $\begin{array}{l}\text { Xylariaceae, Sordariomycetes, } \\
\text { Ascomycota }\end{array}$ & $\begin{array}{l}\text { fruits } \\
\text { fruits }\end{array}$ & $\begin{array}{l}\text { Magnolia } \\
\text { dodecapetala } \\
\text { Magnolia sp. }\end{array}$ & $\begin{array}{l}\text { French West } \\
\text { Indies } \\
\text { French West } \\
\text { Indies }\end{array}$ & $\begin{array}{l}\text { Ju et al. } 2018 \\
\text { Ju et al. } 2018\end{array}$ \\
\hline \multirow[t]{8}{*}{$\begin{array}{l}\text { Xylaria xanthinovelutina } \\
\text { (Mont.) Mont. } \\
\text { [as ‘ianthino-velutina'] }\end{array}$} & $\begin{array}{l}\text { Xylariaceae, Sordariomycetes, } \\
\text { Ascomycota }\end{array}$ & $\begin{array}{l}\text { fruit } \\
\text { fruits }\end{array}$ & $\begin{array}{l}\text { Couroupita } \\
\text { guianensis } \\
\text { Swietenia } \\
\text { macrophylla }\end{array}$ & $\begin{array}{l}\text { Brazil } \\
\text { French West Indies }\end{array}$ & $\begin{array}{l}\text { Ju et al. } 2018 \\
\text { Ju et al. } 2018\end{array}$ \\
\hline & & $\begin{array}{l}\text { fruits } \\
\text { pod }\end{array}$ & $\begin{array}{l}\text { Talauma sp. } \\
\text { Ebenopsis ebano } \\
\text { [= Pithecellobium } \\
\text { flexicaule] }\end{array}$ & $\begin{array}{l}\text { Brazil } \\
\text { Mexico }\end{array}$ & $\begin{array}{l}\text { Ju et al. } 2018 \\
\text { Trierveiler-Pereira et al. } \\
2009\end{array}$ \\
\hline & & pod & Fabaceae plant & Trinidad & $\begin{array}{l}\text { San Martín González \& } \\
\text { Rogers } 1989\end{array}$ \\
\hline & & pod & Guazuma ulmifolia & Mexico & $\begin{array}{l}\text { Trierveiler-Pereira et al. } \\
2009\end{array}$ \\
\hline & & pod & undetermined plant & $\begin{array}{l}\text { Mexico, Brazil, } \\
\text { USA, Philippines, } \\
\text { USA, Venezuela }\end{array}$ & $\begin{array}{l}\text { Rogers 1979a, San } \\
\text { Martín González \& } \\
\text { Rogers 1989, } \\
\text { Trierveiler-Pereira et al. } \\
2009\end{array}$ \\
\hline & & pods & Acacia sp. & Brazil & Ju et al. 2018 \\
\hline & & pods & Eperua sp. & Guyana & Ju et al. 2018 \\
\hline & & pods & Legume plant & $\begin{array}{l}\text { Brazil, Congo, } \\
\text { France [as French } \\
\text { Guiana], } \\
\text { Venezuela }\end{array}$ & Ju et al. 2018 \\
\hline Xylaria luzonensis Henn. & $\begin{array}{l}\text { Xylariaceae, Sordariomycetes, } \\
\text { Ascomycota }\end{array}$ & pods & $\begin{array}{l}\text { Bauhinia } \\
\text { cumingiana }\end{array}$ & Philippines & Ju et al. 2018 \\
\hline $\begin{array}{l}\text { Xylaria magnoliae var. } \\
\text { magnoliae J.D. Rogers }\end{array}$ & $\begin{array}{l}\text { Xylariaceae, Sordariomycetes, } \\
\text { Ascomycota }\end{array}$ & fruits & Magnolia sp. & USA & Rogers 1979a \\
\hline Xylaria oxyacanthae Tul. \& & Xylariaceae, Sordariomycetes, & fruits & Cornus florida & USA & Ju et al. 2018 \\
\hline C. Tul. & Ascomycota & fruits & Cornus sanguinea & France & Ju et al. 2018 \\
\hline
\end{tabular}


Table 2 Continued.

\begin{tabular}{|c|c|c|c|c|c|}
\hline Species & Taxonomy & Substrate & Host & Country & References \\
\hline & & seeds & Carpinus betulus & France & Ju et al. 2018 \\
\hline & & seeds & Carpinus sp. & France & Ju et al. 2018 \\
\hline \multirow[t]{4}{*}{ Xylaria palmicola G. Winter } & Xylariaceae, Sordariomycetes, & fruits & Patrisia sp. & Brazil & Ju et al. 2018 \\
\hline & Ascomycota & seeds & Euterpe globosa & USA & Ju et al. 2018 \\
\hline & & seeds & Euterpe sp. & Brazil & Ju et al. 2018 \\
\hline & & seeds & Palm & Brazil & Ju et al. 2018 \\
\hline \multirow[t]{3}{*}{ Xylaria warburgii Henn. } & Xylariaceae, Sordariomycetes, & fruits & Sloanea berteroana & USA & Ju et al. 2018 \\
\hline & Ascomycota & fruits & Sloanea sp. & Papua New Guinea & Ju et al. 2018 \\
\hline & & fruits & undetermined plant & Taiwan & Ju et al. 2018 \\
\hline Xylariales spp. & Sordariomycetes, Ascomycota & seed capsules & Eucalyptus grandis & South Africa & Jimu et al. 2016 \\
\hline $\begin{array}{l}\text { Xylohyphites verrucosa } \\
\text { Kalgutkar \& Sigler }^{\# * *}\end{array}$ & Fossil fungus & $\begin{array}{l}\text { permineralized } \\
\text { fruit }\end{array}$ & Viracarpon sp. & India & $\begin{array}{l}\text { Kalgutkar \& Sigler } \\
1995\end{array}$ \\
\hline $\begin{array}{l}\text { Xylosphaera carpophila } \\
\text { (Pers.) Dumort. }\end{array}$ & $\begin{array}{l}\text { Xylariaceae, Sordariomycetes, } \\
\text { Ascomycota }\end{array}$ & fruit cupules & Fagus sylvatica & UK & Carré 1964 \\
\hline Yeats & - & seeds & Pinus ponderosa & USA & $\begin{array}{l}\text { James \& Genz 1982, } \\
\text { Anderson 1986a }\end{array}$ \\
\hline $\begin{array}{l}\text { Yelsemia arthropodii J. } \\
\text { Walker }^{\#}\end{array}$ & $\begin{array}{l}\text { Melanotaeniaceae, Ustilaginomycetes, } \\
\text { Basidiomycota }\end{array}$ & $\begin{array}{l}\text { acorns } \\
\text { capsules }\end{array}$ & $\begin{array}{l}\text { Quercus robur } \\
\text { Arthropodium } \\
\text { minor }\end{array}$ & $\begin{array}{l}\text { Poland } \\
\text { Australia }\end{array}$ & $\begin{array}{l}\text { Jankowiak } 2008 \\
\text { Walker } 2001\end{array}$ \\
\hline $\begin{array}{l}\text { Yelsemia droserae R.G. } \\
\text { Shivas, Vánky \& Athip." }\end{array}$ & $\begin{array}{l}\text { Melanotaeniaceae, Ustilaginomycetes, } \\
\text { Basidiomycota }\end{array}$ & capsules & Drosera burmanni & Thailand & Vánky et al. 2006 \\
\hline $\begin{array}{l}\text { Zeloasperisporium } \\
\text { pterocarpi Jayasiri, E.B.G. } \\
\text { Jones \& K.D. Hyde }{ }^{\#}\end{array}$ & $\begin{array}{l}\text { Zeloasperisporiaceae, Dothideomycetes, } \\
\text { Ascomycota }\end{array}$ & pod & Pterocarpus sp. & Thailand & Jayasiri et al. 2018b \\
\hline \multirow[t]{5}{*}{$\begin{array}{l}\text { Zoophagus insidians } \\
\text { Sommerst. }\end{array}$} & $\begin{array}{l}\text { Zoopagaceae, Zoopagomycetes, } \\
\text { Mucoromycota }\end{array}$ & seeds & Atropa belladonna & $\begin{array}{l}\text { Poland or/ } \\
\text { imported to Poland }\end{array}$ & Czeczuga et al. 2009 \\
\hline & & seeds & Bryonia cretica & $\begin{array}{l}\text { Poland or/ } \\
\text { imported to Poland }\end{array}$ & Czeczuga et al. 2009 \\
\hline & & seeds & Canna indica & $\begin{array}{l}\text { Poland or/ } \\
\text { imported to Poland }\end{array}$ & Czeczuga et al. 2009 \\
\hline & & seeds & Ficus pumila & $\begin{array}{l}\text { Poland or/ } \\
\text { imported to Poland }\end{array}$ & Czeczuga et al. 2009 \\
\hline & & seeds & Kalmia latifolia & $\begin{array}{l}\text { Poland or/ } \\
\text { imported to Poland }\end{array}$ & Czeczuga et al. 2009 \\
\hline
\end{tabular}


Table 2 Continued.

\begin{tabular}{|c|c|c|c|c|c|}
\hline Species & Taxonomy & Substrate & Host & Country & References \\
\hline & & seeds & $\begin{array}{l}\text { Rhododendron } \\
\text { smirnowii }\end{array}$ & $\begin{array}{l}\text { Poland or/ } \\
\text { imported to Poland }\end{array}$ & Czeczuga et al. 2009 \\
\hline $\begin{array}{l}\text { Zygorhynchus heterogamus } \\
\text { (Vuill.) Vuill. }\end{array}$ & $\begin{array}{l}\text { Mucoraceae, Mucoromycetes, } \\
\text { Mucoromycota }\end{array}$ & seeds & Rosa mollis & $\begin{array}{l}\text { Poland or/ } \\
\text { imported to Poland }\end{array}$ & Czeczuga et al. 2009 \\
\hline $\begin{array}{l}\text { Zygorhynchus vuilleminii } \\
\text { Namysł. }\end{array}$ & $\begin{array}{l}\text { Mucoraceae, Mucoromycetes, } \\
\text { Mucoromycota }\end{array}$ & seeds & Pinus sylvestris & USSR & $\begin{array}{l}\text { Prisyazhnyuk } 1960 \text {, } \\
\text { Mittal et al. } 1990\end{array}$ \\
\hline $\begin{array}{l}\text { Zygosporium gibbum (Sacc., } \\
\text { M. Rousseau \& E. Bommer) }\end{array}$ & $\begin{array}{l}\text { Zygosporiaceae, Sordariomycetes, } \\
\text { Ascomycota }\end{array}$ & fruits & $\begin{array}{l}\text { Cleistocalyx } \\
\text { operculatus }\end{array}$ & Hong Kong & Tang et al. 2003a \\
\hline \multirow[t]{4}{*}{ S. Hughes } & & fruits & Ilex pubescens & Hong Kong & Tang et al. 2003a \\
\hline & & fruits & Litsea rotundifolia & Hong Kong & Tang et al. 2003a \\
\hline & & fruits & Psychotria asiatica & Hong Kong & Tang et al. 2003a \\
\hline & & fruits & $\begin{array}{l}\text { Rhodomyrtus } \\
\text { tomentosa }\end{array}$ & Hong Kong & Tang et al. 2003a \\
\hline
\end{tabular}

\section{Discussion}

\section{Diversity of fungi on wild seeds and fruits}

In this paper we review the fungal taxa described from wild fruit substrates until December 2019 (Table 2). Currently 300 new fungal species have been described from wild seeds/fruit substrates, from 54 countries (Index Fungorum 2020). Fungi reported from wild seeds and fruits belong to 609 fungal genera of different fungal families and included 28 novel genera (Table 2). Ascomycota and Basidiomycota are the major fungal phyla reported from wild seeds and fruits with significantly fewer Mucoromycota, Chytridiomycota, and Blastocladiomycota reported (Fig. 58). The highest number of fungi on wild seed/fruit substrates, belong to Ascomycota with 82\% reported (Fig. 58). The dominant genera include: Alternaria, Aspergillus, Candida, Chaetomium, Cladosporium, Colletotrichum, Curvularia, Diaporthe, Drechslera, Fusarium, Mucor, Penicillium, Pestalotiopsis, Restiosporium, Rhizopus, Talaromyces, Trichoderma and Xylaria. (Fig. 60). Most were recorded as asexual morphic taxa, with fewer sexual morphs. Wild seed and fruit fungi belong to 27 fungal classes (Fig. 59). The number of Sordariomycetes taxa (30\%) and Dothideomycetes taxa (26\%) associated with forest seeds and fruits are significantly higher than other classes (Fig. 59). Lichenization is one of the major lifestyles among fungi, and are classified in the Ascomycota and Basidiomycota (Lücking et al. 2017). There are three novel lichen fungi, Cladonia lacryma, Lecanora shangrilaensis and Lecanora substrobilina, described from wild seed/fruit substrates (Table 2).

Forest tree seeds have vast diversity in their size, shape and texture (Sutherland et al. 2002). The size and texture of tree seeds ranging from small and hard to the relatively large and fleshy (Sutherland et al. 2002) may influence the fungal diversity. The highest number of new wild seed/fruit fungi are from Thailand (18\%), while Australia, China, USA and India also have significant numbers of novel species (Fig. 62). Fruit diversity in tropical regions is higher than in temperate regions which may lead to an increase in the availability and variety of resources or substrates for fungal 
colonisation (Hyde et al. 2005). Mittal et al. (1990) indicated that the climate conditions in the tropics also facilitate the development of seed-borne fungi in the region. Most of the new fungi have been described from wild fruits and coniferous cones (246 taxa), when compared to seeds (54 taxa).

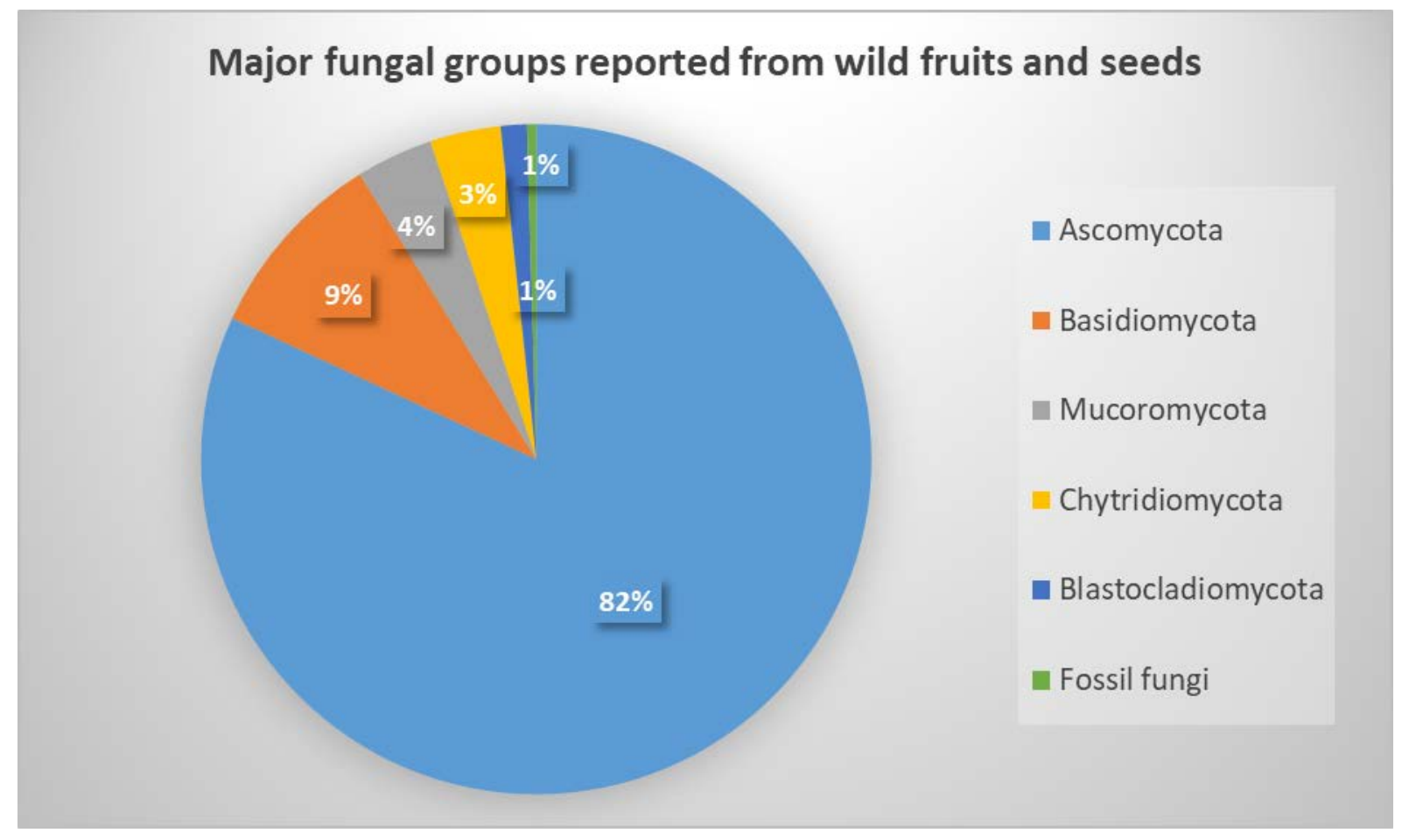

Figure 58 - Percentage of species belonging to major fungal phyla reported from wild seeds and fruits.

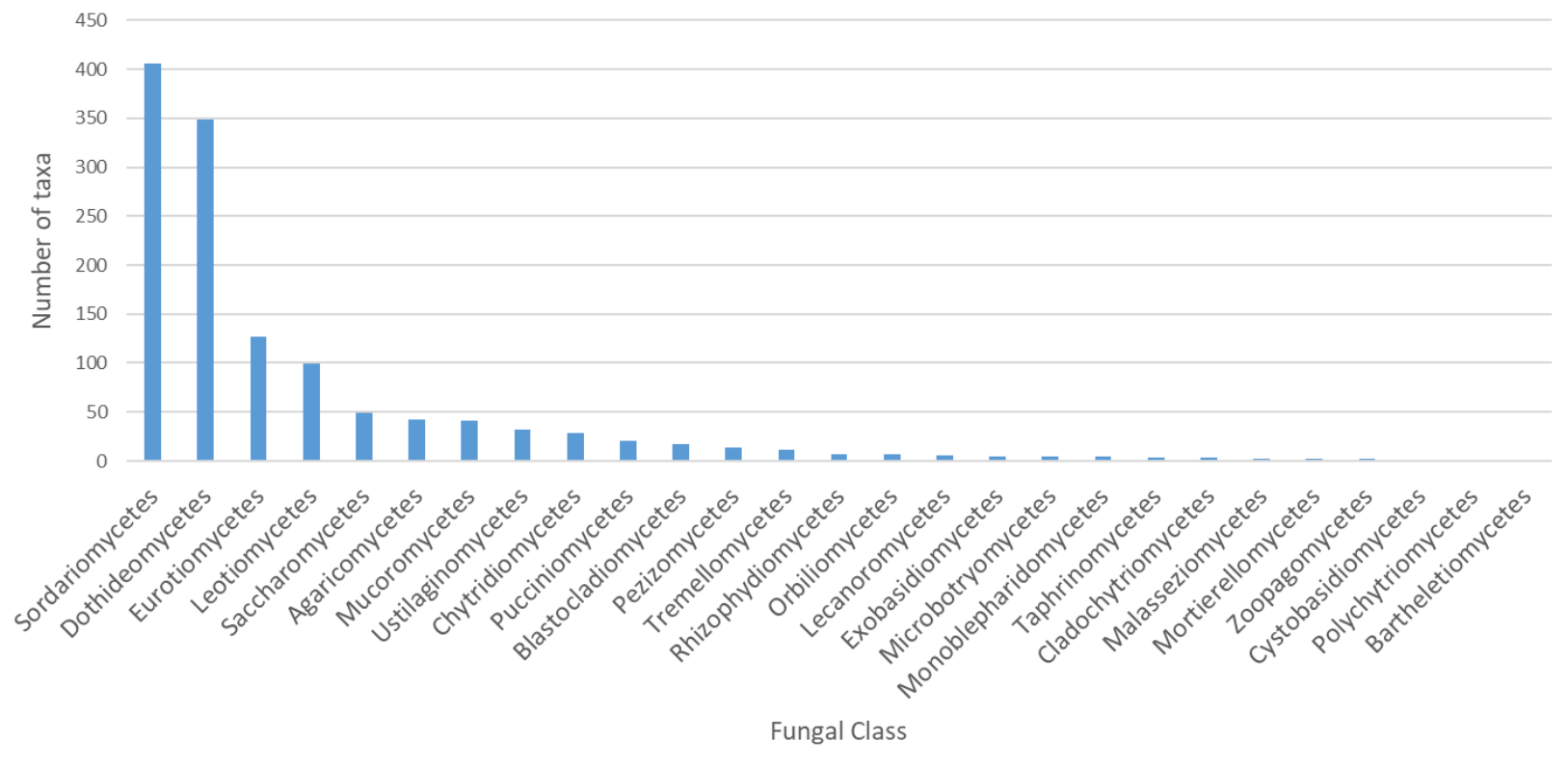

Figure 59 - Number of wild seeds/fruit inhabiting fungi belonging to different fungal classes.

Wild seed and fruit fungi have been described from 80 plant families, although most were collected from the legume family, Fabaceae (Fig. 63, Table 2). Fabaceae is the third largest 
flowering plant family with worldwide distribution (Gepts et al. 2005). Pinaceae, Fagaceae, Restionaceae, and Poaceae are the other plant host families from where the novel wild seed/fruit fungi were mostly described (Fig. 63).

Fusarium species isolated from wild fruits in our study are mainly belonging to Fusarium lateritium species complex (Fig. 24). Species of this complex prefer seed and fruit substrates. They are commonly reported from fruits of commercially important plants, for example, Fusarium stilboides associated with fruits of banana, bell pepper (Capsicum annum) and Coffea arabica (Geiser et al. 2005, Sandoval-Chávez et al. 2011, Zeng et al. 2013), while Fusarium lateritium was isolated from fruits of banana, Coffea arabica, dragon plant (Cereus undatus) and hazelnut (Corylus avellana) (Abo-El-Dahab \& El-Goorani 1969, To et al. 1999, Geiser et al. 2005, Vitale et al. 2011). Fusarium lateritium, F. sarcochroum and F. stilboides are also reported from seeds of various forest plants and acorns of Quercus robur (Table 2).

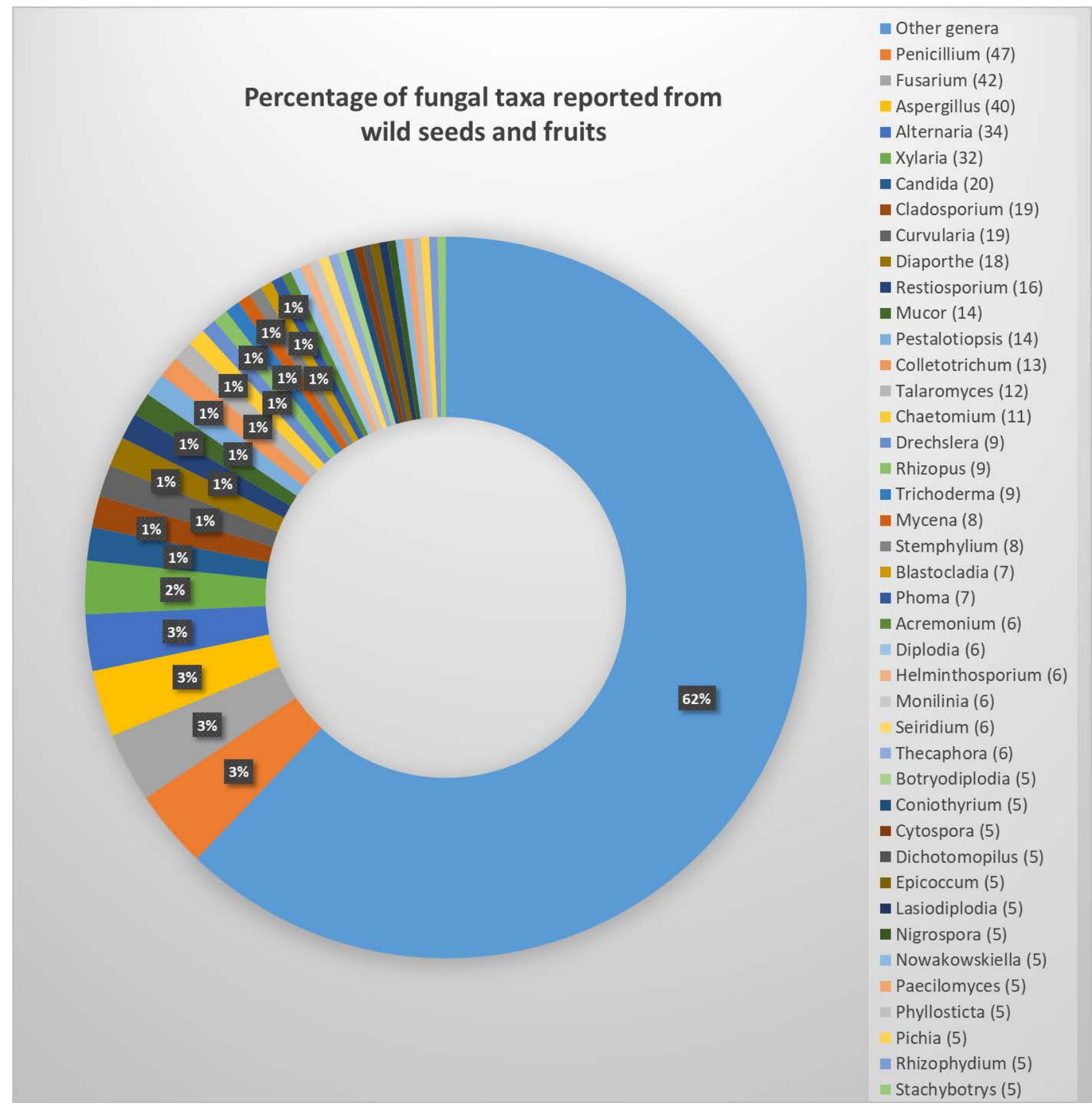

Figure 60 - Percentage of fungal species in different genera reported from wild seeds and fruits (number of species reported for each genus are given in brackets). 


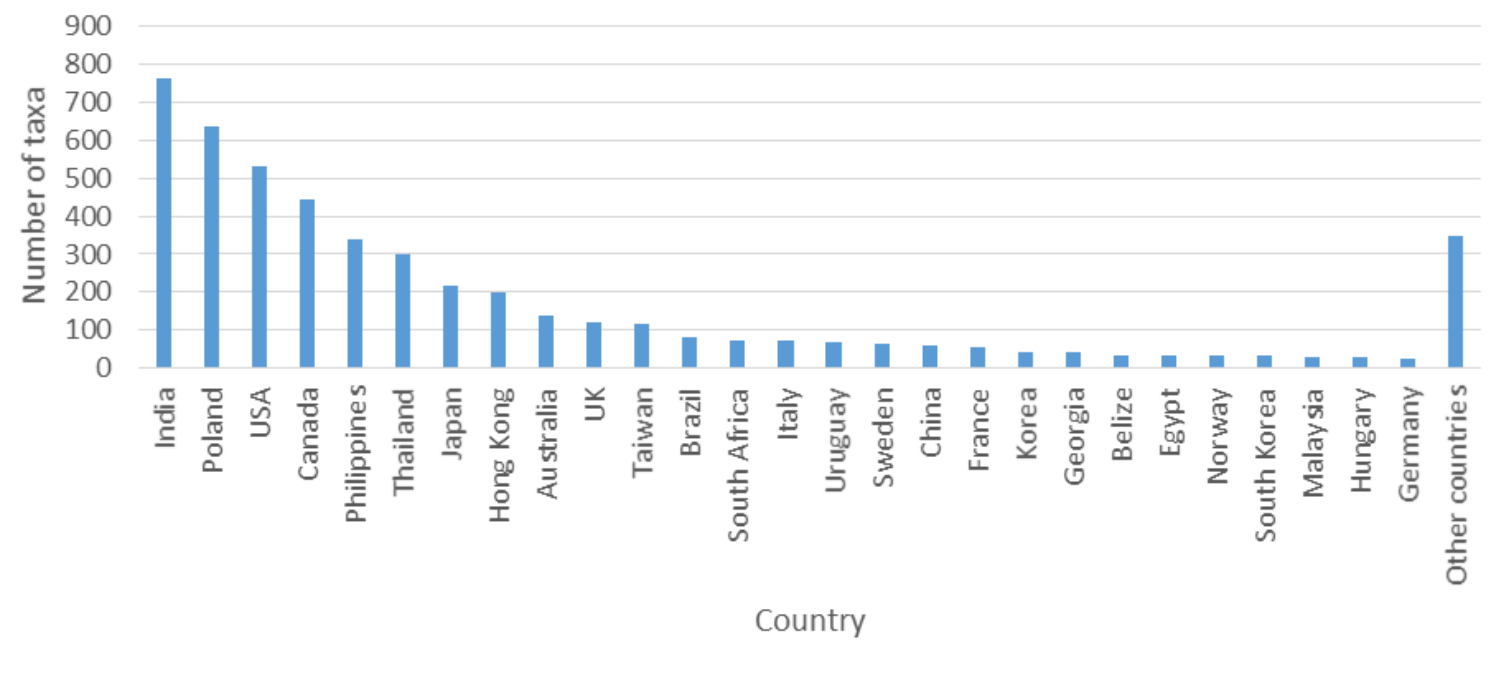

Figure 61 - Number of wild seeds/fruit inhabiting fungi reported from different countries.

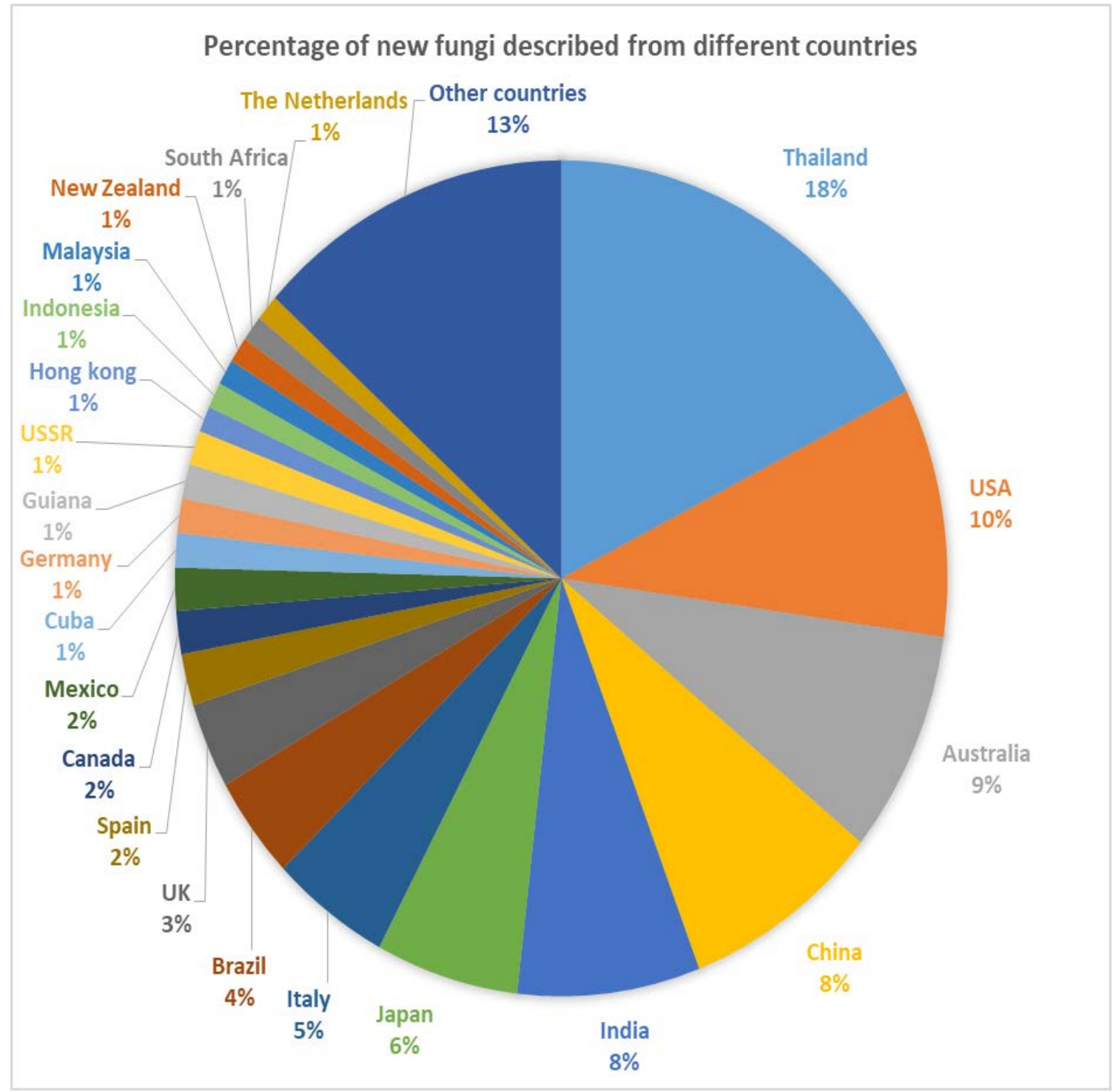

Figure 62 - Percentage of new seed and fruit fungi described from different countries. 


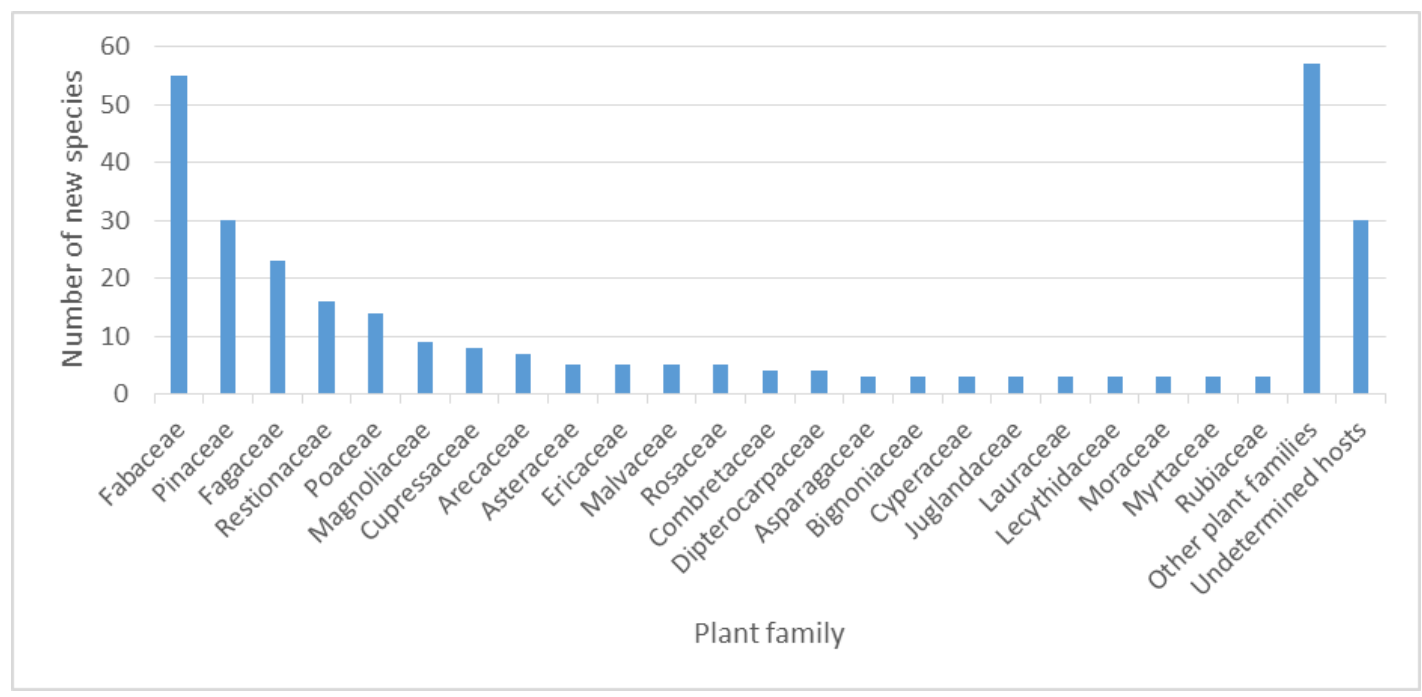

Figure 63 - Number of new wild seed and fruit fungi reported from different plant host families.

\section{Basidiomycota}

The second largest group of fungi reported from wild seeds and fruits is Basidiomycota (9\%), including 65 new species (Fig. 58). This group includes several classes such as Agaricomycetes, Cystobasidiomycetes, Exobasidiomycetes, Microbotryomycetes, Pucciniomycetes and Ustilaginomycetes.

\section{Discomycetes}

Discomycetes are a group of apothecia-producing fungi, having operculate or inoperculate asci, in the phylum Ascomycota (Ekanayaka et al. 2017). There were 20 discomycete species newly introduced from wild seeds and fruits (Table 2).

\section{Rust fungi}

Rust fungi (Pucciniales, Pucciniomycetes) are biotrophic pathogenic fungi with a narrow host range (Niks 1987). They are less likely to infect seeds/fruits however, they can also be found from fruits (Walker \& van der Merwe 2009, Berndt 2011). Aecidium acanthocarpi and Endoraecium kauaianum were described from fruits of Acanthocarpus verticillatus and Acacia koa, respectively (Walker \& van der Merwe 2009, Berndt 2011). We list four rust fungi described from wild seeds/fruits substrates including the above two species (Table 2).

\section{Smut fungi}

Many smuts produce spores in seeds (Table 2). We list 36 new smut fungi reported from wild seed and fruits worldwide (Table 2). They belong in Urocystidales, Ustilaginales (Ustilaginomycetes), Microbotryales (Microbotryomycetes) and Tilletiales (Exobasidiomycetes) (Table 2). Centrolepidosporium was described from fruits of Centrolepis exserta in Australia (Shivas \& Vánky 2007). Restionaceae plants are the most common hosts for many seed/fruit inhabiting smut fungi, while others were found on Fabaceae, Cyperaceae, Poaceae, Araliaceae, Amaranthaceae, Primulaceae, Hypoxidaceae, Asparagaceae, Asteraceae, Droseraceae, Primulaceae and Montiaceae hosts (Fig. 60).

\section{Clavicipitaceous fungi}

Five new clavicipitaceous parasites have been described from seeds and fruits since 1981 and, three (Claviceps bothriochloae, C. microspora and C. panicoidearum) were from noncommercial grass seeds collected in Japan (Tanda 1985, 1991, Tanda \& Harada 1989). Generally, clavicipitaceous species have a narrow host range (Rodriguez et al. 2009). 


\section{Yeasts}

We listed 13 new yeast species belonging to 8 genera namely Candida, Cyberlindnera, Hanseniaspora, Hyphopichia, Kuraishia, Metschnikowia, Saccharomycopsis and Wickerhamiella described from wild seeds and fruits (Table 2). The succession of yeast species colonizing the fallen ripe fruit of amapa (Parahancornia amapa) and Clusia grandiflora in tropical forests was examined by Morais et al. (1995). Fruits with sugary, soft, fleshy inner layer are prone to yeast colonisation during the first few days of fruit fall (Miller \& Phaff 1962, Morais et al. 1995).

\section{Host-specificity}

It has been reported that xylariaceous taxa have specific and distinct relationships with their hosts (Rogers 1979b, 2000, Laessøe \& Lodge 1994, Ju et al. 2018). Some of the seed/fruit associated species of Xylaria appear to be host-specific, as they are restricted to one host species, genus or family (Husbands et al. 2018, Ju et al. 2018). Xylaria carpophila occurs only on Fagus sylvatica; X. karyophthora on Chlorocardium; X. liquidambar on Liquidambar; X. magnoliae var. magnolia and var. microspora on Magnolia, and X. warburgii on Sloanea (Rogers et al. 1992, 2002, Husbands et al. 2018, Ju et al. 2018). Xylaria palmicola and X. rhizocola are probably associated only with palm seeds (Ju et al. 2018). In contrast, Ju et al. (2018) collected $X$. oxyacanthae from fruits/seeds of plants in various families, such as Carpinus (Betulaceae), Carya (Juglandaceae), and Cornus (Cornaceae), Crataegus (Rosaceae), and Prunus (Rosaceae). However, whether seed and fruit fungi are host-specific is doubtful for most species associated with seeds and fruits, especially if they have been collected only once or twice (Ju et al. 2018). There are other species such as $X$. clusiae, $X$. duranii, and $X$. heloidea not only grow on fallen fruits or seeds, but also can be found on fallen leaves as well (Ju et al. 2018). Xylaria xanthinovelutina $(=X$. ianthinovelutina), probably the most commonly found fruit inhabiting Xylaria species, usually occurs on woody pods of legumes but has also been found on dead woody substrates (Martín 1970, Ju et al. 2018). Clavicipitaceous grass fungi are known to be specific at the family level (Petrini 1996, Zhou \& Hyde 2001). Most Epichloë and Neotyphodium species are host-specific and show symbiotic relationships with C3 grasses (Bacon \& White 2000).

\section{Production of bioactive compounds}

Shen et al. (2014) revealed that some wild seed fungi can be potential sources of bioactive compounds and plant defense activators. They screened 49 endophytic fungal strains from seeds of moso bamboo (Phyllostachys edulis) in China for production of bioactive substances and identified Cladosporium sp., Curvularia sp., undefined fungal genus 1, Penicillium sp. and Shiraia sp. with antimicrobial activity against clinical bacteria and yeasts. Shiraia sp. produced hypocrellin which has promising antileishmanial properties (Ma et al. 2004, Shen et al. 2014).

\section{Decomposition of wild seeds and fruits and fungal succession}

Decomposition and nutrient cycling play a crucial role in the balance of ecosystems. Saprobic fungi are vital for decomposition and nutrient cycling processes on the forest floor (Somrithipol et al. 2002a, Bucher et al. 2004, Fukasawa et al. 2012). Fungi secrete extracellular enzymes and play an important role in decomposition of lignocellulose substrates in forest ecosystems (Pointing et al. 2005, Fukasawa et al. 2012). In forest ecosystems the wild seed/fruit litter (especially the woody seed covering organs) is quantitatively important, since it contributes to total carbon in forest soils (Fukasawa et al. 2012). Mast-seeding (masting), the periodic synchronous production of seed crops by a plant population (Kelly 1994), can be observed in many forest tree species (Suzuki et al. 2005) (Fig. 60). Mast seeding of Fagus crenata (Japanese beech) occurs every 5-7 years (Hiroki \& Matsubara 1995, Suzuki et al. 2005, Fukasawa et al. 2012). Cupules are the second largest component of beech litter and comprises up to $28 \%$ of total annual litter fall in mast-seeding years (Kawada \& Maruyama 1986). Pine cones are one component of coniferous litter (Kasai et al. 1995). A study conducted in a coniferous forest (giant sequoia, white fir and sugar pine) in USA revealed $4-10 \%$ of the total litter fall was cones (Stohlgren 1988). 
Nutrient rich substrates (cereals, fruits, berries) are usually colonised by rapidly growing asexual filamentous fungi such as Colletotrichum spp., Fusarium spp., Diaporthe spp. (Marasas et al. 1998, Somrithipol et al. 2002b, Torp \& Nirenberg 2004, Prihastuti et al. 2009, Weir et al. 2012, Udayanga et al. 2013, Crous et al. 2015b). Woody substrates such as cupules of beech, legume pods, cones and woody seeds are rich in lignin and celluloses and provide substrates for saprobic fungi (eg. Xylaria carpophila, Pseudohalonectria fagicola, P. hampshirensis on cupules of Fagus spp.; Thozetella nivea on seed pods of Delonix regia; Lecanora substrobilina on cones of Pinus; Xylaria magnoliae on magnolia fruits (Rogers 1979a, Rogers et al. 1992, Printzen 2001, Somrithipol et al. 2002b, Fukasawa et al. 2012, Perera et al. 2016a). Furthermore, all Helotiales sexual morphic taxa collected from wild fruits/seeds are reported from woody substrates (Table 2).

The fungal assemblages associated with seeds/fruits, in different stages of decomposition also vary (Somrithipol et al. 2002b, Fukasawa et al. 2012). Fungi with different decay abilities successively colonise fruits/seeds and therefore enhance the decay process and is time dependent (Rayner \& Boddy 1988, Somrithipol et al. 2002b, Fukasawa et al. 2012). Loss of weight as fruits/seeds are decomposed can be observed during the fungal succession process (Somrithipol et al. 2002b, Fukasawa et al. 2012). Few fungal succession studies have focused on seeds and fruits (Carré 1964, Kuthubutheen 1984, Somrithipol et al. 2002b, Fukasawa et al. 2012). Fukasawa et al. (2012) studied litter decomposition patterns by fungal colonisers on beech cupules, while Somrithipol et al. (2002b) reported succession on seed pods of Delonix regia.

Fukasawa et al. (2012) found that the major and early stage holocellulose decomposers in beech cupule litter are Xylaria spp. Xylariaceous taxa are recognised as endophytes of live beech plants (Chapela 1989, Sahashi et al. 1999, Osono \& Mori 2003) including cupules (Fukasawa et al. 2012). Following the selective holocellulose decay process, acid-unhydrolyzable residues are decomposed by Basidiomycetes that replaced Xylaria spp. (Fukasawa et al. 2012). In contrast, fallen fruits of Delonix regia were dominated by litter fungi such as Dictyochaeta, Helicosporium, Phaeoisaria, Phoma and Sporoschisma species (Somrithipol et al. 2002b). Surprisingly, Aspergillus, Chaetomium, Penicillium, and Rhizopus species, were the main groups of fungi on D. regia seed pods when samples were collected directly from the tree (with no contact with soil). Similar fungi (Aspergillus niger, Aspergillus flavus, Penicillium sp., and Rhizopus sp.) were associated with decaying Artocarpus communis (breadfruit) kept in storage (Amusa et al. 2002). Kasai et al. (1995) observed that Xylaria sp. and Phomopsis sp. are frequently associated with Pinus densiflora cones when attached to the tree. Conversely Mortierella spp. and Trichoderma spp. were the early stage decomposers of cones on the ground (Kasai et al. 1995).

Resin acids, an important group of extractives found in conifers, were identified in seed cones of Picea glauca and Pinus spp. (Hafizoglu \& Holmbom 1987, Eberhardt et al. 1994). Some of these resin acids can inhibit fungal decay of seed cones of Pinus spp. by imparting water repellency or by inhibiting white rot-fungi (Eberhardt et al. 1994). Takahashi et al. (2010) studied the effects of tannin (polyphenolic compounds) content in Quercus serrata acorns, on the pathogenic fungus Ciboria batschiana. They revealed acorns with higher tannin contents were more resistant to Ciboria batschiana infection (Takahashi et al. 2010). Collectively, the determining factors for the fungal community after death of fallen seed/fruit tissues include the size of the tissue, nutrients, secondary metabolites (antimicrobial compounds such as resin acids, polyphenols), contents of the tissues, moisture content, existing endophytic fungi, and also the life cycles of fungal decomposers (Rayner \& Boddy 1988, Somrithipol et al. 2002b, Tang et al. 2003a, Fukasawa et al. 2012).

Sarmiento et al. (2017) mentioned that communities of fallen seed-associated fungi are structured predominantly by host plant species other than by soil type, forest characteristics, or time in the soil. Carré (1964) indicated that the occurrence pattern of fruiting bodies of fungi on fallen fruits was mostly affected by the weather rather than accumulation and availability of food materials. However, the pattern of occurrence of fruiting bodies was not considered as a proper index for fungal succession in internal tissues (Rayner \& Boddy 1988, Fukasawa et al. 2012). Osono (2011) observed that decomposition rate of fungal species was higher in warmer climates than in cooler locations. Tang et al. (2003a) indicated that the surface area of the fruit does not 
significantly correlate with the number of fungal species. Tang et al. (2005) also observed less diversity and different groups of fungi occurring on persistent fruits of Ardisia spp., Dichroa febrifuga, Sarcandra glabra, and Wikstroemia nutans that cling to the branches and do not drop, when compared to non-persistent fruits.

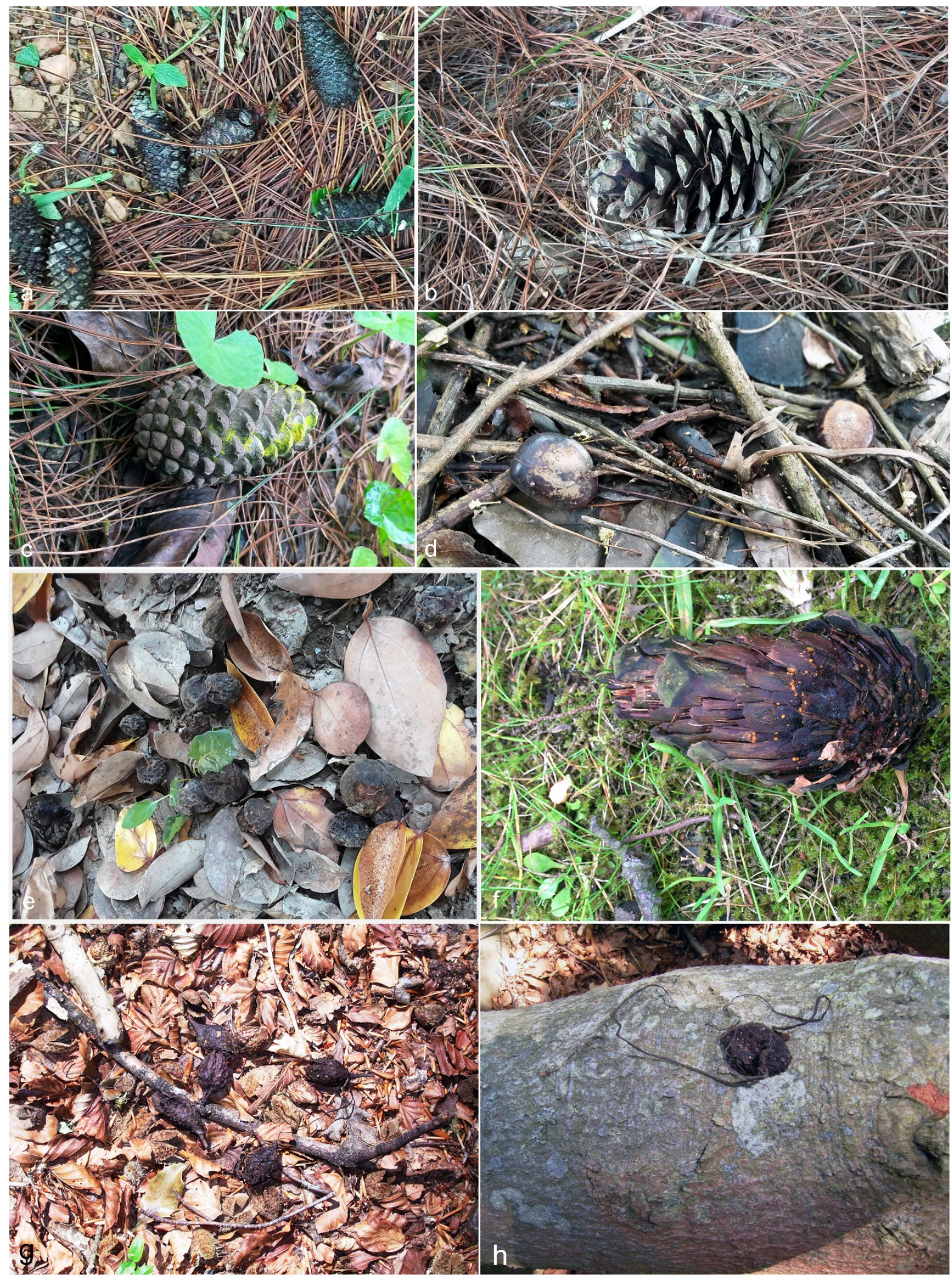

Figure 62 - Fruits on the forest floor (a, b in Sri Lanka, c, d in Thailand, e Taiwan, f-h UK). a-c Pinus spp. d, e Unidentified fruits. f Fir cone with a bright coloured discomycete. g, h Fagus sylvatica (h. with a Xylaria sp.). 

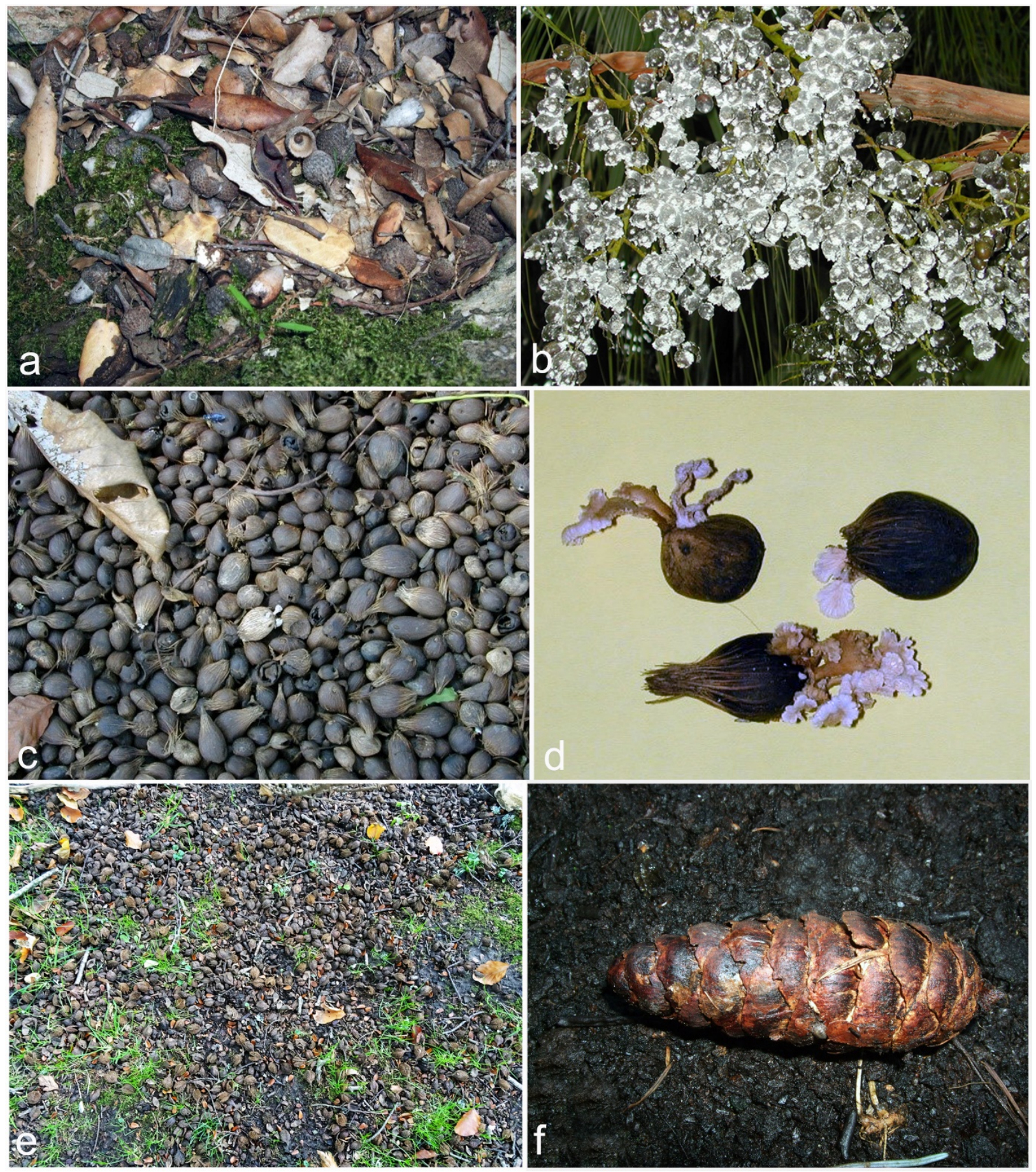

Figure 63 - Fruits on the forest floor (a, b, e, f in UK, c, d in Singapore). a Quercus fruits on forest floor. b Fruits infect by a fungus while still on the palm tree. c Fallen Terminalia catappa (sea alomond) fruits on forest floor colonised by Schizophyllum commune. d Close up of Schizophyllum commune colonising Terminalia catappa fruits. e Fagus sylvatica (beech) cupules and seeds on forest floor. f Pseudotsuga menziesii cone.

A study of succession of yeast species colonizing Parahancornia amapa fruits found that the common fruit-associated genera Kloeckera and Hanseniaspora spp., Candida guilliermondii, and Candida krusei colonised fruits during the first three days after fruit fall (Morais et al. 1995). They also reported that the succession of yeasts species on fallen fruits is determined by both competitive interactions of yeast species (such as production of killer toxins) and the selective dispersion of yeast species by vectors such as Drosophila spp. 


\section{Effects of fungi on seed germination and survival of forest plants}

Biological factors causing mortality of buried seeds in natural forest communities are not widely understood (Blaney \& Kotanen 2001). Few field experiments have addressed this topic although fungi are believed to be most responsible cause of buried seed mortality (Kremer 1993, Blaney \& Kotanen 2001). Soil fungi such as Fusarium spp., Lasiodiplodia spp. and Trichoderma spp. infect forest seeds (Gallery et al. 2007, Sarmiento et al. 2017). Knowledge of host-specific nature of fungi that infect seeds of tropical trees is limited (Gallery et al. 2007, Sarmiento et al. 2017).

Different assemblages of fungi usually colonise pine cone scales and seeds on the forest floor (Lilja et al. 1995, Vujanovic et al. 2000). Anderson et al. (1984) reported that some of these fungi destroy embryos and gametophyte tissues of the pine seeds, kill seeds, or damage cones. This damage happens in both mature and premature cones in natural forests and as well as cone collections in storage conditions (Fraedrich \& Miller 1995, Vujanovic et al. 2000). Sphaeropsis sapinea often infects cones and seeds, and is considered one of the most destructive pathogens of Pinus causing die-back and canker diseases (Smith et al. 1996, Vujanovic et al. 2000). Cram \& Fraedrich (2010) listed several seed-borne pathogens of coniferous forest trees in North America. Caloscypha fulgens, Fusarium circinatum, F. oxysporum, F. moniliforme var. moniliforme, F. proliferatum, Lasiodiplodia theobromae, Sirococcus conigenus, Diplodia pinea and Trichothecium roseum are reported as seed-borne pathogens of Abies grandis, Picea spp., Pinus spp., Pseudotsuga menziesii, Tsuga heterophylla and are responsible for seed diseases, cotyledon blight, damping-off, shoot dieback, cankers and root rot of coniferous plants in North America (Fraedrich et al. 1994, Cram \& Fraedrich 2010).

Pathogenic fungi damage fruits/cones, infect seeds, destroy internal tissues, endosperm and the embryo leading to reduction in storage lifespan, seed vigour and seedling germination in forests and nurseries (Anderson et al. 1984, Cram \& Fraedrich 2010). Moreover, some fungi can weaken and make seeds susceptible to a number of soil-borne pathogens (Mamatha et al. 2000, Gure 2004). Mittal \& Wang (1987) indicated that saprobic fungi do not affect seed viability or vigor as they colonise dead plant material. However, saprobic fungi also can behave as seed pathogens under some conditions, following seed or seed coat injuries, favorable moisture and temperature conditions in seeds or fruits/cones (Singh \& Mittal 1989, Gure 2004). These fungi can impact on the reproduction of forest trees and potentially contribute to maintaining diversity in terrestrial communities (Sarmiento et al. 2017). In vitro experiments found that most of the fungi associated with Quercus seeds inhibited their germination (Sahai \& Otra 1982). The incubation time period of fungal culture filtrates and different fungal strains have different inhibitory effects on seed germination (Armolik \& Dickson 1956, Sahai \& Otra 1982). Sahai \& Otra (1982) attributed that to the amount of toxin production by fungi in different incubation stages.

Some seed-inhabiting fungi have a positive effect on seed germination. Mittal \& Wang (1993) and Gure et al. (2005b) observed a significant increase in germination in seeds of Pinus strobus by Alternaria alternata, Cladosporium cladosporioides, Epicoccum purpurascens and Mucor hiemalis; and seeds of Podocarpus falcatus by Diaporthe sp.

\section{Seed/fruit fungi dispersal and quarantine aspects}

Fruits play an important role in seed dispersal (Tang et al. 2003a). Various agents are involved in the dispersal of seeds and fruits, such as animals, wind, water, self-dispersal and creeping diaspores (creeping diaspores occur with variation in humidity) (Howe \& Smallwood 1982). Dispersal of seeds/fruits also brings about dispersal of their inhabiting microbes. Infected plant seeds might introduce plant diseases to a new area by long distance dispersal of pathogens (Gure 2014).

Unintentional or deliberate introduction of fungi through human activities leads to extensive spread of plant diseases, sometimes causing epidemics (Blaney \& Kotanen 2001, Palm 2001, Wingfield et al. 2001, Desprez-Loustau 2009). Many plant diseases often emerge during the initial stages of establishment of exotic species (Palm 2001, Wingfield et al. 2001, Desprez-Loustau 
2009). Fungal pathogens are most likely introduced with planting stock, seeds, or cones (Wingfield et al. 2001). Alien fungal invasions through seeds is poorly researched, hence the need for greater future attention (Palm 2001, Wingfield et al. 2001, Desprez-Loustau 2009). Repeated introductions of Sphaeropsis sapinea infected seeds of different pine species from a wide range of countries, apparently explains the high genetic diversity of this fungus in South Africa (Smith et al. 2000, Wingfield et al. 2001, Burgess \& Wingfield 2002). Exotic Pinus species were introduced to New Zealand with the implementation of afforestation projects at the end of the $19^{\text {th }}$ Century (Burgess \& Wingfield 2002). However, comparatively low genetic diversity of Sphaeropsis sapinea was observed in New Zealand as they reduced the import of pine seeds after the local seeds became abundant (Wingfield et al. 2001). The quantity of imported pine seeds to South Africa was higher and it occurred repeatedly over a longer period of time leading to a higher genotypic diversity of Sphaeropsis sapinea (Burgess \& Wingfield 2002). Western Australia also imported pine seeds for plantations mostly from other Australian states and New Zealand. Very low genotypic diversity of Sphaeropsis sapinea in South and Western Australia was observed due to a small amount of exotic pine seeds importation over a shorter period (Burgess \& Wingfield 2002). Wernham (1942) reported an incident of introduction of Epichloë typhina infected seeds of Festuca rubra from Hungary to Pennsylvania, USA. Dogwood anthracnose caused by Discula destructiva may have entered USA through imported seeds of kousa dogwood (Cornus kousa) from Asia (native region) (Palm 2001). Pitch canker disease of wild pines (Pinus radiata) caused by the seed-borne fungus Fusarium circinatum was first discovered in California in 1986 (Dick 1998, Gordon 2006). Infected pinus forestry seeds imported from California are one of the pathways the disease was introduced into North America, New Zealand and many other countries (Dick 1998, Gordon 2006). The seed-borne fungus Fusarium subglutinans f. sp. pini, the causative agent of pine pitch canker disease (Dick \& Dobbie 2002), has the potential of becoming a serious threat to Pinus radiata plantations if introduced to New Zealand (Storer et al.1998, Dick \& Dobbie 2002).

Transmission of diseases through international transport of seeds is of concern and preventive measures are needed (Neergaard 1977). Hence, quarantine regulations have been introduced by governments to control or prevent the spread of pests and diseases entering through its borders via seeds (Tanner 1997, Wingfield et al. 2001, Sharma \& Thakur 2007). The risk of introducing harmful pathogens through transport of seeds, and the need of take action to overcome these risks must be recognised (Cleary et al. 2013). Even though governments have introduced quarantine regulations, they need the support of the public and industry to fulfill their aims (Wingfield et al. 2001, Cleary et al. 2013). Morphological and molecular identification of wild seed/fruit fungi and knowledge of the fungal biodiversity will lead to better assessment of risks associated with seed transport and plant diseases. Such databases, as provided in this paper, will be advantageous for effective quarantine decisions and policies in trading agricultural commodities between countries, and in order to safeguard plant resources (Palm 2001, Burgess \& Wingfield 2002).

\section{Acknowledgements}

The Research of Featured Microbial Resources and Diversity Investigation in Southwest Karst area (project no. 2014FY120100) is thanked for financial support. This work was also supported by the Thailand Research Fund, 'The future of specialist fungi in a changing climate: baseline data for generalist and specialist fungi associated with ants, Rhododendron species and Dracaena species' (grant no. DBG6080013). Kevin D. Hyde thanks the Foreign Experts Bureau of Yunnan Province, Foreign Talents Program (2018; grant no. YNZ2018002), the 2019 high-end foreign expert introduction plan to Kunming Institute of Botany (granted by the Ministry of Science and Technology of the People's Republic of China, grant number G20190139006) and, a Visiting Professorship at Chiang Mai University, Thailand. E. B. Gareth Jones acknowledges the award of a Distinguished Scientist Fellowship Program (DSFP), King Saud University, Kingdom of Saudi Arabia and a Visiting Professorship at Chiang Mai University, Thailand. Rekhani H. Perera offers her gratitude to Samantha C. Karunarathne, Rungtiwa Phookamsak and Jianchu Xu for financial support on molecular work under the National Science Foundation of China (NSFC) project no: 
31750110478, The Research Fund from China Postdoctoral Science Foundation (grant no. Y71B283261) and Key Research Program of Frontier Sciences, CAS (grant no. QYZDY-SSWSMC014). H. B. Lee acknowledges the BK21 plus program through the National Research Foundation (NRF) funded by the Ministry of Education of Korea. We are also immensely grateful to Paul Kirk for providing fungal lists of Index Fungorum. Rekhani H. Perera would like to thank Shaun Pennycook for his essential nomenclatural review. Kevin Becker is thanked for the HPLC analysis of the Hypoxylon species. Dhanushka N. Wanasinghe, Chamath Dinuka, Vinodhini Thiyagaraja, Wei DP, Danushka S. Tennakoon, Anuruddha Karunarathna, Ishani Goonasekara, Anupama Daranagama and Diana Sandamali are thanked for their support.

\section{References}

Abdollahzadeh J, Javadi A, Zare R, Phillips AJL. 2014 - A phylogenetic study of Dothiorella and Spencermartinsia species associated with woody plants in Iran, New Zealand, Portugal and Spain. Persoonia 32, 1-12.

Abdullah SK, Descals E, Webster J. 1981 - Teleomorphs of three aquatic hyphomycetes. Transactions of the British Mycological Society 77, 475-483.

Abo-El-Dahab MK, El-Goorani MA. 1969 - A Fusarium fruit rot of banana. Phytopathologia Mediterranea 1, 83-86.

Agmata AL. 1979 - Seed-borne organisms in some forest tree seeds in the Philippines: a preliminary survey. Sylvatrop; The Philippine Forest Research Journal 4, 215-222.

Akhundov TM, Papusha YZ. 1981 - Pestalotia parrotiae Achund., pathogen of seed disease of the Persian ironwood tree [Parrotia persica]. Mikologiia I Fitopatologiia 15, 463-467.

Amusa NA, Kehinde IA, Ashaye OA. 2002 - Bio-deterioration of breadfruit (Artocarpus communis) in storage and its effects on the nutrient composition. African Journal of Biotechnology 1, 57-60.

Anderson RL, Belcher E, Miller T. 1984 - Occurrence of seed fungi inside slach pine seeds produced in seed orchards in the United States. Seed Science and Technology 42, 795-799.

Anderson RL. 1986a - Checklist of micro-organisms associated with tree seeds in the world, 1985. General Technical Report, Southeastern Forest Experiment Station, USDA Forest Service.

Anderson RL. 1986b - New method for assessing contamination of slash and loblolly pine seeds by Fusarium moniliforme var. subglutinans. Plant Disease 70, 452-453.

Anita DD, Sridhar KR, Bhat R. 2009 - Diversity of fungi associated with mangrove legume Sesbania bispinosa (Jacq.) W. Wight (Fabaceae). Livestock Research for Rural Development 21, 1-5.

Anita DD, Sridhar KR. 2009 - Assemblage and diversity of fungi associated with mangrove wild legume Canavalia cathartica. Tropical and subtropical agroecosystems 10, 225-235.

Anju K, Sharma SK, Rao VM. 2012 - Microflora associated with the seeds of Casuarina equisetifolia. Asian Journal of Biochemical and Pharmaceutical Research 2, 101-104.

Antonín V, Ryoo R, Ka KH. 2014 - Marasmioid and gymnopoid fungi of the Republic of Korea. 7. Gymnopus sect. Androsacei. Mycological Progress 13, 703-718.

Arambarri AM, Cabello M, Mengascini A. 1987 - Estudio sistematico de los hyphomycetes del Rio Santiago (Prov. Buenos Aires, Argentina). Darwiniana 28, 293-301.

Aravindakshan DM, Kumar TKA, Manimohan P. 2012 - A new bioluminescent species of Mycena from Kerala State, India. Mycosphere 3, 556-561.

Ariyawansa HA, Hyde KD, Jayasiri SC, Buyck B et al. 2015 - Fungal diversity notes 111-252 taxonomic and phylogenetic contributions to fungal taxa. Fungal Diversity 75, 27-274.

Armolik N, Dickson JG. 1956 - Minimum humidity requirement for germination of conidia of fungi associated with storage of grain. Phytopathology. 46, 462-465.

Arthur JC, Kern FD. 1906 - North American species of Peridermium. Bulletin of the Torrey Botanical Club 33, 403-438. 
Ashaeer F. 2000 - Mycoflora associated with blue pine seed. Pakistan Journal of Forestry 50, 2531.

Axelrood PE, Neumann M, Trotter D, Radley R et al. 1995 - Seedborne Fusarium on Douglas-fir: Pathogenicity and seed stratification method to decrease Fusarium contamination. New Forests 9, 35-51.

Bacon CW, White J. 2000 - Microbial endophytes. CRC press.

Baral HO. 1996 - Hymenoscyphus seminis-alni, a new species of the $H$. fructigenus-complex. Mycotaxon 60, 249-256.

Barrows-Broaddus J, Dwinell LD. 1985 - Branch dieback and cone and seed infection caused by Fusarium moniliforme var. subglutinans in a loblolly pine seed orchard in South Carolina. Phytopathology 75, 1104-1108.

Batko S. 1956 - Report on forest research, H.M. Stationary office. London.

Batko S. 1959 - Report on forest research, H.M. Stationary office. London.

Batra LR, Segal RH, Baxter RW. 1964 - A new Middle Pennsylvanian fossil fungus. American Journal of Botany 51, 991-995.

Batra LR. 1988 - Monilinia gaylussaciae, a new species pathogenic on huckleberries (Gaylussacia) in North America. Mycologia 80, 653-659.

Batra LR. 1991 - World species of Monilinia (Fungi): Their ecology, biosystematics and control. Mycologia Memoirs 16, 1-246.

Bayman P, Angulo-Sandoval P, Báez-ortiz Z, Lodge DJ. 1998 - Distribution and dispersal of Xylaria endophytes in two tree species in Puerto Rico. Mycological Research 102, 944-948.

Beimforde C, Feldberg K, Nylinder S, Rikkinen J et al. 2014 - Estimating the phanerozoic history of the Ascomycota lineages: combining fossil and molecular data. Molecular Phylogenetics and Evolution 78, 386-398.

Bello GD. 2008 - First report of Trichothecium roseum causing postharvest fruit rot of tomato in Argentina. Australasian Plant Disease Notes 3, 103-104.

Benny GL, Samuelson DA, Kimbrough JW. 1985 - Studies on the Coryneliales, I. Fitzpatrickella, a monotypic genus on the fruits of Drimys. Botanical Gazette Crawfordsville 146, 232-237.

Bensch K, Groenewald JZ, Braun U, Dijksterhuis J et al. 2015 - Common but different: The expanding realm of Cladosporium. Studies in Mycology 82, 23-74.

Bentele M, Morgenstern K, Krabel D. 2014 - Lophodermium seditiosum Minter, Staley \& Millar seed-borne on Pinus sylvestris. Journal of Forest and Landscape Research 1, 1-8.

Berndt R. 2010 - Uromycladium naracoortensis, a new species of rust fungi (Uredinales) from Australia, with new observations on described Uromycladium species. Polish Botanical Journal 55, 299-308.

Berndt R. 2011. Taxonomic revision of Endoraecium digitatum (rust fungi, Uredinales) with description of four new species from Australia and Hawaii. Mycological Progress 10, 497517.

Berney C, Pawlowski J. 2006 - A molecular time-scale for eukaryote evolution recalibrated with the continuous microfossil record. Proceedings of the Royal Society B: Biological Sciences 273, 1867-1872.

Bewley JD, Black M. 1994 - Seeds, physiology of development and germination. New York: Plenum Press.

Blaney CS, Kotanen PM. 2001 - Effects of fungal pathogens on seeds of native and exotic plants: a test using congeneric pairs. Journal of Applied Ecology 38, 1104-1113.

Bloomberg WJ. 1966 - The occurrence of endophytic fungi in Douglas-fir seedlings and seed, Canadian Journal of Botany 44, 413-420.

Bloomberg WJ. 1969 - Diseases of Douglas-fir seeds during cone storage. Forest Science 15, 176181.

Boidin J, Lanquetin P. 1987 - Le genre Scytinostroma Donk. Bibliotheca Mycologica 114, 1-130.

Booth C. 1971 - The genus Fusarium. International Mycological Institute, Kew 1-234. 
Bose SR. 1944 - Hereditary (seed-borne) symbiosis in Casuarina equisetifolia Forst. In Proceedings of the Indian Science Congress 31: Part III. 62-63.

Bucher VVC, Hyde KD, Pointing SB, Reddy CA 2004 - Production of wood decay enzymes, mass loss and lignin solubilization in wood by marine ascomycetes and their anamorphs. Fungal Diversity 15, 1-14.

Buchwald NF. 1947 - Sclerotiniaceae of Denmark, A floristic systematic survey of the sclerotial cup fungi found in Denmark, Part 1. Ciboria, Rutstroemia, Myriosclerotinia and Sclerotinia. Friesia 3, 235-330.

Burgess T, Wingfield MJ. 2002 - Quarantine is important in restricting the spread of exotic seedborne tree pathogens in the southern hemisphere. The International Forestry Review 1, 5665.

Byler JW, Platt WD. 1972 - Cone infection by Peridermium harknessii. Canadian Journal of Botany 50, 1429-1430.

Cadez N, Poot GA, Raspor P, Smith MT. 2003 - Hanseniaspora meyeri sp. nov., Hanseniaspora clermontiae sp. nov., Hanseniaspora lachancei sp. nov. and Hanseniaspora opuntiae sp. nov., novel apiculate yeast species. International Journal of Systematic and Evolutionary Microbiology 53, 1671-1680.

Calduch M, Gené J, Stchigel AM, Guarro J. 2002 - New species of Dictyochaetopsis and Paraceratocladium from Brazil. Mycologia 94, 1071-1077.

Cannon PF. 1997 - Two new genera of Ascomycota, and other new or interesting fungi from Slapton Ley National Nature Reserve and its environs. Systema Ascomycetum 15, 121-138.

Carbone I, Kohn LM. 1999 - A method for designing primer sets for speciation studies in filamentous ascomycetes. Mycologia 91, 553-556.

Carré CG. 1964 - Fungus decomposition of beech cupules. Transactions of the British Mycological Society 47, 437-444.

Castañeda-Ruíz RF, Guarro J. 1998 - Two new hyphomycetes from rainforests of Cuba. Canadian Journal of Botany 76, 1584-1588.

Castañeda-Ruiz RF, Gusmão LF, Abarca GH, Saikawa M. 2006 - Some hyphomycetes from Brazil. Two new species of Brachydesmiella, two new combinations for Repetophragma, and new records. Mycotaxon 95, 261-270.

Castañeda-Ruíz RF, Kendrick B. 1991 - Ninety-nine conidial fungi from Cuba and three from Canada. University of Waterloo Biology Series 35, 1-132.

Castañeda-Ruiz RF, Minter DW, Stadler M, Gené J et al. 2010 - Two new anamorphic fungi from Cuba: Endophragmiella profusa sp. nov. and Repetoblastiella olivacea gen. and sp. nov. Mycotaxon 113, 415-422.

Castañeda-Ruíz RF, Saikawa M, Guarro J. 1999 - A new species of Heteroconium from a tropical rainforest. Mycotaxon 71, 295-300.

Castlebury LA, Carris LM. 1999 - Tilletia walkeri, a new species on Lolium multiflorum and L. perenne. Mycologia. 91, 121-131.

Chalermpongse A, Pongpanich K, Boonthavikoon T. 1984 - Seed-borne fungi and diseases of tropical forest tree seeds in Thailand. Forest Pest Control Branch, Thailand Royal Forest Department, Bangkok, Thailand.

Chapela IH. 1989 - Fungi in healthy stems and branches of American beech and aspen: a comparative study, New Phytologist 113, 65-75.

Charlton ND, Craven KD, Mittal S, Hopkins AA et al. 2012 - Epichloë canadensis, a new interspecific epichloid hybrid symbiotic with Canada wildrye (Elymus canadensis). Mycologia 104, 1187-1199.

Chen Q, Hou LW, Duan WJ, Crous PW et al. 2017 - Didymellaceae revisited. Studies in Mycology 87, 105-159.

Cheng D, Igarashi T. 1987 - Fungi associated with natural regeneration of Picea jezoensis Carr. in seed stage: their distribution on forest floors and pathogenicity to the seeds. Research bulletins of the college experiment forests Hokkaido university 44, 175-188. 
Cheplick GP, Clay K. 1988 - Acquired chemical defenses of grasses: the role of fungal endophytes. Oikos 52, 309-318.

Chlebická M. 2014 - Propolis rhodoleuca (Leotiomycetes, inc. sed.) compared with P. farinosa, $P$. occulta sp. nov. and P. strobilina. Nova Hedwigia 98, 491-506.

Cho H, Miyamoto T, Takahashi K, Hong S et al. 2007 - Damage to Abies koreana seeds by soilborne fungi on Mount Halla, Korea. Canadian Journal of Forest Research 37, 371-382.

Cho HK, Miyamoto T, Takahashi K, Kim DW et al. 2005 - First report on Racodium therryanum associated with seed infection of Abies koreana in Korea. The Plant Pathology Journal 21, 297-300.

Chomnunti P, Hongsanan S, Hudson BA, Tian Q et al. 2014 - The sooty moulds. Fungal Diversity $66,1-136$.

Chung KR, Schardl CL. 1997 - Sexual cycle and horizontal transmission of the grass symbiont, Epichloë typhina. Mycological Research 101, 295-301.

Cilliers AJ, Swart WJ, Wingfield MJ. 1995 - The occurrence of Lasiodiplodia theobromae on Pinus elliottii seeds in South Africa. Seed Science and Technology (Switzerland) 23, 851860.

Clay K. 1990 - Fungal endophytes of grasses. Annual Review of Ecology and Systematics 21, 275-297.

Cleary MR, Arhipova N, Gaitnieks T, Stenlid J et al. 2013 - Natural infection of $F$ raxinus excelsior seeds by Chalara fraxinea. Forest Pathology 43, 83-85.

Conners IL. 1967 - An annotated index of plant diseases in Canada and fungi recorded on plants in Alaska, Canada and Greenland. Canada Department of Agriculture Publication 1251.

Cooke MC. 1884 - Synopsis pyrenomycetum. Grevillea 13, 8-16.

Cookson IC. 1947 - Fossil fungi from Tertiary deposits in the Southern Hemisphere. Part I. Proceedings of the Linnean Society of New South Wales 72, 207-214.

Corner EJH. 1996 - The agaric genera Marasmius, Chaetocalathus, Crinipellis, Heimiomyces, Resupinatus, Xerula and Xerulina in Malesia. Beihefte zur Nova Hedwigia 111, 1-175.

Covington SA, Mstretta PA, Affeltranger PA. 1982 - Fungi found on Texas slash and lobolly seed. USDA Forest Service Southeastern Area.

Cram M, Fraedrich S. 2010 - Seed diseases and seedborne pathogens of North America. Tree Planters' Notes 53, 35-44.

Crosier W, Heit CE. 1948 - Some seed-borne fungi of flowers. In Proceedings of the Association of Official Seed Analysts.The Association of Official Seed Analysts. 73-76.

Crous PW, Groenewald JZ, Risède JM, Simoneau P et al. 2004 - Calonectria species and their Cylindrocladium anamorphs: species with sphaeropedunculate vesicles Studies in Mycology 50, 415-430.

Crous PW, Groenewald JZ. 2016 - They seldom occur alone. Fungal Biology 120, 1392-1415.

Crous PW, Peerally A. 1996 - Gliocladiopsis irregularis sp. nov. and notes on Cylindrocladium spathiphylli. Mycotaxon 58, 119-128.

Crous PW, Schumacher RK, Akulov A, Thangavel R et al. 2019 - New and interesting fungi. 2. Fungal Systematics and Evolution 3, 57-134.

Crous PW, Wingfield MJ. 1993 - A re-evaluation of Cylindrocladiella, and a comparison with morphologically similar genera. Mycological Research 97, 433-448.

Crous PW, Wingfield MJ, Burgess TI, Hardy GS et al. 2016 - Fungal planet description sheets: 469-557. Persoonia: Molecular Phylogeny and Evolution of Fungi 37, 218-403.

Crous PW, Wingfield MJ, Guarro J, Hernández-Restrepo M et al. 2015a - Fungal planet description sheets: 320-370. Persoonia 34, 167-266.

Crous PW, Wingfield MJ, Le Roux JJ, Richardson DM et al. 2015b - Fungal planet description sheets: 371-399. Persoonia: Molecular Phylogeny and Evolution of Fungi 35, 264 -327.

Crous PW, Wingfield MJ, Schumacher RK, Summerell BA et al. 2014 - Fungal planet description sheets: 281-319. Persoonia. 33, 212-289. 
Cruz AC, Leao-Ferreira SM, Barbosa FR, Fernando L et al. 2009 - Conidial fungi from semi-arid Caatinga biome of Brazil. New and interesting Dictyochaeta species. Mycotaxon 106, 15-27.

Cruz AC, Marques MF, Gusmão LF. 2014 - Conidial fungi from the semi-arid Caatinga biome of Brazil: The genus Menisporopsis. Acta Botanica Brasilica 28, 339-345.

Cruz BPB, Figueiredo MB. 1961 - Importance of the fungus Cylindrocladium in Eucalyptus growing. Biologico 27, 106-108.

Currie, CR, Wong B, Stuart AE, Schultz TR et al. 2003 - Ancient tripartite coevolution in the attine ant-microbe symbiosis. Science 299, 386-388.

Czeczuga B, Muszyńska E, Godlewska A, Mazalska B. 2009 - Aquatic fungi and fungus-like organisms growing on seeds of 131 plant taxa. Nova Hedwigia 89, 451-467.

Damm U, Cannon PF, Woudenberg JH, Crous PW. 2012 - The Colletotrichum acutatum species complex. Studies in Mycology 73, 37-113.

Daranagama DA, Camporesi E, Jeewon R, Liu XZ et al. 2016 - Taxonomic rearrangement of Anthostomella (Xylariaceae) based on a multigene phylogeny and morphology. Cryptogamie, Mycologie 37, 509-538.

Daranagama DA, Camporesi E, Tian Q, Liu XZ et al. 2015 - Anthostomella is polyphyletic comprising several genera in Xylariaceae. Fungal Diversity 73, 203-238.

Daranagama DA, Hyde KD, Sir EB, Thambugala KM et al. 2018 - Towards a natural classification and backbone tree for Graphostromataceae, Hypoxylaceae, Lopadostomataceae and Xylariaceae. Fungal Diversity 88, 1-165.

Das-Gupta SN, John R. 1988 - A contribution to our knowledge of the genus Blastocladia. Indian Phytopathology 41, 521-547.

Davidson JGN. 1970 - Seed and cone mortality of coast Redwood. Phytopathology 60, 1533.

Dayan MP. 1986 - Fungi associated with different forest tree seeds of the Forest Research Institute seed bank. Embryon 2, 28-39.

Dayo-Owoyemi I, Rosa CA, Rodrigues A, Pagnocca FC. 2014 - Wickerhamiella kiyanii f.a., sp. nov. and Wickerhamiella fructicola f.a., sp. nov., two yeasts isolated from native plants of Atlantic rainforest in Brazil. International Journal of Systematic and Evolutionary Microbiology 64, 2152-2158.

Decourcelle T, Piou D, Desprez-Loustau ML. 2015 - Detection of Diplodia sapinea in Corsican pine seeds. Plant pathology 64, 442-449.

Delatour C, Morelet M. 1979 - Black rot of acorns [Quercus; Ciboria]. Revue Forestiere Francaise (France) 31, 101-115.

Denchev CM, Kakishima M. 2007 - Notes on some Japanese smut fungi. IV. Mundkurella japonica, sp. nov. Mycotaxon 102, 9-16.

Dennis RWG. 1971 - New or interesting British microfungi. Kew Bulletin 25, 335-374.

Desjardin DE. 2000 - Strobilurus diminutivus, a new species of Strobilurus from montane California, USA. Micologia 137-142.

Desprez-Loustau ML. 2009 - Alien Fungi of Europe. In Handbook of alien species in Europe 1528.

Dharkar N, Subhedar A, Hande D, Shahezad MA. 2010 - Two new fungal species from Vidarbha, India. Journal of Mycology and Plant Pathology 40, 235-237.

Dick M. 1998 - Pine pitch canker-the threat to New Zealand. New Zealand Forestry 42, 30-34.

Dick MA, Dobbie K. 2002 - Species of Fusarium on Pinus radiata in New Zealand. New Zealand Plant Protection 55, 58-62.

Dilcher DL. 1965 - Epiphyllous fungi from Eocene deposits in western Tennessee, USA. Palaeontographica 116B, 1-54.

Dissanayake AJ, Camporesi E, Hyde KD, Zhang Wei et al. 2017a - Molecular phylogenetic analysis reveals seven new Diaporthe species from Italy. Mycosphere 8, 853-877.

Dissanayake AJ, Phillips AJL, Hyde KD, Yan JY et al. 2017b - The current status of species in Diaporthe. Mycosphere 8, 1106-1156. 
Döbbeler P. 1995 - Burenia myrrhidendri spec. nov. (Protomycetales), ein bemerkenswerter biotropher Ascomycet in den Früchten einer baumförmigen Umbellifere aus Costa Rica. Nova Hedwigia 60, 171-177.

Dolejs K. 1964 - Fungal diseases of seeds and cones of Pinus sylvestris. Abstract, Review of Applied Mycology 46, 462.

Drake DW. 1974 - Fungal and insect attack of seeds in unopened Eucalyptus capsules. Search, Science, Technology \& Society 5, 444.

Dwinell LD. 1998 - Association of the pitch canker fungus with cones and seeds of pines. In Current and potential impacts of pitch canker in radiata pine: Proceedings of the IMPACT Monterey Workshop, Monterey, California.

Dwinell LD. 1999 - Contamination of Pinus radiata seeds in California by Fusarium circinatum. In Proceedings of the Annual International Research Conference on Methyl Bromide Alternatives and Emissions Reductions, San Diego, CA, USA 1-3.

Eberhardt TL, Han JS, Micales JA, Young RA. 1994 - Decay resistance in conifer seed cones: role of resin acids as inhibitors of decomposition by white-rot fungi. Holzforschung-International Journal of the Biology, Chemistry, Physics and Technology of Wood 48, 278-284.

Edwards DGW, Sutherland JR. 1979 - Hydrogen peroxide treatment of Abies seeds. Bi-monthly Research Notes 35, 3-4.

Ekanayaka AH, Ariyawansa HA, Hyde KD, Jones EBG et al. 2017 - Discomycetes: the apothecial representatives of the phylum Ascomycota. Fungal Diversity 87, 237-298.

Ekanayaka AH, Hyde KD, Gentekaki E, McKenzie EHC et al. 2019 - Preliminary classification of Leotiomycetes. Mycosphere 10, 310-489.

El-Kady IA, El-Maghraby OMO, Saber S. 1986 - Halophilic or halotolerant fungi of four seeds from Egypt. Cryptogamie, Mycologie 7, 289-293.

Epners Z. 1964 - A new psychrophilic fungus causing germination failure of conifer seeds. Canadian Journal of Botany 42, 1589-1604.

Ernst M, Mendgen KW, Wirsel SGR. 2003 - Endophytic fungal mutualists: Seed-borne Stagonospora spp. enhance reed biomass production in axenic microcosms. Molecular PlantMicrobe Interactions 16, 580-587.

Evans HC, Carrión G, Guzmán G. 1993 - A new species of Sphaerulina and its Phloeospora with potential for biological control of Mimosa pigra. Mycological Research 97, 59-67.

Fakir GA, Welty RE, Cowling EB. 1977 - Prevalence and pathogenicity of fungi associated with achenes of sycamore in the field and in storage. Phytopathology 61, 660-668.

Farag SA, Shehata MRA, Omran TA. 1977 - Studies on damping-off of Cupressus sempervirens seedlings in Egypt. 1.-Seed borne fungi associated with damping-off cypress seedlings [Egypt]. Alexandria Journal of Agricultural Research (Egypt) 25, 319-322.

Fernández FA, Huhndorf SM. 2005. New species of Chaetosphaeria, Melanopsammella and Tainosphaeria gen. nov. from the Americas. Fungal Diversity 18, 15-57.

Fogel R, Cromack K Jr. 1977 - Effect of habitat and substrate quality on Douglas fir litter decomposition in western Oregon. Canadian Journal of Botany 55, 1632-1640.

Fournier J, Flessa F, Peršoh D, Stadler M. 2010a - Three new Xylaria species from southwestern Europe. Mycological Progress 10, 33-52.

Fournier J, Ju Y-M, Hsieh H-M, Lindemann U. 2018 - Xylaria aethiopica sp. nov. - a new podinhabiting species of Xylaria (Xylariaceae) from Ethiopia. Ascomycete.org 10, 209-215.

Fournier J, Köpcke B, Stadler M. 2010b - New species of Hypoxylon from Western Europe and Ethiopia. Mycotaxon 113, 209-235.

Friedrich JH. 1969 - Fungi isolated from fruits and seeds of some northern hard wood trees. Master's Thesis, University of New Hampshire.

Friedrich JH, Rich AE, Shigo AE. 1971 - Fungi isolated from fruits and seeds of some northern hardwood trees. Rhodora 73, 306-307. 
Fraedrich SW, Miller T. 1995 - Mycoflora associated with slach-pine seeds from cones collected at seed orchards and cone-processing facilities in the south-eastern USA. European Journal of Forest Pathology 25, 73-82.

Fraedrich SW, Miller T, Zarnoch SJ. 1994 - Factors affecting the incidence of black seed rot in slash pine. Canadian Journal of Forest Research 24, 717-725.

Fukasawa Y, Tateno O, Hagiwara Y, Hirose D et al. 2012 - Fungal succession and decomposition of beech cupule litter. Ecological Research 27, 735-43.

Fuller LR, Hildebrand DM. 1985 - Effects of cold stratification and hydrogen peroxide treatments on seeds of three Rocky Mountain conifer species. Technical report R2-United States Forest Service, Forest Pest Management (USA).

Galán R, Raitviír A, Ayala N, Ochoa C. 1994 - First contribution to the knowledge of the Leotiales of Baja California and adjacent areas. Mycological Research 98, 1137-1152.

Gallery RE, Dalling JW, Arnold AE. 2007 - Diversity, host affinity, and distribution of seed-infecting fungi: a case study with Cecropia. Ecology 88, 582-588.

Gamundí IJ, Spinedi HA. 1988 - Ascomycotina from Antarctica. Mycotaxon 33, 467-482.

Ganley RJ, Newcombe G. 2006 - Fungal endophytes in seeds and needles of Pinus monticola. Mycological Research 110, 318-327.

Garbowski L. 1936 - Contribution to the knowledge of the fungal microflora of forest tree seeds. Review of Phytopathology 15, 5.

Gardner DE. 1980 - Acacia koa seedling wilt caused by Fusarium oxysporum f. sp. koae, f. sp. nov. Phytopathology 70, 594-597.

Geesteranus RAM, de Meijer AAR. 1998 - Further mycenas from the State of Paraná, Brazil. Persoonia 17, 29-46.

Geesteranus RAM, Horak E. 1995 - Mycena and related genera from Papua New Guinea and New Caledonia. Bibliotheca Mycologica 159, 143-229.

Geiser DM, Lewis-Ivey ML, Hakiza G, Juba JH et al. 2005 - Gibberella xylarioides (anamorph: Fusarium xylarioides), a causative agent of coffee wilt disease in Africa, is a previously unrecognized member of the G. fujikuroi species complex. Mycologia, 97, 191-201.

Gelyuta VP. 1990 - Taxa of powdery mildew fungi new for science and for the USSR from Reserve 'Kedrovaya Pad' (the Primoriye Territory). Ukrainskiy Botanichnyi Zhurnal 47, 7883.

Georgescu CC, Petrescu M. 1954 - A parasite of elm fruits: Gloeosporium ulmicola Miles. Rev. Padurilor 69, 106.

Gepts P, Beavis WD, Brummer EC, Shoemaker RC et al. 2005 - Legumes as a model plant family. Genomics for food and feed report of the cross-legume advances through genomics conference. Plant Physiology 137, 1228-1235.

Gibson IAS. 1957 - Saprophytic fungi as destroyers of germinating pine seeds. The East African Agricultural Journal 22, 203-206.

Giraldo A, Gené J, Cano J, de Hoog S et al. 2014 - Acremonium with catenate elongate conidia: phylogeny of Acremonium fusidioides and related species. Mycologia 106, 328-338.

Glass NL, Donaldson GC. 1995 - Development of primer set designed for use with the PCR to amplify conserved genes from filamentous ascomycetes. Applied Environmental Microbiology 61, 1323-1330.

Gloyer WO. 1931 - China aster seed treatment and storage. New York State Agricultural Experiment Station Bulletin 177, 3-41.

Gomes RR, Glienke C, Videira SIR, Lombard L et al. 2013 - Diaporthe: a genus of endophytic, saprobic and plant pathogenic fungi. Persoonia: Molecular Phylogeny and Evolution of Fungi 31, 1-41.

Gómez LD, Henk DA. 2004 - Validation of the species of Septobasidium described by John N. Couch. Lankesteriana 4, 75-96.

Goos RD, Pirozynski KA. 1975 - Fungi of Barro Colorado Island: new and interesting Hyphomycetes. Canadian Journal of Botany 53, 2927-2932. 
Gordon AG, Salt GA, Brown RM. 1976 - Effect of pre-sowing moist-chilling treatments on seedbed emergence of sitka spruce seed infected by Geniculodendron pyriforme, salt. Forestry: An International Journal of Forest Research 49, 143-152.

Gordon LR. 1967 - Fungi associated with Douglas-fir seed during cone development, seed processing, and storage. M.S. Thesis, Oregon State University.

Gordon TR. 2006 - Pitch canker disease of pines. Phytopathology 96, 657-659.

Goswami MS, Ojha BM. 2004 - Fungi of India 1989-2001. Scientific Publishers.

Gräfenhan T, Schroers HJ, Nirenberg HI, Seifert KA. 2011 - An overview of the taxonomy, phylogeny, and typification of nectriaceous fungi in Cosmospora, Acremonium, Fusarium, Stilbella, and Volutella. Studies in Mycology 68, 79-113.

Groves JW, Skolko AJ. 1944 - Notes on seed-borne fungi. II. Alternaria. Canadian Journal of Research 22, 217-234.

Gure A, Slippers B, Stenlid J. 2005a - Seed-borne Botryosphaeria spp. from native Prunus and Podocarpus trees in Ethiopia, with a description of the anamorph Diplodia rosulata sp. nov. Mycological Research 109, 1005-1014.

Gure A. 2004 - Seed-borne fungi of the Afromontane tree species Podocarpus falcatus and Prunus africana in Ethiopia. PhD thesis. Swedish University of Agricultural Sciences, Uppsala.

Gure A, Wahlstrom K, Stenlid J. 2005b - Pathogenicity of seed-associated fungi to Podocarpus falcatus in vitro. Forest Pathology 35, 23-35.

Hafizoglu H, Holmbom B. 1987 - Studies on the Chemistry of Cedrus libani A. Rieh. III. Oleoresin composition of cones and cark from Cedrus libani. Holzforschung 41, 141-145.

Hall TA. 1999 - BioEdit: a user-friendly biological sequence alignment editor and analysis program for Windows 95/98/NT. Nucleic Acids Symposium Series 41, 95-98.

Hall TJ, Taylor GS. 1983 - Aerated-steam treatment for control of Alter nana tenuis on lobelia seed. Annals of Applied Biology 103, 219-228.

Hamamoto M, Nakase T. 1996 - Ballistosporous yeasts found on the surface of plant materials collected in New Zealand. Antonie van Leeuwenhoek 69, 279-291.

Hammer S. 2001 - A New Cladonia from California. The Bryologist 104, 226-229.

Han JG, Shin HD. 2007 - New record of Xylaria persicaria on Liquidambar fruits in Korea. Mycobiology 35, 171-173.

Hangyal W. 1973 - Microflora examinations on Scots and black pine seeds. Erdeszeti Kutatasok 69, 171-179.

Hangyal-Balul W. 1983 - Fungus diseases of Robinia seeds and seedlings and possibilities of their control. Erdeszeti Kutatosok 74, 343-349.

Harada Y, Sasaki M, Sasaki Y, Ichihashi Y et al. 2005 - Monilinia ssiori sp. nov. in the Sclerotiniaceae, causing leaf blight and young fruit rot of Prunus ssiori in Japan Mycoscience 46, 376-380.

Harvey RD Jr. 1980 - Mortality from Caloscypha fulgens and other fungi on spruce seed in Oregon and Washington. Plant Disease (formerly Plant Disease Reporter) 64, 223-224.

Hayatgheibi H. 2013 - Studies on the microflora associated with the seeds of European ash (Fraxinus excelsior) and the infection biology of the pathogen Hymenoscyphus pseudoalbidus causing ash dieback. MSc. Thesis. Department of Forest Mycology and Plant Pathology, Uppsala, Sweden.

Heit CE, Natti JJ. 1969 - Accurate germination of Abies balsamea and Abies fraseri in laboratory tests by control of Rhizoctonia solani and other fungi with PCNB. In Proceedings of the Association of Official Seed Analysts 148-153.

Henkel TW, Aime MC, Largent DL, Baroni TJ. 2014 - The Entolomataceae of the Pakaraima Mountains of Guyana 6: Ten new species and one combination of Nolanea. Mycotaxon 129, 119-148.

Hennebert GL. 1963 - Un Hyphomycète nouveau, Arachnophora fagicola gen. nov. spec. nov. Canadian Journal of Botany 41, 1165-1169. 
Heredia G, Arias RM, Reyes M, Castañeda-Ruíz R. 2002 - New anamorph fungi with rhombic conidia from Mexican tropical forest litter. Fungal Diversity 11, 99-107.

Hernandez-Restrepo M, Schumacher RK, Wingfield MJ, Ahmad I et al. 2016 - Fungal Systematics and Evolution: FUSE 2. Sydowia 68, 193-230.

Hernández-Terán A, Wegier A, Benítez M, Lira R et al. 2017 - Domesticated, genetically engineered, and wild plant relatives exhibit unintended phenotypic differences: a comparative meta-analysis profiling rice, canola, maize, sunflower, and pumpkin. Frontiers in plant science 8, 1-10.

Hiroki S, Matsubara T. 1995 - Fluctuation of nut production and seedling appearance of a Japanese beech (Fagus crenata Blume). Ecological Research 10, 161-169.

Hirooka Y, Rossman AY, Samuels GJ, Lechat C et al. 2012 - A monograph of Allantonectria, Nectria, and Pleonectria (Nectriaceae, Hypocreales, Ascomycota) and their pycnidial, sporodochial, and synnematous anamorphs. Studies in Mycology 71, 1-210.

Hocking D. 1968 - Fungi associated with damped-off and healthy pine seedlings and with seed in East African pine nurseries. Transactions of the British Mycological Society 51, 221-226.

Höhnel FV. 1909 - Fragmente zur mykologie. Javanische Myxomyceten. Sitzungsber. Kaiserl Akademie Der Wissenschaften 118, 427-442.

Homechin M, Pizzinatto MA, Menten JOM. 1986 - Seed health of Pinus elliottii var. elliottii and Pinus taeda and pathogenicity of Fusarium oxysporum in seedlings of Pinus elliottii var. elliottii. Summa Phytopathologica 12, 102-112.

Hong LT. 1981 - A note on some seed fungi of Dipterocarpus. Malaysian Forester 44, 163-166.

Howe HF, Smallwood J. 1982 - Ecology of seed dispersal. Annual Review of Ecology and Systematics 13, 201-228.

Huang G, Guo L, Liu N. 2014a - Two new species of Xylaria and X. diminuta new to China. Mycotaxon 129, 149-152.

Huang G, Guo L, Liu N. 2014b - Xylaria byttneriae sp. nov. from Yunnan Province in China. Mycosystema 33, 567-570.

Huang JW, Kuhlman EG. 1990 - Fungi associated with damping-off of slash pine seedlings in Georgia. Plant Disease 74, 27-30.

Hughes SJ. 1952 - Fungi from the Gold Coast I. Mycological Papers 48, 1-91.

Huhtinen S. 1990 - A monograph of Hyaloscypha and allied genera. Karstenia 29, 45-252.

Husbands DR, Urbina H, Lewis SM, Aime MC. 2018 - Xylaria karyophthora: A new seedinhabiting fungus of Greenheart from Guyana. Mycologia 110, 434-447.

Hyde KD. 1995 - Measuring biodiversity of microfungi in the wet tropics of north Queensland. In: Measuring and monitoring biodiversity of tropical and temperate forests (eds. T.J.B. Boyle and B. Boontawee) CIFOR, Indonesia, Bogor, Indonesia 271-286.

Hyde KD, Cai L, Jeewon R. 2005 - Tropical fungi. Mycology Series 23, 93-116.

Hyde KD, Chaiwan N, Norphanphoun C, Boonmee S et al. 2018 - Mycosphere notes 169-224, Mycosphere 9, 271-430.

Hyde KD, Norphanphoun C, Abreu VP, Bazzicalupo A et al. 2017 - Fungal diversity notes 603708: taxonomic and phylogenetic notes on genera and species, Fungal Diversity 87, 1-235.

Ibáñez C, Zamuner AB. 1996 - Presence of hyphomycetes (Deuteromycetes) in cones of Araucaria mirabilis (Spegazzini) Windhausen, Middle Jurassic of Patagonia. Mycotaxon 59, 137-143.

Islam MR, Akther QY, Yeasmin MN, Ullah MS et al. 2008 - Study on seed borne fungi of ipil-ipil (Leucaena leucocephala) and their control by plant extracts. Journal of Agroforestry and Environment 2, 193-196.

Iturriaga T, Korf RP. 1997 - A preliminary discomycete flora of Macaronesia: Part 10a, Dermateaceae. Mycotaxon 61, 223-242.

Jamaluddin VS, Dadwal VS, Soni KK. 1983 - Studies on pod rot of Pongamia pinnata and its control. Indian Journal of Forestry 6, 287-288. 
James RL. 1983a - Fungal contamination of ponderosa pine cones and seed from the Coeur d'Alene Nursery, Idaho. Missoula, MT: US Department of Agriculture, Forest Service, Northern Region.

James RL. 1983b - Occurrence of Fusarium on Douglas-fir seed from the Coeur d'Alene Nursery. USDA Forest Service, Northern Region Report. 11 p.

James RL. 1984 - Fungi colonizing Douglas-fir seed at the Champion Timberlands Nursery, Plains, Montana. USDA Forest Service, Northern Region Report. 84-13. 3 p.

James RL. 1985a - Fusarium associated with seedborne diseases of ponderosa pine seedlings at the Montana State Nursery, Missoula. USDA Forest Service, Northern Region Report. 5 p.

James RL. 1985b - Pathogenic Fusarium on spruce seed from the Towner Nursery, North Dakota. USDA Forest Service, Northern Region Report. 85-23. 9 p.

James RL. 1986a - Occurrence of Fusarium on Douglas-fir seed and containerized seedlings at the Plum Creek Nursery, Pablo, Montana. USDA Forest Service, Northern Region Report. 86-4. $10 \mathrm{p}$.

James RL. 1986b - Occurrence of Fusarium on western larch seed from the Nishek Nursery, Bonners Ferry, Idaho. USDA Forest Service, Northern Region Report. 3 p.

James RL. 1986c - Occurrence of Fusarium oxysporum on ponderosa pine seed from the USDA Forest Service Nursery, Coeur d'Alene, Idaho. USDA Forest Service, Northern Region Report. 3 p.

James RL. 1995 - Fungi on Douglas-fir and ponderosa pine cones from the USDA Forest Service Nursery, Coeur d'Alene, Idaho.

James RL, Dumroese RK, Wenny DL. 1996 - Western larch seed-contaminating fungi and treatments to reduce infection and improve germination. Forest Health Protection. Report 9697.

James RL, Genz D. 1982 - Evolution of fungal populations on ponderosa pine seeds. USDA Forest Service, Northern Region, Forest Pest Management.

Janerette CA. 1979 - Pathogenicity of fungi isolated from sugar maple seeds. Tree planters' Notes 30, 12-14.

Jankowiak R. 2008 - Fungi occurring in acorn Quercus robur L. infested by insects. Acta Scientiarum Polonorum Silvarum Colendarum Ratio et Industria Lignaria 7, 19-29.

Jayasiri SC, Hyde KD, Ariyawansa HA, Bhat J et al. 2015 - The Faces of Fungi database: fungal names linked with morphology, phylogeny and human impacts. Fungal Diversity 74, 3-18.

Jayasiri SC, Hyde KD, Jones EBG, Ariyawansa HA et al. 2017a - A new hysteriform Dothideomycete (Gloniaceae, Pleosporomycetidae Incertae sedis), Purpurepithecium murisporum gen. et sp. nov. on pine cone scales. Cryptogamie Mycologie 38, 241-251.

Jayasiri SC, Hyde KD, Jones EBG, Lu YZ. 2017b - Neohelicosporium fusisporum sp. nov. (Tubeufiaceae) and a first record of a sexual morph within Neohelicosporium. Studies in Fungi 2, 210-217.

Jayasiri SC, Hyde KD, Jones EBG, McKenzie EHC et al. 2019 - Diversity, morphology and molecular phylogeny of Dothideomycetes on decaying wild seed pods and fruits. Mycosphere $10,1-186$.

Jayasiri SC, Hyde KD, Jones EBG, Peršoh D et al. 2018a - Taxonomic novelties of hysteriform Dothideomycetes. Mycosphere 9, 803-837.

Jayasiri SC, Hyde KD, Jones EBG, Xu JC et al. 2018b - Seed decaying Dothideomycetes in Thailand: Zeloasperisporium pterocarpi sp. nov., (Zeloasperisporiaceae, Zeloasperisporiales) on carpel of Pterocarpus sp. (Fabaceae) seed pod. Asian Journal of Mycology 1, 106-113.

Jeewon R, Hyde KD. 2016 - Establishing species boundaries and new taxa among fungi: recommendations to resolve taxonomic ambiguities. Mycosphere 7, 1669-1677.

Jimu L, Kemler M, Wingfield MJ, Mwenje E et al. 2016 - The Eucalyptus stem canker pathogen Teratosphaeria zuluensis detected in seed samples. Forestry 89, 316-324.

Jong SC, Chen S. 1966 - Microflora of coniferous seeds in Taiwan. Botanical Bulletin-Academia Sinica Taipei 7, 75-81. 
Jordaan A, Taylor JE, Rossenkhan R. 2006 - Occurrence and possible role of endophytic fungi associated with seed pods of Colophospermum mopane (Fabaceae) in Botswana. South African Journal of Botany 72, 245-55.

Ju YM, Rogers JD. 1996 - A revision of the genus Hypoxylon. American Phytopathological Society, APS Press.

Ju YM, Rogers JD, Hsieh HM. 2018 - Xylaria species associated with fallen fruits and seeds. Mycologia 110, 726-749.

Kahn RP, Wheeler WH, Monroe RL, Watson A. 1965 - A re-evaluation of the quarantine significance of Cryptospora longispora Servazzi on imported seed of Norfolk Island Pine, Araucaria excelsa. Plant Disease Reporter 49, 656-659.

Kaitera J. 2013 - Thekopsora and Chrysomyxa cone rusts damage Norway spruce cones after a good cone crop in Finland. Scandinavian Journal of Forest Research 28, 217-222.

Kaitera J, Hiltunen R, Kauppila T, Pitkäranta M et al. 2014 - Fruiting and sporulation of Thekopsora and Chrysomyxa cone rusts in Picea cones and Prunus leaves. Forest Pathology 44, 387-395.

Kaitera J, Tillman-Sutela E, Kauppi A. 2009 - Seasonal fruiting and sporulation of Thekopsora and Chrysomyxa cone rusts in Norway spruce cones and alternate hosts in Finland. Canadian Journal of Forest Research 39, 1630-1646.

Kalgutkar RM, Nambudiri EMV, Tidwell WD. 1993 - Diplodites sweetii sp. nov. from the Late Cretaceous (Maastrichtian) Deccan Intertrappean beds of India. Review of Palaeobotany and Palynology 77, 107-118.

Kalgutkar RM, Sigler L. 1995 - Some fossil fungal form-taxa from the Maastrichtian and Palaeogene ages. Mycological Research 99, 513-522.

Kamara AM, El-Lakany MH, Badran OA, Attia YG. 1981 - Seed pathology of Araucaria spp. I. A survey of seed-borne fungi associated with four Araucaria spp. Australian Forest Research 11, 269-274.

Kasai K, Morinaga T, Horikoshi T. 1995 - Fungal succession in the early decomposition process of pine cones on the floor of Pinus densiflora forests. Mycoscience 36, 325-334.

Kavosi MR, Faridi F, Hajizadeh G. 2013 - Fungal species isolated from Quercus castaneifolia in Hyrcanian Forests, North of Iran. Biodiversitas Journal of Biological Diversity 14, 61-66.

Kawada H, Maruyama K. 1986 - Effects of seed bearing of a natural beech (Fagus crenata Blume) forest on amount of litter fall and its nutrients. Japan Journal Ecology 36, 3-10. (in Japanese with English abstract).

Kelly D. 1994 - The evolutionary ecology of mast seeding. TREE 9, 465-470.

Kilic A, Hafizoglu H, Tümen I, Dönmez IE et al. 2010 - Polysaccharides in cones of eleven coniferous species growing in Turkey. Wood Science and Technology 44, 523-529.

Kirbag S, Aime MC, Kursat M. 2011 - A new Puccinia on Thymelaea from Turkey. Mycotaxon 115, 501-504.

Kirk PM. 1986 - New or interesting microfungi. Transactions of the British Mycological Society 86, 409-428.

Kiziewicz B. 2005 - Aquatic fungi growing on seeds of plants in various types of water bodies of Podlasie Province. Polish Journal of Environmental Studies 14, 49-55.

Kohlmeyer J, Volkmann-Kohlmeyer B. 1991 - Illustrated key to the filamentous higher marine fungi. Botanica Marina 34, 1-61.

Kong HZ. 1997 - Stachybotrys yunnanensis sp. nov. and Neosartorya delicata sp. nov. isolated from Yunnan, China. Mycotaxon 62, 427-434.

Konta S, Maharachchikumbura SS, Senanayake IC, McKenzie EH. 2020 - A new genus Allodiatrype, five new species and a new host record of diatrypaceous fungi from palms (Arecaceae). Mycosphere 11, 239-268.

Kozlowska O. 1968 - Investigations on pathogenic fungi on seeds of forest trees (pinus, larch, oak, birch). Forest Research Institute, Warsaw. 
Kremer RJ. 1993 - Management of weed seed banks with microorganisms. Ecological Applications 3, 42-52.

Krings M, Taylor TN, Dotzler N, Persichini G. 2012 - Fossil fungi with suggested affinities to the Endogonaceae from the Middle Triassic of Antarctica. Mycologia 104, 835-844.

Krol ED, Machowicz-Stefaniak Z, Zimowska B, Abramczyk BA et al. 2015 - Fungi inhabiting seeds of selected forest tree species. Sylwan 159, 135-141.

Kuhnert E, Sir EB, Lambert C, Hyde KD et al. 2017 - Phylogenetic and chemotaxonomic resolution of the genus Annulohypoxylon (Xylariaceae) including four new species. Fungal Diversity 85, 1-43.

Kumar A, Kumar A, Kharwar RN. 2006 - Two new phytoparasitic hyphomycetes from Varanasi, India. Indian Phytopathology 59, 85-90.

Kumar N. 2014 - Post harvest mycobiota of sissoo (Dalbergia sissoo Roxb) grown in north Eastern U.P and their culture filterate potential. IOSR Journal of Environmental Science 8, 101-105.

Kurtzman CP. 2000 - Four new yeasts in the Pichia anomala clade. International Journal of Systematic and Evolutionary Microbiology 50, 395-404.

Kurtzman CP. 2001 - Six new anamorphic yeasts near Candida tanzawaensis. FEMS Yeast Research 1, 177-185.

Kurtzman CP, Robnett CJ. 2014 - Description of Kuraishia piskuri f.a., sp. nov., a new methanol assimilating yeast and transfer of phylogenetically related Candida species to the genera Kuraishia and Nakazawaea as new combinations. FEMS Yeast Research 14, 1028-1036.

Kuthubutheen AJ. 1984 - Effect of pesticides on the seed-borne fungi and fungal succession on rice in Malaysia. Journal of Stored Products Research 20, 31-39.

Kuthubutheen AJ, Nawawi A. 1991 - Key to Dictyochaeta and Codinaea species. Mycological Research 95, 1224-1229.

Labuda L, Eliás P, Sert H, Sterflinger K. 2008 - Alternaria jesenskae sp. nov., a new species from Slovakia on Fumana procumbens (Cistaceae). Microbiological Research 163, 208-214.

Laessøe T, Lodge JD. 1994 - Three host specific Xylaria species. Mycologia 86, 436-446.

Latch GCM, Christensen MJ, Samuels GJ. 1984 - 5 endophytes of Lolium and Festuca in New Zealand. Mycotaxon 20, 535-550.

Latha KPD, Paramban NK, Manimohan P. 2016 - Tubaria keralensis, a new species of T. furfuracea complex from tropical India. Phytotaxa 278, 287-293.

Leão-Ferreira SM, Gusmão LFP. 2010 - Conidial fungi from the semi-arid Caatinga biome of Brazil. New species of Endophragmiella and Spegazzinia with new records for Brazil, South America, and Neotropica. Mycotaxon 111, 1-10.

Leão-Ferreira SM, Gusmão LFP, Castañeda-Ruiz RF. 2018 - Anisogenispora insignissima gen. \& sp. nov. from the Brazilian semi-arid region. Mycotaxon 132, 977-983.

Lechat C, Rossman AY. 2017 - A new species of Fusicolla (Hypocreales), F. ossicola, from Belgium. Ascomycete.org 9, 225-228.

Lee SS, Ahmad AM. 1982 - Cylindrocladium scoparium Morgan-A new pathogen of some forest trees species in Peninsular Malaysia. Pertanika 5, 72-75.

Lennox CL, Serdani M, Groenewald JZ, Crous PW. 2004 - Prosopidicola mexicana gen. et. sp. nov., causing a new pod disease of Prosopis species. Studies in Mycology 50, 187-194.

LePage BA, Currah RS, Stockey RA. 1994 - The fossil fungi of the Princeton Chert. International Journal of Plant Sciences 155, 828-836.

Li GJ, Hyde KD, Zhao RL, Hongsanan S, et al. 2016 - Fungal diversity notes 253-366: taxonomic and phylogenetic contributions to fungal taxa. Fungal Diversity 78, 1-237

Li YL, Wu WS. 2002 - A new seed-borne pathogen, Alternaria dianthicola, on Dianthus in Taiwan. Plant Pathology Bulletin 11, 165-167.

Lilja A. 1979 - Fungi on birch seeds and their pathogenicity. Folia Forestry, Helsinki.

Lilja A, Hallaksela AM, Heinonen R. 1995 - Fungi colonizing Scots-pine cone scales and seedsand their pathogenicity. European Journal of Forest Pathology 25, 43-46. 
Limtong S, Jindamorakot S, Am-in S, Kaewwichian R et al. 2011 - Candida uthaithanina sp. nov., an anamorphic yeast species in Nakaseomyces clade isolated in Thailand. Antonie van Leeuwenhoek 99, 865-871.

Lin CG, Hyde KD, Lumyong S, Mckenzie EH. 2017 - Beltrania-like taxa from Thailand. Cryptogamie, Mycologie 38, 301-319.

Lindemann U, Vega M, Alvarado P. 2015 - Revision der Gattung Kotlabaea: K. deformis, K. delectans und K. benkertii. Zeitschrift für Mykologie 81, 373-402.

Liu JK, Yang J, Maharachchikumbura SS, McKenzie EH et al. 2016 - Novel chaetosphaeriaceous hyphomycetes from aquatic habitats. Mycological Progress 15, 1157-1167.

Liu YJ, Whelen S, Hall BD. 1999 - Phylogenetic relationships among ascomycetes: evidence from an RNA polymerase II subunit. Molecular Biology and Evolution 16, 1799-1808.

Locke T, Colhoun J. 1973 - Fusarium oxysporum f. sp. elaeidis as a seed-borne pathogen. Transactions of the British Mycological Society 60, 594-595.

Lodhi SA, Naeem A. 1955 - Some seed-borne fungi from Pakistan, Transactions of the British Mycological Society 38, 240-242.

Lombard L, Crous PW. 2012 - Phylogeny and taxonomy of the genus Gliocladiopsis, Persoonia: Molecular Phylogeny and Evolution of Fungi 28, 25-33.

Lombard L, Houbraken J, Decock C, Samson RA et al. 2016 - Generic hyper-diversity in Stachybotriaceae. Persoonia 36, 156-246.

Lombard L, Van der Merwe NA, Groenewald JZ, Crous PW. 2015 - Generic concepts in Nectriaceae. Studies in Mycology 80, 189-245.

Lori GA, Salerno MI. 2003 - Fusarium species on seeds of Pinus taeda L. and Pinus elliottii Engelm. in Argentina. Journal of Plant Diseases and Protection 110, 437-443.

Lu HZ, Jia JH, Wang QM, Bai FY. 2004 - Candida asparagi sp. nov., Candida diospyri sp. nov. and Candida qinlingensis sp. nov., novel anamorphic, ascomycetous yeast species. International Journal of Systematic and Evolutionary Microbiology 54, 1409-1414.

Lü L, Zhao ZT. 2017 - Lecanora shangrilaensis sp. nov., on pine cones from China. Mycotaxon 132, 441-444.

Lücking R, Hodkinson BP, Leavitt SD. 2017 - The 2016 classification of lichenized fungi in the Ascomycota and Basidiomycota-Approaching one thousand genera. The Bryologist 119, 361-416.

Lücking R, Huhndorf S, Pfister DH, Plata ER et al. 2009 - Fungi evolved right on track. Mycologia 101, 810-822.

Lupo S, Tiscornia S, Bettucci L. 2001 - Endophytic fungi from flowers, capsules and seeds of Eucalyptus globulus. Revista Iberoamericana de Micologia 18, 38-41.

Ma G, Khan SI, Jacob MR, Tekwani BL et al. 2004 - Antimicrobial and antileishmanial activities of hypocrellins A and B. Antimicrobial Agents and Chemotherapy 48, 4450-4452.

Maciel CG, Muniz MF, Mezzomo R, Reiniger LRS. 2015 - Lasiodiplodia theobromae associated with seeds of Pinus spp. originated from the northwest of Rio Grande do Sul, Brazil. Scientia Forestalis 43, 639-646.

Maharachchikumbura SSN, Hyde KD, Groenewald JZ, Xu J et al. 2014 - Pestalotiopsis revisited. Studies in Mycology 79, 121-186.

Malia ME, Tattar TA. 1978 - Electrical resistance, physical characteristics, and cation concentrations in xylem of sugar maple infected with Verticillium dahliae. Canadian Journal of Forest Research 8, 322-327.

Mamatha T, Lokesh S, Rai VR. 2000 - Impact of seed mycoflora of forest tree seeds on seed quality and their management. Seed Research 28, 59-67.

Manoharachary C, Agarwal DK, Rao NK. 2004 - A new anamorphic genus from India. Indian Phytopathology 57, 161-163.

Manoharachary C, Rao KM, Bhadraiah B. 1978 - Seed rot of Artocarpus integrifolia Linn. Geobios 5, 164. 
Marasas WFO, Rheeder JP, Logrieco A, van Wyk PS et al. 1998 - Fusarium nelsonii and F. musarum: two new species in section Arthrosporiella related to F. camptoceras. Mycologia 90, 505-513.

Marčiulynienė D, Davydenko K, Stenlid J, Shabunin D et al. 2017 - Fraxinus excelsior seed is not a probable introduction pathway for Hymenoscyphus fraxineus. Forest Pathology 48, e12392.

Marmolejo JG, Butin H. 1990 - New conifer-inhabiting species of Ophiostoma and Ceratocystiopsis (Ascomycetes, Microascales) from Mexico. Sydowia 42, 193-199.

Martín P. 1970 - Studies on the Xylariaceae. VIII. Xylaria and its allies. Journal of South African Botany 36, 73-137.

Mason GN, van Arsdel EP. 1978 - Fungi associated with Pinus taeda seed development. Plant Disease Reporter (USA) 62, 864-868.

Mathur SB. 1974 - Fungi recorded in seeds of forest tree species at Danish Government Institute of Seed Pathology. Copenhagen, Denmark.

Matsushima T. 1993 - Matsushima mycological memoirs 7. Matsushima Mycological Memoirs 7, $1-141$.

Mbenoun M, De Beer ZW, Wingfield MJ, Wingfield BD et al. 2014 - Reconsidering species boundaries in the Ceratocystis paradoxa complex, including a new species from oil palm and cacao in Cameroon. Mycologia 106, 757-784.

Medardi G. 2007 - Una nuova Peziza dall'Italia: Peziza sciophila. Rivista Micologia 50, 333-343.

Mehdi FS, Saifullah SM. 2000 - Species diversity and seasonal occurrence of fungi on seedlings of Avicennia marina (Forssk.) Vierh. (Forsk.) Vierh. Pakistan Journal of Biological Sciences 3, 265-268.

Miller MA, Pfeiffer W, Schwartz T. 2010 - Creating the CIPRES Science Gateway for inference of large phylogenetic trees. In Proceedings of the gateway computing environments workshop (GCE) 14 Nov 2010. Institute of Electrical and Electronics Engineers, New Orleans, LA 1-8.

Miller MW, Phaff HJ. 1962 - Successive microbial populations in Calimyrna figs. Applied Microbiology 10, 394-400.

Miller T, Bramlett DL. 1979 - Damage to reproductive structures of slash pine by two seed-borne pathogens: Diplodia gossypina and Fusarium moniliforme var. subglutinans. In Proceedings Flowering and Seed Development in Trees: A Symposium. 1978. USDA Forest Service 347355.

Millspaugh CF, Nuttall LW. 1923 - Flora of Santa Catalina Island 1-413.

Mindell RA, Stockey RA, Beard G, Currah RS. 2007 - Margaretbarromyces dictyosporus gen. sp. nov.: a permineralized corticolous ascomycete from the Eocene of Vancouver Island, British Columbia. Mycological Research 111, 680-684.

Minter DW. 1986 - Spring Foray 1985 - Watersfield, near Pulborough, West Sussex. 24-30 May 1985. Bulletin of the British Mycological Society 20, 82-88.

Mittal RK, Anderson RL, Mathur SB. 1990 - Mico-organisms associated with tree seeds, world checklist 1990. Petawawa National Forestry Institute, PI-X-96E.

Mittal RK, Sharma MR. 1981a - Seed mycoflora of Cassia fistula L. Indian Journal of Forestry 4, 70.

Mittal RK, Sharma MR. 1981b - Seed mycoflora of Dalbergia sisso Roxb. Environ. India 4, 9495.

Mittal RK, Sharma MR. 1982a - Seed microflora of Albezia lebbek Benth. Indian Journal of Forestry 5, 156-157.

Mittal RK, Sharma MR. 1982b - Studies of the mycoflora and its control on the seeds of some forest trees V. Pinus wallichiana. Indian Journal of Plant Pathology 12, 142-147.

Mittal RK, Sharma MR. 1982c - Studies on the mycoflora and its control on the seeds of some forest trees II. Shorea robusta. Indian Journal of Mycology and Plant Pathology 12, 170-174.

Mittal RK, Sharma MR. 1984 - Studies on the mycoflora and its control on the seeds of Eucalyptus citriodora Hook. Vegetational wealth of the Himalayas/editor, GS Paliwal 543-552. 
Mittal RK, Wang BSP. 1986 - Emergence failure and top decay in white spruce germinants due to three fungi. Canadian Plant Disease Survey 66, 5-7.

Mittal RK, Wang BSP. 1987 - Fungi associated with seeds of eastern white pine and white spruce during cone processing and seed extraction. Canadian Journal of Forest Research 17, 10261034.

Mittal RK, Wang BSP. 1993 - Effects of some seed-borne fungi on Picea glauca and Pinus strobes seeds. European Journal of Forest Pathology 23, 138-146.

Mittal RK. 1983 - Studies on the mycoflora and its control on the seeds of some forest trees. I. Cedrus deodara. Canadian Journal of Botany 61, 197-201.

Mittal RK. 1986 - Studies on the mycoflora and its control on the seeds of some forest trees: III. Eucalyptus hybrid. Studies 49, 151-159.

Mohanan C, Chacko KC, Chandran A, Varma G. 2005 - Seed health problems in tropical forest tree seeds and their impact on seedling production. Diseases and Insects in Forest Nurseries 83-93.

Morais PB, Martins MB, Klaczko LB, Mendonça-Hagler LC et al. 1995 - Yeast succession in the Amazon fruit Parahancornia amapa as resource partitioning among Drosophila spp. Applied and Environmental Microbiology 61, 4251-4257.

Moreau PA. 2003 - Mycena plumipes, un nom oublié pour une espèce bien connue. Bulletin Mycologique et Botanique Dauphiné-Savoie 171, 5-11.

Morgan-Jones G, White JF Jr, Piontelli EL. 1990 - Endophyte-host associations of forage grasses. XIII. Acremonium chilense, an undescribed endophyte occurring in Dactylis glomerata in Chile. Mycotaxon 39, 441-454.

Morris EF, Finley DE. 1956 - Tropical fungi imperfecti. Mycologia 48, 728-737.

Motta E, Annesi T, Balmas V. 1996 - Seedborne fungi in Norway spruce: testing methods and pathogen control by seed dressing. European Journal of Forest Pathology 26, 307-314.

Motta E, Saponaro A. 1983 - Mycoflora of the seeds of Cupressaceae. Annali dell Instituto Sperimentale per la Patología Vegetóle 8, 71-75.

Motta E. 1984 - Seiridium cardinale; establishment of the pathogen on seeds of Cupressaceae and possibilities of chemical control. Annali dell’ Istituto Sperimentale per la Patologia Vegetale Roma 9, 205-210.

Motta E. 1986 - Fungal pathogens of forest trees. Bulletin OEPP / EPPO Bulletin 16, 565-569.

Muñiz D, Hladun NL. 2007 - Mycocalicium llimonae, a new species from the Iberian Peninsula. The Lichenologist 39, 205-210.

Munjal RL, Sharma AD. 1975 - Mycoflora of conifer seeds. Indian Journal of Mycology and Plant Pathology 5, 145-148.

Nagao H, Ogawa S, Sato T, Kakishima M. 2003 - Exobasidium symploci-japonicae var. carpogenum var. nov. causing Exobasidium fruit deformation on Symplocos lucida in Japan. Mycoscience 44, 369-375.

Nagaraju D, Kunwar IK, Kumar GS, Manoharachary C. 2011a - A new synnematous hyphomycetous fungus Bhadradriella gen. nov. from India. Journal of Mycology and Plant Pathology 4, 238-240.

Nagaraju D, Kunwar IK, Kumar GS, Manoharachary C. 2011b - Custingophora lignicola sp. nov. and Chaetopsina indica sp. nov. from India. Journal of Mycology and Plant Pathology 41, 610.

Nagaraju D, Kunwar IK, Kumar GS, Manoharachary C. 2011c - Hyalocephalotrichum, a new hyphomycetous genus from India. Journal of Mycology and Plant Pathology 41, 589-591

Narula AM, Rawla GS, Kaushal SC. 1984 - Two new species of Xylaria (Pyrenomycetes) from India. Willdenowia 14, 409-411.

Natarajan S, Vanangamudi K, Jerlin R, Prabakar K. 2003 - Standardisation of seed health testing in Albizia lebbeck. Madras Agricultural Journal 90, 301-386.

Naz H, Shukla AN, Naz A, Pandey A et al. 2015 - Studies on fungi associated with Dalbergia sissoo Roxb. Ex. DC. Journal of Crop Science Research and Technology 1, 1-4. 
Neergaard P. 1945 - Danish species of Alternaria and Stemphylium. Taxonomy, parasiticism, economical significance. Einar Munksgaard, Copenhagen, Denmark.

Neergaard P. 1958 - The saprophytic fungus flora of some horticultural seed species. Science studies 201-207.

Neergaard P. 1977 - Quarantine for seed. Seed Pathology 681-711.

Neergaard P. 2017 - Seed pathology: Volumes 1 and 2. Macmillan International Higher Education.

Neill JC. 1941 - The endophytes of Lolium and Festuca. New Zealand Journal of Science and Technology 23, 1 85-193.

Nelson DL, Krebill RG. 1970 - Effect of Chrysomyxa pirolata cone rust on dispersal and viability of Picea pungens seeds. Phytopathology 60, 1305.

Nelson DL, Krebill RG. 1982 - Occurrence and effect of Chrysomyxa pirolata cone rust on Picea pungens in Utah. The Great Basin Naturalist 42, 262-272.

Nelson SC, Abad ZG. 2010 - Phytophthora morindae, a new species causing black flag disease on noni (Morinda citrifolia L.) in Hawaii. Mycologia 102, 122-134.

Nik WZ, Parbery DG. 1977 - Studies of seed-borne fungi of tropical pasture legume species. Australian Journal of Agricultural Research 28, 821-841.

Niks RE. 1987 - Nonhost plant species as donors for resistance to pathogens with narrow host range I. Determination of nonhost status. Euphytica 36, 841-852.

Nirenberg HI, Aoki T. 1997 - Fusarium nisikadoi, a new species from Japan. Mycoscience 38, 329-333.

Noble M, De Temple J, Neergaard P. 1958 - An annotated list of seed-borne diseases. Commonwealth Mycological Institute, UK.

Nong Y, Zhuang WY. 2005 - Preliminary survey of Bionectriaceae and Nectriaceae (Hypocreales, Ascomycetes) from Jigongshan, China. Fungal Diversity 19, 95-107.

Norphanphoun C, Doilom M, Daranagama DA, Phookamsak R. et al. 2017 - Revisiting the genus Cytospora and allied species. Mycosphere 8, 51-97.

O’Donnell K, Cigelnik E. 1997 - Two divergent intragenomic rDNAITS2 types within a monophyletic lineage of the fungus Fusarium are nonorthologous. Molecular Phylogenetics and Evolution 7, 103-116.

O'Donnell K, Kistler HC, Cigelnik E, Ploetz RC. 1998 - Multiple evolutionary origins of the fungus causing Panama disease of banana: Concordant evidence from nuclear and mitochondrial gene genealogies: In Proceedings of the National Academy of Science of the United States of America 95, 2044-2049.

Oliva J, Boberg J, Stenlid J. 2013 - First report of Sphaeropsis sapinea on Scots pine (Pinus sylvestris) and Austrian pine (P. nigra) in Sweden. New Disease Reports 27, 23

Oliver FW. 1903 - Notes on fossil fungi. New Phytologist 2, 49-53.

Ono K. 1974 - Studies on'Ezo-raigan'disease, sclerotium germination-loss. of Todo-fir seeds. Bulletin of the Government Forest Experiment Station (Japan) 268, 49-80.

Ono Y, Hosoya T. 2001 - Hyaloscyphaceae in Japan (5): some Lachnum-like members. Mycoscience 42, 611-622.

Oskay F, Imal B, Orhan F, Meşe Ö et al. 2018 - Preliminary results on the fungi damaging pedunculate oak (Quercus Robur L.) acorns. Conference: Diseases and Insects in Forest Nurseries 7.03.04 Working Party Meeting 21-26.

Osono T. 2011 - Diversity and functioning of fungi associated with leaf litter decomposition in Asian forests of different climatic regions. Fungal Ecology 4, 375-385.

Osono T, Mori A. 2003 - Colonization of Japanese beech leaves by phyllosphere fungi. Mycoscience 44, 437-441.

Osorio JA, Wingfield MJ, de Beer ZW, Roux J. 2015 - Pseudocercospora mapelanensis sp. nov., associated with a fruit and leaf disease of Barringtonia racemosa in South Africa. Australasian Plant Pathology 44, 349-359. 
Paden JW, Sutherland JR, Woods TAD. 1978 - Caloscypha fulgens (Ascomycetidae, Pezizales): the perfect state of the conifer seed pathogen Geniculodendron pyriforme (Deuteromycotina, Hyphomycetes). Canadian Journal of Botany 56, 2375-2379.

Padovan ACB, Sanson GF, Brunstein A, Briones MR. 2005 - Fungi evolution revisited: application of the penalized likelihood method to a Bayesian fungal phylogeny provides a new perspective on phylogenetic relationships and divergence dates of Ascomycota groups. Journal of Molecular Evolution 60, 726-735.

Palm ME. 2001 - Systematics and the impact of invasive fungi on agriculture in the United States. BioScience 51, 141-147.

Pande A, Waingankar V. 2004 - Four new taxa in Xylaria from Western India. Journal of Economic and Taxonomic Botany 28, 610-613.

Patil MS, Yadav US, Patil SD. 1991 - Contribution to the leaf litter fungi from Maharashtra II. Indian Phytopath 44, 308-313.

Paula LE, Trugilho PF, Napoli A, Bianchi ML. 2011 - Characterization of residues from plant biomass for use in energy generation. Cerne 17, 237-46.

Pawuk WH. 1978 - Damping off of container grown longleaf pine seedlings by seedborne Fusaria. Plant Disease Reporter 62, 82-84.

Penzig AJO, Saccardo PA. 1897 - Diagnoses fungorum novorum in insula Java collectorum. Ser. II. Malpighia 11, 491-530.

Perera RH, Hyde KD, Dissanayake AJ, Jones EBG et al. 2018a - Diaporthe collariana sp. nov., with prominent collarettes associated with Magnolia champaca fruits in Thailand. Studies in Fungi 3, 141-151.

Perera RH, Hyde KD, Jones EBG, Liu JK et al. 2018b - Additions to wild seed and fruit fungi 2: Parascedosporium putredinis: a new Thailand record from Delonix regia seed pods. Studies in Fungi 3, 192-201.

Perera RH, Hyde KD, Peršoh D, Jones EBG et al. 2018c - Additions to wild seed and fruit fungi 1: The sexual morph of Diaporthe rosae on Magnolia champaca and Senna siamea fruits in Thailand. Mycosphere 9, 256-270.

Perera RH, Maharachchikumbura SS, Ariyawansa H, Bahkali AH et al. 2016a - Two new Pseudohalonectria species on beech cupules (Fagus sylvatica) and a new genus to accommodate $P$. suthepensis. Phytotaxa 278, 115-31.

Perera RH, Maharachchikumbura SSN, Bhat JD, Al-Sadi AM et al. 2016b - New species of Thozetella and Chaetosphaeria and new records of Chaetosphaeria and Tainosphaeria from Thailand. Mycosphere 7, 1301-1321.

Perera RH, Maharachchikumbura SSN, Jones EBG, Bahkali et al. 2017 - Delonicicola siamense gen. and sp. nov. (Delonicicolaceae fam. nov., Delonicicolales ord. nov.), a saprobic species from Delonix regia seed pods. Cryptogamie, Mycologie 38, 321-340.

Petrak F. 1953 - Beiträge zur Kenntnis der Pilzflora Irans. Sydowia 7, 50-78.

Petrini O. 1996 - Ecological and physiological aspects of host specificity in endophytic fungi. In endophytic fungi in grasses and woody plants. American Phytopathological Society Press: St Paul MN 87-100.

Phillips AJ, Alves A, Abdollahzadeh J, Slippers B et al. 2013 - The Botryosphaeriaceae: genera and species known from culture. Studies in Mycology 76, 51-167.

Phookamsak R, Hyde KD, Jeewon R, Bhat DJ et al. 2019 - Fungal diversity notes 929-1035: taxonomic and phylogenetic contributions on genera and species of fungi. Fungal Diversity 95, 1-273.

Piepenbring M. 2006 - PB Checklist of Fungi in Panama. Puente Biológico 1, 1-96.

Pirozynski KA, Dalpé Y. 1989 - Geological history of the Glomaceae with particular reference to mycorrhizal symbiosis. Symbiosis 7, 1-36. 
Pointing SB, Pelling AL, Smith GJD, Hyde KD et al. 2005 - Screening of basidiomycetes and xylariaceous fungi for lignin peroxidase and laccase gene-specific sequences. Mycological Research 109, 115-124.

Preuss CG. 1855 - Übersicht untersuchter Pilze, besonders aus der Umgegend von Hoyerswerda (Fortsetzung). Linnaea 26, 705-725.

Prihastuti H, Cai L, Chen H, McKenzie EH et al. 2009 - Characterization of Colletotrichum species associated with coffee berries in northern Thailand. Fungal Diversity 39, 89-109.

Printzen C. 2001 - Corticolous or lignicolous species of Lecanora (Lecanoraceae, Lecanorales) with usnic or isousnic acid in the Sonoran Desert region. Bryologist 104, 382-409.

Prisyazhnyuk AA. 1960 - Fungal diseases of seeds and cones of conifers. Lesnoi Zhurnal, Arkhangel'sk 3, 31-37.

Priya KS, Nagveni HC. 2012 - A new species Penicilliopsis indicus, holomorph of Sarophorum on seeds of Dysoxylum malabaricum. Indian Phytopathology 65, 312-313.

Priya SW, Nagaveni HC, Kunwar IK, Manoharachary IK. 2011 - A new pathogenic species of Beltraniella from India. Journal of Mycology and Plant Pathology 41, 20.

Quiniones SS. 1985 - List of tree seed borne diseases. Unnumbered report. Philippines Forest Research Institute, Laguna.

Quiniones SS. 1987 - Fungi associated with forest tree seeds in the Philippines. Journal of Seed Technology 1, 144-450.

Ragaee S, Abdel-Aal ES, Noaman M. 2006 - Antioxidant activity and nutrient composition of selected cereals for food use. Food Chemistry 98, 32-38.

Raitviír A, Galán R. 1993 - Notes on Spanish glassy-haired Hyaloscyphaceae. Sydowia 45, 34-54.

Raitviír A, Galán R. 1995 - The genus Polydesmia in Spain. Mycotaxon 53, 447-454.

Rashmi M, Kushveer JS. Sarma VV. 2019 - A world-wide list of endophytic fungi with notes on ecology and diversity. Mycosphere 10, 798-1079.

Rayachhetry MB, Webb RS, Kimbrough JW, Miller T. 1995a - Haustorial morphology of Cronartium conigenum in naturally infected cones of three Pinus species from Guatemala. European Journal of Forest Pathology 25, 152-158.

Rayachhltry MB, Webb RS, Miller T, Kimbrough JW. 1995b - Histology of Pinus maximinoi cones infected by Cronartium conigenum. Forest Pathology 25, 100-108.

Rayner ADM, Boddy L. 1988 - Fungal decomposition of wood: its biology and ecology. Wiley, Chichester.

Rayner RW. 1970 - A mycological colour chart. Commonwealth Mycological Institute and British Mycological Society. Kew.

Reddy BS, Sehgal HS, Manoharachary C. 1982 - Studies on seed mycoflora of certain species of Eucalyptus. Acta Botanica Indica 10, 302-303.

Rediske JH, Shea KR. 1965 - Loss of Douglas-fir seed viability during cone storage. Forest Science 11, 463-472.

Rees AA. 1982 - Tree seed pathology - Overseas Development Administration Contract. Report on Forest Research 28-29.

Rees AA. 1988 - Infection of Pinus caribaea seed by Lasiodiplodia theobromae. Transactions of the British Mycological Society 90, 321-324.

Rees AA, Webber JF. 1988 - Pathogenicity of Sphaeropsis sapinea to seed, seedlings and saplings of some Central American pines. Transactions of the British Mycological Society 91, 273277.

Rehm H. 1901 - Beiträge zur Pilzflora von Südamerika. XIII. Xylariaceae. Hedwigia 40, 141-170.

Rehner S. 2001 - Primers for Elongation Factor 1-a (EF1-a) http://ocid.nacse.org/research/deephyphae/EF1primer.pdf

Rehner SA, Samuels GJ. 1994 - Taxonomy and phylogeny of Gliocladium analyzed from nuclear large subunit ribosomal DNA-sequences. Mycological Research 98, 625-34. 
Renault B, Bertrand CE. 1895 - Premieres observations sur des bacteries coprophiles de l'epoque permienne. The French Association for the Advancement of Science. Paris, $23^{\text {rd }}$ session. Notes and Memory 583-587.

Révay Á. 1987 - New or interesting hyphomycetes on forest litter from Hungary. Acta Botanica Hungarica 33, 67-73.

Ribeiro LR, Santos ARO, Groenewald M, Smith MTH et al. 2017 - Description of Hyphopichia buzzinii f.a., sp. nov. and Hyphopichia homilentoma comb. nov., the teleomorph of Candida homilentoma. Antonie van Leeuwenhoek 110, 985-994

Richardson MJ. 1979 - An annotated list of seed-borne diseases. 3rd ed. International Seed Testing Association, Zurich, Switzerland.

Richardson MJ. 1983 - An annotated list of seed-borne diseases. Supplement 2. An annotated list of seed-borne diseases. 3rd ed. International Seed Testing Association, Zurich, Switzerland.

Robich G. 2003 - Mycena d'Europa 728.

Robich G. 2016 - Mycena d'Europa 2, 732-1527.

Rodriguez RJ, White Jr JF, Arnold AE, Redman AR. 2009 - Fungal endophytes: diversity and functional roles. New Phytologist 182, 314-330.

Rogers JD. 1979a - Xylaria magnoliae sp. nov. with comments on several other fruit inhabiting species. Canadian Journal of Botany 57, 941-945.

Rogers JD. 1979b - The Xylariaceae: systematic, biological and evolutionary aspects. Mycologia 71, 1-42.

Rogers JD, San Martín F, Ju Y-M. 2002 - A reassessment of the Xylaria on Liquidambar fruits and two new taxa on Magnolia fruits. Sydowia 54, 91-97.

Rogers JD, Yeomans R, Adams MJ. 2008 - The relationship of Xylaria oxyacanthae to seeds of Crataegus monogyna. North American Fungi 3, 1-5.

Rogers JD, Yu YM, Hemmes DE. 1992 - Hypoxylon rectangulosporum sp. nov., Xylaria psidii sp. nov., and comments on taxa of Podosordaria and Stromatoneurospora. Mycologia 84, 166172.

Ronquist F, Huelsenbeck JP. 2003 - MRBAYES 3: Bayesian phylogenetic inference under mixed models. Bioinformatics 19, 1572-1574.

Rossman AY, Castlebury LA, Farr DF, Stanosz GR. 2008 - Sirococcus conigenus, Sirococcus piceicola sp. nov. and Sirococcus tsugae sp. nov. on conifers: anamorphic fungi in the Gnomoniaceae, Diaporthales. Forest Pathology 38, 47-60.

Rossman AY, Samuels GJ, Rogerson CT, Lowen R. 1999 - Genera of Bionectriaceae, Hypocreaceae and Nectriaceae (Hypocreales, Ascomycetes). Centraalbureau voor Schimmelcultures (CBS).

Rowan SJ, Debarr GL. 1974 - Moldy seed and poor germination linked to seedbug damage in slash pine. Tree planters' notes 25, 25-27.

Ruivo CCC, Lachance MA, Bacci Jr M, Rosa CA et al. 2004 - Candida leandrae, an asexual ascomycetous yeast species isolated from tropical plants. International Journal of Systematic and Evolutionary Microbiology 54, 2405-2408.

Rycyk Jr F, Sharpe D. 1984 - Infection of tall fescue seed in Missouri by the endophyte Epichloë typhina. Plant Disease 68, 1099.

Saccardo PA. 1878 - Fungi Veneti novi vel critici vel mycologiae Venetae addendi. Series VIII. Michelia 1, 239-275.

Sahai A, Otra BSM. 1982 - Mycoflora associated with the seeds of forest trees and their effect on germination. Proceedings of the Indian National Science Academy 48, 706-713.

Sahashi N, Kubono T, Miyasawa Y, Ito S. 1999 - Temporal variations in isolation frequency of endophytic fungi of Japanese beech. Canadian Journal of Botany 77, 197-202.

Sahu RK, Agarwal VK, Sachan IP. 2003 - Detection of fungi associated with the seeds of gulmohur (Delonix regia Boj. Hook.) Raf., Bioved 14, 37-40. 
Saikkonen K, Ion D, Gyllenberg M. 2002 - The persistence of vertically transmitted fungi in grass metapopulations. Proceedings of the Royal Society of London. Series B: Biological Sciences 269, 1397-1403.

Salgado-Salazar C, Rossman AY, Chaverri P. 2016 - The genus Thelonectria (Nectriaceae, Hypocreales, Ascomycota) and closely related species with cylindrocarpon-like asexual states. Fungal Diversity 80, 411-455.

Salisbury PJ. 1955 - Molds of stored douglas fir seed in British Columbia. Government of Canada, Department of Northern Affairs and National Resources, Forestry Branch, Victoria, BC.

Salt GA. 1964 - Pathology experiments on Sitka spruce seedlings. Report on Forest Research for 89-95.

Salt GA. 1974 - Etiology and morphology of Geniculodendron pyriforme gen. et sp. nov., a pathogen of conifer seeds. Transactions of the British Mycological Society 63, 339-351.

Samson RA, Seifert KA. 1986 - The ascomycete genus Penicilliopsis and its anamorphs. In Advances in Penicillium and Aspergillus systematics, Springer, Boston, MA 397-428

Samson RA, Stolk AC, Frisvad JC. 1989 - Two new synnematous species of Penicillium. Studies in Mycology 31, 133-144.

Samuels GJ, Barr ME, Rogerson CT. 1988 - Xenomeris saccifolii and Gibbera sphyrospermi, new tropical species of the Venturiaceae (Fungi, Pleosporales). Brittonia 40, 392-397.

Samuels GJ, Doi Y, Rogerson CT. 1990 - Hypocreales. Memoirs of the New York Botanical Garden 59, 6-108.

Samuels GJ, Dumont KP. 1982 - The genus Nectria (Hypocreaceae) in Panama. Caldasia 13, 379423.

San Martín F, Lavín P, Rogers JD. 2001 - Some species of Xylaria (Hymenoascomycetes, Xylariaceae) associated with oaks in México. Mycotaxon 79, 337-360.

San Martín González F, Rogers JD. 1989 - A preliminary account of Xylaria of Mexico. Mycotaxon 34, 283-373.

Sandoval-Chávez RA, Martínez-Peniche RÁ, Hernández-Iturriaga M, Fernández-Escartín E et al. 2011 - Postharvest biological and chemical control of Fusarium stilboides on bell pepper. Revista Chapingo. Serie Horticultura 17, 161-172.

Sandoval-Denis M, Crous PW. 2018 - Removing chaos from confusion: assigning names to common human and animal pathogens in Neocosmospora. Persoonia: Molecular Phylogeny and Evolution of Fungi 41, 109-129.

Sandoval-Denis M, Guarnaccia V, Polizzi G, Crous PW. 2017 - Symptomatic Citrus trees reveal a new pathogenic lineage in Fusarium and two new Neocosmospora species. Persoonia 40, 125.

Saponaro A, Motta E. 1984 - Seiridium cardinale and other fungus species on seeds of Cupressaceae. Report, Commission of the European Communities 57-63.

Sarmiento C, Zalamea PC, Dalling JW, Davis AS et al. 2017 - Soilborne fungi have host affinity and host-specific effects on seed germination and survival in a lowland tropical forest. Proceedings of the National Academy of Sciences 114, 11458-11463.

Sato K, Shoji T. 1960 - Primary infection of anthracnose on black locust seedlings caused by the seed transmission of Guignardia robinae K. Ito et T. Kobayahi and the control by seed treatments. Bulletin of the Government Forest Experiment Station, Ringyo Shikenjo Kenkyu Hokoku 119, 1-15.

Saxena RM. 1985 - Seedling mortality of Eucalyptus spp. caused by seed mycoflora. Indian Phytopathology 38, 151-152.

Schmidt JC, Kunze G. 1823 - Mykologische Hefte 2, 1-176.

Schmitt JP, Shearer CA. 2003 - A checklist of mangrove-associated fungi, their geographical distribution and known host plants. Mycotaxon 85, 423-477.

Schröder T, Kehr R, Hüttermann A. 2002 - First report of the seed-pathogen Geniculodendron pyriforme, the imperfect state of the ascomycete Caloscypha fulgens, on imported conifer seeds in Germany. Forest Pathology 32, 225-230. 
Schroder T, Kehr R, Prochazkova Z, Sutherland JR. 2004 - Practical methods for estimating the infection rate of Quercus robur acorn seedlots by Ciboria batschiana. Forest Pathology 34, 187-196.

Schroers H-J, Samuels GJ, Seifert KA, Gams W. 1999 - Classification of the mycoparasite Gliocladium roseum in Clonostachys as C. rosea, its relationship to Bionectria ochroleuca, and notes on other Gliocladium-like fungi. Mycologia 91, 365-385.

Schroers HJ. 2001 - A monograph of Bionectria (Ascomycota, Hypocreales, Bionectriaceae) and its Clonostachys anamorphs. Studies in Mycology 46, 1-214.

Schubert GH. 1961 - Fungi associated with viability losses of sugar pine seed during cold storage. In Proceedings of the Society of American Foresters.

Schüßler A, Schwarzott D, Walker C. 2001 - A new fungal phylum, the Glomeromycota: phylogeny and evolution. Mycological Research 105, 1413-1421.

Seaver FJ. 1909 - The Hypocreales of North America-II. Mycologia 1, 117-207.

Seena S, Sridhar KR. 2004 - Endophytic fungal diversity of 2 sand dune wild legumes from the southwest coast of India. Canadian Journal of Microbiology 50, 1015-1021.

Seifert KA, Boulay H. 2004 - Hirsutella uncinata, a new hyphomycete from Australia. Mycologia 96, 929-934

Selosse MA, Strullu-Derrien C, Martin FM, Kamoun S et al. 2015 - Plants, fungi and oomycetes: a 400-million-year affair that shapes the biosphere. New Phytologist 206, 501-506.

Shang QJ, Hyde KD, Camporesi E, Maharachchikumbura SSN 2020 - Additions to the genus Cytospora with sexual morph in Cytosporaceae. Mycosphere 11, 189-224.

Sharma JK, Mohanan C. 1980 - Spermoplane microflora of stored seeds of Tectona grandis, Bombax ceiba and Eucalyptus spp. in relation to germinability. In Proceedings of the International Symposium on Forest Tree Seed Storage.

Sharma MP. 1991 - Diversity in the Himalayan Hymenoscyphus S.F. Gray: an overview. In Khullar SP Sharma MP (eds), Himalayan Botanical Researches, New Delhi: Ashish Publishing House 107-211.

Sharma S, Thakur M. 2007 - Role of plant quarantine in the management of pest organisms - A review. Agricultural Reviews 28, 235-244.

Shen XY, Cheng YL, Cai CJ, Fan L et al. 2014 - Diversity and antimicrobial activity of culturable endophytic fungi isolated from moso bamboo seeds PloS one 9, e95838.

Shigo AL, Yelenosky G. 1963 - Fungus and insect injury to yellow birch seeds and seedlings. Northeastern Forest Experiment Station, Forest Service, US Department of Agriculture.

Shimada T. 2001 - Nutrient compositions of acorns and horse chestnuts in relation to seedhoarding. Ecological Research 16, 803-808.

Shivas RG, Vánky K. 2007 - Centrolepidosporium sclerodermum, gen. et sp. nov. (Ustilaginomycetes) from Australia. Mycologia Balcanica 4, 1-4.

Simmons EG. 1997 - Alternaria themes and variations (151-223). Mycotaxon 65, 1-91.

Simmons EG. 2002 - Alternaria themes and variations (287-304). Species on Caryophyllaceae. Mycotaxon 82, 1-40.

Simmons EG. 2007 - Alternaria: an identification manual. CBS Biodiversity Series 6, 1-775.

Singer R. 1989 - New taxa and new combinations of Agaricales (Diagnoses fungorum novorum Agaricalium 4). Fieldiana Botany 21, 1-133.

Singh P, Mittal RK. 1989 - Influence of seed-borne fungi on the nutrient composition and growth of conifer seedlings. European Journal of Forest Pathology 19, 65-77.

Singh S, Khan SN, Misra BM. 1983 - Gummosis, brown spot and seedling mortality in Su-babul 2. Epidemiology and Control of the Disease. Indian Forester 109, 810-821.

Singh S, Verma VP, Suri RK. 1979 - Protection of sal seeds in storage against moulds. Indian Forester 105, 811-815.

Sipiczki M. 2014 - Metschnikowia laotica f.a., sp. nov., a dimorphic, pigment-producing yeast species isolated from fruit. International Journal of Systematic and Evolutionary Microbiology 64, 1847-1852. 
Smith H, Wingfield MJ, Coutinho TA, Crous PW. 1996 - Sphaeropsis sapinea and Botryosphaeria dothidea endophytic in Pinus spp. and Eucalyptus spp. in South Africa. South African Journal of Botany 62, 86-88.

Smith H, Wingfield MJ, De Wet J, Coutinho TA. 2000 - Genotypic diversity of Sphaeropsis sapinea from South Africa and Northern Sumatra. Plant Disease 84, 139-142.

Smoot JJ, Segall RH. 1963 - Hot water as a postharvest control of mango anthracnose. Plant Disease Reporter 47, 739-742.

Somrithipol S, Chatmala I, Jones EBG. 2002a - Cirrenalia nigrospora sp. nov. and C. tropicalis from Thailand. Nova Hedwigia 75, 477-485.

Somrithipol S, Jones EBG, Hywel-Jones NL. 2002b - Fungal diversity and succession on seed pods of Delonix regia (Leguminosae) exposed in a tropical forest in Thailand. Fungal Diversity 10, 131-139.

Somrithipol S, Jones GEB. 2007 - Lauriomyces cylindricus and Lauriomyces ellipticus spp. nov., two new hyphomycetes from tropical forest of Thailand. Nova Hedwigia 84, 479-486.

Somrithipol S, Kosol S, Jones EBG. 2006 - Lauriomyces sakaeratensis sp. nov., a new hyphomycete on decaying Dipterocarpus costatus fruits from Sakaerat Biosphere Reserve, Thailand. Nova Hedwigia 82, 209-215.

Song Y, Tangthirasunun N, Maharachchikunmbura SSN, Jiang YL et al. 2014 - Novel Pestalotiopsis species from Thailand point to the rich undiscovered diversity of this chemically creative genus. Cryptogamie, Mycologie 35, 139-149.

Spiers GA, Wenilam HT. 1983 - Fungicidal control of Marssonina brunnea on poplar seed. European Journal of Forest Pathology 13, 344-348.

Spooner BM. 1987 - A new Coccomyces (Rhytismataceae) from Picea cones in Austria. Transactions of the British Mycological Society 88, 426-428.

Srinivasan KV. 1952 - Seedling blight of Sesbania grandiflora Pers. Current Science 21, 318-318.

Srivastava SNS. 1956a - A new species of Haplosporella on Dryobalanops aromatica Gaertn. Sydowia 10, 236-238.

Srivastava SNS. 1956b -Two fungal infections of Hevea seeds. Current Science 28, 157-158.

Srivastava SNS. 1964 - Seed rot of Hevea brasiliensis caused by Botryodiplodia theobromae Pat. Indian Phytopathology 17, 172-177.

Stakhov VL, Gubin SV, Maksimovich SV, Rebrikov DV et al. 2008 - Microbial communities of ancient seeds derived from permanently frozen Pleistocene deposits. Microbiology 77, 348355.

Stamatakis A. 2006 - RAxML-VI-HPC: maximum likelihood-based phylogenetic analyses with thousands of taxa and mixed models. Bioinformatics 22, 2688-2690.

Stohlgren TJ. 1988 - Litter dynamics in two Sierran mixed conifer forests. I. Litterfall and decomposition rates. Canadian Journal of Forest Research 18, 1127-1135.

Storer AJ, Gordon TR, Clark SL. 1998 - Association of the pitch canker fungus, Fusarium subglutinans f.sp. pini, with Monterey pine seeds and seedlings in California. Plant Pathology 47, 649-656.

Surup F, Narmani A, Wendt L, Pfütze S et al. 2018 - Identification of fungal fossils and novel azaphilone pigments in ancient carbonised specimens of Hypoxylon fragiforme from forest soils of Châtillon-sur-Seine (Burgundy). Fungal Diversity 92, 345-356.

Sutherland JR. 1979 - The pathogenic fungus Caloscypha fulgens in stored conifer seeds in British Columbia and relation of its incidence to ground and squirrel-cache collected cones. Canadian Journal of Forest Research 9, 129-132.

Sutherland JR, Diekmann M, Berjak P. 2002 - Forest tree seed health for germplasm conservation. IPGRI Technical Bulletin no. 6.

Sutherland JR, Hopkinson SJ, Farris SH. 1984 - Inland spruce cone rust, Chrysomyxa pirolata, in Pyrola asarifolia and cones of Picea glauca, and morphology of the spore stages. Canadian Journal of Botany 62, 2441-2447. 
Sutherland JR, Lock W, Farris SH. 1981 - Sirococcus blight: a seed-borne disease of containergrown spruce seedlings in coastal British Columbia forest nurseries. Canadian Journal of Botany 59, 559-562.

Sutherland JR, Woods TA. 1978 - The fungus Geniculodendron pyriforme in stored Sitka spruce seeds: effects of seed extraction and cone collection methods on disease incidence. Phytopathology 68, 747-750.

Sutton BC 1981 - Sarcopodium and its synonyms. Transactions of the British Mycological Society 76, 97-102.

Suwannasai N, Rodtong S, Thienhirun S, Whalley AJS. 2006 - New species and phylogenetic relationships of Hypoxylon species found in Thailand inferred from the internal transcribed spacer regions of ribosomal DNA sequences. Mycotaxon 94, 303-324.

Suzuki W, Osumi K, Masaki T. 2005 - Mast seedling and its spatial scale in Fagus crenata in northern Japan. Forest Ecology and Management 205, 105-116.

Svrcek M. 1986 - New or less known Discomycetes. XIV. Ceská Mykologie 40, 203-217.

Svrcek M. 1987 - New or less known Discomycetes. XV. Ceská Mykologie 41, 16-25.

Svrcek M. 1989 - New or less known Discomycetes. XX. Ceská Mykologie 43, 215-226.

Takahashi A, Ichihara Y, Isagi Y, Shimada T. 2010 - Effects of acorn tannin content on infection by the fungus Ciboria batschiana. Forest Pathology 40, 96-99.

Takahashi H. 2001 - Notes on new Agaricales of Japan 2. Mycoscience 42, 347-353.

Takahashi Y, Ichihashi Y, Sano T, Harada Y. 2005 - Monilinia jezoensis sp. nov. in the Sclerotiniaceae, causing leaf blight and mummy fruit disease of Rhododendron kaempferi in Hokkaido, Northern Japan. Mycoscience 46, 106-109.

Talgø V, Brodal G, Klemsdal SS, Stensvand A. 2010 - Seed borne fungi on Abies spp. Seed science and Technology 38, 477-493.

Tanda S. 1985 - Mycological studies on the ergot in Japan (XXI). A new species of Claviceps parasitic on Arundinella hirta (Thunb.) C. Tanaka. Journal of Agricultural Science Tokyo Nogyo Daigaku 30, 94-99.

Tanda S. 1991 - Mycological studies on the ergot in Japan (XXVI). A new ergot, Claviceps bothriochloae parasitic on Bothriochloa parviflora. Journal of Agricultural Science Tokyo Nogyo Daigaku 36, 36-42.

Tanda S, Harada Y. 1989 - Mycological studies on the ergot in Japan (XXII). A new ergot parasitic on Isachne globosa. Transactions of the Mycological Society of Japan 30, 105-109.

Tang AM, Corlett RT, Hyde KD. 2005 - The persistence of ripe fleshy fruits in the presence and absence of frugivores. Oecologia 142, 232-237.

Tang AMC, Hyde KD, Corlett RT. 2003a - Diversity of fungi on wild fruits in Hong Kong. Fungal Diversity 14, 165-185.

Tang AMC, Hyde KD, Tsui KM, Corlett RT. 2003b - A new species of Lophiotrema from wild fruit in Hong Kong. Persoonia 18, 265-269.

Tanner C. 1997 - Principles of Australian quarantine. Australian Journal of Agricultural and Resource Economics 41, 541-558.

Tateno O, Hirose D, Osono T, Takeda H. 2015 - Beech cupules share endophytic fungi with leaves and twigs. Mycoscience 56, 252-256.

Taylor TN, Hass H, Kerp H. 1999 - The oldest fossil ascomycetes. Nature 399, 648.

Taylor TN, Hass H, Kerp H, Krings M et al. 2005 - Perithecial ascomycetes from the 400 million years old Rhynie chert: an example of ancestral polymorphism. Mycologia 97, 269-285.

Taylor TN, Krings M, Taylor EL. 2014 - Fossil fungi. Academic Press.

Taylor TN, Taylor EL, Krings M. 2009 - Palaeobotany. The biology and evolution of fossil plants. Elsevier publication, Amsterdam.

Tibpromma S, Hyde KD, Jeewon R, Maharachchikumbura SSN et al. 2017 - Fungal diversity notes 491-602: taxonomic and phylogenetic contributions to fungal taxa. Fungal Diversity 83, 1261. 
Tibpromma S, Hyde KD, McKenzie EH, Bhat DJ et al. 2018 - Fungal diversity notes 840-928: micro-fungi associated with Pandanaceae. Fungal Diversity 93, 1-60.

Tillman-Sutela E, Kauppi A, Hilli A, Kaitera J. 2004 - Fungal injury to seed tissues of Norway spruce, Picea abies (L.) Karst. Trees 18, 151-156.

To LV, Ngu N, Duc ND, Trinh DT et al. 1999 - Quality assurance system for dragon fruit, ACIAR proceeding, no.100, 101-114.

Torp M, Nirenberg HI. 2004 - Fusarium langsethiae sp. nov. on cereals in Europe. International Journal of Food Microbiology 95, 247-256.

Torruella G, Derelle R, Paps J, Lang BF et al. 2012 - Phylogenetic relationships within the opisthokonta based on phylogenomic analyses of conserved single-copy protein domains. Molecular Biology and Evolution 29, 531-544.

Trierveiler-Pereira L, Romero AI, Baltazar JM, Loguercio-Leite C. 2009 - Addition to the knowledge of Xylaria (Xylariaceae, Ascomycota) in Santa Catarina, Southern Brazil. Mycotaxon 107, 139-156.

Trindade RC, Resende MA, Pimenta RS, Lachance MA et al. 2004 - Candida sergipensis, a new asexual yeast species isolated from frozen pulps of tropical fruits. Antonie van Leeuwenhoek 86, 27-32.

Tubaki K, Booth C, Harada T. 1976 - A new variety of Fusarium merismoides, Transactions of the British Mycological Society 66, 355-356.

Turchetti T. 1982 - Antagonism of some Bacillus species to a Rhizoctonia solani Kuhn isolate and its effect on the germination of Pinus nigra Arn. seed. European Journal of Forest Pathology 12, 36-41.

Turner PD. 1981 - Oil palm diseases and disorders. Oxford University Press. Kuala Lumpur Malaysia.

Udayanga D, Castlebury LA, Rossman AY, Chukeatirote E et al. 2015 - The Diaporthe sojae species complex, phylogenetic re-assessment of pathogens associated with soybean, cucurbits and other field crops. Fungal Biology 119, 383-407.

Udayanga D, Castlebury LA, Rossman AY, Hyde KD 2014 - Species limits in Diaporthe: molecular re-assessment of D-citri, D-cytosporella, D-foeniculina and D-rudis. Persoonia 32, 83-101.

Udayanga D, Manamgoda DS, Liu X, Chukeatirote E et al. 2013 - What are the common anthracnose pathogens of tropical fruits? Fungal Diversity 61, 165-179.

Urosevic B. 1959 - The influence of pests and fungus disease on acorn harvest. Communicationes Instituti Forestalis Čechosloveniae 1, 39-54.

Urosevic B. 1961 - The influence of saprophytic and semi-parasitic fungi on the germination of Norway spruce and Scot pine seeds. In Proceedings of the International Seed Testing Association 6, 537-555.

Urosevic B. 1962 - Diseases of acorns found in Czechoslovakia. In Proceedings of $15^{\text {th }}$ World Forestry Congress, Seattle, Washington 910-911.

Urosevic B. 1964 - More important seed borne diseases of Czechoslovak forest trees. In FAOIUFRO Symposium on Internationally Dangerous Forest Diseases and Insects, at Oxford.

Urosevic B. 1983 - Tracheomycotic diseases in oak. Communicationes Instituti Forestalis Cechosloveniae 13, 85-100.

Vaartaja O, Wilner J, Cram WH, Salisbury PJ et al. 1964 - Fungicide trials to control damping-off of conifers. Plant Disease Reporter 48, 12-15.

Vaasma M, Kalamees K, Raitviír A. 1986 - Macrofungi of the caucasian state nature reserve. Scripta Mycologica Tartu 13, 1-106.

Vánky K, Berner D. 2003 - Microbotryum silybum sp. nov. Mycotaxon 85, 307-311.

Vánky K, Guo L. 1987 - Ustilaginales from China. Acta Mycologica Sinica, Supplement 1, 227250.

Vánky K, Iqbal SH, Khalid AN. 2007 - Thecaphora pakistanica sp. nov. (Ustilaginomycetes) on Androsace (Primulaceae). Mycologia Balcanica 4, 83-85. 
Vánky K, Salo V. 2011 - Two new species of Anthracoidea (Ustilaginales) on Carex from North America. Mycologia Balcanica 7, 105-109.

Vánky K, Shivas RG, Athipunyakom P. 2006 - New smut fungi (Ustilaginomycetes) from Thailand. Mycologia Balcanica 3, 107-118.

Vánky K, Shivas RG. 2003 - Further new smut fungi (Ustilaginomycetes) from Australia. Fungal Diversity 14, 243-264.

Vánky K, Shivas RG. 2006 - The smut fungi (Ustilaginomycetes) of Restionaceae s. lat. Mycologia Balcanica 3, 19-46.

Vánky K. 1988 - Taxonomical studies on Ustilaginales. II. Mycotaxon 32, 245-251.

Vánky K. 1995 - Taxonomical studies on Ustilaginales. XIII. Mycotaxon 56, 197-216.

Vánky K. 1998 - Taxonomical studies on Ustilaginales. XVIII. Mycotaxon 69, 93-116.

Vánky K. 2003 - Taxonomical studies on Ustilaginales. XXIII. Mycotaxon 85, 1-66.

Vánky K. 2004 - Taxonomic studies on Ustilaginomycetes - 24. Mycotaxon 89, 55-118.

Vánky K. 2006 - Taxonomic studies on Ustilaginomycetes - 26. Mycotaxon 95, 1-65.

Vánky K. 2007 - Taxonomic studies on Ustilaginomycetes - 27. Mycotaxon 99, 1-70.

Vánky K. 2008a - Restilago capensis gen. et sp. nov., an ascomycetous smut fungus. Mycologia Balcanica 5, 69-72.

Vánky K. 2008b - Two new Thecaphora species, T. ulicis and T. hosackiae (Ustilaginomycetes) on Fabaceae. Mycologia Balcanica 5, 129-133.

Vellingiri V, Amendola D, Spigno G. 2014 - Screening of four different agro-food by-products for the recovery of antioxidants and cellulose. Chemical Engineering 37, 757-762.

Verma RK, Rai ANK. 1987 - Agarwalomyces indicus gen. et sp. nov., a fructicolous synnematous hyphomycete from Uttar Pradesh. Transactions of the British Mycological Society 89, 596599.

Vilgalys R, Hester M. 1990 - Rapid genetic identification and mapping of enzymatically amplified ribosomal DNA from several Cryptococcus species. Journal of Bacteriology 172, 4238-4246.

Visagie CM, Renaud JB, Burgess KMN, Malloch DW et al. 2016 - Fifteen new species of Penicillium. Persoonia 36, 247-280.

Vishnu A, Khan MA, Bera M, Dilcher DL et al. 2017 - Fossil Asterinaceae in the phyllosphere of the eastern Himalayan Neogene Siwalik forest and their palaeoecological significance. Botanical Journal of the Linnean Society 185, 147-167.

Vitale S, Santori A, Wajnberg E, Castagnone-Sereno P et al. 2011 - Morphological and molecular analysis of Fusarium lateritium, the cause of gray necrosis of hazelnut fruit in Italy. Phytopathology 101, 679-86.

von Arx JA, Guarro J, Figueras MJ. 1986 - The ascomycete genus Chaetomium. Beihefte zur Nova Hedwigia 84, 1-162.

Vozzo JA. 1984 - Insects and fungi associated with acorns of Quercus sp. In Proceedings of the cone and seed insects working party conference, Working Party S2. 07.01, Southeastern Forest Experiment Station 40-43.

Vujanovic V, St-Arnaud M, Neumann PJ. 2000 - Susceptibility of cones and seeds to fungal infection in a pine (Pinus spp.) collection. Forest Pathology 30, 305-320.

Wainio WW, Forbes EB. 1941 - The chemical composition of forest fruits and nuts from Pennsylvania. Journal of Agricultural Research 62, 627-635.

Walker J. 2001 - Yelsemia arthropodii gen. et sp. nov. (Tilletiales) on Arthropodium in Australia. Mycological Research 105, 225-232.

Walker J, van der Merwe MM. 2009 - Two previously undescribed rusts of Acanthocarpus and Lomandra (Lomandraceae) in Australia. Australasian Plant Pathology 38, 525-532.

Wanasinghe DN, Phukhamsakda C, Hyde KD, Jeewon R et al. 2018 - Fungal diversity notes 709839: taxonomic and phylogenetic contributions to fungal taxa with an emphasis on fungi on Rosaceae. Fungal Diversity 89, 1-236.

Wang L, Kong HZ. 2000 - Penicillium ellipsoideosporum, a new species isolated from China. Mycosystema 19, 463-465. 
Wang SR, Zeng CY. 2006 - A new species and a new record of smut fungi from Northwestern China. Mycotaxon 96, 9-12.

Wang XY, Zhang ZY, Zhang LX. 1995 - Studies on Botrytis from China III. Two new species of Botrytis. In Anon. (eds), Advances in Plant Pathology Proceedings of the 2nd Young Phytopathologists' Conference on Plant Pathology, Beijing: China Agricultural Science \& Technology Press 264-267.

Watanabe T. 1989 - Three species of Sordaria, and Eudarluca biconica from cherry seeds. Transactions of the Mycological Society of Japan 30, 395-400.

Watanabe T. 1992 - A new species of Pyrenochaeta from Japanese black pine seeds. Transactions of the Mycological Society of Japan 33, 21-24.

Watanabe T. 1995 - Naranus cryptomeriae gen. et sp. nov. from Japanese cedar seed. Mycological Research 99, 806-808.

Watanabe T. 2010 - Pictorial atlas of soil and seed fungi: morphologies of cultured fungi and key to species. Third Edition. CRC press.

Watanabe K, Nishida H, Kobayashi T. 1999 - Cretaceous deuteromycetes on a cycadeoidalean bisexual cone. International Journal of Plant Sciences 160, 435-443.

Weber E. 1992 - Untersuchungen zu Fortpflanzung und Ploidie verschiedener Ascomyceten. Bibliotheca Mycologica 140, 1-186.

Weber NS, Denison WC. 1995 - Western American Pezizales. Strobiloscypha keliae gen. and sp. nov. (Pezizales, Sarcosomataceae) from Oregon. Mycotaxon 54, 129-135.

Weir BS, Johnston PR, Damm U. 2012 - The Colletotrichum gloeosporioides species complex. Studies in Mycology 73, 115-180.

Wendt L, Sir EB, Kuhnert E, Heitkämper S et al. 2017 - Resurrection and emendation of the Hypoxylaceae, recognised from a multigene phylogeny of the Xylariales. Mycological Progress, 17, 115-154.

Wernham CC. 1942 - Epichloë typhina on imported fescue seed. Phytopathology 32, 1093.

White JF Jr, Cole GT, Morgan-Jones G. 1987 - Endophyte-host associations in forage grasses. VI. A new species of Acremonium isolated from Festuca arizonica. Mycologia 79, 148-152.

White TJ, Bruns TD, Lee S, Taylor JW. 1990 - Amplification and direct sequencing of fungal ribosomal RNA for phylogenetics. In: Innis MA, Gelfand DH, Sninsky JJ, White TJ (eds) PCR protocols: a guide to methods and applications. Academic, San Diego 315-322.

Whittle AM. 1977 - Mycoflora of cones and seeds of Pinus sylvestris. Transactions of the British Mycological Society 69, 47-57.

Whitton SR. 2000 - Dictyochaeta and Dictyochaetopsis species from the Pandanaceae. Fungal Diversity 4, 133-158.

Wicklow-Howard MC, Skujins J. 1980 - Infection of Engelmann-spruce seed by Geniculodendron pyriforme in western North America. Mycologia 72, 406-410.

Wijayawardene NN, Hyde KD, Al-Ani LKT, Tedersoo L et al. 2020 - Outline of fungi and funguslike taxa. Mycosphere 11, 1060-1456.

Wijayawardene NN, Hyde KD, Bhat DJ, Camporesi E et al. 2014 - Camarosporium-like species are polyphyletic in Pleosporales; introducing Paraconiothyrium and Pseudoconiothyrium gen. nov. in Montagnulaceae. Cryptogamie, Mycologie 35, 177-198.

Wijayawardene NN, Hyde KD, Lumbsch T, Liu JK et al. 2018 - Outline of Ascomycota - 2017. Fungal Diversity 88, 167-263.

Wijayawardene NN, Hyde KD, Wanasinghe DN, Papizadeh M et al. 2016 - Taxonomy and phylogeny of dematiaceous coelomycetes. Fungal Diversity 77, 1-316

Wingfield MJ, Slippers B, Roux J, Wingfield BD. 2001 - Worldwide movement of exotic forest fungi, especially in the tropics and the southern hemisphere. BioScience 51, 134-140.

Wood AR, Damm U, van der Linde EJ, Groenewald JZ et al. 2015 - Finding the missing link: Resolving the Coryneliomycetidae within Eurotiales. Persoonia 37, 37-56.

Wood C. 1986 - Distribution maps of common tree diseases in British Columbia. Information report BC-X-Canadian Forestry Service, Pacific Forestry Centre. 
Woods TA, Farris SH, Sutherland JR. 1982 - Penetration of Sitka spruce seeds by the pathogenic fungus Caloscypha fulgens. Canadian Journal of Botany 60, 544-548.

Wu WS, Li YL. 2005 - A new species of Alternaria on cosmos seeds. Mycotaxon 91, 15-20.

Wu WS, Li YL, Wu HC. 2006 - Seed-borne fungi of ornamental flower plants. Australasian Plant Pathology 35, 373-375.

Wu WS, Wu HC. 2005 - A new species of Alternaria on seeds of French marigold. Mycotaxon 91, 21-25.

Wu ZW, Bai FY. 2006 - Candida tibetensis sp. nov. and Candida linzhiensis sp. nov., novel anamorphic, ascomycetous yeast species from Tibet. International Journal of Systematic and Evolutionary Microbiology 56, 1153-1156.

Yli-Mattila T, Gagkaeva T, Ward TJ, Aoki T et al. 2009 - A novel Asian clade within the Fusarium graminearum species complex includes a newly discovered cereal head blight pathogen from the Russian Far East. Mycologia 101, 841-852.

Yorou NS, Gardt S, Guissou ML, Diabaté M et al. 2011 - Three new Tomentella species from West Africa identified by anatomical and molecular data. Mycological Progress 11, 449-462.

Zelmer CD, Cuthbertson L, Currah RS. 1996 - Fungi associated with terrestrial orchid mycorrhizas, seeds and protocorms. Mycoscience 37, 439-448.

Zeng LS, Zhao ZH, Lu S, Xi ZJ et al. 2013 - The Fusarium species isolated from banana and their phylogenetic relationships. Mycosystema 32, 617-632.

Zhai M, Shi G, Wang Y, Mao G et al. 2015 - Chemical compositions and biological activities of pyroligneous acids from walnut shell. BioResources 10, 1715-1729.

Zhang G, Lin G, Deng J. 1995 - A new species of the genus Tilletia. Acta Mycologica Sinica 14, 17-19.

Zhou DQ, Hyde KD. 2001 - Host-specificity, host-exclusivity, and host-recurrence in saprobic fungi. Mycological Research 105, 1449-1457.

Zhuang WY. 2002 - Some new species and new records of discomycetes in China. X. Mycosystema 21, 475-479.

Zhuang WY. 2013 - The genus Scutellinia (Pyronemataceae) from China with a key to the known species of the country. Mycosystema 32, 429-447.

Zhuang WY, Nong T, Luo J. 2007 - New species and new Chinese records of Bionectriaceae and Nectriaceae (Hypocreales, Ascomycetes) from Hubei, China. Fungal Diversity 24, 347-357. 